\title{
micromachines
}

\section{Microscale Surface}

Tension and Its Applications

Edited by

Pierre Lambert and Massimo Mastrangeli Printed Edition of the Special Issue Published in Micromachines 
Microscale Surface Tension and Its Applications 



\section{Microscale Surface Tension and Its Applications}

Special Issue Editors

Pierre Lambert

Massimo Mastrangeli 
Special Issue Editors

Pierre Lambert

Massimo Mastrangeli

Université Libre de Bruxelles

Delft University of Technology

Belgium

The Netherlands

\section{Editorial Office}

MDPI

St. Alban-Anlage 66

4052 Basel, Switzerland

This is a reprint of articles from the Special Issue published online in the open access journal Micromachines (ISSN 2072-666X) from 2017 to 2019 (available at: https:/ /www.mdpi.com/journal/ micromachines/special_issues/Microscale_Surface_Tension_Its_Applications)

For citation purposes, cite each article independently as indicated on the article page online and as indicated below:

LastName, A.A.; LastName, B.B.; LastName, C.C. Article Title. Journal Name Year, Article Number, Page Range.

ISBN 978-3-03921-564-5 (Pbk)

ISBN 978-3-03921-565-2 (PDF)

(C) 2019 by the authors. Articles in this book are Open Access and distributed under the Creative Commons Attribution (CC BY) license, which allows users to download, copy and build upon published articles, as long as the author and publisher are properly credited, which ensures maximum dissemination and a wider impact of our publications.

The book as a whole is distributed by MDPI under the terms and conditions of the Creative Commons license CC BY-NC-ND. 


\section{Contents}

About the Special Issue Editors $\ldots \ldots \ldots \ldots \ldots \ldots \ldots$ vii

Pierre Lambert and Massimo Mastrangeli

Microscale Surface Tension and its Applications

Reprinted from: Micromachines 2019, 10, 526, doi:10.3390/mi10080526 . . . . . . . . . . . 1

Di Sun and Karl F. Böhringer

Self-Cleaning: From Bio-Inspired Surface Modification to MEMS/Microfluidics System Integration

Reprinted from: Micromachines 2019, 10, 101, doi:10.3390/mi10020101 . . . . . . . . . . . 2

\section{David Needham, Koji Kinoshita and Anders Utoft}

Micro-Surface and -Interfacial Tensions Measured Using the Micropipette Technique: Applications in Ultrasound-Microbubbles, Oil-Recovery, Lung-Surfactants, Nanoprecipitation, and Microfluidics

Reprinted from: Micromachines 2019, 10, 105, doi:10.3390/mi10020105 . . . . . . . . . . . 28

Sam Dehaeck, Marco Cavaiani, Adam Chafai, Youness Tourtit, Youen Vitry and Pierre Lambert

Hybrid Two-Scale Fabrication of Sub-Millimetric Capillary Grippers

Reprinted from: Micromachines 2019, 10, 224, doi:10.3390/mi10040224 . . . . . . . . . . . . . 85

Hsin-Yi Tsai, Yu-Chen Hsieh, Yu-Hsuan Lin, Han-Chao Chang, Yu-Hsiang Tang and Kuo-Cheng Huang

Fabrication of Hydrophilic Surface on Rigid Gas Permeable Contact Lenses to Enhance the Wettability Using Ultraviolet Laser System

Reprinted from: Micromachines 2019, 10, 394, doi:10.3390/mi10060394 _ . . . . . . . . . . . 96

Hal R. Holmes and Karl F. Böhringer

Vibration Induced Transport of Enclosed Droplets

Reprinted from: Micromachines 2019, 10, 69, doi:10.3390/mi10010069 . . . . . . . . . . . 110

Zhengyong Huang, Wenjie Xu, Yu Wang, Haohuan Wang, Ruiqi Zhang, Ximing Song and Jian Li

One-Step Preparation of Durable Super-Hydrophobic $\mathrm{MSR} / \mathrm{SiO}_{2}$ Coatings by Suspension Air Spraying

Reprinted from: Micromachines 2018, 9, 677, doi:10.3390/mi9120677 . . . . . . . . . . . 120

Sebastian Aland, Dmitry Bratsun, Konstantin Kostarev, Alexey Mizev, Marcel Mokbel, Karin Schwarzenberger and Kerstin Eckert

Adaptive Micromixer Based on the Solutocapillary Marangoni Effect in a Continuous-Flow Microreactor

Reprinted from: Micromachines 2018, 9, 600, doi:10.3390/mi9110600 . . . . . . . . . . . 131

Guang Liu, Pengfei Zhang, Yang Liu, Deyuan Zhang and Huawei Chen

Self-Lubricanting Slippery Surface with Wettability Gradients for Anti-Sticking of Electrosurgical Scalpel

Reprinted from: Micromachines 2018, 9, 591, doi:10.3390/mi9110591 _ . . . . . . . . . . . . 146 
Samira Shiri, Armela Murrizi and James C. Bird

Trapping a Hot Drop on a Superhydrophobic Surface with Rapid Condensation or Microtexture Melting

Reprinted from: Micromachines 2018, 9,566, doi:10.3390/mi9110566 . . . . . . . . . . . . . 158

\section{Christina Barth and Carl Knospe}

Actuation of Flexible Membranes via Capillary Force: Single-Active-Surface Experiments

Reprinted from: Micromachines 2018, 9,545, doi:10.3390/mi9110545 . . . . . . . . . . . . . . . . 169

Kei Nitta and Takahiro Tsukahara

Numerical Demonstration of In-Tube Liquid-Column Migration Driven by Photoisomerization

Reprinted from: Micromachines 2018, 9, 533, doi:10.3390/mi9100533 . . . . . . . . . . . . . 180

Qi Ni and Nathan Crane

Controlling Normal Stiffness in Droplet-Based Linear Bearings

Reprinted from: Micromachines 2018,9,525, doi:10.3390/mi9100525 . . . . . . . . . . . . . . . . 195

Hal R. Holmes, Ana E. Gomez and Karl F. Böhringer

Enabling Droplet Functionality on Anisotropic Ratchet Conveyors

Reprinted from: Micromachines 2017, 8, 363, doi:10.3390/mi8120363

Bo Chang, Zhaofei Zhu, Mikko Koverola and Quan Zhou

Laser-Assisted Mist Capillary Self-Alignment

Reprinted from: Micromachines 2017, 8,361, doi:10.3390/mi8120361 . . . . . . . . . . . . . 220 


\section{About the Special Issue Editors}

Pierre Lambert received his $\mathrm{PhD}$ degree in engineering sciences from the Universite Libre de Bruxelles, Belgium in 2004. He is Professor at Université Libre de Bruxelles, in the field of microengineering and microfluidics. He was the coordinator of the Belgian thematic network on Microfluidics and Micromanipulation: Multiscale Applications of Surface Tension (https://micromast2016.ulb.be/). His current research interests are in the fields of soft robotics (tunable stiffness mechanisms, smart catheters) and of surface tension effects in microsystems (capillary gripping, capillary self-alignment, thermocapillary micromanipulation).

Massimo (Max) Mastrangeli is Assistant Professor (tenure track) at TU Delft's ECTM laboratory, where he is developing novel silicon/polymer-based organ-on-chip and nanoparticle-based devices. He acts as guest editor, editorial board member and reviewer for several technical and scientific journals, and is steering committee member and technical program committee member for several international conferences. Prior to joining TU Delft, Dr. Mastrangeli held research appointments at the Max Planck Institute for Intelligent Systems (Stuttgart, Germany) for soft microrobotics and granular matter, at the Universite' Libre de Bruxelles (ULB, Belgium) for micromechanics and capillary micromanipulation, at École Polytechnique Fédérale de Lausanne (EPFL, Switzerland) for micro/nanofabrication and distributed robotics, and at imec Belgium (Leuven, Belgium) for fluidic microsystems integration and microelectronic packaging. Dr. Mastrangeli holds a BSc and MSc degree (both cum laude) in Electronic Engineering from University of Pisa (Italy) and a PhD degree in Materials Engineering from University of Leuven (Belgium). 



\title{
Editorial
}

\section{Microscale Surface Tension and its Applications}

\author{
Pierre Lambert ${ }^{1, *(\mathbb{D})}$ and Massimo Mastrangeli ${ }^{2, * \mathbb{C}}$ \\ 1 TIPs, Université Libre de Bruxelles, 1050 Bruxelles, Belgium \\ 2 ECTM, Delft University of Technology, 2628CT Delft, The Netherlands \\ * Correspondence: pierre.lambert@ulb.ac.be (P.L.); m.mastrangeli@tudelft.nl (M.M.)
}

Received: 2 August 2019; Accepted: 8 August 2019; Published: 9 August 2019

check for updates

Keywords: contact angle; droplets; liquid bridge; microfabrication; micromanipulation; pick-and-place; soft robotics; surface tension; wetting

More than 200 years since the earliest scientific investigations by Young, Laplace and Plateau, liquid surface tension is still the object of thriving fundamental and applied research. Partly inspired by nature's evolutionary designs exploiting physical properties inherent to liquids, this research is enabling a rich and ever expanding set of technological applications. Micromachines' Special Issue on "Microscale Surface Tension and its Applications" was therefore conceived to present fundamental knowledge, showcase relevant ongoing works and highlight prospective research directions regarding capillarity, wetting, and collateral topics.

Building on significant advances in miniaturization and soft matter, as well as in metrology and interfacial engineering, surface tension effects are indeed a major key to current developments in soft and fluidic microrobotics, precision micromanipulation and fluid/solid interactions. Benefiting from scaling laws, surface tension and capillary effects are expected to enable and support sensing, actuation, adhesion, confinement, compliance, and other structural and functional properties necessary in microand nanosystems.

This Special Issue successfully gathered novel and multidisciplinary contributions on capillary micromechatronics (capillary grippers, vibration-induced transport of droplets, capillary actuators, self-alignment), superhydrophobic and self-lubricating surfaces, soluto-capillary Marangoni-based micromixing, and droplets micromanipulation. Worth highlighting are also two reviews on interfacial tension measurement and self-cleaning surfaces.

This varied and stimulating ensemble of contributions echoes many of the interests and directions identified during the 1st International Conference on Multiscale Applications of Surface Tension (microMAST 2016), organized in September 2016 by the Belgian thematic network on Microfluidics and Micromanipulation (www.micromast2016.be). The goal of that conference and network was, and remains, to bring together various, interrelated or complementary research communities to collectively address up-to-date and unsolved questions concerning the broad field of surface tension effects.

In recapitulating the spirit of that ongoing enterprise, we hope that the interplay between fundamental questions and relevant applications driven by the downscaling of capillary effects captured in this Special Issue will provide an inspiring point of view for the readership of Micromachines.

(C) 2019 by the authors. Licensee MDPI, Basel, Switzerland. This article is an open access article distributed under the terms and conditions of the Creative Commons Attribution (CC BY) license (http://creativecommons.org/licenses/by/4.0/). 
Review

\title{
Self-Cleaning: From Bio-Inspired Surface Modification to MEMS/Microfluidics System Integration
}

\author{
Di Sun and Karl F. Böhringer *(D) \\ Department of Electrical \& Computer Engineering, University of Washington, Seattle, WA 98105, USA; \\ dxs535@uw.edu \\ * Correspondence: karlb@uw.edu; Tel.: +1-206-221-5177
}

Received: 26 November 2018; Accepted: 28 January 2019; Published: 30 January 2019

\begin{abstract}
This review focuses on self-cleaning surfaces, from passive bio-inspired surface modification including superhydrophobic, superomniphobic, and superhydrophilic surfaces, to active micro-electro-mechanical systems (MEMS) and digital microfluidic systems. We describe models and designs for nature-inspired self-cleaning schemes as well as novel engineering approaches, and we discuss examples of how MEMS/microfluidic systems integrate with functional surfaces to dislodge dust or undesired liquid residues. Meanwhile, we also examine "waterless" surface cleaning systems including electrodynamic screens and gecko seta-inspired tapes. The paper summarizes the state of the art in self-cleaning surfaces, introduces available cleaning mechanisms, describes established fabrication processes and provides practical application examples.
\end{abstract}

Keywords: self-cleaning surface; superhydrophobic; superhydrophilic; superomniphobic; microfluidics; electrodynamic screen; gecko setae

\section{Introduction}

A self-cleaning surface is defined as a surface that prevents or reduces surface contamination such as dust, water condensation, stains, or organic matter [1,2]. Self-cleaning surfaces have been under development at least since the late twentieth century. Related research involves multi-disciplinary backgrounds and aims at a broad range of applications including skyscraper windows, car windshields, solar panel cover glass, surveillance camera lenses, and water drag reduction on ship hulls [3]. Scientists have been inspired by nature to modify the microscopic structural and chemical properties of surfaces based on discoveries from plants, insects, and reptiles [4-7]. The approach is termed "biomimetics" as it mimics the micro/nano structures on plant leaves, insect wings, and animal skins.

Self-cleaning surfaces in nature rely often on water droplets (rain or condensation) and gravity to wash away surface contaminants. Such surfaces require to be positioned at a tilted angle, and the path that the droplet follows during cleaning is not precisely defined. Considering these drawbacks, more systematic designs have been proposed employing micro-electro-mechanical systems (MEMS) and microfluidics approaches, in combination with surface modifications for better cleaning effects. Many innovative designs have been implemented aiming at reducing labor and the overall maintenance cost for clean surfaces.

In this review paper, we discuss the working principles of different self-cleaning surfaces and systems, including both passive surface structure design and active microsystems. The design strategies and fabrication processes are introduced, as well as application examples. The paper provides guidelines for self-cleaning surface design and implementation. 


\section{Passive Self-Cleaning Surfaces}

Passive self-cleaning surfaces rely on surface modifications, combining both physical and chemical changes of their surface properties. The surface energy will be altered accordingly to reduce the adhesion of a water droplet to the surface. The droplet can slide off or roll off the surface under gravity when tilted to clean the contaminants along its path. No other external physical fields are involved in dislodging the contaminants [8]. In this section, we will discuss the fundamental surface wettability theory and different surface modification approaches, including superhydrophobic surfaces, superomniphobic surfaces, superhydrophilic surfaces, and liquid infused porous surfaces.

\subsection{Surface Wettability Theory Review}

To describe the wettability properties of a surface, the static and dynamic contact angles of a sessile droplet are commonly characterized. As depicted in Figure 1a, the static contact angle (CA), $\theta$, is determined by the tangent angle between the smooth solid surface and the liquid meniscus outline [9]. The basic law for surface wettability was first derived by Thomas Young in 1805, known as Young's Equation [10]:

$$
\cos \theta=\frac{\gamma_{\mathrm{SG}}-\gamma_{\mathrm{SL}}}{\gamma_{\mathrm{LG}}}
$$

where $\gamma_{\mathrm{SG}}, \gamma_{\mathrm{SL}}$, and $\gamma_{\mathrm{LG}}$ are, respectively, the interfacial surface tension at the solid/gas, solid/liquid, and liquid/gas interfaces. The model is based on the thermodynamic equilibrium approach between the three phases. Surface wettability is described as hydrophobic $\left(\mathrm{CA}>90^{\circ}\right)$ when the solid surface free energy in air is lower than in liquid, and hydrophilic $\left(\mathrm{CA}<90^{\circ}\right)$ when the solid surface free energy in air is higher than with liquid on top [9,11,12]. The suffix of "-philic" or "-phobic" describes whether the liquid has affinity or lacks affinity to the solid. A variety of contact angle measurement methods have been proposed, including direct measurement by goniometer [13], captive bubble method [14], Wilhelmy method [15], capillary tube [16], and capillary bridge [17-19], among others. These approaches rely on Young's Equation and the interfacial surface tension remains unchanged during the measurement. The goniometer is the most widely used tool to measure a static contact angle. The profile of a sessile droplet silhouette is captured, and the droplet contact angle is determined by aligning the tangent of the droplet profile at the liquid/solid contact point. To analyze the droplet contact angle, we cannot cover all the methods but briefly introduce axisymmetric drop shape analysis (ADSA) [20-24], theoretical image fitting analysis (TIFA) [25], and high-precision droplet shape analysis (HPDSA) [26,27]. ADSA was first developed by Y. Rotenberg, et al. to minimize the squares of normal distances between the droplet sideview profile and theoretical capillary curve based on the Laplacian Equation [20]. The surface tension is an adjustable parameter and droplet profile coordinates are determined by edge detection techniques. Instead of knowing the coordinates along the droplet profile, F. K. Skinner, et al. modified the ADSA by measuring the droplet diameter from the top view [24]. The modified approach can measure low contact angles $\left(\mathrm{CA}<30^{\circ}\right)$. ADSA uses a one-dimensional profile curve and requires edge detection. The TIFA method determines the droplet surface tension by two-dimensional fitting between the pendant droplet image and the theoretically calculated profile without the need of edge detection. M. Schmitt and F. Heib developed the HPDSA methods to analyze droplets on inclined surfaces [26,27], using localized ellipse fitting to determine the contact angles separately for non-axisymmetric drop shapes. Sequential images of dynamic droplet contact angle change can be extracted by this method.

As the droplet dynamically wets or dewets the surface, the liquid-air-solid three phase contact line (TPL) starts to advance or recede. More than one state can exist. The interfacial energies at the TPL will have multiple energy equilibrium states [28] caused by surface imperfections such as local defects or roughness. Macroscopically, we can monitor a minimum CA value, called receding angle, $\theta_{\text {rec }}$, as the TPL recedes and a maximum CA value, called advancing angle, $\theta_{\mathrm{adv}}$, as the TPL advances. The difference between the advancing and receding angle is called contact angle hysteresis $(\mathrm{CAH}$, $\left.\theta_{\mathrm{CAH}}=\theta_{\mathrm{adv}}-\theta_{\text {rec }}\right)$, shown in Figure $1 \mathrm{~b}$,c. Due to contact angle hysteresis, a droplet can be pinned on 
inclined surfaces, as shown in Figure 1d. Sliding angle (SA), $\alpha$, is defined as the angle between the tilted substrate and the horizontal plane when a sessile droplet starts to move down the surface due to gravity [29]. The relationship describing the sliding angle on a smooth surface with contact angle hysteresis can be described as [30]:

$$
\mathrm{mg} \sin \alpha / \mathrm{w}=\gamma_{\mathrm{LG}}\left(\cos \theta_{\text {rec }}-\cos \theta_{\text {adv }}\right)
$$

where $m$ is the droplet mass, $g$ is the gravity constant, and $w$ is the droplet width in contact with the surface.

Large contact angle hysteresis implies strong pinning or stiction of the liquid to the surface [31]. Consequently, K.Y. Law proposed a definition of surface hydrophobicity based on the receding CA $\theta_{\text {rec }}$ instead of the static CA $\theta$ [11]. A more distinct difference between the measured wetting force and $\theta_{\text {rec }}$ could be observed when $\theta_{\text {rec }}>90^{\circ}$ or $\theta_{\text {rec }}<90^{\circ}$. On the basis of the surface affinity measurements, the author proposed that the surface was hydrophilic when $\theta_{\text {rec }}<90^{\circ}$ and the surface was hydrophobic when $\theta_{\text {rec }}>90^{\circ}$.

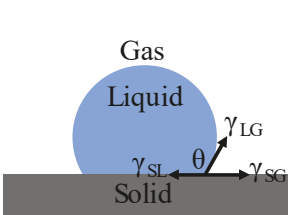

(a)

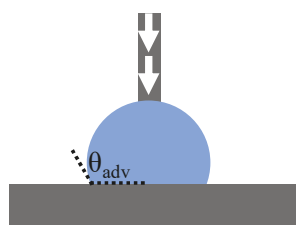

(b)

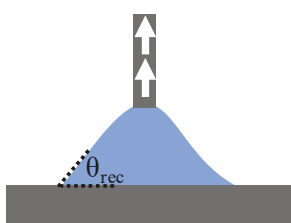

(c)

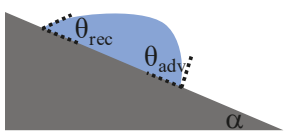

(d)

Figure 1. Schematics of contact angle types. The grey region represents the solid surface and the blue color represents the liquid on top. (a) Static contact angle $\theta$ and interfacial surface tension $\gamma$ according to Young's Equation. $(\mathbf{b}, \mathbf{c})$ represent a method to measure the advancing and receding contact angle. The arrow represents the direction of external pressure to dispense water onto or retreat water from the solid surface through a dispensing needle. (d) Inclination angle $\alpha$, advancing angle $\theta_{\mathrm{adv}}$, and receding angle $\theta_{\text {rec }}$.

Young's Equation does not take the influence of surface roughness into consideration. Wenzel (1936) [32] and Cassie-Baxter (1944) [33] proposed models to study the water droplet apparent CA on a rough surface. For homogeneous wetting conditions, the CA can be estimated using the Wenzel model as in (Figure 2a):

$$
\cos \theta^{*}=r \cos \theta
$$

where $\theta^{*}$ is the apparent CA on a rough surface, $r$ is the surface roughness defined as the ratio of total rough surface area over the projected flat region (always $\geq 1$ ), and $\theta$ is the Young (intrinsic) CA as defined on a flat surface. The Wenzel Equation shows that surface roughness amplifies the wetting on originally flat surfaces [34]. On hydrophilic rough surfaces, the apparent CA $\theta^{*}$ becomes smaller than the intrinsic $\mathrm{CA} \theta$, while on hydrophobic rough surfaces, the apparent $\mathrm{CA} \theta^{*}$ becomes larger as compared to the intrinsic CA on flat surfaces.

However, on rough hydrophobic surfaces, the surface energy of a dry solid surface is lower compared to a wet liquid/solid interface. Instead wetting all solid surface asperities, the water droplet often forms composite interfaces with air pockets and solid surfaces underneath [35,36]. A model that captures this more complex heterogeneous scenario was proposed by Cassie and Baxter to predict water droplet contact angle on composite surfaces (in particular, solid and air, see Figure 2b):

$$
\cos \theta^{*}=\phi_{\text {air }} \cos \theta_{\text {air }}+\phi_{\text {solid }} \cos \theta_{\text {solid }}
$$

where $\phi_{\text {air }}$ and $\phi_{\text {solid }}$ are area fractions of the air and solid surface and $\phi_{\text {air }}+\phi_{\text {solid }}=1 . \theta_{\text {air }}$ and $\theta_{\text {solid }}$ are water CAs when in contact with air or a solid surface. From Young's Equation, it follows that the 
contact angle of water with air is $180^{\circ}$, thus $\cos \theta_{\text {air }}=-1$, and we can derive the relationship between the apparent $\mathrm{CA} \theta^{*}$ and the Young $\mathrm{CA} \theta=\theta_{\text {solid }}$ on the composite surface as:

$$
\cos \theta^{*}=-1+\phi_{\text {solid }}(1+\cos \theta) .
$$

In this case, the solid surface region fraction $\phi_{\text {solid }}$ represents the portion of the heterogeneous surface in contact with liquid, as opposed to the surface roughness $r$, which is the key parameter to determine the contact angle on homogeneously wetted rough surfaces.

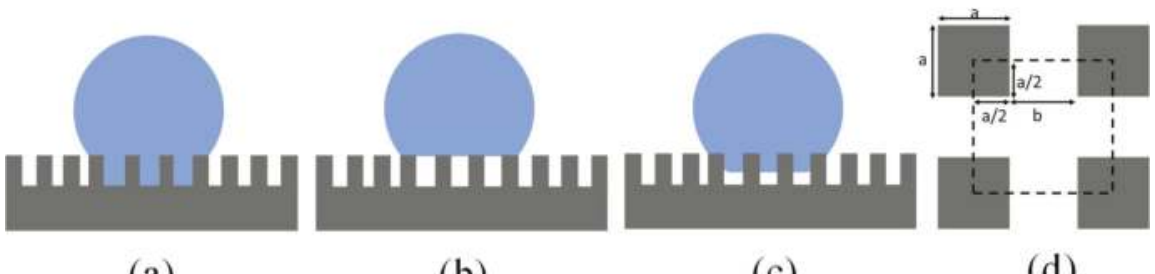

(a)

(b)

(c)

(d)

Figure 2. Schematics of different wetting states. (a) Wenzel state. (b) Cassie-Baxter state. (c) Transitional state between the Wenzel and Cassie-Baxter state, including the "petal effect" with simultaneously high contact angles (CA) and high Sliding angle (SA). (d) Top view of a typical artificial superhydrophobic surface design by creating surface roughness with pillars. The pillar height is $h$, the pillar breadth and width are $a$ and the distance between adjacent pillars edges is $b$. The dotted square shows a periodic structure for calculation with a quarter of pillar surface counted at each corner.

By studying CAs or CAHs on chemically heterogeneous surfaces, the Wenzel and Cassie-Baxter model is accurate only along the contact TPL instead of the whole contact region between droplet and surface. Experiments on chemically heterogeneous surfaces were performed by C.W. Extrand [37] and L. Gao and T. McCarthy [38]. In Gao and McCarthy's experiments, a circular spot with different surface finish was patterned on the substrate, e.g., a hydrophilic spot on a hydrophobic field, or a flat hydrophobic spot on a rough field. By continuously expanding or retrieving the droplet, the advancing $\mathrm{CA}$, receding $\mathrm{CA}$ and the $\mathrm{CAH}$ were all determined by the surface condition on the homogeneous periphery at the TPL instead of the average surface conditions beneath the droplet away from the TPL.

On a flat surface with the known lowest surface energy coatings based on the hexagonal close alignment of $-\mathrm{CF}_{3}$ groups, the highest contact angle of a sessile water droplet can only be approximately $120^{\circ}$ [12]. With surface roughness, according to the Cassie-Baxter model, when $\phi_{\text {solid }}$ is close to zero, the apparent contact angle $\theta^{*}$ approaches $180^{\circ}$. However, as shown in Figure $2 \mathrm{c}$, the water can impregnate into the surface roughness structures. Studied by Miwa, et al. [39], the Cassie-Baxter Equation may be modified as:

$$
\cos \theta^{*}=-1+\phi_{\text {solid }}(1+r \cos \theta)
$$

where $r$ is the analogous surface roughness term as in Wenzel's Equation and $r \phi_{\text {solid }}$ represents the ratio of the substrate-water contact area to the projected surface area. Interaction energy between the liquid and solid is $r \phi_{\text {solid }}$ times higher when compared to a flat surface. A low SA $\left(\sim 1^{\circ}\right)$ is achieved only with a high trapped air ratio and reduced $r$, meaning the droplet needs to rest at the tip of the roughness structures with small impregnation regions into the roughness, close to perfect Cassie-Baxter state. The water impregnation level was further studied with atomic force microscopy (AFM) on hierarchical structures together with Miwa's model by N. Okulova, et al. [40]. Because of the water impregnation, a strong liquid-solid surface adhesion can coexist with high contact angle of the droplet on the surface, named "rose petal effect" [41]. The surface roughness in this case will increase the CA hysteresis [28]. The water droplet keeps a high CA $\left(153^{\circ}\right)$ but meanwhile exhibits a high CA hysteresis by pinning to the substrate even when the substrate is placed vertically or upside down. 
Water droplets on top of surfaces with a high CA $\left(>150^{\circ}\right)$, low SA $\left(<10^{\circ}\right)$ and low CAH $\left(<10^{\circ}\right)$ are most favorable for self-cleaning. This property is termed superhydrophobicity [42,43]. On superhydrophobic surfaces, a water droplet can roll off the surface by gravity easily when the surface is slightly titled and pick up dust particles along its path. The adhesion force of dust to the superhydrophobic substrate is several times lower than on hydrophilic or even hydrophobic surfaces [44]. We term such a cleaning strategy as passive [45] and the cleaning process will happen only when the water droplet is dispensed on the tilted surfaces.

\subsection{Superhydrophobic Surfaces}

Two botanists, Barthlott and Neihuis [46], studied the microrelief of plant surfaces and discovered the papillose epidermal surface roughness and epicuticle wax coatings were the two key factors for self-cleaning mechanisms. Water droplets on top of lotus leaves kept high contact angles $\left(\sim 160^{\circ}\right)$ and low sliding angles $\left(<5^{\circ}\right)$, promoting the motion of the water droplets under gravity when the surface was tilted. Due to the surface roughness, dust particles on top of the leaves had reduced contact regions to the surface, which decreased the adhesion forces and were much easier to be cleaned away. A number of review articles have been published related with superhydrophobic surface fabrication processes and applications [3,47-49]. In this section, we have a concise discussion on the superhydrophobic surface design parameters and artificial superhydrophobic surface examples.

Inspired by the lotus leaf in nature, scientists have explored ways to mimic the lotus effect by designing micro-sized surface roughness and low surface energy coatings. Figure $2 \mathrm{~d}$ shows the top view of a typical artificial superhydrophobic surface with square pillars. The Wenzel Equation (3) and the Cassie-Baxter Equation (4) now become [50,51]:

$$
\begin{gathered}
\cos \theta_{\mathrm{W}}{ }^{*}=\left(1+\frac{4 \phi_{\text {solid }}}{(\mathrm{a} / \mathrm{h})}\right) \cos \theta \\
\cos \theta_{\mathrm{c}}^{*}=-1+\phi_{\text {solid }}(1+\cos \theta) \\
\phi_{\text {solid }}=\frac{1}{(\mathrm{~b} / \mathrm{a}+1)^{2}} .
\end{gathered}
$$

From the Equations, the Wenzel state is dependent on the pillar height while the Cassie-Baxter state is not. In both states, the droplet is in a stable thermodynamic equilibrium. An energy barrier exists to prevent the transition between these two states. To be in Wenzel or Cassie-Baxter state is determined by how the droplet is formed. By calculating the energy of a drop of given volume in equilibrium on a substrate, a small a/h value (slender pillars) is suggested to obtain a robust state. A periodical (b/a) is recommended to make the droplet insensitive to energy state change. A two-tier surface roughness design with both microscale and nanoscale roughness is recommended, which provides more stable superhydrophobic state and lower contact angle hysteresis [52].

Figure 3 presents some examples. R. Furstner, et al. came up with strategies to create multiple types of superhydrophobic surfaces [53]. Shown in Figure 3a-c, silicon micro-sized pillars fabricated with X-ray lithography and followed by reactive ion etching processes, microstructured copper foil surfaces and a replica of lotus leaves using silicone molding were fabricated and characterized. All the surface designs had superhydrophobic properties. For instance, on a replica of plant surfaces, water droplets kept high contact angle $\left(>150^{\circ}\right)$ and low sliding angle $\left(\sim 7^{\circ}\right)$. Cleaning efficiency was defined by checking the number of SEM images without contamination particles after surface cleaning with water droplets divided by the total number of SEM images taken. A cleaning efficiency of $90-95 \%$ could be achieved.

$\mathrm{K}$. Koch, et al. created two-tier hierarchical structures of roughness by depositing lotus wax tubules on top of Si or lotus leaf replicas (Figure 3d) [54], achieving larger water droplet contact angle $\left(\sim 170^{\circ}\right)$ and smaller sliding angle $\left(1^{\circ}-2^{\circ}\right)$ compared with one-tier roughness structures like Si micropillars. 
Figure 3 e shows a nano-cone structure on a flexible Teflon substrate by oxygen plasma etching of a colloidal monolayer of polystyrene beads [55]. The wettability of the surface was controlled geometrically based on plasma treatment time as well as chemically by further gold nanoparticle deposition and silanization.

Figure $3 \mathrm{f}$ shows a low-cost porous structure of isostatic polypropylene (i-PP) [56]. i-PP was dissolved in the solvent mixture consisting of methyl ethyl ketone (MEK), cyclohexanone, and isopropyl alcohol, and dropped on a glass substrate. The solvent was further dried in a vacuum oven. The remaining i-PP formed a porous "bird's nest" morphology. From atomic force measurements, the roughness of pure thin i-PP film was $10 \mathrm{~nm}$ RMS with a water contact angle of $104^{\circ}$, while the porous coating had $300 \mathrm{~nm}$ RMS and improved water droplet contact angle from $104^{\circ}$ to $149^{\circ}$.

K. Lau, et al. [57] developed superhydrophobic surfaces by growing vertical carbon nanotube forests with a plasma-enhanced chemical vapor deposition (PECVD) process, shown in Figure 3g. To provide the stable high water droplet contact angle, the carbon nanotubes were coated with thin conformal hydrophobic poly(tetrafluoroethylene) (PTFE) by a hot filament chemical vapor deposition (HFCVD) process. Most of the superhydrophobic surfaces were made of fragile microstructures or polymeric materials, where durability could be an issue for field applications because of the harsh environment.

Y. $\mathrm{Lu}$, et al. created a mechanically strong coating using an ethanolic suspension of perfluorosilane-coated titanium dioxide nanoparticles (shown in Figure 3h) [58]. Two dimensions of $\mathrm{TiO}_{2}$ nanoparticles ( $200 \mathrm{~nm}$ diameter and $20 \mathrm{~nm}$ diameter) were mixed and suspended in the ethanolic solution. The coating was able to be applied to various types of substrates like clothes, paper, or steel by spray, dip or extrusion coating processes and kept superior high water repellency after 40 cycles of sandpaper abrasion. The robustness of coating processes, substrate choice, and high mechanical strength allowed the paint to have potential applications in harsh environments.

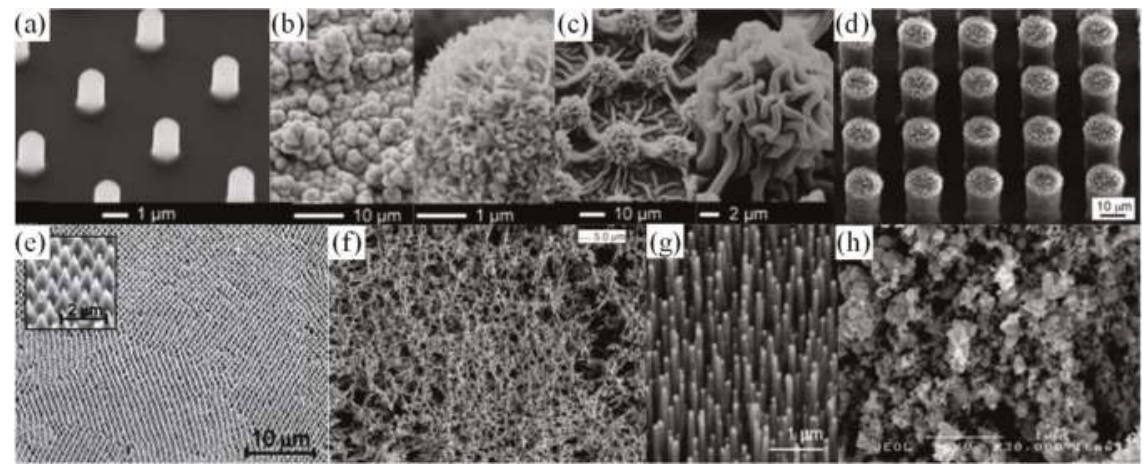

Figure 3. Artificial superhydrophobic surface examples imaged by scanning electron microscopy (SEM). (a) Micro-spikes on Si substrates. Reproduced with permission from [53], published by ACS Publictions, 2005. (b) Heavily structured copper film surface [53]. (c) Silicone rubber replicates of Alocasia structure through molding [53]. (d) Hierarchical structures using Si micropillars covered with lotus wax tubules. Reproduced with permission from [54], published by Royal Society of Chemistry, 2009. (e) Teflon nano cone arrays. Reproduced with permission from [55], published by ACS Publications, 2014. (f) Porous isostatic polypropylene (i-PP) structures from solution drying. Reproduced with permission from [56], published by Science, 2003. (g) Carbon nanotube forest grown by plasma-enhanced chemical vapor deposition (PECVD). Reproduced with permission from [57], published by ACS Publications, 2003. (h) $\mathrm{TiO}_{2}$ particles paint. Reproduced with permission from [58], published by Science, 2015.

Because of the droplet repellency and low adhesion, a condensed droplet on a chilled superhydrophobic substrate can be spontaneously removed. When the tiny droplets coalesce, 
the released energy can power the out-of-plane jumping of the droplet $[59,60]$. Such a jumping condensate process was applied for surface cleaning mechanism [61]. Inspired by cicada wings, K. Wisdom, et al. studied their wing structures and found the self-cleaning mechanism by jumping condensate process [61]. The cicada wing cuticle surface consisted of conical hydrophobic arrays, resulting in super-hydrophobicity with a water contact angle in the range of $148^{\circ}-168^{\circ}$ depending on the location. When the wing surfaces were exposed to vapor flow, the adhering particles or contaminants could be cleaned because of the water condensation process. Shown in Figure 4, the particles were detached from the surface by the water droplet's out-of-plane jumping upon coalescence. The capillary-inertial oscillation of the merged droplet provided the required kinematic energy. The force between the jumping droplet and the particles in contact scaled with the capillary force: $f \sim \gamma R_{\mathrm{p}}$, where $\gamma$ is the surface tension and $R_{\mathrm{p}}$ is the droplet radius of curvature. Due to the scaling law, for small particles, it was less favorable to remove the droplet by inertial forces like gravity, vibration, and centrifugal forces (scaled with $R_{\mathrm{p}}{ }^{2}$ ) or by hydrodynamic forces like wind blowing (scaled with $R_{\mathrm{p}}{ }^{3}$ ). The jumping condensate processes (scaled with $R_{\mathrm{p}}$ ) provided an advantageous mechanism to dislodge particles from the surface by overcoming adhesion forces (van der Waals force and capillary bridging force) to the substrate.
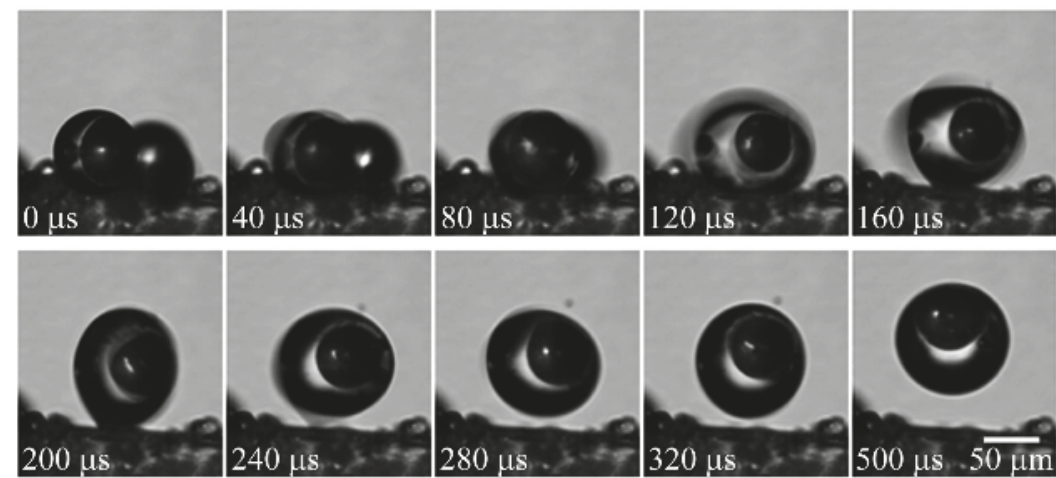

Figure 4. Water vapor condenses and spontaneously jumps off a cicada wing surface, encapsulating $50 \mu \mathrm{m}$ glass beads. Reproduced with permission from [61], published by PNAS, 2013.

\subsection{Omniphobic Surfaces}

Water possesses a high surface tension compared with most other liquids (except for mercury). Low surface tension liquids rarely exist in nature so the naturally evolved surfaces can barely repel artificial low surface tension liquids in our daily lives [62]. According to the simple theoretical derivation, by combining the Wenzel model and Cassie-Baxter model Equations (3) and (5), we obtain the transitioning critical angle between the two states expressed as:

$$
\cos \theta_{\mathrm{c}}=\left(\phi_{\text {solid }}-1\right) /\left(r-\phi_{\text {solid }}\right)
$$

where $\theta_{\mathrm{c}}$ is the critical transition contact angle for a droplet from Wenzel state to Cassie-Baxter state [63]. By definition, we have $r \geq 1 \geq \phi_{\text {solid }}$, and $\theta_{\mathrm{c}}$ is required to be at least $90^{\circ}$ to make the transition happen because the right-hand side of Equation (10) cannot be positive [62]. For low surface tension liquids like hexane and decane, no existing natural or artificial surface coatings can achieve such a high contact angle of the liquids [64,65].

Researchers have successfully created artificial superomniphobic surfaces with the assistance of re-entrant structures [62] or doubly re-entrant structures [66,67], in which curvature is another key factor other than surface chemical composition and roughness. The key to realizing superomniphobic surfaces is that the liquid hanging between surface asperities cannot have higher contact angles 
than given by the intrinsic material wettability [68,69]. More specially, as shown in Figure 5a, if the advancing TPL forms a smaller contact angle, then an equilibrium state can be reached that prevents the droplet from further impalement [70]. The liquid-air interface inside the re-entrant or doubly re-entrant structure remains convex and the net capillary force generated is upward. According to Equation (4), when $\phi_{\text {solid }}$ is small $(<6 \%)$, the surface can repel extremely wetting liquids $\left(\theta_{c}{ }^{*}>150^{\circ}\right.$ with $\theta \sim 0^{\circ}$ ). However, the liquid is difficult to maintain in suspension with small $\phi_{\text {solid }}$ because the liquid will impregnate into the rough structures without enough solid support. A doubly re-entrant structure is thus necessary with vertical, thin, and short overhangs to minimize the projected solid areas while increasing the solid fraction by vertical surfaces (side wall angle $\sim 90^{\circ}$ ). As demonstrated in Figure 5b, on a conventional pillar-like superhydrophobic surface, a water droplet is suspended on the micropillar structure when the pillars are hydrophobic. However, for low surface tension liquid, the liquid-solid contact line overcomes this barrier and reaches the lower edge of the re-entrant structure, as shown in Figure 5c. For a completely wetting liquid, the contact line further wets down the overhang and reaches the tip of the curvature (Figure $5 \mathrm{~d}$ ). Because of the doubly re-entrant structure, the liquid-solid contact line stops wetting at the interior edge of the vertical overhangs while keeping ultra-low contact angle.

To fabricate the superomniphobic surfaces, efforts have been made to explore re-entrant and doubly re-entrant microstructure arrays. Figure $6 \mathrm{a}-\mathrm{c}$ show different types of re-entrant designs. The micro hoodoo structure in Figure 6a was made by reactive ion etching of the $\mathrm{SiO}_{2}$ layer on top of a $\mathrm{Si}$ substrate followed by isotropic etching of the $\mathrm{Si}$ substrate using $\mathrm{XeF}_{2}$. The process resulted in Si pillars with $\mathrm{SiO}_{2}$ caps [71]. Figure $6 \mathrm{~b}$ started with lithographic patterning on a copper substrate, followed by through-mold and over-mold electroplating to form hemispherical mound copper structures atop a photoresist layer [72]. After photoresist strip, the mushroom-like copper structure was created. Figure $6 \mathrm{c}$ demonstrates a nano-nail structure by using a deep reactive ion etching process to fabricate tall silicon pillars with $\mathrm{SiO}_{2}$ nail caps atop [73]. All the three designs required a fluoro-polymer coating as a finishing step to maintain the low surface tension required for stable fluid suspension. A vapor phase immersing deposition process was usually applied on $\mathrm{SiO}_{2}$ surfaces and a solution soaking process could be applied on metal surfaces. The self-assembled monolayer, terminated with the tricholorosilane group or thiol head group, formed stable covalent bond and modified the surface energy with a fluorinated tail group [74]. The silanization process was widely used for many surfaces to adjust the surface wetting behaviors [75-78].

As an alternative to lithography processes, A. Tuteja, et al. synthesized a class of fluoropolymers (polyhedral oligomeric silsesquioxane (POSS) shown in Figure 6d), with which the substrate was coated by electrospinning. The surface tension of the electrospun fiber mat could be altered by changing the mass fraction ratio of fluoro-POSS and a mildly hydrophilic polymer, thus systematically tuning the water contact angle [62,71].

Deng, et al. created a transparent superomiphobic surface using candle soot as a template, shown in Figure 6e $[79,80]$. The soot consisted of piles of nano carbon spheres with a diameter range of $30-40 \mathrm{~nm}$. After depositing the soot on the glass substrate, a layer of silica shell was formed utilizing chemical vapor deposition (CVD) of tetraethoxysilane (TES) catalyzed by ammonia. The sample was sintered in the oven for $2 \mathrm{~h}$ at $600{ }^{\circ} \mathrm{C}$ to burn away the carbon cores and link the silica nano shells. The surface kept good transparency and superomiphobicity up to $400^{\circ} \mathrm{C}$.

Besides the re-entrant structures, doubly re-entrant structures have been fabricated, presenting superior surface properties as compared to re-entrant structures. Learning from smart springtail skins [66], T. Liu, et al. microfabricated structures with doubly re-entrant overhangs, shown in Figure 6f [67]. Due to its particular geometry, the surface could repel any of the existing fluids even without fluoro-polymer treatment of the final surface. Because of a pure combination of $\mathrm{SiO}_{2}$ and $\mathrm{Si}$, the surface would also withstand high-temperature environments over $1000{ }^{\circ} \mathrm{C}$. Derived from this process flow, metal or polymeric doubly re-entrant omniphobic surfaces were successfully fabricated as well. 

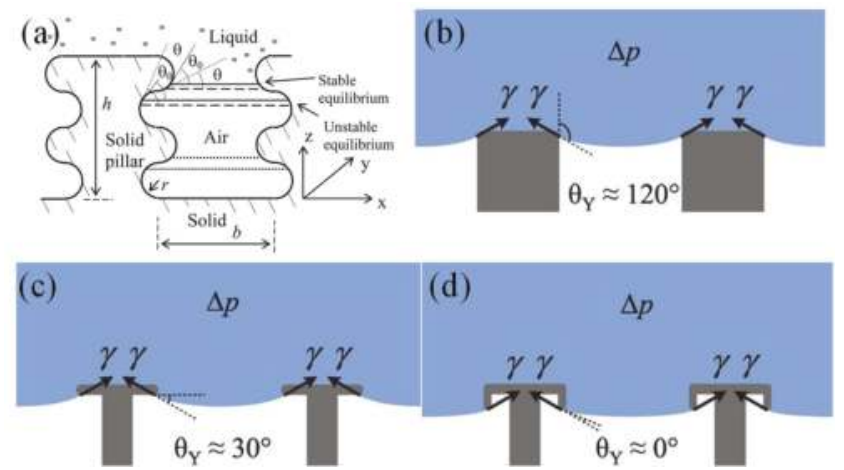

Figure 5. Liquid-solid contact angle required for stable liquid suspension on (a) Surfaces with both semicircular bumps and grooves. Reproduced with permission from [70], published by ACS Publications, 2007. (b) micro-pillar structures, (c) re-entrant structures, (d) doubly re-entrant structures. Reproduced with permission from [67], published by Science, 2014.

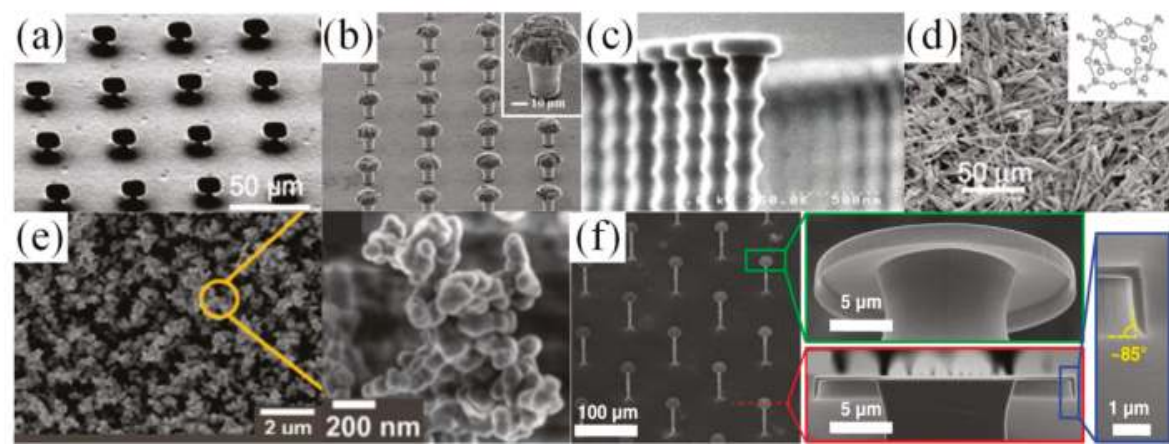

Figure 6. Examples of superomniphobic surface designs. (a) Micro hoodoo structures with a rectangular $\mathrm{SiO}_{2}$ cap on Si micro-pillars. Reproduced with permission from [71], published by PNAS, 2008. (b) Mushroom structure of copper surfaces. Reproduced with permission from [72], published by Nature, 2015. (c) Nano-nail structures. Reproduced with permission from [73], published by ACS Publications, 2008. (d) Fluorinated electrospun fibers [71]. (e) Candle soot structure after being coated with silica nanoshell and after carbon core removal by high-temperature sintering. Reproduced with permission from [79], published by Science, 2012. (f) Microposts with doubly re-entrant overhangs. Reproduced with permission from [67], published by Science, 2014.

\subsection{Superhydrophilic Surfaces}

Superhydrophobicity is not the exclusive strategy to realize self-cleaning functionality, which can also be realized while the water droplet contact angle atop a surface is extremely low (close to zero). The simplest way to increase the surface hydrophilicity is by oxygen plasma treatment, as demonstrated by B. Gupta, et al. [81]. Their process only modified the surface properties without altering the bulk substrate material. Experiments proved that the treated surface had anti-fogging and anti-fouling properties, but the hydrophilicity would decrease over time [82].

Another approach was to take advantage of both the light-induced superhydrophilicity [83-85] and the photocatalytic properties of $\mathrm{TiO}_{2}$ thin films, namely the "Photo-Kolbe" reaction [82,86]. The as-prepared $\mathrm{TiO}_{2}$ surface water contact angle is $\sim 72^{\circ}$. The $\mathrm{UV}$ exposure creates oxygen vacancies at bridging sites favorable for dissociative water adsorption $\left(\mathrm{Ti}^{3+}\right.$ sites instead of $\mathrm{Ti}^{4+}$ sites $)$, making the water contact angle close to $0^{\circ}$. Microscopically, after UV radiation, the $\mathrm{TiO}_{2}$ surface wettability is not 
heterogeneous anymore, and the hydrophilic regions are distributed across the surface with area sizes in the sub-micrometer range, based on measurements by friction force microscopy. Macroscopically, the water will spread on the surface instead of forming droplets, to wash away surface contaminants easily [83].

The photo induced oxidation/decarboxylation/fragmentation of organic acids is well-known for photo-semiconductors like $\mathrm{TiO}_{2}$ or $\mathrm{ZnO}$ [87-92]. The $\mathrm{TiO}_{2}$ preparation can use wet chemical processes like sol-gel, dip-coating, or spin-coating processes $[93,94]$. A post calcination process is usually required to improve the adhesion between the $\mathrm{TiO}_{2}$ film and the substrate [95]. Upon UV radiation $(<385 \mathrm{~nm})$ of the $\mathrm{TiO}_{2}$, the proton with an energy exceeding the bandgap would excite an electron $\left(\mathrm{e}^{-}\right)$from the valence band to the conduction band, leaving a hole $\left(\mathrm{h}^{+}\right)$on the valence band. Valence band holes react with the water through a strong oxidization process on the surface to produce reactive hydroxyl radicals $(\cdot \mathrm{OH})$ and convert surface contaminants, especially organic residues, into byproducts like water or $\mathrm{CO}_{2}$ [94]. Because of the weakening of the bonding, the surface contaminants are easily washed away by rain.

\subsection{Slippery Liquid-Infused Porous Surface (SLIPS) Surfaces}

Solid substrates have been modified to create superhydrophobic or superhydrophilic surfaces by etching of physically rough texture or by chemical modification. However, Wong, et al. developed a system to create a liquid repellant surface, naming it "slippery liquid-infused porous surface" (SLIPS) [96]. Inspired by the Nepenthes pitcher plant [97], Figure 7a shows the fabrication process of the SLIPS surface. A porous solid surface was infused with low surface tension and chemically inert lubricating liquid, which wicked into the porous substrate while being immiscible and repelling to the test liquids applied to the surface. The contact angle hysteresis for sessile water drops was as low as $2.5^{\circ}$ and the sliding angle was smaller than $5^{\circ}$. Figure $7 \mathrm{~b}, \mathrm{c}$ demonstrate the outstanding anti-fouling performance of the SLIPS surface by applying crude oil and human blood. In comparison with superhydrophobic surfaces and hydrophilic surfaces, no stains were left on the surface. Both oil and blood would quickly slip away from the SLIPS surface. Besides the superb repellency, the SLIPS surfaces also have self-healing properties [96]. Because of the surface ultra-smoothness and lack of nucleation sites [98-101], no frost formation or a reduced ice adhesion were observed on cold SLIPS surfaces.

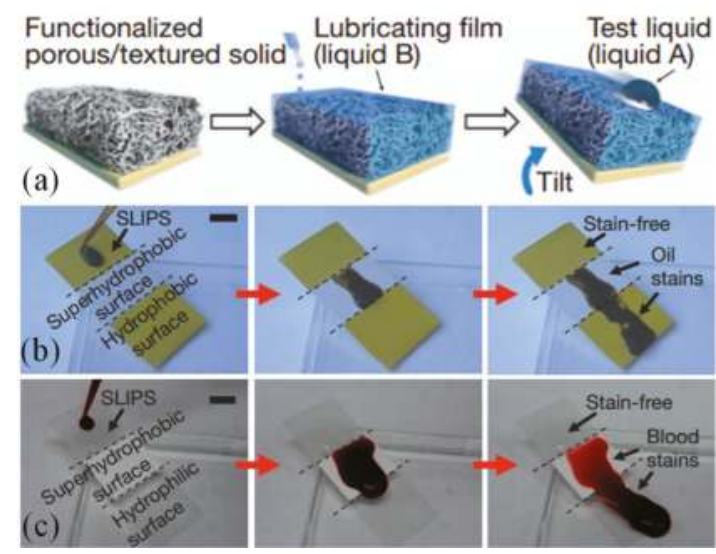

Figure 7. (a) Slippery liquid-infused porous surface (SLIPS) fabrication process flow. Low surface energy, chemically inert fluid was infused into the porous solid substrate. The surface remained smooth with lubricating film between the substrate and the applied liquid. (b) Crude oil and (c) blood movement on SLIPS, superhydrophobic and superhydrophilic surfaces. Reproduced with permission from [96], published by Nature, 2011. 
With regards to bio-fouling applications, extensive studies have been performed on superhydrophobic surfaces [102-107]. However, the anti-biofouling property of superhydrophobic surfaces could be short-lived as the air-bubble layer trapped between the liquid and the rough surface is not stable and may disappear within several hours [108]. More bacterial adhesion could end up on the superhydrophobic surfaces due to the high surface roughness when compared with intact smooth surfaces. Extensive work has been explored by adopting SLIPS surfaces to prevent bio-fouling issues by various fabrication methods, which were more promising and with better performance than superhydrophobic surfaces. A. Epstein, et al. adopted SLIPS surfaces to prevent surface bio-film attachment [109]. Shown in Figure 8a, the SLIPS surfaces were fabricated with porous fluoropolymer substrates (with pore size of $0.2 \mu \mathrm{m}$ ). By staining the surface with bacterial culture solution, the SLIPS surface can reduce the cell attachment compared with superhydrophobic surfaces. The coffee ring effect of the biofilm was suppressed on SLIPS by leaving only a pellet of bio-stains after evaporation. Similar liquid infused porous substrate structures were obtained by phosphoric acid etching of enamels [110].

D. Leslie, et al. created a SLIPS surface with self-assembled monolayers (SAM), shown in Figure 8. The structure was applied on a wide range of smooth medical device surfaces, which repelled flowing blood and prevented thrombosis [111]. A molecular tethered perfluorocarbon (TP) layer was first coated on the smooth surfaces by soaking the plasma treated surface in liquid solution. Then a mobile layer of perfluorodecalin (LP) was applied, forming a tethered-liquid perfluorocarbon (TLP) surface. By exposing the uncoated and TLP coated acrylic surfaces to fresh human blood, the TLP surface had 27-fold less platelet adhesion and platelets were considered as one of the major components causing thrombosis. Both in vitro and in vivo experiments showed promising results, demonstrating that the TLP surfaces were resistant to the physiological shear stress brought by the blood flow while reducing the protein adhesion and thrombosis for at least $8 \mathrm{~h}$.

Beyond the silane liquid soaking process to create the TP layer, M. Badv, et al. improved the hydrophobic salinization process with a more robust, reproducible and less disruptive chemical vapor deposition (CVD) process in vacuum [112,113] (Figure 8c). Coronary catheters were treated by both two silanization processes, followed by adding perfluorodecalin or perfluoroperhydrophenanthrene to make TLP surfaces. The CVD treated surfaces provided better anti-thrombotic performance compared with silane liquid solution soaking processes. As shown in Figure 8c, CVD treated catheters surfaces found no blood clot or protein adhesion after blood immersion. By mixing different self-assembled monolayer silanes (aminosilane and fluorosilane) during the surface treatment, tunable cell repellency and selective binding of antibodies can be realized. The target anti-bodies would be anchored by the aminosilanes while the fluorosilane will repel the non-desired cells, proteins or plasma clotting assays, creating the bio-functional lubricant-infused surfaces (BLPS) [114].

SLIPS can be fabricated on porous micropillar arrays with sharp overhang structures [115]. As shown in Figure 8d, the liquid on top of such surfaces meets a new liquid-air interface, compared with solid-air interfaces of the normal superhydrophobic or superomniphobic designs without liquid infusion as discussed above. The micropillar arrays with sharp overhang structures and nano-porous micropillar top surface finish were created by direct laser writing, which can process any arbitrary $3 \mathrm{D}$ components with sub-micrometer resolution. A layer of $\mathrm{Al}_{2} \mathrm{O}_{3}$ by atomic layer deposition was coated on the outer layer of polymeric micropillars and fluorinated by SAMs. Low surface tension fluid was dropped directly on the micro-pillar porous surfaces and confined by the micropillar surface roughness as well as the overhangs. The composite surface designs can repel low surface tension fluids while reducing more than twice of the adhesion force, as measured with scanning droplet adhesion microscopy. 


\section{(a)}

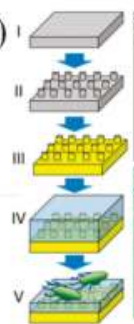
(al)

(c)

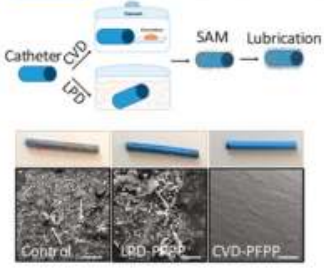

(b)
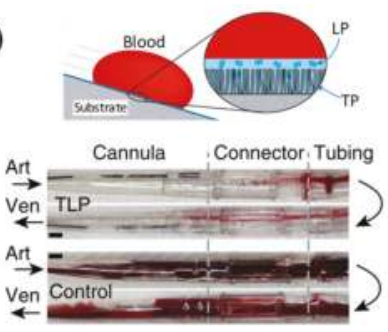

(d)

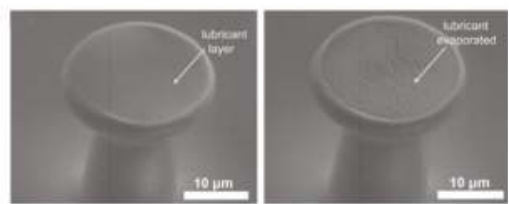

Figure 8. (a) Fabrication process of SLIPS surface using porous substrate and bio-fouling experiment with and without liquid infusion. Reproduced with permission from [109], published by PNAS, 2012. (b) SLIPS surface fabricated with liquid soaking deposited self-assembled monolayers (SAM). Reproduced with permission from [111], published by Nature, 2014. The experimental results showed TLP modified tubing and control tubing after $8 \mathrm{~h}$ of blood flow. The blood flow through arterial (Art) or venous (Ven) cannula was indicated by the black arrow. (c) SLIPS surface fabricated with both liquid soaking and vapor deposited SAM. Reproduced with permission from [112], published by Nature, 2017. (d) SEM images of SLIPS and doubly re-entrant superomniphobic composite structures. The left image shows the surface with lubrication and the right image shows the surface after lubricant evaporation. Reproduced with permission from [115], published by Wiley Online Library, 2018.

\section{Active Self-Cleaning Microsystems}

Besides employing passive surface modification techniques, microsystems can be designed to actively remove unwanted surface contaminants or fluids [8]. Many strategies have been tested using surface tension gradients, electrostatic fields, and vibrations. Moreover, geckos can clean their feet dynamically while naturally walking with hyperextension. In such systems, water droplet movement or dust removal can be accomplished in a systematic way while applying more controlled forces. Thus, the active self-cleaning approach can be utilized in combination with passive surface modification to improve cleaning efficiency. In this part, we will first introduce the surface cleaning strategies by combining the superhydrophobic and SLIPS surface design with droplet manipulation. Then we will discussion surface dust removal techniques by electro-dynamic screen, repelling surface contaminants by high alternating voltage. At last, self-cleaning synthetic adhesives inspired by gecko setae structures are discussed.

\subsection{Self-Cleaning Surfaces by Water Droplet Transport}

Microfluidic systems have been developed using MEMS technology and widely applied for biomedical and chemical applications. The recent development of microfluidic systems using microor nano-liter water droplet transport, commonly known as digital microfluidics (DMF), offers the potential for a wide range of applications [116]. To control the water droplet transport, researchers have focused on creating surface tension anisotropy at the interface of gas, liquid and solid, defined as the three-phase contact line (TPL). DMF systems can be used to direct water droplet transport along the surface using chemical gradients [117], thermal gradients [118], electrowetting-on-dielectric (EWOD) [119-121], surface acoustic waves [122], and micro textures [123,124]. Dust particles or 
undesired fluids along the path of the water droplet movement can be carried away to other locations, leaving the desired surface regions clean and functional.

A typical EWOD setup is shown in Figure 9a. A water droplet is initially placed on a hydrophobic insulator surface. When a voltage is applied between the droplet and the electrode underneath, the electrostatic field will significantly modify the solid-liquid interfacial tension, leading to a reduction of contact angle and an improved wetting of the droplet on the solid surface. This effect of the voltage can be quantified by the following Equation:

$$
\gamma_{\mathrm{SL}, V}=\gamma_{\mathrm{SL}}-\frac{1}{2} C V^{2}
$$

where the original solid-liquid surface energy $\gamma_{\mathrm{SL}}$ is modulated by the electrostatic field (given by the normalized capacitance $C$, measured in $C / \mathrm{m}^{2}$, and the applied voltage $V$ ) to produce the effective surface energy $\gamma_{S L, V}$. This leads to a generalized form of Young's Equation (1):

$$
\gamma_{\mathrm{SG}}=\gamma_{\mathrm{SL}}-\frac{1}{2} C V^{2}+\gamma_{\mathrm{LG}} \cos \theta_{V}
$$

and the Young-Lippmann Equation:

$$
\gamma_{\mathrm{LG}} \cos \theta_{V}-\gamma_{\mathrm{LG}} \cos \theta=\frac{1}{2} C V^{2}
$$

where $\theta_{V}$ is the effective contact angle under an applied voltage $V$.

Asymmetric interfacial surface tension change at the droplet-substrate interface can be introduced by energizing different electrodes, and the surface on top of the energized electrodes tend to be more hydrophilic. The droplet can be transported precisely controlled by sequentially enabling different electrodes. Depending on the application, two popular EWOD configurations are often used, shown in Figure 9b, the parallel-plate system, and Figure 9c, the co-planar system. For the parallel-plate system, the water droplet was sandwiched in between the top and bottom electrodes, insulated by dielectric layer (SU8, $\mathrm{SiO}_{2}[120]$ or parylene [125]) and hydrophobic (Teflon ${ }^{\mathrm{TM}} \mathrm{AF}[126,127]$ or Cytop ${ }^{\circledR}[128,129]$ coatings. The electrode on one plate was patterned and the electrode on the other was fully grounded. Once the electrode was energized, the droplet was first deformed by the electrostatic field and driven by pressure gradient inside the droplet [130]. The parallel-plate system can prevent droplet evaporation and is less sensitive to gravity influence, compared with the co-planar system, where the cover plate is removed. However, the co-planar system has broad applications and can be integrated into many other systems which do not permit a top cover plate [131]. Meanwhile, the dielectrics and top coatings of the EWOD system can be easily integrated with superhydrophobic surface or SLIPS surface designs to enable the active cleaning capabilities by systematically controlling the droplet.

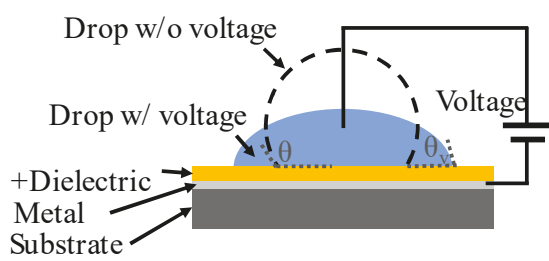

(a)

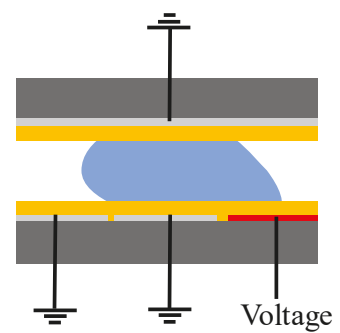

(b)

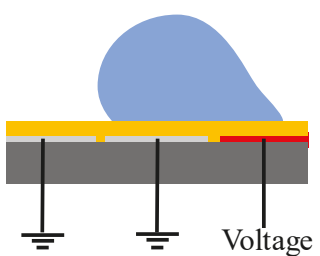

(c)

Figure 9. Electrowetting-on-dielectric (EWOD) setup: (a) Typical EWOD setup to measure contact angle change by external voltage. (b) Parallel-plate EWOD design to transport droplet. (c) Open coplanar EWOD design. The energized electrodes are marked as red color in $(\mathbf{b}, \mathbf{c})$. 
Latip, et al. explored the anti-fouling properties by applying EWOD top coatings with hydrophobic (Cytop) or superhydrophobic (NeverWet ${ }^{\circledR}$ ) materials [132]. Different concentrations of protein solutions were prepared. A similar test bench setup as shown in Figure 9a was performed to test the contact angle hysteresis by gradually increasing and then reducing the voltage within a period of time. Compared with superhydrophobic surfaces, the contact angle hysteresis greatly increased on Cytop surfaces with increased protein concentration, maximum applied voltage and the period of time with voltage applied. Higher roll-off angle and afterwards higher fluorescence intensity with labelled protein were observed on the Cytop surface, showing a stronger protein adhesion to the Cytop. As for the droplet transport, both closed parallel plate configuration (Figure 9b) and open coplanar configuration were tested with superhydrophobic coatings. On the coplanar system, a droplet of $35 \mu \mathrm{L}$ was applied, and the actuation was difficult to control since the droplet continued to roll on the surface due to low friction. However, in the parallel plate system, a droplet with only $5 \mu \mathrm{L}$ was needed and was successfully transported, merged or mixed.

M. Jönsson-Niedziółka et al. showed droplet transport with a parallel plate system configuration to remove bio-particles [133]. The top and counter electrodes were separated by $300 \mu \mathrm{m}$ spacers. A square wave voltage was applied to the selected base electrodes at the frequency of $1 \mathrm{kHz}$ and the switching time between adjacent electrodes was adjusted based on droplet movement speed. The water droplet displacement was driven by the surface wettability change induced by the voltage. The cleaning efficiency was defined as: \%efficiency $=\left(1-N_{\text {in }} / N_{\text {out }}\right) \times 100$, where $N_{\text {in }}$ is the average number of particles inside the water droplet pathway and $N_{\text {out }}$ is the average number of particles outside the water droplet pathway. Examples of water droplet transport along the electrodes and cleaning of the surface are presented in Figure 10. Synthetic particles like polystyrene latex microspheres and bio-particles, including proteins, bacterial spores, and viral simulant were tested with the system. When the substrate surface was designed to be superhydrophobic, more than $90 \%$ of cleaning efficiency could be reached with water droplets even for protein particles, which usually have high adhesion to the substrate and are hard to clean.

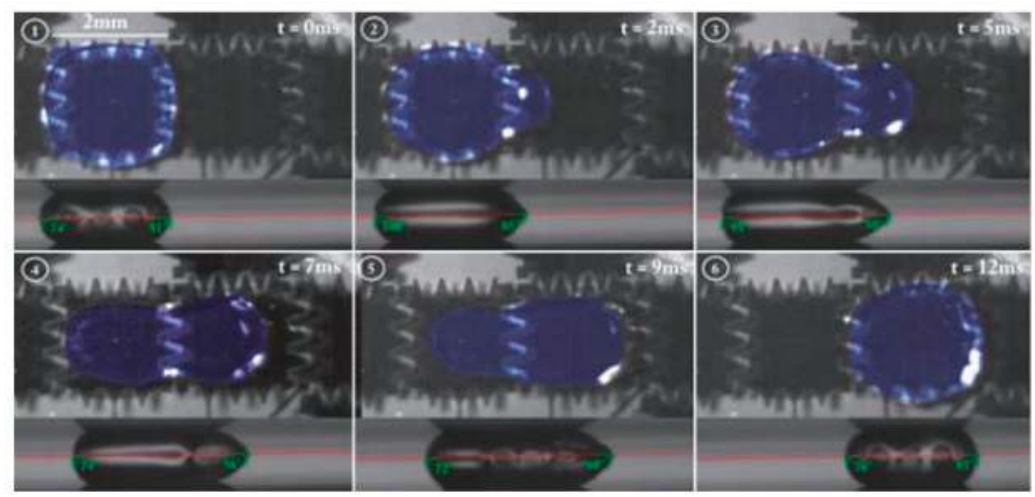

Figure 10. Both top and side views of water droplet transport on the superhydrophobic surface at different times. Reproduced with permission from [133], published by Royal Society of Chemistry, 2011.

Y. Zhao, et al. developed a similar EWOD system for sampling of micro particles (Figure 11) [134]. The actuation electrodes were insulated with a dielectric layer $\left(\mathrm{SiO}_{2}\right)$ and coated with hydrophobic (Teflon) coatings. Driven by sequentially actuated electrodes, the water droplet swept along the surface and picked up particles. The path covered by the water droplet became clear to visual inspection, meaning that most of the particles were collected by the moving water droplet. 


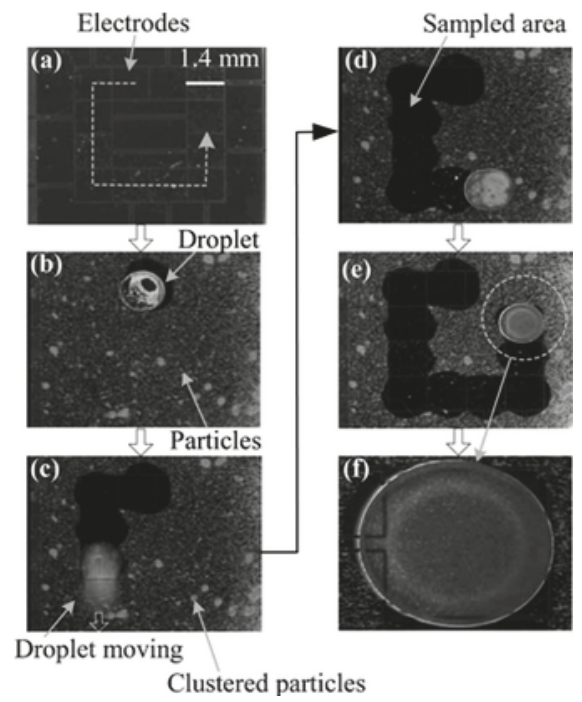

Figure 11. (a-e) Glass beads are collected sequentially using water droplets. The glass beads are suspended inside the sweeping water droplets. The dashed line in (a) indicates the path of the droplet for particle sampling and cleaning. Reproduced with permission from [134], published by Royal Society of Chemistry, 2006. (f) shows a close-up view of the droplet with suspended sampled particles.

An EWOD system can also be designed to remove unwanted small amounts of water residue adhering to surfaces. K. Y. Lee et al. developed an open coplanar EWOD system without a top cover plate targeting miniature camera surfaces for automobiles [135]. The electrodes were fabricated with indium tin oxide (ITO), which is transparent and can be integrated with the camera lens as a lens cover. 1-70 $\mu \mathrm{L}$ water droplet sizes were tested with different threshold voltages under surface inclination angles from $0^{\circ}$ to $180^{\circ}$. Figure 12 shows a demonstration of water droplet removal as well as micro-particle removal on the camera lens cover.
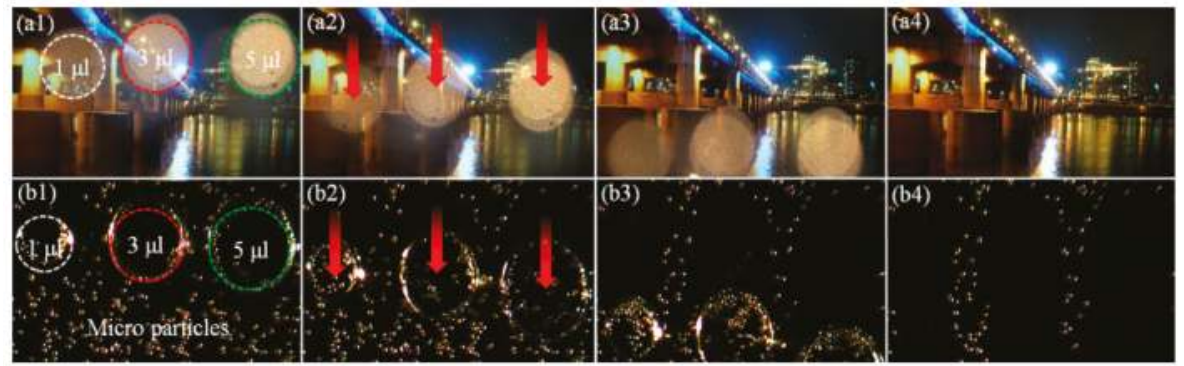

Figure 12. Sequential images of cleaning of (a) water droplets and (b) particles with different volumes on the lens cover of a smartphone camera. Reproduced with permission from [135], published by Elsevier, 2017.

Besides superhydrophobic surfaces, H. Geng and S.K. Cho combined the SLIPS with an open coplanar EWOD system [136]. The dielectric layer was SU8 and the top coating was replaced with porous fluoropolymer film infused with lubricating fluid in this configuration. Droplets can be transported along the SLIPS under voltage actuation. Bovine serum albumin (BSA) protein solution 
left tiny stains after evaporation on SLIPS while a large "coffee ring" bio-stain pattern was left on hydrophobic coatings. The bio-stain could be cleaned by droplet actuation as shown in Figure 13.
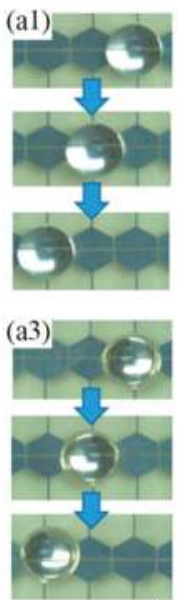

(a)
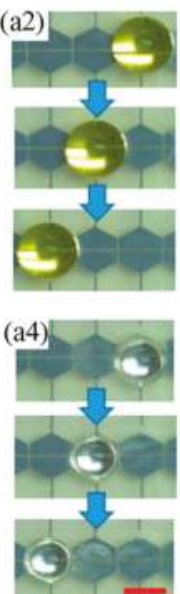

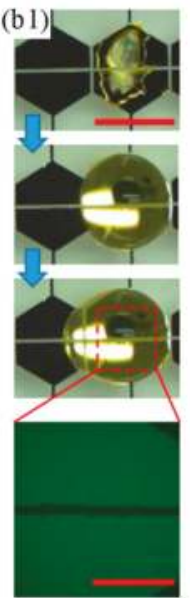

(b)

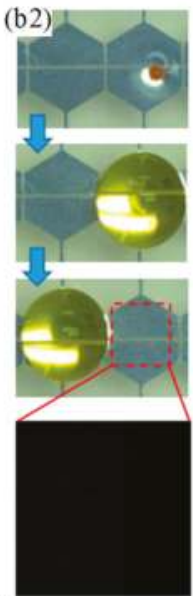

Figure 13. (a) Different liquids transported on EWOD-SLIPS surfaces: (a1) Deionized (DI) water, (a2) Bovine serum albumin (BSA) solution, (a3) propylene carbonate, (a4) isopropyl alcohol. (b) A droplet cleaning bio-stains left by evaporation: (b1) droplet fails to move on hydrophobic coatings due to bio-fouling, (b2) droplet moves and cleans the bio-stain. The green in the fluorescent images indicate the BSA residues on the surfaces. Reproduced with permission from [136], published by IEEE, 2018.
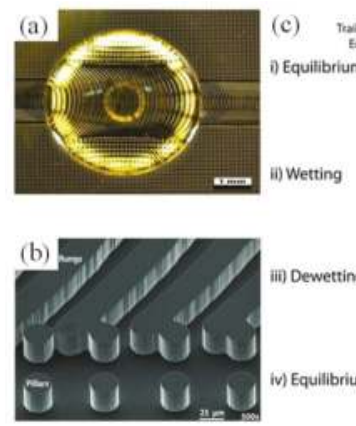

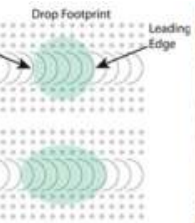

iii) Dewetting

iv) Equilibrium

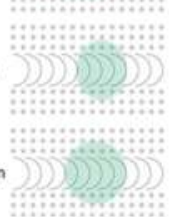

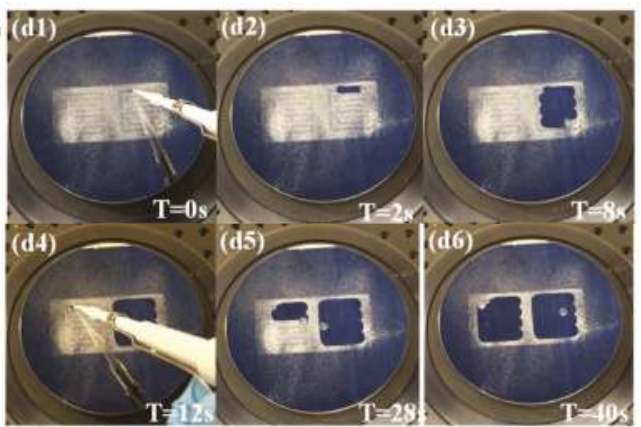

Figure 14. (a) A water droplet resting on the anisotropic ratchet conveyors (ARC) surface with etched

pillars. (b) SEM image of the ARC surface. (c) Schematic of droplet interaction with droplet under vibration. The leading edge conforms to the semi-circular rung, which acts as a wetting barrier, while the trailing edge has only intermittent contacts with the rung pattern. Reproduced with permission from [124], published by ACS Publications, 2012. Reproduced with permission from [137], published by Wiley Online Library, 2012. (d) Surface cleaning performance for powdered sweetener (dextrose, maltodextrin, and sucralose) contamination on a chemically flat ARC surface consisting of two loop tracks. Reproduced with permission from [138], published by IEEE, 2017.

As an alternative to the EWOD approach, self-cleaning surface systems using water droplet transport have been realized by anisotropic ratchet conveyors (ARC) under orthogonal vibration [123,137]. Micro-scale hydrophilic semi-circular rungs are patterned on a hydrophobic background, as shown in Figure 14 above [124]. The portion of the water droplet edge that aligns with the rung curvature, which has a mostly continuous TPL, is denoted as the leading edge of the 
droplet, while the other portion, which has only intermittent TPLs across different rungs, is called the trailing edge of the droplet. During each vibration cycle, the leading edge provides higher pinning force than the trailing edge as the footprint of the water droplet expands and recesses. This asymmetry in pinning forces causes water droplets to move toward the direction of the rung curvature. For a surface cleaning demonstration based on the ARC approach [138], two ARC tracks were laid out in a zig-zag pattern. The white contaminant on the surface consists of powdered sweetener. $10 \mu \mathrm{L}$ water droplets are applied to the surface and remove all the powder along their paths. Most water-soluble materials (like salt and sweetener) plus low surface adhesion insoluble particles (like sand) can be effectively cleaned from the self-cleaning ARC surface with water droplets.

\subsection{Self-Cleaning Surfaces by Electro-Static Charge}

Electrodynamic screen devices have been developed to remove dust particles for scenarios where the water resource is scarce or not available, as in desert regions. The concept of transporting particles using an electrostatic traveling wave was first developed by Masuda [139], where a series of electrodes were connected to the AC source to serve as contactless conveyors. Mazumder, et al. developed an electrodynamic screen (EDS) with traveling-wave AC field to create a self-cleaning system for the problem of dust accumulation both on Mars missions [140-142] and on terrestrial solar panels [143]. Figure 15 demonstrates a typical EDS design with dust accumulation and cleaning effect before and after the AC voltage was supplied [144,145]. Interdigitated electrodes were fabricated on printed circuit board or glass substrates. The electrodes were insulated with a layer of transparent polymer. As AC voltage was applied (700 1000 V peak-to-peak), the electrodynamic force applied to the particles overcame gravity and the viscous force of air to lift the particles from the surface and transported them to different locations. Over $90 \%$ of cleaning efficiency could be achieved by optimizing the frequency, voltage, and signal shape. The power consumption and the cleaning time were only in the order of milliwatts and tens of seconds. Other EDS systems were designed employing a standing-wave AC field, with simplified electrical circuit designs and high voltage resources. Bing Guo, et al. systematically studied the EDS efficiency in terms of EDS dimension size, dielectric cover thickness, dust loading level, dust deposition methods, and particle size distribution for solar energy applications [146,147]. A dust removal efficiency of $90 \%$ could be achieved within $10 \mathrm{~s}$ of energizing at dust loading level of $100 \mathrm{~g} \cdot \mathrm{m}^{-2}$ with a voltage level of $6 \mathrm{kV}$ p. Dust removal efficiency improved with increased dust loading levels, reduced dielectric cover thickness and large dust agglomerations.

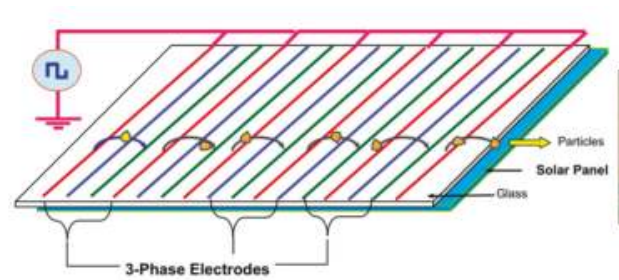

(a)

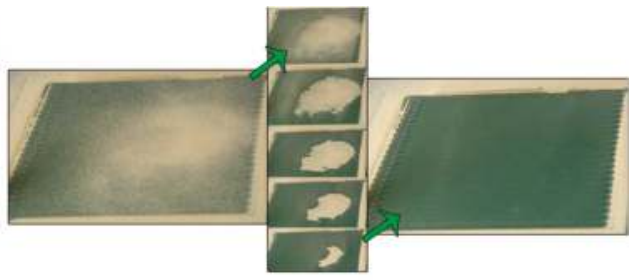

(b)

Figure 15. (a) Schematic view of a typical electrodynamic screen (EDS) design. Reproduced with permission from [144], published by Elsevier, 2013. (b) Sequential images of the dust removal processes on top of the EDS panel by electrodynamic force. Reproduced with permission from [143], published by IEEE, 2013.

\subsection{Self-Cleaning Surfaces by Gecko Tape}

Geckos have attracted the attention of researchers for many years due to their ability to climb up smooth vertical surfaces. The gecko's foot has millions of hairs, named keratinous setae, providing large van der Waals adhesive forces $[148,149]$ that prevent the gecko from falling off from smooth 
vertical surfaces. Geckos have intimate contact on various surfaces with their sticky toes but their setae virtually always keep clean and dry [150]. W. R. Hansen and K. Autumn [151] studied the gecko's keratinous setae and found the self-cleaning mechanism: Each of the millions of setae on the gecko's toe pads has hundreds of spatulae, sub-micron triangular structures aligned in parallel with each other but not normal to the toes. An imbalance exists between the adhesive force of one or more spatulae to the dirt particles and the dirt particles to the substrate surface. When touching the clean substrate surface, the dirt has higher contact areas to the surface and tends to stick to the surface rather than the gecko's spatulae. The adhesive and shear force of a contaminated gecko's foot is recovered gradually by successive steps on a clean surface. By comparing the SEM images of spatula arrays after dirtying with microspheres and after several simulated cleaning steps, most of the spatula surfaces were free of micro sphere contamination. The shear force measurement also showed the gradual restoration as the simulated step numbers increased.

Self-cleaning adhesive tapes have been developed using carbon nanotubes [152] and polymer microfibrillars [153] mimicking gecko setae. Figure 16a shows contaminated polypropylene fibrillars fabricated by a thermal casting process. An estimated 42 million fibrillars were created per square centimeter with an average length of $18 \mu \mathrm{m}$ and average radius of $18 \mathrm{~nm}$. After 30 contacts on a clean glass substrate with standard simulated gecko steps (Figure 17), 60\% of the Au microspheres were removed from the tip of the micropillars (Figure 16b). The sheer force could be restored by $33 \%$ after 20-25 cleaning steps. As a comparison, a conventional pressure sensitive adhesive (PSA) went through the simulated steps. The PSA surface was almost completely covered by the Au microspheres, shown in Figure 16d.

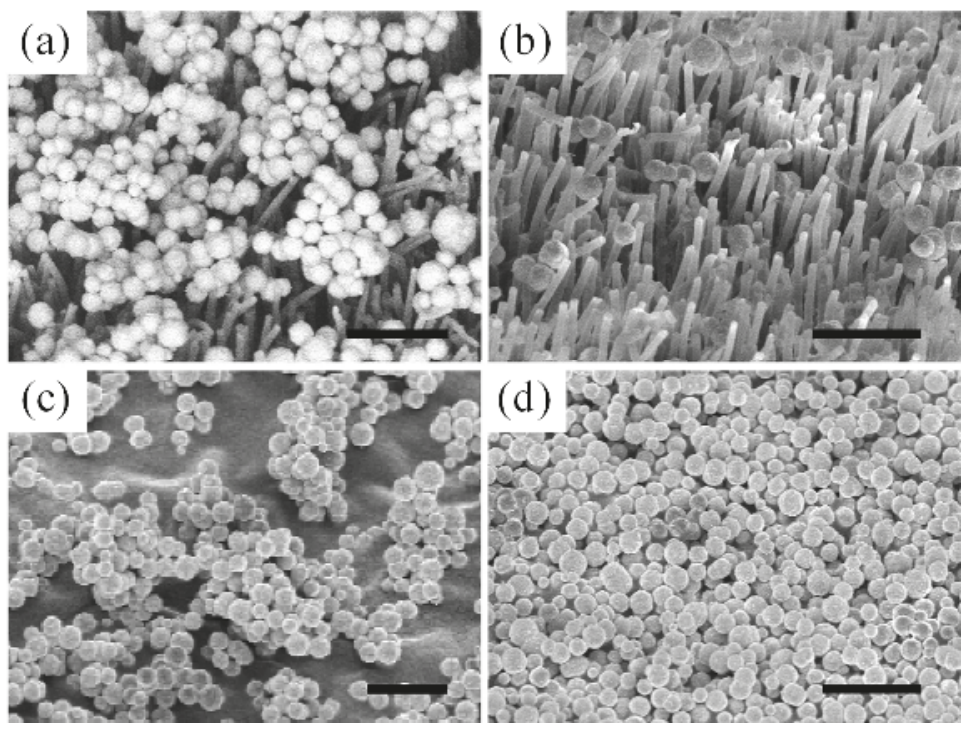

Figure 16. (a) Micro fiber adhesive contaminated with gold microspheres. (b) Micro fiber adhesive after 30 contacts (simulated steps) on a clean glass substrate. Some of the microspheres were trapped inside the micro fibers. (c) Conventional pressure sensitive adhesive (PSA) surface contaminated with microspheres. (d) PSA surface fully covered by the Au microparticles after the same simulated steps. Reproduced with permission from [153], published by ACS Publications, 2008. 

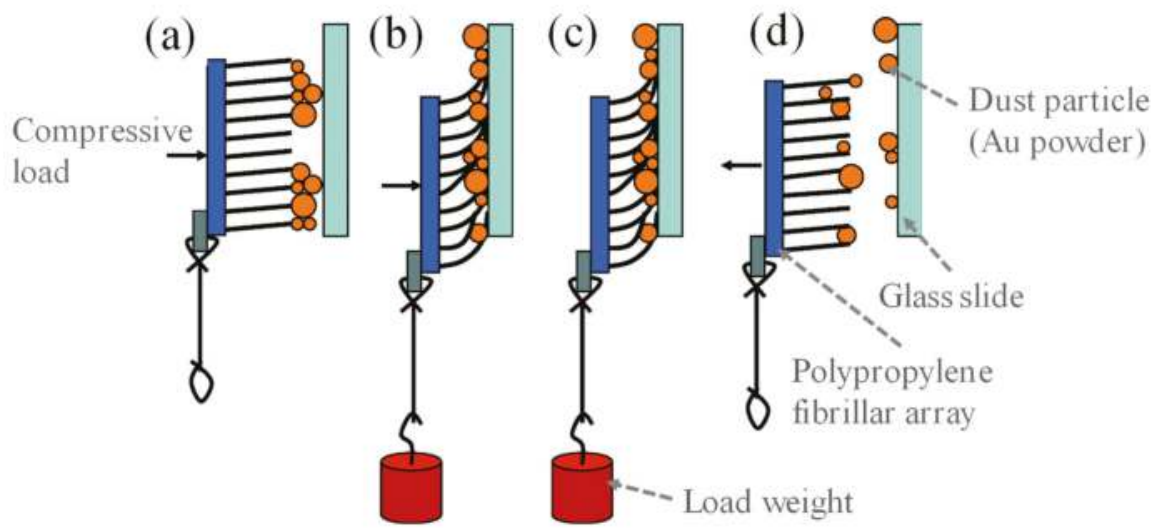

Figure 17. Standard protocol of mimicked gecko step cycle: (a) Normal compressive force was applied on the back side of the fiber substrate. (b) Applying shear load added to the compressive force. (c) Removing the compressive force to make the load a pure shear force. (d) Detaching the sample from the clean surface. Reproduced with permission from [153], published by ACS Publications, 2008.

\section{Conclusions}

Self-cleaning surfaces can have a broad range of applications from bio-fouling in medical instrumentation to building and vehicle windows to solar panel cover glass in the outdoor environment. The examples mentioned in this review article provide suggestions and protocols for designing and characterizing self-cleaning surfaces and systems. Compared with passive superhydrophobic or superhydrophilic surface designs, active cleaning systems can perform the cleaning with more delicate control of water movement, and more efficient use of water resources. Both dust and unwanted water residue can be removed at the same time. However, more complex mechanical components or control circuitry are often required for active self-cleaning systems, leading to higher initial hardware investment, larger maintenance costs, and longer payback time.

Many commercial products have emerged in the market including superhydrophobic coating sprays, photocatalyst coated window glass, and solar electrodynamic shields using EDS designs, among others. The obstacles and challenges for self-cleaning surfaces currently involve poor durability and high cost in terms of scaling and mass production. The fine micro or nano structures often cannot withstand the harsh outdoor environment for an extended period of time while the polymeric coating or surface infusion fluid will age and decay over time under solar radiation. The practical lifetime for a self-cleaning surface or system might only last from months to 1 or 2 years but the requirement is usually in the 10-20 year range, especially for applications in outdoor environments, for example in dry desert regions to reduce dust accumulation, or under water to prevent bio-fouling on a ship hull. We need to strive for the creation of self-cleaning coatings for surfaces or systems with multiple merits including low cost, good scalability, durability, transparency, and antireflection.

Author Contributions: D.S. and K.F.B. wrote the original manuscript draft; K.F.B. performed the final editing and reviewing.

Funding: This research was funded in part by Amazon Catalyst, the University of Washington CoMotion Innovation Fund, and the National Science Foundation (grant number ECCS-1308025). Part of this work was conducted at the Washington Nanofabrication Facility/Molecular Analysis Facility, a National Nanotechnology Coordinated Infrastructure (NNCI) site at the University of Washington, which is supported in part by funds from the National Science Foundation (grant numbers NNCI-1542101, 1337840 and 0335765), the National Institutes of Health, the Institute for Nano-engineered Systems, the Molecular Engineering \& Sciences Institute, the Clean Energy Institute, the Washington Research Foundation, the M. J. Murdock Charitable Trust, Altatech, ClassOne Technology, GCE Market, Google and SPTS. 
Acknowledgments: Di Sun received support from the University of Washington NSF I-Corps site, a graduate fellowship from the UW Clean Energy Institute, and prizes from the Smukowski Family in the UW Business Plan Competition and from the Alaska Airlines Environmental Innovation Challenge.

Conflicts of Interest: The authors declare no conflict of interest. The funders had no role in the design of the study; in the collection, analyses, or interpretation of data; in the writing of the manuscript, and in the decision to publish the results.

\section{References}

1. Nakajima, A.; Hashimoto, K.; Watanabe, T.; Takai, K.; Yamauchi, G.; Fujishima, A. Transparent superhydrophobic thin films with self-cleaning properties. Langmuir 2000, 16, 7044-7047. [CrossRef]

2. Paz, Y.; Luo, Z.; Rabenberg, L.; Heller, A. Photooxidative self-cleaning transparent titanium dioxide films on glass. J. Mater. Res. 1995, 10, 2842-2848. [CrossRef]

3. Bhushan, B.; Jung, Y.C. Natural and biomimetic artificial surfaces for superhydrophobicity, self-cleaning, low adhesion, and drag reduction. Prog. Mater. Sci. 2011, 56, 1-108. [CrossRef]

4. Blossey, R. Self-cleaning surfaces-Virtual realities. Nat. Mater. 2003, 2, 301-306. [CrossRef] [PubMed]

5. Comanns, P.; Buchberger, G.; Buchsbaum, A.; Baumgartner, R.; Kogler, A.; Bauer, S.; Baumgartner, W. Directional, passive liquid transport: The texas horned lizard as a model for a biomimetic 'liquid diode'. J. R. Soc. Interface 2015, 12. [CrossRef]

6. Comanns, P.; Esser, F.J.; Kappel, P.H.; Baumgartner, W.; Shaw, J.; Withers, P.C. Adsorption and movement of water by skin of the australian thorny devil (agamidae: Moloch horridus). R. Soc. Open Sci. 2017, 4, 170591. [CrossRef] [PubMed]

7. Nishimoto, S.; Bhushan, B. Bioinspired self-cleaning surfaces with superhydrophobicity, superoleophobicity, and superhydrophilicity. RSC Adv. 2013, 3, 671-690. [CrossRef]

8. Khalil, K.S.; Mahmoudi, S.R.; Abu-Dheir, N.; Varanasi, K.K. Active surfaces: Ferrofluid-impregnated surfaces for active manipulation of droplets. Appl. Phys. Lett. 2014, 105, 041604. [CrossRef]

9. Yuan, Y.; Lee, T.R. Contact angle and wetting properties. In Surface Science Techniques; Springer: Berlin, Germany, 2013; pp. 3-34.

10. Young, T. III. An essay on the cohesion of fluids. Philos. Trans. R. Soc. Lond. 1805, 95, 65-87.

11. Law, K.-Y. Definitions for Hydrophilicity, Hydrophobicity, and Superhydrophobicity: Getting the Basics Right; ACS Publications: Washington, DC, USA, 2014.

12. Nishino, T.; Meguro, M.; Nakamae, K.; Matsushita, M.; Ueda, Y. The lowest surface free energy based on -cf3 alignment. Langmuir 1999, 15, 4321-4323. [CrossRef]

13. Smithwick, R.W., III; Boulet, J.A. Vibrations of microscopic mercury droplets on glass. J. Colloid Interface Sci. 1989, 130, 588-596. [CrossRef]

14. Carr, J.S. Studies of Contact Angles on Oxidized Copper Minerals. Master's Thesis, Missouri School of Mines and Metallurgy, Rolla, MO, USA, 1948.

15. Furlong, D.; Hartland, S. Wall effect in the determination of surface tension using a wilhelmy plate. J. Colloid Interface Sci. 1979, 71, 301-315. [CrossRef]

16. Weiss, P. Wetting, spreading and adhesion, jf padday, ed., academic, london, 1978, 498 pp. Price: $\$ 48.90$. J. Polym. Sci. Polym. Lett. Ed. 1979, 17, 463-464. [CrossRef]

17. Marmur, A. Equilibrium contact angles: Theory and measurement. Colloids Surf. A Physicochem. Eng. Asp. 1996, 116, 55-61. [CrossRef]

18. Restagno, F.; Poulard, C.; Cohen, C.; Vagharchakian, L.; Léger, L. Contact angle and contact angle hysteresis measurements using the capillary bridge technique. Langmuir 2009, 25, 11188-11196. [CrossRef] [PubMed]

19. Vagharchakian, L.; Restagno, F.; Léger, L. Capillary bridge formation and breakage: A test to characterize antiadhesive surfaces. J. Phys. Chem. B 2008, 113, 3769-3775. [CrossRef]

20. Cabezas, M.G.; Bateni, A.; Montanero, J.M.; Neumann, A.W. Determination of surface tension and contact angle from the shapes of axisymmetric fluid interfaces without use of apex coordinates. Langmuir 2006, 22, 10053-10060. [CrossRef]

21. Cheng, P.; Li, D.; Boruvka, L.; Rotenberg, Y.; Neumann, A. Automation of axisymmetric drop shape analysis for measurements of interfacial tensions and contact angles. Colloids Surf. 1990, 43, 151-167. [CrossRef] 
22. Del Rio, O.; Neumann, A. Axisymmetric drop shape analysis: Computational methods for the measurement of interfacial properties from the shape and dimensions of pendant and sessile drops. J. Colloid Interface Sci. 1997, 196, 136-147.

23. Moy, E.; Cheng, P.; Policova, Z.; Treppo, S.; Kwok, D.; Mack, D.; Sherman, P.; Neuman, A. Measurement of contact angles from the maximum diameter of non-wetting drops by means of a modified axisymmetric drop shape analysis. Colloids Surf. 1991, 58, 215-227. [CrossRef]

24. Skinner, F.; Rotenberg, Y.; Neumann, A. Contact angle measurements from the contact diameter of sessile drops by means of a modified axisymmetric drop shape analysis. J. Colloid Interface Sci. 1989, 130, 25-34. [CrossRef]

25. Cabezas, M.; Bateni, A.; Montanero, J.; Neumann, A. A new method of image processing in the analysis of axisymmetric drop shapes. Colloids Surf. A Physicochem. Eng. Asp. 2005, 255, 193-200. [CrossRef]

26. Schmitt, M.; Heib, F. High-precision drop shape analysis on inclining flat surfaces: Introduction and comparison of this special method with commercial contact angle analysis. J. Chem. Phys. 2013, 139, 134201. [CrossRef] [PubMed]

27. Heib, F.; Schmitt, M. Statistical contact angle analyses with the high-precision drop shape analysis (hpdsa) approach: Basic principles and applications. Coatings 2016, 6, 57. [CrossRef]

28. Bhushan, B.; Nosonovsky, M. The rose petal effect and the modes of superhydrophobicity. Philos. Trans. R. Soc. Lond. A Math. Phys. Eng. Sci. 2010, 368, 4713-4728. [CrossRef] [PubMed]

29. Frenkel, Y.I. On the behavior of liquid drops on a solid surface. 1. The sliding of drops on an inclined surface. arXiv 2005, arXiv:physics/0503051.

30. Ford, R.; Furmidge, C. Studies at phase interfaces: II. The stabilization of water-in-oil emulsions using oil-soluble emulsifiers. J. Colloid Interface Sci. 1966, 22, 331-341. [CrossRef]

31. Larsen, S.T.; Andersen, N.K.; Søgaard, E.; Taboryski, R. Structure irregularity impedes drop roll-off at superhydrophobic surfaces. Langmuir 2014, 30, 5041-5045. [CrossRef]

32. Wenzel, R.N. Resistance of solid surfaces to wetting by water. Ind. Eng. Chem. 1936, 28, 988-994. [CrossRef]

33. Cassie, A.; Baxter, S. Wettability of porous surfaces. Trans. Faraday Soc. 1944, 40, 546-551. [CrossRef]

34. Quéré, D. Rough ideas on wetting. Phys. A Stat. Mech. Its Appl. 2002, 313, 32-46. [CrossRef]

35. Bico, J.; Thiele, U.; Quéré, D. Wetting of textured surfaces. Colloids Surf. A Physicochem. Eng. Asp. 2002, 206, 41-46. [CrossRef]

36. Bico, J.; Marzolin, C.; Quéré, D. Pearl drops. EPL (Europhys. Lett.) 1999, 47, 220-226. [CrossRef]

37. Extrand, C. Contact angles and hysteresis on surfaces with chemically heterogeneous islands. Langmuir 2003, 19, 3793-3796. [CrossRef]

38. Gao, L.; McCarthy, T.J. How wenzel and cassie were wrong. Langmuir 2007, 23, 3762-3765. [CrossRef] [PubMed]

39. Miwa, M.; Nakajima, A.; Fujishima, A.; Hashimoto, K.; Watanabe, T. Effects of the surface roughness on sliding angles of water droplets on superhydrophobic surfaces. Langmuir 2000, 16, 5754-5760. [CrossRef]

40. Okulova, N.; Johansen, P.; Christensen, L.; Taboryski, R. Effect of structure hierarchy for superhydrophobic polymer surfaces studied by droplet evaporation. Nanomaterials 2018, 8, 831. [CrossRef]

41. Feng, L.; Zhang, Y.; Xi, J.; Zhu, Y.; Wang, N.; Xia, F.; Jiang, L. Petal effect: A superhydrophobic state with high adhesive force. Langmuir 2008, 24, 4114-4119. [CrossRef]

42. Wang, S.; Jiang, L. Definition of superhydrophobic states. Adv. Mater. 2007, 19, 3423-3424. [CrossRef]

43. Gao, L.; McCarthy, T.J. The "lotus effect" explained: Two reasons why two length scales of topography are important. Langmuir 2006, 22, 2966-2967. [CrossRef]

44. Yu, M.; Chen, S.; Zhang, B.; Qiu, D.; Cui, S. Why a lotus-like superhydrophobic surface is self-cleaning? An explanation from surface force measurements and analysis. Langmuir 2014, 30, 13615-13621. [CrossRef]

45. Pechook, S.; Sudakov, K.; Polishchuk, I.; Ostrov, I.; Zakin, V.; Pokroy, B.; Shemesh, M. Bioinspired passive anti-biofouling surfaces preventing biofilm formation. J. Mater. Chem. B 2015, 3, 1371-1378. [CrossRef]

46. Barthlott, W.; Neinhuis, C. Purity of the sacred lotus, or escape from contamination in biological surfaces. Planta 1997, 202, 1-8. [CrossRef]

47. Quéré, D. Non-sticking drops. Rep. Prog. Phys. 2005, 68, 2495. [CrossRef]

48. Roach, P.; Shirtcliffe, N.J.; Newton, M.I. Progess in superhydrophobic surface development. Soft Matter 2008, 4, 224-240. [CrossRef] 
49. Zhang, X.; Shi, F.; Niu, J.; Jiang, Y.; Wang, Z. Superhydrophobic surfaces: From structural control to functional application. J. Mater. Chem. 2008, 18, 621-633. [CrossRef]

50. Patankar, N.A. On the modeling of hydrophobic contact angles on rough surfaces. Langmuir 2003, 19, 1249-1253. [CrossRef]

51. Patankar, N.A. Transition between superhydrophobic states on rough surfaces. Langmuir 2004, 20, 7097-7102. [CrossRef]

52. Zhu, L.; Xiu, Y.; Xu, J.; Tamirisa, P.A.; Hess, D.W.; Wong, C.-P. Superhydrophobicity on two-tier rough surfaces fabricated by controlled growth of aligned carbon nanotube arrays coated with fluorocarbon. Langmuir 2005, 21, 11208-11212. [CrossRef]

53. Fürstner, R.; Barthlott, W.; Neinhuis, C.; Walzel, P. Wetting and self-cleaning properties of artificial superhydrophobic surfaces. Langmuir 2005, 21, 956-961. [CrossRef]

54. Koch, K.; Bhushan, B.; Jung, Y.C.; Barthlott, W. Fabrication of artificial lotus leaves and significance of hierarchical structure for superhydrophobicity and low adhesion. Soft Matter 2009, 5, 1386-1393. [CrossRef]

55. Toma, M.; Loget, G.; Corn, R.M. Flexible teflon nanocone array surfaces with tunable superhydrophobicity for self-cleaning and aqueous droplet patterning. ACS Appl. Mater. Interfaces 2014, 6, 11110-11117. [CrossRef]

56. Erbil, H.Y.; Demirel, A.L.; Avc1, Y.; Mert, O. Transformation of a simple plastic into a superhydrophobic surface. Science 2003, 299, 1377-1380. [CrossRef] [PubMed]

57. Lau, K.K.; Bico, J.; Teo, K.B.; Chhowalla, M.; Amaratunga, G.A.; Milne, W.I.; McKinley, G.H.; Gleason, K.K. Superhydrophobic carbon nanotube forests. Nano Lett. 2003, 3, 1701-1705. [CrossRef]

58. Lu, Y.; Sathasivam, S.; Song, J.; Crick, C.R.; Carmalt, C.J.; Parkin, I.P. Robust self-cleaning surfaces that function when exposed to either air or oil. Science 2015, 347, 1132-1135. [CrossRef] [PubMed]

59. Boreyko, J.B.; Chen, C.-H. Self-propelled dropwise condensate on superhydrophobic surfaces. Phys. Rev. Lett. 2009, 103, 184501. [CrossRef]

60. Miljkovic, N.; Enright, R.; Nam, Y.; Lopez, K.; Dou, N.; Sack, J.; Wang, E.N. Jumping-droplet-enhanced condensation on scalable superhydrophobic nanostructured surfaces. Nano Lett. 2012, 13, 179-187. [CrossRef] [PubMed]

61. Wisdom, K.M.; Watson, J.A.; Qu, X.; Liu, F.; Watson, G.S.; Chen, C.-H. Self-cleaning of superhydrophobic surfaces by self-propelled jumping condensate. Proc. Natl. Acad. Sci. USA 2013, 110, 7992-7997. [CrossRef]

62. Tuteja, A.; Choi, W.; Ma, M.; Mabry, J.M.; Mazzella, S.A.; Rutledge, G.C.; McKinley, G.H.; Cohen, R.E. Designing superoleophobic surfaces. Science 2007, 318, 1618-1622. [CrossRef]

63. Lafuma, A.; Quéré, D. Superhydrophobic states. Nat. Mater. 2003, 2, 457-460. [CrossRef]

64. Shibuichi, S.; Yamamoto, T.; Onda, T.; Tsujii, K. Super water-and oil-repellent surfaces resulting from fractal structure. J. Colloid Interface Sci. 1998, 208, 287-294. [CrossRef]

65. Coulson, S.R.; Woodward, I.; Badyal, J.; Brewer, S.A.; Willis, C. Super-repellent composite fluoropolymer surfaces. J. Phys. Chem. B 2000, 104, 8836-8840. [CrossRef]

66. Helbig, R.; Nickerl, J.; Neinhuis, C.; Werner, C. Smart skin patterns protect springtails. PLoS ONE 2011, 6, e25105. [CrossRef] [PubMed]

67. Liu, T.; Kim, C.-J. Turning a surface superrepellent even to completely wetting liquids. Science 2014, 346, 1096-1100. [CrossRef] [PubMed]

68. Butt, H.-J.; Semprebon, C.; Papadopoulos, P.; Vollmer, D.; Brinkmann, M.; Ciccotti, M. Design principles for superamphiphobic surfaces. Soft Matter 2013, 9, 418-428. [CrossRef]

69. Zhang, B.; Zhang, X. Elucidating nonwetting of re-entrant surfaces with impinging droplets. Langmuir 2015, 31, 9448-9457. [CrossRef]

70. Nosonovsky, M. Multiscale roughness and stability of superhydrophobic biomimetic interfaces. Langmuir 2007, 23, 3157-3161. [CrossRef] [PubMed]

71. Tuteja, A.; Choi, W.; Mabry, J.M.; McKinley, G.H.; Cohen, R.E. Robust omniphobic surfaces. Proc. Natl. Acad. Sci. USA 2008, 105, 18200-18205. [CrossRef] [PubMed]

72. Chen, X.; Weibel, J.A.; Garimella, S.V. Water and ethanol droplet wetting transition during evaporation on omniphobic surfaces. Sci. Rep. 2015, 5, 17110. [CrossRef]

73. Ahuja, A.; Taylor, J.; Lifton, V.; Sidorenko, A.; Salamon, T.; Lobaton, E.; Kolodner, P.; Krupenkin, T. Nanonails: A simple geometrical approach to electrically tunable superlyophobic surfaces. Langmuir 2008, 24, 9-14. [CrossRef]

74. Schreiber, F. Structure and growth of self-assembling monolayers. Prog. Surf. Sci. 2000, 65, 151-257. [CrossRef] 
75. Gan, S.; Yang, P.; Yang, W. Photoactivation of alkyl $\mathrm{c}-\mathrm{h}$ and silanization: A simple and general route to prepare high-density primary amines on inert polymer surfaces for protein immobilization. Biomacromolecules 2009, 10, 1238-1243. [CrossRef] [PubMed]

76. Bormashenko, E.; Grynyov, R.; Chaniel, G.; Taitelbaum, H.; Bormashenko, Y. Robust technique allowing manufacturing superoleophobic surfaces. Appl. Surf. Sci. 2013, 270, 98-103. [CrossRef]

77. Nagappan, S.; Ha, C.-S. Superhydrophobic and self-cleaning natural leaf powder/poly (methylhydroxysiloxane) hybrid micro-nanocomposites. Macromol. Res. 2014, 22, 843-852. [CrossRef]

78. Lu, X.; Munief, W.M.; Heib, F.; Schmitt, M.; Britz, A.; Grandthyl, S.; Müller, F.; Neurohr, J.U.; Jacobs, K.; Benia, H.M. Front-end-of-line integration of graphene oxide for graphene-based electrical platforms. Adv. Mater. Technol. 2018, 3, 1700318. [CrossRef]

79. Deng, X.; Mammen, L.; Butt, H.-J.; Vollmer, D. Candle soot as a template for a transparent robust superamphiphobic coating. Science 2012, 335, 67-70. [CrossRef] [PubMed]

80. Deng, X.; Paven, M.; Papadopoulos, P.; Ye, M.; Wu, S.; Schuster, T.; Klapper, M.; Vollmer, D.; Butt, H.J. Solvent-free synthesis of microparticles on superamphiphobic surfaces. Angew. Chem. Int. Ed. 2013, 52, 11286-11289. [CrossRef] [PubMed]

81. Gupta, B.; Hilborn, J.; Hollenstein, C.; Plummer, C.; Houriet, R.; Xanthopoulos, N. Surface modification of polyester films by rf plasma. J. Appl. Polym. Sci. 2000, 78, 1083-1091. [CrossRef]

82. Bates, G.W.; Boyer, J.; Hegenauer, J.C.; Saltman, P. Facilitation of iron absorption by ferric fructose. Am. J. Clin. Nutr. 1972, 25, 983-986. [CrossRef]

83. Wang, R.; Hashimoto, K.; Fujishima, A.; Chikuni, M.; Kojima, E.; Kitamura, A.; Shimohigoshi, M.; Watanabe, T. Light-induced amphiphilic surfaces. Nature 1997, 388, 431-432. [CrossRef]

84. Piispanen, M.; Hupa, L. Comparison of self-cleaning properties of three titania coatings on float glass. Appl. Surf. Sci. 2011, 258, 1126-1131. [CrossRef]

85. De Jesus, M.A.M.L.; da Silva Neto, J.T.; Timò, G.; Paiva, P.R.P.; Dantas, M.S.S.; de Mello Ferreira, A. Superhydrophilic self-cleaning surfaces based on tio 2 and tio 2 / sio 2 composite films for photovoltaic module cover glass. Appl. Adhes. Sci. 2015, 3, 5. [CrossRef]

86. Kraeutler, B.; Bard, A.J. Photoelectrosynthesis of ethane from acetate ion at an n-type titanium dioxide electrode. The photo-kolbe reaction. J. Am. Chem. Soc. 1977, 99, 7729-7731. [CrossRef]

87. Hoffmann, M.R.; Martin, S.T.; Choi, W.; Bahnemann, D.W. Environmental applications of semiconductor photocatalysis. Chem. Rev. 1995, 95, 69-96. [CrossRef]

88. Vautier, M.; Guillard, C.; Herrmann, J.-M. Photocatalytic degradation of dyes in water: Case study of indigo and of indigo carmine. J. Catal. 2001, 201, 46-59. [CrossRef]

89. Dolamic, I.; Bürgi, T. Photocatalysis of dicarboxylic acids over tio2: An in situ atr-ir study. J. Catal. 2007, 248, 268-276. [CrossRef]

90. Yang, D.; Ni, X.; Chen, W.; Weng, Z. The observation of photo-kolbe reaction as a novel pathway to initiate photocatalytic polymerization over oxide semiconductor nanoparticles. J. Photochem. Photobiol. A Chem. 2008, 195, 323-329. [CrossRef]

91. Schmitt, M. Zno nanoparticle induced photo-kolbe reaction, fragment stabilization and effect on photopolymerization monitored by raman-uv-vis measurements. Macromol. Chem. Phys. 2012, 213, 1953-1962. [CrossRef]

92. Schmitt, M. Synthesis and testing of zno nanoparticles for photo-initiation: Experimental observation of two different non-migration initiators for bulk polymerization. Nanoscale 2015, 7, 9532-9544. [CrossRef]

93. Meilert, K.; Laub, D.; Kiwi, J. Photocatalytic self-cleaning of modified cotton textiles by tio2 clusters attached by chemical spacers. J. Mol. Catal. A Chem. 2005, 237, 101-108. [CrossRef]

94. Damodar, R.A.; You, S.-J.; Chou, H.-H. Study the self cleaning, antibacterial and photocatalytic properties of tio2 entrapped pvdf membranes. J. Hazard. Mater. 2009, 172, 1321-1328. [CrossRef]

95. Hugenschmidt, M.B.; Gamble, L.; Campbell, C.T. The interaction of h2o with a tio2 (110) surface. Surf. Sci. 1994, 302, 329-340. [CrossRef]

96. Wong, T.-S.; Kang, S.H.; Tang, S.K.; Smythe, E.J.; Hatton, B.D.; Grinthal, A.; Aizenberg, J. Bioinspired self-repairing slippery surfaces with pressure-stable omniphobicity. Nature 2011, 477, 443-447. [CrossRef] [PubMed] 
97. Bohn, H.F.; Federle, W. Insect aquaplaning: Nepenthes pitcher plants capture prey with the peristome, a fully wettable water-lubricated anisotropic surface. Proc. Natl. Acad. Sci. USA 2004, 101, 14138-14143. [CrossRef] [PubMed]

98. Kim, P.; Wong, T.-S.; Alvarenga, J.; Kreder, M.J.; Adorno-Martinez, W.E.; Aizenberg, J. Liquid-infused nanostructured surfaces with extreme anti-ice and anti-frost performance. ACS Nano 2012, 6, 6569-6577. [CrossRef] [PubMed]

99. Wilson, P.W.; Lu, W.; Xu, H.; Kim, P.; Kreder, M.J.; Alvarenga, J.; Aizenberg, J. Inhibition of ice nucleation by slippery liquid-infused porous surfaces (slips). Phys. Chem. Chem. Phys. 2013, 15, 581-585. [CrossRef] [PubMed]

100. Urata, C.; Dunderdale, G.J.; England, M.W.; Hozumi, A. Self-lubricating organogels (slugs) with exceptional syneresis-induced anti-sticking properties against viscous emulsions and ices. J. Mater. Chem. A 2015, 3, 12626-12630. [CrossRef]

101. Irajizad, P.; Hasnain, M.; Farokhnia, N.; Sajadi, S.M.; Ghasemi, H. Magnetic slippery extreme icephobic surfaces. Nat. Commun. 2016, 7, 13395. [CrossRef] [PubMed]

102. Khalil-Abad, M.S.; Yazdanshenas, M.E. Superhydrophobic antibacterial cotton textiles. J. Colloid Interface Sci. 2010, 351, 293-298. [CrossRef]

103. Crick, C.R.; Ismail, S.; Pratten, J.; Parkin, I.P. An investigation into bacterial attachment to an elastomeric superhydrophobic surface prepared via aerosol assisted deposition. Thin Solid Film. 2011, 519, 3722-3727. [CrossRef]

104. Mahalakshmi, P.; Vanithakumari, S.; Gopal, J.; Mudali, U.K.; Raj, B. Enhancing corrosion and biofouling resistance through superhydrophobic surface modification. Curr. Sci. 2011, 101, 1328-1336.

105. Privett, B.J.; Youn, J.; Hong, S.A.; Lee, J.; Han, J.; Shin, J.H.; Schoenfisch, M.H. Antibacterial fluorinated silica colloid superhydrophobic surfaces. Langmuir 2011, 27, 9597-9601. [CrossRef] [PubMed]

106. Moreno-Couranjou, M.; Mauchauffé, R.; Bonot, S.; Detrembleur, C.; Choquet, P. Anti-biofouling and antibacterial surfaces via a multicomponent coating deposited from an up-scalable atmospheric-pressure plasma-assisted cvd process. J. Mater. Chem. B 2018, 6, 614-623. [CrossRef]

107. Sun, K.; Yang, H.; Xue, W.; He, A.; Zhu, D.; Liu, W.; Adeyemi, K.; Cao, Y. Anti-biofouling superhydrophobic surface fabricated by picosecond laser texturing of stainless steel. Appl. Surf. Sci. 2018, 436, 263-267. [CrossRef]

108. Hwang, G.B.; Page, K.; Patir, A.; Nair, S.P.; Allan, E.; Parkin, I.P. The anti-biofouling properties of superhydrophobic surfaces are short-lived. ACS Nano 2018, 12, 6050-6058. [CrossRef] [PubMed]

109. Epstein, A.K.; Wong, T.-S.; Belisle, R.A.; Boggs, E.M.; Aizenberg, J. Liquid-infused structured surfaces with exceptional anti-biofouling performance. Proc. Natl. Acad. Sci. USA 2012, 109, 13182-13187. [CrossRef]

110. Yin, J.; Mei, M.L.; Li, Q.; Xia, R.; Zhang, Z.; Chu, C.H. Self-cleaning and antibiofouling enamel surface by slippery liquid-infused technique. Sci. Rep. 2016, 6, 25924. [CrossRef]

111. Leslie, D.C.; Waterhouse, A.; Berthet, J.B.; Valentin, T.M.; Watters, A.L.; Jain, A.; Kim, P.; Hatton, B.D.; Nedder, A.; Donovan, K. A bioinspired omniphobic surface coating on medical devices prevents thrombosis and biofouling. Nat. Biotechnol. 2014, 32, 1134-1140. [CrossRef]

112. Badv, M.; Jaffer, I.H.; Weitz, J.I.; Didar, T.F. An omniphobic lubricant-infused coating produced by chemical vapor deposition of hydrophobic organosilanes attenuates clotting on catheter surfaces. Sci. Rep. 2017, 7, 11639. [CrossRef]

113. Hosseini, A.; Villegas, M.; Yang, J.; Badv, M.; Weitz, J.I.; Soleymani, L.; Didar, T.F. Conductive electrochemically active lubricant-infused nanostructured surfaces attenuate coagulation and enable friction-less droplet manipulation. Adv. Mater. Interfaces 2018, 5, 1800617. [CrossRef]

114. Badv, M.; Imani, S.M.; Weitz, J.I.; Didar, T.F. Lubricant-infused surfaces with built-in functional biomolecules exhibit simultaneous repellency and tunable cell adhesion. ACS Nano 2018, 12, 10890-10902. [CrossRef]

115. Dong, Z.; Schumann, M.F.; Hokkanen, M.J.; Chang, B.; Welle, A.; Zhou, Q.; Ras, R.H.; Xu, Z.; Wegener, M.; Levkin, P.A. Superoleophobic slippery lubricant-infused surfaces: Combining two extremes in the same surface. Adv. Mater. 2018, 30, 1803890. [CrossRef] [PubMed]

116. Holmes, H.R.; Böhringer, K.F. Transporting droplets through surface anisotropy. Microsyst. Nanoeng. 2015, 1, 15022. [CrossRef]

117. Chaudhury, M.K.; Whitesides, G.M. How to make water run uphill. Science 1992, 256, 1539-1541. [CrossRef] [PubMed] 
118. Kataoka, D.E.; Troian, S.M. Patterning liquid flow on the microscopic scale. Nature 1999, 402, $794-797$. [CrossRef]

119. Pollack, M.G.; Shenderov, A.D.; Fair, R. Electrowetting-based actuation of droplets for integrated microfluidics. Lab A Chip 2002, 2, 96-101. [CrossRef]

120. Cho, S.K.; Moon, H.; Kim, C.-J. Creating, transporting, cutting, and merging liquid droplets by electrowetting-based actuation for digital microfluidic circuits. J. Microelectromech. Syst. 2003, 12, 70-80.

121. Park, S.-Y.; Teitell, M.A.; Chiou, E.P. Single-sided continuous optoelectrowetting (scoew) for droplet manipulation with light patterns. Lab A Chip 2010, 10, 1655-1661. [CrossRef]

122. Tan, M.K.; Friend, J.R.; Yeo, L.Y. Microparticle collection and concentration via a miniature surface acoustic wave device. Lab A Chip 2007, 7, 618-625. [CrossRef]

123. Shastry, A.; Case, M.J.; Böhringer, K.F. Directing droplets using microstructured surfaces. Langmuir 2006, 22, 6161-6167. [CrossRef]

124. Duncombe, T.A.; Parsons, J.F.; Böhringer, K.F. Directed drop transport rectified from orthogonal vibrations via a flat wetting barrier ratchet. Langmuir 2012, 28, 13765-13770. [CrossRef]

125. Van Grinsven, K.L.; Ousati Ashtiani, A.; Jiang, H. Fabrication and actuation of an electrowetting droplet array on a flexible substrate. Micromachines 2017, 8, 334. [CrossRef]

126. Fan, S.-K.; Yang, H.; Wang, T.-T.; Hsu, W. Asymmetric electrowetting-Moving droplets by a square wave. Lab A Chip 2007, 7, 1330-1335. [CrossRef]

127. Kim, J.-H.; Lee, J.-H.; Kim, J.-Y.; Mirzaei, A.; Wu, P.; Kim, H.W.; Kim, S.S. Electrowetting on the dielectric (ewod) properties of teflon-coated electrosprayed silica layers in air and oil media and the influence of electric leakage. J. Mater. Chem. C 2018, 6, 6808-6815. [CrossRef]

128. Crane, N.B.; Volinsky, A.A.; Mishra, P.; Rajgadkar, A.; Khodayari, M. Bidirectional electrowetting actuation with voltage polarity dependence. Appl. Phys. Lett. 2010, 96, 104103. [CrossRef]

129. Li, C.; Jiang, H. Fabrication and characterization of flexible electrowetting on dielectrics (ewod) microlens. Micromachines 2014, 5, 432-441. [CrossRef]

130. Lee, J.; Moon, H.; Fowler, J.; Schoellhammer, T.; Kim, C.-J. Electrowetting and electrowetting-on-dielectric for microscale liquid handling. Sens. Actuators A Phys. 2002, 95, 259-268. [CrossRef]

131. Yi, U.-C.; Kim, C.-J. Characterization of electrowetting actuation on addressable single-side coplanar electrodes. J. Micromech. Microeng. 2006, 16, 2053. [CrossRef]

132. Latip, E.A.; Coudron, L.; McDonnell, M.; Johnston, I.; McCluskey, D.; Day, R.; Tracey, M. Protein droplet actuation on superhydrophobic surfaces: A new approach toward anti-biofouling electrowetting systems. RSC Adv. 2017, 7, 49633-49648. [CrossRef]

133. Jönsson-Niedziółka, M.; Lapierre, F.; Coffinier, Y.; Parry, S.; Zoueshtiagh, F.; Foat, T.; Thomy, V.; Boukherroub, R. Ewod driven cleaning of bioparticles on hydrophobic and superhydrophobic surfaces. Lab A Chip 2011, 11, 490-496. [CrossRef]

134. Zhao, Y.; Cho, S.K. Microparticle sampling by electrowetting-actuated droplet sweeping. Lab A Chip 2006, 6 , 137-144. [CrossRef]

135. Lee, K.Y.; Hong, J.; Chung, S.K. Smart self-cleaning lens cover for miniature cameras of automobiles. Sens. Actuators B Chem. 2017, 239, 754-758.

136. Geng, H.; Cho, S.K. In Anti-biofouling droplet manipulation by slippery liquid infused porous surface (slips) integrated with electrowetting and liquid-dielectrophoresis. In Proceedings of the 2018 IEEE Micro Electro Mechanical Systems (MEMS), Belfast, UK, 21-25 January 2018; pp. 261-264.

137. Duncombe, T.A.; Erdem, E.Y.; Shastry, A.; Baskaran, R.; Böhringer, K.F. Controlling liquid drops with texture ratchets. Adv. Mater. 2012, 24, 1545-1550. [CrossRef]

138. Sun, D.; Böhringer, K.F. Self-cleaning surfaces using anisotropic ratchet conveyors. In Proceedings of the 2017 19th International Conference on Solid-State Sensors, Actuators and Microsystems (TRANSDUCERS), Kaohsiung, Taiwan, 18-22 June 2017; pp. 1773-1776.

139. Masuda, S.; Washizu, M.; Kawabata, I. Movement of blood cells in liquid by nonuniform traveling field. IEEE Trans. Ind. Appl. 1988, 24, 217-222. [CrossRef]

140. Sharma, R.; Wyatt, C.A.; Zhang, J.; Calle, C.I.; Mardesich, N.; Mazumder, M.K. Experimental evaluation and analysis of electrodynamic screen as dust mitigation technology for future mars missions. IEEE Trans. Ind. Appl. 2009, 45, 591-596. [CrossRef] 
141. Biris, A.; Saini, D.; Srirama, P.; Mazumder, M.; Sims, R.; Calle, C.; Buhler, C. Electrodynamic removal of contaminant particles and its applications. In Proceedings of the Conference Record of the 2004 IEEE Industry Applications Conference, Seattle, WA, USA, 3-7 October 2004; pp. 1283-1286.

142. Sharma, R.; Wyatt, C.; Zhang, J.; Mazumder, M.; Calle, C.; Mardesich, N. Performance analysis of electrodynamic self-cleaning transparent films for its applications to mars and lunar missions. In Proceedings of the 2007 IEEE Industry Applications Annual Meeting, New Orleans, LA, USA, 23-27 September 2007; pp. 434-437.

143. Mazumder, M.; Horenstein, M.N.; Stark, J.W.; Girouard, P.; Sumner, R.; Henderson, B.; Sadder, O.; Hidetaka, I.; Biris, A.S.; Sharma, R. Characterization of electrodynamic screen performance for dust removal from solar panels and solar hydrogen generators. IEEE Trans. Ind. Appl. 2013, 49, 1793-1800. [CrossRef]

144. Horenstein, M.N.; Mazumder, M.; Sumner, R.C., Jr. Predicting particle trajectories on an electrodynamic screen-theory and experiment. J. Electrost. 2013, 71, 185-188. [CrossRef]

145. Horenstein, M.N.; Mazumder, M.K.; Sumner, R.C.; Stark, J.; Abuhamed, T.; Boxman, R. Modeling of trajectories in an electrodynamic screen for obtaining maximum particle removal efficiency. IEEE Trans. Ind. Appl. 2013, 49, 707-713. [CrossRef]

146. Guo, B.; Javed, W. Efficiency of electrodynamic dust shield at dust loading levels relevant to solar energy applications. IEEE J. Photovolt. 2018, 8, 196-202. [CrossRef]

147. Guo, B.; Javed, W.; Pett, C.; Wu, C.-Y.; Scheffe, J.R. Electrodynamic dust shield performance under simulated operating conditions for solar energy applications. Sol. Energy Mater. Sol. Cells 2018, 185, 80-85. [CrossRef]

148. Autumn, K.; Liang, Y.A.; Hsieh, S.T.; Zesch, W.; Chan, W.P.; Kenny, T.W.; Fearing, R.; Full, R.J. Adhesive force of a single gecko foot-hair. Nature 2000, 405, 681-685. [CrossRef]

149. Autumn, K.; Sitti, M.; Liang, Y.A.; Peattie, A.M.; Hansen, W.R.; Sponberg, S.; Kenny, T.W.; Fearing, R.; Israelachvili, J.N.; Full, R.J. Evidence for van der waals adhesion in gecko setae. Proc. Natl. Acad. Sci. USA 2002, 99, 12252-12256. [CrossRef]

150. Stark, A.Y.; Wucinich, N.A.; Paoloni, E.L.; Niewiarowski, P.H.; Dhinojwala, A. Self-drying: A gecko's innate ability to remove water from wet toe pads. PLoS ONE 2014, 9, e101885. [CrossRef]

151. Hansen, W.R.; Autumn, K. Evidence for self-cleaning in gecko setae. Proc. Natl. Acad. Sci. USA 2005, 102, 385-389. [CrossRef]

152. Sethi, S.; Ge, L.; Ci, L.; Ajayan, P.M.; Dhinojwala, A. Gecko-inspired carbon nanotube-based self-cleaning adhesives. Nano Lett. 2008, 8, 822-825. [CrossRef]

153. Lee, J.; Fearing, R.S. Contact self-cleaning of synthetic gecko adhesive from polymer microfibers. Langmuir 2008, 24, 10587-10591. [CrossRef]

(C) 2019 by the authors. Licensee MDPI, Basel, Switzerland. This article is an open access article distributed under the terms and conditions of the Creative Commons Attribution (CC BY) license (http:/ / creativecommons.org/licenses/by/4.0/). 
Review

\title{
Micro-Surface and -Interfacial Tensions Measured Using the Micropipette Technique: Applications in Ultrasound-Microbubbles, Oil-Recovery, Lung-Surfactants, Nanoprecipitation, and Microfluidics
}

\author{
David Needham ${ }^{1,2,3, *(\mathbb{C}, \text { Koji Kinoshita }}{ }^{1}$ and Anders Utoft ${ }^{1}$ \\ 1 Institute for Molecular Medicine, University of Southern Denmark, Odense 5230, Denmark; \\ koji@health.sdu.dk (K.K.); aum@health.sdu.dk (A.U.) \\ 2 Department of Mechanical Engineering and Material Science, Duke University, Durham, NC 27708, USA \\ 3 School of Pharmacy, University of Nottingham, Nottingham, NG7 2RD, UK \\ * Correspondence: d.needham@duke.edu; Tel.: 919-660-5355
}

Received: 14 December 2018; Accepted: 25 January 2019; Published: 1 February 2019

check for updates

\begin{abstract}
This review presents a series of measurements of the surface and interfacial tensions we have been able to make using the micropipette technique. These include: equilibrium tensions at the air-water surface and oil-water interface, as well as equilibrium and dynamic adsorption of water-soluble surfactants and water-insoluble and lipids. At its essence, the micropipette technique is one of capillary-action, glass-wetting, and applied pressure. A micropipette, as a parallel or tapered shaft, is mounted horizontally in a microchamber and viewed in an inverted microscope. When filled with air or oil, and inserted into an aqueous-filled chamber, the position of the surface or interface meniscus is controlled by applied micropipette pressure. The position and hence radius of curvature of the meniscus can be moved in a controlled fashion from dimensions associated with the capillary tip $(\sim 5-10 \mu \mathrm{m})$, to back down the micropipette that can taper out to $450 \mu \mathrm{m}$. All measurements are therefore actually made at the microscale. Following the Young-Laplace equation and geometry of the capillary, the surface or interfacial tension value is simply obtained from the radius of the meniscus in the tapered pipette and the applied pressure to keep it there. Motivated by Franklin's early experiments that demonstrated molecularity and monolayer formation, we also give a brief potted-historical perspective that includes fundamental surfactancy driven by margarine, the first use of a micropipette to circuitously measure bilayer membrane tensions and free energies of formation, and its basis for revolutionising the study and applications of membrane ion-channels in Droplet Interface Bilayers. Finally, we give five examples of where our measurements have had an impact on applications in micro-surfaces and microfluidics, including gas microbubbles for ultrasound contrast; interfacial tensions for micro-oil droplets in oil recovery; surface tensions and tensions-in-the surface for natural and synthetic lung surfactants; interfacial tension in nanoprecipitation; and micro-surface tensions in microfluidics.
\end{abstract}

Keywords: micropipette-technique; air-water surface; oil-water interface; soluble surfactant; insoluble lipids; "black lipid films"; "droplet-interface-bilayers"; equilibrium; dynamic; adsorption; gas-microbubbles; oil-microdroplets; lung-surfactants; nanoprecipitation; microfluidics 


\section{Graphical Abstract}

\section{The versatile micropipette manipulation technique 1983-2018}

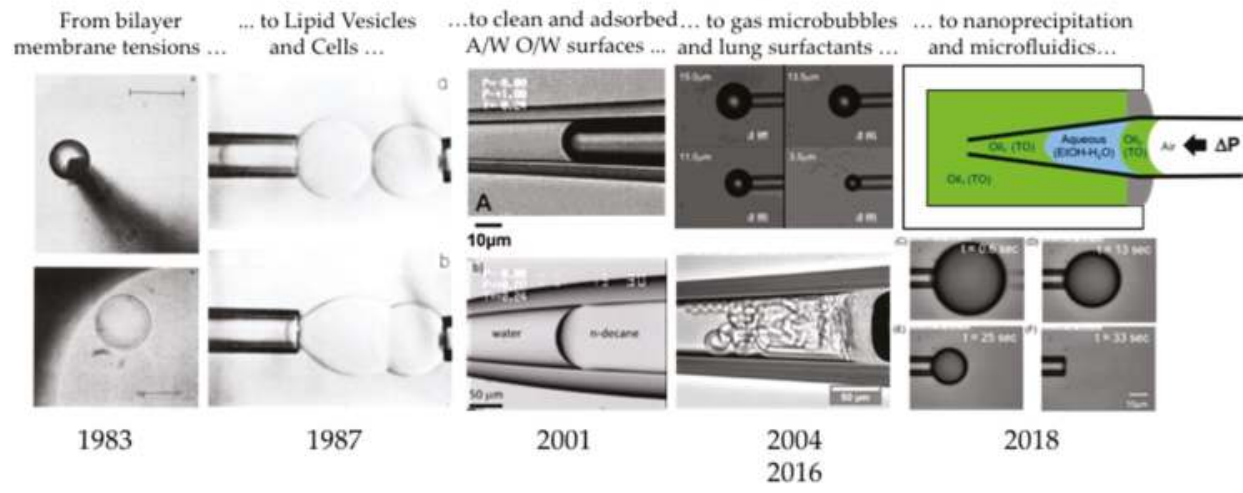

\section{Introduction}

In this contribution to the Special Issue "Microscale Surface Tension and Its Applications," we give an up-to-date review from 1983 [1] to the present day, of our wide range of micropipette-techniques utilised for measurements of surface and interfacial tensions, droplet dissolution, and molecular adsorption in air-water, oil-water, water-oil systems. While there are many other techniques and methodologies associated with microsurfaces including microfluidic tensiometry, capillarity of gas bubbles, and other micro techniques involving "Laplace sensors" [2-5] we take the liberty to limit the scope of this review to our own studies. Micropipettes are glass capillaries that are custom cut to have tip diameters $\sim 5 \mu \mathrm{m}$ and can taper out to $450 \mu \mathrm{m}$, and so all measurements are actually made on surface and interfacial menisci, gas microbubbles or liquid microdroplets at the microscale. As motivated in the special issue, surface tension and capillary effects enable many of the applications in micro- and nano-systems. So here, by utilising a micropipette technique, we provide direct measures of surface and interfacial tensions at the same scales as microfluidic, lab-on-chip, and other devices. The goal is to provide the readership with a comprehensive review of many of the surface and interfacial tension measurements we have been able to make using the micropipette technique, including equilibrium measurements of the clean air-water surface and oil-water interface [6], as well as equilibrium and dynamic adsorption of water-soluble surfactants $[7,8]$ and water-insoluble lipids $[9,10]$ that required the development of a new technique, the Micropipette Interfacial Area-Expansion Method (MIAM) [7]. We also give examples of where our measurements and those of others [11] have had a direct impact on at least five applications. These selected applications include: gas microbubbles for ultrasound contrast [12-14]; interfacial tensions of micro-oil droplets for oil recovery [11]; surface tensions and tensions-in-the surface of natural and synthetic lung surfactants [10]; interfacial tension in nanoprecipitation [15-17]; and micro-surface tensions in microfluidics [18].

We start though by presenting a few short stories behind some aspects of surfactancy we find interesting and/or have made contributions to. This historical-perspective briefly describes certain collaborations, personal contacts, and friendships that often underlie, or have even enabled, surfactancy R\&D. It takes us on a potted personalised journey that includes: Franklin and his "cruet of oil"; the importance and role of Unilever scientists in generating much of the fundamental studies at the time; an academic-industrial friendship that came out of those studies; an early micropipette technique for studying fundamentals of "Black Lipid Films" (BLMs) and their interfacial tension, and how this system helped to generate a new, and currently very active, field of "Droplet Interface Bilayers" (DIBs). 


\subsection{Franklin and Friends at the Royal Society}

As is well known, when an oil droplet is introduced at the air-water surface, an oil film can spontaneously spread producing eventually a monomolecular film. Franklin communicated his famous experiment to his friend William Brownrigg, and it was read and published in the Royal Society in 1774 [19]. While Clapham Common may have been one site for "smoothing the waves," their friendship extended to Franklin and John Pringle visiting Brownrigg at his home in Ormathwaite in the English Lake District. As recounted by Mertons [20], "The three of them went to nearby Derwent Water where Franklin demonstrated the wave-stilling effect of a little oil he kept in the hollow upper joint of his bamboo cane."

Having observed that the wakes of two ships were remarkably smooth, while all the others were ruffled by the wind, Franklin asked the captain, who told him that the cooks on those two ships had probably just emptied their greasy water. As recounted by Franklin in his letter to the Royal Society $[19,20]$.

"At length being at Clapham where there is, on the common, a large pond, which I observed to be one day very rough with the wind, I fetched out a cruet of oil, and dropt a little of it on the water. I law it spread itself with surprizing swiftness upon the surface; but the effect of smoothing the waves was not produced; for I had applied it first on the leeward side of the pond, where the waves were largest and the wind drove my oil back upon the shore. I then went to the windward side, where they began to form; and there the oil, though not more than a tea spoonful, produced an instant calm over a space several yards square, which spread amazingly, and extended itself gradually till it reached the lee side, making all that quarter of the pond, perhaps half an acre, as smooth as a looking-glass."

This spreading of an oil film is one of the characteristics of amphiphilic molecules having hydrophilic and hydrophobic parts. As Tanford mentioned in his book in 1980 [21], if the oil molecule has only pure hydrocarbon chains, the film-spreading phenomena will not happen, i.e., the oil stays at the surface as a droplet; but, if it is an amphiphile, a monolayer of just one molecule thick can be obtained, and this monolayer reduces the clean surface or interfacial tension. Hardy, a British biologist and food scientist [22], in his 1912 paper to the Royal Society, entitled "The tension of composite fluid surfaces and the mechanical stability of films of fluid" [23] found that without a polar group (hydrophilic part) in the molecule, there indeed was no driving force for surface adsorption and so no surface tension reduction. Since then, more than one century has passed. Currently, liquid-liquid and liquid-gas interfacial phenomena with various monolayer-forming amphiphilic compounds have been extensively investigated in order to understand the mechanism of spreading, wetting, and dynamic and equilibrium adsorption that change surface and interfacial tension. These fundamental studies have been applied mainly for product development in drug and food industries, which brings us to our next story.

\subsection{Surfactant-Colleagues at Unilever and Cambridge}

It could be argued that one of the biggest driving influences on the basics of surfactancy was in the development of margarine. In relating this story, we have an opportunity to point out that, as is often forgotten when we now so easily collect papers and references from search engines, talented and hardworking people are behind those studies, and it is their legacy from which we now benefit.

The story starts with the establishment of one of the largest industrial mergers of its time; in 1929, Margarine Unie teamed up with Lever Brothers to create Unilever [24]. Unilever scientists were at the forefront of fundamental surface and interfacial chemistry research that underlies the performance of their, now over- 400 products in food and beverages (about $40 \%$ of its revenue), cleaning agents, and personal care products. One particular group of researchers requires special mention. Brian Pethica and James Mingins, working at Unilever Research, Port Sunlight Laboratory, Port Sunlight, Wirral, Cheshire L62 4XN, England, were particularly prolific from the late 1950s through the 1980s [25-35] (and actually beyond [36]). They published papers on such topics as "The Properties of Ionized Monolayers" [25]; 
"Phase-changes and mosaic formation in single and mixed phospholipid monolayers at the oil-water interface" [28]; "Entropies of Compression of Charged Monolayers at Aqueous Interfaces" [29]; "Phospholipid interactions in monolayers" [31]; and "Intermolecular forces in monolayers at air/water interfaces" [35]. These articles appeared in journals like, Trans Faraday Soc, Journal of Colloid and Interface Science and the edited book, "Monolayers" [29]. These are just a few of their publications on the most fundamental topics in surface science, carried out in a commercial company initially founded on making margarine. We would encourage interested readers to examine these "industrial" papers.

Interestingly, the "human factor" of research and development is perhaps no better exemplified than in the friendship between the "academic," Dennis Haydon FRS in the Physiological Laboratories in Cambridge, studying surfactancy of black-lipid films and anaesthesia in biological membranes, and the "industrialist" Jim Mingins at Unilever. They were good friends and colleagues, often referencing each other's work, and enjoyed especially hiking, and snow- and ice-climbing together. One can only imagine the discussions of monolayers and bilayers, surfactants and margarine, that might have ensued in the crags of Snowdonia in North Wales or Ben Nevis in Scotland [37]. It is therefore an important point to make and recognise that, not only were fundamentals applied in a range of new products since the early part of the 20th century, the development of these applications necessitated advancement in fundamentals of surface and interfacial chemistry. Fundamentals and applications, "academics and industry" went hand-in-hand in those days.

\subsection{From BLMs in Cambridge (1983) to DIBs in Oxford (2005) and Beyond}

One of us, (Needham) was lucky enough to do a post doc with Dennis Haydon FRS from 1980 to 1983. In one project, we evaluated the interfacial tension of a bilayer membrane against water (made from Glyceryl Mono-Oleate (GMO)) and a series of alkanes and squalene [1]. These bilayer membranes were called "Black Lipid Membranes" (BLM), because their thickness is below the wavelength of light and they are only "visible" microscopically at reflected glancing angles. The goal of the experiment was to evaluate the interfacial tension of a bilayer membrane $(\sigma)$ by measuring the interfacial tension of the stabilising monolayer $(\gamma)$ and the contact angle $(\theta)$ between a lens trapped in the bilayer, and with it, determine the free energy of formation of the newly discovered solvent-free films in equilibrium with Squalene, as first introduced by Stephen White [38]. In the methods previously developed by Haydon et al., the black lipid membranes were formed in a tiny $1 \mathrm{~mm}$-diameter hole drilled in a Teflon support [39] and GMO-decane solution was introduced into the hole via painting across the hole with a small paint-brush. Normally, for BLMs formed by solutions like GMO-decane, hydrocarbon-lenses were readily trapped in the bilayer during the electrical compression that triggers bilayer formation and the angle was found to be $\sim 2^{\circ}$ [39]. The contact angle was simply measured from the ringed-interference pattern of the lens, (or torus) when viewed in incident light [39]. However, for systems where the difference in bilayer to monolayer tension was greater, we expected a much greater contact angle. It was nevertheless thought that the interference fringes could be observed and measured using a high resolution interferometer, in the labs at Port Sunlight. However, with the GMO-Squalene systems, we found that it was not possible to even trap lenses during this process associated with bilayer formation. Attempts failed to form a lens. Repeated failed attempts, one after the other, were followed by a cleaning of the hole in which the GMO solution was introduced in the Teflon support, (i.e., quick "suck-blow" with a pasteur pipette), ready for the next attempt. One night, a surprising (and as it turned out fortuitous) observation was made; when the next bilayer was formed, (without a trapped lens), over the next few minutes, tiny spots of light appeared in the membrane when view in incident light. Remarkably, what had happened was that, the "suck-blow-cleaning" with the pasteur pipette had formed small emulsion droplets, that were driven downwards.

Then, when a new bilayer was formed, and as the emulsion droplets rose due to their buoyancy-effect, they touched and fused with the new bilayer! Tiny lenses had formed! A new apparatus had to be designed, built, and tested. 
A more controlled technique was therefore developed in which a single, new lens could be formed in the bilayer by introducing it to the bilayer interface with a micropipette using an in-house built contraption [1], as shown in Figure 1A. When the lens was viewed with incident interference optics, the interference fringe-rings were not resolvable, implying that the contact angle was indeed quite large. However, as shown in Figure 1C, the hydrocarbon-filled-torus that supports the bilayer can be viewed in transmitted light, as could the lens. And so, the new method that was developed as a consequence of this "happy accident," allowed a lens of known volume to be ever-so-gently, introduced from underneath these, notoriously fragile ("2-molecule-thin") bilayers by the micropipette (Figure 1B). Then, by viewing in transmitted light (Figure 1C), we could visualise (and photograph) the new lens, and use its geometry to calculate its curvature and hence contact angle with the bilayer [1].

Thus, this apparatus and technique [1] allowed us to measure the actual membrane tension, $\sigma$, given by

$$
\sigma=2 \gamma \cos \theta
$$

Then, from the area of the film A, we determine the Helmholtz free energy of formation of a black lipid film, $\Delta A$, from the relationship,

$$
\Delta A=2 \gamma A(\cos \theta-1) .
$$

For the solvent-free films in equilibrium with squalene, [38] (or with Triolein [1]), the contact angles were much higher than for the solvent containing decane films (of only $2^{\circ}$ ). The squalene monolayer tension was measured by the drop-volume method to be $2.4 \mathrm{mN} / \mathrm{m}$, its contact angle was $26.5^{\circ}$, giving a bilayer tension of $2.2 \mathrm{mN} / \mathrm{m}$ and a Helmholtz free energy of formation per unit area $\Delta \mathrm{A}^{*}$ of $-511+134 \mu \mathrm{J} / \mathrm{m}^{2}$ which was $\sim 100$ times that of the decane film (only $-4.5 \mu \mathrm{J} / \mathrm{m}^{2}$ ). For GMO bilayers in equilibrium with Triolein, the difference between the tensions of the monolayer $(1.8 \mathrm{mN} / \mathrm{m})$ and bilayer $(1.0 \mathrm{mN} / \mathrm{m})$ were even larger, showing an even larger contact angle $\left(57^{\circ}\right)$ and concomitantly larger free energy of formation, of $-1673 \mu \mathrm{J} / \mathrm{m}^{2}$. Thus, for these solventless bilayers of only $2.3 \mathrm{~nm}$ thickness (essentially twice the length of the GMO hydrocarbon oleate chains), the free energies of formation and hence stability turned out to be very high. Previous explanations for the free energy of formation of membranes made from GMO-decane to -hexadecane measured previously by Requena et al. [40], had focused on simply the thinning energy associated with the Lifshitz theory of van der Waals attraction of water across the membrane. However, the newly-measured values supported the molecular-exclusion, mean field theory predictions of David Gruen, (who was also a post doc of Haydon's) 3 years earlier working on theory [41,42]. These solventless bilayers then provided artificial, solvent-free model membranes [38] that more closely modelled natural membranes. Thus, in 1981, Needham was already working with, and exploring the use of, a micro-manipulated-micropipette to deliver oil droplets for interfacial tension measurements long-before working with the current more advanced micropipette manipulation set up (see later, Figure 4). In fact, it was this ability to innovate, develop and perform these very delicate experiments that prompted Haydon to suggest and recommend Needham (for his next post doc) to Evan Evans, who had been pioneering the micropipette manipulation techniques for evaluation of red blood cell membranes since the early 1970s. And, as they say, the rest is history. 
A)

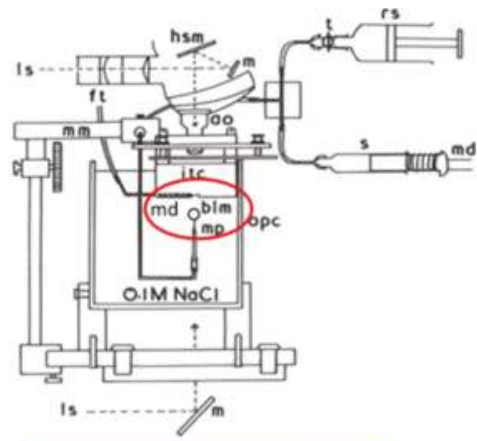

B)

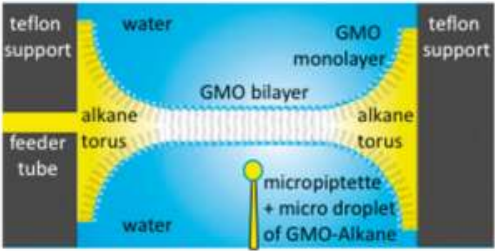

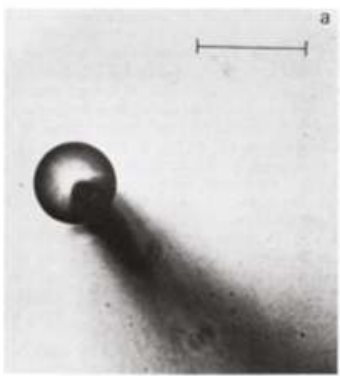

C)

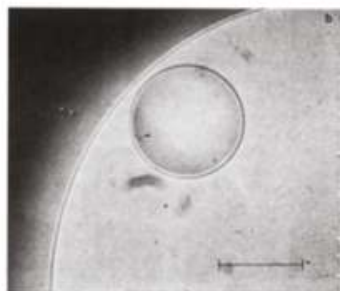

Figure 1. Measurement of high contact angles in black lipid membranes. (A) Diagram of the apparatus showing the micropipette ( $\mathrm{mp}$ ) with microdroplet (md) of Glyceryl Mono-Oleate (GMO)-alkane placed just beneath the black lipid membrane (blm) circled in red. (B) Schematic of the BLM formed in a Teflon support using a feeder tube, showing the GMO bilayer in the hole, in equilibrium with the GMO monolayer on the alkane torus, and the positioning of the micropipette with microdroplet ready to be inserted into the bilayer. (C) Photographic images of: (a) a droplet of monoolein $8.4 \mathrm{mM}$ in squalene under $0.1 \mathrm{M} \mathrm{NaCl}$ on the end of a micropipette of tip external diameter $13.6 \mu \mathrm{m}$; (b) the lens that was formed by touching the droplet to the black lipid film formed from the same solution. Bar equals $100 \mu \mathrm{m}$. Adapted from [1].

Finally, it was recognising this new-found bilayer stability that led Needham, in the summer of 2005, to suggest to Hagan Bayley, Professor of Chemical Biology at Oxford University, to flip the BLM-system and use two opposing water droplets under oil and so form a similar bilayer between them [43]. Bayley had been studying single-channel conductance of hemolysin channels in BLMs, but was having trouble stabilising the films. As illustrated in Figure 2, Needham had shown in, as then, unpublished work, that when two water droplets were formed on the ends of two micropipettes in solutions of GMO-alkane and then brought gently into contact, they spread on each other but did not fuse into one droplet. It was clear that a "BLM" had been formed between the two microdroplets (Figure 2A) and was stabilised in this droplet-droplet system as shown in the microscope image and schematic bilayer overlay in Figure 2B. 

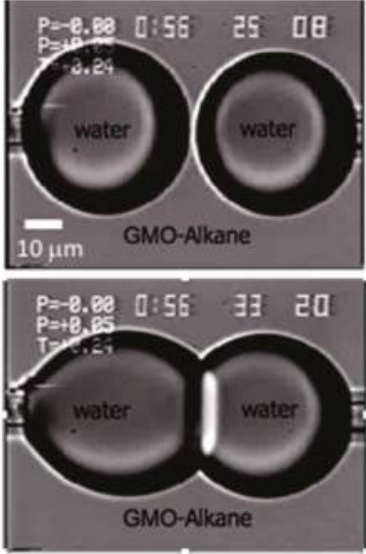

B)

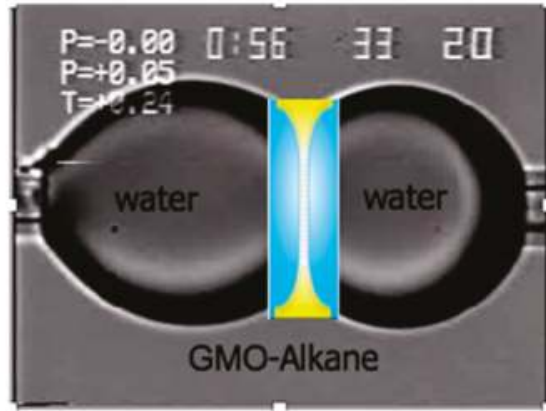

Figure 2. Two opposing water droplets under oil. (A) Two water droplets formed (top) in GMO-Alkane solution have a monolayer of GMO at their interfaces. When presented to each other (bottom) using micropipette manipulation they form a GMO-bilayer between them; (B) the same image as in (A) (bottom) but now with an overlaid-schematic of the Droplet Bilayer Interface (DIB) showing that a bilayer is formed between them as in the black lipid film.

Upon learning this, Bayley's post doc, Mathew Holden, rapidly and successfully implemented the idea [44] and this two-droplet system joined by a black lipid film became known as the Droplet Interface Bilayer (DIB). Since then the DIB has been the basis for and invention [45], and multiple new applications involving membrane-stabilised protein channels [43], and the development of a new class of active material based on the ion-transport properties of functional biomolecules [46]. Adding more and more droplets together, Gabriel Villar then created new droplet networks as "Multisomes" [47], that were shown to make tissue-like printed-droplet-networks [48,49] of 35,000 droplets and their stabilising interface bilayers [48]. As Villar concluded, (these networks) "might be interfaced with tissues, used as tissue engineering substrates, or developed as mimics of living tissue" [48]. These systems have also been extended by others into droplet microfluidics for the construction of compartmentalised model membranes [50], and organogels [51]. Here then, this simple BLM, when flipped to be a bilayer between water droplets in oil (Figure 2B), has now spawned over 1000 entries listed on Google Scholar. Once again, this kind of innovation, research, and development exemplifies the power of developing new techniques to allow new measurements-in this case of bilayer tensions, understanding fundamentals of free energies of formation, that are picked up by other talented and driven scientists (perhaps our friends, or friends to be) for subsequent development and new applications. We hope you enjoyed this little potted-history of just some aspects of surfactancy and appreciated the "human factor" at the root of all published research that we perhaps too often take for granted.

\section{Basic Micropipette Manipulation Techniques for Surface and Interfacial Tension Measurement}

The micropipette manipulation technique is based on the principles of capillary action. As is well-known, capillary action is the tendency of a fluid to be raised in a narrow tube, as the result of the positive adhesion and wetting of the tube by the liquid. (Note: Non-wetting can produce the opposite effect and suppress capillarity, e.g., mercury-air-glass). The classic observation is that, when a narrow glass tube, with a radius of a few hundred microns, is dipped into water, the water rises up the tube to such an extent that its wetting-adhesion around the circumference of the glass opposes the gravitational force on its raised mass. In the micropipette technique, we use similar glass capillary tubes mounted horizontally (hence, no gravity effects) where the capillary action is now precisely controlled by the application of often delicate, applied micropipette pressures ( $10 \mathrm{~s}$ of micro-metres of 
water) to sometimes quite forceful (10 s of centimetres of water), all viewed under an inverted optical microscope. While initially developed in its current form in the early 1970s and used for studying the micromechanics of red blood cells [52-60] white blood cells, [61-71], and lipid vesicles [72-84], here, we review its adaptation for measurements of surface and interfacial tension at air-water surfaces, oil-water interfaces, and the equilibrium and dynamic adsorption of surfactants and lipids.

\subsection{Principles of Capillary Action and the Micropipette}

At its essence then, the micropipette technique is one of capillary-action, glass-wetting and applied pressure. Classical capillary rise is a well-known physical phenomenon associated with the surface tension forming inside a capillary [85-87]. It relies on gravity as the opposing force on the water that wets the glass capillary at its air-water surface meniscus. In general, when one end of a vertical capillary is immersed in a liquid to from the air-liquid surface, the liquid comes into the capillary. Figure 3a shows a schematic image of this capillary action.

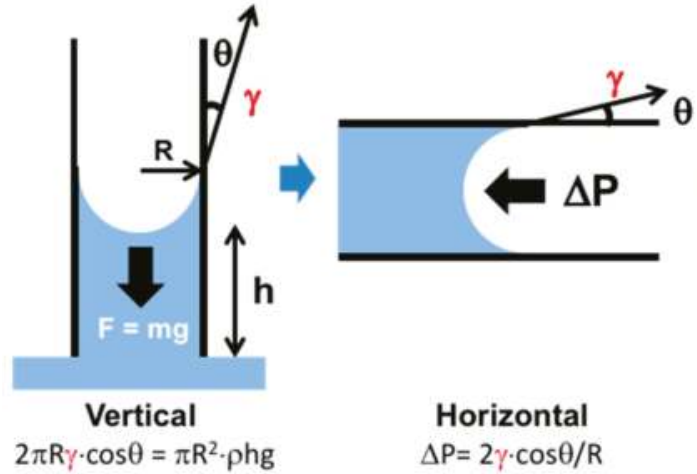

(a)

(b)

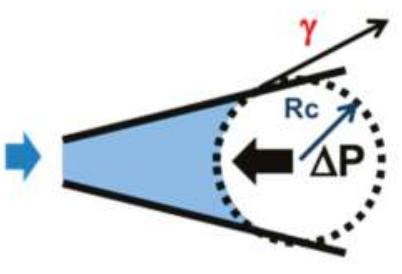

Tapered micropipette $\Delta \mathrm{P}=2 \gamma / \mathrm{R}_{\mathrm{c}}$

(c)

Figure 3. Capillary actions of the air-water surface. (a) Capillary-rise of water inside a vertical capillary. Upon dipping the capillary tube into the liquid, meniscus rises against gravity a distance $h$ above the outside bulk surface. At equilibrium, this height reaches a force balance that follows the Young-Laplace equation between gravity and wetting (mg versus $2 \pi R \gamma \cos \theta$ ). (b) In the horizontal capillary immersed in an aqueous-filled microscope chamber, the water comes inside the horizontal capillary until opposed by a counter force such as applying a pressure to the back-end of the pipette to hold it in position or push it back. Again, the force balance follows the Young-Laplace equation of pressure opposed by the surface tension scaled by the reciprocal of the single radius. (c) Similarly, for a tapered micropipette where the meniscus position is now opposed requiring a series of increasing applied pressure to move the surface down the taper. It is this tapered pipette that has been the most useful and is the basis for our tension measurements.

In the vertical set up (Figure 3a), the liquid-rise is due to wetting of the glass (at some wetting contact angle), providing a concave surface. This means that the pressure just below the surface is less than the ambient pressure above it. The height of the meniscus $(h)$, from the bulk surface is thus the equilibrium between this reduced pressure scaled by the interfacial tension-acting at the capillary circumference effectively "pulling" the meniscus upwards and gravitational force of the mass of liquid "pulling" downwards. Thus, the interfacial tension force $\left(F_{i}\right)$ is expressed by the equation, $F_{i}=2 \pi R \gamma \cos \theta_{c}$, where $R$ is the inner radius of the capillary, and $\theta_{c}$ is the contact angle between the liquid and the capillary surface material. The gravitational force, as a counter balance 
force, is expressed by, $F_{g}=\pi R^{2} \rho h$, where $\rho$ is the density of the liquid, and $g$ is the gravitational constant. Using these equations, the interfacial tension is given by the following relationship [87],

$$
\gamma=\frac{R \rho h g}{2 \cos \theta_{c}} .
$$

From the proportional relations of $R$ and $h$ in this equation, the capillary rise becomes higher, when the capillary radius becomes smaller. The relation shows that for a given liquid, material of the capillary (e.g., glass), and air-water surface tension, the capillary radius is a key factor in controlling the capillary rise of the meniscus surface.

Using these principles, micropipette capillary techniques were developed to observe the gas-liquid surface or liquid-liquid interface in an inverted microscope. Micropipettes are mounted horizontally as a parallel shaft (Figure 3b) or tapered shaft (Figure 3c). Now, the position of the surface or interface meniscus is controlled by the applied micropipette pressure, and can be moved in a controlled fashion all the way to dimensions associated with the capillary tip $(\sim 5-10 \mu \mathrm{m})$ (and even blow out a bubble, see later, Figure 21). Figure $3 \mathrm{~b}$ shows the capillary in a horizontal position and thus there is no gravitational force acting on the meniscus or liquid in this position. In this case, the liquid comes inside the capillary by capillary action unopposed and would move throughout the whole length of the capillary tube since there is no counter balance against $F_{i}$. To resist the liquid flowing into the capillary, a counter balancing force can be initiated inside the micropipette by applying a positive (blowing) pressure, which, when scaled by the surface tension and reciprocal of the radius, is again the Laplace pressure. The relation is thus described with the Young-Laplace equation,

$$
\Delta=\frac{2 \gamma \cos \theta_{c}}{R} \text {. }
$$

The Laplace pressure required to stop the liquid flow is proportional to the reciprocal of the capillary radius, and so, again, smaller diameter capillaries require higher opposing pressures to create smaller radii of curvature. The other important factor is the contact angle $\theta_{c}$ between the liquid and the glass-surface material of the capillary. The contact angle of the air-water surface at a clean glass surface is about $5^{\circ}$. To apply this technique for all liquids including mixed solutions against glass surfaces and other surfaces, the $\theta_{c}$ values have to be measured independently. However, because of the constant diameter of the parallel capillary there is only a single pipette pressure for any given tension, and so statistical averaging is somewhat limited. To allow multiple pressure-radius measurements and so provide self-consistent (same system) values for the surface or interfacial tension, in 2001 a new capillary-action-based technique, the tapered micropipette, was developed and applied to a series of clean surfaces and interfaces as well as soluble- and insoluble-surface active materials [6,9].

Figure $3 \mathrm{c}$ shows the schematic image of the tapered micropipette for a surface tension measurement. The tapered capillary is again set in a horizontal position. Following the Young-Laplace equation and geometry of the capillary, the Laplace pressure controlling the liquid flow shows the more simple relation (Young-Laplace equation),

$$
\Delta=\frac{2 \gamma}{R_{c}}
$$

where, again, $R_{c}$ is the radius of curvature of the interface inside the capillary. Using the tapered shape for the capillary, the factor of $\theta_{c}$ is cancelled out in the equation, as discussed below in association with Equation (6).

Therefore, the surface or interfacial tension value is obtained by knowing the applied pipette pressure $\Delta P$, required to keep the meniscus at a geometry of $R_{c}$, and does not require knowledge of the contact angle of the meniscus at the capillary surface. 


\subsection{Micropipette Manipulation Apparatus}

The interfacial tension measurement with the tapered micropipette is achieved by using a bright field microscope system with one or more micropipette micro-manipulators mounted on the microscope stage $[7,9]$. Other accessary equipment, such as a pressure transducer and camera are also required. Figure 4a shows a photographic image of the micropipette manipulation system with a tapered micropipette mounted in a microscope chamber filled with the test liquid (Figure $4 \mathrm{~b}$ ).

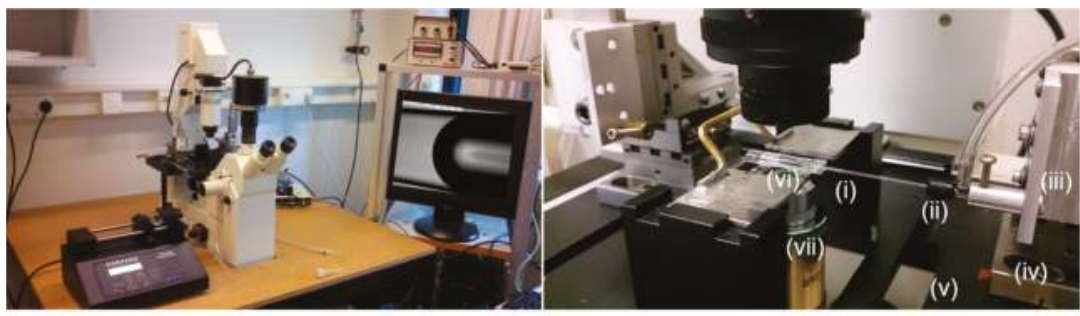

(a)

(b)

Figure 4. Our Signature Micropipette Manipulation Platform provides an ability to establish a well-defined interface (air-water, oil-water, with or without adsorbed material). (a) The system is comprised of: micropipette pressure control from micro-atm to milli-atm; a microchamber for the test solution; temperature control: 5 to $50{ }^{\circ} \mathrm{C}$; manipulators with fine positional control of pipette. Microscope images are recorded digitally for analysis. (b) Micropipette (i) is mounted via a chuck (ii) in a custom-built holder (iii), mounted on a stage micrometre (iv) bolted firmly to the microscope platform (v). The image of the micropipette in the microchamber (vi) is viewed via a $40 \times$ and $20 \times$ objective lens for equilibrium and dynamic surface tension measurement, respectively (vii). With permission from Elsevier [18].

Figure 4 a shows the overall microscopy system. The bright-field microscope with Köhler illumination (Zeiss) is used to observe the geometrical shape of the surface or interface inside a micropipette, as for example, the water-air surface displayed on the monitor in Figure $4 \mathrm{a}$. Figure $4 \mathrm{~b}$ shows an enlarged view around the glass cuvette sample-chamber $(2 \mathrm{~mm} \times 3 \mathrm{~mm} \times 10 \mathrm{~mm})$ with a micropipette inserted. Tapered micropipettes (taper angle $\theta_{p} \sim 3-4^{\circ}$ ) are custom-made de novo by using a pipette puller (Shutter instrument, Novato, CA, USA), and cut to the desired tip diameter $(\sim 5-10 \mu \mathrm{m})$ with a micro-forge (Narishige) [18]. To manipulate the tapered micropipette inside the chamber, the pipette is attached to a Newport 3D mechanical micromanipulator, which is firmly bolted to the microscopy stage. To monitor and record the geometrical shapes at the tip and the interface inside the tapered micropipette, a CCD-camera with 30 frames per second (DAGE-MIT, Michigan, IN, USA) is attached to the system. In-line pressure transducers (Validyne Engineering Corp., Northridge, CA, USA) measure the applied pipette pressure, in the plastic tubing that connects to the micropipette via an "L"-junction chuck. Precise pressure control by a syringe or manometer allows the system to be set to zero flow and hence zero applied pressure. Surfaces, interfaces and formed gas microbubbles or liquid microdroplets are monitored in real time and recorded as digitalised information on a computer by using a home-built LabVIEW program. The digitised image is analysed with ImageJ software provided by National Institute of Health [88].

\subsection{Gas-Liquid Interfaces}

The simplest measurement that can be made using the micropipette technique is to validate the well-established clean air-water surface tension using the tapered micropipette [9]. (This is actually an experiment we use to train new researchers on the micropipette manipulation system). Figure 5 shows a series of typical air-water surface images inside a micropipette corresponding to four different applied positive Laplace pressures [9]. 
decreasing applied pipette pressures allow meniscus to recede down tapered pipette
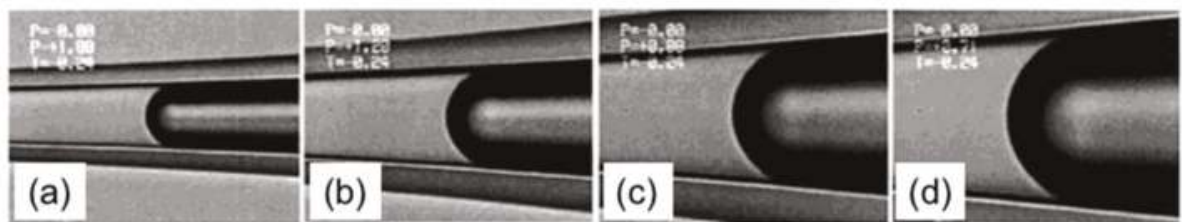

$\overline{10 \mu \mathrm{m}}$

Figure 5. Microscope images of an air-water surface inside a tapered micropipette. (a) The air-filled micropipette was inserted into the surfactant solution under relatively high positive applied pipette pressure $18.8 \mathrm{kPa}$. The meniscus came to equilibrium such that the interface meniscus (diameter $\sim 15 \mu \mathrm{m}$ ) was close to pipette tip. (b-d) The applied positive pressure was then decreased to, (b) $12 \mathrm{kPa}$, (c) $8.8 \mathrm{kPa}$, and (d) $7.1 \mathrm{kPa}$ respectively allowing the meniscus to recede down the tapered pipette to new equilibrium curvatures. In the experiment this is followed by a series of increasing pressures that move the meniscus back down the pipette and so is advancing. There was no hysteresis in the positions or contact angle for these receding or advancing contact angles. With permission from American Chemical Society [9].

As described previously (Section 2.1., Principles of Capillary Action and the Micropipette), water enters and flows continually into a horizontal micropipette by capillary action if we do not apply any positive, opposing pressure inside the pipette. In the experiment the micropipette was inserted into the surfactant solution under an initially relatively high pre-set positive applied pipette pressure of $18.8 \mathrm{kPa}$. As seen in Figure $5 \mathrm{a}$, the meniscus came to equilibrium with a diameter of $\sim 15 \mu \mathrm{m}$ such that it was close to pipette tip. A subsequent decrease of the applied pressure from $18.8 \mathrm{kPa}$ (Figure 5a) to $7.1 \mathrm{kPa}$ (Figure 5d), resulted in the movement of the air-water meniscus to larger and larger radii in the tapered pipette. This control of the meniscus position (and hence radius of curvature of the interface) inside the tapered micropipette, provides the surface tension measurement by simple application of the Laplace equation, Equation (5). Figure 6 shows how to calculate the radius of curvature $R_{c}$, from the air-water surface geometry, defined by the vertical distance $(Y)$ and the horizontal distance $(X)$ that correspond, respectively, to the chord between the ends of an arc spanning the cap and the height of the cap.

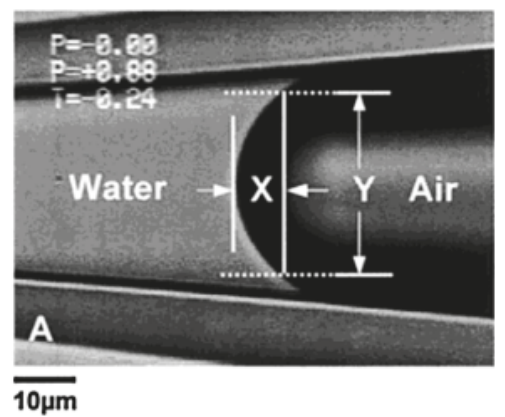

Figure 6. Calculation of radius of curvature $R_{\mathcal{C}}$ from the geometry of the surface image. With permission from American Chemical Society [9].

From the geometry of the interface inside the capillary, the following relation is obtained,

$$
R_{c}=\frac{\left(\frac{\gamma}{2}\right)^{2}+X^{2}}{2 X} \text {. }
$$


As mentioned above, the tapered micropipette manipulation technique solved the $\theta_{c}$ problem by using $R_{c}$ in the equation rather than using $R$, thus not requiring any factor of $\theta_{c}$ at the point where the three phases (air-water-glass) meet. In order to check this, we measured the air-water surface tension for two different contact angles. A glass pipette surface coating of 3-Cyanopropyltrichloro silane (CTPCS) produced a much higher contact angle of $54^{\circ}$ compared to that for a clean glass surface of $5^{\circ}$ [7]. Figure 7a (CTPCS-coated) and Figure $7 \mathrm{~b}$ (non-silane coated) show the images of the air-water surface with different contact angles at $\sim 1.4 \mathrm{kPa}$ applied pressure.
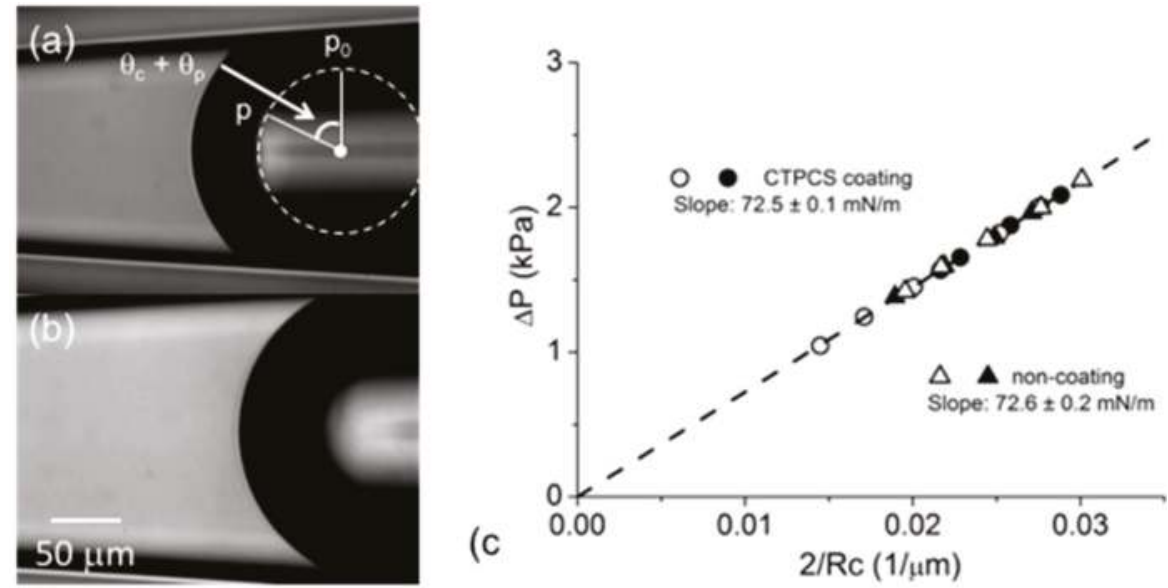

Figure 7. Contact angle change with and without silane coating at the glass surface of the micropipette at $20^{\circ} \mathrm{C}$ and corresponding surface tension plot. (a) 3-Cyanopropyltrichloro silane (CTPCS) coated micropipette, having a contact angle of $54^{\circ}$ and applied pressure of $\sim 1.4 \mathrm{kPa}$ inside the pipette. Using the fitting of curvature (dashed circle) at the diffraction pattern at the air surface area, the contact angle is calculated by considering the point " $\mathrm{p}$ " and " $\mathrm{p}_{0}$ " giving $54 \pm 7^{\circ}$. Note this circle is not at the interface but at the point at which the meniscus leaves the glass surface. Point " $\mathrm{p}$ " shows the grey and black pattern boundary spot on the circle, and $\mathrm{p}_{0}$ shows a crossing point of the circle and a vertical line crossing the circle centre. (b) Non-silane coated (just glass) micropipette having $\sim 1.4 \mathrm{kPa}$ applied pressure inside the pipette with the much smaller contact angle of $5^{\circ}$. (c) Equilibrium air-water surface tension measured by the micropipette manipulation method with increasing pressure (hollow symbols: interfacial area decreasing) and decreasing pressure (solid symbols: interfacial area increasing) comparing with and without CTPCS coating. The surface tension was calculated from the slope of a plot of applied pressure $\Delta P$ vs. the reciprocal radius of curvature $2 / R_{C}$ at each applied pressure. With permission from Elsevier [7].

From the fitting circle at the edge of the diffraction pattern (Figure $7 \mathrm{a}$, white dashed circle), the contact angle $\theta_{c}$ of the air-water surface against the hydrophobic CTPCS-coated micropipette glass surface was estimated to be $\theta_{c}=54 \pm 7^{\circ}$. This showed good agreement with other literature data of $56.3 \pm 2.2^{\circ}$ [89]. We therefore confirmed that the estimation of $\theta_{c}$ from the diffraction pattern was a reliable method for the air-water surface tension measurement. By comparison, the contact angle against the clean glass surface of $5^{\circ}$, shown in Figure $7 \mathrm{~b}$ is, as expected, much smaller than the CTPCS-coated micropipette. Satisfyingly, in Figure 7c, both measurements of $\Delta P$ vs. $2 / R_{c}$ plotted for a series of different applied $\Delta P$ showed the same linear fitting slope. Then, using Young-Laplace, Equation (5), the slope expressed the $\gamma$ values, and these were, $\gamma=72.5 \pm 0.1$ (CTPCS coating) and $72.6 \pm 0.2 \mathrm{mN} / \mathrm{m}$ (non-coating), at $20^{\circ} \mathrm{C}$. Thus, even though the two glass surfaces were of completely different hydrophobic or hydrophilic character, the measurement of the clean air-water surface tension was self-consistent in excellent and accurate agreement with reference data from the literature [90]. 
What these simple experiments show is that the radius of curvature $R_{c}$ is accurately determined from a segment of the interface and the contact angle is not required in order to measure the surface tension with the tapered micropipette system.

\subsection{Liquid-Liquid Interfaces}

We have also established the technique for making equilibrium interfacial tension measurements at oil-water interfaces [9]. By partially filling the pipette with oil and placing water in the microchamber, a curved interface can be formed in the micropipette, much like the one between air and water. Since oil is hydrophobic the curvature of the meniscus in contact with the glass pipette surface is again dependent on water-wetting of the hydrophilic glass. As with the simple air-water system, as shown in Figure 8, the application of positive pipette pressure moves the interface position down the pipette (Figure $8 \mathrm{a}, \mathrm{b}$ ) changing its radius. The application of negative pressures then allows it to recede back up the pipette for reversible measurement of advancing and receding positions.

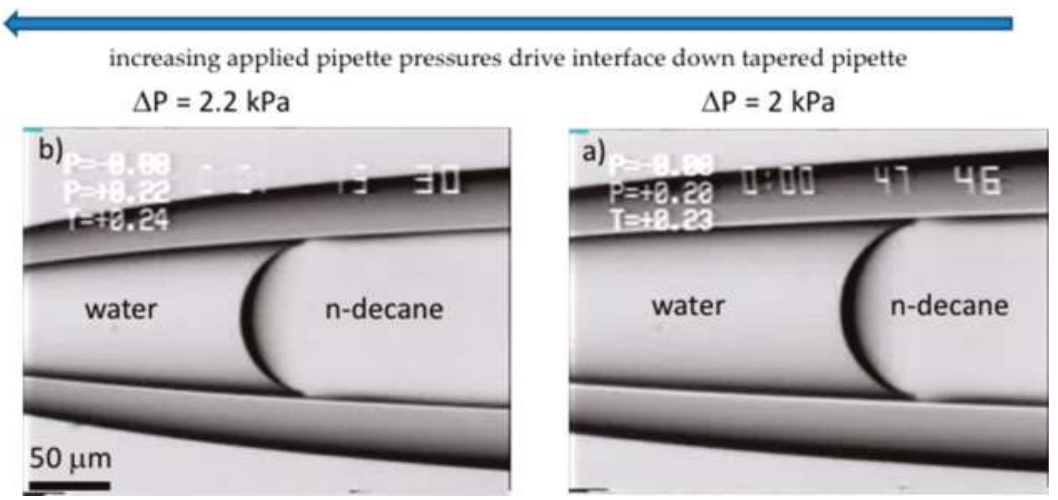

Figure 8. Equilibrium interfacial tension for the simple oil-water system. Shown are video-micrographs from an experiment where n-decane filled the micropipette and water was placed in the microchamber. (a) (right-hand micrograph) As in Figure 5, the (partially) oil-filled micropipette was inserted into the water under positive applied pipette pressure $\Delta P$ of $2 \mathrm{kPa}$ to give an interface meniscus in the pipette taper with a diameter $\sim 100 \mu \mathrm{m}$. The applied positive pressure was increased to (b) $2.2 \mathrm{kPa}$, and the oil-water interface was moved to a new position with a smaller diameter of $93 \mu \mathrm{m}$. This was repeated to obtain the advancing and receding meniscus positions and corresponding radii used to plot the data in Figure 9.

As before (Figures 5-7 and associated text), this allows the interfacial tension to be measured using the Laplace equation, Equation (5). From the best fitting slope to a plot in Figure 9 of applied pressure $\Delta P$ vs. $2 / R_{c}$ at each applied pressure, gives the interfacial tension for the decane-water interface as $51.2 \pm 0.4 \mathrm{mN} / \mathrm{m}$ [9]. Additionally shown on the plot is plot and surface tension for the decane-air interface as $23.6 \pm 0.5 \mathrm{mN} / \mathrm{m}$ [9]. These values were in good reasonable agreement with literature values [91]. In general, the oil-air interfacial tension has a lower value compared with the oil-water interfacial tension. For completion, and to summarise this section on gas-liquid and liquid-liquid systems, Table 1 shows the list of different alkane or alkanol interfacial and surface tensions against water $\gamma_{\mathrm{OW}}$ or air $\gamma_{\mathrm{OA}}$. The results from the tapered micropipette manipulation technique (bold letters) are combined with those from the literature [91,92]. Interestingly, for the completely non-polar alkanes the alkane-water surface tension $(\sim 51 \mathrm{mN} / \mathrm{m})$ is always larger than the alkane-air tension $(\sim 21 \mathrm{mN} / \mathrm{m})$. The alkanol-air tensions $(\sim 27 \mathrm{mN} / \mathrm{m})$ are similar to those of the alkane-air due to the presentation of their own alkane chains to air. Conversely, at the alkanol-water interface, the polar head group of the alkanol can orient towards the water (see later, Figure 11a) and provides a lower interfacial tension 
$(\sim 8 \mathrm{mN} / \mathrm{m})$ than to air. This result clearly suggests that alkanols have a monolayer-forming capacity and can act as surface active compounds, an effect that we examined for 1-Octanol [7] and present and discuss later as equilibrium and dynamic adsorption (see Section 4. Equilibrium and Dynamic Surface Tension: Adsorption of Soluble Surfactants). As mentioned at the beginning of this review, a pure hydrocarbon chain molecule, like decane cannot spread at the air-water surface because of a lack of molecule polarity [21].

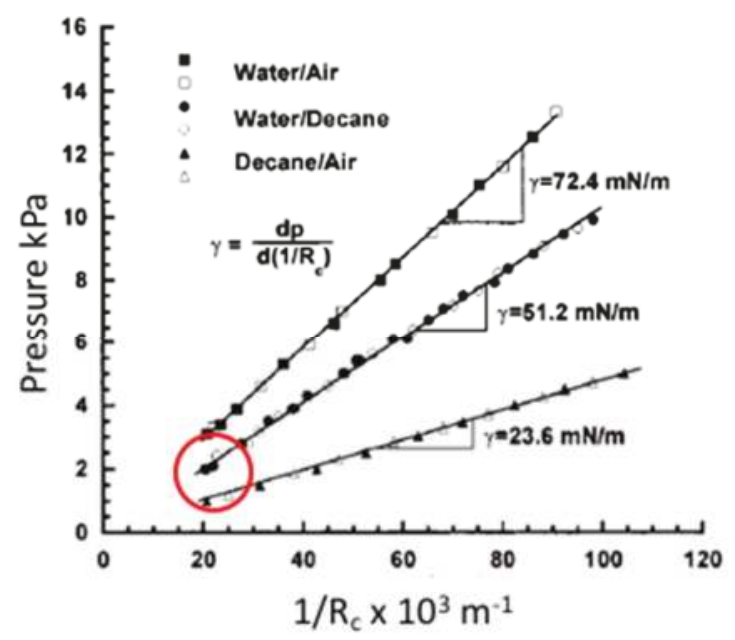

Figure 9. Plot of Equilibrium surface and interfacial tensions measured by the tapered micropipette manipulation technique. Data for applied micropipette pressure $\Delta P(\mathrm{kPa})$ is plotted in this particular paper [9] against the reciprocal of the radius of curvature $1 / R c\left(\times 10^{3} \mathrm{~m}^{-1}\right)$ for Water/Air, Water/Decane and Decane/Air. Meniscus position was measured for both increasing pressure (hollow symbols) and decreasing pressure (solid symbols). As before (Figure 7), the surface tension was calculated from the slope of the plot. With permission from Elsevier [9]. The circled data correspond to the menisci shown in Figure 8, i.e., at the lowest pressures and largest radii of curvature.

Table 1. Alkane and alkanol interfacial and surface tensions against water or air. Micropipette aspiration technique results are shown in bold letters. Data are at $20^{\circ} \mathrm{C}$, except, micropipette aspiration technique results $\left(23^{\circ} \mathrm{C}\right)$, row of Decane $\left(24.5^{\circ} \mathrm{C}\right)$, and both 1 -Hexanol interfacial tensions $\left(25^{\circ} \mathrm{C}\right)$. The literature data is obtained from $[91,92]$.

\begin{tabular}{|c|c|c|}
\hline Alkane & $\begin{array}{c}\text { Alkane-Water } \\
\text { row }(\mathrm{mN} / \mathrm{m})\end{array}$ & $\begin{array}{c}\text { Alkane-Air } \\
\gamma_{\mathrm{OA}}(\mathrm{mN} / \mathrm{m})\end{array}$ \\
\hline Hexane (C6) & 51.1 & 18.4 \\
\hline Octane (C8) & 50.8 & 21.6 \\
\hline Decane (C10) & $51.2(52.0)$ & $23.6(23.8)$ \\
\hline Alkanol & $\begin{array}{c}\text { Alkanol-Water } \\
\gamma_{\text {OW }}(\mathrm{mN} / \mathrm{m})\end{array}$ & $\begin{array}{l}\text { Alkanol-Air } \\
\gamma_{\mathrm{OA}}(\mathrm{mN} / \mathrm{m})\end{array}$ \\
\hline 1-Hexanol (C6) & 6.8 & 25.8 \\
\hline 1-Octanol (C8) & 8.52 & 27.5 \\
\hline 1-decanol (C10) & 8.97 & 28.9 \\
\hline
\end{tabular}

In order to finish this section on the development of the micropipette techniques, we present now our most recent Micropipette Interfacial Area-expansion Method (MIAM) that has allowed us to make Dynamic Surface Tension (DST) measurements. 


\subsection{More Advanced Techniques: Micropipette Interfacial Area-Expansion Method (MIAM)}

The initial tapered micropipette technique was developed and introduced by Lee et al. in 2001 [6,9]. It was used to measure equilibrium tensions of clean and surfactant-adsorbed surfaces and also to make dynamic surface tension measurements for the adsorption of phospholipids (as described later, see Section 5. Equilibrium and Dynamic Surface Tension: Adsorption of Insoluble Surfactants). The system was able to track dynamic surface tension changes for times on the order of $\sim 15 \mathrm{~s}$. It was successful in that it demonstrated the formation of lipid monolayers from adsorbing lipid vesicles in the aqueous phase, that came to equilibrium in times on the order of $2 \mathrm{~min}$. While successful for lipid adsorption, it clearly had limits for faster-transport-surfactants. In the dynamic process of lipid or surfactant adsorption to a clean surface or interface, there is a decrease in tension and so a meniscus-interface in the tapered micropipette would move to smaller radii for a constant applied pipette pressure. While we did manage to blow surfactant solution at a clean interface using a smaller delivery pipette inserted into the larger tapered pipette (see Figures 6 and 7 in Lee et al. [9]) and observe the rapid movement of the meniscus to correspondingly smaller radii, the limits of this early technique were simply due to not being able to move the micropipette inside the chamber fast enough to observe the meniscus position (and measure its radius). Therefore, for more advanced applications (see later in Section 6 Applications) and indeed for more accurate and sensitive fundamental studies in dynamic tension measurement for surfactants, we developed the Micropipette Interfacial Area-expansion Method (MIAM) [7].

In order to improve the time-lag, any need for micropipette movement was eliminated by fixing the micropipette a priori at a position that represented the expected meniscus diameter for a clean air-water surface (i.e., $\sim 72 \mathrm{mN} / \mathrm{m}$ tension). This surface was then ready for the initial adsorption and therefore setting a diameter for time zero [7,8]. Figure 10 shows schematic images of the Micropipette Interfacial Area-expansion Method as used in this technique and a description of the sequence of events.

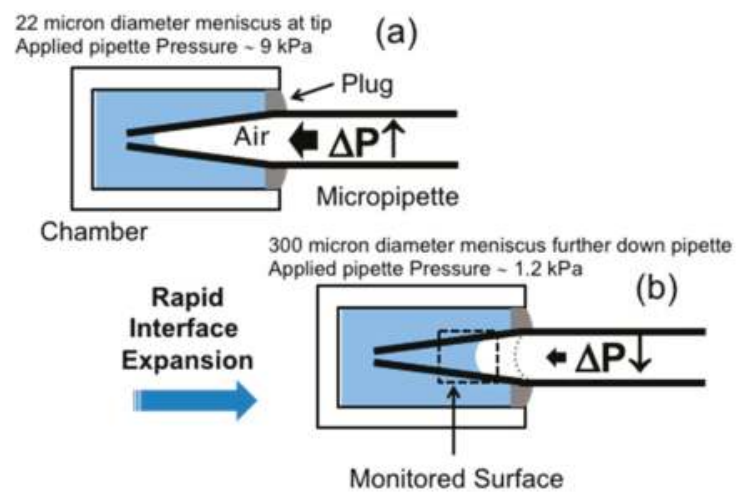

Figure 10. Schematic image of the Dynamic Surface Tension (DST) measurement using the Micropipette Interfacial Area-expansion Method. (a) The micropipette is inserted into the surfactant solution under high positive applied pipette pressure such that the new interface meniscus (diameter $\sim 22 \mu \mathrm{m}$ ) is close to the pipette tip. This is observed by initially positioning the micropipette tip in the field of view. To avoid convective flow inside the chamber, the microchamber is gently sealed with a plug of hexadecane after the pipette is inserted and the pipette moved to a new viewing position where a clean surface is expected to locate when a pre-set lower pipette pressure is applied. (b) Following a 15-fold decrease of the applied pipette pressure, $\Delta P$, (from the high value of $\sim 9 \mathrm{kPa}$, that held the interface close to the pipette tip, to $0.6 \mathrm{kPa}$ ), the surface area quickly expands to a new diameter of $\sim 300 \mu \mathrm{m}$ in 0.1 to $0.3 \mathrm{~s}$. The pipette pressure is rapidly fixed to a constant reset pressure of $1.2 \mathrm{kPa}$, and the surface meniscus moves to the observed region (dotted box). The movement of the surface meniscus is tracked as surfactant adsorbs to the water-air surface and reduces the tension to the new equilibrium. With permission from Elsevier [7]. 
The method follows a protocol of these three steps:

Step 1: Set the position of the micropipette inside the microchamber and apply a high positive pressure $(\sim 9 \mathrm{kPa})$ by using a syringe pump. This pressure minimises the air-liquid meniscus area and makes it almost coincident with the micropipette tip (radius $\sim 11 \mu \mathrm{m}$ ) (Figure 10a). The end of the microchamber is sealed with a small volume of hexadecane to avoid water evaporation and limit convective flow during the experiment.

Step 2: Quickly release the applied pressure from 9 to $0 \mathrm{kPa}$ in 0.1 to $0.3 \mathrm{~s}$. This achieves a 200-fold interfacial area expansion, i.e., from 700 to $140,000 \mu \mathrm{m}^{2}$, as shown in Figure 10b, and moves the meniscus to the position indicated by the grey-dashed meniscus in the pipette. This rapid surface area expansion creates an essentially clean air-water surface in the micropipette (i.e., with only $\sim 0.5 \%$ of the initial concentration of the surfactant that would have been adsorbed at the initial small interface).

Step 3: After the rapid expansion of the surface area, quickly apply a reset pressure $\sim 1.0 \mathrm{kPa}$, observe the position of the surface meniscus (dotted box) and proceed with the $R_{c}$ measurement of its gradual movement down the micropipette taper to smaller radii at this constant applied pipette pressure. That is, the maximum surface area becomes smaller when surface active agents adsorb at the relatively clean air-water surface (Figure 10b, monitored area). Thus, the dynamic change of the position of the meniscus in the tapered micropipette and hence its corresponding surface curvature is monitored and recorded in real time for later analysis.

This technique then was developed to capture the fastest times possible for surface and interfacial movements in response to surfactant adsorption. Of course, this process includes the diffusion of surfactants to (and from) the surface and so embedded in it, and obtainable from the results, is the diffusion coefficient of the adsorbing species. All this will be presented and shown later for both non-ionic and ionic test surfactants (Sections 4 and 5) and for an application studying lung surfactants (Section 6.3.). First, though, we consider the entities involved in the equilibrium and dynamic surfactancy that we have studied.

\section{Entities: Soluble and Insoluble Surfactants as Monolayers, Micelles, Vesicles and Emulsions}

By way of a more technical introduction to the systems we have studied, we now briefly describe the surfactant- and lipid-systems in which our micropipette manipulation experimentation has made some important contributions. Amphiphilic compounds are well-known surface-active agents at the air-water surface and also form association colloids. The schematic images in Figure 11 show typical behaviours for some common examples of these compounds at the air-water surface as soluble monolayers and in bulk solution as micelles, as well as insoluble monolayers and membrane vesicles of the relatively more-insoluble lipids. In our earlier micropipette work in 2001, we established the rudimentary methods for measuring equilibrium tensions of clean and surfactant-adsorbed surfaces [9] as well as the equilibrium and dynamic tensions due to adsorption of phospholipids [6]. In 2017 we have now improved on, and extended, these techniques for both equilibrium and shorter-time dynamic behaviour of the surface-active agents Octanol [7] and sodium dodecyl sulphate [8], and as described later in Section 6.3., for lung surfactant formulations [10].

Simple amphiphilic compounds can create monolayers at the air-water surface by orienting their monomers, i.e., hydrophilic polar head groups towards the water and hydrophobic chain(s) towards the air. Upon formation of a monolayer of these compounds, the air-water surface tension is decreased from $72 \mathrm{mN} / \mathrm{m}\left(\right.$ at $\left.20^{\circ} \mathrm{C}\right)$ to much lower values depending on the surface concentration $(\Gamma)$, and characteristics of these compounds, but can be on the order of $30 \mathrm{mN} / \mathrm{m}$. As depicted in Figure 11, amphiphilic compound-monolayers can be separated into two groups, i.e., soluble and insoluble monolayers [93]. When monomers of the monolayer have significant solubility in the aqueous phase and can exchange with excess monomers in the equilibrium bulk solution, it is called a soluble monolayer. These monomers rapidly come to equilibrium and maintain a relatively constant surface pressure due to rapid desorption or adsorption when compressed or expanded, respectively. If the monomers are relatively insoluble in water and remain at the surface rather than 
exchanging during any monolayer expansion or compression, it is called an insoluble monolayer. These different characteristics of monomers can also result in different aggregation behaviour in bulk aqueous media. For example, soluble surfactants tend to form micelles (Figure 11b) that can exchange with soluble monolayers, while lipids form bilayer-membrane vesicles (Figure 11c) that can form insoluble monolayers but are less likely to exhibit rapid exchange. In all cases, above the solubility limit of the monomers, they coexist with these self-assembled-aggregates in the bulk water. Interestingly, the surfactants themselves, e.g., octanol, even in the absence of a second oil phase, can form their own "oil droplets" as microscopic emulsions and as nanoparticles in aqueous suspension (as shown in Figure 11c). Next, we consider the physicochemical characteristics of each amphiphilic compound and how it influences the micropipette manipulation-measurements we have made.

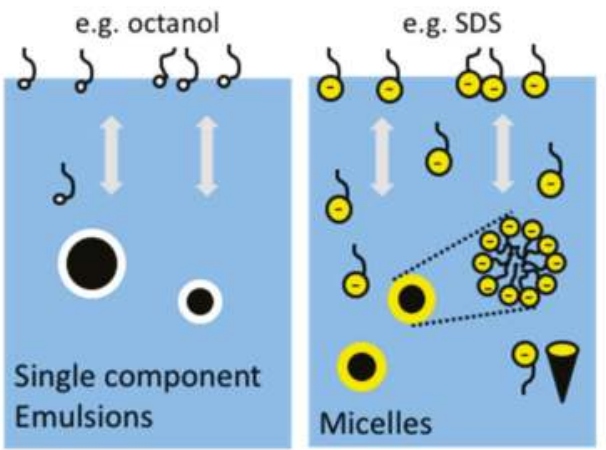

Soluble Monolayer

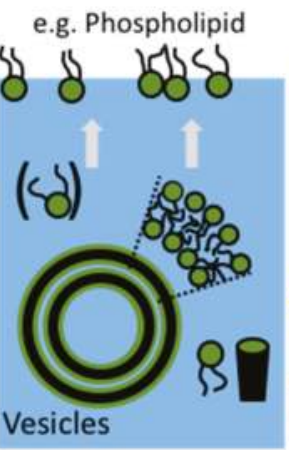

Insoluble Monolayer

(a)

(c)

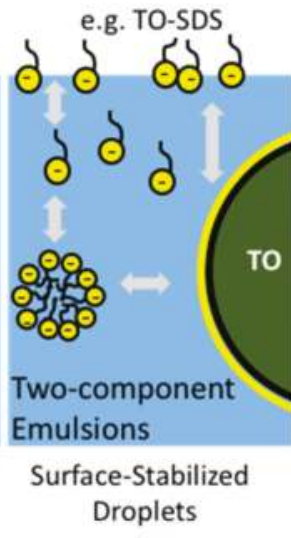

(d)

Figure 11. Surface active compounds at the air-water surface and in bulk aqueous phase. (a) Soluble Monolayer: Long-chain alcohols, such as Octanol, can spread at the air-water (blue background) surface to make a soluble monolayer (monomer can transfer between monolayer and bulk). The monolayer has a certain orientation, i.e., hydrophilic $\mathrm{OH}$-headgroup (white circle) towards the water and hydrophobic C8 alkyl chain (black curvy line) towards the air. Above its solubility limit in bulk aqueous solution, octanol can behave like pure hydrophobic oil, i.e., forming its own oil emulsion with octanol also adsorbed at this interface. (b) Soluble Monolayer: Detergents, such as the anionic sodium dodecyl sulphate (SDS), can spread at the air-water surface to form a soluble monolayer. The orientation is the same as the long-chain alcohol with the negatively charged hydrophilic polar group facing the aqueous phase, (yellow circle) and the hydrophobic C12 alkyl chain towards the air (black curvy line). Above its solubility limit in bulk, monomers form micelles. (c) Insoluble Monolayer: Lipids, such as phospholipids, can spread at the air-water surface to form an insoluble monolayer, again with headgroups in the water phase (green circles) and double acyl chains in air (double black curvy lines), with relatively little molecular lipid (bracketed) in solution (water solubility, $S_{w} \sim 10 \mathrm{nM}$ or less). In the bulk aqueous phase monomer lipids self-assemble into vesicles. (d) Surface-Stabilised-Droplets. Microor nano-emulsion droplets can be stabilised with, for example, the anionic detergent SDS.

\subsection{Long-Chain Alcohols form Soluble Monolayers and Emulsions}

In general, water-miscible, short-chain alcohols decrease the surface tension by forming a monolayer at the air-water surface. For example, by increasing the concentration of the water-miscible solvents methanol, ethanol, and propanol in an aqueous solution, there is a concomitant decrease in the air-water surface tension from 71 to $\sim 23 \mathrm{mN} / \mathrm{m}$ at $20^{\circ} \mathrm{C}$ [94]. In the case of the longer-chain, water-immiscible alcohols, such as 1-Octanol (see below, Section 4.1.) they can also decrease the air-water surface tension to $\sim 25-30 \mathrm{mN} / \mathrm{m}$ [7], by forming a soluble monolayer at the surface, and so not quite as much as for short-chain alcohols. A special case for longer-chain alcohols is their ability to 
form emulsion droplets (Figure 11a) and hence an immiscible interface. Interestingly, the long-chain alcohol molecules start aggregating into emulsion droplets before reaching the lowest surface tension. This behaviour correlates with the solubility of the carbon chain, i.e., the longer the carbon chain length, the lower its solubility in water [21] and the greater its tendency to form the emulsion droplet. As mentioned in the Introduction 1.1, non-polar, pure hydrocarbon chains, such as the n-alkane homologous series, cannot spread as monomolecular films at the air-water surface. Therefore, just the addition of one terminal-OH group converts the relatively insoluble and surface inactive octane to a more soluble and surface-active octanol. As described in more detail below, (Figures 12 and 13) our micropipette experiments have measured the rapid and dynamic adsorption of Octanol to otherwise clean air water interfaces and shown their rapid approach to equilibrium tensions [7]. These measurements provided a value for the diffusion coefficient of 1-Octanol, of $7.2 \pm 0.8 \times 10^{-6} \mathrm{~cm}^{2} / \mathrm{s}$. While highly related, but beyond the main scope of this review, once we obtained a microdroplet of octanol in water, the micropipette technique was further developed (along the lines as for gas bubble dissolution, see Section 6.1.1.) to measure the dissolution of octanol $[7,16]$ into water, as well as a series of organics and their mixtures [95]. Briefly, the dissolution of a $50 \mu \mathrm{m}$ diameter microdroplet of octanol into water takes a relatively long time, $3000 \mathrm{~s}$. This is because, as we can see according to the Epstein Plesset equation (see later Equation (8) [96]), the rate of loss of material, dm/dt, is a function of the product of the diffusion coefficient $\mathrm{D}$ (of octanol in water) and its water-saturation concentration $C_{s}$. In contrast to a dissolving gas bubble (Section 6.1.1.) that has a finite surface tension and hence a Laplace pressure, for a liquid microdroplet the interfacial tension and the Laplace pressure play virtually no role because of the incompressibility of the octanol. Thus, the very low solubility of octanol (and other organics) in water increases their microdroplet dissolution time. This experiment has thus allowed us to actually measure the diffusion coefficients for octanol to be $7.3 \pm 0.1 \times 10^{-6} \mathrm{~cm}^{2} / \mathrm{s}$, in excellent agreement with the result from the dynamic adsorption technique [7]. Dissolution is also proportional to $1 / R$ and so large droplets on the order of millimetres might appear to "never" dissolve. This is another key aspect of being able to directly observe and measure microdroplet behaviour with the micropipette technique and our single microparticle micropipette experiments, i.e., for micron-scale dimensions (e.g., $x=100 \mu \mathrm{m}$ ) and diffusion coefficients on the order of $5 \times 10^{-6} \mathrm{~cm}^{2} / \mathrm{s}$, Einstein's mean-square displacement diffusion equation [97], $x^{2}=2 D t$, puts the time $t=10 \mathrm{~s}$, whereas for 10 millimetre-sized particles, $t$ is on the order of 100,000 s ( 28 h). Thus, because of the scale of our experimentation, the results of micropipette manipulation experiments are observable in our laboratory time frame.

\subsection{Soluble Monolayer: Detergents form Micelles}

More common than alcohols are the well-known ionic surfactants such as sodium dodecyl sulphate (SDS). This anionic detergent can decrease the air-water surface tension from $71 \mathrm{mN} / \mathrm{m}$ to around $40 \mathrm{mN} / \mathrm{m}$ by forming a soluble monolayer at the air-water surface (see Section 4.1.) [8]. One of characteristics of this compound is that it readily forms micelles at its limit of monomer-solubility $(8.1 \mathrm{mM}$ ) in bulk water (Figure 11b)—its critical micelle concentration, CMC. (Note, this micelle is also in solution and has its own dynamic, solubilised interface). Compared with the self-assembly of long-chain alcohols into actual emulsion droplets, SDS forms micelles in water where 30 to 70 monomers are oriented per micelle depending on concentrations in excess of the CMC $[98,99]$. Interestingly, in $\mathrm{NaCl}$ solution, the aggregation number is increased to 130 (in $0.4 \mathrm{M} \mathrm{NaCl}$ ) because of electrostatic shielding of the sulphate negative charge so allowing a more close-packed arrangement of the sulphate headgroups. As described in more detail below (Figures 12 and 13), using similar techniques as developed for octanol, our micropipette experiments have also measured the rapid and dynamic adsorption of SDS to otherwise clean air-water interfaces and have shown their rapid approach to equilibrium tensions [8]. 


\subsection{Insoluble Monolayer: Lipids form Vesicles}

The most well-known insoluble monolayer is the phospholipid monolayer. Actually, the study of insoluble monolayers was first established by studying fatty acids [100]. Unlike the rapidly-exchanging, soluble monolayers, insoluble monolayers are characterised by complex 2-dimensional phase behaviour as surface pressure-molecular area (П-A) diagram, nicely reviewed by [101]. Such pressure vs. area curves characteristically include two-dimensional analogues of solid, liquid, and gaseous states along with intermediate phases such as the liquid-expanded and liquid-condensed regions appearing on occasion between the main states [102]. The addition of a small amount of a phospholipid to a clean air water interface results in a rapidly spread monolayer of the material and, again, a reduction in surface tension to values that can be $\sim 20-25 \mathrm{mN} / \mathrm{m}$ at the air-water surface (again measured by our micropipette technique [10], see later Section 6.3. Lung Surfactants) and as low as $1-2 \mathrm{mN} / \mathrm{m}$ at the oil-water interface (see Section 6.4. Nanoprecipitation) [17].

The unique measure of lipid self-association is characterised by the Critical Bilayer Concentration (CBC). In general, the "CBC" of such insoluble monolayer compounds is extremely low compared with soluble monolayer compounds such as SDS (CMC $8.1 \mathrm{mM})$. For example, the CBC for dipalmitoyl-PC (DPPC, two carbon chains of C16:0) is $0.46 \mathrm{nM}$ [103] — seven orders of magnitude smaller than the CMC of SDS! This is because of the greater hydrophobicity of DPPC's two long hydrocarbon chains compared to SDS's one. These lipids form vesicles as shown in Figure 11c, where simple rehydration of dried lipid can form multi-lamellar vesicles (MLV) having bilayer membranes in a sphere shell shape [104-106]. Vesicles can also be made as small (100 nm) unilamellar vesicles (SUVs) with special extrusion processing [107] or can be large enough as so called Giant Unilamellar Vesicles (GUVs) to make microscale measurements on them with the micropipette by gentle rehydration. The mechanochemistry, thermal and interactive properties of these GUVs also have been studied extensively by the micropipette technique as reviewed many times $[73,80,108]$, and also recently by Parra and Needham [109].

Interestingly, since air is relatively hydrophobic, a similar phenomenon occurs at the oil-water interface and a spread monolayer is formed. As established by Mingins [110], such materials can also be described by isotherms of surface pressure $(\pi)$ against area per molecule (A) reported for a homologous series of pure synthetic saturated 1,2-di-acyl glycerophosphocholines (lecithins) (C14 to $\mathrm{C2}$,) spread at n-heptane/aqueous electrolyte interfaces. Haydon also measured lipid spreading on monolayers and the surface-potential changes in lipid monolayers and the 'cut-off' in anaesthetic effects of N-alkanols [111]. Therefore, here we have a situation where a phospholipid monolayer is now a solvent for n-alkanols, and is actually an interesting test system for anaesthetic absorption into bilayer membranes and the proteins they contain in nerve cells. In another anomaly, as presented in the introduction, a single chain surfactant like Glycerol MonoOleate (GMO) can actually form bilayers if its molecular volume is enhanced with adsorbed alkanes, and so forms the so called "black lipid films" [39,112]. As described above (Section 1.3) these bilayer films [47] have been characterised in terms of free-energies of formation from solvent-containing to solvent free, $[1,38]$ and used extensively as model biological membranes, for anaesthetic adsorption and channel formation and activity $[113,114]$.

Thus, lipid monolayers have been studied extensively in their own right, and interestingly, when literally pushed to their limit, i.e., when the monolayer is compressed above a certain surface pressure limit, they collapse into vesicles rather than reverting to monomers $[115,116]$. They can even exist in equilibrium with vesicles that are placed in the aqueous sub-phase, as shown by MacDonald and Simon [117]. Here, the collapse pressure was similar to the internal pressure of lipid bilayers $(\sim 50 \mathrm{mN} / \mathrm{m})$, which corresponds to a true equilibrium for unstressed liposomes. Such monolayers of DMPC also underwent the same phase transitions as would a vesicle. Their data agreed well with Needham and Evan's data [76] on the same DMPC lipid as a GUV. Thus, the mechanical and thermodynamic properties of bilayers, particularly phase-transition parameters, have corresponded closely to those of monolayers with which they are in equilibrium. In the context of lipid bilayer vesicles, while we are mainly focused here on surface and interfacial tensions at interfaces, for completion, the 
micropipette technique has been also used extensively to apply well defined tensions and measure the mechanical $[73,78,80,83,108,109,118]$, thermal [72,76,77], molecular exchange and inter-bilayer interaction properties [73,119] of giant (20-30 $\mu$ m-diameter) phospholipid vesicles [120].

Returning to lipid monolayers, some of our first measurements were made while developing and using the micropipette technique for measuring the dynamic and equilibrium surface tensions of adsorbed phospholipid monolayers from aqueous suspensions of uni-lamellar lipid vesicles to clean air water interfaces [6,9] (see later Section 5, Adsorption of Insoluble Surfactants). These were followed 18 years later by using the improved micropipette methods to observe and measure the adsorption and complex multi-bilayer forming mechanism of lung surfactants, seen earlier by others [10,121-125].

Thus, it is generally accepted that phospholipids form bilayers and soluble surfactants form micelles. However, when the carbon chains of the phospholipids are made extremely short, these compounds can also make micelles rather than bilayer vesicles [100]. Such short chain phosphatidyl-cholines (PC) are structurally phospholipids, but their short fatty acyl chains of 6-8 C-atoms endow the molecule with detergent-like properties [126]. Since surfactants are usually single chain and the lipids are usually double chain, there is a relationship between the molecular volume, its headgroup area at the interface and the length of the hydrocarbon chain [93], and so micelle- or vesicle-formation can be described by a packing parameter model [127].

\subsection{Surfactants Can Adsorb at Oil-Water Interfaces and the Oil Can Swell Micelles}

Finally, just as they can adsorb at air-water surfaces, surfactants can adsorb at oil-water interfaces. Air is hydrophobic, and so just like at the air water interface, the hydrocarbon chains of a surfactant partition in the same way at oil-water interfaces. As shown by example, in Figure 11d, micro- or nano-emulsion droplets of Triolein can be stabilised with the anionic detergent SDS. For this situation, several equilibria are set up: SDS molecules in solution are in equilibrium with the monolayer at the air-water surface, with micelles if above the CMC, and with the oil-water interface of the emulsion. Interestingly, the micelle is also in equilibrium with the bulk phase of the emulsion, and can contain a number of oil molecules that are in equilibrium with the oil in the bulk phase. In unpublished studies (Needham, undergraduate projects) we hypothesized in 2000, that this represents just enough molecules to create bulk matter [128]. We found that, indeed, small alkanes (pentane to decane) would swell micelles of Tween 20 (hydrogen-saturated C12 monolaurate chain) showing a statistically significant increase in hydrodynamic radius within the first $48 \mathrm{~h}$ from the initial micelle radius of $4.42 \pm 0.08 \mathrm{~nm}$ to $6.5 \pm 0.09 \mathrm{~nm}$ for the pentane system, to $7.84 \pm 0.33 \mathrm{~nm}$ for the decane system. If the radius of the micelle is equivalent to the size of the Tween 20 molecule, and we assume that this represents the thickness dimension of the Tween 80 monolayer on the swollen micelle, then the alkane-core-radius for decane is simply $7.84 \mathrm{~nm}-4.42 \mathrm{~nm}=3.42 \mathrm{~nm}$, which gives a core-volume of decane of $\sim 168 \mathrm{~nm}^{3}$. Since decane has a molar volume of $195 \mathrm{~cm}^{3} / \mathrm{mol}$, and so volume per molecule of $0.32 \mathrm{~nm}^{3} /$ molecule, the micelle core would contain 525 molecules. Here the molecules in the swollen micelle are expected to be in equilibrium with, and so at the same chemical potential as, the bulk emulsion. Is this how many molecules of decane it takes to create bulk matter? On-going experiments in our lab are now exploring this further.

As is often said by our friend and collaborator Evan Evans, there are only two things we need to know in characterising any system scientifically, "where it is going" (equilibrium thermodynamics) and "how long it takes to get there" (kinetics or dynamics). Therefore, following this maxim, next, we give examples of using these techniques for equilibrium and dynamic surface tension measurements on soluble surface active materials (Octanol and SDS) and insoluble phospholipids (the Phosphatidylcholines).

\section{Equilibrium and Dynamic Surface Tension: Adsorption of Soluble Surfactants}

In 2017, we presented new measurements of the equilibrium and dynamic surface tensions of soluble monolayers of 1-Octanol and SDS at the air-water surface using the tapered micropipette 
manipulation technique $[7,8]$. Since SDS is anionic, we would expect shielding of the electrostatic interactions between molecules at the surface for SDS in $\mathrm{NaCl}$, reflected in a lower CMC. Experimental conditions therefore included milli-pure water and high ionic strength $\mathrm{NaCl}$ solutions in order to evaluate the effect of charge of the SDS molecule on adsorption rates and monolayer formation. It was these measurements that necessitated the development of the more advanced Micropipette Interfacial Area-expansion Method (MIAM) (see previous Section 2.5.) [7,8].

\subsection{Equilibrium Surface Tension for Adsorption of Soluble Surfactants: Octanol and Sodium Dodecyl Sulfate}

As mentioned above (Figure 11a) [129], the long-chain alcohol, 1-Octanol has a limiting solubility of $C_{S}=3.53 \mathrm{mM}$ in aqueous media. Above this solubility limit concentration, its molecules can self-assemble as oil emulsions. Therefore surface tension measurements were done at much lower solution concentrations than the $C_{S}$ of 1-Octanol i.e., 0, 0.3, 0.5, 1.0 and $2.0 \mathrm{mM}$ [7]. Figure 12a shows plots of the applied micropipette pressure $\Delta \mathrm{P}$ vs. the reciprocal radius of curvature as $2 / \underline{R_{c}}$ for the air-water meniscus in the pipette at each applied pressure for each 1-Octanol solution concentration.

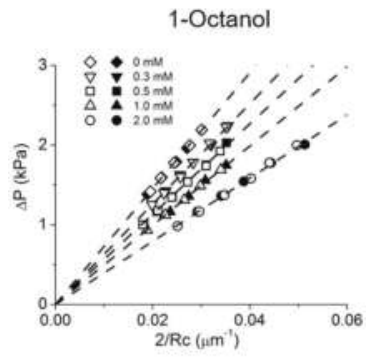

(a)

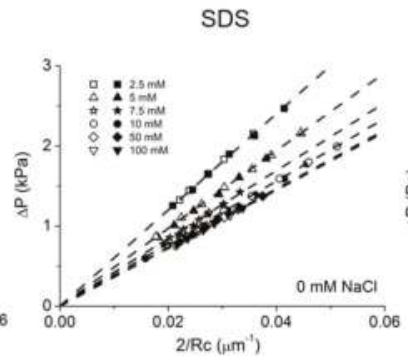

(b)

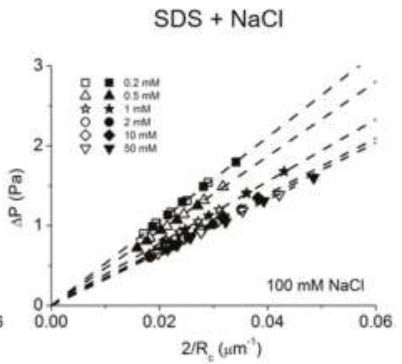

(c)

Figure 12. Equilibrium surface tension measurements for soluble surfactants. Pipette pressure vs. $2 / R_{c}$ plots for test surfactants Octanol and SDS to give equilibrium surface tension of soluble monolayers at each of the test concentrations in aqueous solution for (a) 1-Octanol, (b) SDS and (c) SDS with $100 \mathrm{mM}$ $\mathrm{NaCl}$. Measurements were made for both increasing and decreasing applied pressure at $20^{\circ} \mathrm{C}$. Hollow and solid symbols show the direction of applied pressure-hollow symbols: pressure increasing, interfacial area decreasing; and solid symbols: pressure decreasing, interfacial area increasing. Each surface tension was calculated from fitting slope as mentioned in the text corresponding to the plots in Figure 7c. Reproduced from Kinoshita et al. [7,8], with permission from Elsevier.

The equilibrium surface tension was calculated from the best fitting linear slope of the plots at each concentration. As we saw for the clean air-water surface tension in Figure 7c, the linear slope at each concentration did not show any significant difference between advancing, and so shrinking the area of the meniscus (hollow symbols) and receding, and so expanding the area of the meniscus (solid symbols) with applied pressure control. This clearly demonstrates that the transfer of octanol monomers between the monolayer and in solution (Figure 11a) is rapidly reversible showing slow smoothly-performed area change at the air-water surface under applied pressure control. As shown in Figure 11b, SDS can make micelles above its CMC of $8 \mathrm{mM}$ in water or $1.4 \mathrm{mM}$ in $100 \mathrm{mM} \mathrm{NaCl}$. Thus, SDS measurements were made in Milli-Q-purified water and in $100 \mathrm{mM} \mathrm{NaCl}$ (Figure 12b,c) and the obtained radius of curvature values at each pressure were again plotted in the $2 / R_{c}$ vs. $\Delta \mathrm{P}$ plot at each SDS concentration and found to be linear.

As expected and shown in Figure 13a the octanol data showed a linear relationship between surface tension and aqueous concentration of Octanol (on a logarithmic scale), consistent with previously published data measured by Du Noüy ring and Pulsating Bubble Method (PBS) methods $[130,131]$. As also expected for SDS, below its CMC the plots showed a similar linear behaviour between surface tension and log concentration and then levelled out as the CMC was approached and 
exceeded. For SDS, in Figure 13b, the effect of charge shielding in the presence of $\mathrm{NaCl}$ shifted the curve to a lower concentration range consistent with its lower CMC. In fact, the change in slope for surface tension vs. concentration, is actually used to define the CMC and gives CMC values of $8.1 \mathrm{mM}$ for SDS in pure water and $1.4 \mathrm{mM}$ for SDS in $100 \mathrm{mM} \mathrm{NaCl}$ (Figure 13b, dashed lines).

1-Octanol

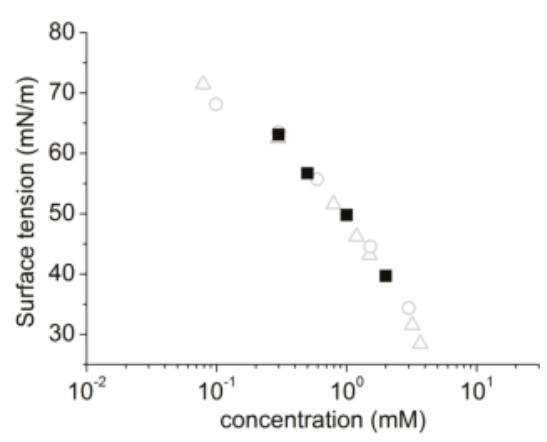

(a)

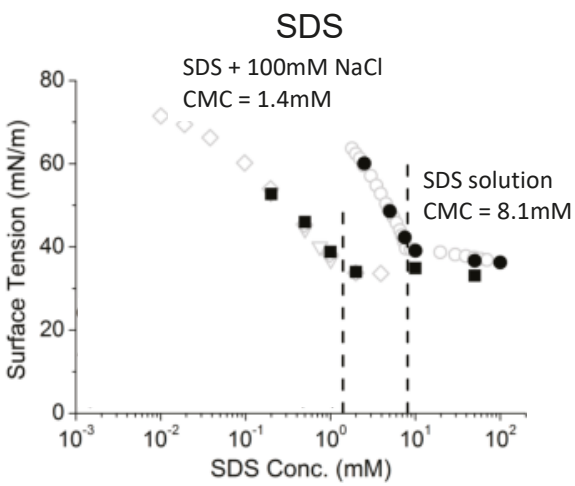

(b)

Figure 13. Equilibrium surface tension vs. soluble surface active compound concentrations measured by the tapered micropipette manipulation technique at $20^{\circ} \mathrm{C}$. (a) 1-Octanol equilibrium surface tension made by the micropipette technique (solid squares). The obtained data was compared to literature values, which were measured by Du Noüy's ring (open triangles) and Pulsating Bubble Method (PBS) methods (open circles) [130,131]. (b) SDS equilibrium surface tension in the absence (solid circles) and presence (solid squares) of $100 \mathrm{mM} \mathrm{NaCl}$ again made by the micropipette technique [7,8]. Literature results from the Wilhelmy plate method (open circles), drop volume (open diamonds) and maximum bubble method (inverted triangles) are also added for comparison [25,132,133]. The dashed lines show the critical micelle concentration $(\mathrm{CMC})$ values, $8.1 \mathrm{mM}$ (pure water) and $1.4 \mathrm{mM}(100 \mathrm{mM}$ $\mathrm{NaCl})$, which agree with literature $[9,25,98]$. Reproduced from Kinoshita et al. $[7,8]$, with permission from Elsevier.

Thus, Octanol shows a monotonic decrease in surface tension with increasing log of octanol concentration up to its solubility limit. For SDS, when micelles form they compete with the monolayer and the surface adsorption is limited and actually produces a change in slope for surface tension vs. concentration. Above the CMC, SDS monomers still continue to adsorb at the air-water surface as shown by the slightly decreasing surface tension. As a prelude to making dynamic tension measurements, these systems allowed us to validate that the tapered micropipette manipulation technique was capable of making accurate and reproducible measurements of soluble monolayer equilibrium surface tensions $[7,8]$.

\subsection{Dynamic Surface Tension for Adsorption of Soluble Surfactants}

One of the biggest challenges of interfacial tension measurements is how to measure a precise dynamic surface tension change in such short times that are associated with molecular diffusion to the surface [134]. Until now, only a few techniques have been successful and achieved reliable data that could be analysed in terms of theoretical models. For technical reasons, the majority of interfacial tension measurement techniques were not fast and/or sensitive enough to detect dynamic surface tension changes during, for example, initial surfactant adsorption to a clean interface and de novo monolayer formation. In order to bring a new technique to the field and see how far we could take this micropipette technique, in 2017 we developed and the tapered pipette methodology Micropipette Interfacial Area-expansion Method (MIAM) [7,8]. Table 2 shows the short time adsorption limits for a 
given interfacial area (technique specific) and the time range that is possible for each dynamic surface tension measurement as reviewed by Eastoe [135] and now including our new MIAM technique. Currently, the Maximum Bubble Pressure Method (MBPM) provides the fastest time scale for the measurement of dynamic surface tension-less than milliseconds [136-138].

Table 2. Dynamic surface tension measurement techniques and their possible time-ranges for surfactant adsorption and area characteristics (adapted from Eastoe [135] and now including our new Micropipette Interfacial Area-expansion Method (MIAM) technique).

\begin{tabular}{|c|c|c|c|c|}
\hline Techniques & $\begin{array}{c}\text { Short-Time } \\
\text { Adsorption (s) }\end{array}$ & $\begin{array}{l}\text { Interfacial Area } \\
\left(\mu \mathrm{m}^{2}\right)\end{array}$ & Functions and Drawbacks & Ref. \\
\hline MIAM & $<1$ & $10^{3}-10^{5}$ & $\begin{array}{l}\text { Interfacial area expansion } \\
\text { 150-200 times } \\
\text { No requirement of contact angle } \\
\text { information } \\
\text { Leakage for high viscous and } \\
\text { sticky materials for glass surface }\end{array}$ & [6-9] \\
\hline PBT & $10^{-1}$ & $10^{7}$ & $\begin{array}{l}\text { Reliable data in the middle time } \\
\text { range }(0.1 \mathrm{~s} \text { to mins) } \\
\text { Limit of long-time adsorption } \\
\text { (bubble detachment) } \\
\text { Bubble expansion rate } \\
\text { increasing cause error }\end{array}$ & {$[139,140]$} \\
\hline Growing-drop & $10^{-2}$ & $10^{7}$ & $\begin{array}{l}\text { Wide range (milliseconds to } \\
\text { more than hours) dynamic } \\
\text { surface tension measurement } \\
\text { Need to keep constant flow, } \\
\text { material loss from tip climbing }\end{array}$ & {$[141,142]$} \\
\hline Oscillating Jet & $10^{-3}-10^{-2}$ & $10^{5}$ & $\begin{array}{l}\text { Short time adsorption } \\
\text { measurement } \\
\text { Not suitable for the long-time } \\
\text { adsorption range of ( }>10 \mathrm{~s}) \text {, no } \\
\text { equilibrium tension }\end{array}$ & {$[87,143,144]$} \\
\hline MBPM & $<10^{-3}$ & $10^{5}$ & $\begin{array}{l}\text { Wide range, short-time and } \\
\text { long-time, adsorption } \\
\text { measurement } \\
\text { Leakage of material, } \\
\text { requirement of hydrophobic } \\
\text { treatment inner wall and } \\
\text { hydrophilic tip }\end{array}$ & {$[133,136,145]$} \\
\hline Langmuir-Wilhelmy & $>20$ & $\sim 10^{2} \mathrm{~cm}^{2}-\mathrm{m}^{2}$ & $\begin{array}{l}\text { Easily assembles with } \\
\text { microscopy and spectroscopy } \\
\text { methods } \\
\text { Not suitable for fast area } \\
\text { exchange because of leakage of } \\
\text { material } \\
\text { Contact angle requirement }\end{array}$ & {$[146,147]$} \\
\hline CBS & $\sim 10^{-1}$ & $10^{7}$ & $\begin{array}{l}\text { Leakage proof, mimic alveolar } \\
\text { environment } \\
\text { Limited interfacial area } \\
\text { expansion (two times) range for } \\
\text { adsorption dynamic }\end{array}$ & {$[148,149]$} \\
\hline PBS & 1 & $10^{6}$ & $\begin{array}{l}\text { Mimic breath control, easy to } \\
\text { operate } \\
\text { Material leakage, lacks } \\
\text { operational flexibility }\end{array}$ & {$[122,150]$} \\
\hline
\end{tabular}

MIAM: Micropipette Interfacial Area-expansion Method; PBT: Pendant Bubble Tensiometer; MBPM: Maximum Bubble Pressure Method; CBS: Captive Bubble Surfactometer; PBS: Pulsating Bubble Surfactometer. 
The other methods, Oscillating jet [143,144], Growing-drop [141,142], Pendant bubble tensiometry (PBT) [139] achieve times down to $10^{-3}-10^{-1} \mathrm{~s}$. The Pulsating Bubble Surfactometer is limited to times $\sim 1 \mathrm{~s}$, and the Langmuir Trough/Wilhelmy plate is best used for equilibrium tensions, and only has dynamic times on the order of $>20 \mathrm{~s}$ because of its large interfacial area $\geq 10^{2} \mathrm{~cm}^{2}$. In comparison, our new Micropipette Interfacial Area-expansion Method technique can make sub-second measurements, but still, there is a three-orders-of-magnitude range difference between the best MBPM and the MIAM $[7,8]$. However, the slightly slower measurement time for dynamic surface tension for MIAM does not result in a lack of sensitivity. In fact, sensitivity is the other important factor for dynamic adsorption measurements. Since dynamic surface tension measurements monitor the adsorption of the surface active molecular species at the interfacial area, our smaller (microscale) surface and interfacial areas do show higher sensitivity of the dynamic surface tension change at these short times. Compared with the other methods, MIAM, has a meniscus-area in the micropipette of $10^{3}-10^{5} \mu \mathrm{m}^{2}$, $\left(10^{-9}-10^{-7} \mathrm{~m}^{2}\right)$, which is two to four orders of magnitude smaller area than the other measurement methods (Table 2). Therefore, we can see that MIAM has the highest sensitivity on the list for dynamic surface tension measurements and, consistent with the scope of this special issue, we are making tension measurements at microscales.

\subsection{Practical Limits of Theoretical Analysis}

For theoretical analysis of the first events in the adsorption of surfactants to a clean surface or interface, in 1946 Ward and Tordai introduced a fairly complex model [151]. It was subsequently developed as asymptotic solutions by Fainerman et al. [152]. A numerical solution by Li et al. [153] describes how the adsorption kinetics of a non-ionic soluble surfactant is governed by a two-step process: (1) the diffusion of molecules from the bulk solution to the subsurface (i.e., the layer immediately below the surface layer, at a thickness of only a few molecular diameters); (2) the molecular diffusion and adsorption from the subsurface layer to the interface $[135,154]$. It was this model that we used for linearly fitting our experimental dynamic surface tension data for adsorption [7]. The main result was that we could calculate the diffusion coefficient for octanol in this process. Then, using a second micropipette technique, we carried out a droplet dissolution experiment and provide a corroborative measure of octanol diffusion [7]. Therefore, here two micropipette techniques were combined to measure and validate fundamental properties that govern the dynamics of adsorption to surfaces, change of surface tension, and also droplet dissolution.

For practical purposes, it is now recognised that the Ward Tordai short-time approximation method is valid only over a very specific ranges of time intervals or surface tensions. There is however a "long time" analysis (from sub-second to minutes (or hours)) that can give dynamic data. Addressing these issues, MIAM was developed to take advantage of the highest sensitivity for long-time adsorption measurements [7,8]. As described above (Section 2.5. and Figure 10), MIAM is a new tool for the "long-time" adsorption measurements, (that are nevertheless still relatively short at $0.5-1 \mathrm{~s}$ ) and was used for measuring both equilibrium and dynamic surface tensions for water-soluble surfactants and (later) water-insoluble lipids.

\subsection{Dynamic Surface Tension: 1-Octanol and SDS Adsorption}

\subsubsection{1-Octanol}

MIAM was used to measure the dynamic surface tension associated with the formation of a 1-Octanol monolayer at the air-water surface [7]. Taking the area changes first, Figure 14a shows the measured area of the small initial air-water surface positioned close to the micropipette tip at a few seconds before time zero (under high $9 \mathrm{kPa}$ applied pipette pressure). This is followed by the rapid area expansion due to the rapid drop in pipette pressure (to $0 \mathrm{kPa}$ ), immediately followed by the re-set pressure of $1 \mathrm{kPa}$ (see Figure 10 and Section 2.5 for the protocol). 


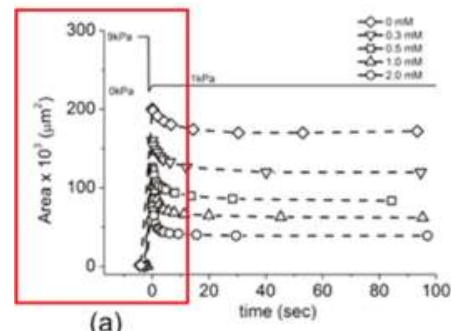

(a)

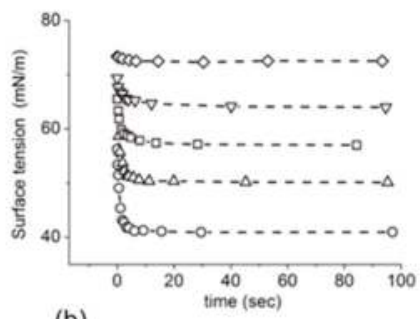

(b)

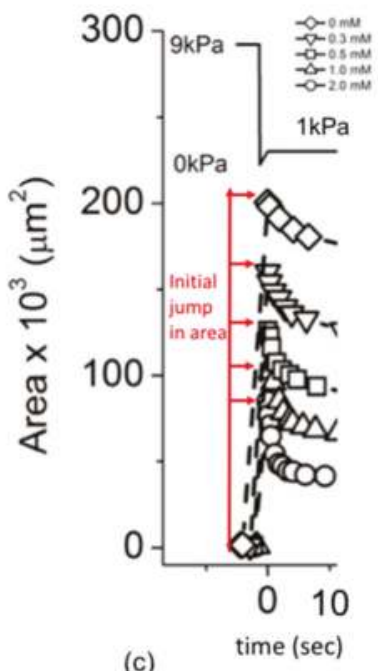

(c)

Figure 14. Dynamic surface area and corresponding surface tension of 1-Octanol aqueous solutions at various concentrations measured by the MIAM at $20^{\circ} \mathrm{C}$. (a) Meniscus surface-area change vs. time; also shown are the step changes in pipette pressure in sequence, 9, 0 and $1 \mathrm{kPa}$, and (b) derived dynamic surface tension plots also as a function of time. (c) Expanded view of the first $10 \mathrm{~s}$ for the area vs. time plot, showing more clearly how the meniscus area jumps to smaller and smaller distances, and falls much more precipitously with increasing octanol concentration. With permission from Elsevier [7].

For zero octanol concentration, (i.e., pure water), there is a rapid increase in meniscus area from the $1000 \mu \mathrm{m}^{2}$ at the micropipette tip as it shoots back down the micropipette when the holding high pressure $(9 \mathrm{kPa})$ is taken off to $200,000 \mu \mathrm{m}^{2}$ further down the taper. As mentioned previously in the protocol, this is a 200-fold area expansion that creates an essentially clean air-water surface in the micropipette. This technique then effectively dilutes any initial concentration of the surfactant adsorbed material at the small surface to $\sim 0.5 \%$ and provides essentially a clean surface for subsequent adsorption. Upon application of the new reset pressure of $1 \mathrm{kPa}$, the meniscus moves to a new position down the pipette, and so there is small decrease in the meniscus area that stabilises within $\sim 5 \mathrm{~s}$. The same measurement was then made for four different 1-Octanol concentrations, 0.3, 0.5, 1.0 and $2.0 \mathrm{mM}$. Now, in Figure 14a, and expanded in Figure 14c, we see how, with increasing 1-Octanol concentration, the initial jump in area expansion became smaller. As the red arrows indicate, for $0.3 \mathrm{mM}$

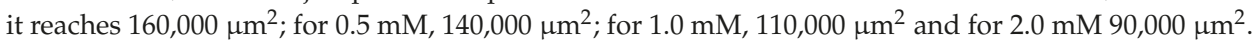
This was because of a faster and greater adsorption of 1-Octanol to the surface at higher 1-Octanol concentrations in the short-time of the pressure change. Then, the meniscus area falls much more precipitously with increasing octanol concentration.

Since this area reduction following the initial rapid expansion was carried out at a constant applied pipette pressure, these meniscus radii were readily converted to surface tensions by applying the Laplace equation to the measured meniscus radii. Analysis of this dynamic area change in terms of a surface tension change is thus shown in Figure $14 \mathrm{~b}$ for each concentration over the same time period. The pure water control ( $0 \mathrm{mM}$, diamonds) showed a slight apparent decrease in surface tension, which would not be expected to occur for a clean air water surface. In the paper we interpreted this as due to a transient heating of the air in the tube connecting the micropipette to the syringe pump, causing slight friction from a rapid plunging of the piston that was nevertheless self-correcting. Then, for the octanol solutions, Figure 14b shows that once the maximum area was attained for this relatively clean surface, the surface tension quickly dropped in a few seconds from $\sim 72 \mathrm{mN} / \mathrm{m}$ (pure water surface 
tension against air, Figure 7c) to succeedingly lower values depending on the degree of 1-Octanol adsorption. It then slowly decreased toward the minimum surface tension, i.e., the equilibrium surface tension, for each concentration. These data then show that octanol rapidly adsorbs to a clean interface and can produce equilibrium tensions within 2-3 s. The data were then analysed by the Ward-Tordai model (as described later in Section 4.5) to obtain the diffusion coefficient for octanol in water.

\subsubsection{Sodium Dodecyl Sulfate}

Similarly, dynamic adsorption of SDS at the air-water surface was also investigated by using the same MIAM technique [8]. As mentioned earlier (Section 3, Figure 11b), SDS is in the form of micelles at and above its $\mathrm{CMC}(=8.1 \mathrm{mM}$ in water, and $1.4 \mathrm{mM}$ in $100 \mathrm{mM} \mathrm{NaCl})$. Therefore, a series of dynamic surface tension with different SDS concentrations, 2.5, 5, 7.5, 10, 50 and $100 \mathrm{mM}$, in water bracketed the CMC. As above described for octanol, based on the area changes for the meniscus, in Figure 15a, where solutions were made up in milli-pure water, we obtained the dynamic surface tension change versus time for each SDS concentration. Figure $15 b$ shows the dynamic surface tension for SDS concentrations of 0.2 to $50 \mathrm{mM}$ made up in $100 \mathrm{mM} \mathrm{NaCl}$.

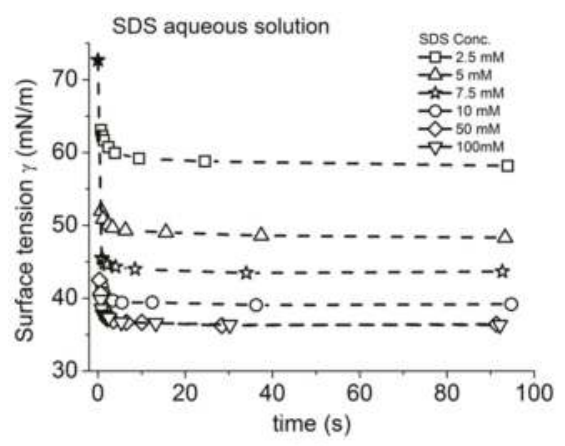

(a)

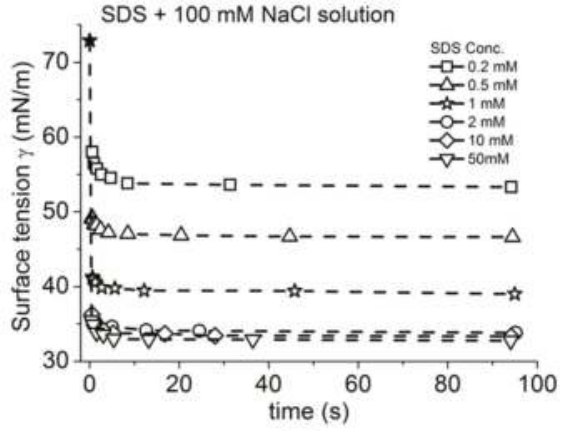

(b)

Figure 15. Dynamic surface tension of SDS in aqueous solution and in $100 \mathrm{mM} \mathrm{NaCl}$ solution measured by the MIAM at $20^{\circ} \mathrm{C}$. (a) SDS adsorption in aqueous solutions for SDS concentrations from 2.5 to $100 \mathrm{mM}$ made up in milli-pure water. (b) SDS adsorption in the presence of $100 \mathrm{mM} \mathrm{NaCl}$ for SDS concentrations from 0.2 to $50 \mathrm{mM}$. The solid star symbol $(\star)$ shows the initial clean water or $100 \mathrm{mM}$ $\mathrm{NaCl}$ surface tensions $\gamma_{0}$, at time $0 \mathrm{~s}$, obtained from independent measurements. With permission from Elsevier [8].

Just like for 1-Octanol, and as shown in Figure 15a, upon rapid expansion of the initial surface, the dynamic surface tension quickly dropped in a few seconds and slowly approached the equilibrium surface tension for each SDS concentration. Again, this was because of increasing SDS adsorption at the air-water surface toward the equilibrium surface concentration. Initial area jumps were again corelated and the surface tension became measurably less with each increase in SDS concentration. When carried out in $\mathrm{NaCl}$ solution, MIAM could also detect the effect of ionic strength. Figure 15b shows the dynamic surface tension change of each SDS concentration mad up in $100 \mathrm{mM} \mathrm{NaCl}$ versus time, again decreased in the first few seconds toward the equilibrium. The equilibrium values agreed with the known lowering of the CMC from 8.1 to $1.4 \mathrm{mM}$ by adding $\mathrm{NaCl}$ in agreement with Figure 13 . The Ward-Tordai analysis then allowed us to estimate the diffusion coefficients of these amphiphilic molecules $[7,8]$.

\subsection{Ward-Tordai Long-Time Adsorption Analyses for 1-Octanol and SDS Adsorption}

As mentioned above (Section 2.5.), the MIAM is designed to measure the surface tension in the relatively "long-time" adsorption regime, albeit with high sensitivity. Therefore, the obtained 
dynamic surface tension data (Figures 14 and 15) was analysed by using the long-time adsorption approximation of the Ward-Tordai model [7], combined with an adsorption equation to give the surface excess concentration $\Gamma_{\text {eq }}$. Thus, the dynamic surface tension change $\gamma(t)$ is given by this Ward-Tordai long-time adsorption approximation as,

$$
\gamma(t)=\gamma_{\mathrm{eq}}+\frac{n R T \Gamma_{\mathrm{eq}}^{2}}{c_{0}} \sqrt{\frac{\pi}{4 D t}},
$$

where, $n$ is a constant, the value of which depends on the amphiphilic compound and salt concentration (if the compound has a charge). In the case of 1-Octanol (non-ionic compound) or univalent ionic surfactant with excess electrolyte with common counterion, $n=1$; for a univalent ionic compound in the absence of supporting electrolyte, (e.g., in water) it takes on the maximum value, i.e., $n=2$. $R$ is the gas constant, $T$ is temperature, $C_{0}$ is the bulk concentration. Here, the surface tension is proportional to $t^{-1 / 2}$ by taking a limit of subsurface concentration to bulk concentration in this long-time approximation. Following Equation (7), the surface tension data were replotted as in Figure 16 for 1 -Octanol as $\gamma(t)$ vs. $t^{-1 / 2}$.

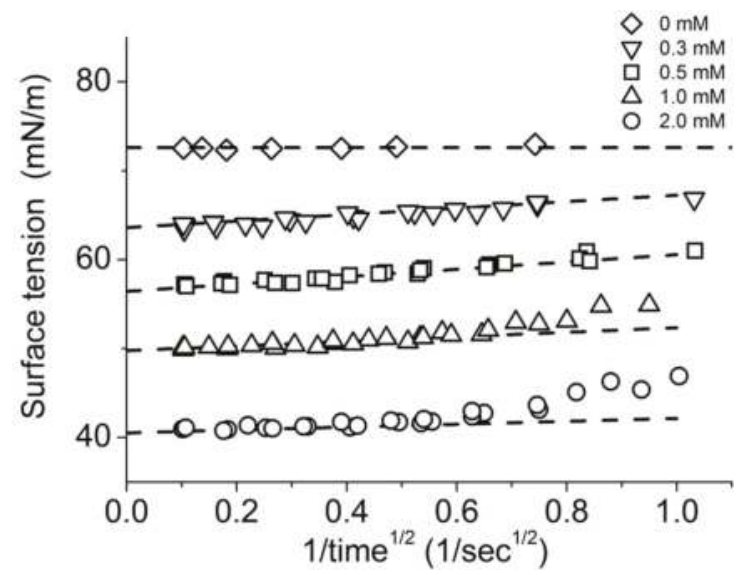

Figure 16. 1-Octanol dynamic surface tension at $20^{\circ} \mathrm{C}$ plotted according to the Ward-Tordai long-time approximation model Equation (7). 1-Octanol surface tensions from Figure 14b are plotted as a function of $t^{-1 / 2}$. The dashed lines show the best fitting line at each concentration. With permission from Elsevier [7].

The data show fairly linear regimes of surface tension versus $t^{-1 / 2}$ for each concentration. Following Equation (7), the linear slope depends on two parameters, i.e., the surface excess concentration at equilibrium, $\Gamma_{\mathrm{eq}}$, and the diffusion coefficient, $D$, of the compound in aqueous solution. Therefore, to estimate the $\mathrm{D}$ value from the slope of dynamic surface tension change, we needed to determine the $\Gamma_{\text {eq }}$ value at each concentration of the surface active compound. Unfortunately, the $\Gamma_{\text {eq }}$ value is not obtainable for our measurements. Although the $\Gamma_{\text {eq }}$ value is measurable directly by using other techniques, e.g., neutron reflection, the technique needs further development for direct dynamic $\Gamma(t)$ measurements [155-157]. Alternatively, we can estimate the maximum surface excess concentration, $\Gamma_{\mathrm{m}}$, value from fitting models, such as Langmuir isotherm and Frumkin isotherm models, to equilibrium surface tension plotted against the logarithm of concentration. The maximum surface excess concentration, $\Gamma_{\mathrm{m}}$, values were in turn obtained from fitting adsorption isotherm models to equilibrium surface tension plotted against the logarithm of concentration. We found that the best isotherm was the extended Frumkin isotherm adsorption model with a deal ionic activity correction factor. The estimated $\Gamma_{\mathrm{m}}\left(=7.75 \times 10^{-6} \mathrm{~mol} / \mathrm{m}^{2}\right)$ from MIAM [7] was in the same range 
as other methods, $\sim 6-9 \times 10^{-6} \mathrm{~mol} / \mathrm{m}^{2}[139,143,150,158]$, giving diffusion coefficient values from the slopes in Figure 16 of $7.2 \pm 0.8 \times 10^{-6} \mathrm{~cm}^{2} / \mathrm{s}$ for the Frumkin model. Interestingly, using a different micropipette technique we could also check this value by carrying out a droplet dissolution experiment for single 1-Octanol microdroplets that we reported in the same paper [7]. The diffusion coefficient $\mathrm{D}$ value of 1-Octanol was also directly measured by droplet dissolution and analysed by the Epstein-Plesset model giving $D=7.3 \pm 0.1 \times 10^{-6} \mathrm{~cm}^{2} / \mathrm{s}$, which showed excellent agreement between these dynamic surface tension data. Thus, for non-ionic surface active compounds, the adsorption barrier and activation energy derived from the adsorption dynamic models (especially the Frumkin model) worked well to explain the obtained dynamic surface tension data and also the diffusion coefficient $[7,139]$. Both obtained D values showed excellent agreement compared with other dynamic surface tension measurement methods, such as pendant bubble method [139].

We also applied the long-time adsorption approximation model to the dynamic surface tension for SDS [8]. Again, the dynamic tension data was replotted with the time axis of $t^{-1 / 2}$. Figure 17 shows the replotted results of SDS in the absence and presence of salt.

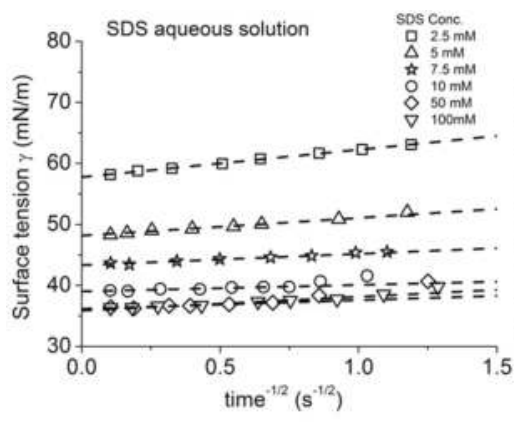

(a)

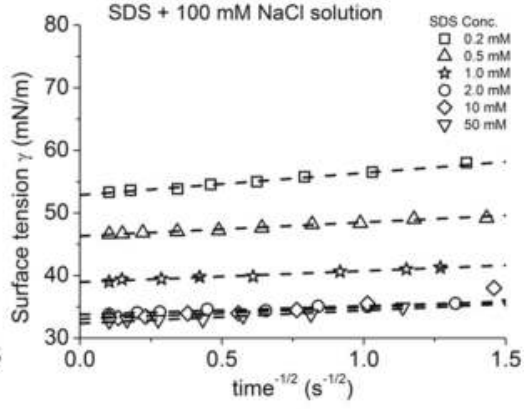

(b)

Figure 17. SDS dynamic surface tension at $20^{\circ} \mathrm{C}$ plotted according to the Ward-Tordai long-time approximation model Equation (7). SDS surface tensions are plotted as a function of $t^{-1 / 2}$. (a) SDS surface tensions for different SDS aqueous solution concentrations made up in milli pure water from 2.5 to $100 \mathrm{mM}$. (b) SDS surface tensions for different SDS solution concentrations from 0.2 to $50 \mathrm{mM}$, made up in $100 \mathrm{mM} \mathrm{NaCl}$. The intercept of each fitting line showed agreement with the equilibrium surface tension at each concentration. With permission from Elsevier [8].

In both cases, whether in the absence (Figure 17a) or presence (Figure 17b) of salt, the obtained dynamic surface tension data followed a linear slope for each concentration. Analysis for this case of an ionic surfactant was much more complicated than the non-ionic octanol and required corrections to several parameters used in the analyses. First, as described in more detail in the paper [8], the maximum surface excess concentration, $\Gamma_{\mathrm{m}}$, according to the Frumkin isotherm did not account for the adsorption activation energy $\Delta \mathrm{E}$ of ionic surfactant. This inconsistency was solved by applying a mean ionic activity correction to the fitting [159]. Then, an ideal ionic activity correction factor $\mathrm{A}_{ \pm \mathrm{i}}$ $(=0.29)$ for SDS was required and was obtained from the plot of mean ionic activity coefficient vs. $\Gamma_{\mathrm{m}}$ measured at different salt concentrations. This treatment of $\mathrm{A}_{ \pm \mathrm{i}}$ provided a means to normalise to a condition representative of a non-ionic interaction for an ideal electrolyte solution of SDS, i.e., the SDS molecules would be able to diffuse and adsorb at the air-water surface just like a non-ionic surfactant in this condition [8]. After applying the $\mathrm{A}_{ \pm \mathrm{i}}$ correcting factor, the mean ionic activity became the "ideal" ionic activity, $\mathrm{A}_{ \pm \mathrm{i}} \mathrm{C}^{*}$, and gave a more consistent $D$ value, $D=5.3 \pm 0.3 \times 10^{-6} \mathrm{~cm}^{2} / \mathrm{s}$ for the diffusion coefficient of single SDS molecules in aqueous media. 


\section{Equilibrium and Dynamic Surface Tension: Adsorption of Insoluble Surfactants}

In this final fundamental section, we review the measurement of equilibrium and dynamic surface tensions that we have made using the micropipette technique for insoluble lipid monolayers. Here, again using the tapered micropipette manipulation techniques [6,9], we chose to study a homologous series of phospholipids as our test materials. Later, in Section 6 Applications, we also include an interesting application that was initiated based on these earlier experiments-measuring the equilibrium and dynamic surface tensions and observing the kinds of multi-layered structures that occur at the air-water surface for natural and synthetic lung surfactants composed of mixed lipid and protein/peptide surfactant [10].

\subsection{Equilibrium Surface Tension for Adsorption of Phospholipids vs. Temperature}

In the early work by Lee et al. [6], we used that original tapered micropipette manipulation technique to study the homologous series of phosphatidylcholines, increasing chain length (e.g., diC12-diC18) produces an increase in their main acyl chain melting transition temperatures $\left(\mathrm{T}_{\mathrm{m}}\right)$ [160]. Figure 18 shows the equilibrium surface tension of four different saturated phospholipids, dilauroyl-phosphatidylcholine (DLPC, C12:0), dimyristoyl-phosphatidylcholine (DMPC, C14:0), dipalmitoyl-phosphatidylcholine (DPPC, C16:0), and distearoyl-phosphatidylcholine (DSPC, C18:0) in phosphate buffer (pH 7.4), PBS, with increasing temperature [6].

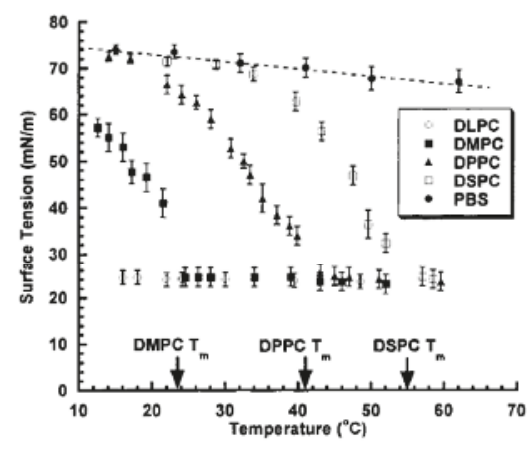

(a)

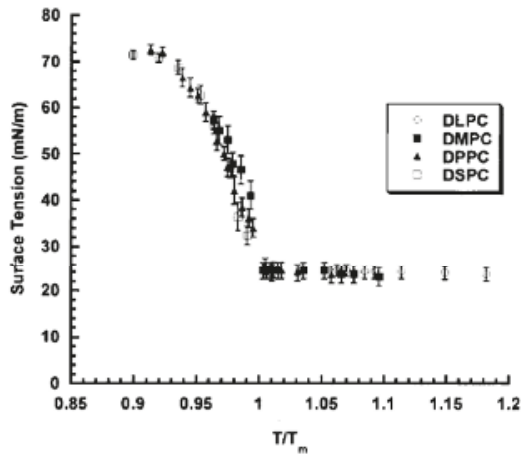

(b)

Figure 18. Equilibrium surface tension of four different phospholipids, i.e., dilauroyl- phosphatidylcholine (DLPC), dimyristoyl-phosphatidylcholine (DMPC), dipalmitoyl-phosphatidylcholine (DPPC) and distearoyl-phosphatidylcholine (DSPC) monolayers. (a) Equilibrium surface tension of each phospholipid monolayer at each temperature. Arrows mark the gel-to-liquid crystalline phase transition temperature for each phospholipid. (b) The equilibrium surface tension values are plotted as a function of their relative phase transition temperature $\left(\mathrm{T} / \mathrm{T}_{\mathrm{m}}\right)$, and the behaviour is collapsed to a single curve. Reproduced from Lee et al. [6], with permission from American Chemical Society.

As a control, it is important to recognise that the clean PBS-interface has a slight temperature dependence, decreasing from $74 \mathrm{mN} / \mathrm{m}$ at $13{ }^{\circ} \mathrm{C}$ to $66 \mathrm{mN} / \mathrm{m}$ at $62{ }^{\circ} \mathrm{C}$, but this in no way can account for the decreases seen in the presence of the phospholipids. Starting at low temperatures relative to their respective gel-to-liquid crystalline phase transition temperature $T_{m}$, the measured equilibrium surface tension (Figure 18a) of each phospholipid progressively decreased with increasing temperature. The surface tensions for all phospholipids reached a lower minimum constant value of $\sim 24 \mathrm{mN} / \mathrm{m}$, coinciding with each $T_{m}\left(23.5,41.4\right.$ and $55.1^{\circ} \mathrm{C}$ for DMPC, DPPC, and DSPC, respectively). Above this temperature, the surface tensions were relatively constant for these now liquid phase monolayers. 
This constant value above $T_{m}$ suggests there is no influence of carbon chain length between C12 and C18 on the $\gamma_{m}$ value for the liquid crystalline phase. Moreover, when the same plots were replotted scaling the temperature as a function of their relative phase transition temperature $\left(T / T_{m}\right)$, Figure $18 \mathrm{~b}$ showed that all the data from all four different lipids collapsed onto a single curve. Thus, the temperature-dependent change in surface tension (decreasing with increasing temperature, until a minimum value is reached) only depends on the relative phase transition temperature, and not on the total carbon chain length. What this data then shows is that the limiting surface tension is governed by the hydrocarbon-air interface of the outer parts of the chain and terminal methyls. In fact, the common value of $\gamma_{m} \sim 24 \mathrm{mN} / \mathrm{m}$ is essentially the same as that measured for liquid hydrocarbons like $\mathrm{n}$-decane which has a decane-air surface tension of $23.7 \mathrm{mN} / \mathrm{m}$ at $\mathrm{T}=22^{\circ} \mathrm{C}$ [161].

\subsection{Dynamic Surface Tension for Adsorption of Phospholipids}

We also made dynamic surface tension measurements for these same insoluble lipid monolayers. Here, only the simple area-expansion method was used and so we only observed the interface after about $15 \mathrm{~s}$ of initial monolayer formation [6]. However, this did not pose too much of a problem in time resolution because, unlike the molecular species of octanol and SDS, the adsorbing species were liposomes, and so the rate of monolayer formation reflected the diffusion (Brownian motion) of these much larger, $100 \mathrm{~nm}$ entities. Diffusion coefficients for liposomes ( $250 \mathrm{~nm}$ ) have been measured in water to be $\sim 8.8 \times 10^{-8} \mathrm{~cm}^{2} / \mathrm{s}$ [162], and so liposome diffusion is over 100 times slower than simple single molecules (7.3 and $2-6 \times 10^{-6} \mathrm{~cm}^{2} / \mathrm{s}$ for octanol and SDS respectively). Such entities could be subject to Ward-Tordai analyses (but have not been done so far). In any event, the process of adsorption and monolayer formation from liposomes is not just governed solely by their diffusion to the interface; it also includes their collapse and spreading, and so there are potentially multiple rate determining steps. In this experiment then, we could still observe the monolayer formation rate as liposomes spread and lowered the interfacial tension. Figure 19a shows the dynamic surface tension change for just one of the lipids, DPPC, with increasing temperature.

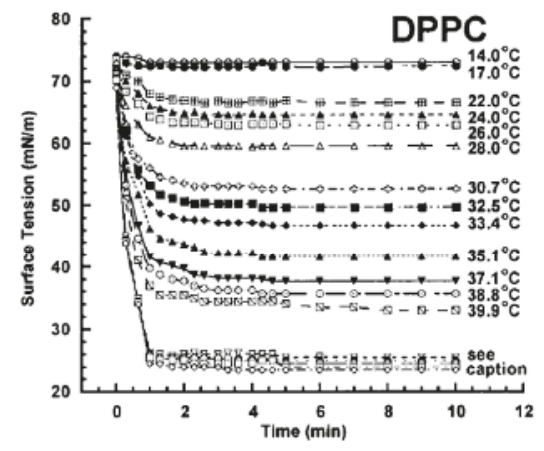

(a)

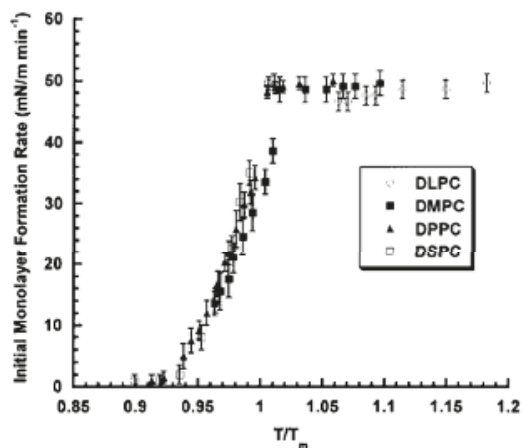

(b)

Figure 19. Dynamic surface tension change and initial monolayer formation rates. (a) Effect of temperature on adsorption kinetics at the water-air interface of DPPC monolayers, as monitored by change in surface tension with elapsed time of exposure of the interface to DPPC lipid aqueous suspensions $(1.0 \mathrm{mM})$ at various temperatures from 14 to $59.6^{\circ} \mathrm{C}$. Note: the surface tensions at $\left(43^{\circ} \mathrm{C}\right.$ $(\nabla), 45^{\circ} \mathrm{C}(\bullet), 47^{\circ} \mathrm{C}(\bigcirc), 51.1^{\circ} \mathrm{C}(\square)$, and $59.6^{\circ} \mathrm{C}(\diamond)$ all rapidly reached an equilibrium value of $25 \mathrm{mN} / \mathrm{m}$ within 1 to $2 \mathrm{~min}$, and so the data effectively lies on top of each other. (b) Plot of the initial monolayer formation rates of four different lipids, i.e., DLPC, DMPC, DPPC, and DSPC, at the air-water surface as a function of their relative phase transition temperature $\left(T / T_{m}\right)$. The four different types of lipid data were collapsed to a single curve. Reproduced from Lee et al. [6], with permission from American Chemical Society. 
DPPC has a main acyl meting temperature $T_{m}$ of $41.3{ }^{\circ} \mathrm{C}$ when it enters the liquid L $\alpha$ phase. Below this temperature, the liposomes are in their so called "gel" phase, where, for DPPC, there are also several sub-phase transitions with distinct temperature ranges [160]:

- $\quad \mathrm{P}_{\beta}{ }^{\prime}$ or ripple phase from $34.45-41.3^{\circ} \mathrm{C}$

- $\quad \mathrm{L}_{\beta}$ planar gel phase from $18-34.4^{\circ} \mathrm{C}$

Therefore, the temperature ranges in Figure 19 were designed to capture these phases up to and including the melted liquid $\mathrm{L}_{\alpha}$ phase.

As is shown by the Figure $19 \mathrm{a}$, at $14{ }^{\circ} \mathrm{C}$, which is below its $\mathrm{L}_{\mathrm{c}}$ phase, DPPC liposomes do not hardly, if at all, even spread on the clean $72 \mathrm{mN} / \mathrm{m}$ air-water surface. With successively increasing temperatures, the equilibrium surface tension is lowered at faster and faster rates through each bilayer sub-gel-phase until the liquid state is reached and the minimum surface tension is obtained. As concluded by Lee et al., in order for rapid spreading on the clean interface to occur the lipid vesicles are required to be in the liquid state. Additionally, the equilibrium surface tension and the monolayer formation rate depend on the relative phase transition temperature of the lipid. Thus, we see that, in agreement with MacDonald and Simon's study on a similar lipid DMPC [117], the monolayers follow the bilayer systems (liposomes) with which they are in equilibrium.

As with the surface tension data vs. reduced temperature $(\mathrm{T} / \mathrm{Tm})$, the monolayer formation rate (in units of $\mathrm{mN} / \mathrm{m} \cdot \mathrm{min}$ ), shown in Figure 19b, also collapsed onto a single curve for all lipid systems. Interestingly, at a reduced temperature of 0.9 to 0.93 for all lipid systems, none of the lipids would spread on a clean interface. Then, from 0.93 to 1.0 there was an increase in the spreading rate until $\mathrm{T}_{\mathrm{m}}$, at which point the initial monolayer formation rate reached a common maximum of $\sim 50 \mathrm{mN} / \mathrm{m} \cdot \mathrm{min}$. Comparisons with previously reported data using the Langmuir trough can be made with this micropipette-lipid-adsorption data. The Langmuir trough produces surface pressure isotherms, and so we can convert the surface tension values of the lipid-adsorbed interface $(\gamma)$ and the clean interface $\left(\gamma_{0}\right)$ into surface pressure values $(\pi)$ via the relation $\pi=\gamma_{0}-\gamma$. Converting the data in Figure 18b, to surface pressure (surface pressure is negative) a nearly linear increase in equilibrium spreading pressure for the monolayer material was observed as the temperature was raised to the transition temperature.

Thus, using the tapered micropipette manipulation techniques, Lee et al. (2001) found that both equilibrium and dynamic surface tensions depend on the relative phase transition temperature $T / T_{m}$ of the lipid [6]. One interesting observation here is that for all lipid systems, for a reduced temperature of 0.9 to 0.93 , lipids actually have zero spreading pressure on a clean interface. One could imagine a practical application of this result where such highly solidified lipid vesicles could be used in aqueous suspension to actually keep interfaces clean by perhaps acting as sinks for other impurity materials.

\section{Applications in Medical Imaging (Ultrasound), Oil Recovery, Nanoprecipitation, the Biology of the Lung Interface, and Microfluidics}

Finally, we give examples of where our measurements of micro-surface tensions, and those of others (notable Tony Yeung [11]), for clean and adsorbed surfaces, and gas- and droplet-dissolution have had an impact on five applications. These selected applications include: (1) gas microbubbles for ultrasound contrast; (2) interfacial tensions for micro-oil droplets in oil recovery; (3) surface tensions and tensions-in-the surface for natural and synthetic lung surfactants; (4) interfacial tension in nanoprecipitation; and (5) micro-surface tensions and droplet dissolution in microfluidics.

6.1. Gas Micro-Bubbles for Ultrasound Contrast: Surface Tension and their Dissolution into Water at the Scale of the Microbubble

Measuring the surface tension of an air-water surface $[6,9]$ and then being motivated by industrial interest to working with micro bubbles [12] was actually the first time we developed the micropipette technique from its original applications in studying cells and vesicles to studying colloids and surfaces 
of gas, liquid and solid microsystems. As we saw in Figures 5 and 7, just by placing an air-filled pipette in a water filled chamber it is simply a matter of increasing the positive pipette pressure to drive the gas interface to the tip of the pipette. Applying a bit more pressure then blows out a gas microbubble bubble. The additional pressure required to do this is relatively small compared to the large pressure required to get the meniscus to such a small radius of curvature. As described by Tony Yeung [11] (see Section 6.2. where we give the equation for aspirating a droplet, but now consider the process is in reverse), when an air-water surface inside the pipette is driven by positive pressure to emerge from the pipette tip into the unconstrained environment of the chamber, the formation of a bubble of diameter greater than the pipette tip diameter results in the rapid growth of the gas bubble. As can now be appreciated, this pressure is determined by the level of surface tension of the surface itself, and, if it is a clean air-water surface of $72 \mathrm{mN} / \mathrm{m}$, the pressure needed to blow out the bubble through the relatively small micropipette tip, becomes a large driving force for microbubble expansion. The force balance associated with the buoyancy of air will pull the forming bubble off the pipette at some point, but microbubbles can grow to hundreds of micrometres before they detach. One technique we developed to control the formed microbubble size was to simply angle the pipette at the top of the chamber so that its tip was 10-20 $\mu \mathrm{m}$ below the glass surface such that as the microbubble rapidly emerged, it was forced off the pipette. We could therefore "manufacture" a series of gas microbubbles by this angled-pipette technique and then pick single microbubbles for study.

In this application the goal was to stabilise gas microbubbles against dissolution for an important medical application-ultrasound contrast. While gassed-up salt solution had been an industry standard, at the time new contrast agents were being formed using proteins [163]. One was called Albunex (Molecular Biosystems, Inc, San Diego). It was a commercially prepared contrast agent made from sonicated $5 \%$ human serum albumin. The mean microsphere size used in these experiments was $4.0 \mu \mathrm{m}$ with a concentration of 437 million $/ \mathrm{mL}$. The (competing) company who contracted us, was interested in, if, and to what extent, lipids could be used as the stabilising monolayer. As with Albunex, the idea was that following intravenous injection, they could survive long enough in the blood stream to provide blood-pool contrast using ultrasound for myocardial contrast echocardiography (MCE) and the like. As was obvious from Laplace surfaces, surface tension is a key parameter that determines the dissolution rate of air into water because the surface tension creates an above-ambient pressure on the gas inside the microbubble, as given by the Laplace equation, Equation (5). The influence of surface tension and the role of microbubble size, and the diffusion coefficient of air in water were the key parameters that Epstein and Plesset [96] brought together in their 1950 paper that described gas bubble dissolution (or growth). Therefore, we started our product development by testing and validating this equation using gas microbubbles made and observed using the micropipette technique. As we will see, this is again where, working at the microscale meant we could observe and measure all dissolution processes for single individual microbubbles in a convenient lab time frame of just a few seconds.

The basic Epstein-Plesset (EP) equation for the dissolution rate of a free gas microparticle considering only the gas concentration in an unsaturated solution (surface tension is not yet included) is given by,

$$
\frac{d R}{d t}=-\frac{D\left(C_{s}-C_{o}\right)}{\rho}\left[\frac{1}{R}+\frac{1}{\sqrt{\pi D t}}\right]
$$

where $R$ is the diameter of the microbubble, $C_{s}$ is the saturation concentration, $C_{0}$ is the concentration of gas in the bulk solution, $\rho$ is the density of the gas, and $t$ is time. The model assumes that there is a large volume of surrounding solution relative to the volume of the bubble, the gas concentration at the bubble's surface is in equilibrium with the gas in the bubble and is considered saturated, $C_{S}$, and the concentration at infinity is the initial gas concentration in solution $C_{0}$.

The inclusion of surface tension enters the analysis through of the curvature of the bubble's surface and the fact that a surface tension at the interface creates an over pressure inside the bubble according to the Laplace equation, (given earlier, Equation (5)). The total pressure in the bubble therefore increases as $R$ decreases. Thus, the influence of surface tension is introduced into the dynamic 
equation, assuming ideal gas, via a recalculation of the gas density with increasing over-pressure as given by Epstein and Plesset [96] and detailed in Duncan and Needham [12].

Until we did the experiment over 50 years later using the micropipette technique [12], this equation, and the dissolution rate as influenced by the surface tension, had never really been tested at the scale of gas microbubbles. As already mentioned, it was the ability to work at this scale of $10 \mathrm{~s}$ of microns using the micropipette technique, that allowed the gas bubble size and dissolution rate to be measured, whereas all previous attempts using millimetre sized bubbles took days to dissolve, making it difficult to readily test this important model for gas-dissolution. We have also now extended these studies and this model fully characterises the dissolution of immiscible liquid microdroplets of oil into water and water into oil, [95,164,165].

\subsubsection{Test of the Epstein-Plesset Model for Gas Microparticle Dissolution in Aqueous Media}

The experiment then was to simply form a 10-20 $\mu$ m diameter air microbubble using the micropipette, and, as shown in Figure 20A, hold it on the tip of the pipette, in a static position, in infinite dilution, in an isotropic diffusion field, in the middle of the aqueous filled chamber.

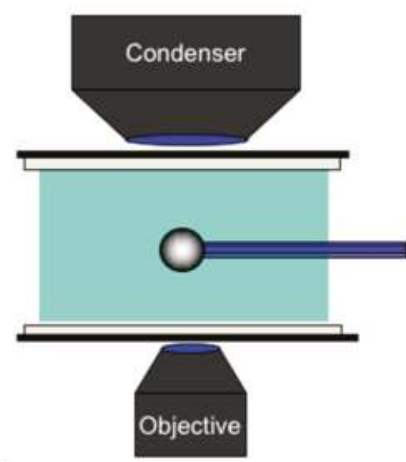

A) isotropic diffusion field

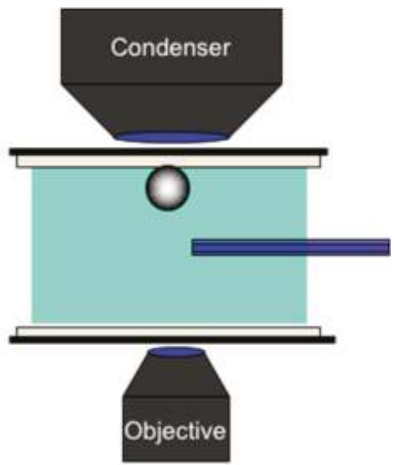

B) impermeable boundary

Figure 20. Schematics of pipette and gas microbubble in microchamber. (A) Microbubble held in infinite dilution and isotropic diffusion field; (B) microbubble allowed to rise to the top of the chamber, also in infinite dilution but now air-diffusion is limited by the impermeable boundary of the wall of the glass microchamber.

As shown in Figure 20A, we could then observe its gradual dissolution into saturated or undersaturated water, and fit this data to the EP model knowing the surface tension of the air-water surface. Air bubbles were better stabilised (against adhesion to the glass pipette) by the adsorption of an SDS monolayer and so were formed in a $10 \mathrm{mM}$ SDS solution giving an air-water SDS monolayer surface tension of $40 \mathrm{mN} / \mathrm{m}$. In Figure 20B, the microbubble is released from the micropipette and allowed to rise to the top surface of the chamber. There is now an impermeable boundary that limits the air diffusion away from the microbubble into the aqueous phase, and so the microbubble is no longer in an isotropic diffusion field, but this can be accounted for by an empirical model [12,166].

Figure 21 shows the data along with the EP model with no free parameters, thereby validating this model for an air-water surface stabilised by the soluble surfactant SDS. Thus, a $30 \mu \mathrm{m}$ diameter air microbubble dissolves in water in $50 \mathrm{~s}$, in almost complete agreement with the EP model. Imagine now how compromised the original contrast agent systems were of gassed up salt solution! How long would a $5 \mu \mathrm{m}$ diameter gas bubble would last in the blood stream? Just a few seconds! Of course, if it adsorbed any protein, that would reduce its surface tension (see next Section 6.1.2.), then it might last a little longer. However, initially-uncoated gas microparticles were extremely difficult to work with and not very effective contrast agents. Now we know why, quantitatively. 
A released microbubble though (Figure 20B) rises to the top of the chamber and comes to rest against the glass surface. The result is that air saturation of the solution can build up around the microbubble and so its dissolution is $\sim 44 \%$ longer. The data is fit to an empirical model by Wise et al., [166] in Figure 21B. We mention this boundary condition here because it may be of interest and important to the current "Micromachine" audience since microbubbles near surfaces or in bulk-isotropy occur in one or more of the in micro- and nano-systems (that might include, microfluidic and lab-on-chip devices, soft gripping and manipulation of particles, colloidal and interfacial assemblies, fluidic/droplet mechatronics).

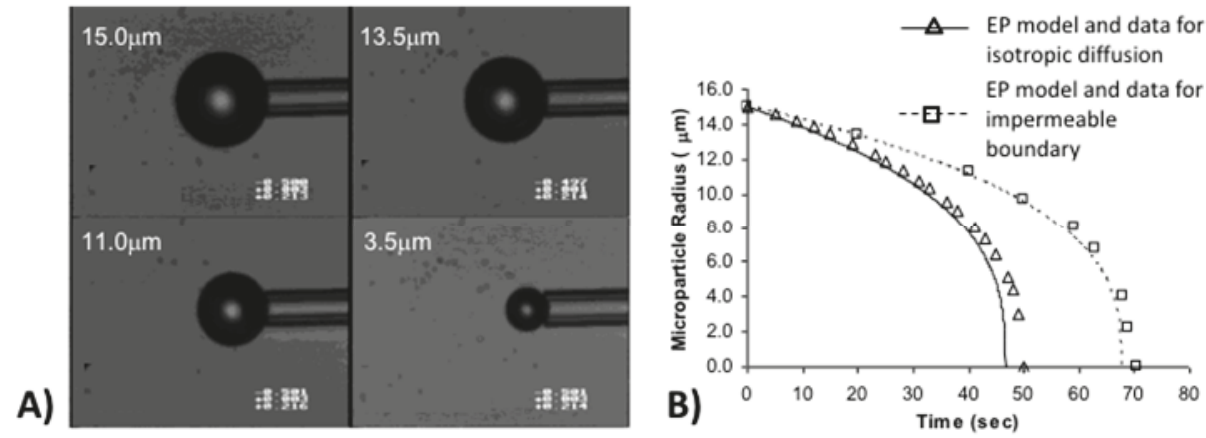

Figure 21. Dissolution of a $30 \mu \mathrm{m}$ diameter air bubble into water. (A) A series of video images of the dissolution of an air microbubble in $10 \mathrm{mM}$ SDS solution held in the center of the chamber by a pipet with a very low suction pressure of $500 \mathrm{~Pa}$ and $21.5^{\circ} \mathrm{C}$. The air bubble is shown at times $0 \mathrm{~s}$ (top left), $10 \mathrm{~s}$ (top right), $30 \mathrm{~s}$ (bottom left), and $48 \mathrm{~s}$ (bottom right) respectively, completely dissolving in $\sim 50 \mathrm{~s}$. (B) Plot of microparticle radius versus time for dissolution in isotropic and boundary-limited conditions where the data are fitted by the Epstein-Plesset (EP) model and an empirical model by Wise et al. [166].

Gas microbubble-lifetimes depend on both the dissolution-driving over pressure due to their surface tension and the diffusion field that surrounds the microbubble. This boundary condition will also apply to dissolving micro and nano-scale liquid-in-liquid emulsions, but here the over pressure is negligible because of the incompressibility of liquids as we have also shown for an aniline-water micro-system [164]. Additionally, as is clear from the EP model, an increase in surface tension results in a faster dissolution and so shorter dissolution time. For example, the same $30 \mu \mathrm{m}$ diameter air bubble for a clean air-water surface tension of $72 \mathrm{mN} / \mathrm{m}$ would take $\sim 30 \mathrm{~s}$ to dissolve compared to $50 \mathrm{~s}$ for the lower surface tension $(40 \mathrm{mN} / \mathrm{m})$ of the SDS-coated bubble.

6.1.2. When the Tension-in-the-Surface is Zero: Effect of Gas Saturation in Solution on Microbubble Dissolution

Since the surface tension creates the over pressure, gas bubbles will always dissolve even in saturated solution. However, what would happen without this driving force? That is, what if there was no surface tension at the gas bubble surface? Clearly, for an air-water surface or any interface to exist we cannot have a "zero surface tension." However, we can have "zero-tension-in-the-surface" if that surface is now a monolayer of an insoluble material, like, for example, a phospholipid. Basically we "coated-air" and, in the same paper [12], we measured the rates of dissolution, in the absence of the Laplace overpressure, for increasing gas saturation in the aqueous medium. In order to create a "zero-tension-in-the-surface" we utilised the same gel-phase DSPC lipid as a monolayer shell adsorbed on the air microparticle, as was used and shown to adsorb by Lee et al. [6] (and described earlier in Figures 18 and 19). This adsorbed, solid-phase, lipid monolayer permits the assumption to be satisfied of zero tension in the gas microparticle surface, and with it, a condition of zero Laplace pressure. 
Gas dissolution is then only driven by the level of gas under-saturation in the surrounding aqueous phase placed in the microchamber. (Note this is now not a gas-bubble per se with an exchangeable interface composed of a soluble surfactant or just water; it is gas encapsulated in a monomolecular insoluble shell).

Starting with a maximally undersaturated solution, and carrying out dissolution-experiments much faster than the undersaturated solution re-saturates with air, we could see that as the air escaped from the gas microparticle (Figure 22), the solid-shelled monolayer was observed to be misshapen (crinkle) and, at excess crumpling, reshaped ("popped") back to spherical caused by a shedding of the lipid monolayer while still remaining contiguous with the surface material. Under this microscopic resolution the shell is not visible, but was seen with interference optics. This return to a spherical shape allowed us to quantify the gas-loss as a change in radius, measured at each spherical-reshaping, versus time as a function of the initial degree of air-water saturation, $\mathrm{f}$. We confirmed that the particle dissolved at slower and slower rates the closer the degree of under-saturation, $f$, got to unity. At $f=1$, conditions essentially represented an infinite dissolution time, demonstrating that the solid shell indeed provided zero tension in the surface and zero Laplace pressure.

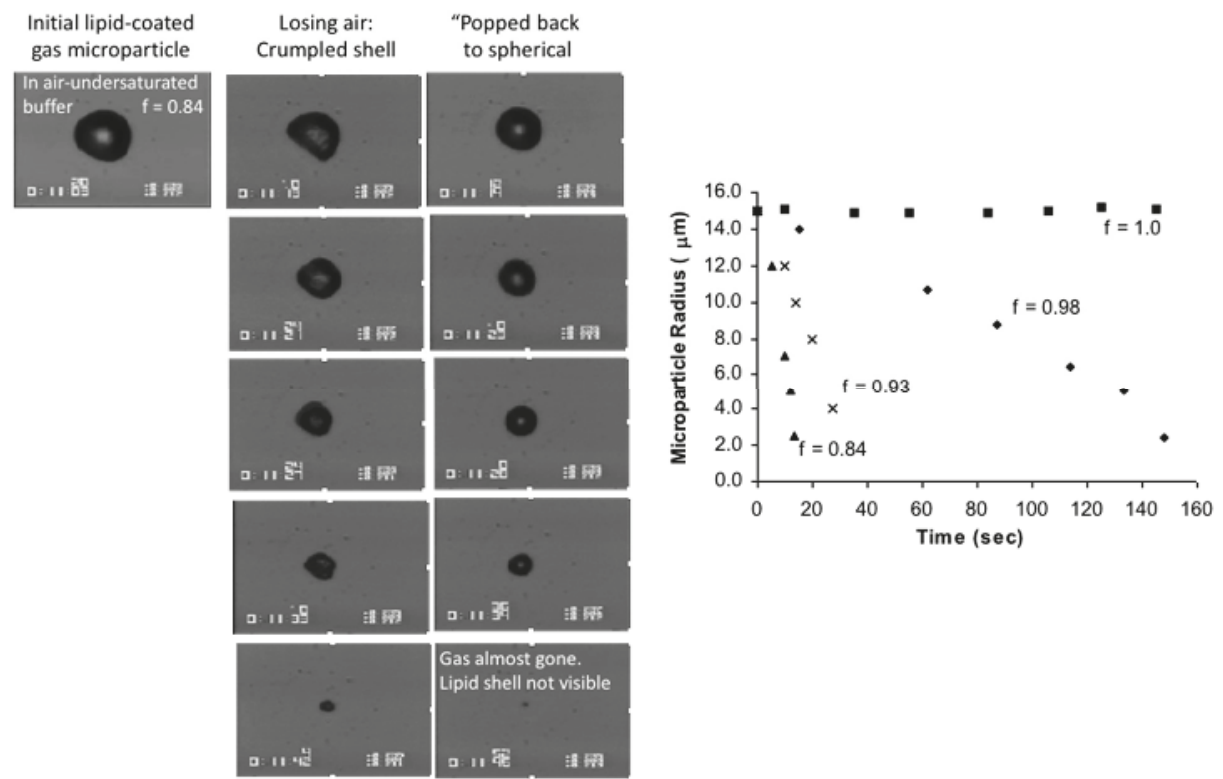

Figure 22. Lipid-coated gas microparticle losing air in undersaturated solution. The degree of air-water saturation, $\mathrm{f}$, is the ratio of the initial air concentration in surrounding water to the saturated concentration of air in water. (Left) Videomicrograph of the initial lipid-coated gas microparticle in $\mathrm{f}=0.84$. (Middle) Series of lipid-coated gas-microparticles losing air, the shell crumples, and then pops back to spherical as lipid is shed until there is no gas left; (Right) plot of microparticle radius vs. time for gas microparticles losing air at increasing air saturation. The microparticle in the saturated solution $(\mathrm{f}=1)$ is stable.

While beyond the scope of this paper to go into too much detail, it is worth mentioning briefly that, having formed these "zero-tension" shells, the micropipette technique was also used to characterise the mechanical (viscoelastic) nature of lipid shells on gas microbubble surfaces. Interested readers can learn of these experiments and results in a series of experiments on lipid-coated single gas microparticles $[13,14]$, including the process of making the lipid-coated microparticles in which air micro-bubbles were formed by sonication in the presence of lipid as liposomes above their phase 
transition temperature. Following the data in Figures 18 and 19, upon formation of the clean gas microbubble air-water surface, lipid rapidly adsorbed (to equilibrium in $2 \mathrm{~min}$ ) to form monolayer above $\mathrm{T}_{\mathrm{m}}$, and the final coated microbubble suspension was obtained when it was cooled to solidify the monolayers. Aspiration of the shells demonstrated that they deformed in shear at room temperature and that the values of their yield shear and shear viscosity were dependent on the composition, grain microstructure, and thermal processing of the material $[13,14]$. For example, the $2 \mathrm{D}$ viscosity for the series of diC18-diC24 phosphatidylcholines was dependent on their relative transition temperature, characterising such surface monolayers with values of yield shear $\left(1-6 \mathrm{mN} \cdot \mathrm{m}^{-1}\right)$ and shear viscosity $\left(5-25 \mathrm{mN} \cdot \mathrm{s} \cdot \mathrm{m}^{-1}\right)$ obtained by the micromanipulation technique for these phospholipid shells. These values of yield shear and shear viscosity can be converted to comparative bulk values by dividing by the monolayer thickness of $3 \mathrm{~nm}$, giving values of $1.7-8.3 \times 10^{6} \mathrm{Ns} / \mathrm{m}^{2},(1.7-8.3 \mathrm{MPa})$ and $0.3-2 \times 10^{6} \mathrm{~N} / \mathrm{m}^{2},(0.3-2 \mathrm{MPa} \cdot \mathrm{s})$ which interestingly are comparable to the properties of common plastics like high density polyethylene [167]—tensile yield (10 Mpa) and melt-viscosity (0.3 MPa.s). This again demonstrates that the intermolecular interactions (largely van der Waals-bonding) between lipid acyl chains dominate micromechanical and surface-properties of such monomolecular materials.

\subsection{Surface Tension Measurements of Microdroplets for Oil Recovery}

Instead of making an interface inside the tapered micropipette, we have shown that an isolated single oil droplet can be formed in water [16-18,95], or water microdroplet can be formed in oil [165,168] and held at the tip of the micropipette thereby creating a micro-interface. Then, by applying a controlled suction pressure to the oil droplet, it is possible to expand the interface and measure the critical tensile yield (interfacial tension) of the immiscible interface. This unique droplet interfacial tension measurement was used by Yeung's group in 2000 [11]. Figure 23 shows a single water droplet in heptol (1:1 mixture, by volume, of $n$-heptane and toluene).

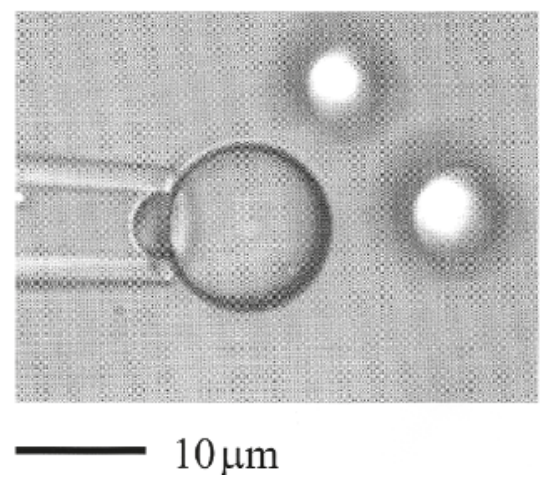

Figure 23. Single water microdroplet held by the micropipette in heptol solution (1:1 mixture, by volume, of $n$-heptane and toluene). The water-oil interfacial tension of $40.3 \pm 0.6 \mathrm{mN} / \mathrm{m}$ was directly measured from the geometrical shape of the microdroplet and the minimum pressure required to draw in the droplet to give a projection length inside the pipette of one pipette radius. With permission from Elsevier [11].

For this water-microdroplet-aspiration technique, the interfacial tension (symbolised now in Yeung's equations by $\sigma$ ), of $40.3 \pm 0.6 \mathrm{mN} / \mathrm{m}$ was calculated from the geometrical shape of the deformed microdroplet with critical pressure, $p_{c r}$, following the relation [11,169],

$$
\sigma=\frac{p_{c r} R_{p}}{2\left(1-\frac{R_{p}}{R_{o}}\right)},
$$


where $R_{p}$ is the inner radius of the pipette tip, and $R_{o}$ is the radius of the exterior drop segment. The equation was originally developed by Evans to measure the lipid bilayer membrane tension in mechanical characterisations of the red blood cell membrane $[56,58,63,170]$ and was also extensively applied to characterise the mechanochemistry of synthetic of phospholipid membranes as giant unilamellar vesicles $[73,75-80,83,108,118,169,171]$. It is thus a main-stay of the micropipette technique. In order to calibrate and validate the droplet-tension technique, interfacial tensions were measured by Yeung et al. for water vs. ethyl acetate, dichloromethane, toluene, carbon tetrachloride and showed fairly good agreements with the literature values. In Yeung's experience, some oils such as crude oil showed high viscosity and were sticking against the glass pipette walls. To avoid measurement errors, the micropipette surface was salinized [11]. We have also used such salinization when dealing with lung surfactants [10] and these experiments on lung surfactant properties are given next.

\subsection{Surface Tension or "Tension-in-the Surface": The Biology of the Lung-Air Surface}

Taking the basic and fundamental measurements of lipid monolayer surface tensions we made in 2001 [6] into an application, we recently carried out a series of interesting and illuminating new studies on natural and synthetic lung surfactants [10]. Surfactant formulations are used for the medical treatment of a range of lung conditions including, neonatal respiratory distress syndrome (NRDS), Acute Lung Injury (ALI) and Acute Respiratory Distress Syndrome (ARDS) patients [172-174]. The new micropipette studies show not only the level of surface tension achieved by these components of lipid and protein at the air-water surface, but have also allowed us to visually observe and measure the assembly of new multi-lamellar structures-all by viewing the material adsorption in the tapered-micropipette. Again, with an average diameter of 100-200 $\mu \mathrm{m}$, our micropipette techniques provide surface tension and structural data at the same scale as the phenomenon under study. In this case adsorption and spreading on alveolar surfaces, that have, themselves, an average diameter of $200 \mu \mathrm{m}$, with an increase in diameter and hence expansion in surface area during inhalation-all the parameters (radius of curvature, surface area and applied pressure across and tension in the surface) we can control and apply in the micropipette manipulation techniques.

\subsubsection{Lung Surfactant Components}

The major components of natural lung surfactant are lipids ( $\sim 90 \%$ by mass: phosphatidylcholines, phosphatidylglycerol, and cholesterol) and three proteins ( 10\%: hydrophilic SP-A and SP-D proteins, and hydrophobic SP-B and SP-C proteins) [147,175]. Of these proteins, SP-B (79 amino acids; monomer MW of $8.7 \mathrm{kDa}$ ), is strictly required for the assembly of pulmonary surfactant and the formation of stable surface-active films at the air-liquid alveolar interface, making SPB essential for lung expansion function [176]. Nowhere is this more critical than in NRDS in premature babies, [177] who are born without lung surfactant; an absence of lung surfactant is incompatible with life itself.

Currently, there are several commercialised lung surfactant-products available on the market. These include: the animal lung-derived surfactant products, Curosurf, Survanta, Infasurf, Alveofact, and BLES; and synthetically developed products like Lucinactant (Surfaxin), (consisting of phospholipids, a fatty acid, and sinapultide (—a 21-amino acid hydrophobic synthetic peptide called KL4 peptide), and Colfosceril (exosurf) (consisting of just the synthetic lipid Colfosceril Palmitate (DPPC), plus-Cetyl Alcohol, and Tyloxapol-a non-ionic liquid polymer of the alkyl aryl polyether alcohol. In addition to these more traditional animal and synthetic surfactant formulations, Molecular Express and their academic collaborators (Walther and Waring) in California have developed a new, completely synthetic, formulation that mimics the Surfactant Protein (SP)-B, called Mini-B [178], that was recently modified further into a Super Mini-B construct [179]. The Super Mini-B (SMB) analogue, together with a second peptide construct SM-C [180] showed that this combination was actually superior to single-peptide formulations in rabbits with chemical acute lung injury.

Thus, as introduced by Walther et al. [179], Mini-B, (34 amino acid sequence) is a disulphide-linked construct based on the N- and C-terminal regions of SP-B (i.e., residues 8-25 and 63-78). Mini-B 
retains critical in vitro and in vivo surfactant functions of the native protein, and the Super Mini-B construct has native SP-B residues (1-7) attached to the N-terminus of Mini-B. These peptides are known to rearrange lipid molecules in the fluid lining the lung so that alveoli can more easily inflate. The new synthetic lung surfactant formulation then comprises a host phospholipid mixture (PL = DPPC:POPC:POPG 50:30:20 molar ratio) and the $4 \mathrm{wt} \%$ SMB lung surfactant protein peptide. In keeping with the personalised-introduction, the lung surfactant projects started when we were asked by our friend, collaborator and CEO/President of Molecular Express Inc, CA, Gary Fujji, "Can you measure surface tensions?" .. "Of course we can, see our papers from 2001" . . and this launched another industrial project. While other techniques, such as the pulsating bubble surfactometer had been used in the past to measure lung surfactant tensions [122], we applied the tapered micropipette manipulation techniques to investigate lung surfactant air-water surface tensions and the multi-layered and complex structures they produced [10]. The hypothesis was that the inclusion of the SMB peptide would induce lipid fusion and massive reassembly of multilamellar structures, and that we could observed this at the microscopic interface in the micropipette. This was indeed the case.

\subsubsection{Equilibrium and Dynamic Surface Tensions for the Lung Surfactant Formulations}

Firstly then, Figure 24 shows both equilibrium and dynamic surface tension data of the various different types of lung surfactants at the air water interface in the tapered micropipette [10]. The equilibrium surface tension values obtained from the slope of a linear fit to the $\Delta P$ vs. $2 / R_{c}$ plots are shown numerically in the legend (Figure 20a).

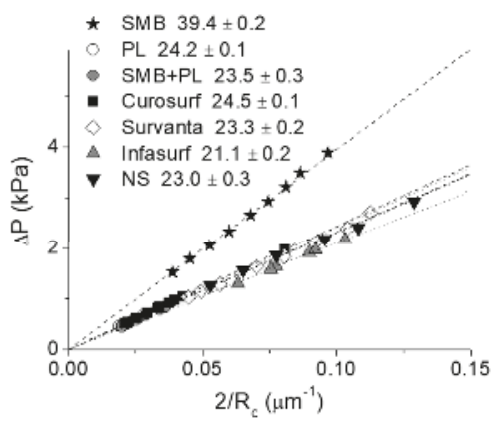

(a)

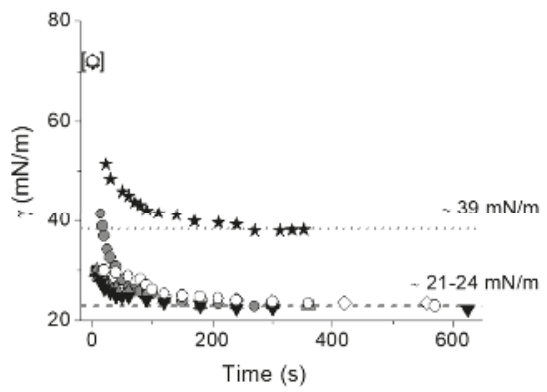

(b)

Figure 24. Equilibrium air-water surface tensions for lung surfactants in Tris buffer $(\mathrm{pH} 7)$ at $37^{\circ} \mathrm{C}$. The data include $1 \mathrm{mg} / \mathrm{mL}$ suspensions of three different commercialised lung surfactants and extracted native surfactant (NS) from porcine lungs and the measurement of a synthetic lung surfactant formulation: 4\% Super Mini-B (SMB) lung surfactant protein peptide in a phospholipid mixture (PL = DPPC:POPC:POPG 50:30:20 molar ratio), and the pure SMB peptide itself. (a) Equilibrium surface tension plot from the applied pressure vs. $2 / R_{c}$ plot. The surface tension for each sample are shown in the legend derived as usual form linear fitting of the slopes. (b) Dynamic surface tension change of each sample by using the first-generation area expansion method developed by Lee et al. [9]. The symbols correspond between these two graphs. With permission from American Chemical Society [10].

Interestingly, the animal-derived lung surfactants (protein-free Curosurf, Survanta, and protein-containing Infasurf [147]) and extracted native porcine lung surfactant (NS) all gave surface tension values in the range $21-25 \mathrm{mN} / \mathrm{m}$. Importantly, theses surfactants showed the same equilibrium surface tension values as the pure phospholipid mixture (PL = DPPC:POPC:POPG 50:30:20 molar ratio), $\sim 24 \mathrm{mN} / \mathrm{m}$ (see earlier Figures 18 and 19). Consistent with this data, mixing the synthetic peptide SMB $[10,179]$ in with the artificial lipid mixture (PL) did not change the equilibrium surface tension, which was $23.5 \mathrm{mN} / \mathrm{m}$. In the absence of lipids, Figure 20a also shows that the peptide itself had a slightly higher surface tension of $39.4 \mathrm{mN} / \mathrm{m}$. Thus, even though they each contain either some 
of the native proteins or the synthetic peptide for all compositions, the equilibrium surface tensions were all dominated by the lipid fraction.

The question then was, is there an effect of the inclusion of protein or peptide on the dynamic surface tension? As shown in Figure 20b, the dynamic surface tension measurements (indicated by the time-rates of change of surface tension and so in units of $\mathrm{mN} / \mathrm{m} / \mathrm{min}$ ) for each of the surfactants gave initial adsorption rates in the range of $60-240 \mathrm{mN} / \mathrm{m} / \mathrm{min}$, over the first $30 \mathrm{~s}$. These values were again in the same range as that of the pure phospholipid mixture (PL), and in agreement with the single pure phospholipid solutions, maximum $\sim 50 \mathrm{mN} / \mathrm{m} / \mathrm{min}$ (Figure 19). Interestingly, as shown in Figure 20b, the SMB peptide itself also showed a similar dynamic adsorption speed, $\sim 60 \mathrm{mN} / \mathrm{m} / \mathrm{min}$.

\subsubsection{Morphological Changes of Membranes Formed at the Interface}

The most interesting and biologically-relevant aspect of the study was when we then explored the morphological changes that were observed during some of these equilibrium and dynamic measurements. We had seen not only adsorption but actual growth of structures from the lung surfactant-adsorbed surface. These studies, carried out for pure phospholipid and the various protein and peptide containing formulations, demonstrated quite categorically that, especially the SMB peptide was causing massive rearrangement of adsorbed lipid layers at the air-water surface.

As the interface was trapped inside the tapered pipette and could be viewed under high magnification, we observed dramatic and complex morphological changes of the monolayers and subsequent membranes formed at the interface for the SMB + PL mixture. Figure 25 shows the kind of microtubule-formation and growth at the interface of this totally synthetic SMB formulation that was triggered by compression of the interface. Under increasing compression $(0.7,1$ and $2 \mathrm{kPa})$, microtubule-growth was only observed from SMB-containing lipid samples. The pure lipid $(0 \mathrm{wt} \%$ SMB) maintained a clean monolayer surface. However then, with increasing SMB concentration above $0.1 \mathrm{wt} \% \mathrm{SMB}$ in the mixture suspension $(1,2$, and $4 \mathrm{wt} \%)$, the lung surfactant showed a stronger and stronger tendency for tube-formation-activity and dynamics. Although not as dramatic, similar microtubule-forming behaviour was observed for the protein-containing Infasurf and NS, but not for the protein-free liposomes Survanta and Curosurf $[147,181]$.

Thus, the inclusion of protein in the commercial formulations, and especially the SMB peptide in Super Mini-B, was shown to be a key factor for the microtubule formation. While previous analysis had yielded some understanding [182], these new micropipette data gave a more detailed knowledge of how certain peptides (and proteins) interact (adsorb, intercalate, bond, and fuse) with lipids within lung surfactant multilayers. They provided a deeper physico-chemical understanding of mechanisms that drive their biological function.

Length and volumetric growth rates were calculated from the averaged slopes of plots of tube diameters, lengths, and volumes versus time of the microtubes growing from the surface lipid layers containing increasing SMB concentrations, from 0.1 to $4 \mathrm{wt} \%$. Data from this experiment are presented in Table 3. Measurements were made of several tubular structures whose growth from the surface membrane layers was visualised in real time inside the micropipette and are listed as a function of SMB content in the aqueous phase suspension of DPPC:POPC:POPG (50:30:20) liposomes. As seen in Table 3 , the length- and volume-growth rates were all positive and relatively consistent for each SMB concentration, at $2-3 \mu \mathrm{m} / \mathrm{s}$ in length and $20-30 \mu \mathrm{m}^{3} / \mathrm{s}$ in volume. However, there were relatively large standard deviations on the same order as their average sizes. Tube-growth measurements were made over time-periods of 60-120 s, and then, interestingly, the tubes tended to retract back and aggregate as more spherical structures. Tube-volumes were estimated by multiplying the instantaneous length by the apparent cross-sectional area of the tube, calculated from the measured diameters at the equatorial plane of each structure. 


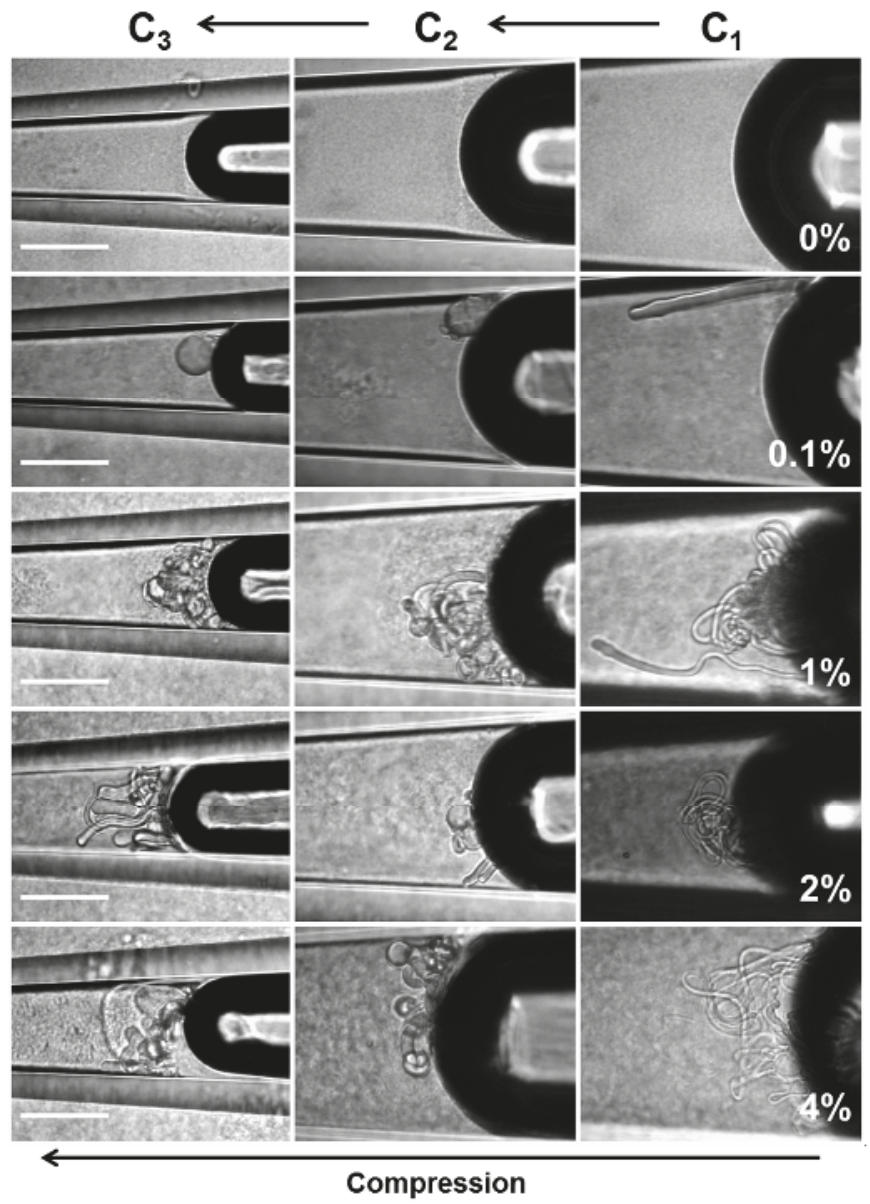

Figure 25. Microtubules formation and growth at $37^{\circ} \mathrm{C}$. Air-water surfaces are shown in the tapered micropipette for a series of increasing SMB concentrations from 0 to $4 \mathrm{wt} \%$ in the Phospholipid (PL) mixture solution. Each meniscus is moved down the micropipette taper by increasing the micropipette pressure from $0.7,1$ and $2 \mathrm{kPa}$, to give the sequential surface compressions $\mathrm{C} 1, \mathrm{C} 2$ and $\mathrm{C} 3$, respectively. In each case, compressions were made, and images were obtained after a $10 \mathrm{~min}$ waiting period. The scale bar is $50 \mu \mathrm{m}$. With permission from American Chemical Society [10].

Table 3. Diameters and linear and volumetric growth rates of the tubes in SMB + PL Samples. All the results are shown as the mean $\pm \mathrm{SD}$, calculated from all the tubes analysed for each composition. From [10].

\begin{tabular}{|c|c|c|c|c|}
\hline$\%$ SMB & \# Analysed Tubes & Thickness $(\mu \mathrm{m})$ & $\begin{array}{c}\text { Linear Growth } \\
\text { Rate }(\mu \mathrm{m} / \mathrm{s})\end{array}$ & $\begin{array}{l}\text { Volume Growth } \\
\text { Rate }\left(\mu \mathrm{m}^{3} / \mathrm{s}\right)\end{array}$ \\
\hline 0.1 & 5 & $4.46 \pm 1.85$ & $1.76 \pm 1.24$ & $28.37 \pm 28.37$ \\
\hline 1 & 15 & $3.41 \pm 1.07$ & $2.68 \pm 3.84$ & $22.38 \pm 25.11$ \\
\hline 2 & 20 & $4.73 \pm 2.19$ & $2.23 \pm 2.34$ & $26.65 \pm 14.10$ \\
\hline 4 & 21 & $3.48 \pm 1.24$ & $2.73 \pm 1.70$ & $22.14 \pm 12.95$ \\
\hline
\end{tabular}

One of the most astonishing observations in the micropipette experiments was how the adsorption of the SMB peptide-lipid system became a "living" multilamellar structure [10]. As shown in Figure 25, 
and in additional images in Figure 26, we saw inside the pipette that new tubular and then helical structures could grow out of the multilamellar stacks driven, we assume, by the peptide-lipid causing massive membrane membrane-adhesion, fusion and aggregation
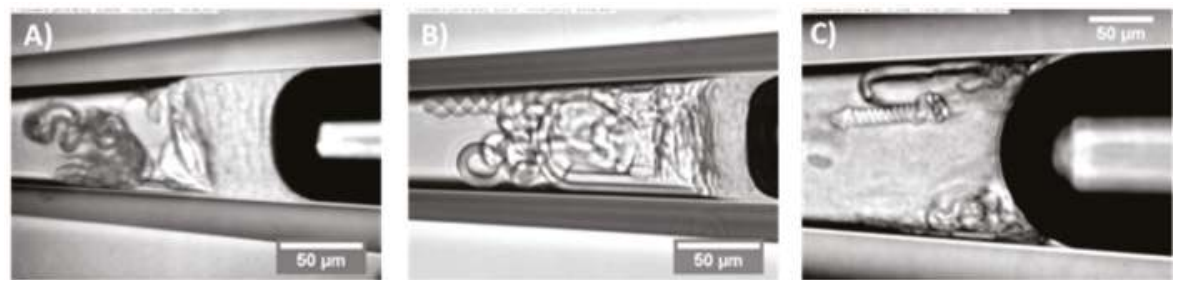

Figure 26. Video Micrographs showing presentative membrane structures observed in lipid-Minisurf samples. (A) Stacked and collapsed membrane multilayer layers beneath the interface; (B) protrusions and tubules growing from the surface layers, $(\mathbf{C})$ independent helical structures resulting from the coiling of membrane microtubes. Higher levels of material accumulation next to the interface led to an increased number of tubes and helixes developing towards the water side (see Supporting Information and videos [183]).

Interestingly, the peptide is overall positively charged and the inclusion of the negatively charged lipid POPG brings the possibility of close electrostatic interaction, charge-neutralisation between membranes and the distinct potential of bilayer-bilayer fusion. Thus, these peptide-lipid mixtures when presented to an air-water surface were indeed able to illicit membrane-membrane fusion and the complete rearrangement of the multi-bilayers into such relatively dehydrated and complex membrane structures. The reader is encouraged to go to the online version of the paper [10] and view the available videos [184,185] in Supporting Information [183].

The presence and dynamic-growth of these new lipid-peptide multilamellar and tubular structures could well be foundational to their action in vivo at the lung-air surface. Our observations show that the tubules and multilayers grow into the aqueous space from the air-side of the surface and, so in vivo, they could perhaps integrate and embed themselves into the lung epithelium. Their active growth could actually be the basis for new innovation for drug delivery that could penetrate into the lung epithelium and so deliver any encapsulated drug deeper into this interfacial tissue.

\subsection{Interfacial Tension is a Key Parameter in Nanoprecipitation}

This next application of interfacial tension measurement inside the tapered pipette was motivated by the need to measure the interfacial tensions of Triolein (TO) against ethanol-water mixtures, including in the presence of phospholipid, palmitoyl-oleoyl-phosphatidylcholine (POPC, C16:0-18:1). In a series of separate projects, we have been studying and characterising the nanoprecipitation of Triolein from organic solvent into the anti-solvent water. This rapid solvent-shifting technique forms the basis for our new thrust in anti-cancer drug delivery to metastatic tumours [186]. Cancers have an altered lipid-metabolic-reprogramming [187], over-express Low Density Lipoprotein Receptors (LDLR) [188] and take in more LDLs and albumin than normal cells, to the extent that a cancer patient's LDL- and albumin-counts can even go down [189]; LDL-uptake promotes aggressive phenotypes [190] resulting in proliferation and invasion in breast cancer [191], and an abundance of LDL-Receptors is a prognostic indicator of metastatic potential [192]. Inspired by the LDL and its biology-of-uptake by cancer cells, Needham's lab has developed a strategy we call, "Make the drug look like the cancer's food" [186] and are currently developing and testing new prodrug nanoparticles in cell and preclinical animal studies.

We have used Triolein and the POPC lipid as test materials with which to understand the fundamentals of such nanoprecipitation techniques. The key to understanding the process is to start with Classical Nucleation Theory (see excellent review by Karthika et al. [193] and applications to 
hydrophobic materials by Horn and Reiger [194]) and to measure the two main free parameters for a particular system. These are: the degree of supersaturation $(S)$ of TO in the EtOH/Water mixture in which it becomes supersaturated and therefore precipitates out; and the interfacial tension $(\gamma)$ of the $\mathrm{TO}$ at the same concentration. These two parameters, along with the molecular volume of $\mathrm{TO}\left(V_{m}\right)$ (and $k_{b} \mathrm{~T}$ ) give a direct measure of the critical radius $\left(r_{c}\right)$ of the precipitated nucleus material, such that,

$$
r_{c}=\frac{2 \gamma V_{m}}{k_{b} \operatorname{Tln} S} .
$$

In our drug delivery design, we coat the nanoparticle with a monolayer of lipid that stabilises the new nucleate and kinetically traps it at the minimum size. Interestingly, just like TO, the phospholipid is soluble in pure ethanol and in high \% ethanol in the ethanol-water mixture. At some point in the solvent exchange (i.e., exchange of the solvent ethanol for anti-solvent water), it also comes out of solution to form the monolayer around the precipitated TO (or drug) nucleate. This micropipette experiment then was designed to measure the interfacial tension of a series of $\mathrm{EtOH} /$ Water mixtures without and with the presence of the phospholipid POPC; the challenge was to measure the interfacial tension in the tapered micropipette for this liquid-liquid system where one of the components (ethanol) is volatile.

6.4.1. Tapered Micropipette Manipulation Technique for Volatile Oil-Water System (Water-Ethanol Mixtures)

In this experiment, we managed to maintain the starting ethanol concentrations in the ethanol water mixtures by producing liquid "plugs" that sealed the phase interfaces being measured inside the tapered pipette [16,17]. Figure 27 shows a schematic of the liquid-liquid equilibrium interfacial tension measurement system for Triolein measured against water-ethanol mixtures that also contains phospholipid. Thus, following the procedure in the figure legend, the aqueous solution is "trapped" between two oil solutions, $\mathrm{Oil}_{1}$ in the chamber and $\mathrm{Oil}_{2}$, by using a plug that is preloaded into the pipette to prevent evaporation of the ethanol component during the measurement. As illustrated, such a set-up contains at least three different interfaces, i.e., $\mathrm{Oil}_{1}$-water, water-Oil ${ }_{2}$, and $\mathrm{Oil}_{2}$-air that each can influence the way the applied pressure is dropped across the system, depending on their interfacial tensions and their respective radii. Therefore, dealing with this kind of system is not trivial (experimentally as well as theoretically).

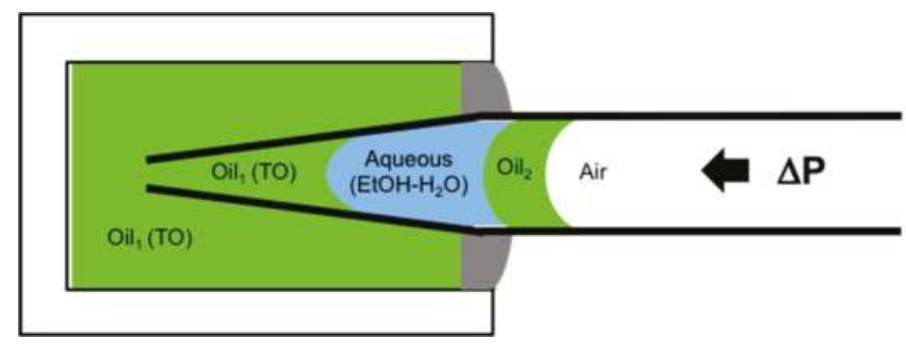

Figure 27. Schematic illustration of the equilibrium interfacial tension measurement of an oil-water interface in which one component is volatile $[16,17]$. In the set-up, the chamber is filled with the oil solution $\left(\mathrm{Oil}_{1}\right)$ Triolein. Then, the tapered micropipette, is prefilled with the same oil $\left(\mathrm{Oil}_{2}\right)$ and the water solution under investigation is also aspirated into the tip. The pipette is then inserted into the $\mathrm{Oil}_{1}$ under positive applied pipette-pressure in order to maintain the water and $\mathrm{Oil}_{2}$ plug inside the tapered micropipette. The $\mathrm{Oil}_{2}$ solution is used to avoid evaporation of water solution. Once again, control of applied pipette pressure drives the interface meniscus to measurable radii in the tapered pipette, and the application of the Laplace equation, Equation (5), gives the interfacial tensions. 
As reported in Utoft's thesis [16], and described in detail in [17], to obtain the interfacial tension of this particular Oil $1_{1}$-water interface, we needed to know the pressure difference for each of the three interfaces: Oil 1 -Water, $(O 1, W)$; Water-Oil $2 ;(\mathrm{W}, \mathrm{O} 2)$; and at the back of the micropipette, $\mathrm{Oil}_{2}$-Air $(O 2, A)$, and where, the corresponding pressure differences are $\Delta P_{\mathrm{O} 1, W}, \Delta P_{\mathrm{W}, \mathrm{O} 2}, \Delta P_{\mathrm{O} 2, A}$

It is instructive to go through this analysis for any application where multiple interfaces and components are included, such as may occur in a microfluidic system.

The pressure differences at each interface then are:

$$
\begin{gathered}
\mathrm{Oil}_{1}-\text { Water }: \Delta P_{\mathrm{O} 1, W}=P_{\mathrm{W}}-P_{\mathrm{O} 1}, \\
\text { Water }-\mathrm{Oil}_{2}: \Delta P_{W, \mathrm{O} 2}=P_{\mathrm{O} 2}-P_{W}, \\
\text { Oil }{ }_{2}-\text { Air }: \Delta P_{\mathrm{O}, A}=P_{A}-P_{\mathrm{O} 2} .
\end{gathered}
$$

Therefore, starting at the back of the pipette, the applied pressure going from the air inside of micropipette all the way to $\mathrm{Oil}_{1}$ in the chamber is the sum of these pressure differences as follows,

$$
\Delta P=\Delta P_{\mathrm{O} 2, A}+\Delta P_{W, O 2}+\Delta P_{\mathrm{O} 1, W}=P_{A}-P_{O 1}=\Delta P_{\mathrm{O} 1, A} .
$$

Following the Laplace equation for the tapered micropipette, Equation (5), the surface tension between water and $\mathrm{Oil}_{1}, \gamma_{\mathrm{O}, \mathrm{W}}$, is thus expressed as,

$$
\Delta P_{\mathrm{O}, W}=\Delta P-\left(\Delta P_{W, O 2}+\Delta P_{O 2, A}\right)=\frac{2 \gamma_{O 1, W}}{R_{O 1, W}} .
$$

Therefore, to obtain the $\gamma_{O 1, W}$ value for a single surface tension measurement, requires measuring the interfacial pressure of $\Delta P_{W, O 2}$ and $\Delta P_{\mathrm{O} 2, A}$. However, if the sum value, $\Delta P_{W, O 2}+\Delta P_{\mathrm{O} 2, \mathrm{~A}}$, of each pressure is constant, the surface tension of $\gamma_{\mathrm{O}, \mathrm{W}}$ can be calculated from the slope of $\Delta P$ vs. $2 / R_{O 1, W}$ plot (see Figures $7 \mathrm{c}$ and 9). The intercept at the Y-axis (at $2 / R_{O 1, W}=0$ ) is equal to the constant value of $\Delta P_{W, O 2}+\Delta P_{\mathrm{O} 2, \mathrm{~A}}$. Thus, if the intercept is zero, it means that $\Delta P_{W, O 2}+\Delta P_{\mathrm{O} 2, \mathrm{~A}}$ become zero, as in the liquid-gas interfacial tension result in Figure 7c. Therefore, to keep the value of $\Delta P_{\mathrm{W}, \mathrm{O} 2}+\Delta P_{\mathrm{O} 2, \mathrm{~A}}$ constant during the measurement, the two interfaces of water-Oil 2 , and $\mathrm{Oil}_{2}$-air must be kept in the non-tapered (large-diameter-bore, $450 \mu \mathrm{m}$ ) micropipette section during the measurement. Hence, the non-tapered part is quite large compared to the tapered pipette where the measurements are made, making the pressure drop at this diameter comparatively low to maintain an interface in this region of the pipette. Therefore, both interfaces are kept in the constant inner diameter section by adjusting the volume of water solution in the pipette and applying the appropriate pressure. As is quite obvious then, in order to obtain the $\gamma_{O 1, W}$ value, the interface under measurement, of $\mathrm{Oil}_{1}$-water, has to always be in the tapered section of the micropipette tip.

\subsubsection{Lipid Adsorption at the Triolein-Ethanol/Water Interface}

This new advanced tapered micropipette manipulation technique was then used to measure the Triolein-water interfacial tension and to investigate palmitoyl-oleoyl-phosphatidylcholine (POPC, C16:0-18:1) monolayer formation at the same Triolein-water interface. Triolein is an unsaturated triglyceride, having three oleic acid (C18:1) acyl chains and so forms an immiscible interface with water with perhaps an oriented monolayer of Triolein at this interface, but itself it is not that surface active and so can easily be replaced by adsorbing phospholipid. Figure 28 shows the interfacial tension between Triolein and ethanol-water mixtures in the absence and presence of $1 \mathrm{mM}$ POPC.

For pure Triolein against increasing ethanol concentration in the ethanol-water mixture, the interfacial tension of the oil-water interface showed a smooth decrease from $\gamma=31.1 \pm 0.1 \mathrm{mN} / \mathrm{m}$ for the pure water to $1.3 \pm 0.1 \mathrm{mN} / \mathrm{m}$ for pure ethanol. Hence, Triolein forms an immiscible phase boundary with a finite surface tension against water, and against ethanol. As mentioned in Section 3, short-chain alcohols can themselves decrease the interfacial tension by forming a monolayer that then reduces the Triolein interfacial tension. 


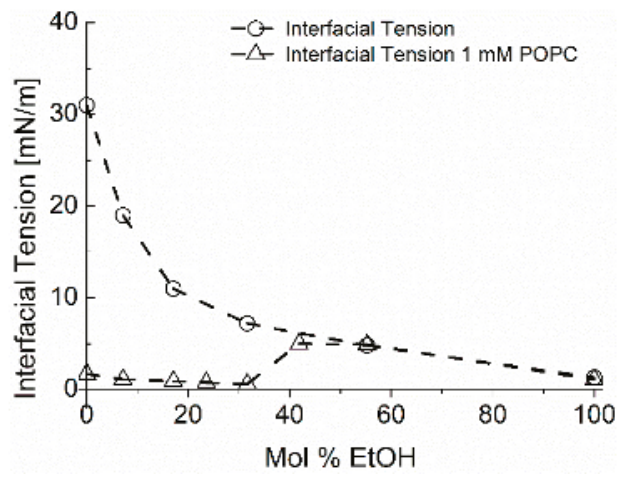

Figure 28. Interfacial tension of Triolein vs. ethanol-water mixtures across the entire binary regime in absence and presence of $1 \mathrm{mM}$ palmitoyl-oleoyl-phosphatidylcholine (POPC). Open circles show the interfacial tension of Triolein against water-ethanol with increasing ethanol $\mathrm{mol} \%$. Open triangles show the measured interfacial tension of Triolein against water-ethanol in the presence of $1 \mathrm{mM}$ POPC, present as liposomes in the aqueous solution. The sharp bend for the interfacial tension in the presence of POPC at around $40 \mathrm{~mol} \%$ ethanol represents the limit of solubility of POPC in the ethanol-water mixture.

When POPC was mixed in the water, the interfacial tension was dramatically reduced from $31 \mathrm{mN} / \mathrm{m}$ for the pure Triolein-water interface to $1.7 \mathrm{mN} / \mathrm{m}$ for the formed POPC monolayer. Thus, as we have seen before for lipids at air-water and now oil-water interfaces, POPC is a very effective way to reduce the oil-water interfacial tension by forming a POPC monolayer. This value is similar to that measured by Needham and Haydon for GMO at the water-Triolein interface of $1.82 \mathrm{mN} / \mathrm{m}$ [1]. With increasing ethanol concentration in the water-ethanol mixture the interfacial tension was even further decreased to just $0.6 \mathrm{mN} / \mathrm{m}$, showing that there was some influence of adsorbed ethanol along with the phospholipid. Then, at a critical ethanol concentration of between $32-42 \mathrm{~mol} \%$, the interfacial tension actually rose to a value of $5.0 \pm 0.1 \mathrm{mN} / \mathrm{m}$, coincident with the lipid-free interface. Above $55 \mathrm{~mol} \%$ ethanol, the interfacial tension followed the exact same trend as in the system without POPC present.

What this interfacial tension behaviour therefore seems to indicate is a limiting solubility of POPC in ethanol water, i.e., at ethanol concentrations below $42 \mathrm{~mol} \%$ the POPC is still insoluble in this mainly aqueous mixture, but above 42 to $45 \mathrm{~mol} \%$ ethanol, the liposomes are solubilised in the monomer state [195]. Once dissolved, the POPC does not account for any further adsorption to the interface. Rather, ethanol, having a much higher surface activity than the dissolved lipids [196], replaces POPC molecules at the Triolein surface and take over the role of the main surface-active compound by this exchange. Thus, in any microfluidic or other interfacial systems, it is necessary to quantify these tensions as a function of solvent mixtures and determine which of the potentially surface active components is actually adsorbed and determining the surface or interfacial tension. Once again, this is where the micropipette technique can isolate individual components and make these crucial measurements.

\subsection{Microsurface Tensions and Droplet Dissolution for Microfluidics}

Finally, not only can we use the pipettes as a tapered tube for surface and interfacial tension measurements, the micropipette manipulation technique is capable of forming and making fundamental measurements and analyses for single (individual) particles. Such an example was given previously for the water-in-oil experiments of Yeung, but we have many studies and papers on this aspect of the pipette technique including droplet dissolution of one immiscible phase into another, as well as precipitation and crystallisation from such concentrating solutions [12,95,164,165,168,197-199]. These 
kinds of experiments are critical for establishing processing parameters in systems such as microfluidics and homogenisation. In order to demonstrate what can be achieved at the single particle level in the case of microfluidic processing, we present here a brief description of some experiments we carried out and reported in a paper directed at this same audience, entitled, "From Single Microparticles to Microfluidic Emulsification: Fundamental Properties (Solubility, Density, Phase Separation) from Micropipette Manipulation of Solvent, Drug and Polymer Microspheres" [7], as well as in other studies and reports that form a series of industrial collaborations (not published). It is here that interfacial tensions can change as a result of adsorption of surfactants and polymers, or drugs, and these materials can themselves dissolve into the second (usually) aqueous phase. These experiments also give a measure of the diffusion coefficients of each component, and provide a correlative measure of diffusion that has agreed with dynamic surface tension measurements we have also made with the micropipette technique, as described earlier in Section 4.4.1. for octanol.

One good example where interfacial tensions, droplet dissolution, and drug and polymer precipitation are combined is microfluidic processing of polymer microspheres for drug encapsulation. In these systems one of the most used and studied materials is that of a biodegradable PLGA polymer particle, where, in processing, drug is co dissolved in the PLGA-solvent (usually Dichloromethane) solution. What we have provided with the micropipette technique [18], is:

- An ability to measure all relevant interfacial tensions for each component that tend to be unique to the industrial process and so are not readily obtained from the literature,

- Make individual particles as a function of composition,

- Observe any emerging microstructures inside or on the particles,

- Make measurements of fundamental properties like mechanical deformation of these materials at the single microparticle level and,

- Measure any dissolution (e.g., diffusion coefficients) into the suspending medium.

As discussed by de Bruijn et al. [200], an accurate measurement of interfacial tension is important for setting the right parameters for microfluidic droplet formation. The process of microfluidic emulsification involves the injection of two solutions: the dispersed solvent phase (the liquid to form the droplet containing the polymer and any API, flow rate Qd); and the aqueous continuous phase (the carrier liquid surrounding the droplet, also called the dissolution medium, flow rate Qc). As shown in Figure 29, the dispersed phase is injected through the central inlet, indicated as (ii), which is continuous with a cross-junction geometry that connects it to the outer cross-inlet, labelled as (i), through which the continuous phase flows.

The dispersed phase is pumped into a micro-channel and enters the cross junction, 'hydrodynamic flow focusing' of the continuous phase breaks the polymer solution into microdroplets. Thus, parameters such as viscosity, $\mu$, flow rate, $Q$, and interfacial tension, $\gamma$, become important in how and at what rate droplets form. The key is a precise control over the flow rate $Q_{c}$ and $Q_{d}$ from the inlet channels (Figure 29a), and control of the process lies in the force-balances at the intersection: the balance between interfacial and viscous forces (characterised by the capillary number of dispersion phase, $C_{a d}$ ); and the balance between the dispersed phase and the continuous phase that is forced into the same outlet (characterised by the ratio of flow rates $Q_{d} / Q_{c}$ ). The dimensionless parameter of $Q_{d} / Q_{c}$ is often cited to show the profile of characterised microdroplet distribution such as the flow map (Figure 29b). In region A, the produced microparticles become nicely spherical. However, in region B the microdroplets become non-uniform and in $\mathrm{C}$ can be larger than the exit channel.

It is here where a knowledge of the interfacial tension $\gamma$ is required for the two solutions used in the device. For the process of creating an emulsion it is preferred that the ratio of the dispersed phase flow rate $Q_{d}$ to the continuous phase flow rate $Q_{c}$ is $\leq 0.00272 \mathrm{Oh}^{*}$, where $\mathrm{Oh}^{*}$ is the Ohnesorge number of the system, and is inversely proportional to the interfacial tension $\gamma$. 


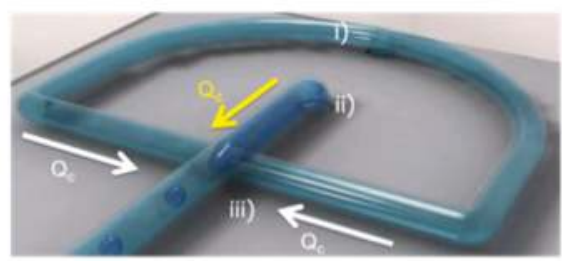

(a)

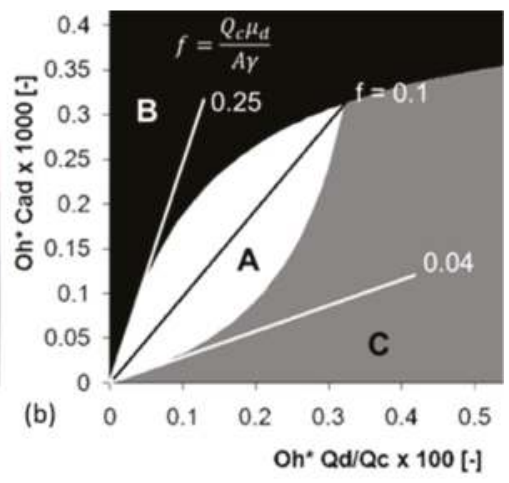

Figure 29. The microfluidic system and flow parameters that determine effective droplet formation. (a) Schematic 3D picture of droplet formation showing the arrangement of the two inlet tubes: (i) continuous phase inlet that then splits into two tubes; (ii) dispersed phase inlet; (iii) mixing head. (bottom) High-speed microscope image of the single-channel production window. (b) Flow map, depicting all combinations of liquid properties, microchannel size and flow rates. Combinations in the white area (region A) yield the desired process, i.e., spherical particles; combinations in the black area (region B) lead to instabilities and non-uniform droplets; and, in the grey area (region C), to droplets larger than the exit channel. From Kinoshita et al. [7].

The interfacial tension is clearly expected to depend on the nature and the concentrations of each component and so needs to be measured at their microscale interfaces for each solvent-polymer-drug-aqueous solution systems including with and without the stabilising surfactant polyvinyl pyrrolidine (PVA). For example, for control systems of pure solvents against the aqueous phase, such as DCM vs. water, the interfacial tension was measured to be $31.4 \mathrm{mN} / \mathrm{m}$. The inclusion of $1 \mathrm{mM}$ SDS in the aqueous phase to help with droplet colloid-stability, as expected, lowered this interfacial tension to $16.9 \mathrm{mN} / \mathrm{m}$. For example, the inclusion of a particular drug (proprietary Pharma compound) at $25 \mathrm{mg} / \mathrm{mL}$ in the DCM vs. $1 \mathrm{mM}$ SDS showed an average surface tension, of $13.4 \mathrm{mN} / \mathrm{m}$. The interfacial tension of a $20 \mathrm{mg} / \mathrm{mL}$ PLGA and $2 \mathrm{mg} / \mathrm{mL}$ of an AI compound mixed in the final solvent mixture of DCM:DMSO (8:2) against water was $7.0 \pm 0.2 \mathrm{mM} / \mathrm{m}$ but $10.5 \pm 0.2 \mathrm{mN} / \mathrm{m}$ with $0.1 \%$ PVA added. These are the interfacial measurements then that allow parameters to be set to achieve effective microdroplet formation in the microfluidic device.

As mentioned, a knowledge of the dissolution time of microdroplets is also important in the latter stages of microsphere formation. Our micropipette techniques have also therefore been used to determine this time, as well as measures of the diffusion coefficients of solvents and also drugs. For example, the diffusion coefficient of DCM in the aqueous phase was measured by droplet dissolution and applying the EP Equation (8), to be $D=1.77 \pm 0.01 \times 10^{-5} \mathrm{~cm}^{-2} / \mathrm{s}$, while the diffusion coefficient of the drug Ibp in PBS ( $\mathrm{pH}$ 7.4) at room temperature, was three times smaller at $D=5.5 \pm 0.2 \times 10^{-6} \mathrm{~cm}^{2} / \mathrm{s}$. That is, for a DCM-Ibp solution microdroplet, once the DCM had all dissolved, the pure drug continued to dissolve but at a much slower rate. Additionally, the time, $t D$, for a given diameter of a spherical microparticle of Ibp to dissolve in an infinite medium is given by the following equation derived from the EP equation $[12,18]$,

$$
t_{D}=\frac{R_{o}^{2}}{2 D} \frac{\rho}{C_{s}},
$$

where, $R_{o}$ is the initial radius of the particle, $\rho$ is the density of the $\mathrm{Ibp}(1030 \mathrm{mg} / \mathrm{mL}), D$ is the diffusion coefficient of the molecule in aqueous media $\left(5.5 \pm 0.2 \times 10^{-6} \mathrm{~cm}^{2} / \mathrm{s}\right)$, and $C_{S}$ is its solubility limit in PBS (pH7.4) buffer, $0.825 \mathrm{mg} / \mathrm{mL}(4 \mathrm{mM})$. This equation successfully predicted the measured dissolution time of a pure Ibp microparticle of diameter of $16.4 \mu \mathrm{m}$, as $\sim 75 \mathrm{~s}$ and dissolution rate of 
30 femtogram/s or 1.9 picograms/min [18]. This calculation shows how important it is in applications of pure drug dissolution to not only know the size of the drug microparticles but to actually control it. Using the micropipette technique, the dissolution rates for pure drug microparticles can be measured in water, buffer, surfactant solution or biological fluids (e.g., synovial fluid or blood plasma).

Thus, by simply using the dissolution time equation derived from the EP model, we can provide our microfluidic collaborators with a measure of the time needed in the microfluidic processes for solvent, drug, or polymer dissolution and how this changes as a function of any processing parameter. These may include any composition change in the organic-polymer-drug solution or the addition of stabilising surfactants in the aqueous phase. It can be done for a range of microparticle diameters from 5 to over $100 \mu \mathrm{m}$. In one application, we showed that the dissolution time of a very hydrophobic drug meant that the formulation did not in fact need a polymer-releasing system. EP and $t_{D}$ calculations simply showed that for a microsphere diameter size of $2 \mu \mathrm{m}$, it would take only $2.1 \mathrm{~min}$ for complete dissolution. However, for a $600 \mu \mathrm{m}$ diameter microsphere (300 times larger diameter) it would take a much longer time, on the order of 6 months. The required specifications of the formulation was a release (or now dissolution) time of $\sim 6$ months, and so this was achieved with just drug microparticles. Such a formulation was superior to any PLGA encapsulation where the PLGA itself would be degraded in few weeks to a month.

\section{Summary and Conclusions}

In this review paper then, we have presented a series of the micropipette techniques that we have developed and used to make measurements of the surface and interfacial tensions for clean air-water surfaces and oil-water interfaces. By using a tapered micropipette and introducing rapid image monitoring of the position of meniscus in the micropipette and hence its radius of curvature for a given applied micropipette pressure, we have presented a series of equilibrium tensions as well as equilibrium and dynamic tensions due to the adsorption of water-soluble surfactants and water-insoluble and lipids. Micropipette dimensions associated with the capillary tip are $\sim 5-10 \mu \mathrm{m}$, and the micropipette can taper out to $450 \mu \mathrm{m}$, thus, importantly all measurements are actually made at the microscale. Following the Young-Laplace equation and geometry of the capillary, the surface or interfacial tension value is simply obtained from the radius of the meniscus in the tapered pipette and the applied pressure to keep it there.

As an interesting prelude to the comprehensive description of these experimental techniques and their theoretical analysis we also provided a brief potted-historical perspective that included Franklin's early experiments that demonstrated molecularity and monolayer formation, fundamental surfactancy driven by margarine, the first use of a micropipette to (circuitously) measure bilayer membrane tensions and free energies of formation, and how this black lipid film concept formed basis for study and applications of membrane ion-channels in Droplet Interface Bilayers and beyond, into printed microdroplet "tissues."

To address the goals of the special issue we selected five examples of where our measurements have had an impact on applications in micro-surfaces and microfluidics, including gas microbubbles for ultrasound contrast; interfacial tensions for micro-oil droplets in oil recovery; surface tensions and tensions-in-the surface for natural and synthetic lung surfactants; interfacial tension in nanoprecipitation; and micro-surface tensions in microfluidics. The micropipette technique that was originally designed and built to study the properties of biological cells (red and white blood cells and Giant Unilamellar-Lipid Vesicles) by Evans, Kwok and Needham, and Chien, Skalak, Schmidt-Shoenbein and co-workers, has now found new and myriad utility in making as well as characterising individual and pairs of micro colloids and surfaces, and is an essential tool for anyone studying, designing or using Micromachines for science and technology of small structures, devices and systems. We have tried to show how important and versatile this micropipette technology can be especially as soft matter is miniaturised, surface areas become huge, and their surface energies become major drivers of structural and material transformations in micro- and nano-systems. As we have 
also tried to show, equilibrium surface and interfacial tension data has also to be coupled to dynamic processes, such as adsorption of simple surfactants or more complex insoluble materials like lipids (and this would also include future studies on surface and interfacial polymers and proteins), and the dissolution by diffusion (or convection) of the tiny microscale materials (often in the volumes of picoand femto-litres), characterised by diffusion in unique and perhaps not-already-known solvent systems.

Even though it is a very long document, we think that the whole structure and flow brings a readable and educational/informative review of these important techniques. It takes us from a historical perspective that includes a personalised approach to some aspects of surfactancy, through the basic techniques we have developed and a series of five applications that we hope are of interest to the Micromachines readership (and beyond). We therefore offer this unique capillaryand single-particle-manipulating techniques to the Micromachines, and Microsurfaces community to help enhance the development, design, and testing of various applications such as the stated: "microfluidic and lab-on-chip devices, soft gripping and manipulation of particles, colloidal and interfacial assemblies, fluidic/droplet mechatronics," and the fundamentals or applications yet to be explored.

Acknowledgments: We gratefully acknowledge the generous support of the Danish National Research Foundation through a Niels Bohr Professorship Award to D. Needham, Centre for Single Particle Science and Engineering, grant project No. 232149.

Dedication: This article is dedicated to Needham's two most influential mentors, D. A. Haydon FRS and E. A. Evans. Evans was also mentor to Kinoshita. We owe them so much. Thank you.

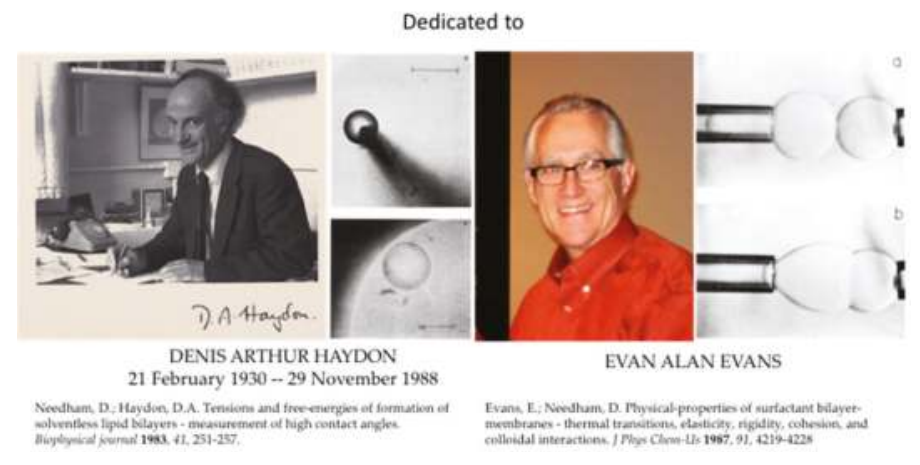

Funding: This research was funded in part by the Danish National Research Foundation through a Niels Bohr Professorship Award to David Needham, Center for Single Particle Science and Engineering, grant project no. 232149 (2013-2017). The rest, from 1982-2013, were funded by various grants including: Oppenheimer Research Fellowship, Physiological Laboratory, Cambridge University (1982-1983); NIH (FIRST Award) GM 40162 (1998-1999); NSF, NSF-BCS-91-06452 (1991-1993); and industrial contracts from Molecular Express Incorporated, Los Angeles, CA, USA; EmulTech, Eindhoven, NL.

Author Contributions: D.N. wrote and finalised the paper with assistance from K.K., and A.U.

Conflicts of Interest: The authors declare no commercial associations or any conflicts of interest.

\section{References}

1. Needham, D.; Haydon, D.A. Tensions and free-energies of formation of solventless lipid bilayersmeasurement of high contact angles. Biophys. J. 1983, 41, 251-257. [CrossRef]

2. Honaker, L.W.; Lagerwall, J.P.F.; Jampani, V.S.R. Microfluidic Tensiometry Technique for the Characterization of the Interfacial Tension between Immiscible Liquids. Langmuir 2018, 34, 2403-2409. [CrossRef] [PubMed]

3. Metz, T.; Paust, N.; Zengerle, R.; Koltay, P. Capillary driven movement of gas bubbles in tapered structures. Microfluid. Nanofluidics 2010, 9, 341-355. [CrossRef]

4. Renvoisé, P.; Bush, J.W.M.; Prakash, M.; Quéré, D. Drop propulsion in tapered tubes. Epl (Europhys. Lett.) 2009, 86, 64003 . 
5. Zhou, H.; Yao, Y.; Chen, Q.; Li, G.; Yao, S. A facile microfluidic strategy for measuring interfacial tension. Appl. Phys. Lett. 2013, 103, 234102. [CrossRef]

6. Lee, S.; Kim, D.H.; Needham, D. Equilibrium and Dynamic Interfacial Tension Measurements at Microscopic Interfaces Using a Micropipet Technique. 2. Dynamics of Phospholipid Monolayer Formation and Equilibrium Tensions at the Water-Air Interface. Langmuir 2001, 17, 5544-5550. [CrossRef]

7. Kinoshita, K.; Parra, E.K.; Needham, D. New Sensitive Micro-Measurements of Dynamic Surface Tension and Diffusion Coefficients: Validated and Tested for the Adsorption of 1-Octanol at a Microscopic Air-Water Interface and its Dissolution into Water. J. Colloid Interface Sci. 2017, 488, 166-179. [CrossRef]

8. Kinoshita, K.; Parra, E.; Needham, D. Adsorption of ionic surfactants at microscopic air-water interfaces using the micropipette interfacial area-expansion method: Measurement of the diffusion coefficient and renormalization of the mean ionic activity for SDS. J. Colloid Interface Sci. 2017, 504, 765-779. [CrossRef]

9. Lee, S.; Kim, D.H.; Needham, D. Equilibrium and Dynamic Interfacial Tension Measurements at Microscopic Interfaces Using a Micropipet Technique. 1. A New Method for Determination of Interfacial Tension. Langmuir 2001, 17, 5537-5543. [CrossRef]

10. Parra, E.; Kinoshita, K.; Needham, D. Micropipette Technique Study of Natural and Synthetic Lung Surfactants at the Air-Water Interface: Presence of a SP-B Analog Peptide Promotes Membrane Aggregation, Formation of Tightly Stacked Lamellae, and Growth of Myelin Figures. Langmuir 2016, 32, 10570-10581. [CrossRef]

11. Yeung, A.; Dabros, T.; Masliyah, J.; Czarnecki, J. Micropipette: A new technique in emulsion research. Colloid Surf. A 2000, 174, 169-181. [CrossRef]

12. Duncan, P.B.; Needham, D. Test of the Epstein-Plesset model for gas microparticle dissolution in aqueous media: Effect of surface tension and gas undersaturation in solution. Langmuir 2004, 20, 2567-2578. [CrossRef] [PubMed]

13. Kim, D.H.; Costello, M.J.; Duncan, P.B.; Needham, D. Mechanical properties and microstructure of polycrystalline phospholipid monolayer shells: Novel solid microparticles. Langmuir 2003, 19, 8455-8466. [CrossRef]

14. Kim, D.H.; Klibanov, A.L.; Evans, E.A.; Needham, D. Viscoelastic properties of phospholipid monolayer shells on air microbubbles. Biophys. J. 1998, 74, A313.

15. Needham, D.; Walke, P.; Utoft, A.; Hervella, P.; Kinoshita, K. Nucleation, Growth and Characterization of Triolein Nanoparticles Prepared in the Absence and Presence of Phospholipids by Rapid Solvent Shifting Technique. 2019; unpubished data, in preparation.

16. Utoft, A. Micropipette Manipulation Studies: Material Characterization of Multiphase, Multicomponent Systems; University of Southern Denmark: Odense, Denmark, 2017.

17. Utoft, A.; Kinoshita, K.; Needham, D. Characterization of the ternary triolein-water-ethanol system: Solubility, Interfacial tension and supersaturation. Langmuir 2018, submitted.

18. Kinoshita, K.; Parra, E.; Hussein, A.; Walke, P.; Bruijn, R.D.; Needham, D. Single Particle Science and Engineering Guiding Scale-Up: Microparticle Formation and Dissolution of Solvent-Emulsion, PLGA, Ibuprofen, and Ibuprofen/PLGA Microspheres. Processes 2016, 4, 49. [CrossRef]

19. Franklin, B.; Brownrigg, W. Of the stilling of waves by means of oil. Extracted from sundry letters between Benjamin Franklin, LL. D. F. R. S. William Brownrigg, M.D.F.R.S. and the Reverend Mr. Farish. Phil. Trans. 1774, 64, 445-460.

20. Mertens, J. Oil on troubled waters: Benjamin Franklin and the honor of Dutch Seamen. Phys. Today 2006, 59, 36. [CrossRef]

21. Tanford, C. The Hydrophobic Effect: Formation of Micelles \& Biological Membranes; John Wiley and Sons, Inc.: New York, NY, USA, 1980.

22. Wikipedia. William Bate Hardy. Available online: https://en.wikipedia.org/wiki/William_Bate_Hardy (accessed on 30 January 2019).

23. Hardy, W.B. The tension of composite fluid surfaces and the mechanical stability of films of fluid. PR Soc Lond A-Conta 1912, 86, 610-635. [CrossRef]

24. Unilever. Our History. Available online: https://www.unilever.co.uk/about/who-we-are/our-history/ (accessed on 30 January 2019).

25. Matijevic, E.; Pethica, B.A. The Properties of Ionized Monolayers. 1. Sodium Dodecyl Sulphate at the Air-Water Interface. Trans. Faraday Soc. 1958, 54, 1382-1389. [CrossRef] 
26. Mingins, J.; Zobel, F.G.; Pethica, B.A.; Smart, C. Potential differences due to spread monolayers at the polar oil/water interface. Proc. R. Soc. Lond. A. Math. Phys. Sci. 1971, 324, 99. [CrossRef]

27. Mingins, J.; Pethica, B.A. Properties of Monolayers at the Air/Water Interface. Part 2? Effect of Temperature on the Double Layer and Water Dipole Contributions to the Surface Potential of Sodium Octadecyl Sulphate. J. Chem. Soc. Faraday Trans. 1 1973, 69, 500-513. [CrossRef]

28. Taylor, J.A.G.; Mingins, J.; Pethica, B.A.; Tan, B.Y.J.; Jackson, C.M. Phase changes and mosaic formation in single and mixed phospholipid monolayers at the oil-water interface. Biochim. Et Biophys. Acta (Bba) Biomembr. 1973, 323, 157-160. [CrossRef]

29. Mingins, J.; Owens, N.F.; Taylor, J.A.; Brooks, J.H.; Pethica, B.A. Entropies of Compression of Charged Monolayers at Aqueous Interfaces. In Monolayers; Goddard, E.D., Ed.; ACS: Washington, DC, USA, 1975; Volume 144.

30. Pethica, B.A.; Standish, M.M.; Mingins, J.; Smart, C.; Iles, D.H.; Feinstein, M.E.; Hossain, S.A.; Pethica, J.B. The Significance of Volta and Compensation States and the Measurement of Surface Potentials of Monolayers. Monolayers 1975, 144, 123-134. [CrossRef]

31. Pethica, B.A.; Mingins, J.; Taylor, J.A.G. Phospholipid interactions in monolayers. J. Colloid Interface Sci. 1976, 55, 2-8. [CrossRef]

32. Taylor, J.A.G.; Mingins, J.; Pethica, B.A. Phospholipid monolayers at the n-heptane/water interface. Part 2.-Dilute monolayers of saturated 1,2-diacyl-lecithins and -cephalins. J. Chem. Soc. Faraday Trans. 1 Phys. Chem. Condens. Phases 1976, 72, 2694-2702. [CrossRef]

33. Yue, B.; Jackson, C.; Taylor, J.A.G.; Mingins, J.; Pethica, B.A. Phospholipid monolayers at nonpolar oil/water interfaces: Phase transition in distearoyl-lecithin films at the n-heptane aqueous sodium chloride interface. J. Chem. Soc. Faraday Trans. 1976, 72, 2685-2693. [CrossRef]

34. Hall, D.G.; Pethica, B.A. Thermodynamics of the Volta effect for surface films. Proc. R. Soc. Lond. A. Math. Phys. Sci. 1977, 354, 425. [CrossRef]

35. Pethica, B.A.; Glasser, M.L.; Mingins, J. Intermolecular forces in monolayers at air/water interfaces. J. Colloid Interface Sci. 1981, 81, 41-51. [CrossRef]

36. Middleton, S.R.; Pallas, N.R.; Mingins, J.; Pethica, B.A. Thermodynamics of Ionized Monolayers: Surface Manometry on Very Low Density Spread Monolayers of Sodium Octadecyl Sulfate at the Air/Water Interface and Analysis of Ionic Double Layer Contributions to the Isotherms. J. Phys. Chem. C 2011, 115, 8056-8063. [CrossRef]

37. Keynes, R.D. Denis Arthur Haydon, 21 February 1930-29 November 1988. Biogr. Mems Fell. R. Soc. 1990, 36, 199-216.

38. White, S.H. Formation of "solvent-free" black lipid bilayer membranes from glyceryl monooleate dispersed in squalene. Biophys. J. 1978, 23, 337-347. [CrossRef]

39. Taylor, J.; Haydon, D.A. Stabilization of thin films of liquid hydrocarbon by alkyl chain interaction. Disc. Faraday Soc. 1966, 42, 51. [CrossRef]

40. Requena, J.; Billett, D.F.; Haydon, D.A. Van der Waals forces in oil-water systems from the study of thin lipid films. I. Measurement of the contact angle and the estimation of the van der Waals free energy of thinning of a film. Proc. Roy. Soc. Lond. A. Math. Phys. Sci. 1975, 347. [CrossRef]

41. Gruen, D.W.R. A mean-field model of the alkane-saturated lipid bilayer above its phase transition. I. Development of the model. Biophys. J. 1981, 33, 149-166. [CrossRef]

42. Gruen, D.W.R.; Haydon, D.A. A mean-field model of the alkane-saturated lipid bilayer above its phase transition. II. Results and comparison with experiment. Biophys. J. 1981, 33, 167-188. [CrossRef]

43. Bayley, H.; Cronin, B.; Heron, A.; Holden, M.A.; Hwang, W.L.; Syeda, R.; Thompson, J.; Wallace, M. Droplet interface bilayers. Mol. Biosyst. 2008, 4, 1191-1208. [CrossRef] [PubMed]

44. Holden, M.A.; Needham, D.; Bayley, H. Functional bionetworks from nanoliter water droplets. J. Am. Chem. Soc. 2007, 129, 8650-8655. [CrossRef] [PubMed]

45. Bayley, H.; Holden, M.; Heron, A.; Needham, D. Formation of Bilayers of Amphpathic Molecules. WO 2008/012552, 2008.

46. Sarles, S.A.; Leo, D.J. Tailored Current-Voltage Relationships of Droplet-Interface Bilayers Using Biomolecules and External Feedback Control. J. Intell. Mater. Syst. Struct. 2009, 20, 1233-1247. [CrossRef]

47. Needham, D. Lipid structures: A brief history of multisomes. Nat. Nanotechnol. 2011, 6, 761-792. [CrossRef] [PubMed] 
48. Villar, G.; Graham, A.D.; Bayley, H. A tissue-like printed material. Science 2013, 340, 48-52. [CrossRef] [PubMed]

49. Villar, G.; Heron, A.J.; Bayley, H. Formation of droplet networks that function in aqueous environments. Nat. Nanotechnol. 2011, 6, 803-808. [CrossRef] [PubMed]

50. Trantidou, T.; Friddin, M.S.; Salehi-Reyhani, A.; Ces, O.; Elani, Y. Droplet microfluidics for the construction of compartmentalised model membranes. Lab A Chip 2018, 18, 2488-2509. [CrossRef] [PubMed]

51. Challita, E.J.; Najem, J.S.; Monroe, R.; Leo, D.J.; Freeman, E.C. Encapsulating Networks of Droplet Interface Bilayers in a Thermoreversible Organogel. Sci. Rep. 2018, 8, 6494. [CrossRef] [PubMed]

52. Hochmuth, R.M.; Mohandas, N. Uniaxial loading of the red-cell membrane. J. Biomech. 1972, 5, 501-509. [CrossRef]

53. Evans, E.A. A new material concept for the red cell membrane. Biophys. J. 1973, 13, 926. [CrossRef]

54. Evans, E.A. New Membrane Concept Applied to the Analysis of Fluid Shear- and Micropipette-Deformed Red Blood Cells. Biophys J. 1973, 13, 941-954. [CrossRef]

55. Skalak, R.; Tozeren, A.; Zarda, R.P.; Chien, S. Strain Energy Function of Red Blood Cell Membranes. Biophys. J. 1973, 13, 245-264. [CrossRef]

56. Evans, E.; Hochmuth, R.M. A solid-liquid composite model of the red blood cell membrane. J. Membr. Biol. 1977, 30, 351-362. [CrossRef] [PubMed]

57. Chien, S.; Sung, K.L.; Skalak, R.; Usami, S.; Tözeren, A. Theoretical and experimental studies on viscoelastic properties of erythrocyte membrane. Biophys. J. 1978, 24, 463-487. [CrossRef]

58. Evans, E.; Hochmuth, R.M. Mechanochemical Properties of Membranes. In Current Topics in Membranes and Transport; Bonner, F., Kleinzeller, A., Eds.; Academic Press: New York, NY, USA, 1978; Volume 10, p. 1.

59. Evans, E.; Skalak, R. Mechanics and Thermodynamics of Biomembranes; CRC: Boca Raton, FL, USA, 1980.

60. Tözeren, H.; Chien, S.; Tözeren, A. Estimation of viscous dissipation inside an erythrocyte during aspirational entry into a micropipette. Biophys. J. 1984, 45, 1179-1184. [CrossRef]

61. Meiselman, H.J.; Lichtman, M.A.; LaCelle, P.L. White cell mechanics: basic science and clinical aspects. In Proceedings of the a Symposium Held at the Kroc Foundation, Santa Ynez Valley, CA, USA, 2-6 May 1984.

62. Dong, C.; Skalak, R.; Sung, K.L.P.; Schmid-Schonbein, G.W.; Chien, S. Passive deformation analysis of human leukocytes. J. Biomech. Eng. 1988, 110, 27-36. [CrossRef] [PubMed]

63. Evans, E. Micromethods for measurement of deformability and adhesivity properties of blood cells and synthetic membrane vesicles. In Physical Basis of Cell-Cell Adhesion; Bongrand, P., Ed.; CRC: Boca Raton, FL, USA, 1988.

64. Sung, K.; Dong, C.; Schmid-Schoenbein, G.W.; Chien, S.; Skalak, R. Leukocyte relaxation properties. Biophys. J. 1988, 54, 331. [CrossRef]

65. Needham, D.; Armstrong, M.; Hatchell, D.L.; Nunn, R.S. Rapid deformation of passive polymorphonuclear leukocytes-The effects of pentoxifylline. J. Cell. Physiol. 1989, 140, 549-557. [CrossRef] [PubMed]

66. Hochmuth, R.M.; Needham, D. The viscosity of neutrophils and their transit times through small pores. Biorheology 1990, 27, 817-828. [CrossRef] [PubMed]

67. Needham, D.; Hochmuth, R.M. Rapid flow of passive neutrophils into a 4 mu-m pipette and measurement of cytoplasmic viscosity. J. Biomech. Eng. Trans. Asme 1990, 112, 269-276. [CrossRef]

68. Needham, D. Possible role of cell cycle-dependent morphology, geometry, and mechanical properties in tumor cell metastasis. Cell Biophys. 1991, 18, 99-121. [CrossRef]

69. Transontay, R.; Needham, D.; Yeung, A.; Hochmuth, R.M. Time-dependent recovery of passive neutrophils after large deformation. Biophys. J. 1991, 60, 856-866. [CrossRef]

70. Sonkin, P.L.; Freedman, S.F.; Needham, D.; Rao, K.M.K.; Hatchell, D.L. Pentoxifylline modulates deformability, f-actin content, and superoxide anion production of polymorphonuclear leukocytes from diabetic cats. Exp. Eye Res. 1992, 55, 831-838. [CrossRef]

71. Hochmuth, R.M.; Tingbeall, H.P.; Beaty, B.B.; Needham, D.; Transontay, R. Viscosity of passive human neutrophils undergoing small deformations. Biophys. J. 1993, 64, 1596-1601. [CrossRef]

72. Evans, E.; Kwok, R. Mechanical calorimetry of large dimyristoylphosphatidylcholine vesicles in the phase transition region. Biochemistry 1982, 21, 4874-4879. [CrossRef]

73. Evans, E.; Needham, D. Physical-properties of surfactant bilayer-membranes-Thermal transitions, elasticity, rigidity, cohesion, and colloidal interactions. J. Phys. Chem-Us 1987, 91, 4219-4228. [CrossRef] 
74. Evans, E.; Rawicz, W. Entropy-driven tension and bending elasticity in condensed-fluid membranes. Phys. Rev. Lett. 1990, 64, 2094-2097. [CrossRef]

75. Evans, E.; Rawicz, W.; Hofmann, A.F. Lipid bilayer expansion and mechanical disruption in solutions of water soluble bile acid. In Proceedings of the XIII International Bile Salt Meeting. Bile Acids in Gastroenterology: Basic \& Clinical AdvancesFalk Symposium, San Diego, CA, USA, 30 September-2 October 1994.

76. Needham, D.; Evans, E. Structure and Mechanical-Properties of Giant Lipid (DMPC) Vesicle Bilayers from $20{ }^{\circ} \mathrm{C}$ below to $10{ }^{\circ} \mathrm{C}$ above the liquid crystal-crystalline phase transition at $24^{\circ} \mathrm{C}$. Biochemistry 1988,27 , 8261-8269. [CrossRef]

77. Needham, D.; McIntosh, T.J.; Evans, E.A. Thermomechanical and transition properties of dimyristoylphosphatidylcholine/cholesterol bilayers. Biochemistry 1988, 27, 4668-4673. [CrossRef]

78. Needham, D.; Nunn, R.S. Elastic deformation and failure of lipid bilayer membranes containing cholesterol. Biophys. J. 1990, 58, 997-1009. [CrossRef]

79. Needham, D.; Stoicheva, N.; Zhelev, D.V. Exchange of monooleoylphosphatidylcholine as monomer and micelle with membranes containing poly(ethylene glycol)-lipid. Biophys. J. 1997, 73, 2615-2629. [CrossRef]

80. Needham, D.; Zhelev, D. The mechanochemistry of lipid vesicles examined by micropipet manipulation techniques. In Vesicles; Rosoff, M., Ed.; Marcell Dekker: New York, NY, USA, 1996; Volume 62, p. 373.

81. Noppl-Simson, D.; Needham, D. Avidin-Biotin interactions at vesicle surfaces: surface binding, cross-bridge formation and lateral interactions. Biophys. J. 1996, 70, 1391-1401. [CrossRef]

82. Olbrich, K.C. Water Permeability and Mechanical Properties of Unsaturated Lipid Membranes and Sarcolemmal Vesicles; Duke University: Durham, UK, 1997.

83. Rawicz, W.; Olbrich, K.C.; McIntosh, T.; Needham, D.; Evans, E. Effect of chain length and unsaturation on elasticity of lipid bilayers. Biophys. J. 2000, 79, 328-339. [CrossRef]

84. Zhelev, D.V.; Needham, D.; Hochmuth, R.M. A novel micropipet method for measuring the bending modulus of vesicle membranes. Biophys. J. 1994, 67, 720-727. [CrossRef]

85. Walton, A. Surface tension and capillary rise. Phys. Educ. 1972, 7, 491. [CrossRef]

86. Birdi, K.S.; Vu, D.T.; Winter, A.; Norregard, A. Capillary Rise of Liquids in Rectangular Tubings. Colloid Polym. Sci. 1988, 266, 470-474. [CrossRef]

87. Adamson, A.W.; Gast, A.P. Physical Chemistry of Surface; John Wiley and Sons: New York, NY, USA, 1997

88. Schneider, C.A.; Rasband, W.S.; Eliceiri, K.W. NIH Image to ImageJ: 25 years of image analysis. Nat. Methods 2012, 9, 671-675. [CrossRef] [PubMed]

89. Cox, J.R.; Ferris, L.A.; Thalladi, V.R. Selective growth of a stable drug polymorph by suppressing the nucleation of corresponding metastable polymorphs. Angew. Chem. Int. Ed. 2007, 46, 4333-4336. [CrossRef] [PubMed]

90. Vargaftik, N.B.V.; Voljak, L.D. International Table of the Surface Tension of Water. J. Phys. Chem. Ref. Data 1983, 12, 817-820. [CrossRef]

91. Demond, A.H.; Lindner, A.S. Estimation of Interfacial-Tension between Organic Liquids and Water. Environ. Sci. Technol. 1993, 27, 2318-2331. [CrossRef]

92. U.S. Coast Guard. CHRIS—Hazardous Chemical Data; U.S. Government Printing Office: Washington, DC, USA, 1978.

93. Israelachvili, J.N. Intermolecular and Surface Force, 3rd ed.; Academic Press: New York, NY, USA, 2011.

94. Vazquez, G.; Alvarez, E.; Navaza, J.M. Surface-Tension of Alcohol Plus Water from 20-Degrees-C to 50-Degrees-C. J. Chem. Eng. Data 1995, 40, 611-614. [CrossRef]

95. Su, J.T.; Needham, D. Mass Transfer in the Dissolution of a Multicomponent Liquid Droplet in an Immiscible Liquid Environment. Langmuir 2013, 29, 13339-13345. [CrossRef] [PubMed]

96. Epstein, P.S.; Plesset, M.S. On the Stability of Gas Bubbles in Liquid-Gas Solutions. J. Chem. Phys. 1950, 18, 1409-1505. [CrossRef]

97. Einstein, A. On the Motion of Small Particles Suspended in Liquids at Rest Required by the Molecular-Kinetic Theory of Heat. Ann. Der Phys. 1905, 17, 549-560. [CrossRef]

98. Phillips, J.N. The Energetics of Micelle Formation. T Faraday Soc 1955, 51, 561-569. [CrossRef]

99. Pisárčik, M.; Devínsky, F; Pupák, M. Determination of micelle aggregation numbers of alkyltrimethylammonium bromide and sodium dodecyl sulfate surfactants using time-resolved fluorescence quenching. Open Chem. 2015, 13, 922-931. [CrossRef] 
100. Tausk, R.J.; Karmiggelt, J.; Oudshoorn, C.; Overbeek, J.T. Physical chemical studies of short-chain lecithin homologues. I. Influence of the chain length of the fatty acid ester and of electrolytes on the critical micelle concentration. Biophys. Chem. 1974, 1, 175-183. [CrossRef]

101. Mohwald, H. Phospholipid Monolayers. In Handbook of Biological Physics; Lipowsky, R., Sackmann, E., Eds.; Elsevier Science B.V.: Amsterdam, The Netherlands, 1995; Volume 1.

102. Albrecht, O.; Gruler, H.; Sackmann, E. Polymorphism of phospholipid monolayers. J. Phys. 1978, 39, $301-313$. [CrossRef]

103. Smith, R.; Tanford, C. The critical micelle concentration of L-dipalmitoylphosphatidylcholine in water and water-methanol solutions. J. Mol. Biol. 1972, 67, 75-83. [CrossRef]

104. Kinoshita, K.; Yamazaki, M. Organic solvents induce interdigitated gel structures in multilamellar vesicles of dipalmitoylphosphatidylcholine. Bba-Biomembranes 1996, 1284, 233-239. [CrossRef]

105. Kinoshita, K.; Yamazaki, M. Phase transition between hexagonal II (H-II) and liquid-crystalline phase induced by interaction between solvents and segments of the membrane surface of dioleoylphosphatidylethanolamine. Bba-Biomembranes 1997, 1330, 199-206. [CrossRef]

106. Kinoshita, K.; Furuike, S.; Yamazaki, M. Intermembrane distance in multilamellar vesicles of phosphatidylcholine depends on the interaction free energy between solvents and the hydrophilic segments of the membrane surface. Biophys. Chem. 1998, 74, 237-249. [CrossRef]

107. Cullis, P.R.; Mayer, L.D.; Bally, M.B.; Madden, T.D.; Hope, M.J. Generating and loading of liposomal systems for drug-delivery applications. Adv. Drug Deliv. Rev. 1989, 3, 267-282. [CrossRef]

108. Needham, D.; Zhelev, D. Chapter 9. Use of Micropipet Manipulation Techniques to Measure the Properties of Giant Lipid Vesicles. In Giant Vesicles; Luisi, P.L., Walde, P., Eds.; John Wiley \& Sons Ltd.: Chichester, UK, 2000; pp. 103-147.

109. Parra, E.; Needham, D. Mechanic assays based on micropipette aspiration. In The Giant Vesicle Book; Marques, C., Dimova, R., Eds.; CRC Press-Taylor \& Francis: Boca Raton, FL, USA, 2018; in press.

110. Mingins, J.; Taylor, J.A.G.; Pethica, B.A.; Jackson, C.M.; Yue, B.Y.T. Phospholipid monolayers at non-polar oil/water interfaces. Part 3.-Effect of chain length on phase transitions in saturated di-acyl lecithins at the n-heptane/aqueous sodium chloride interface. J. Chem. Soc. Faraday Trans. 1 Phys. Chem. Condens. Phases 1982, 78, 323-339. [CrossRef]

111. Haydon, D.A.; Elliott, J.R. Surface potential changes in lipid monolayers and the 'cut-off' in anaesthetic effects of N-alkanols. Biochim. Et Biophys. Acta (Bba)-Biomembr. 1986, 863, 337-340. [CrossRef]

112. Mueller, P.; Rudin, D.O.; Ti Tien, H.; Wescott, W.C. Reconstitution of Cell Membrane Structure in vitro and its Transformation into an Excitable System. Nature 1962, 194, 979. [CrossRef] [PubMed]

113. Elliott, J.R.; Needham, D.; Dilger, J.P.; Haydon, D.A. The effects of bilayer thickness and tension on gramicidin single-channel lifetime. Biochim. Et Biophys. Acta (Bba)-Biomembr. 1983, 735, 95-103. [CrossRef]

114. Hladky, S.B.; Haydon, D.A. Ion transfer across lipid membranes in the presence of gramicidin A: I. Studies of the unit conductance channel. Biochim. Et Biophys. Acta (Bba)-Biomembr. 1972, 274, 294-312. [CrossRef]

115. Baoukina, S.; Monticelli, L.; Risselada, H.J.; Marrink, S.J.; Tieleman, D.P. The molecular mechanism of lipid monolayer collapse. Proc. Natl. Acad. Sci. USA 2008, 105, 10803-10808. [CrossRef] [PubMed]

116. Girard-Egrot, A.; Blum, L. Langmuir-Blodgett Technique for Synthesis of Biomimetic Lipid Membranes. In Nanobiotechnology of Biomimetic Membranes, Martin, D., Ed.; Springer: Basel, Switzerland, 2007; pp. 23-74.

117. MacDonald, R.C.; Simon, S.A. Lipid monolayer states and their relationships to bilayers. Proc. Natl. Acad. Sci. USA 1987, 84, 4089-4093. [CrossRef] [PubMed]

118. Evans, E.; Needham, D. Giant vesicle bilayers composed of mixtures of lipids, cholesterol and polypeptides-Thermomechanical and (mutual) adherence properties. Faraday Discuss. 1986, 81, 267-280. [CrossRef]

119. Needham, D. Measurement of interbilayer adhesion energies. Methods Enzymol. 1993, 220, 111-129. [CrossRef] [PubMed]

120. Evans, E.; Rawicz, W.; Smith, B.A. Concluding remarks back to the future: Mechanics and thermodynamics of lipid biomembranes. Faraday Discuss. 2013, 161, 591-611. [CrossRef]

121. Casals, C.; Canadas, O. Role of lipid ordered/disordered phase coexistence in pulmonary surfactant function. Bba-Biomembranes 2012, 1818, 2550-2562. [CrossRef] 
122. Zuo, Y.Y.; Veldhuizen, R.A.; Neumann, A.W.; Petersen, N.O.; Possmayer, F. Current perspectives in pulmonary surfactant-inhibition, enhancement and evaluation. Biochim. Et Biophys. Acta 2008, 1778, 1947-1977. [CrossRef] [PubMed]

123. Keating, E.; Zuo, Y.Y.; Tadayyon, S.M.; Petersen, N.O.; Possmayer, F.; Veldhuizen, R.A. A modified squeeze-out mechanism for generating high surface pressures with pulmonary surfactant. Biochim. Et Biophys. Acta 2012, 1818, 1225-1234. [CrossRef] [PubMed]

124. Serrano, A.G.; Perez-Gil, J. Protein-lipid interactions and surface activity in the pulmonary surfactant system. Chem. Phys. Lipids 2006, 141, 105-118. [CrossRef] [PubMed]

125. Taeusch, H.W.; Bernardino de la Serna, J.; Perez-Gil, J.; Alonso, C.; Zasadzinski, J.A. Inactivation of pulmonary surfactant due to serum-inhibited adsorption and reversal by hydrophilic polymers: Experimental. Biophys. J. 2005, 89, 1769-1779. [CrossRef] [PubMed]

126. Hauser, H. Short-chain phospholipids as detergents. Biochim. Et Biophys. Acta (Bba)-Biomembr. 2000, 1508, 164-181. [CrossRef]

127. Israelachvili, J. The Science and Applications of Emulsions-An Overview. Colloid Surf. A 1994, 91, 1-8. [CrossRef]

128. Lee, J.; Needham, D. Swollen Micelles: When does Bulk Matter Appear? Duke University: Durham, NC, USA, 2000.

129. Haynes, W.M. CRC Handbook of Chemistry and Physics; Haynes, W.M., Ed.; CRC Press: Boca Raton, FL, USA, 2012.

130. Hommelen, J.R. The elimination of errors due to evaporation of the solute in the determination of surface tensions. J. Colloid Sci. 1959, 14, 385-400. [CrossRef]

131. Chang, C.H.; Wang, N.H.L.; Franses, E.I. Adsorption Dynamics of Single and Binary Surfactants at the Air-Water-Interface. Colloid Surf. 1992, 62, 321-332. [CrossRef]

132. Elworthy, P.H.A.K.J.M. The surface tension of sodium dodecylsulfate solutions and the phase separation model of micelle formation. J. Colloid Interface Sci. 1966, 21, 331-347. [CrossRef]

133. Christov, N.C.; Danov, K.D.; Kralchevsky, P.A.; Ananthapadmanabhan, K.P.; Lips, A. Maximum bubble pressure method: Universal surface age and transport mechanisms in surfactant solutions. Langmuir 2006, 22, 7528-7542. [CrossRef] [PubMed]

134. Casandra, A.; Ismadji, S.; Noskov, B.A.; Liggieri, L.; Lin, S.Y. A study on the method of short-time approximation-Criteria for applicability. Int. J. Heat Mass. Trans. 2015, 90, 752-760. [CrossRef]

135. Eastoe, J.; Dalton, J.S. Dynamic surface tension and adsorption mechanisms of surfactants at the air-water interface. Adv. Colloid Interface Sci. 2000, 85, 103-144. [CrossRef]

136. Fainerman, V.B.; Miller, R. Dynamic Surface-Tension Measurements in the Submillisecond Range. J. Colloid Interface Sci. 1995, 175, 118-121. [CrossRef]

137. Fainerman, V.B.; Miller, R. Adsorption kinetics of short-chain alcohols at the water/air interface: Diffusion-controlled adsorption under the conditions of a nonequilibrium surface layer. J. Colloid Interface Sci. 1996, 178, 168-175. [CrossRef]

138. Fainerman, V.B.; Mys, V.D.; Makievski, A.V.; Petkov, J.T.; Miller, R. Dynamic surface tension of micellar solutions in the millisecond and submillisecond time range. J. Colloid Interface Sci. 2006, 302, 40-46. [CrossRef] [PubMed]

139. Lin, S.Y.; Wang, W.J.; Hsu, C.T. Adsorption kinetics of 1-octanol at the air-water interface. Langmuir 1997, 13, 6211-6218. [CrossRef]

140. Ozdemir, O.; Karaguzel, C.; Nguyen, A.V.; Celik, M.S.; Miller, J.D. Contact angle and bubble attachment studies in the flotation of trona and other soluble carbonate salts. Miner. Eng. 2009, 22, 168-175. [CrossRef]

141. Macleod, C.A.; Radke, C.J. A Growing Drop Technique for Measuring Dynamic Interfacial-Tension. J. Colloid Interface Sci. 1993, 160, 435-448. [CrossRef]

142. Zhang, X.G.; Harris, M.T.; Basaran, O.A. Measurement of Dynamic Surface-Tension by a Growing Drop Technique. J. Colloid Interface Sci. 1994, 168, 47-60. [CrossRef]

143. Bleys, G.; Joos, P. Adsorption-Kinetics of Bolaform Surfactants at the Air Water Interface. J Phys Chem-Us 1985, 89, 1027-1032. [CrossRef]

144. Defay, R.; Hommelen, J.R.I. Measurement of Dynamic Surface Tensions of Aqueous Solutions by the Oscilating Jet Method. J. Colloid Sci. 1958, 13, 553-564. [CrossRef] 
145. Horozov, T.S.; Dushkin, C.D.; Danov, K.D.; Arnaudov, L.N.; Velev, O.D.; Mehreteab, A.; Broze, G. Effect of the surface expansion and wettability of the capillary on the dynamic surface tension measured by the maximum bubble pressure method. Colloids Surf. A Physicochem. Eng. Asp. 1996, 113, 117-126. [CrossRef]

146. Ma, G.; Allen, H.C. DPPC Langmuir monolayer at the air-water interface: Probing the tail and head groups by vibrational sum frequency generation spectroscopy. Langmuir 2006, 22, 5341-5349. [CrossRef] [PubMed]

147. Zhang, H.; Fan, Q.H.; Wang, Y.E.; Neal, C.R.; Zuo, Y.Y. Comparative study of clinical pulmonary surfactants using atomic force microscopy. Bba-Biomembranes 2011, 1808, 1832-1842. [CrossRef] [PubMed]

148. Putz, G.; Walch, M.; Van Eijk, M.; Haagsman, H.P. A spreading technique for forming film in a captive bubble. Biophys. J. 1998, 75, 2229-2239. [CrossRef]

149. Schurch, S.; Bachofen, H.; Goerke, J.; Possmayer, F. A Captive Bubble Method Reproduces the Insitu Behavior of Lung Surfactant Monolayers. J. Appl. Physiol. 1989, 67, 2389-2396. [CrossRef] [PubMed]

150. Chang, C.H.; Franses, E.I. Dynamic Tension Behavior of Aqueous Octanol Solutions under Constant-Area and Pulsating-Area Conditions. Chem. Eng. Sci. 1994, 49, 313-325. [CrossRef]

151. Ward, A.F.H.; Tordai, L. Time-Dependence of Boundary Tensions of Solutions. 1. The Role of Diffusion in Time-Effects. J. Chem. Phys. 1946, 14, 453-461. [CrossRef]

152. Fainerman, V.B.; Makievski, A.V.; Miller, R. The Analysis of Dynamic Surface-Tension of Sodium Alkyl Sulfate-Solutions, Based on Asymptotic Equations of Adsorption Kinetic-Theory. Colloid Surf. A 1994, 87, 61-75. [CrossRef]

153. Li, X.; Shaw, R.; Evans, G.M.; Stevenson, P. A simple numerical solution to the Ward-Tordai equation for the adsorption of non-ionic surfactants. Comput. Chem. Eng. 2010, 34, 146-153. [CrossRef]

154. Chien-Hsiang Chang, E.I.F. Adsorption dynamics of surfactants at the air/water interface: A critical review of mathematical models, data, and mechanisms. Colloid Surf. 1995, 100,1-45. [CrossRef]

155. Lu, J.R.; Purcell, I.P.; Lee, E.M.; Simister, E.A.; Thomas, R.K.; Rennie, A.R.; Penfold, J. The Composition and Structure of Sodium Dodecyl-Sulfate Dodecanol Mixtures Adsorbed at the Air-Water-Interface-A Neutron Reflection Study. J. Colloid Interface Sci. 1995, 174, 441-455. [CrossRef]

156. Li, P.X.; Li, Z.X.; Shen, H.H.; Thomas, R.K.; Penfold, J.; Lu, J.R. Application of the Gibbs Equation to the Adsorption of Nonionic Surfactants and Polymers at the Air-Water Interface: Comparison with Surface Excesses Determined Directly using Neutron Reflectivity. Langmuir 2013, 29, 9324-9334. [CrossRef]

157. Xu, H.; Li, P.X.; Ma, K.; Thomas, R.K.; Penfold, J.; Lu, J.R. Limitations in the Application of the Gibbs Equation to Anionic Surfactants at the Air/Water Surface: Sodium Dodecylsulfate and Sodium Dodecylmonooxyethylenesulfate Above and Below the CMC. Langmuir 2013, 29, 9335-9351. [CrossRef] [PubMed]

158. Firooz, A.; Chen, P. Surface tension and adsorption kinetics of amphiphiles in aqueous solutions: The role of carbon chain length and temperature. J. Colloid Interface Sci. 2012, 370, 183-191. [CrossRef]

159. Fainerman, V.B.; Lucassen-Reynders, E.H. Adsorption of single and mixed ionic surfactants at fluid interfaces. Adv. Colloid Interface Sci. 2002, 96, 295-323. [CrossRef]

160. Koynova, R.; Caffrey, M. Phases and phase transitions of the phosphatidylcholines. Biochim. Et Biophys. Acta (Bba)-Rev. Biomembr. 1998, 1376, 91-145. [CrossRef]

161. Goebel, A.; Lunkenheimer, K. Interfacial Tension of the Water/n-Alkane Interface. Langmuir 1997, 13, 369-372. [CrossRef]

162. Cooper, V.G.; Yedgar, S.; Barenholz, Y. Diffusion coefficients of mixed micelles of Triton X-100 and sphingomyelin and of sonicated sphingomyelin liposomes, measured by autocorrelation spectroscopy of Rayleigh scattered light. Biochim. Et Biophys. Acta (Bba)-Biomembr. 1974, 363, 86-97. [CrossRef]

163. Keller, M.W.; Glasheen, W.; Kaul, S. Albunex: A Safe and Effective Commercially Produced Agent for Myocardial Contrast Echocardiography. J. Am. Soc. Echocardiogr. 1989, 2, 48-52. [CrossRef]

164. Duncan, P.B.; Needham, D. Microdroplet dissolution into a second-phase solvent using a micropipet technique: Test of the Epstein-Plesset model for an aniline-water system. Langmuir 2006, 22, 4190-4197. [CrossRef] [PubMed]

165. Su, J.T.; Duncan, P.B.; Momaya, A.; Jutila, A.; Needham, D. The Effects of Hydrogen Bonding on the Diffusion of Water in n-Alkanes and n-Alcohols Measured with a Novel Single Microdroplet Method. J. Chem. Phys. 2010, 132, 044506. [CrossRef] [PubMed]

166. Wise, D.L.; Houghton, G. Effect of an impermeable wall on bubble collapse in diffusion coefficient measurements. Chem. Eng. Sci. 1968, 23, 1502-1503. [CrossRef] 
167. Ram, A.; Narkis, M. Melt Viscosity of Polyethylene: Shear Dependence. J. Polym. Sci. 1966, 10, 481-489. [CrossRef]

168. Rickard, D.L.; Duncan, P.B.; Needham, D. Hydration Potential of Lysozyme: Protein Dehydration Using a Single Microparticle Technique. Biophys. J. 2010, 98, 1075-1084. [CrossRef] [PubMed]

169. Kwok, R.; Evans, E. Thermoelasticity of Large Lecithin Bilayer Vesicles. Biophys. J. 1981, 35, 637-652. [CrossRef]

170. Waugh, R.; Evans, E.A. Thermoelasticity of red blood cell membrane. Biophys. J. 1979, 26, 115-131. [CrossRef]

171. Needham, D.; Zhelev, D.V. Lysolipid exchange with lipid vesicle membranes. Ann. Biomed. Eng. 1995, 23, 287-298. [CrossRef] [PubMed]

172. Raghavendran, K.; Willson, D.; Notter, R.N. Surfactant Therapy for Acute Lung Injury and Acute Respiratory Distress Syndrome. Crit. Care Clin. 2011, 27, 525-529. [CrossRef]

173. Ainsworth, S.B. Pathophysiology of neonatal respiratory distress syndrome: Implications for early treatment strategies. Treat. Respir. Med. 2005, 4, 423-437. [CrossRef]

174. Moya, F.R.; Gadzinowski, J.; Bancalari, E.; Salinas, V.; Kopelman, B.; Bancalari, A.; Kornacka, M.K.; Merritt, T.A.; Segal, R.; Schaber, C.J.; et al. A multicenter, randomized, masked, comparison trial of lucinactant, colfosceril palmitate, and beractant for the prevention of respiratory distress syndrome among very preterm infants. Pediatrics 2005, 115, 1018-1029. [CrossRef]

175. Agassandian, M.; Mallampalli, R.K. Surfactant phospholipid metabolism. Biochim. Et Biophys. Acta 2013, 1831, 612-625. [CrossRef]

176. Olmeda, B.; García-Álvarez, B.; Pérez-Gil, J. Structure-function correlations of pulmonary surfactant protein SP-B and the saposin-like family of proteins. Eur. Biophys. J. 2013, 42, 209-222. [CrossRef]

177. Merrill, J.D.; Ballard, R.A. Pulmonary surfactant for neonatal respiratory disorders. Curr. Opin. Pediatrics 2003, 15, 149-154. [CrossRef]

178. Waring, A.J.; Walther, F.J.; Gordon, L.M.; Hernandez-Juviel, J.M.; Hong, T.; Sherman, M.A.; Alonso, C.; Alig, T.; Braun, A.; Bacon, D. The role of charged amphipathic helices in the structure and function of surfactant protein B. J. Pept. Res. 2005, 66, 364-374. [CrossRef]

179. Walther, F.J.; Waring, A.J.; Hernandez-Juviel, J.M.; Gordon, L.M.; Wang, Z.D.; Jung, C.L.; Ruchala, P.; Clark, A.P.; Smith, W.M.; Sharma, S.; et al. Critical Structural and Functional Roles for the N-Terminal Insertion Sequence in Surfactant Protein B Analogs. PLoS ONE 2010, 5, e8672. [CrossRef]

180. Walther, F.J.; Hernández-Juviel, J.M.; Gordon, L.M.; Waring, A.J. Synthetic surfactant containing SP-B and SP-C mimics is superior to single-peptide formulations in rabbits with chemical acute lung injury. PeerJ 2014, 2, e393. [CrossRef]

181. Alonso, C.; Bringezu, F.; Brezesinski, G.; Waring, A.J.; Zasadzinski, J.A. Modifying calf lung surfactant by hexadecanol. Langmuir 2005, 21, 1028-1035. [CrossRef]

182. Parra, E.; Moleiro, L.H.; Lopez-Montero, I.; Cruz, A.; Monroy, F.; Perez-Gil, J. A combined action of pulmonary surfactant proteins SP-B and SP-C modulates permeability and dynamics of phospholipid membranes. Biochem. J. 2011, 438, 555-564. [CrossRef]

183. Parra, E.; Kinoshita, K.; Needham, D. Supporting Information: Micropipette Technique Study of Natural and Synthetic Lung Surfactants at the Air-Water Interface: Presence of a SP-B Analog Peptide Promotes Membrane Aggregation, Formation of Tightly Stacked Lamellae, and Growth of Myelin Figures. Langmuir 2016, 32, 10570-10581. [CrossRef]

184. Parra, E.; Kinoshita, K. Needham. (4.85 MB). Available online: https://pubs.acs.org/doi/suppl/10.1021/ acs.langmuir.6b01420/suppl_file/la6b01420_si_002.mpg (accessed on 30 January 2019).

185. Parra, E.; Kinoshita, K.; Needham, D. (3.69 MB). Available online: https://pubs.acs.org/doi/suppl/10.1021/ acs.langmuir.6b01420/suppl_file/la6b01420_si_003.mpg (accessed on 30 January 2019).

186. Needham, D.; Arslanagic, A.; Glud, K.; Hervella, P.; Karimi, L.; Høeilund-Carlsen, P.-F.; Kinoshita, K.; Mollenhauer, J.; Parra, E.; Utoft, A.; et al. Bottom up design of nanoparticles for anti-cancer diapeutics: "put the drug in the cancer's food". J. Drug. Target. 2016, 24, 836-856. [CrossRef]

187. Beloribi-Djefaflia, S.; Vasseur, S.; Guillaumond, F. Lipid metabolic reprogramming in cancer cells. Oncogenesis 2016, 5, e189. [CrossRef]

188. Firestone, R.A. Low-Density Lipoprotein as a Vehicle for Targeting Antitumor Compounds to Cancer Cells. Bioconjugate Chem. 1994, 5, 105-113. [CrossRef] 
189. Merriel, S.W.D.; Carroll, R.; Hamilton, F.; Hamilton, W. Association between unexplained hypoalbuminaemia and new cancer diagnoses in UK primary care patients. Fam. Pract. 2016, 33, 449-452. [CrossRef]

190. Yue, S.; Li, J.; Lee, S.-Y.; Lee, H.J.; Shao, T.; Song, B.; Cheng, L.; Masterson, T.A.; Liu, X.; Ratliff, T.L.; et al. Cholesteryl Ester Accumulation Induced by PTEN Loss and PI3K/AKT Activation Underlies Human Prostate Cancer Aggressiveness. Cell Metab. 2014, 19, 393-406. [CrossRef]

191. Rodrigues dos Santos, C.; Domingues, G.; Matias, I.; Matos, J.; Fonseca, I.; de Almeida, J.M.; Dias, S. LDL-cholesterol signaling induces breast cancer proliferation and invasion. Lipids Health Dis. 2014, 13, 16. [CrossRef]

192. Gallagher, E.J.; Zelenko, Z.; Neel, B.A.; Antoniou, I.M.; Rajan, L.; Kase, N.; LeRoith, D. Elevated tumor LDLR expression accelerates LDL cholesterol-mediated breast cancer growth in mouse models of hyperlipidemia. Oncogene 2017, 36, 6462-6471. [CrossRef]

193. Karthika, S.; Radhakrishnan, T.K.; Kalaichelvi, P. A Review of Classical and Nonclassical Nucleation Theories. Cryst. Growth Des. 2016, 16, 6663-6681. [CrossRef]

194. Horn, D.; Rieger, J. Organic Nanoparticles in the Aqueous Phase-Theory, Experiment, and Use. Angew. Chem. Int. Ed. 2001, 40, 4330-4361. [CrossRef]

195. Aarts, P.A.; Gijeman, O.L.; Kremer, J.M.; Wiersema, P.H. Dynamics of phospholipid aggregation in ethanol-water solutions. Chem. Phys. Lipids 1977, 19, 267-274. [CrossRef]

196. Dunn, R.O.; Schwab, A.W.; Bagby, M.O. Physical Property and Phase Studies of Nonaqueous Triglyceride Unsaturated Long-Chain Fatty Alcohol Methanol Systems. J. Disper. Sci. Technol. 1992, 13, 77-93. [CrossRef]

197. Bitterfield, D.L.; Utoft, A.; Needham, D. An Activity-Based Dissolution Model for Solute-Containing Microdroplets. Langmuir 2016, 32, 12749-12759. [CrossRef]

198. Parra, E.; Hervella, P.; Needham, D. Real-Time Visualization of the Precipitation and Phase Behavior of Octaethylporphyrin in Lipid Microparticles. J. Pharm. Sci. 2017, 106, 1025-1041. [CrossRef]

199. Utoft, A.; Kinoshita, K.; Bitterfield, D.; David, N. Manipulating Single Microdroplets of NaCl Solutions: Solvent Dissolution, Microcrystallization, and Crystal Morphology. Langmuir 2018, 34, 3626-3641. [CrossRef]

200. De Bruijn, R.; Schaaf, J.V.D.; Patil, N.; Schouten, J. Process for Preparing Monodispersed Emulsions. WO 2010/031709 A1, 2010.

(C) 2019 by the authors. Licensee MDPI, Basel, Switzerland. This article is an open access article distributed under the terms and conditions of the Creative Commons Attribution (CC BY) license (http:/ / creativecommons.org/licenses/by/4.0/). 


\title{
Hybrid Two-Scale Fabrication of Sub-Millimetric Capillary Grippers
}

\author{
Sam Dehaeck ${ }^{1}$,, Marco Cavaiani ${ }^{2}$, Adam Chafai ${ }^{1}$, Youness Tourtit ${ }^{1}$, Youen Vitry ${ }^{1}$ \\ and Pierre Lambert $1, *$ (D) \\ 1 TIPs Department, Université libre de Bruxelles (ULB), 1050 Brussels, Belgium; \\ sam.dehaeck@gmail.com (S.D.); adam.chafai@ulb.be (A.C.); ytourtit@ulb.ac.be (Y.T.); yvitry@ulb.ac.be (Y.V.) \\ 2 Politecnico di Milano, 20133 Milano, Italy; marco.cavaiani@outlook.com \\ * Correspondence: pierre.lambert@ulb.ac.be
}

Received: 22 February 2019; Accepted: 26 March 2019; Published: 29 March 2019

\begin{abstract}
Capillary gripping is a pick-and-place technique that is particularly well-suited for handling sub-millimetric components. Nevertheless, integrating a fluid supply and release mechanism becomes increasingly difficult to manufacture for these scales. In the present contribution, two hybrid manufacturing procedures are introduced in which the creation of the smallest features is decoupled from the macro-scale components. In the first procedure, small scale features are printed directly (by two-photon polymerisation) on top of a 3D-printed device (through stereolithography). In the second approach, directional ultraviolet (UV)-illumination and an adapted design allowed for successful (polydimethylsiloxane, PDMS) moulding of the microscopic gripper head on top of a metal substrate. Importantly, a fully functional microchannel is present in both cases through which liquid to grip the components can be supplied and retracted. This capability of removing the liquid combined with an asymmetric pillar design allows for a passive release mechanism with a placement precision on the order of $3 \%$ of the component size.
\end{abstract}

Keywords: capillary gripper; pick and place; micromanufacturing; two-photon polymerization; stereolithography; polydimethylsiloxane (PDMS) replication

\section{Introduction}

Micromanipulation can be based on a wide variety of physical principles (dielectrophoresis, magnetic, optical tweezers, mechanical tweezers, hydrodynamic flows ... [1]). However, industrial picking-and-placing of sub-millimetric components such as SMD (surface mounted device) components is dominated by vacuum gripping. Very efficient even for components as small as $200 \mu \mathrm{m}$, this technique reaches its limits for smaller components because of scaling laws (adherence force scales as the square of the size [2]). Another challenge for such grippers in handling micro-components comes from the need to keep the collision force minimal. As discussed in literature [3], specialised solutions such as air bearings for the gripper head are necessary to increase the picking frequency. This added complexity is not without its cost.

An emerging alternative is the capillary picking paradigm [4], relying on capillary forces generated by the liquid meniscus between the component and the picking tool. Here, the adhering force is linear with the component size, which scales better than vacuum gripping for smaller components. This liquid layer also acts as a cushion so the component remains scratch free and surface irregularities do not impact the picking force substantially. The main design choices that need to be addressed for such a gripper are the liquid handling strategy (through a channel, dipping or evaporation/condensation) and the releasing mechanism (active/passive) [5-7]. By externalising these two mechanisms, the gripper head can be made quite simple (e.g., a simple polysterene sphere glued to a golden rod [7]) at the 
expense of a more elaborate control of the exterior conditions (temperature control of the gripper and the substrate [7]). In the present contribution, we will demonstrate a gripper design where most of the complexity is integrated in the gripper head so as to minimise the demands on the ambient control. As a result, the manufacturing of such a gripper presents some issues, mainly due to the multiplicity of scales present: the gripper surface will be of a size similar to the component and this will need to be connected to $\mathrm{cm}$-sized components (mechanical stage and liquid supply) and finally, a releasing mechanism or surface texturation might be present which will be an order of magnitude smaller than the component.

One manufacturing technique capable of creating complicated 3D shapes with micro-meter resolution is two-photon polymerisation [8], as was demonstrated by e.g., the manufacturing of a vision-based force-sensor [9]. The challenge here is more in reaching the centimetric scale with this kind of machine. Although 'meso-scale' printing with this technique has been improved with respect to manufacturing time [10] and the removal of defects [11], it remains a point-wise polymerisation technique with a micron-sized point. Therefore, polymerising a centimetric object will inherently require an excessive amount of time.

To overcome this, two different hybrid two-scale manufacturing approaches are suggested. In this way, the gripper itself is created with two-photon polymerisation and the larger scales are handled by other manufacturing techniques. While this is somewhat similar to the work of [12], we will here focus more on the assembling of different manufacturing techniques. In Section 3, we will combine two different 3D printers and in Section 4 a replication strategy for fast serial production of grippers is shown. After the description of the two manufacturing techniques, the correct functioning of the gripper is shown and a novel passive releasing mechanism is explained. Note that the first assembly is based on the work described in [13].

\section{Design Overview}

Before introducing the different hybrid manufacturing techniques that were used for the construction of our capillary grippers, we will first present their global design and working principle. In Figure 1a, a macroscopic sketch is given of the overall design. The part that is performing the capillary gripping is the sub-millimetric component indicated by the letter $\mathrm{A}$ in the figure. This small size is determined by the target that we want to pick up; in this case a 1005 SMD capacitor, which has a $1.0 \times 0.5(\mathrm{~mm})$ surface. In Figure 1b, we can see a simplified version of the gripper in action. A liquid bridge between the two flat surfaces provides the force to lift the component. Releasing of the target should occur upon retraction of the liquid. However, when the target thus comes in contact with the gripper surface, some remaining liquid in the gap will typically prevent the release. Therefore, the unequal side-pillar design shown in Figure $1 \mathrm{c}$ is proposed as a passive release mechanism. As the target has a rectangular shape of $1.0 \times 0.5(\mathrm{~mm})$ and our gripping surface is only $0.5 \times 0.5(\mathrm{~mm})$, the target will have a large overhang in one of the directions. Now, upon withdrawing the liquid into the channel, this overhanging part will be blocked at the location of the two unequal-sized pillars and this leads to a slant of the component. A gentle downward movement of the gripper, will lead to a detaching moment when the corner of the target touches the substrate. This results in the controlled release of the component. More details on the functioning principle and a demonstration will be given in Section 5 .

Although the design would be simplified by the absence of the liquid supply line, it forms an integral part of the operation here. As such, the gripper head needs to be connected to a (larger-scale) liquid injection system. This part, which we will call the tip-holder, is denoted by B in Figure 1a. Finally, part $C$ is necessary to connect the gripper to a pick-and-place machine. As these last two parts (B and C) do not present any microscopic features, they will be created with different manufacturing methods than the microscopic part (A). 

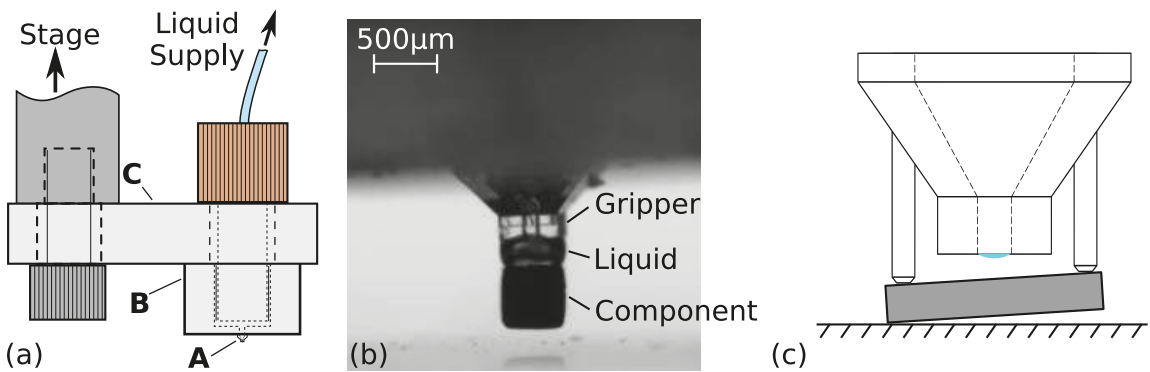

Figure 1. (a) Global overview of design (b) Example of capillary gripping [13] (c) Sketch of releasing mechanism based on unequal pillars design.

\section{Two-Scale 3D Printing}

\subsection{Microscopic Manufacturing}

As is apparent from Figure 1c, there are quite a few sub-millimetric slender features and intricate $3 \mathrm{D}$ shapes present in our design. As a result, a 3D printer capable of printing such features is deemed to be the best candidate. One of the most adequate 3D printing techniques capable of creating such a structure is two-photon polymerization, especially if we would want to shrink the components further in the future. In the present contribution, a Nanoscribe Photonics Professional GT was used to this end. The design shown in Figure 2 is printed in Nanoscribe IP-L 780 resist in dip-in configuration using a $25 \times$ objective.

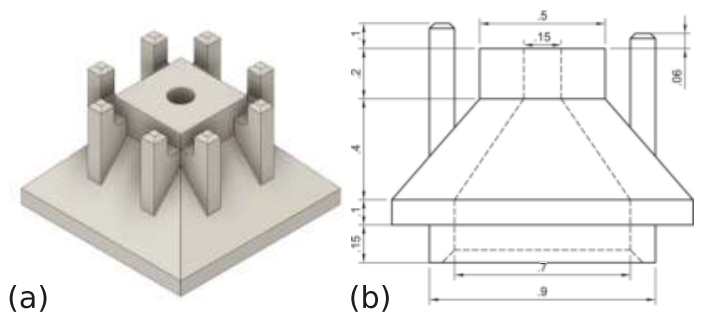

Figure 2. (a) Isometric view and (b) technical drawing of gripper head printed with Nanoscribe [13] (dimensions in $\mathrm{mm}$ ).

\subsection{Macroscopic Manufacturing}

Now, as the microscopic gripper head is already printed in a photo-sensitive resist, it seems advantageous to make the macroscopic parts in the same (or a similar) material by using a stereolithographic 3D printer. In this way, we hope to achieve a perfect adhesion between both parts and avoid (thermal and/or mechanical) property mismatch. In addition, such 3D printers are also capable of intricate 3D designs with sub-millimetric features [14]. To this end, we have here used an Autodesk Ember 3D printer. For this printer, the UV-polymerisation is initiated by a digital projector resulting in a pixel-size of $50 \mu \mathrm{m}$. The resist used is Autodesk PR48 with a slicing distance of $25 \mu \mathrm{m}$.

The design of the tip-holder is shown in Figure 3a. Note how we can print the $1 / 4^{\prime \prime}$ screw-thread directly into the component, allowing to connect the tubing. This tube is then connected to a CETONI Nemesys injection pump for the supply of water. Also note how the central $0.8 \mathrm{~mm}$ opening will be connected to the $0.7 \mathrm{~mm}$ opening in the microscopic component. In the microscopic part (Figure 2), this liquid channel is then further reduced in a funnel-design to a final opening of $0.15 \mathrm{~mm}$.

In Figure $3 \mathrm{~b}$ the supporting structure allowing to connect the gripper to a movement stage is shown. This piece will be clamped between the fitting and Figure 3a. On the other end, it will be 
similarly clamped between a screw and metal rod, which is itself connected to the movement stage. The final composition of all three components is shown in Figure 1a.

(a)

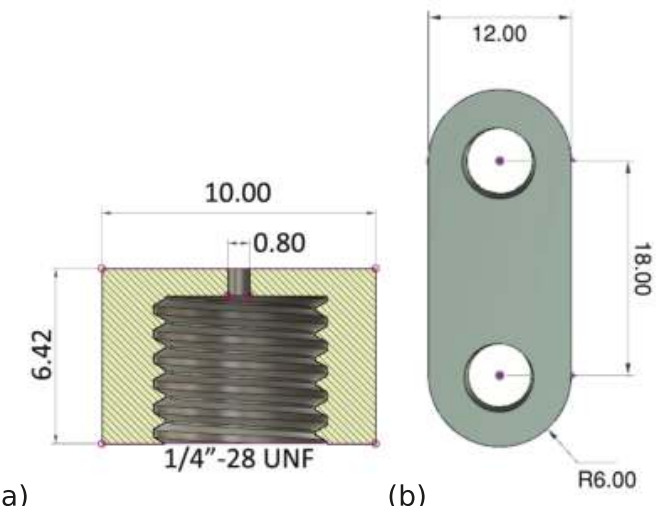

(b)
R6.00

Figure 3. (a) Macroscopic connection part to liquid supply [13] (b) Macroscopic connection to stage [13] (dimensions in $\mathrm{mm}$ ).

\subsection{Bonding of Microscopic and Macroscopic Components}

While the creation of the three pieces described above is quite trivial with the right equipment, the difficulty is in how to place the microscopic component correctly on top of the macroscopic component in Figure $3 \mathrm{a}$ and create a strong bonding. A bonding which should avoid blocking the small liquid channel.

In the current manuscript, the chosen manufacturing procedure was to write the microscopic component directly on top of the macroscopic component (i.e., the tip-holder shown in Figure 3a). As two-photon polymerization is capable of freely selecting the points that need to be polymerized, there is no difficulty in producing an open channel. Yet, in order to do this, we need to be able to insert the printed component into our Nanoscribe Photonics Professional GT. However, it only accepts standard-shaped glass slides or wafers as a target substrate on which to start writing. As such, we have had to design an envelope structure around our tip-holder, so that it conforms to a substrate size that the Nanoscribe sample holder can accept. Its design is shown in Figure 4. To summarize, we have mimicked a 1 inch square glass plate (indicated by (2) in the figure) surrounding the tip-holder (1). The tip-holder is held in place by a series of thin bars (4), which we can easily snap off after manufacturing. Note also that we have had to add extra thickness (3) to component (2) as the thin sheet started to deform after development.

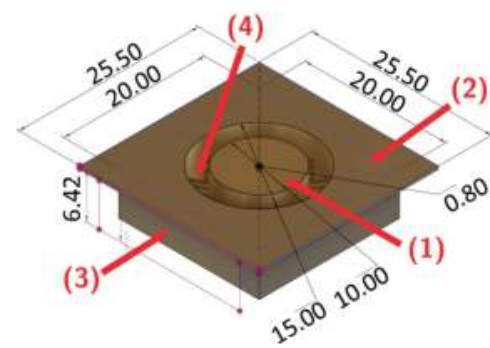

Figure 4. Tip holder design to conform to the Nanoscribe sample holder (dimensions in $\mathrm{mm}$ ). (1) is the component shown in Figure 3a, (2) mimics the common glass substrates used in Dip-in configuration. (3) adds some rigidity as the small thickness of part (2) otherwise leads to a curved interface. (4) are a series of tiny bars connecting (1) to the holding structure [13]. 
Now, the final procedure to manufacture the combined component is as follows. The component in Figure 4 is printed with the Autodesk Ember. This component is then inserted into the sample holder of the Nanoscribe Photonics Professional GT. A drop of IP-L is placed on top of component (1) of Figure 4 . The $25 \times$ objective is then allowed to dip into this drop to start writing the structure of Figure 2. Yet, before we can launch the printing, two more steps need to be performed first.

The first step is to find the liquid-solid interface, so that we can have a z-reference for the print. The in-build interface detection method by Nanoscribe is quite precise $( \pm 1 \mu \mathrm{m})$ but depends on the presence of a sufficiently large contrast in refractive index between the resist and the solid. The necessity of this is exemplified by the fact that even normal glass substrates need to be coated with ITO (Indium Tin Oxyde) to increase this contrast. Therefore it is quite inevitable that the refractive index contrast between the cured resist (Autodesk PR-48) and the liquid Nanoscribe IP-L 780 resist is not large enough to allow for an accurate automatic interface detection, due to the large similarity of the two materials. As adding an intermediate coating step could reduce the adhesion between both components, we chose to perform the interface finding manually based on the optical images of small residues on top of the printed part shown by Nanoscribe in direct imaging mode. As the obtained accuracy in this way is very poor and to account for any remaining non-flatness of the macroscopic component, we therefore added a $0.15 \mathrm{~mm}$ extension of the pyramid structure below the zero-height reference. As re-illumination of already polymerised voxels has no detrimental effects, this allows us to make sure that the pyramid is firmly attached to the tip-holder.

A second reference that is still needed is the position of the centre of the liquid channel. This centering was also performed manually by noting the stage coordinates where the top, bottom, left and right edges of the channel are centered in the camera's field of view. From this, the center point can be calculated. While the precision of this procedure could be as bad as a few tens of microns, this does not impact the final performance of our capillary gripper as the connecting channel has a sufficiently large diameter to be only mildly affected by such a misalignment of the two channels. The full workflow for this manufacturing approach is shown in Figure 5.

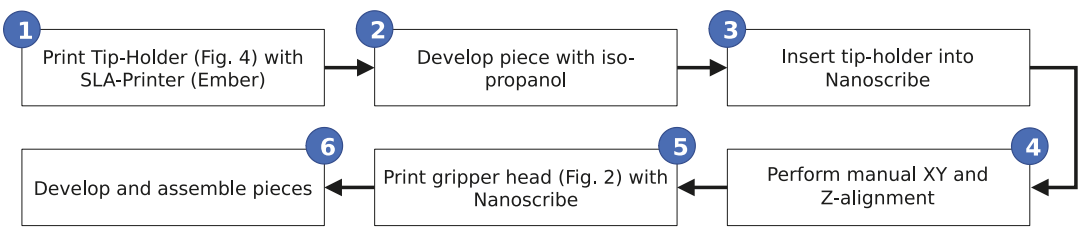

Figure 5. Flow chart for the manufacturing of the two-scale 3D printed capillary gripper.

\subsection{Manufacturing Results and Discussion}

In Figure 6, the micrometric gripper head was measured by a confocal microscope (Keyence VK-X200, Keyence, Osaka, Japan). This clearly demonstrates that the chosen two-photon polymerisation manufacturing method is capable of faithfully reproducing the micrometric features of our design.

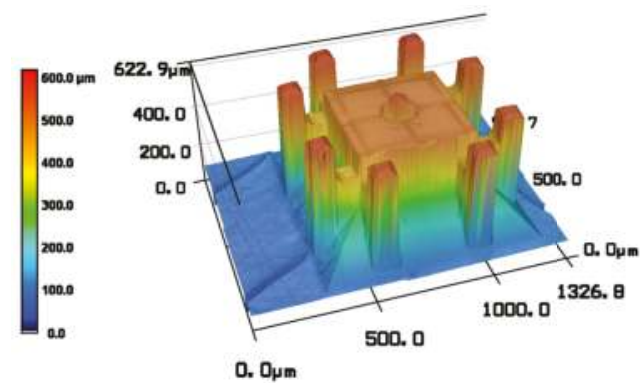

Figure 6. Metrology of the final picking head [13]. 
As will be shown in Section 5, the produced capillary grippers could pick and place the target components correctly. Nevertheless, there were a few manufacturing issues that appeared from time to time. First, we noticed that liquid leaks were sometimes present in the system. These could be due to limits in the manual alignment described above or could be due to cracks growing in the tip holder. Indeed, the resist employed by the Ember (PR-48) suffers of such cracking problems when continuously exposed to day light. A second issue that appeared from time to time was the occurrence of micro-explosions when polymerising with the Nanoscribe. Presumeably, a non-perfect cleaning step of the printed tip-holder could result in some debris floating in the IP-L. Illumination of this debris resulted in the appearance of explosions and bubbles, which resulted in a non-working gripper head. Although no extensive study was performed into these two issues, we do not consider either of them as an intrinsic problem of the proposed hybrid manufacturing method, but rather 'solveable' by optimisation studies (e.g., different resists for the Ember and lower illumination intensities for the Nanoscribe).

With respect to the total manufacturing time, we can note that the production of the tip holder (and its envelope) takes approximately $1.5 \mathrm{~h}$ to print with the Ember, whereas writing the gripper head with the Nanoscribe takes close to $5 \mathrm{~h}$ (including manual preparation tasks and development steps). As such, it is difficult to speed up the production process significantly in a cost-effective manner due to the prohibitive cost of buying additional Nanoscribe Photonics Professional GT units. On the other hand, the proposed two-scale 3D printing approach is optimally suited for complex 3D designs, both in the macroscopic components as in the microscopic components.

\section{Metal+Moulding Manufacturing}

\subsection{Macroscopic Manufacturing}

When looking at the designs in Figure 3, it is clear that none of these macroscopic components really requires the use of a $3 \mathrm{D}$ printer. Simple metal milling and drilling tools can create both components, which is what we have done for the second manufacturing approach. Due to reasons explained in the next subsection, a small modification of the tip-holder was necessary (an extra recess surrounding the microchannel) and the updated design is shown in Figure 7.

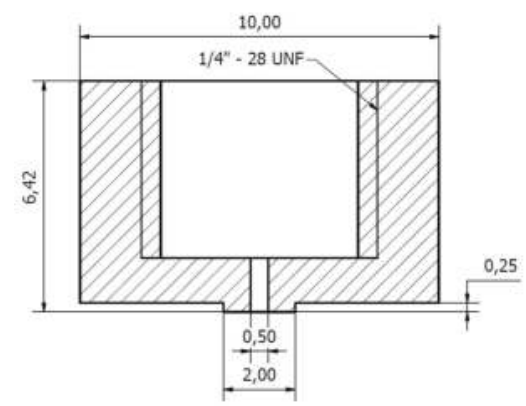

Figure 7. Updated design of the liquid connection device (dimensions in $\mathrm{mm}$ ).

\subsection{Microscopic Manufacturing and Bonding}

The bottleneck in the previous hybrid manufacturing method was the production of the gripper head directly on top of each macroscopic component. The process that we will investigate now is how to use the technique of micromoulding to speed up the manufacturing. Clearly, a straightforward moulding of the complete gripper is not easy due to the complicated 3D design of the gripper with an internal channel. However, as the macroscopic components are easily manufactured using standard metal tooling, we really only need to focus here on the replication of a microscopic gripper head onto the metal tip holder. 


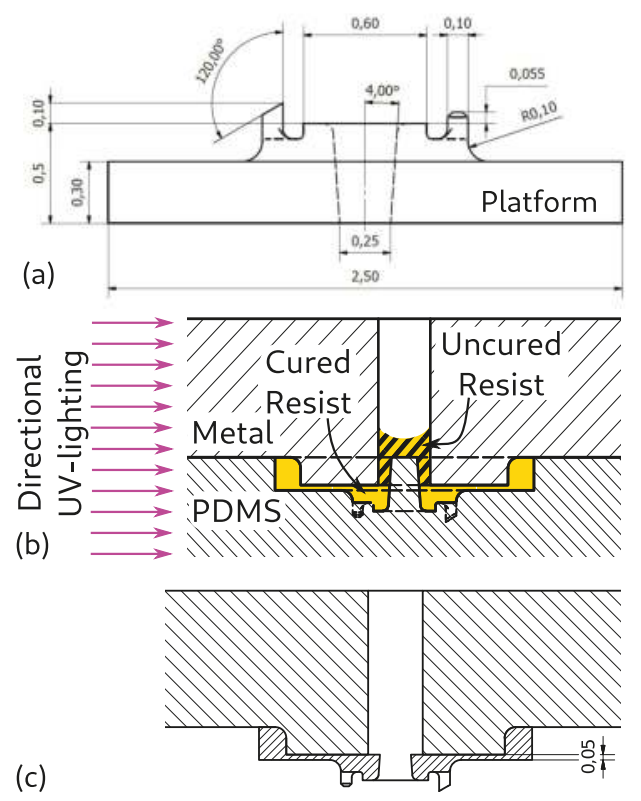

Figure 8. (a) Micromanufacturing design for Nanoscribe Manufacturing (dimensions in mm) (b) Zoom on the moulding composition near the liquid channel. (c) Zoom on the final gripper geometry.

The first concern here is how to preserve the microscopic details of our gripper head in the mould. Fortunately, this problem is already solved as replication with PDMS-moulds has already been shown in literature to faithfully reproduce micron-sized features created using two-photon polymerisation [15]. As such, we can use the same microscopic manufacturing method for the creation of the original gripper head, provided we simplify the design slightly so that moulding and demoulding can occur. This implies, for instance, that the funnel design of the microchannel in Figure 2 is replaced by a straight channel or an inverted funnel. The mould is obtained by casting a mixture of 10:1 PDMS/curing agent over the IP-L gripper in a vacuum environment to remove any bubbles. It is then cured over night at $65^{\circ} \mathrm{C}$ and demoulded. Note that it is vital that the gripper undergoes a silanisation $(1 \mathrm{~h}$ in a vacuum bell with an open reservoir of Hexamethyldisilazane) before the PDMS is poured, to allow for an easy detachment.

Another concern is how to combine the replicated gripper head with the tip-holder and ensure that there is a good adherence between the two components. To this end, replication and bonding is combined into a single step by applying the UV-illumination when the liquid resin is in contact both with the metal tip-holder as with the mould. Not only does this ensure a good bonding but it simplifies the total process significantly. Note that IP-L and Fun-to-do unpigmented standard blend were both tested successfully and never lead to liquid leakage, which qualitatively indicates that the bonding was indeed sufficiently strong.

The remaining issue is how to avoid obtaining a blocked microchannel. This will be achieved by a combination of design modifications and a special UV-illumination technique. The extra recess for the macroscopic component was already described. For the gripper, note how the head is now placed on a small platform in Figure 8a. The central hole for the microchannel goes through this platform and in this case even up to the substrate (see Figure 8a). Upon moulding, the platform becomes a recess and the hole becomes a pillar that sticks out above the level of the recess (Figure 8b). Now, when liquid resist is present in the mould and the metal piece is put in position, the pillar easily fits into the hole of the metal piece (see Figure $8 b$ ). However, if we would now polymerise all the resist, we would end up 
with a blocked channel as liquid resist will creep up into the micro-channel. This is avoided here by using directional UV-lighting that only comes from the side, not from the top or bottom (in practice we rotate the piece to illuminate it from all 4 sides). As the metal piece is non-transparent to UV-rays, the liquid resist inside the microchannel is not polymerised (region indicated by the hatched lines in Figure $8 b$ ). The final geometry of the gripper, upon retraction of the mould, is shown in Figure 8c.

Note that several other minor modifications were also applied to the design with respect to Figure 6, such as the presence of the releasing pillars only in a single direction and a modification of the releasing pillar shape in an attempt to optimise the performance of the gripper. The full workflow for this manufacturing approach is shown in Figure 9.

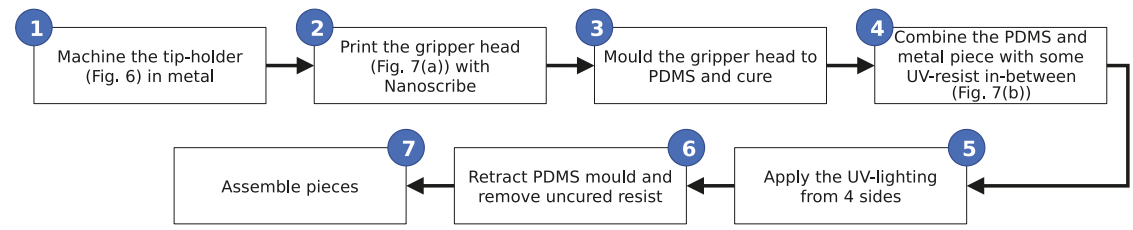

Figure 9. Flow chart for the manufacturing of the moulded gripper head on top of a macroscopic component.

\subsection{Manufacturing Results and Discussion}

In Figure 10, the result of the confocal measurement of the mould is shown. As anticipated from literature [15], all of the sub-millimetric features are correctly preserved. More importantly, no sign of blocked bubbles are seen in the mould, even though there were many 'dead-ends' in the design. This is due to the casting in a vacuum chamber and the gas permeability of PDMS. In our limited testing, we could also easily make up to 7 assembled grippers from a single PDMS mould. In 2 of these cases, there was nevertheless a small membrane blocking the channel, presumeably due to spurious reflections from the UV-light. This blockage was however easily overcome by applying some pressure with the liquid syringe pump. After assembling 7 grippers, the mould was slightly damaged. It is expected that one could increase this number significantly by a careful deposition of a silane layer before each replication step or by the application of a more permanent chemical vapour deposited layer of Parylene-C [16]. By a similar deposition of a Parylene-C coating on the master, the amount of PDMS moulds that can be created per Nanoscribe master was shown to be arbitrarily large [17].

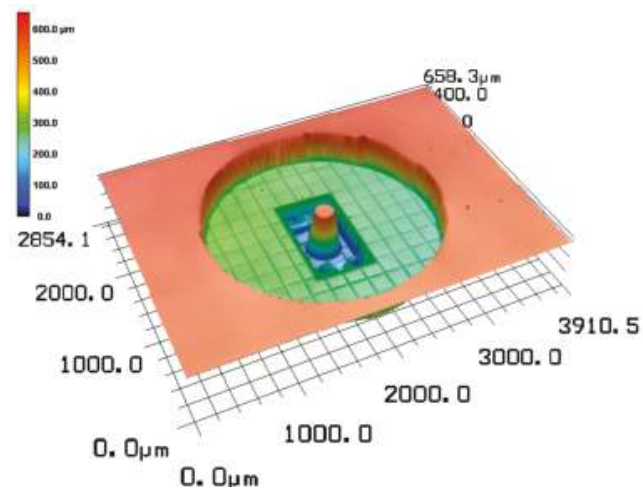

Figure 10. Metrology of the gripper head mould in polydimethylsiloxane (PDMS).

Now, with respect to the manufacturing time required to reach this result, the micro-manufacturing step takes more or less the same time than the previous design, i.e., $5 \mathrm{~h}$. The moulding step in principle should only add about $3 \mathrm{~h}$. However, in our experiments, we have chosen to leave the PDMS to cure 
overnight at $65^{\circ} \mathrm{C}$. In short, the initial tooling step takes about a day. However, once this is completed, creation of a replication head is just a matter of minutes and could easily be automated. As such, the current hybrid manufacturing method could be used in an industrial context and allows for the low-cost replication of microscopic gripper heads. To increase the productivity even further and parallellise the manufacturing, one can always create more high-precision moulds from the same master, as this process is non-destructive.

Another advantage of the present manufacturing approach is that we can easily use different UV-sensitive resists having different mechanical or other properties. For instance, the use of flexible UV-curable materials (such as UV-sensitive PDMS) could prove interesting.

Finally, we would like to mention that this moulding process could also be combined with 3D printing of the tip-holder as in the previous approach, without the need for the envelope structure. Indeed, resists are available that are opaque to UV-rays for the wall thicknesses considered here $(>1 \mathrm{~mm})$ [14]. As such, the directional UV-illumination will not penetrate into the micro-channel and it will remain unobstructed. This combination of manufacturing techniques (3D printing + moulding) would allow for more complex 3D designs in the macroscopic parts than in the microscopic part, but at a much faster total manufacturing speed.

\section{Capillary Gripping}

Figure 11 shows the manipulation sequence with the gripper from Section 3: first, positioning (a) occurs: a droplet of $100 \mathrm{~nL}$ is sent to the picking surface and the gripper is aligned with the SMD component. Then the tool is moved downwards (b) and the component is picked up. To release the component, the working fluid is sucked back into the internal channel (c), the gripper is moved downwards again (d) and the component is released (e). As almost no liquid is left on the SMD component after the releasing operation, the re-picking performance is not influenced (Figure 11f-h). A video showing this sequence is available in the supporting information.

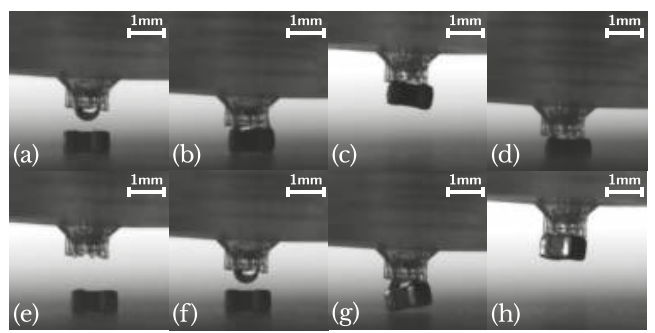

Figure 11. (a-e) -Pick and place sequence with the gripper from Section 3. (e-h) correct repicking of the component [13] (see video S1 in the Supplementary Materials).

With the gripper from Section 4, a preliminary study was performed in order to investigate the positioning precision. In order to be independent of the precision of the used stage, no lateral displacements were performed with the stage, only vertical ones. The substrate was mounted on top of an inverted microscope (Leica microscope with a $2.5 \times$ objective). Using a glass substrate, this allowed a measurement of the absolute displacement of the centre of the component and the angular rotation from 13 subsequent pick and place actions. For the displacement, a systematic bias of $47 \mu \mathrm{m}$ was found combined with a random standard deviation of $29 \mu \mathrm{m}$. For the rotation, we obtained $0.12 \pm 5.5^{\circ}$.

On more than one occasion however, the releasing procedure failed as is shown in Figure 12a-f. Although the component disconnected correctly from the droplet and the shorter pillar, the detachment from the longest pillar was not successful (e). The adherence to this pillar could even be large enough to lift the entire component. But eventually it disconnects and lands in an uncontrolled way (f). This was observed with the flat pillars from Section 3 and with the new slanted pillar from Section 4. A 
possible solution that could be investigated in the future is to increase the redrawal acceleration to overcome this residual adherence.

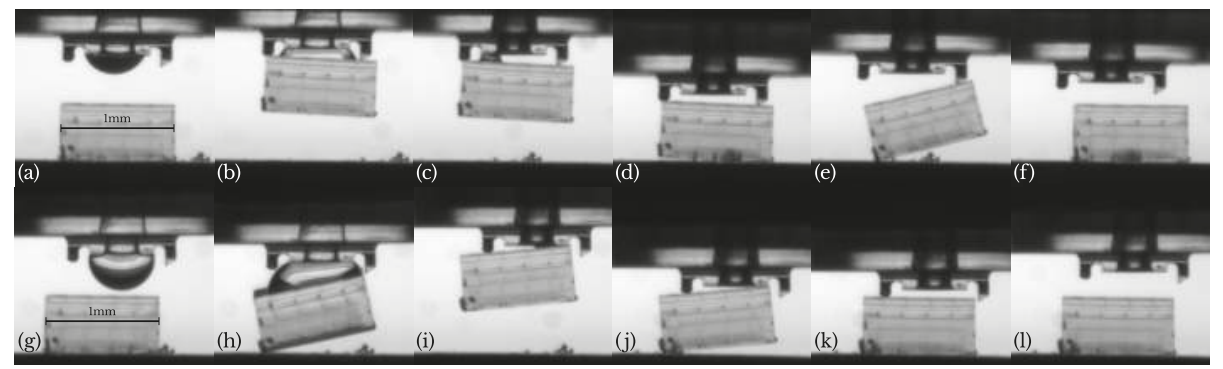

Figure 12. (a-f)—correct picking but faulty release sequence (e,f) with the gripper from Section 4 (see video S2 in the Supplementary Materials). (g-1)—correct single-pillar pick and release sequence (pillars on the right not in contact with the component) (see video S3 in the Supplementary Materials).

An interesting possibility to improve our gripper design, can be seen in Figure $12 \mathrm{~g}-1$. Here, only the shortest pillar is used and the component is aligned with 3 edges of the gripper surface. This leads to a larger inclination and detaching moment for the gripper upon touching the substrate. Due to this, the component disconnects from the gripper surface at a larger height ( $j$ ) and inertia allows it to disconnect from the pillar as well, leading to a clean separation $(k-1)$. The mechanism could be understood better from the video in the Supplementary Materials.

Another advantage of only using a single pillar, is in the time scale needed to release the component. Not yet mentioned, is the fact that not all liquid can be retracted in the tube. As can be clearly seen in Figure 12c, a small liquid bridge remains on the left of the channel. For releasing the component, this should be allowed to evaporate $(\approx 40 \mathrm{~s}$ for water) $(\mathrm{d})$. In comparison, the quantity of liquid after retraction shown in Figure $12 \mathrm{i}$ is clearly much smaller, allowing for a faster placing.

\section{Conclusions}

In the present paper, we have experimentally validated a design for a capillary gripper for submillimetric components. The gripper can be connected to tubing for liquid supply and withdrawal. The release mechanism is completely passive and based on asymmetric pillars with a placement precision of $\pm 29 \mu \mathrm{m}$ (for a $1 \times 0.5$ ( $\mathrm{mm}$ ) component).

A major focus of the paper was on the development of hybrid multi-scale manufacturing methods, capable of integrating micron-sized 3D features into a centimetric component. A first development focussed on ultimate 3D complexity of both the 'macroscopic' component as the 'microscopic' parts by using two 3D printers; an Autodesk Ember (Stereolithographic 3D printer) and a Nanoscribe Photonics Professional GT (two-photon polymerisation) respectively. By printing the gripper head directly on top of the macroscopic part, a good adherence was assured. However, this creates an inherently slow manufacturing process with a maximum of 1 or two pieces produced per day.

The second manufacturing procedure introduced, consisted in replicating the microscopic gripper head directly from a PDMS mould onto a metallic part. Assuming that the metal parts can be produced straightforwardly, this method could produce tens of completed grippers in a day. This is of course at the expense of a higher start-up cost in which an appropriate master and its PDMS-mould needs to be manufactured $(\approx 1$ day). The production of a non-obstructed micro-channel without additional post-processing steps needed an adapted design and use of directional UV-lighting to avoid polymerisation inside the channel, which was successfully demonstrated.

Supplementary Materials: The following videos are available online at http:/ / www.mdpi.com/2072-666X/ 10/4/224/s1. Video S1: PickPlace_EmberNanoscribe.mkv, Video S2: PickPlace_BadRelease.mkv, Video S3: PickPlace_SinglePillar.mkv. 
Author Contributions: Conceptualization, S.D. and P.L.; methodology, M.C., A.C., Y.V. and S.D.; investigation and validation, M.C., A.C. and Y.T.; writing-original draft preparation, S.D.; writing-review and editing, All Authors; supervision, S.D.; Funding acquisition: P.L.

Funding: This work was supported by the F.N.R.S. (Fonds National de la Recherche Scientifique) through an F.R.I.A. (Fonds pour la formation à la Recherche dans l'Industrie et dans l'Agriculture) grant for Adam CHAFAI. We also acknowledge FNRS financial support through research project PDR T.0050.16 (Bioinspired passive liquid dispensing) and FNRS GEQ-3D microstructuration and microengineering of surfaces with 3 photon lithography (2014-2016) (Nanoscribe GT Photonics, co-funding ULB/FNRS Grant UG01415F).

Conflicts of Interest: The authors declare no conflict of interest.

\section{References}

1. Lambert, P. Capillary Forces in Microassembly; Springer: Berlin, Germany, 2007.

2. Lambert, P. Surface Tension Effects in Microsystems; Springer: Berlin, Germany, 2013.

3. Bos, E.J.C.; Bullema, J.E.; Delbressine, F.L.M.; Schellekens, P.H.J.; Dietzel, A. A lightweight suction gripper for micro assembly. Precis. Eng. 2008, 32, 100-105. [CrossRef]

4. Grutzeck, H. Investigations of the capillary effect for gripping silicon chips. Microsyst. Technol. 2005, 11, 194-203. [CrossRef]

5. Fantoni, G.; Hansen, H.N.; Santochi, M. A new capillary gripper for mini and micro parts. CIRP Ann. Manuf. Technol. 2013, 62, 17-20. [CrossRef]

6. Knospe, C.R.; Haj-Hariri, H. Surface Tension in Microsystems: Engineering Below the Capillary Length. Mechatronics 2012, 22, 251-256. [CrossRef]

7. Uran, S.; Safaric, R.; Bratina, B. Reliable and accurate release of micro-sized objects with a gripper that uses the capillary-force method. Micromachines 2017, 8, 182. [CrossRef]

8. Maruo, S.; Ikuta, K.; Korogi, H. Force-Controllable, Optically Driven Micromachines Fabricated by Single-Step Two-Photon Microstereolithography. J. Microelectromech. Syst. 2003, 12, 533-539. [CrossRef]

9. Guelpa, V.; Prax, J.-S.; Vitry, Y.; Lehmann, O.; Dehaeck, S.; Sandoz, P.; Clévy, C.; Le Fort-Piat, N.; Lambert, P.; Laurent, G. 3D-printed vision-based micro-force sensor dedicated to in-situ SEM measurements. In Proceedings of the IEEE International Conference on Advanced Intelligent Mechatroncis (AIM), Munich, Germany, 3-7 July 2017.

10. Dehaeck, S.; Scheid, B.; Lambert, P. Adaptive stitching for meso-scale printing with two-photon lithography. Addit. Manuf. 2018, 21, 589-597. [CrossRef]

11. Jonusauskas, L.; Gailevicius, D.; Baravykas, T.; Juodkazis, S.; Malinauskas, M. Mesoscale ultrafast laser 3D lithography: throughput in voxels-per-second. In Proceedings SPIE 3D Printed Optics and Additive Photonic Manufacturing; International Society for Optics and Photonics: Strasbourg, France, 2018.

12. Fernandez, J.; De Coninck, J. Multiresolution Layered Manufacturing. Rapid Prototyp. J. 2018, 25, 87-94. [CrossRef]

13. Cavaiani, M.; Dehaeck, S.; Vitry, Y.; Lambert, P. Multi-scale 3D printed capillary gripper. In Proceedings of the 2018 International Conference on Manipulation, Automation and Robotics at Small Scales (MARSS), Nagoya, Japan, 4-8 July 2018.

14. Waheed, S.; Cabot, J.; Macdonald, N.; Lewis, T.; Guijt, R.; Paull, B.; Breadmore, M. 3D printed microfluidic devices: Enablers and barriers. Lab Chip 2016, 16, 1993-2013. [CrossRef] [PubMed]

15. Lafratta, C.; Baldacchini, T.; Farrer, R.; Fourkas, J.; Teich, M.; Saleh, B.; Naughton, M. Replication of Two-Photon-Polymerized Structures with Extremely High Aspect Ratios and Large Overhangs. J. Phys. Chem. B 2004, 108, 11256-11258. [CrossRef]

16. Chen, Y.; Pei, W.; Tang, R.; Chen, S.; Chen, H. Conformal coating of parylene for surface anti-adhesion in polydimethylsiloxane (PDMS) double casting technique. Sens. Actuators A Phys. 2013, 189, 143-150. [CrossRef]

17. Heyries, K.; Hansen, C. Parylene C coating for high-performance replica molding. Lab Chip 2011, 11, 4122. [CrossRef] [PubMed] 
Article

\title{
Fabrication of Hydrophilic Surface on Rigid Gas Permeable Contact Lenses to Enhance the Wettability Using Ultraviolet Laser System
}

\author{
Hsin-Yi Tsai $\mathbb{D}$, Yu-Chen Hsieh, Yu-Hsuan Lin, Han-Chao Chang $\mathbb{D}$, Yu-Hsiang Tang \\ and Kuo-Cheng Huang *
}

Taiwan Instrument Research Institute, National Applied Research Laboratories, Hsinchu 30076, Taiwan; kellytsai@narlabs.org.tw (H.-Y.T.); yuchen820606@narlabs.org.tw (Y.-C.H.); marklin@narlabs.org.tw (Y.-H.L.); roman@itrc.narl.org.tw (H.-C.C.); sky520830@narlabs.org.tw (Y.-H.T.)

* Correspondence: huangkc@narlabs.org.tw; Tel.: +886-3-577-9911

Received: 29 March 2019; Accepted: 11 June 2019; Published: 13 June 2019

\begin{abstract}
The widely used rigid gas permeable (RGP) contact lenses provide higher oxygen permeability and tear exchange rate than do soft contact lenses. However, their wettability warrants improvement to enhance the wearing comfort. This study used UV laser (wavelength $=355 \mathrm{~nm}$ ) to modify the surface properties of RGP contact lenses with materials of Boston $X \mathrm{O}^{\circledR}$ (Bausch \& Lomb Incorporated). Briefly, the mesh pattern was fabricated on the RGP contact lens surface by using the laser and smoothed by using oxygen plasma; the enhanced hydrophilic efficiency was analyzed using contact angle measurement. The experiment results indicated that the contact angle of the lens material decreased by approximately $10^{\circ}-20^{\circ}$ when the pitch of mesh pattern was $<50 \mu \mathrm{m}$ under a $500-\mathrm{mm} / \mathrm{s}$ scanning speed. The oxygen plasma enhanced surface wettability with a decreased contact angle $\left(40^{\circ}\right)$. The hydrophilic characteristic of the UV laser and oxygen plasma-treated surface was twice that of oxygen plasma-treated and untreated surfaces. In the future, RGP contact lens edges could be treated with UV laser and oxygen plasma to enhance the tear wettability and wearing comfort.
\end{abstract}

Keywords: rigid gas permeable contact lenses; wettability; hydrophilic; hydrophobic; $355 \mathrm{~nm}$ UV laser; surface treatment; microstructure

\section{Introduction}

Contact lenses are divided into rigid gas permeable (RGP) and soft contact lenses according to the hardness of the lens material. Silicone hydrogel and poly(methyl methacrylate) (PMMA) are commonly used as main material for soft and hard contact lenses, respectively. Two critical characteristics determine the quality of both soft and RGP contact lenses: Permeability $[1,2]$ and wettability $[3,4]$. Soft contact lenses are typically manufactured using water-containing, gel-like plastic materials, which are pliable and comfortable to wear. However, after several hours of wearing these lenses, the water gradually evaporates, impeding oxygen permeation through the lens. Consequently, as the cornea becomes hypoxic, the wearer's eyes begin feeling dry. Compared with general soft contact lenses, RGP contact lenses provide high oxygen permeability and tear exchange rate [5]. For improving their permeability, high oxygen permeable materials such as silicone and fluorine are added to soft and RGP contact lenses, respectively. These materials enable rapid permeation of oxygen toward the cornea. This increases the lenses' wettability to prevent microbial infections [6]. In addition, surface treatment and material modification are commonly used in industries and laboratories to increase the wettability of contact lenses. 
Wettability is one of the most crucial properties determining a material's solid surface and material applications [7]. In recent years, numerous studies have reported on surface hydrophilicity and hydrophobicity [8]. Several methods, such as chemical treatment, mechanical and flame method, ion-assisted reaction, and electron beam irradiation, can be used to transform normal material surfaces into hydrophilic or hydrophobic status. A well-designed microstructure can also be used to control surface roughness and alter its wettability $[9,10]$. The contact angle of the droplet on the surface can be enhanced to $>160^{\circ}$. The most common example of this phenomenon is the lotus-leaf effect; in that, the numerous microstructures present on a lotus leaf render it superhydrophobic, with self-cleaning properties. Laser processing is another common method used in industries to change the surface roughness and wettability of material. By manufacturing different types and depth patterns on material surfaces, we can obtain different levels of material surface wettability. In a study, a femtosecond laser was employed to fabricate the micro or nano pattern on the polytetrafluoroethylene substrate [11]. The process parameters, such as the scanning speed, fluence, and beam overlap were adjusted, and uniaxial and biaxial patterns were produced to investigate the wettability of the patterns [11]. The results revealed that the biaxially scanned samples had superhydrophobicity properties and exhibited high contact angles and low contact angle hysteresis. Cai et al. [12] suggested that the dimensionless functional parameter $R_{\text {hy }}$ and $I_{S}$ are the most sensitive to the water contact angle of the specimen. Herein, the $R_{\text {hy }}$ was the average ratio of the maximum height of the profile to the mean width of the profile element, and $I_{S}$ was the average laser pulse energy per unit area of the specimen. Moreover, the $\mathrm{R}_{\text {hy }}$ and $\mathrm{I}_{\mathrm{S}}$ of 0.41 and $536 \mathrm{~J} / \mathrm{mm}^{2}$, respectively, can aid in ensuring the superhydrophobicity of the specimen during laser ablation. In addition, laser treatment on the surface of the substrate can be used to reduce the contact angle and produce a hydrophilic surface $[13,14]$. Lawrence et al. [15] used $\mathrm{CO}_{2}, \mathrm{Nd}: \mathrm{YAG}$, excimer, and high-power diode laser (HPDL) radiation to treat the surface of the biomaterial PMMA, and their results revealed that the variation in wettability of $\mathrm{CO}_{2-}$, Nd:YAG-, and HPDL-treated PMMA surfaces was small; by contrast, the wettability of the excimer laser radiation-treated PMMA surface considerably improved.

In addition, the effect of functional terminal groups with regard to wettability is widely known [16]. For instance, the terminal trifluoromethyl group $\left(\mathrm{CF}_{3}\right)$ leads to a high contact angle situation, which represents the hydrophobic property of material surfaces. By contrast, some functional terminal groups, such as the hydroxyl group $(\mathrm{SiOH})$, result in low contact angle and hydrophilicity of a material surface. Bodas et al. [17] used the conventional (oxygen plasma), unconventional plasma modification (oxygen and $\mathrm{C}_{2} \mathrm{~F}_{6}$ ), and the oxygen plasma polymerization of 2-hydroxyethyl methacrylate (HEMA) process to modify the surface properties of poly(dimethyl siloxane) (PDMS). The results indicated that the surface with two days of hydrophilic stability was modified by oxygen plasma, and the surface with one week of good hydrophilic stability was modified by oxygen and $\mathrm{C}_{2} \mathrm{~F}_{6}$. In addition, the chemical modification using HEMA provided the most efficient hydrophilic stability over 10 days. Plasma surface treatment is commonly used to form hydrophobic and hydrophilic surfaces [18]. Du et al. [19] coated the polytetrafluoroethylene (PTFE) on high-aspect ratio nanostructures and obtained hydrophobic surfaces. They employed the oxygen plasma etching and laser interference lithography to create hierarchical nanostructures, which resulted in superhydrophobic surfaces. With the aforementioned treatments, the droplet mobility on the surface of a slippery nanostructure was also enhanced by the hierarchical nanostructures. Kim et al. [20] fabricated nanospikes on a polyimide surface through plasma etching and fabricated hydrophilic microgrooves on the hydrophobic nanospike surface through laser ablation. In addition, colloidal silver was dipped in and attached on hydrophilic microgrooves. Thus, the zone affected by the laser heat should be reduced to reduce the line width of hydrophilic patterns, and these hydrophilic patterns of the hydrophobic surface can be used for cell growth, protein manipulation, and microfluidic collection.

For contact lenses, wettability also indicates how easily liquids spread over the lens surface; this can be observed through the interaction between the lens surface and tears and be defined by the contact angle [21]. The sessile drop, captive bubble, and Wilhelmy plate methods can be used to 
measure the contact angle and determine the wettability of a contact lens. Based on the aforementioned methods, measuring the contact angle of a contact lens without the ISO standard is difficult. Therefore, the standardized process of each technique should be built to reduce the measurement variations in contact angles. Cheng et al. [22] used the captive-bubble technique to measure the advancing and receding contact angles of two commercial silicone hydrogel lenses and reported that all the lenses had a considerable contact angle hysteresis with an advancing angle of almost $90^{\circ}$ in the isotonic solution. In addition, when the lysozyme or mucin were added in the solution, the advancing and receding contact angles decreased because of the molecular absorption of proteins on the lens and indicated that the high water wettability of a lens can eliminate the protein absorption. Lin et al. [23] used the pendant-drop technique to measure the air and aqueous surface tension as well as the contact angle of soft contact lenses removed from the blister packs. The influence of the surface-active agents (surfactants) was also analyzed. The results revealed that the surface tension of all blister pack solutions was lower than that of pure water. Moreover, the wettability of most lenses was determined by the surfactants; the corresponding value decreased with the depletion of surfactants. By contrast, the $\mathrm{SiH}$ lenses exhibited a stable and self-sustained surface wettability.

No previous study has focused on enhancing the wettability of RGP contact lenses by fabricating patterns using laser technology. Herein, laser technology offered advantages such as rapidity and pattern flexibility for the Boston $X^{\circledR}{ }^{\circledR}$ (Bausch \& Lomb Incorporated, Rochester, NY, USA) RGP contact lens. On exposure to $\mathrm{CO}_{2}$ and Nd:YAG lasers, Boston $X \mathrm{O}^{\circledR}$ RGP contact lenses absorb energy and melt because of the ablation mechanism which, at this wavelength, is mainly driven by a thermal effect in the microstructure fabrication process. Therefore, we employed an ultraviolet (UV) laser system to modify the surface properties of XO RGP contact lenses. The potential volume of the droplet was selected through patterned and unpatterned RGP contact lens surfaces. In addition, the laser system's processing parameters, such as scanning speed and line pitch of the ablated pattern, were adjusted, and its effect on the surface roughness of RGP contact lenses and contact angle of the droplet were analyzed simultaneously. Therefore, the suitable parameters in the laser ablation process to fabricate a hydrophilic surface can be determined. Based on the experimental results, the hydrophilic surface on the material of RGP contact lenses can be fabricated through laser ablation. This process can aid in enhancing tear exchange intervals and opportunities when the lenses are worn. This method provides fast results, customized patterns, and wearing comfort.

\section{Fundamental Theory}

A tear has a high adhesive force and thus can cause droplets to spread on the surface of the eyes and the surrounding tissue. Although soft contact lenses are made of high water content material, the water content in RGP contact lenses is relatively low, and the hydrophilicity between the RGP contact lens and eyeball surface needed to be improved to ensure that the eyes will not be dehydrated. The contact angle $(\theta)$ represents the angle at the liquid-vapor and solid-liquid interfaces [24,25]. In general, a contact angle of $<90^{\circ}$ indicates that the solid material has a wettable surface and thus is highly hydrophilic, whereas if the contact angle is $>150^{\circ}$, the solid material is highly hydrophobic. In highly hydrophobic materials, liquids cannot enter the microstructure of the solid surface and thus the interface between the solid material and the liquid becomes small. This phenomenon is called the lotus-leaf effect.

Because the RGP contact lens surface is not perfect, contact angle hysteresis occurs from the difference between the advancing and receding contact angles $\left(\theta_{A}\right.$ and $\left.\theta_{R}\right)$. In addition, the equilibrium contact angle $\left(\theta_{e}\right)$ can be described as follows [26-29]:

$$
\theta_{e}=\cos ^{-1}\left(\frac{\gamma_{A} \cos \left(\theta_{A}\right)+\gamma_{R} \cos \left(\theta_{R}\right)}{\gamma_{A}+\gamma_{R}}\right)
$$


where

$$
\begin{aligned}
& \gamma_{A}=\left(\frac{\sin ^{3}\left(\theta_{A}\right)}{2-3 \cos \left(\theta_{A}\right)+\cos ^{3}\left(\theta_{A}\right)}\right)^{\frac{1}{3}} \\
& \gamma_{R}=\left(\frac{\sin ^{3}\left(\theta_{R}\right)}{2-3 \cos \left(\theta_{R}\right)+\cos ^{3}\left(\theta_{R}\right)}\right)^{\frac{1}{3}}
\end{aligned}
$$

Rough surfaces increase hydrophilicity of the contact lens; these rough surface textures can be divided into homogeneous and heterogeneous textures. The current study aims to reduce the contact angle of a droplet on the RGP contact lens by modifying the surface of the RGP contact lens by using a laser system and increase its hydrophilicity. The classic descriptive theories of the contact angle of a droplet on microstructure surfaces include the Wenzel and Cassie-Baxter models [30-33]. The contact angle hysteresis can be ignored because the contact lens is static, and the droplet would not flow when worn. Therefore, the surface of the material treated with the laser system was maintained as a homogeneous surface, and the phenomenon can be described by the Wenzel model, as illustrated in Figure 1 and as written in Equation (4),

$$
\cos \left(\theta^{*}\right)=r \cos (\theta)
$$

where $\theta^{*}$ is the static contact angle and $\mathrm{r}$ is the roughness ratio of the material surface, which is the ratio of the true area of the object to the apparent area. In addition, $\theta$ is the Young contact angle, which represents the contact angle of the ideal surface.

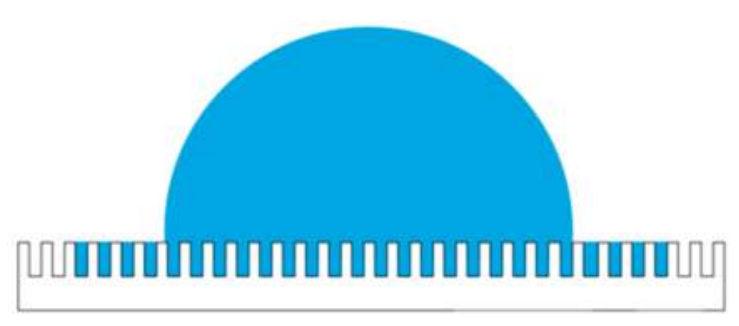

Figure 1. Droplet on microstructures according to the Wenzel model.

When the surface of the material has microstructures, a higher hydrophilicity can be achieved when the surface roughness is finer. The microstructures on the contact lenses provide spreading and imbibition abilities, and the surface is defined as the highest hydrophilicity with a contact angle less than $\pi / 2$ [34]. If it is lower than the critical contact angle of the model, there will be a formation of liquid film on the surface, which is called ultrahigh hydrophilicity.

\section{Materials and Experimental Setup}

\subsection{Materials}

PMMA is a stable material. Its mechanical or optical properties are not significantly affected when it is exposed to a high-humidity environment or is infiltrated with water. Herein, the contact angle of the flat PMMA was $77.5^{\circ}$ when the volume is $3 \mu \mathrm{L}$. Here, the RGP contact lens material XO was purchased from Bausch \& Lomb Incorporated. In addition to the conventional PMMA material, silicon and fluorine were added to the $\mathrm{XO}$ to allow more oxygen to pass through the lens and reach the patient's eye, and the material was defined as the hexafocon $\mathrm{A}$. The Boston $X \mathrm{O}^{\circledR} \mathrm{RGP}$ contact lenses have an ultrasmooth surface and are nonstick; they thus resist dirt and debris. The customized design of the lens provides a perfect fit and natural shape for the patient's eyes. The wetting angle of $\mathrm{XO}$ is $49^{\circ}$, and its oxygen permeability is $100\left(\left(\mathrm{~cm}^{3}\left[\mathrm{O}_{2}\right] \cdot \mathrm{cm}\right) /\left(\mathrm{cm}^{2} \cdot \mathrm{s} \cdot \mathrm{mmHg}\right)\right)[35]$. 


\subsection{Experimental Setup}

\subsubsection{Surface Treatment: UV Laser ( $355 \mathrm{~nm}$ ) Processing and Oxygen Plasma Cleaning}

The laser source was a diode-pumped solid-state UV laser (Coherent, Inc. AVIA 355-14 ${ }^{\mathrm{TM}}$, Santa Clara, CA, USA) with a wavelength of $355 \mathrm{~nm}$ and a maximum average output power of $14 \mathrm{~W}$, operating a pulse repetition frequency range of $1-400 \mathrm{kHz}$. Its each pulse width is of $32 \mathrm{~ns}$ at $40-\mathrm{kHz}$ pulse repetition rate, transverse mode is TEM $^{00}$ with an output beam diameter of $3.5 \mathrm{~mm}$, and beam quality factor (e.g., $\mathrm{M}^{2}$ ) is $<1.3$. Here, its beam diameter was magnified by a $2 \times$ beam expander. High speed galvanometric scanning mirrors were used to transform the direction of the laser beam for laser processing and improve processing efficiency (Figure 2). A telecentric focusing lens module with a $160-\mathrm{mm}$ focal length was used; the theoretical and actual diameters of the focused laser spot on the working plane were approximately 13.5 and approximately $30 \mu \mathrm{m}$, respectively.

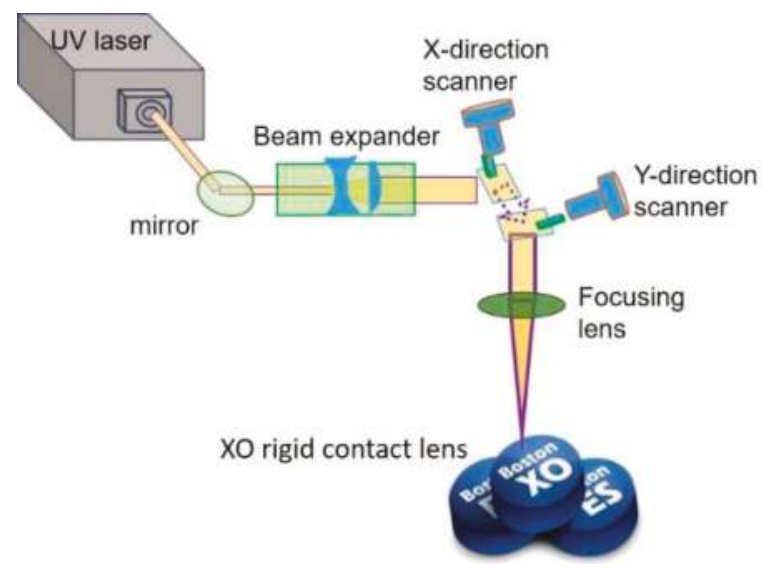

Figure 2. Laser treatment system setup on Boston $X \mathrm{X}^{\circledR}$ rigid gas permeable (RGP) contact lens.

First, we used the UV laser to make patterns on the surface of the material of the RGP contact lens. The scanning speed of the laser spot and spacing of the laser scanning were adjusted and designed to make mesh patterns with various depths, line pitch, and roughness. The output power of the laser system was set at $10 \mathrm{~W}$, and the pulse repetition frequency was fixed at $100 \mathrm{kHz}$. Laser scanning speeds of $500,1000,1500$ and $2000 \mathrm{~mm} / \mathrm{s}$ were used. The line pitch of the mesh pattern ranged from 30 to $110 \mu \mathrm{m}$ at $20-\mu \mathrm{m}$ intervals. The mesh patterns with five line pitches are illustrated in Figure 3 . After the surface treatment with the UV laser, an oxygen plasma cleaner (HARRICK PLASMA, PLASMA CLEANER PDC-32G, Ithaca, NY, USA) was employed to clean the splash on the surface material caused by the laser processing and modify the surface properties of the material of the RGP contact lens. For the oxygen plasma cleaning parameters, the input power was $100 \mathrm{~W}$, the power applied to the RF coil was $11 \mathrm{~W}$, and the cleaning lasted for $10 \mathrm{~min}$. 


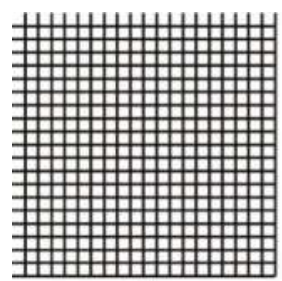

(a)

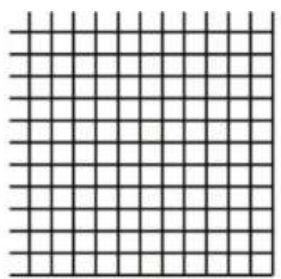

(b)

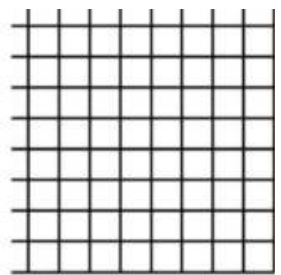

(c)

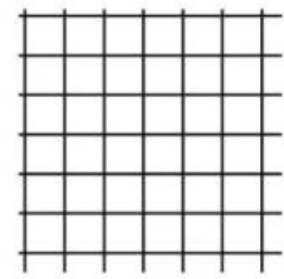

(d)

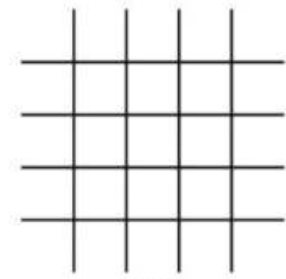

(e)

Figure 3. Mesh patterns with pitch of (a) 30, (b) 50, (c) 70, (d) 90, and (e) $110 \mu \mathrm{m}$.

\subsubsection{Contact Angle Measurement and Surface Morphology Observation}

After the surface treatment of the lens with the laser, the treated lens's surface morphology and roughness were observed and measured using a three-dimensional confocal laser scanning microscope (KEYENCE, VK-X200, Itasca, IL, USA) and its analysis software program (KEYENCE, VK-Analyzer Plus $^{\mathrm{TM}}$, Itasca, IL, USA). In addition, the contact angle of the droplet on the Boston $\mathrm{XO}^{\circledR}$ RGP contact lens related to its wettability was measured through contact angle measurement (FTA 188, First Ten Angstroms Inc, Portsmouth, VA, USA).

\subsubsection{Experimental Process}

Four primary steps were conducted to obtain the contact angle and determine the performance of the treated pattern on the XO RGP contact lens surface; the adjusted and measured target of each step are described in detail as follows:

Step(I): The AutoCAD package was used to design the ablation path of the laser spot. The parameter of the pitch of two ablated lines was adjusted to range from 30 to $110 \mu \mathrm{m}$ with intervals of $20 \mu \mathrm{m}$.

Step (II): The parameters, such as the power and pulse repetition frequency of the laser system, were fixed, and the scanning speed of the laser spot was adjusted to generate various spot overlaps, line widths, and surface roughness.

Step (III): A 3D confocal microscope was used to measure the morphology of the ablated mesh pattern and analyze the relationship of the real line pitch, width, and surface roughness with the scanning speed during laser treatment. Herein, the surface roughness was the mean height $(\mathrm{Ra})$.

Step (IV): The XO RGP contact lens was placed into an ultrasonic oscillator with DI (de-ionized) water for $10 \mathrm{~min}$ to clean its surface, and nitrogen $\left(\mathrm{N}_{2}\right)$ was used to blow dry its surface. The lens was then processed for contact angle measurement, for which a 3- $\mu \mathrm{L}$ droplet of physiological saline was added to the lens. The contact angle was measured. In addition, the relationship between the contact angle and surface roughness of the XO RGP contact lens was analyzed to obtain the best pattern to reduce the contact angle and enhance the wettability of RGP contact lenses. 


\section{Experimental Results and Discussion}

To investigate the wettability of the XO RGP contact lens, affected by the volume of a droplet and the surface properties, after the laser treatment, the contact angle and surface roughness of each lens was analyzed. The measured contact angle was the average of the eight values measured from four directions in a circle at the intervals of $90^{\circ}$. The measurement was repeated twice. Surface roughness was the average value in the full of view of the measured area, which was approximately $1.6 \times 1.0 \mathrm{~mm}^{2}$ under the $10 \times$ objective lens.

\subsection{Analysis of the Contact Angle Affected by the Droplet Volume}

Different volumes of physiological saline droplets have different gravity; therefore, the selectivity of the droplet volume was determined in the subsequent experiments. On the original surface of the XO RGP contact lens, the contact angle of the droplet was only affected by the gravity, and the increase in the droplet volume required a larger area and a higher base width to support the droplet. Therefore, the support of the droplet edge on the material's surface caused the contact angle of the droplet to decrease simultaneously (Table 1). In addition, the mesh pattern with line pitches of $30 \mu \mathrm{m}$, treated with a laser scanning speed of $1000 \mathrm{~mm} / \mathrm{s}$, was fabricated on the lens surface. With the microstructures, the contact angle of the droplet is affected by the gravity, surface roughness, and pattern's height. In this situation, the droplet infiltrated into the valley of microstructures, and the base width of the droplet increased only slightly with the increase in droplet volume, as summarized in Table 2. Therefore, sufficient surface area was generated to support the droplet and caused a similar contact angle when the droplet volume ranged from 3 to $5 \mu \mathrm{L}$ (Table 1). Based on the aforementioned results, a droplet volume of $2 \mu \mathrm{L}$ was too small to be affected by the surface morphology and a volume of $4-5 \mu \mathrm{L}$ was too large and easily affected by the gravity and surface morphology. Moreover, a droplet volume of $3 \mu \mathrm{L}$ started to be affected by the microstructures, indicating that this volume of droplet was significantly affected by the surface properties. Therefore, the droplet volume of $3 \mu \mathrm{L}$ was determined in the following experiments to investigate the contact angle affected by the surface morphology and roughness.

Table 1. Contact angle of the droplet on XO RGP contact lens with and without mesh patterns (Unit: ${ }^{\circ}$ ).

\begin{tabular}{ccccc}
\hline \multirow{2}{*}{ Pattern } & \multicolumn{4}{c}{ Droplet Volume $(\boldsymbol{\mu L})$} \\
\cline { 2 - 5 } & $\mathbf{2}$ & $\mathbf{3}$ & $\mathbf{4}$ & $\mathbf{5}$ \\
\hline Without mesh patterns & $121 \pm 6.1$ & $118 \pm 3.4$ & $112 \pm 3.5$ & $104 \pm 8.9$ \\
$\begin{array}{c}\text { With patterns of line pitch } \\
30 \mu \mathrm{m}\end{array}$ & $118 \pm 6.5$ & $111 \pm 8.7$ & $114 \pm 7.4$ & $110 \pm 6.2$ \\
\hline
\end{tabular}

Table 2. Base width of the droplet on XO RGP contact lens with and without mesh patterns (Unit: $\mathrm{mm}$ ).

\begin{tabular}{ccccc}
\hline \multirow{2}{*}{ Pattern } & \multicolumn{4}{c}{ Droplet Volume $(\mu \mathrm{L})$} \\
\cline { 2 - 5 } & $\mathbf{2}$ & $\mathbf{3}$ & $\mathbf{4}$ & $\mathbf{5}$ \\
\hline Without mesh patterns & $2.08 \pm 0.14$ & $2.35 \pm 0.14$ & $2.62 \pm 0.10$ & $2.98 \pm 0.29$ \\
With patterns of line pitch & $2.14 \pm 0.17$ & $2.53 \pm 0.13$ & $2.55 \pm 0.14$ & $2.77 \pm 0.15$ \\
$30 \mu \mathrm{m}$ & & & & \\
\hline
\end{tabular}

\subsection{Analysis of the Surface Morphology and Roughness}

After the scanning speed of the laser spot was fixed and the scanning path of different line pitches was imported, the surface morphology of the contact lens treated with the laser system could be observed from the optical images (Figure 4). The size of the focused laser spot was approximately $30 \mu \mathrm{m}$, and the line pitches of $30 \mu \mathrm{m}$ caused the laser spot on the XO RGP contact lens to close the next strip. Therefore, the full view of the laser scanning area was treated by the laser spot and caused melting and the rough surface and the largest surface roughness (Figure 4a). 


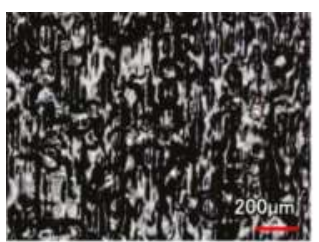

(a)

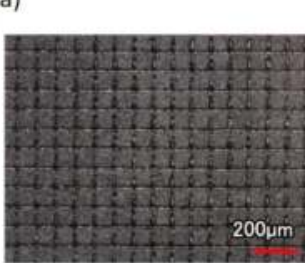

(d)

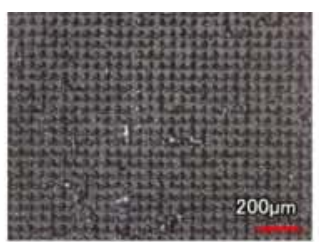

(b)

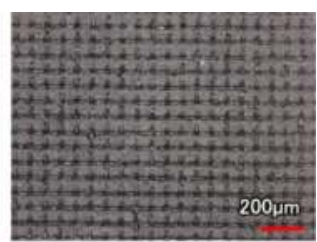

(c)

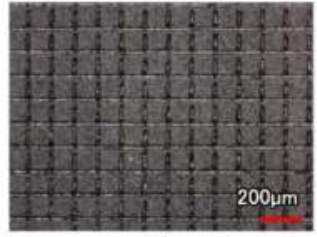

(e)

Figure 4. Optical images of the XO RGP contact lens treated with the laser system with a scanning speed of $500 \mathrm{~mm} / \mathrm{s}$ and line pitches of (a) 30, (b) 50, (c) 70, (d) 90, and (e) $110 \mu \mathrm{m}$.

In addition, in the region where only the laser focused spot was ablated and the checkerboard microstructures were fabricated, the line pitches became wider, and the surface roughness would gradually decrease simultaneously, as summarized in Table 3. When the line pitch of the laser spot was fixed and the scanning speed was adjusted, the scanning speed affected the residence time of the laser spot; the treated depth was also altered (Table 4). The treated depth of the laser spot and the surface roughness decreased with the increase of the scanning speed (Figure 5), even without the ablation trace on the surface of the XO RGP contact lens (Figure 5d). The surface roughness and the root mean square of the height decreased from 13.002 to $2.230 \mu \mathrm{m}$ and from 18.770 to $3.081 \mu \mathrm{m}$, respectively, when the scanning speed increased from 500 to $2000 \mathrm{~mm} / \mathrm{s}$. Herein, the roughness and root mean square height was defined by the average value and root mean square along the sampling length, respectively; there was no overlap in the laser trace when the line pitches of the microstructures were 90 and $110 \mu \mathrm{m}$. Thus, the variation of the surface roughness and root mean square height of mesh patterns in the aforementioned pitches were small when the scanning speed was changed.

Table 3. Surface roughness ( $\mathrm{Ra}$ ) of mesh patterns on the XO RGP contact lens treated by different laser scanning speeds and line pitches (Unit: $\mu \mathrm{m}$ ).

\begin{tabular}{cccccc}
\hline \multirow{2}{*}{$\begin{array}{c}\text { Scanning Speed } \\
(\mathbf{m m} / \mathbf{s})\end{array}$} & $\mathbf{5 0}$ & $\mathbf{5 0}$ & $\mathbf{7 0}$ & $\mathbf{9 0}$ & $\mathbf{1 1 0}$ \\
\cline { 2 - 6 } & 13.002 & 9.912 & 5.503 & 2.593 & 3.266 \\
500 & 6.032 & 2.698 & 2.642 & 2.797 & 2.507 \\
1000 & 2.747 & 2.678 & 2.767 & 2.276 & 2.478 \\
1500 & 2.230 & 2.885 & 2.506 & 2.781 & 2.754 \\
2000 & & & & &
\end{tabular}

Table 4. Root mean square height (Rq) of mesh patterns on the XO RGP contact lens treated by different laser scanning speeds and line pitches (Unit: $\mu \mathrm{m}$ ).

\begin{tabular}{cccccc}
\hline \multirow{2}{*}{$\begin{array}{c}\text { Scanning Speed } \\
(\mathbf{m m} / \mathbf{s})\end{array}$} & $\mathbf{5 0}$ & $\mathbf{5 0}$ & $\mathbf{7 0}$ & $\mathbf{9 0}$ & $\mathbf{1 1 0}$ \\
\cline { 2 - 6 } & 18.770 & 13.215 & 8.300 & 3.569 & 4.222 \\
500 & 7.989 & 3.689 & 3.573 & 3.752 & 3.391 \\
1000 & 3.894 & 3.631 & 3.727 & 3.089 & 3.352 \\
1500 & 3.081 & 3.856 & 3.409 & 3.762 & 3.682 \\
2000 & & & & & \\
\hline
\end{tabular}




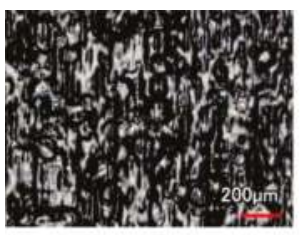

(a)

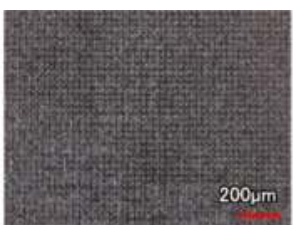

(b)

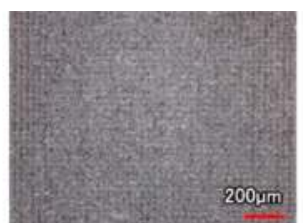

(c)

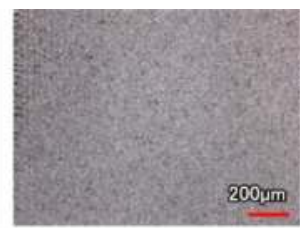

(d)

Figure 5. Optical images of the XO RGP contact lens treated with the laser system with line pitches of (a) $30 \mu \mathrm{m}$ and a scanning speed of (a) 500, (b) 1000, (c) 1500, and (d) $2000 \mathrm{~mm} / \mathrm{s}$.

\subsection{Analysis of the Contact Angle Affected by Line Pitch and Laser Scanning Speed}

When the physiological saline droplet was dropped on the surface of the treated RGP contact lens, the droplet infiltrated the melting surface and caused a lower contact angle (Figure 6a). The checkerboard pattern became clearer with the increase in the line pitch. Subsequently, the droplet was supported on the surface of the XO RGP contact lens, and the contact angle increased and reached $130^{\circ}$ on the pattern with a line pitch of $90 \mu \mathrm{m}$. When the line pitch was $110 \mu \mathrm{m}$, the surface roughness increased again because of the more plate area and relatively higher position than the pattern with a line pitch of $90 \mu \mathrm{m}$ in the same area. The droplet usually exists on the surface and infiltrates the valley of patterns at the droplet edge to cause the lowering of the contact angle of the droplet (Figure 6d,e).

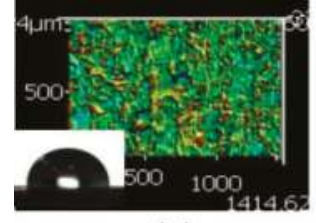

(a)

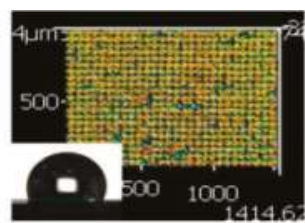

(b)

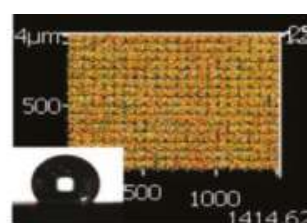

(c)

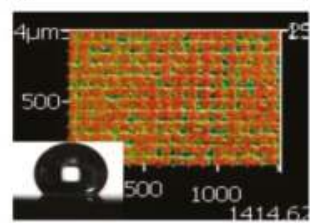

(d)

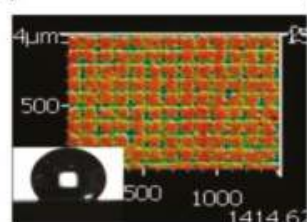

(e)

Figure 6. Three dimensional (3D) images of the XO RGP contact lens treated with the laser system with a scanning speed of $500 \mathrm{~mm} / \mathrm{s}$ and line pitches of (a) 30, (b) 50, (c) 70, (d) 90, and (e) $110 \mu \mathrm{m}$.

When the pattern was fabricated using different scanning speeds and line pitches, the contact angle of the droplet on these patterns was measured; the results are presented in Figure 7. The results indicated that the contact angle of the droplet usually increased with the increase of the line pitch of the patterns treated by the same scanning speed, particularly in the range of 30-70 $\mu \mathrm{m}$. It also represented that the contact angle of the droplet was evidently affected by the pitch of microstructures. When the line pitch of the pattern increased to 90 and $110 \mu \mathrm{m}$, there was sufficient platted region to support the droplet, and the contact angle would be affected by various factors such as the droplet position on the microstructures, surface roughness, and ablated depth of the pattern. Therein, the higher surface roughness was obtained from the pattern with the line pitch of $110 \mu \mathrm{m}$ than $90 \mu \mathrm{m}$ while treated by the laser system with a scanning speed of $500 \mathrm{~mm} / \mathrm{s}$, shown as Table 3 and Figure 6, and the droplet major exists on the platted region and may infiltrate into the valley of patterns when the line pitch of pattern increased. Thus, the contact angle of the droplet has a maximum value on the pattern with a line pitch of $90 \mu \mathrm{m}$. (Figure 7a) Therefore, smaller line pitches were more suitable to be selected to fabricate the 
microstructures and reduce the contact angle of the droplet. In addition, there was no laser trace on the XO RGP contact lens when the scanning speed was higher than $1500 \mathrm{~mm} / \mathrm{s}$. Therefore, the variation of the contact angle of the physiological saline droplet on the XO RGP contact lens was small between the scanning speed parameters of 1500 and 2000 mm/s, as illustrated in Figure 7c,d.

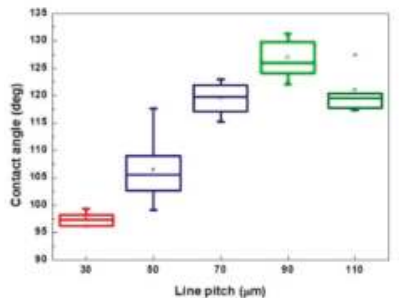

(a)

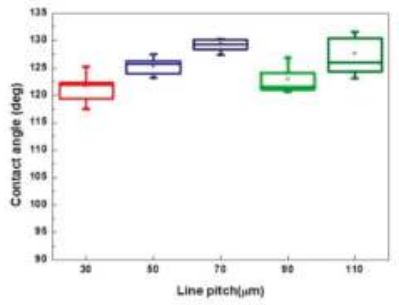

(c)

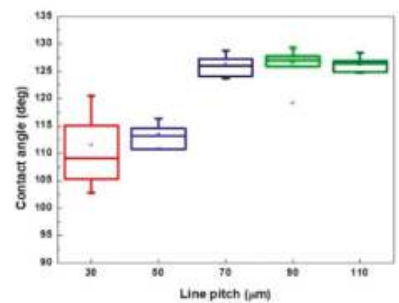

(b)

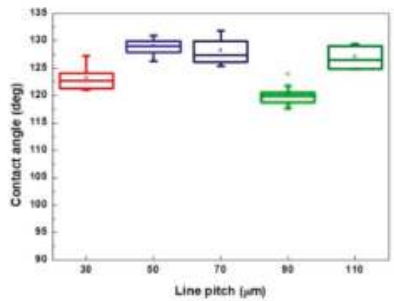

(d)

Figure 7. Contact angle of physiological saline droplet on the XO RGP contact lens with different line pitches under the scanning speed of (a) 500, (b) 1000, (c) 1500, and (d) $2000 \mathrm{~mm} / \mathrm{s}$.

Based on the aforementioned results, the pattern with a line pitch of $30 \mu \mathrm{m}$ was selected to analyze the effect caused by the laser scanning speed. Compared with the 3D profile of the patterns and the measured contact angle of the droplet (Figure 8), it revealed that the droplet infiltrated the melted surface and had the lowest contact angle of approximately $97^{\circ}$ with the largest surface roughness of $13.002 \mu \mathrm{m}$ when the pattern was treated with a scanning speed of $500 \mathrm{~mm} / \mathrm{s}$. By contrast, the surface was relatively smooth and exhibited almost no ablated trace when the scanning speed was $>1500 \mathrm{~mm} / \mathrm{s}$; it had the lowest surface roughness when the pattern was treated with a scanning speed of $2000 \mathrm{~mm} / \mathrm{s}$. Therefore, the contact angle of the droplet was usually $>120^{\circ}$ on the aforementioned patterns. It remains a hydrophobic surface under these treatment parameters, and the wettability was poor than the XO RGP contact lens without laser treatment. 


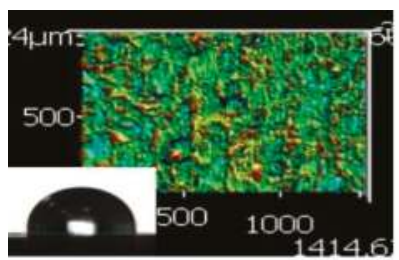

(a)

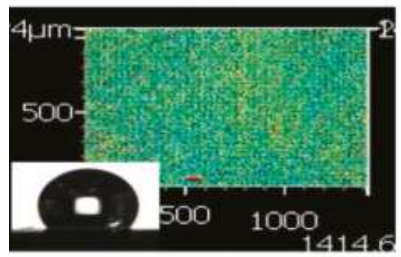

(c)

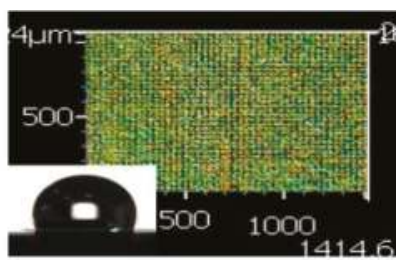

(b)

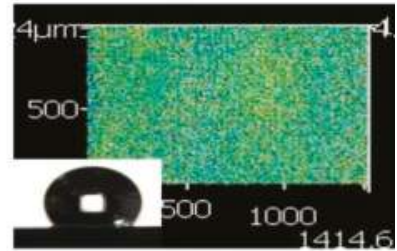

(d)

Figure 8. 3D images of the XO RGP contact lens treated with the laser system with line pitches of (a) 30 $\mu \mathrm{m}$ and a scanning speed of (a) 500, (b) 1000, (c) 1500, and (d) $2000 \mathrm{~mm} / \mathrm{s}$.

From the surface roughness summarized in Table 3 and the contact angle of the droplet presented in Figures 7, 9 and 10, the contact was usually $>110^{\circ}$ and even $>120^{\circ}$ when the surface roughness was $<5 \mu \mathrm{m}$. When the surface roughness ranged from 5 to $10 \mu \mathrm{m}$, the contact angle could be reduced to $100^{\circ}-122^{\circ}$. In addition, the surface of the XO RGP contact lens will gradually become hydrophilic when the surface roughness was enhanced to $>10 \mu \mathrm{m}$, which was approximately in the range of $96^{\circ}-99^{\circ}$ when the surface roughness was approximately $13 \mu \mathrm{m}$. Therefore, a lower scanning speed of the laser system and a pattern with smaller line pitches should be employed to reduce the contact angle of the droplet and enhance its wettability.

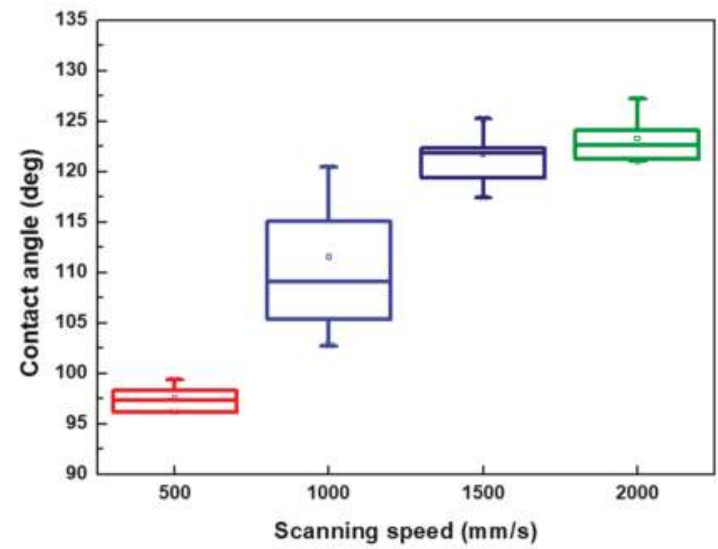

Figure 9. Contact angle of physiological saline droplet on the XO RGP contact lens with line pitch of $30 \mu \mathrm{m}$ under four various laser scanning speeds. 


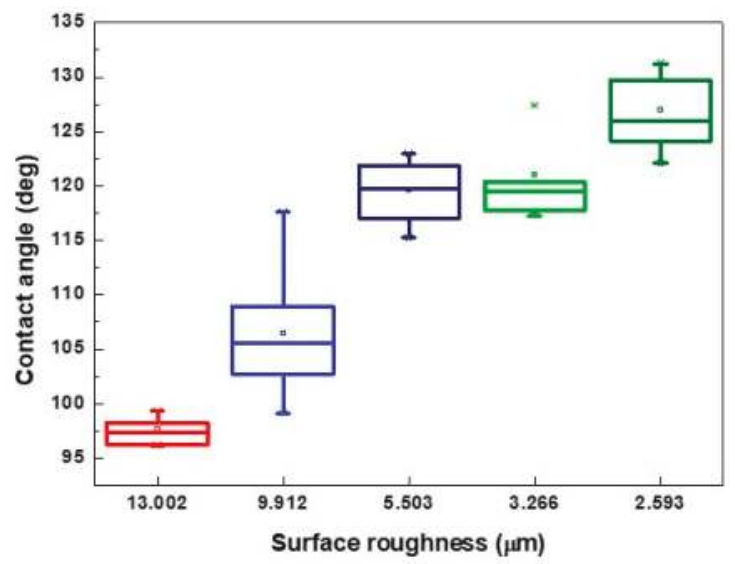

Figure 10. Contact angle of physiological saline droplet on the XO RGP contact lens with five surface roughness under laser scanning speed of $500 \mathrm{~mm} / \mathrm{s}$.

\subsection{Analysis of the Contact Angle Affected by Oxygen Plasma}

Oxygen plasma cleaning is usually employed to remove surface impurities and contaminants by exciting gas atoms with high energy states and ionization. The $\mathrm{OH}$ - group will be introduced by the oxygen plasma and cause the decrease in the contact angle of the droplet on the surface. The lowest contact angle of the droplet was measured from the surface of the RGP contact lens with a line pitch of $30 \mu \mathrm{m}$ UV treated with a laser with a scanning speed of $500 \mathrm{~mm} / \mathrm{s}$, which was approximately $20^{\circ}$ lower than that on the smooth surface without UV laser treatment. To enhance the hydrophilic characteristics, the oxygen plasma was applied to the surface of the XO RGP contact lens, and the measured value of the contact angle was approximately $36^{\circ}$ on both surfaces with and without UV laser treatment. However, the hydrophilic characteristics gradually decreased because the particle was attached on the surface and caused reduced hydrophilic properties when the XO RGP contact lens was used for several hours or days. Therefore, the surface of the RGP contact lens recovered to the hydrophobic surface, and the contact angle of the droplet increased to $101^{\circ}$ after the $110 \mathrm{~h}$ of oxygen plasma treatment, as summarized in Table 5. By contrast, the surface of the RGP contact lens conserved its hydrophilic characteristics, and the contact angle of the droplet on the surface treated with UV laser treatment only increased to $55^{\circ}$ after $110 \mathrm{~h}$. It indicated that the surface of the RGP contact lens that was treated by UV laser and oxygen plasma had good hydrophilic characteristics even after four or five days, and its maintenance was better than the surface only treated with oxygen plasma.

Table 5. Comparison of contact angle of the droplet on the XO RGP contact lens treated by oxygen plasma (Unit: ${ }^{\circ}$ ).

\begin{tabular}{ccc}
\hline \multirow{2}{*}{ Oxygen Plasma Treatment } & \multicolumn{2}{c}{ Pattern } \\
\cline { 2 - 3 } & Without & Line Pitch of $\mathbf{3 0} \boldsymbol{\mu m}$ \\
\hline Before & $118.03 \pm 2.59$ & $97.58 \pm 1.29$ \\
after 1 h & $36.22 \pm 4.41$ & $36.82 \pm 2.28$ \\
after 110 h & $101.18 \pm 1.50$ & $55.82 \pm 3.13$ \\
\hline
\end{tabular}

\section{Conclusions}

To enhance the wettability of RGP contact lenses, a laser treatment was applied in this study to investigate the relationship between the contact angle and the line pitch of the pattern and its ablated trace and depth affected by the laser scanning speed. The results revealed that a lower contact angle and better wettability can be obtained when the surface roughness is increased by reducing the laser 
scanning speed or line pitches. The most hydrophilic surface was fabricated at a laser scanning speed of $500 \mathrm{~mm} / \mathrm{s}$ and a line pitch of $30 \mu \mathrm{m}$, whereas the most hydrophobic surface was obtained when the surface roughness was $<5 \mu \mathrm{m}$ and fabricated from line pitches of $>70 \mu \mathrm{m}$ with all scanning speed and line pitches of 30-50 $\mu \mathrm{m}$ with a scanning speed of $1500-2000 \mathrm{~mm} / \mathrm{s}$. In addition, the oxygen plasma was employed to re-enhance the wettability after the laser treatment, and the contact angle decreased to $40^{\circ}$ compared with that before the oxygen plasma treatment. The contact angle of the droplet on the surface treated with the UV laser and oxygen plasma treatment was half of that of the surface treated with oxygen plasma. In the future, the edge of the RGP contact lens can be treated to enhance the wettability of tears, and different patterns can be directly fabricated by the laser system. It has the advantage of being fast, not requiring a mask, and offering various pattern selectivities. Moreover, the pattern at the edge of the RGP contact lens would not affect the vision area.

Author Contributions: Conceptualization, H.-C.C.; Methodology, K.-C.H.; Validation, Y.-H.L.; Formal Analysis, H.-Y.T.; Investigation, Y.-C.H.; Resources, Y.-H.T. and H.-C.C.; Data Curation, Y.-C.H.; Writing-Original Draft Preparation Y.-C.H. and H.-Y.T.; Writing-Review and Editing, H.-Y.T.; Visualization, Y.-H.L.; Supervision, K.-C.H.; Project Administration, K.-C.H.; Funding Acquisition, H.-C.C. and K.-C.H.

Funding: This work was supported partially by the Ministry of Science and Technology, Taiwan, under grant numbers MOST 106-2221-E-492-017- and MOST 107-2622-E-492-017-CC3.

Acknowledgments: This work was supported partially by the Ministry of Science and Technology, and the experimental and measurement setup was supported by the Taiwan Instrument Research Institute.

Conflicts of Interest: The authors declare no conflict of interest.

\section{References}

1. Bergenske, P.D.; Polse, K.A. The effect of rigid gas permeable lenses on corneal sensitivity. J. Am. Optom. Assoc. 1987, 58, 212-215. [PubMed]

2. Tyanoudis, I.; Efyon, N. Oxygen permeability of rigid contact lens materials. J. Br. Contact Lens Assoc. 1995, 18, 49-53. [CrossRef]

3. Bourassa, S.; Benjamin, W.J. RGP wettability: The first day could be the worst day! J. Br. Contact Lens Assoc. 1992, 19, 25-34. [CrossRef]

4. Guillon, M.; Guillon, J.P.; Shah, D.; Bertrand, S.; Grant, T. In vivo wettability of high Dk RGP materials. J. Br. Contact Lens Assoc. 1995, 18, 9-15. [CrossRef]

5. Ichijima, H.; Cavanagh, H.D. How rigid gas-permeable lenses supply more oxygen to the cornea than silicone hydrogels: A new model. Eye Contact Lens 2007, 33, 212-223. [CrossRef]

6. Harmano, H.; Waranabe, K.; Hamano, T.; Mitsunaga, S.; Kotani, S.; Okada, A. A study of the complications induced by conventional and disposable contact lenses. J. Contact Lens Assoc. Ophthalmol. 1994, 20, 103-108.

7. Samuel, B.; Zhao, H.; Law, K.Y. Study of Wetting and Adhesion Interactions between Water and Various Polymer and Superhydrophobic Surfaces. J. Phys. Chem. C 2011, 115, 14852-14861. [CrossRef]

8. Law, K.Y. Definitions for Hydrophilicity, Hydrophobicity, and Superhydrophobicity: Getting the Basics Right. J. Phys. Chem. Lett. 2014, 5, 686-688. [CrossRef]

9. Zhu, L.; Feng, Y.; Ye, X.; Zhou, Z. Tuning wettability and getting superhydrophobic surface by controlling surface roughness with well-designed microstructures. Sens. Actuators A 2006, 130, 595-600. [CrossRef]

10. Jung, Y.C.; Bhushan, B. Contact angle, adhesion and friction properties of micro- and nanopatterned polymers for superhydrophobicity. Nanotechnology 2006, 17, 4970-4980. [CrossRef]

11. Toosi, S.F.; Moradi, S.; Kamal, S.; Hatzikiriakos, S.G. Superhydrophobic laser ablated PTFE substrates. Appl. Surf. Sci. 2015, 349, 715-723. [CrossRef]

12. Cai, Y.; Luo, X.; Liu, Z.; Qin, Y.; Chang, W.; Sun, Y. Product and process fingerprint for nanosecond pulsed laser ablated superhydrophobic surface. Micromachines 2019, 10, 177. [CrossRef] [PubMed]

13. Qi, H.; Chen, T.; Yao, L.; Zuo, T. Hydrophilicity modification of poly (methyl methacrylate) by excimer laser ablation and irradiation. Microfluid Nanofluid 2008, 5, 139-143. [CrossRef]

14. Marco, C.; De Eaton, S.M.; Martinez-Vazquez, R.; Rampini, S.; Cerullo, G.; Levi, M.; Turri, S.; Osellame, R. Solvent vapor treatment controls surface wettability in PMMA femtosecond-laser-ablated microchannels. Microfluid Nanofluid 2014, 14, 171-176. [CrossRef] 
15. Lawrence, J.; Li, L. Modification of the wettability characteristics of polymethyl methacrylate (PMMA) by means of $\mathrm{CO}_{2}, \mathrm{Nd}$ : YAG, excimer and high power diode laser radiation. Mater. Sci. Eng. A 2001, 303, 142-149. [CrossRef]

16. Aono, Y.; Hirata, A.; Tokura, H. Non-textured laser modification of silica glass surface: Wettability control and flow channel formation. Appl. Surf. Sci. 2016, 371, 530-537. [CrossRef]

17. Bodas, D.; Khan-Malek, C. Formation of more stable hydrophilic surface of PDMS by plasma and chemical treatments. Microelectron. Eng. 2006, 83, 1277-1279. [CrossRef]

18. Tan, S.H.; Nguyen, N.T.; Chua, Y.C.; Kang, T.G. Oxygen plasma treatment for reducing hydrophobicity of a sealed polydimethysiloxane microchannel. Biomicrofluidics 2010, 4, 32204. [CrossRef]

19. Du, K.; Jiang, Y.; Liu, Y.; Wathuthanthri, I.; Choi, C.H. Manipulation of superhydrophobicity of plasma-etched polymer nanostructures. Micormachines 2018, 9, 304. [CrossRef]

20. Kim, M.Y.; Noh, J. Fabrication of a hydrophilic line on a hydrophobic surface by laser ablation processing. Micromachines 2018, 9, 208. [CrossRef]

21. Wong, S. A review of Contact angle technique. In Contact Lens Update Clinical Insights Based in Current Research; Centre for Ocular Research \& Education: Waterloo, ON, Canada, 2017.

22. Cheng, L.; Muller, S.J.; Radke, C.J. Wettability of silicone-hydrogel contact lenses in the presence of tear-film components. J. Curr. Eye Res. 2004, 28, 93-108. [CrossRef] [PubMed]

23. Lin, M.C.; Svitova, T.F. Contact Lenses Wettability In Vitro: Effect of Surface-Active Ingredients. Optom. Vis. Sci. 2010, 87, 440-447. [CrossRef] [PubMed]

24. Eustathopoulos, N.; Nicholas, M.G.; Drevet, B. Wettability at High Temperatures, 1st ed.; Pergamon Materials Series; Elsevier: Oxford, UK, 1999; Volume 3.

25. Schrader, M.E.; Loeb, G.I. Modern Approaches to Wettability. Theory and Applications; Plenum Press: New York, NY, USA, 1992.

26. Young, T. An Essay on the Cohesion of Fluids. Philos. Trans. R. Soc. Lond. 1805, 95, 65-87. [CrossRef]

27. Chow, T.S. Wetting of rough surfaces. J. Phys. Condens. Matter 1998, 10, L445-L451. [CrossRef]

28. Tadmor, R. Line energy and the relation between advancing, receding and Young contact angles. Langmuir 2004, 20, 7659-7664. [CrossRef]

29. Schrader, M.E. Young-Dupre Revisited. Langmuir 1995, 11, 3585-3589. [CrossRef]

30. Marmur, A. Wetting of Hydrophobic Rough Surfaces: To be heterogeneous or not to be. Langmuir 2003, 19, 8343-8348. [CrossRef]

31. Whyman, G.; Bormashenko, E.; Stein, T. The rigorous derivation of Young, Cassie-Baxter and Wenzel equations and the analysis of the contact angle hysteresis phenomenon. Chem. Phys. Lett. 2008, 450, 355-359. [CrossRef]

32. Ishino, C.; Okumura, K. Wetting transitions on textured hydrophilic surfaces. Eur. Phys. J. E 2008, 25, 415-424. [CrossRef] [PubMed]

33. Bico, J.; Thiele, U.; Quéré, D. Wetting of textured surfaces. Colloids Surf. A Phys. Eng. Asp. 2002, 206, 41-46. [CrossRef]

34. Yuan, Y.; Lee, T.R. Contact angle and wetting properties. Surf. Sci. Technol. 2003, 51, 3-34.

35. Domínguez-Vincent, A.; Esteve-Taboada, J.J.; Ferrer-Blasco, T.; García-Lázaro, S.; Montés-Micó, R. Optical quality comparison among different Boston contact lens materials. Clin. Exp. Optom. 2016, 99, 39-46. [CrossRef] [PubMed]

(C) 2019 by the authors. Licensee MDPI, Basel, Switzerland. This article is an open access article distributed under the terms and conditions of the Creative Commons Attribution (CC BY) license (http://creativecommons.org/licenses/by/4.0/). 


\title{
Vibration Induced Transport of Enclosed Droplets
}

\author{
Hal R. Holmes ${ }^{1}$ (D) and Karl F. Böhringer ${ }^{1,2, * \mathbb{C}}$ \\ 1 Department of Bioengineering, University of Washington, Seattle, WA 98105, USA; hrholmes@uw.edu \\ 2 Department of Electrical \& Computer Engineering and Institute for Nano-engineered Systems (NanoES), \\ University of Washington, Seattle, WA 98105, USA \\ * Correspondence: karlb@uw.edu
}

Received: 7 December 2018; Accepted: 16 January 2019; Published: 19 January 2019

\begin{abstract}
The droplet response to vibrations has been well characterized on open substrates, but microfluidic applications for droplets on open systems are limited by rapid evaporation rates and prone to environmental contamination. However, the response of enclosed droplets to vibration is less understood. Here, we investigate the effects of a dual-plate enclosure on droplet transport for the anisotropic ratchet conveyor system. This system uses an asymmetric pattern of hydrophilic rungs to transport droplets with an applied vibration. Through this work, we discovered that the addition of a substrate on top of the droplet, held in place with a 3D printed fixture, extends the functional frequency range for droplet transport and normalizes the device performance for droplets of different volumes. Furthermore, we found that the edge movements are anti-phasic between top and bottom substrates, providing a velocity profile that is correlated to vibration frequency, unlike the resonance-dependent profiles observed on open systems. These results expand the capabilities of this system, providing avenues for new applications and innovation, but also new insights for droplet mechanics in response to applied vibration.
\end{abstract}

Keywords: droplet; vibrations; transport; microfluidics

\section{Introduction}

The response of liquid droplets to a vibration is typically studied with unconfined droplets on a homogenous substrate. In this configuration, researchers have provided thorough characterizations of resonance [1,2], contact angle hysteresis [3-5], and the movement of the contact line [6,7] in response to vibrations. Similar studies have also demonstrated that droplet transport can be induced by asymmetric vibrations [8,9], and sinusoidal vibrations can be converted to horizontal transport on gradient surfaces [10-12], creating the basis for microfluidic systems using these principles [13,14]. More recent work has leveraged this ability to rectify vertical vibrations into horizontal transport by creating patterned asymmetric surfaces that transport droplets over indefinite distances (e.g., transport does not stop at the end of a gradient) [14-19]. These systems present much potential to meet a variety of applications in microfluidics, but, for some applications (particularly those that require heating or long incubation times), are limited by their high evaporation rates compared to enclosed systems [20,21]. Therefore, this work demonstrates the implementation of an enclosed configuration for droplet transport based on the anisotropic ratchet conveyor system.

Anisotropic ratchet conveyors (ARCs) transport droplets through a periodic pattern of curved hydrophilic rungs, defined by a hydrophobic background. The curvature of these rungs divides the footprint of these droplets into leading and trailing edges, that experience a difference in pinning forces, as only the leading edge of the droplet conforms to the curved rungs (Figure 1) [14]. 


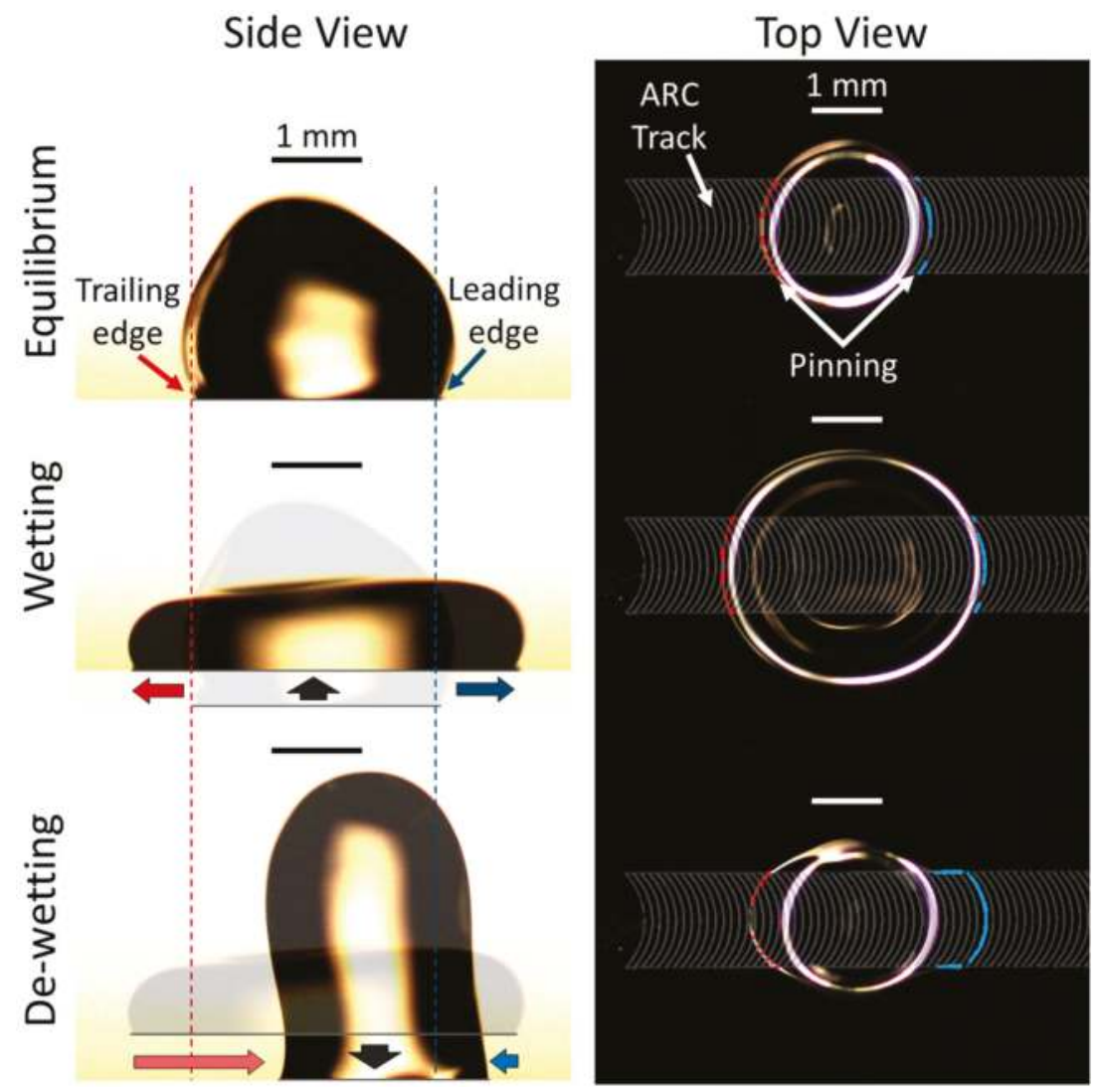

Figure 1. Mechanisms of droplet transport on anisotropic ratchet conveyors (ARCs). Droplets are transported by an ARC pattern (top view) that is composed of periodically spaced, curved rungs. The shape of the rungs creates a difference in pinning forces between the leading and trailing edges of the droplet, as only the front edge of the droplet can conform with the rung curvature. Vibration (vertical movement of the substrate) causes the edges of the droplet to oscillate between wetting and de-wetting phases. The increased pinning on the leading edge of the droplet creates an asymmetric force during the de-wetting phase, which results in a net force in the direction of droplet transport over a complete vibration cycle (side view). On the side view, the blue dashed line and arrows indicate the initial position and movement of the leading edge, and the red dashed line and arrows indicate the initial position and movement of the trailing edge, respectively. On the top view, blue and red dots indicate regions of the leading and trailing edges, respectively, that are pinned to the hydrophilic ARC pattern. Scale bars (black on side view and white on top view) indicate $1 \mathrm{~mm}$.

A sinusoidal vibration, applied vertically, causes the edges of the droplet to oscillate between wetting (edges of the droplet are advancing away from the centroid) and de-wetting (edges are contracting toward the centroid) phases. This combination of edge oscillation and a difference in pinning forces between leading and trailing edges results in a net force that drives droplet transport [14,18,19]. Previous work demonstrated a first principles model that showed droplet transport was enabled by two key anisotropies on ARCs: (1) pinning forces are greater on the leading edge of the droplet, and (2) the droplet is less susceptible to the ARC pattern during wetting (i.e., the difference in contact angle between the leading and trailing edges is larger during de-wetting than wetting) [22]. These characteristics provide for droplet transport with a ratcheting effect, wherein the droplet takes a small 
step backward and a larger step forward through each vibration cycle. From this foundation, additional functional capabilities of the ARC system have also been realized through the development of devices that can selectively pause, switch, and merge transported droplets [23], demonstrating the potential of ARC systems to automate sample handling protocols and processes. However, evaporation was still a major concern for this system. Although it was known that transport of an enclosed droplet is possible [24], the mechanics of vibrated droplets in an enclosed ARC system had yet to be investigated and it remained to be demonstrated that an enclosed ARC system could perform practical microfluidic tasks. In this work, a dual-plate ARC system was created to study how the addition of a top-plate enclosing and contacting the droplet affected the resulting transport of droplets. This system used 3D printed holders to align ARC substrates, allowing for the entire system to be vibrated. The results of this work advance the possibilities of the ARC system, providing new opportunities for innovation, and presenting new insights into droplet mechanics.

\section{Materials and Methods}

ARC fabrication begins by patterning a negative resist (NR9-1000PY) on an oxidized $<100>$ p-type silicon wafer. Following development, the wafers are vapor coated with a hydrophobic silane (fluorooctyltrichlorosilane, FOTS, Sigma-Aldrich, St. Louis, MO, USA) to create the hydrophobic background (Figure 2). Wafers are then diced (Disco DAD321) into $25 \times 75 \mathrm{~mm}$ plates. The photoresist is stripped from the ARC plates with acetone, revealing a transparent pattern of hydrophilic silicon dioxide $\left(\mathrm{SiO}_{2}\right)$ rungs defined by the hydrophobic FOTS background [25]. Plate holders are fabricated through fused deposition molding with a 3D printer (Aleph Objects Lulzbot TAZ-6, Aleph Objects, Loveland, CO, USA) to provide a separation between the plates of 2.0 and $2.5 \mathrm{~mm}$. Separation distances were confirmed through image analysis (Figure S1). This work characterized enclosed droplet transport with two configurations: (1) ARC-ARC, in which both top and bottom plates have the ARC pattern (i.e., they are mirrored), and (2) ARC-FOTS, in which the bottom plate has the ARC pattern and the top plate has a uniform hydrophobic FOTS coating.

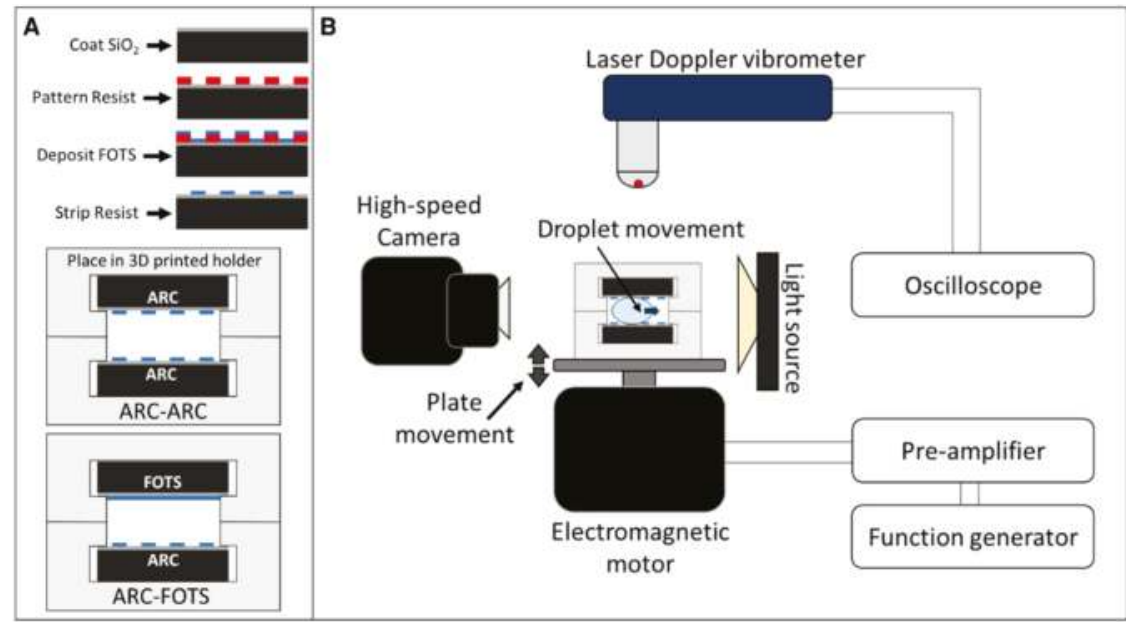

Figure 2. Fabrication process and experimental set-up. Anisotropic ratchet conveyors (ARCs) are fabricated by patterning resist on an oxidized silicon wafer, and coating exposed regions with a hydrophobic silane. (A) Stripping the resist reveals the hydrophilic ARC pattern. Wafers are diced into plates and secured in a 3D printed holder. (B) Droplet transport is examined by driving this system with an electromagnetic motor and function generator. The acceleration of the driving vibration is measured with a laser Doppler vibrometer and droplet motion is recorded with a high-speed camera. 
ARC devices are driven by an electromagnetic motor (Brüel \& Kjær, Nærum, Denmark) controlled by a function generator. The performance of the ARC devices is characterized by the minimum acceleration amplitude of the substrate required to initiate droplet transport (defined as the ARC threshold), which is measured with a laser Doppler vibrometer (Polytec OFV, Polytec Inc., Mooresville, NC, USA). This measurement was performed by increasing the vibration by small increments until transport occurs and recording the reading on the laser doppler vibrometer. The ARC threshold is reported in acceleration rather than displacement because, with a sinusoidal motion, the acceleration of the platform scales with the product of displacement and vibration frequency squared, and acceleration is directly proportional to the energy input of the system, as previously established $[18,19,22,25]$. The movement of droplets on the ARC devices is captured with a high-speed camera (Photron UX50, Photron, Tokyo, Japan), and these frames are analyzed in MATLAB (Matrix Laboratory, MathWorks, Natick, MA, USA) with custom scripts to provide quantitative measurements of transport velocity and edge movement. Error bars represent \pm standard deviation in all quantitative data shown in this work.

\section{Results}

The configurations used in this work consisted of the ARC pattern on bottom and hydrophobic coating on top (ARC-FOTS) and the ARC pattern on both top and bottom (ARC-ARC) substrates. Separations of 2.0 and $2.5 \mathrm{~mm}$ were used for both configurations with 8,13 , and $18 \mu \mathrm{L}$ droplets. Measurements of $8 \mu \mathrm{L}$ droplets were only recorded with the $2.0 \mathrm{~mm}$ plate separation because $8 \mu \mathrm{L}$ droplets did not contact the top plate when separated by $2.5 \mathrm{~mm}$.

\subsection{Effects of Top Plate Enclosure on Droplet Transport}

Qualitatively, droplets transported in this dual-plate configuration appear pillarlike, with leading and trailing edges on both plates and a liquid bulge (the center of mass of the droplet) oscillating back and forth between the two plates (Figure 3A and Video S1). ARC threshold measurements demonstrate that droplets transported in the dual-plate system require a larger vibration amplitude for transport at low frequencies than the open configuration, but at the upper end of the functional frequency range of the open plate system, a cross-over occurs, and the dual-plate system becomes more efficient (Figure 3B). On open ARC systems, the ARC threshold profile is characterized by a frequency where ARC threshold is a minimum (related to the physical properties of the droplet [22] and the pattern of the ARC device [25]) and a sharp increase in ARC threshold at the high frequency end of the functional frequency range. However, the ARC threshold profiles for the dual-plate configuration do not exhibit such pronounced increases in ARC threshold at the high frequency end. The functional frequency range for the dual-plate system is also much broader than that of the open system. As the separation distance is reduced, a similar trend occurs-the ARC threshold is higher at low frequencies but crosses over near the end of the functional frequency range of the dual-plate system with a larger separation. While droplets appeared more stable with the ARC-ARC configuration (de-pinning from the top plate was observed during some vibration cycles only with the ARC-FOTS system), no quantitative difference in performance was observed. These observations are elaborated in the discussion section. 


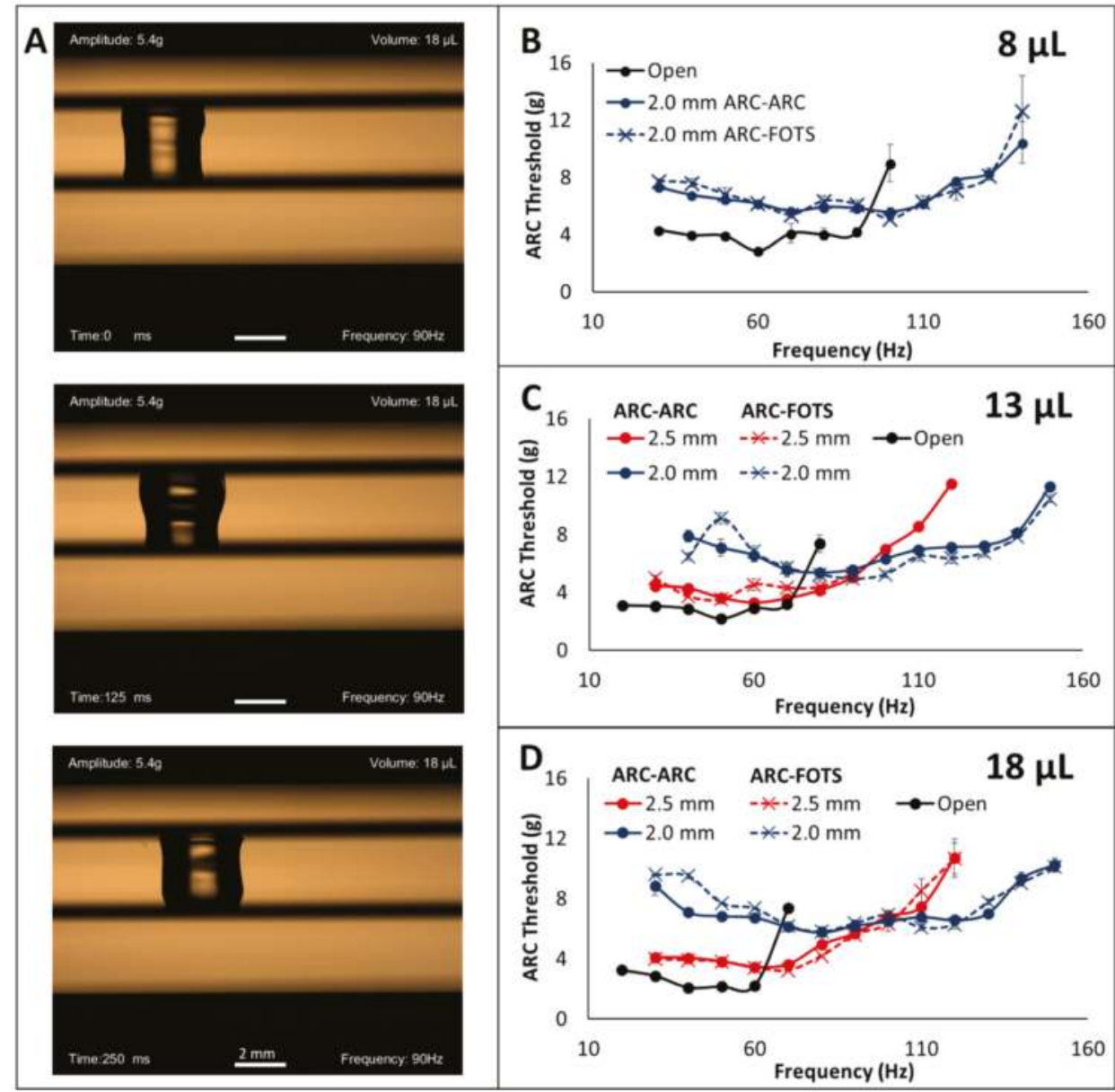

Figure 3. Anisotropic ratchet conveyor (ARC) threshold is dependent on separation distance with a dual-plate system. Droplets transported between two plates (A) exhibit a higher ARC threshold at low frequencies, but lower ARC threshold at higher frequencies, as well as a larger functional frequency range (B-D). This trend also repeats as the separation distance between plates is reduced. These observations are consistent for both ARC-ARC and ARC-FOTS configurations and all droplet volumes. Data are provided for measurements of ARC thresholds on three ARC devices and error bars represent \pm standard deviation. Scale bars show $2 \mathrm{~mm}$ on droplet images.

\subsection{Length Scale Normalization}

Transport on the dual-plate system also appears to be independent of droplet volume. Plotting these measurements on the same axes for each separation distance shows aligned ARC threshold profiles for droplets of each volume interrogated (Figure 4). These data suggest that the dual-plate configuration normalizes the vertical movement of the center of mass of the droplets. In other words, for unconfined droplets in an open system, the vertical movement of the center of mass depends on the droplet volume [22] and vibration frequency [25], but on this enclosed system, the vertical movement of the center of mass is confined to a fixed range between the two plates. 


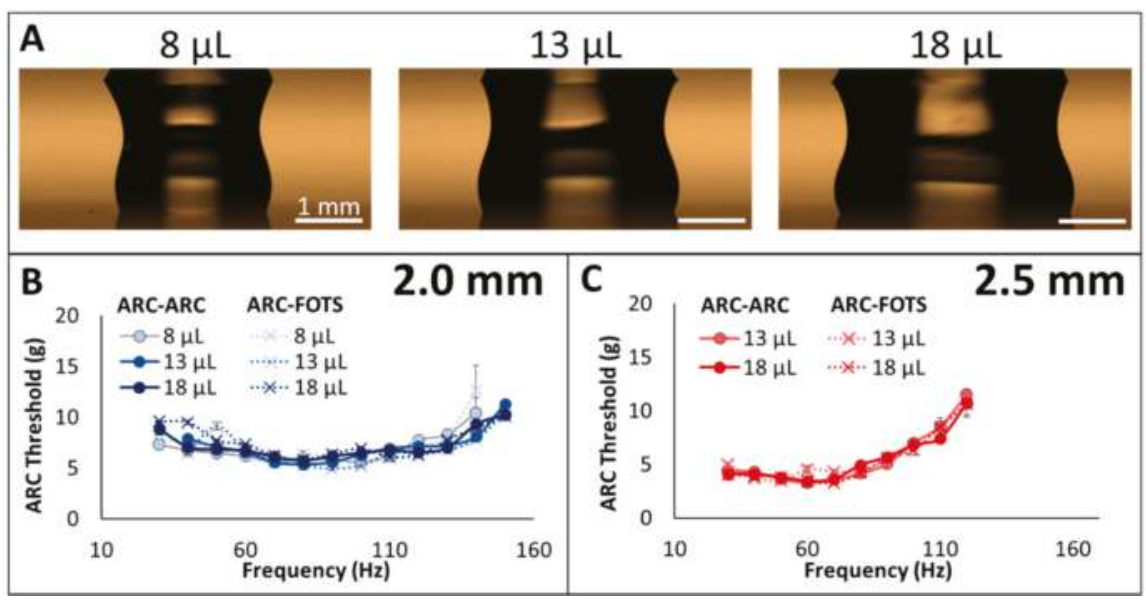

Figure 4. Anisotropic ratchet conveyor (ARC) threshold is independent of droplet volume in dual-plate system. ARC threshold values are similar for droplets of different volume (A) transported in $2.0 \mathrm{~mm}$ (B) and $2.5 \mathrm{~mm}(\mathrm{C})$ tracks. On open ARC systems, ARC threshold profiles are dependent on droplet volume [22], thus these profiles demonstrate that the ARC threshold profiles scale with vertical movement of the center of mass of the droplets. Error bars indicate \pm standard deviation; scale bars on droplet images represent $1 \mathrm{~mm}$.

\subsection{Transport Velocity of Enclosed Droplets}

The velocity of droplets transported under these configurations was also determined (Figure 5). Velocity was recorded at the ARC threshold, which therefore describes the minimum velocity that a droplet can be transported at for each frequency. Previous work examining droplet velocities in an open configuration showed that transport velocity profiles exhibited a large velocity at a specific frequency dependent on droplet volume. This velocity dropped as frequency was increased even though the acceleration (and therefore velocity) of the driving substrate was higher [25]. The peak in this velocity profile provided the appearance of a resonance frequency, but this frequency was higher than the predicted natural frequency of these droplets on a surface $[6,22]$, and was influenced by the pattern of the ARC device [25]. While these peaks appear to be related to resonance of the droplet, additional theoretical modelling is required to more accurately describe these effects.

On the enclosed system, local maxima and minima were observed, but these peaks occurred at higher frequencies than on the open system and, generally, the transport velocity increased with increasing frequency. The velocity was also slightly higher on the ARC-FOTS configuration, which is likely due to the reduced pinning forces acting on the top of the droplet. This result is likely due to the tethering of the droplet to both top and bottom plates. In the dual-plate configuration, the droplet edges are wetting on the top plate and de-wetting on the bottom plate as the droplet center of mass moves upward and vice-versa as the center of mass moves downward. Thus, the movement of droplet edges is anti-phasic (Figure 6), so the leading edge on one plate cannot advance too far ahead of the other in any particular vibration cycle. Thus, unlike an open ARC system [25], the droplet consistently advances by the same number of rungs on each plate throughout each vibration cycle. Therefore, increasing frequency results in the droplets taking regular steps of the same distance more quickly (Video S2). 

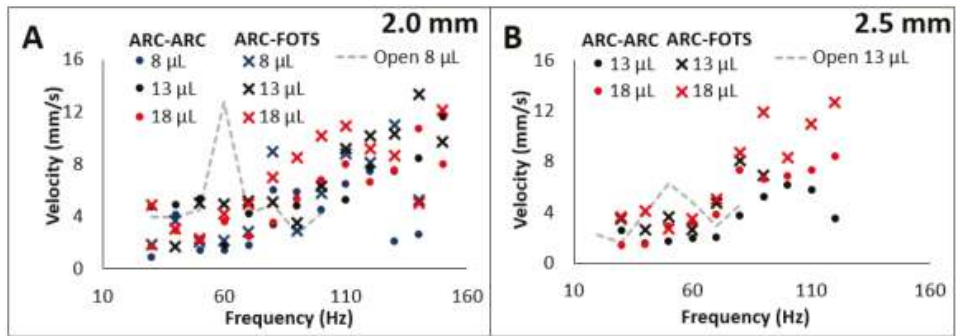

Figure 5. Transport velocity is correlated with vibration frequency on a two-plate system. Transport velocity at the anisotropic ratchet conveyor (ARC) threshold trends upward with increasing frequency on two-plate systems with (A) $2.0 \mathrm{~mm}$ and (B) $2.5 \mathrm{~mm}$ separations. Gray dashed lines indicate the velocity profiles of (A) $8 \mu \mathrm{L}$ and (B) $13 \mu \mathrm{L}$ droplets on open ARC systems with the same pattern. The velocity profiles on open systems are characterized by a peak velocity related to the droplet volume and a sharp drop in velocity at higher frequencies, whereas velocity profiles on the enclosed system generally increase with vibration frequency although volume dependent local maxima and minima are observed.

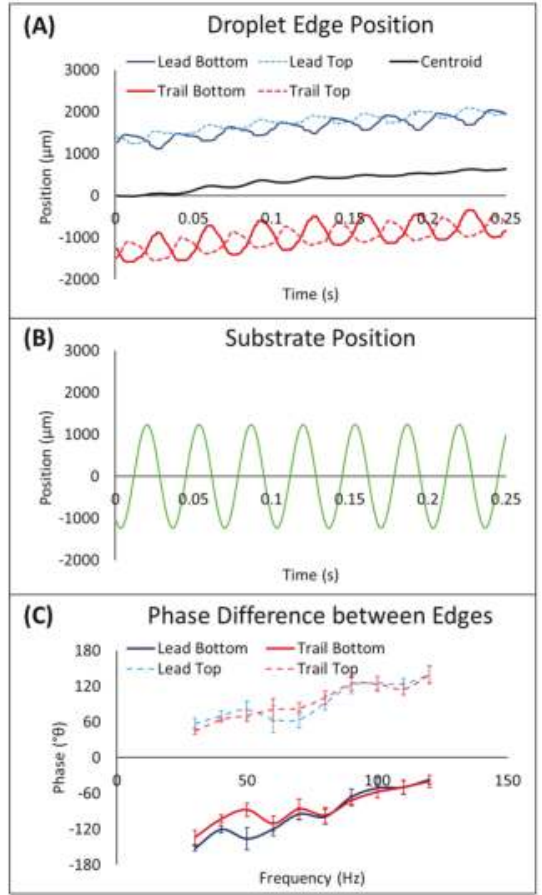

Figure 6. Droplet edges are anti-phasic between substrates. The wetting and de-wetting phases of contact line oscillation alternate between top and bottom edges (A). The movement of the droplet centroid also demonstrates that the ratcheting effect (i.e., small step backward and larger step forward) is still present for droplet transport on the enclosed system. Edge movement and the corresponding position of the driving substrate (B) is shown for a $13 \mu \mathrm{L}$ droplet transported at $30 \mathrm{~Hz}$ in an enclosed ARC-ARC system with a separation of $2.5 \mathrm{~mm}$. The phase difference, defined as the time delay between the highest position of the substrate and the furthest expansion of droplet edges, shows that top and bottom edges are anti-phasic (with a phase difference of $186.8^{\circ} \pm 16.4$ between leading edges and $178.4^{\circ} \pm 13.0$ between trailing edges) throughout the entire functional frequency range for the system (C). Error bars show \pm standard deviation of the phase angle for each frequency. 


\section{Discussion and Conclusion}

In previous work, droplet transport profiles on an open system were non-dimensionalized with respect to the radius of the droplet, as it was assumed that the displacement of the center of mass during vibration scaled with the radius of the droplet (i.e., the vertical oscillation of the center of mass gets larger as the droplets get larger) [22]. The alignment of ARC threshold profiles based on plate separation observed in this work provides further evidence for this scaling argument, as the dual-plate system effectively normalizes the displacement of the center of mass for each droplet. This effect also contributes to the broadening of the functional frequency range observed when the top plate is added and separation distance is reduced. The confined motion of the droplet center of mass emulates the motion of smaller droplets. Future work will look into creating a comprehensive model of these effects, as well as the effects of the droplet interaction with the top-plate. However, there are limits on this normalization effect, and measuring a broader range of droplet volumes would likely show a bandpass (or volume-pass) effect where droplets too small to contact the top-plate or too large to sufficiently respond to the ARC track will not be transported, but all volumes in between will exhibit a similar ARC threshold profile. This volume range is dependent on the plate separation distance and the feature size of the ARC pattern, and therefore could be selected for specific applications. For example, $8 \mu \mathrm{L}$ droplets behave as open droplets while 13 and $18 \mu \mathrm{L}$ droplets behave as confined droplets with a plate separation of $2.5 \mathrm{~mm}$, meaning that 13 and $18 \mu \mathrm{L}$ droplets could be transported at frequencies at $110 \mathrm{~Hz}$ and above on this configuration while $8 \mu \mathrm{L}$ droplets would remain in place. Practically, this attribute could be extremely beneficial for a user-friendly device, as it would allow a large tolerance for the application of droplets by a user that does not have access to precision pipetting equipment. Furthermore, the higher reproducibility of step size on the dual-plate systems could meet applications requiring precise positioning of droplets.

The similarity in ARC threshold and velocity profiles between ARC-ARC and ARC-FOTS configurations indicate that pinning forces acting on the top and bottom plates of the ARC-ARC configuration do not sum. This effect is likely a result of the anti-phasic nature of this system, as these pinning forces will be out of sync. However, if only one plate were moving (e.g., their separation distance was increasing and decreasing), then these phases would be synchronized between the top and bottom plates. This configuration may provide for a lower ARC threshold and could potentially transport droplets vertically, but the changing plate separation would also complicate the formation of a seal to prevent evaporation.

Compared to an open ARC system, a dual-plate configuration expands the functional frequency range and normalizes device performance with regard to droplet volume. The dual-plate configuration also provides a simple means of sealing this system to reduce evaporation rates and offers new opportunities to improve the capabilities of the ARC system — particularly the ability to handle fluids with lower surface tension than pure water, control droplets with smaller volumes, and perform new functions (i.e., droplet fission) that have yet to be achieved on ARC systems, which will be the focus of future work. Overall, these results demonstrate the increasing potential of ARC systems to meet a wider variety of applications in microfluidics.

Supplementary Materials: The following are available online at http:/ /www.mdpi.com/2072-666X/10/1/69/s1, Figure S1: Dual-plate set up; Video S1: ARC transport of enclosed droplet; Video S2: Transport velocity depends on vibration frequency.

Author Contributions: H.R.H. conceived, designed and performed experiments, analyzed the data, and wrote the paper. K.F.B. contributed reagents/materials/analysis tools, analyzed the data, and revised and edited the paper. All authors have given approval to the final version of the manuscript.

Funding: This research was funded by the National Science Foundation grant number ECCS-1308025. 
Acknowledgments: The authors would like to thank Conservation X Labs for use of the 3D printer and filament used in this work. HRH would like to acknowledge support by the Department of Defense (DoD) through the National Defense Science \& Engineering Graduate Fellowship (NDSEG) Program. Fabrication of ARCs was performed at the Washington Nanofabrication Facility (WNF), a National Nanotechnology Coordinated Infrastructure (NNCI) site at the University of Washington, which is supported in part by the National Science Foundation (awards NNCI-1542101, 1337840 and 0335765), the Washington Research Foundation, the M. J. Murdock Charitable Trust, Altatech, ClassOne Technology, Global Capital Equipment (GCE) Market, Google, and SPTS.

Conflicts of Interest: The authors declare no conflict of interest.

\section{References}

1. Perez, M.; Brechet, Y.; Salvo, L.; Papoular, M.; Suery, M. Oscillation of liquid drops under gravity: Influence of shape on the resonance frequency. Europhys. Lett. 1999, 47, 189. [CrossRef]

2. Vukasinovic, B.; Smith, M.K.; Glezer, A. Dynamics of a sessile drop in forced vibration. J. Fluid Mech. 2007, 587, 395-423. [CrossRef]

3. Sharp, J.S. Resonant properties of sessile droplets; contact angle dependence of the resonant frequency and width in glycerol/water mixtures. Soft Matter 2011, 8, 399-407. [CrossRef]

4. Sharp, J.S.; Farmer, D.J.; Kelly, J. Contact angle dependence of the resonant frequency of sessile water droplets. Langmuir 2011, 27, 9367-9371. [CrossRef] [PubMed]

5. Mettu, S.; Chaudhury, M.K. Vibration spectroscopy of a sessile drop and its contact line. Langmuir 2012, 28, 14100-14106. [CrossRef] [PubMed]

6. Noblin, X.; Buguin, A.; Brochard-Wyart, F. Vibrated sessile drops: Transition between pinned and mobile contact line oscillations. Eur. Phys. J. E 2004, 14, 395-404. [CrossRef] [PubMed]

7. Noblin, X.; Buguin, A.; Brochard-Wyart, F. Vibrations of sessile drops. Eur. Phys. J. Spec. Top. 2009, 166, 7-10. [CrossRef]

8. Buguin, A.; Brochard, F.; De Gennes, P.-G. Motions induced by asymmetric vibrations. Eur. Phys. J. E 2006, 19, 31-36. [CrossRef]

9. Mettu, S.; Chaudhury, M.K. Motion of liquid drops on surfaces induced by asymmetric vibration: Role of contact angle hysteresis. Langmuir 2011, 27, 10327-10333. [CrossRef]

10. Daniel, S.; Chaudhury, M.K. Rectified motion of liquid drops on gradient surfaces induced by vibration. Langmuir 2002, 18, 3404-3407. [CrossRef]

11. Daniel, S.; Sircar, S.; Gliem, J.; Chaudhury, M.K. Ratcheting motion of liquid drops on gradient surfaces. Langmuir 2004, 20, 4085-4092. [CrossRef] [PubMed]

12. Shastry, A.; Case, M.J.; Böhringer, K.F. Directing Droplets Using Microstructured Surfaces. Langmuir 2006, 22, 6161-6167. [CrossRef] [PubMed]

13. Daniel, S.; Chaudhury, M.K.; De Gennes, P.-G. Vibration-actuated drop motion on surfaces for batch microfluidic processes. Langmuir 2005, 21, 4240-4248. [CrossRef] [PubMed]

14. Holmes, H.R.; Böhringer, K.F. Transporting droplets through surface anisotropy. Microsyst. Nanoeng. 2015, 1, 15022. [CrossRef]

15. Demirel, M.C. Emergent properties of spatially organized poly(p-xylylene) films fabricated by vapor deposition. Colloids Surf. Physicochem. Eng. Asp. 2008, 321, 121-124. [CrossRef]

16. Malvadkar, N.A.; Hancock, M.J.; Sekeroglu, K.; Dressick, W.J.; Demirel, M.C. An engineered anisotropic nanofilm with unidirectional wetting properties. Nat. Mater. 2010, 9, 1023-1028. [CrossRef] [PubMed]

17. Agapov, R.L.; Boreyko, J.B.; Briggs, D.P.; Srijanto, B.R.; Retterer, S.T.; Collier, C.P.; Lavrik, N.V. Length scale selects directionality of droplets on vibrating pillar ratchet. Adv. Mater. Interfaces 2014, 1. [CrossRef]

18. Duncombe, T.A.; Parsons, J.F.; Böhringer, K.F. Directed drop transport rectified from orthogonal vibrations via a flat wetting barrier ratchet. Langmuir 2012, 28, 13765-13770. [CrossRef]

19. Duncombe, T.A.; Erdem, E.Y.; Shastry, A.; Baskaran, R.; Böhringer, K.F. Controlling liquid drops with texture ratchets. Adv. Mater. 2012, 24, 1545-1550. [CrossRef]

20. Lin, T.-H.; Yao, D.-J. Applications of EWOD Systems for DNA Reaction and Analysis. J. Adhes. Sci. Technol. 2012, 26, 1789-1804. [CrossRef]

21. Zhu, L.; Feng, Y.; Ye, X.; Feng, J.; Wu, Y.; Zhou, Z. An ELISA chip based on an EWOD microfluidic platform. J. Adhes. Sci. Technol. 2012, 26, 2113-2124. [CrossRef] 
22. Dong, Y.; Holmes, H.R.; Böhringer, K.F. Converting vertical vibration of anisotropic ratchet conveyors into horizontal droplet motion. Langmuir 2017, 33, 10745-10752. [CrossRef] [PubMed]

23. Holmes, H.R.; Gomez, A.E.; Böhringer, K.F. Enabling Droplet Functionality on Anisotropic Ratchet Conveyors. Micromachines 2017, 8, 363. [CrossRef] [PubMed]

24. Duncombe, T.A.; Kumemura, M.; Fujita, H.; Böhringer, K.F. Integrating EWOD with surface ratchets for active droplet transport and sorting. In Proceedings of the 2009 IEEE 22nd International Conference on Micro Electro Mechanical Systems, Sorrento, Italy, 25-29 January 2009; pp. 531-534.

25. Holmes, H.R.; Böhringer, K.F. Transport velocity of droplets on ratchet conveyors. Adv. Colloid Interface Sci. 2017. [CrossRef] [PubMed]

(C) 2019 by the authors. Licensee MDPI, Basel, Switzerland. This article is an open access article distributed under the terms and conditions of the Creative Commons Attribution (CC BY) license (http:/ / creativecommons.org/licenses/by/4.0/). 


\title{
One-Step Preparation of Durable Super-Hydrophobic $\mathrm{MSR} / \mathrm{SiO}_{2}$ Coatings by Suspension Air Spraying
}

\author{
Zhengyong Huang ${ }^{1,2, *}$, Wenjie Xu ${ }^{1}$, Yu Wang ${ }^{1}$, Haohuan Wang ${ }^{1}$, Ruiqi Zhang ${ }^{1}$, Ximing Song ${ }^{1}$ \\ and Jian $\mathrm{Li}^{1 \text {,* }}$ \\ 1 State Key Laboratory of Power Transmission Equipment \& System Security and New Technology, \\ Chongqing University, Chongqing 400040, China; 18883878345@163.com (W.X.); wangy@cqu.edu.cn (Y.W.); \\ 20134209@cqu.edu.cn (H.W.); zhangruiqi@cqu.edu.cn (R.Z.); songximingchina@gmail.com (X.S.) \\ 2 Postdoctoral Research Station on Chemical Engineering and Technology, Chongqing University, \\ Chongqing 400040, China \\ * Correspondence: huangzhengyong@cqu.edu.cn (Z.H.); lijian@cqu.edu.cn (J.L.); \\ Tel.: +86-023-6510-2442 (Z.H. \& J.L.)
}

Received: 4 November 2018; Accepted: 14 December 2018; Published: 19 December 2018

\begin{abstract}
In this study, we develop a facial one-step approach to prepare durable super-hydrophobic coatings on glass surfaces. The hydrophobic characteristics, corrosive liquid resistance, and mechanical durability of the super-hydrophobic surface are presented. The as-prepared super-hydrophobic surface exhibits a water contact angle (WCA) of $157.2^{\circ}$ and contact angle hysteresis of $2.3^{\circ}$. Mico/nano hierarchical structures and elements of silicon and fluorine is observed on super-hydrophobic surfaces. The adhesion strength and hardness of the surface are determined to be 1 st level and $4 \mathrm{H}$, respectively. The coating is, thus, capable of maintaining super-hydrophobic state after sand grinding with a load of $200 \mathrm{~g}$ and wear distances of $700 \mathrm{~mm}$. The rough surface retained after severe mechanical abrasion observed by atomic force microscope (AFM) microscopically proves the durable origin of the super-hydrophobic coating. Results demonstrate the feasibility of production of the durable super-hydrophobic coating via enhancing its adhesion strength and surface hardness.
\end{abstract}

Keywords: super-hydrophobic; durable; adhesion; corrosive resistance

\section{Introduction}

Nowadays, with the development of nanotechnology, micro/nano devices have drawn attention to many researchers, and are widely applied in medical, chemistry, biology, electronics, and precise instrument fabricating fields [1-3]. However, most of the precise electronic devices are moisture-sensitive, such that water and moisture greatly influences the longevity and the precision. Also, the bioadhesion of the surface, involving different types of interactions between organisms and the surface, make difficulties in further applications in medical and biology [4].

In recent decades, it was found that the surface of lotus leaves demonstrated strong hydrophobicity, so-called super-hydrophobicity. The super-hydrophobic surface is defined as the one with a water contact angle (WCA) greater than $150^{\circ}$ and water sliding angle (WSA) less than $5^{\circ}$, such that water droplets on it remain spherical and tend to slide off easily [5-7]. The reduced area between the droplet and the super-hydrophobic surface leads to a variety of potential applications in anti-fogging [8], humidity-proof [9], bio-surface [10], anti-icing [11], leakage current suppression [12,13], self-cleaning [14], oil/water separation [15], and corrosion protection [16].

In general, the super-hydrophobic surfaces can be achieved by constructing micro/nano-structures on surfaces with low surface energy. Many methods such as chemical etching [17,18], electrochemical deposition [19,20], electrospinning [21,22], phase separation [23,24], plasma treating [25,26], and sol-gel 
processes $[27,28]$ have been adopted to mimic the "lotus-effect" super-hydrophobic surfaces. The super-hydrophobic surfaces allow a large amount of air entrapment and thus consequently reduce skin friction drag between the surface itself and the droplet. Water droplets tend to roll or slide off the surfaces by tilting or external vibration. The excellent hydrophobicity and self-cleaning properties of super-hydrophobic surfaces are beneficial to reduce water droplets and contamination accumulation, which, therefore, make super-hydrophobic material a prospective candidate to improve anti-pollution performance of outdoor high-voltage insulators in power systems. However, the super-hydrophobic surfaces fail to maintain the super-hydrophobicity because of the fragile micro-structures on them [29-31]. Commonly applied inorganic super-hydrophobic coatings like metal oxides [32-34] and rare-earth oxides [35] are chemically susceptible. Organic super-hydrophobic coatings [4,36] are short of mechanical robustness, although they have good chemical resistance [37].

Most of the robust super-hydrophobic surfaces are prepared with intrinsically durable media, such as metal meshes and fabrics [38]. The abrasion resistance of a super-hydrophobic surface based on polyurethane elastomer and sacrificial aluminum oxide template was maintained after 10,000 cycles, with a water contact angle (WCA) of above $150^{\circ}$ [21]. Furthermore, Alan et al. used a mesh cloth as a reusable template to fabricate a robust super-hydrophobic surface, which remained super-hydrophobic after more than 5500 abrasion cycles at $32.00 \mathrm{kPa}$ [39]. Zhou et al. reported a super-hydrophobic fabric coating made of cross-linked polydimethylsiloxane elastomer, which is capable of withstanding more than 20,000 cycles of abrasion at $12.00 \mathrm{kPa}$ without losing super-hydrophobicity [40]. In addition, Zimmermann et al. prepared durable super-hydrophobic fabrics by a one-step gas phase coating method that maintains the super-hydrophobic property after 1450 cycles of abrasion with the load pressure of $7.8 \mathrm{kPa}$ [41]. Nevertheless, preparing a super-hydrophobic surface with high abrasion resistance without introducing durable media is still challenging.

In this work, we prepared a durable super-hydrophobic coating on glass surfaces by a facial one-step approach and analyzed the hydrophobic properties, mechanical durability, and corrosive liquid resistance. The as-prepared super-hydrophobic surface exhibits a water contact angle (WCA) of $157.2^{\circ}$ and contact angle hysteresis of $2.3^{\circ}$. The hierarchical structures on the surface of super-hydrophobic coatings were observed through scanning electron microscopy (SEM) images. The adhesion strength and the hardness of the super-hydrophobic surface were determined to be first level and $4 \mathrm{H}$, respectively. In addition, observed by the atomic force microscope (AFM), the rough structure remains on the super-hydrophobic surface after wearing for $700 \mathrm{~mm}$ with a mechanical load of $200 \mathrm{~g}$, which shows that the as-prepared surface maintains the super-hydrophobic ability for long periods. The results demonstrate the feasibility of production of durable super-hydrophobic coatings via enhancing their surface hardness and adhesion strength between the coating and the substrates.

\section{Materials and Methods}

The super-hydrophobic coating in this study was fabricated by incorporation of $\mathrm{SiO}_{2}$ nanoparticles

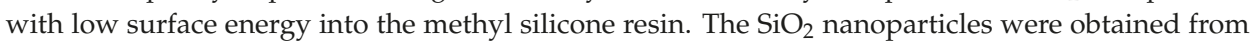
the gelation of the nano-silica sol, formed by the composites of the tetraethyl orthosilicate (TEOS) and ammonium hydroxide. Firstly, $30 \mathrm{~mL}$ of TEOS dissolved in $350 \mathrm{~mL}$ of alcohol was stirred for 24 hours. Then, $18 \mathrm{~mL}$ of ammonium hydroxide (volume ratio of distilled water to ammonia was 2:7) was added for the sol generation. Nano-silica aerogel was obtained by drying the nano-silica sol in vacuum at $120^{\circ} \mathrm{C}$ for 12 hours and purification with high-speed centrifugation. The chemical reaction is shown below:

$$
\left(\mathrm{C}_{2} \mathrm{H}_{5} \mathrm{O}\right)_{4} \mathrm{Si} \stackrel{\mathrm{NH}_{4} \mathrm{OH}}{\rightarrow} \mathrm{SiO}_{2}+\mathrm{C}_{2} \mathrm{H}_{5} \mathrm{OH}
$$

To reduce the surface energy of the $\mathrm{SiO}_{2}$ nanoparticles, $3 \mathrm{~g}$ of $\mathrm{SiO}_{2}$ nanoparticles synthesized above was sequentially mixed with $25 \mathrm{~mL}$ of n-hexyl alcohol and $1 \mathrm{~g}$ of hydroxyl-terminated polydimethylsiloxane (OH-PDMS) by mechanical stirring. After 24 hours of stirring, $0.08 \mathrm{~g}$ of dibutyltindilaurate (DBTD) and $10 \mathrm{~mL}$ of $\mathrm{n}$-hexyl alcohol were further added. The chemical graft of the $\mathrm{OH}-\mathrm{PDMS}$ onto the surface of $\mathrm{SiO}_{2}$ nanoparticles proceeded after a further 30 minutes of mechanical 
stirring. The hydrophobic $\mathrm{SiO}_{2}$ nanoparticles were obtained after drying for $2 \mathrm{~h}$ at $60^{\circ} \mathrm{C}$. The chemical graft of OH-PDMS on the silica surface is shown below:

$$
\mathrm{SiOH}+\mathrm{HO}[\mathrm{SiOSi}]_{\mathrm{n}} \mathrm{OH} \stackrel{\mathrm{DBTD}}{\rightarrow} \mathrm{SiO}[\mathrm{SiOSi}]_{\mathrm{n}} \mathrm{OSi}+\mathrm{H}_{2} \mathrm{O}
$$

Two grams of methyl silicone resins and $10 \mathrm{~g}$ of hydrophobic $\mathrm{SiO}_{2}$ nanoparticles were mixed and ultrasonically dispersed at room temperature for $10 \mathrm{~min}$. The methyl silicone resins $/ \mathrm{SiO}_{2}$ mixtures were sprayed onto the surface of the slide glass by air spraying using an air spray gun with a nozzle diameter of $0.5 \mathrm{~mm}$ and spraying pressure of $0.15 \mathrm{MPa}$. The spraying distance between the glass slide surface and the nozzle tip was kept around $15 \mathrm{~cm}$. The glass slide with methyl silicone resin $/ \mathrm{SiO}_{2}$ composite coating was dried in an oven at $100{ }^{\circ} \mathrm{C}$ for $1 \mathrm{~h}$ and, finally, the super-hydrophobic methyl silicone resin $/ \mathrm{SiO}_{2}$ composite coating was obtained on the surface of the glass slides.

The hydrophobicity, such as the static water contact angle and the water contact angle hysteresis, of the as-prepared super-hydrophobic coating was measured by a contact angle meter (Drop Meter A-20, Maishi, Ningbo, China). The micro-topography of the as-prepared coating was scanned by scanning electron microscopy (TESCAN VEGA, Zeiss, Oberkochen, Germany) and atomic force microscopy (PARK XE7, Park, Suwon, Korea). The root-mean-square roughness of the super-hydrophobic coating after wearing was calculated from the surface topography. The corrosive liquid tolerance of the super-hydrophobic surface was evaluated by hydrophobicity test after immersion in the acidic and basic liquids. The mechanical durability of the super-hydrophobic coating was tested by measuring the water contact angles and observing the microscopic surface topography after sandpaper abrasion with certain mechanical loads and wear distances, similar to the procedure in the ISO 8251-81 [42] standard. The surface adhesion of the super-hydrophobic coating was evaluated by the adhesion test in ASTM D3359 [43], which classifies the adhesion level of the coating according to the integrity of the coating after being scratched by a multi-blade cutter. In addition, the surface hardness of the as-prepared super-hydrophobic coating was determined by scratch testing based on the ASTM D3363 standard [44], which evaluates the surface hardness of the coating by comparing it with that of the given pencils with a certain hardness by scratching the surface of the coating with fixed pressure.

\section{Results and Discussion}

\subsection{Surface Micro-Structure}

Figure $1 \mathrm{a}, \mathrm{b}$ show the scanning electron micrograph (SEM) images of the as-prepared super-hydrophobic surface. In Figure 1a, the surface consists of an irregular three-dimensional (3D) micropapilla with a diameter between $2 \mu \mathrm{m}$ and $4 \mu \mathrm{m}$. Furthermore, in Figure $1 \mathrm{~b}, 43 \mathrm{~nm}$ to $60 \mathrm{~nm}$ nanoparticles are found on the micropapilla. The SEM images further prove that the super-hydrophobic surface has a rough surface.

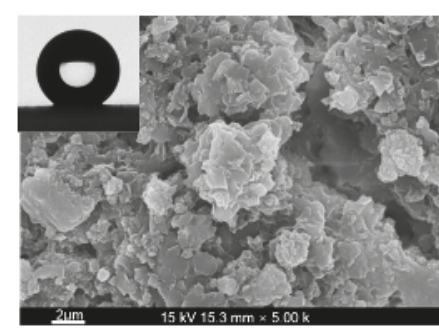

(a)

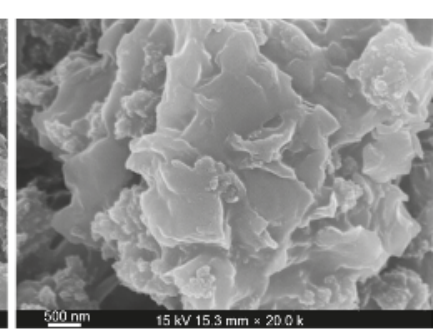

(b)

Figure 1. Scanning electron micrograph (SEM) images of the super-hydrophobic methyl silicone resin $/ \mathrm{SiO}_{2}$ composite surface. 


\subsection{Surface Wettability}

The inset picture in Figure 1 shows the profiles of water droplets on the surface of the methyl silicone resin $/ \mathrm{SiO}_{2}$ composite coatings. The bare glass substrate exhibited a WCA of $37.5 \pm 2^{\circ}$. Compared with the hydrophilic bare glass plate, the water droplets on the surface of methyl silicone resin $/ \mathrm{SiO}_{2}$ composite coatings are approximately spherical. The static water contact angles on the composite surface are between $154.9^{\circ}$ and $160.3^{\circ}$, with an average static water contact angle of $157.2^{\circ}$.

Figure 2 shows the contact angle hysteresis of the methyl silicone resin $/ \mathrm{SiO}_{2}$ composite coating determined via one cycle of addition and depression of a water droplet of $10 \mu \mathrm{L}$. The contact angle hysteresis curve in the shape of "Z" demonstrates the difference between the advancing and receding water contact angles. As the volume of the water droplet increases, the average advancing water contact angle is measured as $160.8^{\circ}$. When the water droplets stop growing and start to shrink, the receding water contact angle initially increases and then becomes steady, with values between $159.6^{\circ}$ and $155.7^{\circ}$, and the average receding water contact angle is $158.5^{\circ}$. The difference between the advancing water contact angle and the receding water contact angle is defined as the water contact angle hysteresis of the methyl silicone resin $/ \mathrm{SiO}_{2}$ composite coating, with an average value of $2.3^{\circ}$. The low value of the contact angle hysteresis and the great water contact angles of the methyl silicone resin $/ \mathrm{SiO}_{2}$ composite coating indicates the super-hydrophobic performance of the as-prepared methyl silicone resin surface.

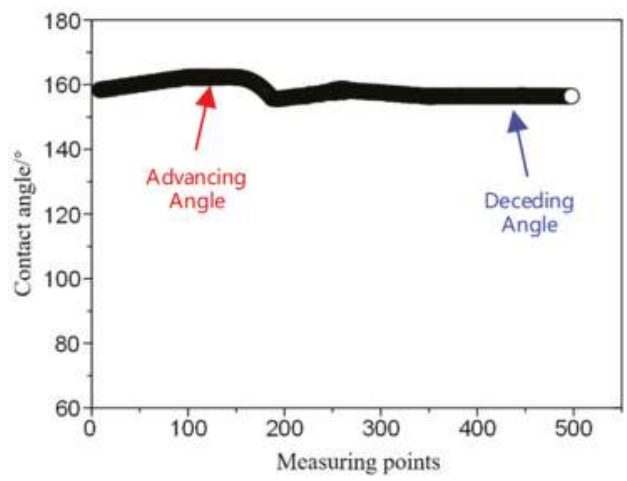

Figure 2. Water contact angle hysteresis of the super-hydrophobic methyl silicone resin $/ \mathrm{SiO}_{2}$ composite surface.

The water droplet stays nearly spherical on the super-hydrophobic surface for the air gaps trapped between the irregular serration of the rough surface and the water droplet. The Cassie Equation [45] was used to quantify the contact area of the super-hydrophobic surface with the air:

$$
\cos \theta=f_{1}\left(1+\cos \theta_{e}\right)-1
$$

where $\theta$ is the static water contact angle of the super-hydrophobic coating; $f_{1}$ is the ratio of the solid-liquid contact area of the super-hydrophobic surface; and $\theta_{\mathrm{e}}$ is the intrinsic contact angle of the methyl silicone resin surface. The static water contact angle of the super-hydrophobic coating is measured as $157 \cdot 2^{\circ}$. The contact angle of the methyl silicone resin surface is found to be $112^{\circ}$. According to the Cassie Equation, the value of $\mathrm{f}_{1}$ can be calculated as $12.49 \%$. Thus, the ratio of the solid-liquid contact area of the super-hydrophobic coating with water droplets can be obtained. Additionally, the proportion of the gas-liquid contact area of the super-hydrophobic coating $f_{2}$ is calculated as $87.51 \%$. The results indicate that the actual contact area of the super-hydrophobic coating with water droplets only accounts for $12.49 \%$ of the total composite contact surface area, and the 
composite contact surface area occupied by air is estimated to be $87.51 \%$. The calculation results indicate the excellent super-hydrophobic properties of the super-hydrophobic coating.

\subsection{Chemical and Mechanical Durability}

The chemical durability of the super-hydrophobic methyl silicone resin/ $\mathrm{SiO}_{2}$ composite coating was investigated. Figure 3 shows the static contact angle of the super-hydrophobic coating wetted by aqueous solutions with different $\mathrm{pH}$ values after $24 \mathrm{~h}$. From the test results, after being wetted by corrosive liquids with $\mathrm{pH}$ values between 1 and 14, the static contact angle of super-hydrophobic surface is always greater than $150^{\circ}$. The minimum and maximum average static contact angle of the corrosive liquid are $152.4^{\circ}$ and $159.5^{\circ}$, respectively.

Figure 4 shows the contact angle hysteresis of the super-hydrophobic coating wetted by corrosive liquids with $\mathrm{pH}$ values ranging from 1 to 14 after $24 \mathrm{~h}$. The contact angle hysteresis of the water droplets stays low with a maximum average of $5.8^{\circ}$ and a minimum average of $1.5^{\circ}$. Apparently, the corrosive liquids with different $\mathrm{pH}$ range have little influence on the static contact angle and contact angle hysteresis of the super-hydrophobic methyl silicone resin $/ \mathrm{SiO}_{2}$ composite coating.

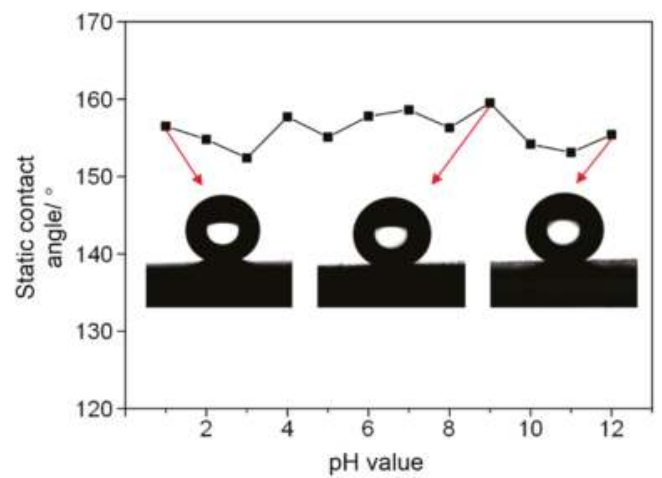

Figure 3. Static contact angle of super-hydrophobic methyl silicone resin $/ \mathrm{SiO}_{2}$ composite coating after infiltrating with corrosive liquids with different $\mathrm{pH}$ values for $24 \mathrm{~h}$.

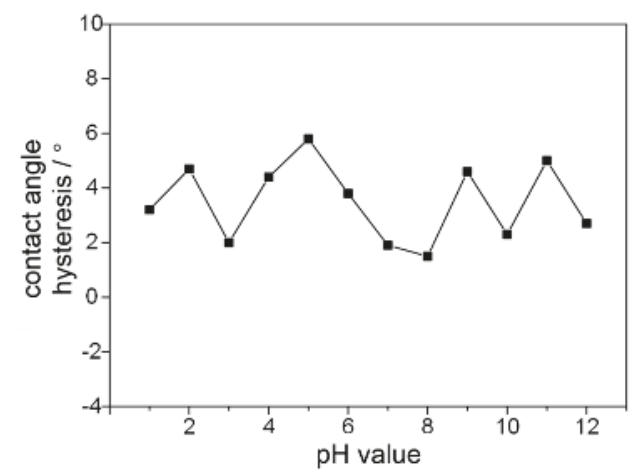

Figure 4. Water contact angle hysteresis of the super-hydrophobic methyl silicone resin $/ \mathrm{SiO}_{2} \operatorname{composite}$ coating after infiltrating with acidic and base liquids for $24 \mathrm{~h}$.

The tests above show that the methyl silicone resin $/ \mathrm{SiO}_{2}$ composite coating maintains super-hydrophobic performance after the corrosion with acidic and alkaline solutions for $24 \mathrm{~h}$, and demonstrates excellent acid and base tolerance. When acidic or basic corrosive liquids are 
dropped on the surface of super-hydrophobic coating, the OH-PDMS molecules and the methyl silicone molecules in the super-hydrophobic coatings come into direct contact with the corrosive liquids. The chemically stable OH-PDMS and methyl silicone molecules cannot be corroded by acidic or alkaline mediums. The great gas-liquid contact area of the super-hydrophobic coating reduces the contact area between the corrosive liquids and the super-hydrophobic methyl silicone resin $/ \mathrm{SiO}_{2}$ composite surface. Both the chemical stability of the low-energy molecules and the low contact area with the corrosive liquids result in the corrosive durability of the super-hydrophobic methyl silicone resin $/ \mathrm{SiO}_{2}$ composite coating.

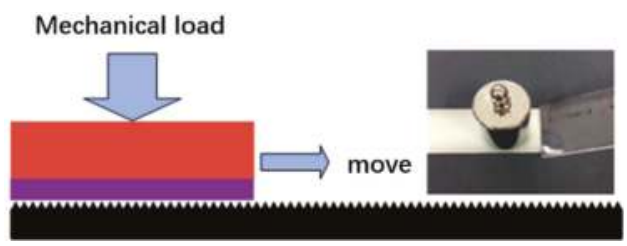

Figure 5. Schematic diagram of the wearing test of the super-hydrophobic surface.

The mechanical durability of the super-hydrophobic methyl silicone resin $/ \mathrm{SiO}_{2}$ composite coating was investigated by sandpaper abrasion. The test process of sandpaper abrasion is shown in Figure 5. The super-hydrophobic coating was worn by a 1500 mesh sandpaper with different mechanical loads on it. After sandpaper abrasion of the super-hydrophobic methyl silicone resin $/ \mathrm{SiO}_{2}$ composite coating at a speed of about $20 \mathrm{~mm} / \mathrm{s}$, the static contact angle and contact angle hysteresis of the worn super-hydrophobic coating were measured. Figure 6a shows the static water contact angle of the super-hydrophobic coatings under different mechanical loads (50 g, $100 \mathrm{~g}$, and $200 \mathrm{~g}$ ) and wearing distances $(350 \mathrm{~mm}$ and $700 \mathrm{~mm}$ ). For super-hydrophobic coatings with a wear distance of $350 \mathrm{~mm}$, the static water contact angle decreases from $156.5^{\circ}$ to $155.4^{\circ}$ while the mechanical load increased from $50 \mathrm{~g}$ to $200 \mathrm{~g}$. With the same wear distance, the static water contact angle of the super-hydrophobic coating gradually decreases with the greater mechanical load. For a super-hydrophobic coating with a mechanical load of $200 \mathrm{~g}$, the static water contact angle decreases from $155.4^{\circ}$ to $154.0^{\circ}$ as the wear distance increases from $350 \mathrm{~mm}$ to $700 \mathrm{~mm}$. With the same mechanical load, the static contact angle of the super-hydrophobic coating decreases as the wearing distance increases. Compared with the static water contact angle of the fresh super-hydrophobic coating (dashed line in the Figure 6a), the static water contact angle of the super-hydrophobic coating decreases slightly because of mechanical wearing, but remains over $150.0^{\circ}$.
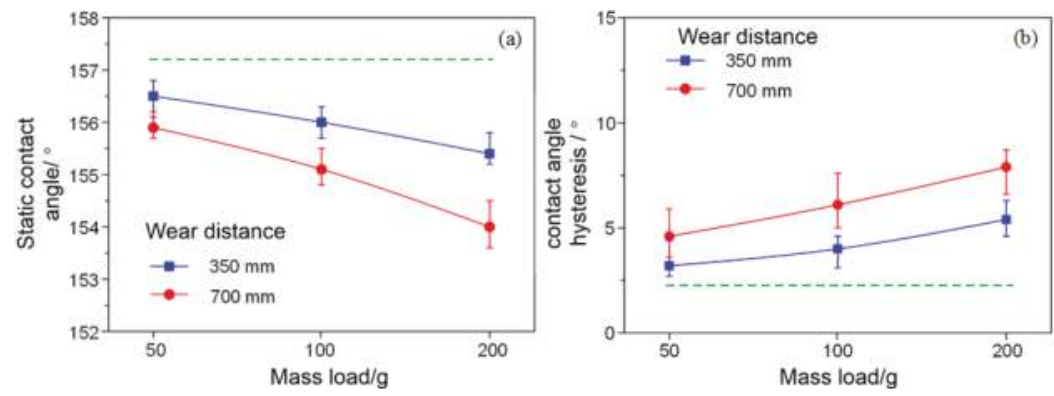

Figure 6. (a) Static water contact angles and (b) water contact angle hysteresis of the worn super-hydrophobic coatings.

Figure $6 \mathrm{~b}$ shows the contact angle hysteresis of the super-hydrophobic coating under different mechanical loads $(50 \mathrm{~g}, 100 \mathrm{~g}$, and $200 \mathrm{~g}$ ) and wearing distances ( $350 \mathrm{~mm}$ and $700 \mathrm{~mm}$ ). For the 
super-hydrophobic coating with the wear distance of $350 \mathrm{~mm}$, the contact angle hysteresis increases from $3.2^{\circ}$ to $5.4^{\circ}$ when the mechanical load increases from $50 \mathrm{~g}$ to $200 \mathrm{~g}$. The contact angle hysteresis of the super-hydrophobic coating gradually increases as the mechanical load increases in the case of the same wear distance. For the super-hydrophobic coating with a mechanical load of $200 \mathrm{~g}$, the contact angle hysteresis increases from $5.4^{\circ}$ to $7.9^{\circ}$ when the wearing distance increases from $350 \mathrm{~mm}$ to $700 \mathrm{~mm}$. The contact angle hysteresis of the super-hydrophobic coating also increases as the wearing distance increases for the same mechanical load. The contact angle hysteresis of the super-hydrophobic coating increases with mechanical wearing, but remains within $10^{\circ}$ in the study, compared with the contact angle hysteresis of $2.3^{\circ}$ for the unworn super-hydrophobic coating. The test results above show that the static water contact angle and the contact angle hysteresis of the super-hydrophobic coating degrades with the enhanced mechanical stress and wear distance. However, after severe mechanical tests, the methyl silicone resin $/ \mathrm{SiO}_{2}$ composite coating stays super-hydrophobic.

Figure 7 demonstrates a representative side view of water droplets on a super-hydrophobic coating after wearing for $100 \mathrm{~mm}$ with a $200 \mathrm{~g}$ mechanical load. The surface of the worn super-hydrophobic coating remains flat and free of visible scratches. The water droplets remain spherical in the worn area of the super-hydrophobic surface. In addition, water droplets easily slide off the slightly inclined super-hydrophobic surface. The results above indicate the great mechanical durability of the super-hydrophobic coating.

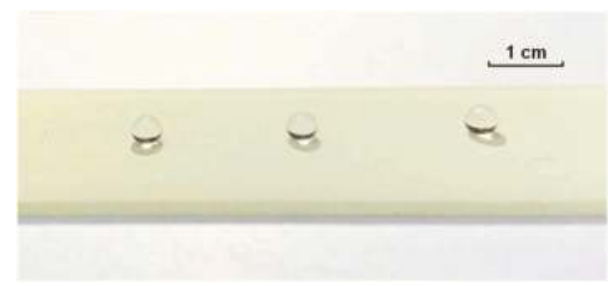

Figure 7. Hydrophobic performances of the super-hydrophobic coating after the sandpaper wearing test.

As shown in Figure 8, compared with the root-mean-square roughness of $213 \mathrm{~nm}$ for the fresh super-hydrophobic coating, the super-hydrophobic surface under the wear distance of $350 \mathrm{~mm}$ reduces the surface roughness from $204.3 \mathrm{~nm}$ to $190.2 \mathrm{~nm}$ with the mechanical load between $50 \mathrm{~g}$ and $200 \mathrm{~g}$. For the super-hydrophobic coating with a mechanical load of $200 \mathrm{~g}$, the surface root-mean-square roughness decreases from $190.2 \mathrm{~nm}$ to $174.5 \mathrm{~nm}$ as the wearing distance increases from $350 \mathrm{~mm}$ to $700 \mathrm{~mm}$.

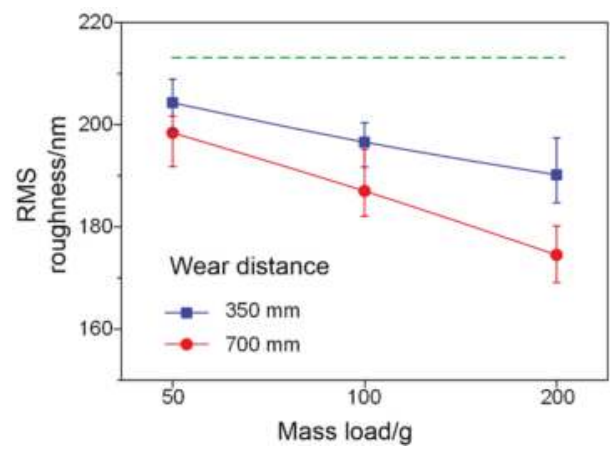

Figure 8. Root-mean-square (RMS) roughness of the worn super-hydrophobic coatings. 
Figure 9 shows the surface topography of the super-hydrophobic coating before and after mechanical abrasion for $700 \mathrm{~mm}$ with a mechanical load of $200 \mathrm{~g}$. Micro-scaled scratches can be observed as shown in blue arrows on the rugged rough surface of the super-hydrophobic coating. Compared with the as-prepared super-hydrophobic surface, certain amounts of micro-protrusions at a size of around $1.2 \mu \mathrm{m}$ disappeared on the worn super-hydrophobic surface. However, irregular micron-scale protrusions of around $0.4 \mu \mathrm{m}$ with embedded nano-scale bumps are still visible on the surface of the super-hydrophobic coating.

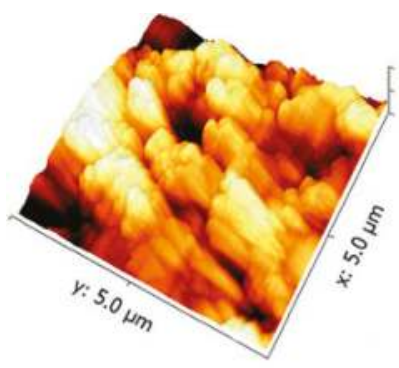

(a)

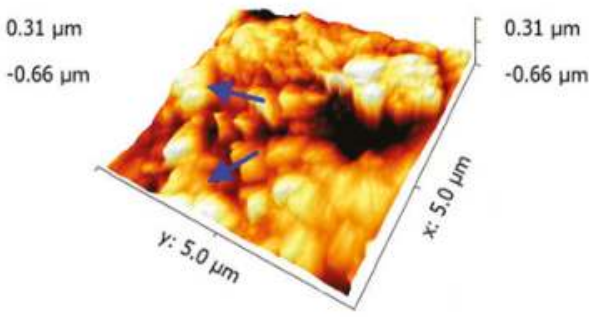

(b)

Figure 9. Atomic force microscope (AFM) images of the (a) as-prepared and (b) worn super-hydrophobic coatings.

For the high activity of the methyl silicone resin, the strong adhesion ability of the methyl silicone resin between the nano- $\mathrm{SiO}_{2}$ particles and the substrate renders the difficulty in the destruction of the rough structures on surface of the super-hydrophobic coating. The maintained binary micro/nano roughness enables the low contact ratio of the droplets with the rough surface, as well as the great air gap between the droplets and the coating. Thus, the worn methyl silicone resin $/ \mathrm{SiO}_{2}$ composite surface demonstrates similar super-hydrophobic performances to the fresh one.

\subsection{Surface Adhesion and Hardness}

Figure 10 shows the adhesion of the super-hydrophobic methyl silicone resin $/ \mathrm{SiO}_{2}$ composite coating on the surface using the method of lattice grid cutting. The square edge has a few peeling marks, and most of the squares are intact without large-area blanks. According to the evaluation criteria of the adhesion of the coating [43], the adhesion of the methyl silicone resin $/ \mathrm{SiO}_{2}$ composite coating with the glass substrate is determined to be grade 1 .

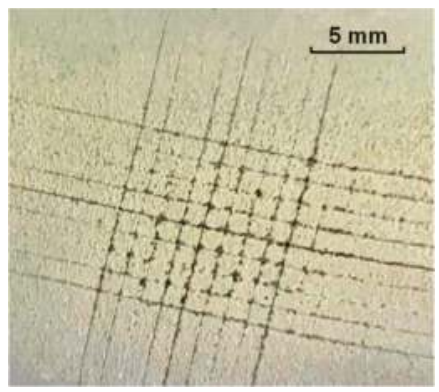

Figure 10. Surface adhesion of the super-hydrophobic surface by cross-cut tester.

Figure 11 shows the surface hardness of the super-hydrophobic composite surface by pencil scratch with different hardness. After the pencil with a hardness of $4 \mathrm{H}$ crosses the surface of the 
super-hydrophobic coating, there is no damage caused on the coating surface. However, the surface of the super-hydrophobic coating gets scratched by the pencil with a hardness of $5 \mathrm{H}$. On the basis of the standard of ASTM D3363 [44], the surface hardness of the super-hydrophobic methyl silicone resin $/ \mathrm{SiO}_{2}$ composite coating is measured as $4 \mathrm{H}$.

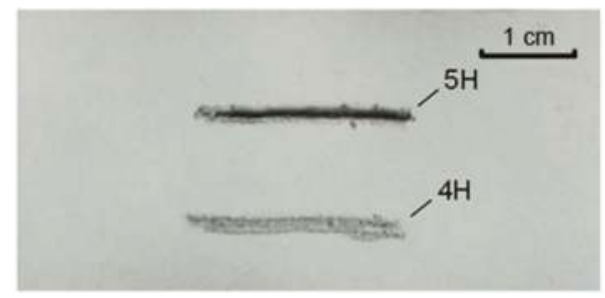

Figure 11. Surface hardness of the super-hydrophobic coating after the pencil test.

\section{Conclusions}

A durable super-hydrophobic coating on the surface of a glass slide is fabricated by air spraying of composites of methyl silicone resins and nano-sized silica $\left(\mathrm{SiO}_{2}\right)$ particles. The as-prepared durable super-hydrophobic surface exhibits a water contact angle (WCA) of $157.2^{\circ}$ and water contact angle hysteresis of $2.3^{\circ}$. SEM images indicate the existence of micro/nano hierarchical structures on super-hydrophobic surfaces. The adhesion strength and hardness of the surface is determined to be grade 1 and $4 \mathrm{H}$, respectively. In the mechanical durability test, the micro/nano hierarchical structures remain on the surface of the super-hydrophobic coating after wearing for $700 \mathrm{~mm}$ with a mechanical load of $200 \mathrm{~g}$. The rough surface retained after severe mechanical abrasion microscopically proves the durable origin of super-hydrophobic coating. The results demonstrate the feasibility of production of durable super-hydrophobic coating via enhancing its surface hardness and adhesion strength between the coating and the substrates.

Author Contributions: The idea was conceptualized by Z.H. and J.L., while preparation was performed by W.X., H.W., and X.S. Z.H. and Y.W. performed the experiments and analyzed the results. The draft was written by R.Z. and Z.H. All authors discussed the results and commented on the manuscript.

Funding: This research was partially funded by National Key Basic Research Program of China (973 Program) (2015CB251003), National Natural Science Foundation of China (No. 51425702 and No. 51321063), China Postdoctoral Science Foundation (No. 2018T110944), National Engineering Laboratory for Ultra High Voltage Engineering Technology (Kunming, Guangzhou, NELUHV211001), and the Science and Technology Project of SGCC (SGTYHT /14-JS-188), Central university fund (106112017CDJZRPY0008), Electric Power Research Institute of State Grid Chongqing Electric Power Company (2017 Yudian Technology 16\#).

Conflicts of Interest: The authors declare no conflict of interest.

\section{References}

1. Wang, Y.; Wu, Y.; Chen, Y.; Zhang, J.; Chen, X.; Liu, P. Nanoliter Centrifugal Liquid Dispenser Coupled with Superhydrophobic Microwell Array Chips for High-Throughput Cell Assays. Micromachines 2018, 9, 286. [CrossRef] [PubMed]

2. Gentile, F.; Das, G.; Coluccio, M.L.; Mecarini, F.; Accardo, A.; Tirinato, L.; Tallerico, R.; Cojoc, G.; Liberale, C.; Candeloro, P.; et al. Ultra low concentrated molecular detection using super hydrophobic surface based biophotonic devices. Microelectron. Eng. 2010, 87, 798-801. [CrossRef]

3. Sun, H.-B.; Kawata, S. Two-Photon Laser Precision Microfabrication and Its Applications to Micro-Nano Devices and Systems. J. Light. Technol. 2003, 21, 624.

4. Zhang, X.; Shi, F.; Niu, J.; Jiang, Y.; Wang, Z. Superhydrophobic surfaces: From structural control to functional application. J. Mater. Chem. 2008, 18, 621-633. [CrossRef]

5. Liu, K.; Li, Z.; Wang, W.; Jiang, L. Facile creation of bio-inspired superhydrophobic Ce-based metallic glass surfaces. Appl. Phys. Lett. 2011, 99, 261905. [CrossRef] 
6. Bhushan, B.; Jung, Y.C. Micro- and nanoscale characterization of hydrophobic and hydrophilic leaf surfaces. Nanotechnology 2006, 17, 2758. [CrossRef]

7. Chen, J.; Liu, J.; He, M.; Li, K.; Cui, D.; Zhang, Q.; Zeng, X.; Zhang, Y.; Wang, J.; Song, Y. Superhydrophobic surfaces cannot reduce ice adhesion. Funct. Ecol. 2012, 101, 111603. [CrossRef]

8. Varshney, P.; Mohapatra, S.S. Durable and regenerable superhydrophobic coatings for brass surfaces with excellent self-cleaning and anti-fogging properties prepared by immersion technique. Tribol. Int. 2018, 123, 17-25. [CrossRef]

9. Samuel, J.D.J.S.; Ruther, P.; Frerichs, H.-P.; Lehmann, M.; Paul, O.; Rühe, J. A simple route towards the reduction of surface conductivity in gas sensor devices. Sensors Actuators B Chem. 2005, 110, $218-224$. [CrossRef]

10. Roach, P.; Shirtcliffe, N.J.; Farrar, D.; Perry, C.C. Quantification of Surface-Bound Proteins by Fluorometric Assay: Comparison with Quartz Crystal Microbalance and Amido Black Assay. J. Phys. Chem. B 2006, 110, 20572-20579. [CrossRef] [PubMed]

11. Nguyen, T.B.; Park, S.; Lim, H. Effects of morphology parameters on anti-icing performance in superhydrophobic surfaces. Appl. Surf. Sci. 2018, 435, 585-591. [CrossRef]

12. Lee, S.G.; Ham, D.S.; Lee, D.Y.; Bong, H.; Cho, K. Transparent superhydrophobic/translucent superamphiphobic coatings based on silica-fluoropolymer hybrid nanoparticles. Langmuir 2013, 29, 15051-15057. [CrossRef] [PubMed]

13. Wang, B.; Guo, Z. Superhydrophobic copper mesh films with rapid oil/water separation properties by electrochemical deposition inspired from butterfly wing. Appl. Phys. Lett. 2013, 103, 63704. [CrossRef]

14. Latthe, S.S.; Terashima, C.; Nakata, K.; Sakai, M.; Fujishima, A. Development of sol-gel processed semi-transparent and self-cleaning superhydrophobic coatings. J. Mater. Chem. A 2014, 2, 5548-5553. [CrossRef]

15. Niu, L.; Kang, Z. Spray deposition process to fabricate $\mathrm{Cu}_{2} \mathrm{O}$ superhydrophobic surfaces on brass mesh for efficient oil-water separation. Mater. Lett. 2018, 210, 97-100. [CrossRef]

16. Wang, H.; Dong, S.; Wang, Z. One-step fabrication of superhydrophobic surface on beryllium copper alloys and corrosion protection application. Colloids Surfaces A Physicochem. Eng. Asp. 2018, 556, 291-298. [CrossRef]

17. Fu, X.; He, X. Applied Surface Science Fabrication of super-hydrophobic surfaces on aluminum alloy substrates. Appl. Surf. Sci. 2008, 255, 1776-1781. [CrossRef]

18. Liu, Y.H.; Wang, X.K.; Luo, J.B.; Lu, X.C. Fabrication and tribological properties of super-hydrophobic surfaces based on porous silicon. Appl. Surf. Sci. 2009, 255, 9430-9438. [CrossRef]

19. Zhai, L.; Cebeci, F.C.; Cohen, R.E.; Rubner, M.F. Stable superhydrophobic coatings from polyelectrolyte multilayers. Nano Lett. 2004, 4, 1349-1353. [CrossRef]

20. Shirtcliffe, N.J.; McHale, G.; Newton, M.I.; Chabrol, G.; Perry, C.C. Dual-scale roughness produces unusually water-repellent surfaces. Adv. Mater. 2004, 16, 1929-1932. [CrossRef]

21. Sarkar, M.K.; Bal, K.; He, F.; Fan, J. Design of an outstanding super-hydrophobic surface by electro-spinning. Appl. Surf. Sci. 2011, 257, 7003-7009. [CrossRef]

22. Zhu, M.; Zuo, W.; Yu, H.; Yang, W.; Chen, Y. Superhydrophobic surface directly created by electrospinning based on hydrophilic material. J. Mater. Sci. 2006, 41, 3793-3797. [CrossRef]

23. Wang, Q.; Hou, W.; Zhang, Y. Superhydrophobic and luminescent methylsilicone resin film. Appl. Surf. Sci. 2009, 256, 664-667. [CrossRef]

24. Wei, Z.J.; Liu, W.L.; Tian, D.; Xiao, C.L.; Wang, X.Q. Preparation of lotus-like superhydrophobic fluoropolymer films. Appl. Surf. Sci. 2010, 256, 3972-3976. [CrossRef]

25. Woodward, I.; Schofield, W.C.E.; Roucoules, V.; Badyal, J.P.S. Super-hydrophobic surfaces produced by plasma fluorination of polybutadiene films. Langmuir 2003, 19, 3432-3438. [CrossRef]

26. Li, J.; Huang, Z.; Wang, F.; Yan, X.; Wei, Y. One-step preparation of transparent superhydrophobic coatings using atmospheric arc discharge. Appl. Phys. Lett. 2015, 107, 51603. [CrossRef]

27. Mahadik, S.A.; Kavale, M.S.; Mukherjee, S.K.; Rao, A.V. Transparent superhydrophobic silica coatings on glass by sol-gel method. Appl. Surf. Sci. 2010, 257, 333-339. [CrossRef]

28. Venkateswara Rao, A.; Latthe, S.S.; Nadargi, D.Y.; Hirashima, H.; Ganesan, V. Preparation of MTMS based transparent superhydrophobic silica films by sol-gel method. J. Colloid Interface Sci. 2009, 332, 484-490. [CrossRef] [PubMed] 
29. Shriram, R.K.A.; Chandrasekar, S.; Karthik, B. PD Signal Time-Frequency Map and PRPD Pattern Analysis

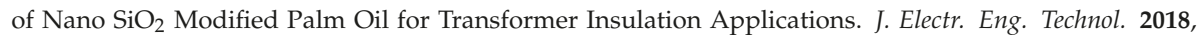
13, 902-910.

30. Kulinich, S.A.; Farhadi, S.; Nose, K.; Du, X.W. Superhydrophobic Surfaces: Are They Really Ice-Repellent? Langmuir 2011, 27, 25-29. [CrossRef] [PubMed]

31. Lazauskas, A.; Guobienè, A.; Prosyčevas, I.; Baltrušaitis, V.; Grigaliūnas, V.; Narmontas, P.; Baltrusaitis, J. Water droplet behavior on superhydrophobic $\mathrm{SiO}_{2}$ nanocomposite films during icing/deicing cycles. Mater. Charact. 2013, 82, 9-16. [CrossRef]

32. Lu, Y.; Sathasivam, S.; Song, J.; Crick, C.R.; Carmalt, C.J.; Parkin, I.P. Robust self-cleaning surfaces that function when exposed to either air or oil. Science 2015, 347, 1132-1135. [CrossRef] [PubMed]

33. Deng, X.; Mammen, L.; Butt, H.-J.; Vollmer, D. Candle Soot as a Template for a Transparent Robust Superamphiphobic Coating. Science 2012, 335, 67-70. [CrossRef] [PubMed]

34. Zhang, X.; Jin, M.; Liu, Z.; Nishimoto, S.; Saito, H.; Murakami, T.; Fujishima, A. Preparation and photocatalytic wettability conversion of $\mathrm{TiO}_{2}$-based superhydrophobic surfaces. Langmuir 2006, 22, 9477-9479. [CrossRef] [PubMed]

35. Azimi, G.; Dhiman, R.; Kwon, H.-M.; Paxson, A.T.; Varanasi, K.K. Hydrophobicity of rare-earth oxide ceramics. Nat. Mater. 2013, 12, 315. [CrossRef] [PubMed]

36. Wang, S.; Feng, L.; Jiang, L. One-step solution-immersion process for the fabrication of stable bionic superhydrophobic surfaces. Adv. Mater. 2006, 18,767-770. [CrossRef]

37. Peng, C.; Chen, Z.; Tiwari, M.K. All-organic superhydrophobic coatings with mechanochemical robustness and liquid impalement resistance. Nat. Mater. 2018, 17, 355-360. [CrossRef] [PubMed]

38. Zhou, X.; Zhang, Z.; Xu, X.; Guo, F.; Zhu, X.; Men, X.; Ge, B. Robust and durable superhydrophobic cotton fabrics for oil/water separation. ACS Appl. Mater. Interfaces 2013, 5, 7208-7214. [CrossRef]

39. Xu, Q.F.; Mondal, B.; Lyons, A.M. Fabricating superhydrophobic polymer surfaces with excellent abrasion resistance by a simple lamination templating method. ACS Appl. Mater. Interfaces 2011, 3, 3508-3514. [CrossRef]

40. Superhydrophobic, R.; Coating, F. Supporting Information Fluoroalkyl Silane Modified Silicone Rubber/Nanoparticle Composite: A Super Durable, Robust Superhydrophobic Fabric Coating. Adv. Mater. 2012, 24, 2409-2412. [CrossRef]

41. Zimmermann, J.; Reifler, F.A.; Fortunato, G.; Gerhardt, L.C.; Seeger, S. A simple, one-step approach to durable and robust superhydrophobic textiles. Adv. Funct. Mater. 2008, 18, 3662-3669. [CrossRef]

42. International Organization for Standardization. Anodizing of Aluminium and its Alloys-Measurement of Abrasion Resistance of Anodic Oxidation Coatings; ISO/TC 79/SC 2 8251:2018; International Organization for Standardization: Geneva, Switzerland, 2018.

43. American Society for Testing and Materials. Standard Test Methods for Measuring Adhesion by Tape Test; ASTM D3359; ASTM International: West Conshohocken, PA, USA, 1997.

44. American Society for Testing and Materials. ASTM International Standard Test Method for Film Hardness by Pencil Test 1; ASTM D3363-05; ASTM International: West Conshohocken, PA, USA, 2010.

45. Ren, S.; Yang, S.; Zhao, Y.; Yu, T.; Xiao, X. Preparation and characterization of an ultrahydrophobic surface based on a stearic acid self-assembled monolayer over polyethyleneimine thin films. Surf. Sci. 2003, 546, 64-74. [CrossRef]

(c) 2018 by the authors. Licensee MDPI, Basel, Switzerland. This article is an open access article distributed under the terms and conditions of the Creative Commons Attribution (CC BY) license (http:/ / creativecommons.org/licenses/by/4.0/). 


\title{
Adaptive Micromixer Based on the Solutocapillary Marangoni Effect in a Continuous-Flow Microreactor
}

\author{
Dmitry Bratsun ${ }^{1}\left(\right.$, Konstantin Kostarev ${ }^{2}$, Alexey Mizev ${ }^{2}{ }^{\circledR}$, Sebastian Aland ${ }^{3}{ }^{\circledR}$, \\ Marcel Mokbel ${ }^{3}$, Karin Schwarzenberger ${ }^{4}$ (i) and Kerstin Eckert $4,5, *$ (i) \\ 1 Department of Applied Physics, Perm National Research Polytechnic University, Perm 614990, Russia; \\ DABracun@pstu.ru \\ 2 Institute of Continuous Media Mechanics, Perm 614013, Russia; kostarev@icmm.ru (K.K.); \\ alex_mizev@icmm.ru (A.M.) \\ 3 Faculty of Informatics/Mathematics, HTW Dresden, 01069 Dresden, Germany; \\ sebastian.aland@htw-dresden.de (S.A.); marcel.mokbel@htw-dresden.de (M.M.) \\ 4 Institute of Fluid Dynamics, Helmholtz-Zentrum Dresden-Rossendorf, 01328 Dresden, Germany; \\ k.schwarzenberger@hzdr.de \\ 5 Institute of Process Engineering and Environmental Technology, TU Dresden, 01062 Dresden, Germany \\ * Correspondence: kerstin.eckert@tu-dresden.de
}

Received: 30 September 2018; Accepted: 13 November 2018; Published: 16 November 2018

\begin{abstract}
Continuous-flow microreactors are an important development in chemical engineering technology, since pharmaceutical production needs flexibility in reconfiguring the synthesis system rather than large volumes of product yield. Microreactors of this type have a special vessel, in which the convective vortices are organized to mix the reagents to increase the product output. We propose a new type of micromixer based on the intensive relaxation oscillations induced by a fundamental effect discovered recently. The mechanism of these oscillations was found to be a coupling of the solutal Marangoni effect, buoyancy and diffusion. The phenomenon can be observed in the vicinity of an air-liquid (or liquid-liquid) interface with inhomogeneous concentration of a surface-active solute. Important features of the oscillations are demonstrated experimentally and numerically. The periodicity of the oscillations is a result of the repeated regeneration of the Marangoni driving force. This feature is used in our design of a micromixer with a single air bubble inside the reaction zone. We show that the micromixer does not consume external energy and adapts to the medium state due to feedback. It switches on automatically each time when a concentration inhomogeneity in the reaction zone occurs, and stops mixing when the solution becomes sufficiently uniform.
\end{abstract}

Keywords: continuous-flow reactor; mixing; solutal Marangoni effect; relaxation oscillations

\section{Introduction}

In the last decades, the interaction between reaction-diffusion phenomena and hydrodynamics has attracted increasing interests both from the fundamental point of view of nonlinear science and from application-oriented aspects in chemical engineering [1-4]. This arises from the fact that the chemically-induced changes of fluid properties such as density, viscosity, thermal conductivity or surface tension may result in flow instabilities, which exhibit a large variety of convective patterns.

From the technological point of view, continuous-flow microreactors are at the center of recent developments in chemical engineering [4]. Significant changes in this area were initiated in the early 2000s with the entrance of new technologies into organic synthesis [5]. Since the pharmaceutical production needs flexibility in reconfiguring the synthesis system rather than high throughput, increasingly miniature reactor design concepts are established $[5,6]$. This new reactor type provides 
several advantages with respect to the traditional batch-reactor. The latter in general is considered as a closed chamber without mass exchange between the reactor and the external environment. Hence, the different design and operation mode leads to following features:

- $\quad$ high productivity due to the absence of the loading-unloading stage, as well as cleaning of the reactor after each batch

- $\quad$ uniformity and stability of the process ensuring easy control

- $\quad$ stable consumption of reagents and energy due to the small volume of the reactor zone

- $\quad$ increase in output by the replication of the production line

This list is not intended to be exhaustive but exemplarily shows prominent benefits of this technology. Consequently, numerous studies have been published in recent years detailing the outcome of continuous-flow chemistry applied to single or multi-step syntheses of target compounds on various reaction schemes and spatial scales [7-12].

Two basic physical principles can be used to efficiently mix reagents in continuous-flow microreactors: diffusion and convection. When using diffusion as the main mixing tool, it is required to maintain high gradients of the diffusing components and to use channels with very small cross sections. Initially, the development of microreactor technology was mainly on this path [5,6]. With the miniaturization of the connecting capillaries, the flow remains laminar. These microreactors are manufactured without a particular reaction zone, since mixing of the reactants occurs directly after the junction of the feed capillaries. However, this approach has some drawbacks. For example, a significant pressure has to be applied to pump liquid through narrow channels involving higher energy costs. Simultaneously, the achievable reactor throughput is reduced. Further limitations arise due to the compatibility for different reaction kinetics. As the mixing time of reagents decreases, only fast reactions, i.e., small reaction times are possible. Other shortcomings, such as reactor fouling and high investment costs for precise manufacturing are discussed in the literature [13].

In the last few years, different types of microreactors and -mixers have been proposed [14-17]. Their operation principle relies not only on diffusion, but also on convective mixing. Microreactors of this type have a specifically designed reaction zone, where the flow forms vortex structures providing an efficient mixing of the reagents. This leads to a considerable increase in the product output and allows to run processes with lower reaction rates. Furthermore, various techniques have been developed for mixing typically laminar flows in narrow channels. Among them are passive methods where the mixing effect is introduced by geometry [18], e.g., using bas-relief structures on the bottom of the channel [19], but also active methods using an external Braille pin actuator array [20]. Further micromixer working principles are based on fluidic dielectrophoresis [21], or centrifugal action [22]. A recent theoretical study [23] investigates the collective behavior of hydrodynamically coupled molecular motors.

In this work, a recently discovered hydrodynamic effect is employed to create a novel, adaptive micromixer. For many years, the authors have been involved in the experimental and theoretical studies of the fundamental properties of soluto-capillary phenomena in different immiscible systems with [24-31] and without chemical reactions [32-36]. Among the various effects studied by the authors, a prominent finding was that solutal Marangoni convection frequently is accompanied by intensive relaxation oscillations. For example, this phenomenon can be observed in the vicinity of an air-liquid interface with inhomogeneous concentration of the soluble surfactant (aqueous solution of acetic acid) along the surface [33,35]. Similar oscillations were obtained in an immiscible liquid-liquid system, consisting of paraffin oil and water, with isopropyl alcohol as surface-active solute [36]. The mechanism of these oscillations was found to be a periodic coupling of the Marangoni effect, buoyancy convection acting in the presence of gravity and the restoring effect of diffusion. This interaction leads to a repeated consumption and regeneration of the Marangoni driving force. Because of that, a regular periodicity of convection is obtained. We now bring this particularly advantageous feature to a technological application. 
Therefore, we show both experimentally and theoretically that the proposed micromixer can effectively work as a basic microreactor module. In this regard, the article is organized as follows. Section 2 presents different experimental observations of the relaxation oscillations at the interface of a single bubble and drop to underline the versatility of this effect with respect to various fluid combinations and geometries. Furthermore, we explain the mechanism of the oscillations used later as operation principle of the micromixer. The results of numerical simulations for a single droplet system are given in Section 3. Various aspects of the micromixer design are discussed in Section 4. A concrete implementation of our micromixer, based on a single bubble placed in the reaction zone, is also demonstrated here. Section 5 summarizes the results and provides some discussion.

\section{Experimental Observations of Relaxation Oscillations}

\subsection{Liquid-Liquid System}

In this section, we describe the main features of the basic hydrodynamic effect of the relaxation oscillations. The experimental system consists of two immiscible liquids placed in a Hele-Shaw cell as described below. The oscillations can be excited either near drops which formed during the filling procedure or near air bubbles produced via a capillary [36]. Here, we focus on the former case, while a second system with air bubbles is analyzed in Section 2.2.

\subsubsection{Experimental Setup}

The liquid-liquid system consists of paraffin oil and water with $4.6 \mathrm{wt}$.\% isopropyl alcohol being dissolved in the organic phase. The material parameters of this system are listed in [36]. All chemical substances are used as purchased. Water and isopropyl alcohol are of HPLC grade, and the paraffin oil is of pharmaceutical quality (Ph.Eur., DAB). The system is placed in a rectangular narrow cuvette of size $L \times H \times W \approx 20 \mathrm{~mm} \times 70 \mathrm{~mm} \times 1 \mathrm{~mm}$ by a special filling procedure described in [26,36] to satisfy the condition for the quasi 2D Hele-Shaw geometry. Due to its higher density, water is filled as the lower layer and paraffin oil with isopropyl alcohol forms the upper layer in the vertically oriented Hele-Shaw cell. We use a short-chain alcohol as surface-active solute to ensure that complex phenomena such as micelle formation or adsorption-desorption kinetics usually connected with strong surfactants only play a minor role. This justifies our simplified model employed in our simulations. Due to the hydrophilic nature of isopropyl alcohol, partition is largely in favour of the aqueous phase. This leads to an intense mass transfer and, accordingly, to a fast depletion of the organic phase in regions near the interface.

The diffusive concentration profile, which establishes at the interface of the two layer system (material parameters according to [36]) after a time $t=600 \mathrm{~s}$ is shown in Figure 1a as a function of the distance from the interface $(y=0)$. The profile is calculated by the well-known analytical formula [37] assuming diffusion between two infinitely extended layers since the layer height (35 $\mathrm{mm})$ is much larger than the distance of the concentration front to the interface. The concentration axis is normalized by the initial concentration in the donating paraffin oil phase. The graph clearly shows the depletion of the organic phase and the concentration profile developing in the lower aqueous phase. Note that the jump in the concentration profile at the interface is caused by the partition coefficient value 0.16 of isopropyl alcohol between paraffin oil and water.

During the filling procedure, drops of paraffin oil can form which remain at the wall of the Hele-Shaw cell in some distance of the interface. The vertical position of the drop, whose relaxation oscillations are plotted in Figure 1d, is marked by a black circle in Figure 1a. The diameter of the drops is typically $0.1-0.5 \mathrm{~mm}$, corresponding to a volume of a few nanoliters. At these drops, pronounced relaxation oscillations occur as described next. To visualize the flow structure at the drops, a shadowgraph optics is employed (construction by TSO, Pulsnitz, Germany), operating in transmission. This technique detects the second derivative of the refractive index field. Hence, 
it qualitatively captures the patterns arising from interfacial convection since the convective mass transfer influences the concentration distribution and, consequently, the refractive index field.

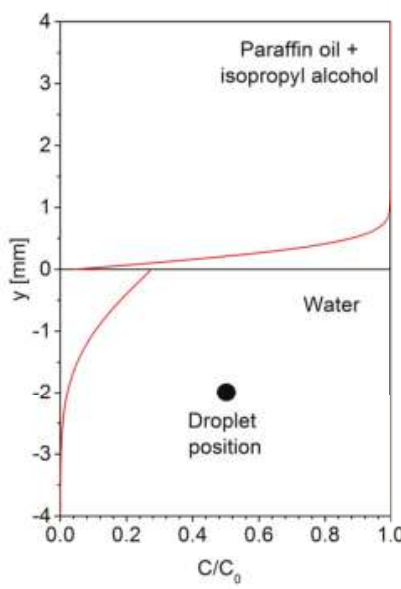

(a)

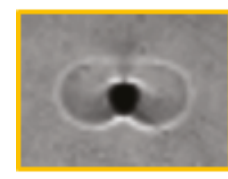

(b)

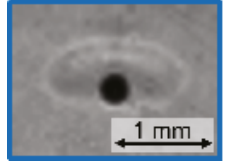

(c)

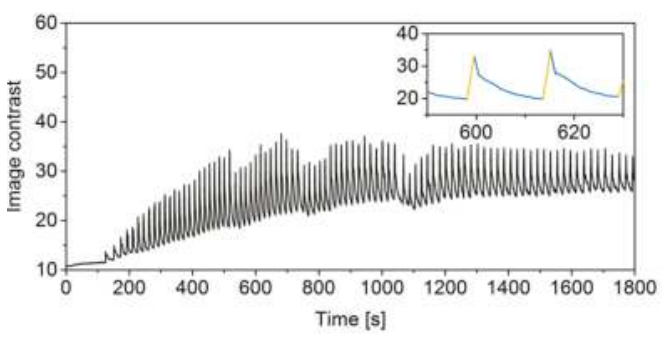

(d)

Figure 1. (a) The two-layer system paraffin oil/water with normalized concentration profile of isopropyl alcohol due to mass transfer out of the organic phase. The vertical position of a paraffin oil drop in the aqueous phase is marked by a black circle. (b) Active stage of relaxation oscillations at paraffin oil drop in the aqueous phase and (c) relaxation stage. (d) Time evolution of shadowgraph image contrast in the surrounding of the drop. The inset details two periods of relaxation oscillations.

\subsubsection{Experimental Results}

Two characteristic stages of the relaxation oscillations are shown in Figure 1b,c. The corresponding drop of paraffin oil is immersed in the lower aqueous phase at a distance of $2 \mathrm{~mm}$ from the interface and sits at the wall of the Hele-Shaw cell. The droplet itself appears as a black circle in the shadowgraph optics due to its high interfacial curvature. In the surrounding of the droplet, the convective structure is visible as bright and dark regions differing from the quiescent fluid with intermediate gray value.

The periodicity of the oscillations can be described as follows. The cycle begins with the start-up of Marangoni convection in the form of two vortices at either side of the drop (this stage is shown in Figure $1 \mathrm{~b}$, orange frame). Due to the mass transfer of isopropyl alcohol across the overlying interface of the two-layer system, the drop is placed in a vertical concentration gradient (cf. Figure 1a). Hence, it is subjected to high solute concentration at the upper side, implying low interfacial tension since isopropyl alcohol makes the surface tension lower, and low concentration at the bottom, i.e., high interfacial tension. Therefore, the Marangoni convection at the drop surface is directed downwards, entraining fluid rich in solute to lower regions. This also affects the density stratification around the droplet. The initial density gradient is buoyantly stable because the solution density of the aqueous phase decreases with increasing isopropyl alcohol concentration. Due to the Marangoni-driven downflow, the stable density stratification locally is inverted. In the presence of gravity, this leads to a buoyancy-driven convection supporting the rise of the fluid in the Marangoni vortex at some distance from the interface. As a result, an intense mixing of the fluid, visible in Figure 1b, takes place which exhausts the vertical concentration gradient in the vicinity of the drop and leads to the breakdown of Marangoni convection.

Figure 1c (blue frame) displays the second characteristic stage, referred to as relaxation phase. During this stage, buoyancy-driven convection carries the mixed fluid upwards, and diffusion further redistributes the solute. After this, the conditions that existed before the active convective mass transfer 
around the drop are sufficiently restored. The vertical concentration gradient at the droplet again is high enough so that the system is ready to repeat the described cycle.

As visible in the comparison of Figure $1 \mathrm{~b}, \mathrm{c}$, the intensity of convection in the surrounding of the drop influences the local contrast of the shadowgraph images. This is used in Figure $1 \mathrm{~d}$ to illustrate the dynamics of the relaxation oscillations. The inset details the characteristic time evolution. The steep rise in image contrast (marked orange) corresponds to the short, impulsive stage of Marangoni convection. The relaxation phase (marked blue) lasts longer due to the slower processes of diffusion and buoyant convection being responsible for the restoration of the Marangoni driving force. The temporal overview shows that the oscillations start when the solute front, which penetrates the aqueous phase during mass transfer from the paraffin oil phase, reaches the droplet. The convection continues as long as the drop is subjected to sufficiently large concentration gradients. The oscillation frequency is highest at $t \approx 400 \mathrm{~s}$ due to the passing solute front. When the aqueous solution near the interface is progressively equilibrated at later times, the concentration gradient diminishes and the oscillation frequency drops. This demonstrates that the mixing by Marangoni convection around such a drop or bubble automatically responds to the process state.

\subsection{Air-Liquid System}

In our second series of experiments, we examine the relaxation oscillations at a different configuration where an air-liquid interface is introduced in a vertical concentration gradient by a specifically designed setup. To further detail the fine structures in the velocity and concentration field during the relaxation oscillations, we now use a Fizeau interferometer for flow visualization.

\subsubsection{Experimental Setup}

The cuvette for these experiments is again a vertically oriented Hele-Shaw cell with an adapted geometry to produce an air-liquid interface. The gap for the fluids is formed by two closely spaced parallel glass plates (Figure 2a). Inside the cuvette, a horizontal channel, which is $35 \mathrm{~mm}$ long, $2.4 \mathrm{~mm}$ high and $1.2 \mathrm{~mm}$ thick, is created by inserts. One end of the channel is open into the working chamber of the cell and the other is blocked off by the air bubble. Before the experiment, the cell is filled with water from the bottom inlet 1 and air is injected into the channel via the inlet 2 to produce the air bubble. The air bubble remains confined in the channel to prevent its buoyancy rise. Then, part of the water is discharged through pipe 1 up to the upper part of the horizontal channel and the vacant space above is filled with an aqueous solution of isopropyl alcohol of a given concentration. This results in the formation of a stably stratified two-layer miscible system consisting of pure water (bottom) and alcohol solution separated by a rather narrow initial diffusion zone. As a final step, a small amount of water again is discharged until the alcohol solution penetrates into the channel.

To visualize the concentration field, a Fizeau interferometer is employed (Figure $2 b$ ). In the resulting interferogram, the distribution of solute along a quasi-two-dimensional space (for example, the Hele-Shaw cell) is visualized as a system of interference fringes representing isolines of equal optical path length provided that the system is isothermal. If the concentration field can be assumed as two dimensional, i.e., concentration changes across the gap width can be neglected, then each interference fringe can be correlated to a certain concentration value. For our cell, a transition from one interference fringe to another corresponds to a change of isopropyl alcohol concentration in water by $0.27 \mathrm{wt} . \%$. The interferometer can also be used to trace the evolution of flows in inhomogeneous solutions. Because of a very slow mass diffusion (the Péclet number is of the order of $10^{4}$ ), changes in the concentration distribution can be directly attributed to the fluid flow. 


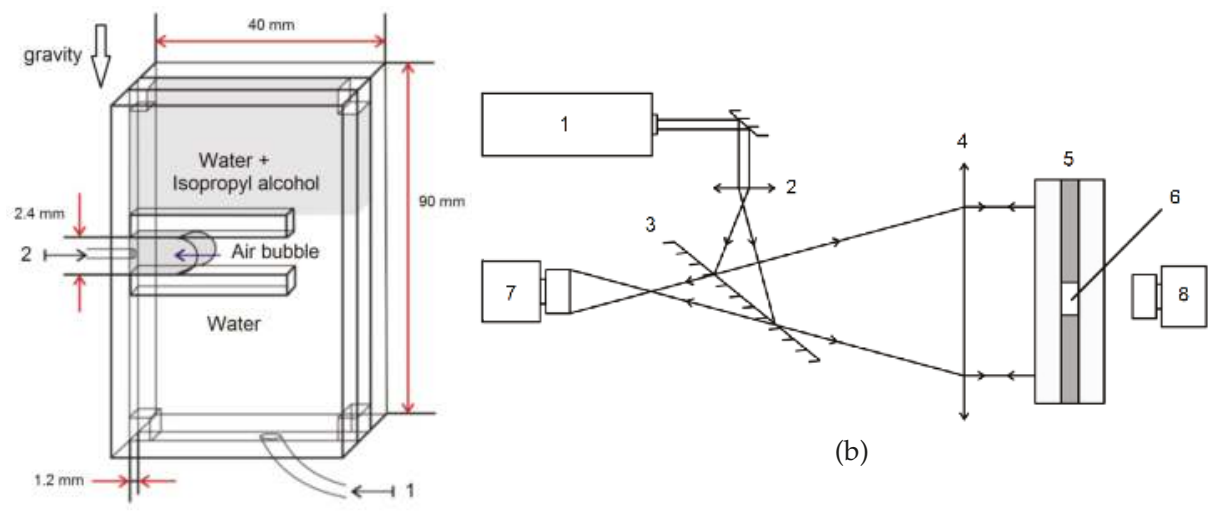

(a)

Figure 2. (a) Scheme of cuvette: the inlet for water during the filling procedure 1; the inlet for air to make the bubble 2. (b) Scheme of the Fizeau interferometer: helium-neon laser 1; microscope objective 2; semi-transparent mirror 3; objective-collimator 4; vertically oriented Hele-Shaw cell 5 with parallel glasses providing the workspace for interferometry; working chamber 6 with bubble or drop in channel; video cameras 7 and 8 .

\subsubsection{Experimental Results}

Figure 3 displays a series of interferograms describing the evolution of the concentration field near the air bubble with an initial concentration of the isopropyl alcohol solution $C_{0}$. The interferograms show a part of the channel in the Hele-Shaw cell sketched in Figure 2a. One can see that the aqueous solution of alcohol gradually advances towards the bubble in the form of a tongue (see, for example, Figure 3a), which finally touches the bubble surface in the upper part. Thereby, it creates a surface tension difference, i.e., a Marangoni stress along the air-liquid surface, providing the conditions for the onset of Marangoni convection.

The experiments revealed that a minimum concentration gradient is required for the onset of pronounced interfacial convection. Hence, the flow only is amplified when the resulting concentration difference at the interface is higher than a certain threshold value $\triangle C^{*}$. Figure 3 illustrates this by comparing snapshots of the time evolution near this threshold. The left-hand column of interferograms corresponds to an initial concentration of $C_{0}=5 \mathrm{wt}$. $\%$ resulting in a concentration difference $\triangle C$, which is slightly lower than the threshold value $\triangle C^{*}$. A slightly higher concentration $C_{0}=6 \mathrm{wt} . \%$ is used for the snapshots in the right-hand column which turned out to be enough to excite the convection. The existence of a clear threshold for the onset of convection indicates the presence of adsorbed trace impurities on the surface which cannot be fully excluded despite our rigorous cleaning procedure. Such surface-active impurities can change the surface rheology or can induce counteracting Marangoni stresses when redistributed by an arising interfacial flow. Thus, to initiate interfacial convection, it is necessary that the Marangoni shear stress by the acting concentration gradient is high enough to overcome these effects. This is the case for the experiment at $C_{0}=6 \mathrm{wt} . \%$ when the tongue of the alcohol solution approaches the bubble surface (Figure 3e). An intensive Marangoni flow appears immediately after the tongue touches the bubble surface at a time $t=15.28 \mathrm{~s}$. In contrast to that, for $C_{0}$ $=5 \mathrm{wt} . \%$, the convection cannot develop even $105 \mathrm{~s}$ after the contact with the alcohol tongue (Figure 3d). In the latter case, the downward propagation of the solute front mainly is caused by diffusion.

Generally, the development of the convection near the bubble for $C_{0}=6 \mathrm{wt} . \%$ is similar to the scenario described in Section 2.1. At the first stage, the fluid flow is directed downward along the bubble surface (the vortex rotates counterclockwise), propagating into the region of lower isopropyl alcohol concentration (Figure 3f). Over time, the vortex entrains more and more solute-rich fluid to 
the lower region (Figure 3g), decreasing the density in comparison to the surrounding. As a result, the lighter liquid begins to rise upward due to buoyancy pushing the alcohol tongue away from the bubble (Figure $3 \mathrm{~h}$ ). The last stage is responsible for the restoration of the Marangoni driving force.

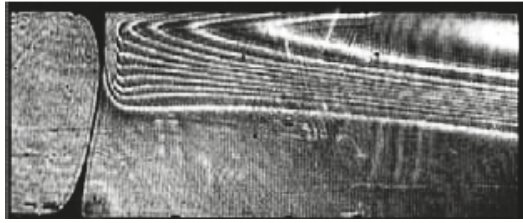

(a)

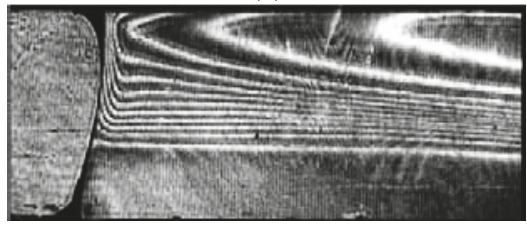

(b)

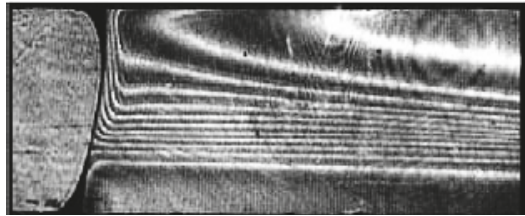

(c)

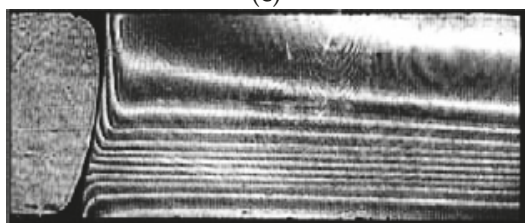

(d)

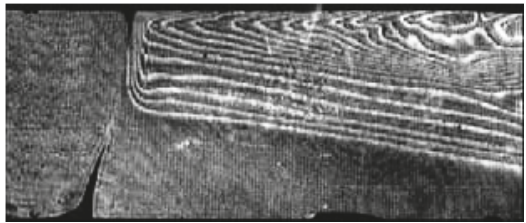

(e)

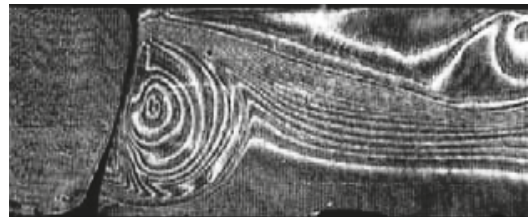

(f)

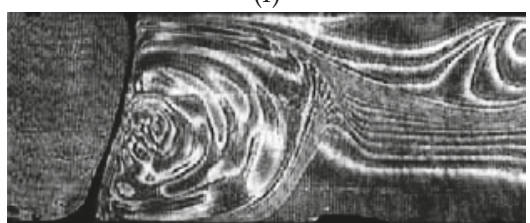

(g)

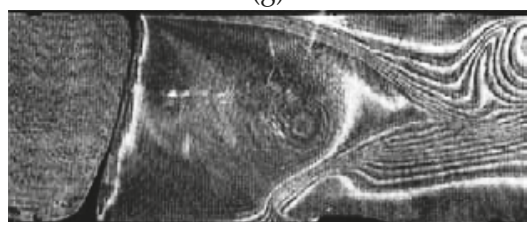

(h)

Figure 3. Evolution of the concentration field near the air bubble in the absence of pronounced convective mass transfer $(\mathbf{a}-\mathbf{d})$ for $C_{0}=5 \mathrm{wt}$. $\%$ and at the onset of relaxation oscillations due to the coupling of solutal Marangoni convection with buoyancy and diffusion (e-h) for $C_{0}=6 \mathrm{wt}$. \%. The left and right frames from up to down correspond to time $t: 0 \mathrm{~s}(\mathbf{a}) ; 13 \mathrm{~s} \mathrm{(b);} 30 \mathrm{~s} \mathrm{(c);} 105 \mathrm{~s}(\mathbf{d}) ; 0 \mathrm{~s}(\mathbf{e}) ; 15.3 \mathrm{~s}$ (f); $17 \mathrm{~s}(\mathrm{~g})$; and $22 \mathrm{~s}(\mathbf{h})$.

Figure 4 shows the time evolution of the oscillation period. Due to the intensive stirring of the solution near the bubble, the regeneration time for the influx of fresh isopropyl alcohol solution increases, thus longer oscillation periods can be observed in the later stage of the experiments. 


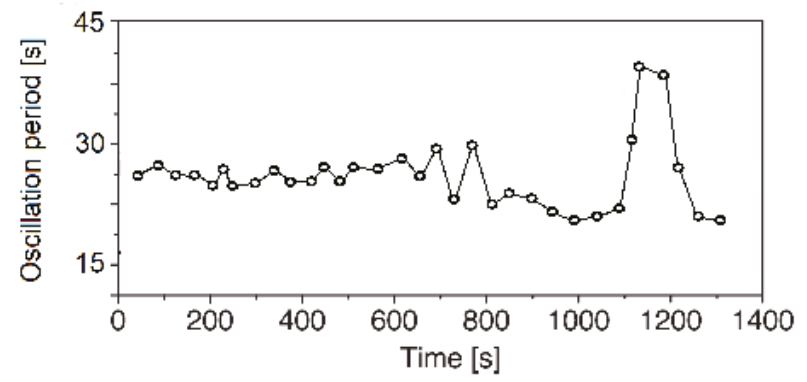

Figure 4. Time evolution of the oscillation period for an aqueous isopropyl alcohol solution at $C_{0}=6 \mathrm{wt} . \%$.

\section{Numerical Simulations}

\subsection{Mathematical Model}

Numerical simulations of the flow around drops or bubbles in a concentration gradient of a surface active solute allow us to estimate the influence of specific material constants by a defined parameter variation. We use a diffuse interface model, whereby the governing equations are formulated according to the Hele-Shaw approximation $[25,27,38,39]$. The Hele-Shaw approximation assumes that the fluids are enclosed in the gap between two parallel plates with a small distance $d$ such that the fluid motion becomes mainly two-dimensional. In line with this, the drop/bubble is modeled as a cylindrical fluid domain between the plates.

In the surrounding aqueous phase, an initially constant vertical concentration gradient of isopropyl alcohol is supposed. That means the non-steady state in the experiment with an evolving concentration profile in the aqueous phase is approximated by a steady-state concentration field with a constant concentration gradient. The solute concentration in the drop/bubble is neglected. According to the diffuse interface model, a phase field $\phi$ is used to represent the phases, with $\phi=0$ in the drop/bubble and $\phi=1$ in the surrounding fluid. We assume diffusion-limited adsorption where local thermodynamic equilibrium exists between the excess concentration $\Gamma$ at the interface and the solute concentration $C$ adjacent to the interface. A linear relation between both quantities given by the Henry isotherm $\Gamma=K C$ ( $K$ stands for the adsorption coefficient) is applied so that interfacial tension $\sigma$ as well depends linearly on $C$. The mass transport is modeled by an advection-diffusion equation and the momentum transport by the incompressible Navier-Stokes equations coupled with the shear stress balance at the interface. The resulting governing equations for velocity $\mathbf{V}$, pressure $p$ and solute concentration $C$ in the aqueous phase are based on $[36,40]$ :

$$
\begin{array}{r}
\rho(\phi, C) \partial_{t} \mathbf{V}-\nabla \cdot\left(\eta(\phi)\left(\nabla \mathbf{V}+\nabla \mathbf{V}^{T}\right)\right)+\frac{3}{d^{2}} \eta(\phi) \mathbf{V}+\nabla p+k \nabla \phi \otimes \nabla \phi \cdot \mathbf{V}= \\
\mathbf{g} \rho(\phi, C)+\sigma_{r e f} \alpha_{C}|\nabla \phi| \mathbf{P} \nabla C, \\
\nabla \cdot \mathbf{V}=0, \\
\phi \partial_{t} C+\phi \mathbf{V} \cdot \nabla C-D \nabla \cdot(\phi \nabla C)-D_{n} \nabla \cdot((\mathbf{n} \cdot \nabla C)|\nabla \phi| \mathbf{n})=0,
\end{array}
$$

where $\mathbf{g}$ is the gravitational acceleration, $\sigma_{\text {ref }}$ is the interfacial tension at zero solute concentration, $\alpha_{C}$ is the interfacial tension coefficient, $D$ is the diffusion coefficient of isopropyl alcohol in the aqueous phase, $\mathbf{P}=\mathbf{I}-\mathbf{n} \otimes \mathbf{n}$ is the operator of the surface projection, $\mathbf{I}$ is the identity matrix, $\mathbf{n}=\nabla \phi /|\nabla \phi|$ is the outer normal to the drop and $k$ is a penalty constant enforcing zero normal velocity at the surface. $D_{n}$ stands for an interfacial normal diffusion ensuring the desired no flux boundary condition at the drop surface. The third term on the left-hand side of Equation (1) depending on the gap width $d$ is 
introduced due to the Hele-Shaw approximation. Assuming a parabolic velocity profile between the plates, it accounts for the friction at the walls. Linear interpolations are used for the density $\rho$ and viscosity $\eta$ in the phase field model:

$$
\begin{array}{r}
\rho(\phi, C)=\phi \rho_{r e f}^{(1)}\left(1-\beta_{C}^{(1)} C\right)+(1-\phi)\left(\rho_{r e f}^{(2)}\left(1-\beta_{C}^{(2)} C\right)\right), \\
\eta(\phi)=\phi\left(\eta^{(1)}\right)+(1-\phi) \eta^{(2)},
\end{array}
$$

where $(i)$ denotes the fluid phase with $i=1$ for water and $i=2$ for paraffin oil/air, $\beta_{c}$ is the volume expansion coefficient and $\rho_{\text {ref }}$ is the density at zero solute concentration.

Simulations are performed for a set of material parameters corresponding to an aqueous solution of isopropyl alcohol surrounding a paraffin oil drop, whereas simulations for an air bubble are shown in [36]. The drop diameter is set to $0.3 \mathrm{~mm}$ which is a typical value according to the experimental observations in Section 2.1. The domain size was chosen $L_{x} \times L_{y}=8 \mathrm{~mm} \times 8 \mathrm{~mm}$ which was verified to be large enough to eliminate finite-size effects. The grid resolution is adapted to capture the fine structures arising during Marangoni convection. The mesh size (longest side of triangles) amounted to $3.906 \times 10^{-5} \mathrm{~m}$ at the interface and $1.5625 \times 10^{-4} \mathrm{~m}$ in the bulk phase. An initial concentration gradient of $d C / d y=25 \mathrm{mmol} /(1 \cdot \mathrm{mm})$ is estimated from the diffusive concentration profile (Figure 1a) in the aqueous phase near the interface at a time of $600 \mathrm{~s}$. For the velocity, the initial and boundary condition is set to $\mathbf{V}=0$. This quiescent initial state is unstable, since the vertical concentration gradient imposes interfacial tension gradients at the drop surface and Marangoni convection sets in as already observed experimentally. Since the experiments indicate that the buoyancy-driven convection plays an important role for the mechanism of the relaxation oscillations, the volume expansion coefficient in the aqueous phase $\beta_{C}^{(1)}$ is varied in relation to the reference value of isopropyl alcohol in steps of $0.1,0.25,0.5,1,2,4,10$. This corresponds to solutes which have a weaker or stronger influence on the solution density in comparison to isopropyl alcohol. Note that the highest values of 4 and 10 rather are of theoretical interest since they strongly exceed the typical volume expansion coefficients of aqueous solutions. Furthermore, we only consider systems with density-lowering solutes, i.e., negative volume expansion coefficients. Otherwise, the system dynamics would be governed by Rayleigh-Taylor instability in the aqueous phase.

\subsection{Numerical Results}

Figure 5 shows snapshots of the concentration distribution around the drop for increasing magnitudes of the volume expansion coefficient $\beta_{C}^{(1)}$ from left to right. The stage of active Marangoni convection is displayed in the upper row and the end of the relaxation stage is depicted in the lower row. The observed structure of the concentration distribution agrees with the mechanism of the relaxation oscillations discussed in Section 2. In the active stage, one can clearly see the strong inflow of solute-rich fluid at the top of the drop and the mixing zone which encloses the drop and finally extinguishes the driving concentration gradient. With a higher magnitude of the volume expansion coefficient, the density stratification in the bulk fluid becomes increasingly stabilizing. This implies that more work is necessary to shift a fluid element vertically by the Marangoni convection what counteracts the mixing effect. As visible in Figure 5, the zone of the mixed fluid strongly shrinks from left to right. This effect is quantified in Figure 6 a by plotting the mixing area $A_{m i x}$ directly after the termination of the active stage over the relative volume expansion coefficient (again normalized by the value for isopropyl alcohol as the reference case). We define $A_{\text {mix }}$ as the area where the concentration $C$ deviates more than $5 \mathrm{mmol} / \mathrm{L}$ from the initial linear concentration profile. The curve in Figure 6a shows that $A_{\text {mix }}$ tends to zero for very high magnitudes of the volume expansion coefficient, but strongly increases if this value is small. 
The buoyancy effects influence not only the structure of the convection, but also the oscillation dynamics. To characterize the time evolution, the mean solute concentration

$$
\langle C\rangle=\frac{1}{2 \pi r} \int_{\Omega}|\nabla \phi| C \mathrm{~d} \mathbf{x}
$$

at the drop surface $\Omega$ is shown in Figure $6 \mathrm{~b}$ for varied volume expansion coefficients. Since the initial concentration gradient is constant throughout the whole domain, the first cycle usually is more intense, while the subsequent periods start from a pre-mixed state. It can be seen that, for a low magnitude of the volume expansion coefficient (almost buoyantly neutral solute), the curve oscillates around a higher concentration value. Without hindrance by a stabilizing density stratification, a more effective inflow of solute-rich fluid from the upper regions can take place. In the quiet relaxation phase, the concentration at the interface only drops to a moderate value. Furthermore, it takes longer to redistribute the larger mixing area so that the oscillation frequency decreases. When the relative volume expansion coefficient is high, the stronger buoyancy quickly restores the vertical concentration gradient and the next short oscillation begins.

The order of magnitude for the oscillation frequency in the simulations satisfactorily agrees with the experiments considering the simplifications inherent in the theoretical model. Besides the applied linearizations for the dependence of interfacial tension and solution density on isopropyl alcohol concentration, and general uncertainties in the material properties, the 2D Hele-Shaw approximation is the main source for the quantitative deviations observed. Since the size of the drop $(0.3 \mathrm{~mm})$ is significantly smaller than the gap width of the Hele-Shaw cell $(1 \mathrm{~mm})$, only one side of the drop touches the wall in the experiments, whereas the Hele-Shaw approximation assumes a cylindrical form of the drop between the glass plates. Therefore, significant 3D effects have to be taken into account, which certainly influence the temporal evolution. However, as shown above, our theoretical approach allows us to study the influence of relevant material parameters and to obtain qualitative trends with the advantage of a strongly reduced computational cost compared to full 3D simulations.

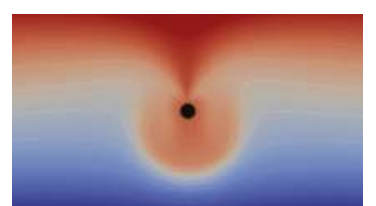

(a)

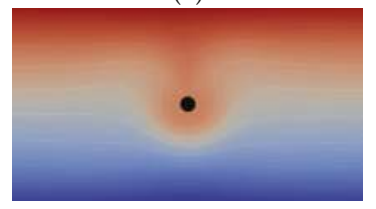

(d)

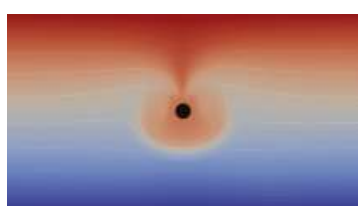

(b)

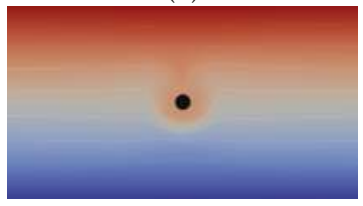

(e)

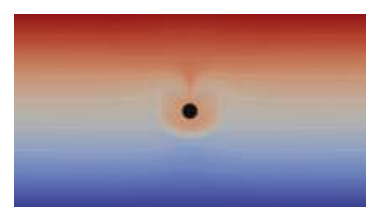

(c)

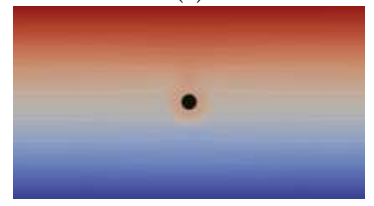

(f)

Figure 5. Concentration distribution from simulations of a paraffin oil drop placed in an aqueous solution with a vertical gradient of surface active solute. The value of the volume expansion coefficient is varied relative to that of isopropyl alcohol as: $0.1(\mathbf{a}, \mathbf{d}) ; 1(\mathbf{b}, \mathbf{e})$; and $10(\mathbf{c}, \mathbf{f})$. Shown is a section of $5.5 \mathrm{~mm} \times 3 \mathrm{~mm}$ of the computational domain. The upper row $(\mathbf{a}-\mathbf{c})$ displays the active stage of Marangoni convection, corresponding to a local maximum in the curves of Figure $6 \mathrm{~b}$, while the lower row (d-f) shows the final part of the relaxation stage, corresponding to a local minimum in the curves of Figure $6 b$. 


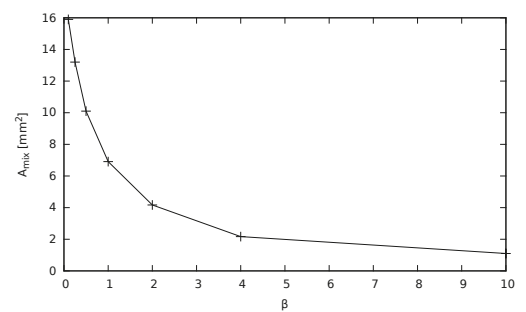

(a)

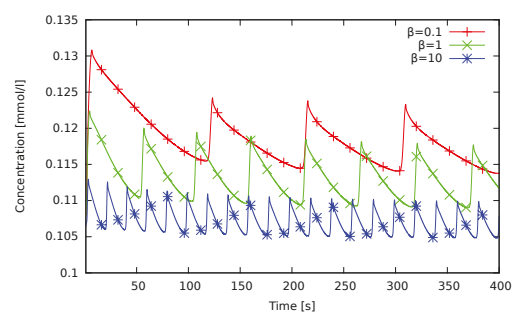

(b)

Figure 6. (a) Area of mixed fluid directly after the termination of the active stage of Marangoni convection from numerical simulations as a function of the volume expansion coefficient. (b) Relaxation oscillations of the mean solute concentration at the drop surface for different values of the volume expansion coefficient.

\section{Micromixer Using a Single Bubble Inside the Reactor}

By taking into account the characteristic properties of the relaxation oscillations discussed in the preceding sections, we propose a novel design of a continuous-flow microreactor based on the mixing effect of the solutal Marangoni convection. The general view and the internal details of the reactor are shown in Figure 7a,b. The working area of the reactor is a quasi-rectangular cell placed between two parallel glasses. A PTFE liner between the glass plates sets the inner dimensions of $L \times H \times W=20 \mathrm{~mm} \times 10 \mathrm{~mm} \times 2 \mathrm{~mm}$. The narrow gap between the glass plates again provides quasi two-dimensionality of the fluid flow. The reactor was used in a vertical position so that the gravity vector is parallel to the front and back glass plates (Figure $7 \mathrm{~b}$ ).

To switch on the Marangoni effect inside the reactor, a free surface is necessary. For this purpose, we have placed a plastic ring with a diameter of $6 \mathrm{~mm}$ in the middle of the cavity. This ring bounds the area in which the air bubble is created and holds the bubble in place during convective mixing of the fluid. To ensure sufficient contact of the bubble with the reacting fluids, symmetrical cutouts are inserted in the ring as shown in Figure $7 \mathrm{~b}$. By this, the bubble is fixed between solid walls from above and below, but the lateral bubble surface is free. The depth of the notch in the plastic ring is $1 \mathrm{~mm}$, i.e., half the gap width between the glass plates. Each of the lateral notches has an angular dimension of $150^{\circ}$. In the middle of one of the glass plates, tube 1 is inserted, through which air can be supplied to form a bubble inside the circular cell 2 . The bubble can be retained in the cell up to a feed inflow velocity of $1 \mathrm{~mm} / \mathrm{s}$. When this critical pumping rate is exceeded, the flow can detach the air bubble from the holding ring. Since flow visualization inside the air bubble is not required, cell 2 is covered by an opaque moisture-repellent film on both sides.

In the reactor, two vertical channels ( 3 and 4 ) are provided, through which the reagent solutions are fed (Figure $7 \mathrm{~b}$ ). The two horizontal channels (5 and 6) serve to withdraw the reaction products from the reactor zone. Such a reactor design can be used, for example, to implement a quite common second-order scheme A $+\mathrm{B} \rightarrow \mathrm{C}$, given that one of the substances is surface-active. To test the operation of the mixer, a non-reacting system is used. The procedure to prepare the device for operation is as follows. At the beginning, the cavity is completely filled with water through tube 3 . Then, the air bubble is injected in the cell 2 via tube 1 . At the next stage, an aqueous solution of isopropyl alcohol, which serves as a working surfactant, is continuously fed into the reactor cavity through tube 4 concurrently with the inflow of pure water from below.

Figure $8 \mathrm{a}-\mathrm{d}$ shows four snapshots of the time evolution in the reactor at the onset of the relaxation oscillations. At the very beginning, there is a stage without intense Marangoni convection along the free surface of the bubble. Consequently, the liquid leaves the reactor via side channels 5 and 6 rather unmixed: the vertical concentration stratification is clearly visible in Figure 8a. This induction period probably is connected to the threshold of Marangoni convection as noted above. At $t=250 \mathrm{~s}$ the 
active phase is initiated and the fluid is mixed by the intense Marangoni convection (Figure $8 b$ ). The first stage of vigorous convective motion lasts about $150 \mathrm{~s}$. This prolongation of the active phase can be explained by the fluid flow which is superposed by the external pumping. It continuously feeds solute-rich fluid supporting the Marangoni vortex at the bubble.

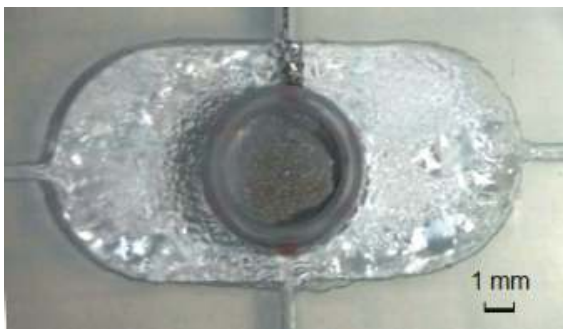

(a)

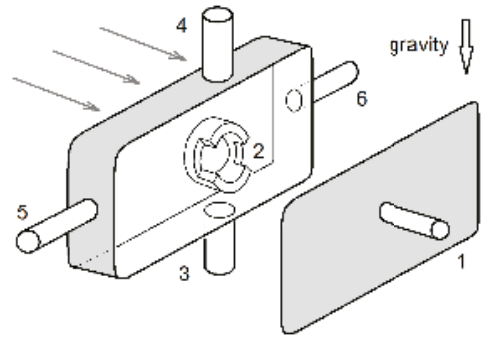

(b)

Figure 7. (a) Photograph as general view of the reaction zone of the continuous-flow microreactor with an adaptive mixing effect due to an air bubble placed in the center. (b) Scheme of the cuvette: inlet for air to produce the bubble 1; plastic insert to hold the bubble 2; inlets for the reagents 3 and 4; outlets for the reaction product 5 and 6 . Arrows indicate the direction of observation with the Fizeau interferometer.

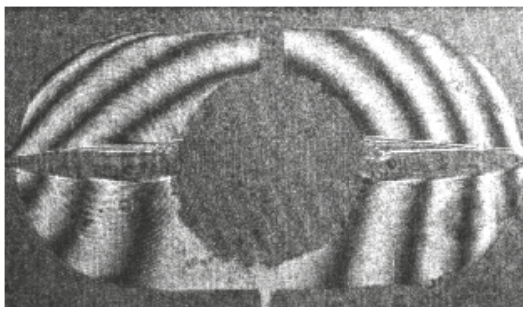

(a)

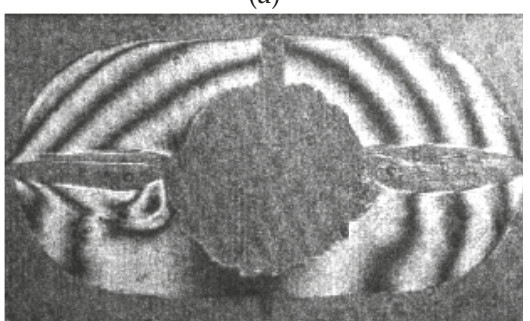

(c)

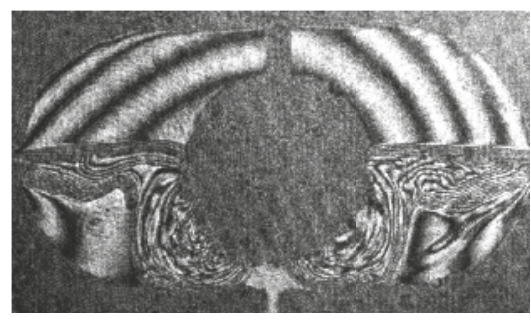

(b)

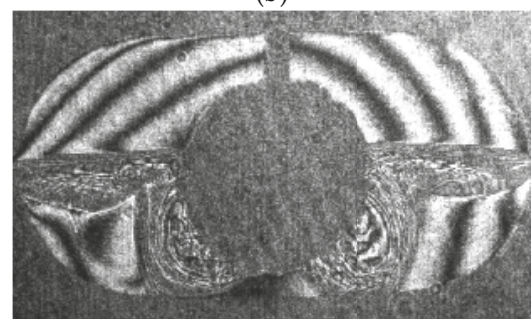

(d)

Figure 8. Evolution of the concentration field of isopropyl alcohol near the air bubble placed in the center of the cavity for $C_{0}=15 \mathrm{wt}$. $\%$ and inflow velocities from both inlets $V=0.02 \mathrm{~mm} / \mathrm{s}: t=240 \mathrm{~s} \mathrm{(a)}$; $t=270 \mathrm{~s}(\mathbf{b}) ; t=420 \mathrm{~s}(\mathbf{c}) ;$ and $t=450 \mathrm{~s}(\mathrm{~d})$.

When the solution in the reactor zone becomes completely mixed so that the concentration difference on the surface of the bubble strongly decreases, the relaxation phase occurs. Nevertheless, according to the structure of the interferogram stripes, one can conclude that a weak convective movement still continues, and the mixed liquid leaves the reactor via lateral channels (Figure 8c). During the relaxation phase, fresh alcohol solution is supplied from the top channel again creating the concentration difference on the bubble surface. The relaxation phase lasts for a relatively short time 
(approximately 20-30 s). Thereafter, the Marangoni convection is excited again, as shown in Figure 8d. Interestingly, the mixing area is located in the lower part of the reactor. Most likely, this is caused by the interaction of the Marangoni convection with the concentration stratification and the superposed feed flow. This is an important observation for optimizing the reactor design in future work.

\section{Discussion and Conclusions}

In this work, we propose a new micromixer design based on the joint action of solutal Marangoni instability, buoyancy convection and diffusion. For this function principle, the presence of a fluidic interface and a surface-active solute is required. The operation of the micromixer does not consume additional external energy, but works due to the repeated consumption and regeneration of the driving concentration gradients. Thus, the micromixer automatically adapts to the conditions in the reactor zone. If the concentration field becomes sufficiently uniform, the oscillations stop. When the concentration inhomogeneities reappear, the system indicates that the reagents in the reactor zone are still not completely mixed and the convection is excited again. For specific applications in chemical engineering, this adaptability will be advantageous, e.g., for the degradation of pollutants, where higher educt concentrations require an intensified process. For common continuous-flow microreactors, where fresh reactants are permanently delivered into the mixing zone, the concentration gradient will be constant in time, providing the necessary inhomogeneity around the bubble. Furthermore, a continuously operating micromixer independent of the medium can be obtained by introducing an inert surfactant (not participating in the reaction) in the feed flow.

Indeed, the application of such a microreactor requires an individual approach to the specific reaction type and the used solutes. Our experiments however show that the relaxation oscillations occur in different geometries and fluid combinations making it versatile for the implementation in various microreactor systems. The formation of bubbles is an inexpensive method to introduce a fluidic interface in a microreactor setup. On the other hand, the liquid-liquid system is relevant for droplet microfluidics where the droplets themselves are used as a spatially bounded reaction zone. Our tested micromixer also works in the presence of slight contaminations if the Marangoni driving force is high enough, which is important under technological conditions.

However, further developments are necessary to approach technological applicability. Our simulations support this by providing a thorough understanding of the basic mechanisms and dependencies of the relaxation oscillations. They suggest that an even more effective mixing can be obtained for solutes with lower volume expansion coefficient or under a reduced influence of gravity, i.e., in a horizontal reactor position. The identification of such determining parameters gives the directions for future experimental work.

Author Contributions: Conceptualization, D.B.; Methodology and Experimental Observations, K.K., A.M. and K.S.; Numerical simulations, S.A. and M.M.; and Writing and Draft Preparation, D.B., K.S. and K.E.

Funding: The reported study was funded by Russian Foundation for Basic Research and Ministry of Science and Education of Perm region according to the research project No. 17-41-590100. Furthermore, we acknowledge financial support provided by the Helmholtz Recruitment Initiative Fellowship (K.E.). The simulations were performed at the Center for Information Services and High Performance Computing (ZIH) at TU Dresden.

Conflicts of Interest: The authors declare no conflict of interest.

\section{References}

1. Avnir, D.; Kagan, M.L. The evolution of chemical patterns in reactive liquids, driven by hydrodynamic instabilities. Chaos 1995, 5, 589-601. [CrossRef] [PubMed]

2. Bees, M.A.; Pons, A.J.; Sorensen, P.G.; Sagues, F. Chemoconvection: A chemically driven hydrodynamic instability. J. Chem. Phys. 2001, 114, 1932-1943. [CrossRef]

3. Almarcha, C.; Trevelyan, P.M.J.; Grosfils, P.; De Wit, A. Chemically Driven Hydrodynamic Instabilities. Phys. Rev. Lett. 2010, 104, 044501. [CrossRef] [PubMed]

4. Reschetilowski, W. (Ed.) Microreactors in Preparative Chemistry; Wiley-VCH: Weinheim, Germany, 2013; p. 352 
5. Jensen, K.F. Microreaction engineering-Is small better? Chem. Eng. Sci. 2001, 56, 293-303. [CrossRef]

6. Hessel, V.L.; Holger-Schonfeld, F. Micromixers-A review on passive and active mixing principles. Chem. Eng. Sci. 2005, 60, 2479-2501. [CrossRef]

7. Mascia, S.; Heider, P.L.; Zhang, H.; Lakerveld, R.; Benyahia, B.; Barton, P.I.; Braatz, R.D.; Cooney, C.L.; Evans, J.M.B.; Jamison, T.F.; et al. End-to-end continuous manufacturing of pharmaceuticals: Integrated synthesis, purification, and final dosage formation. Angew. Chem. Int. Ed. 2013, 52, 12359-12363. [CrossRef] [PubMed]

8. Newman, S.G.; Jensen, K.F. The role of flow in green chemistry and engineering. Green Chem. 2013, 15, 1456-1472. [CrossRef]

9. Filipponi, P.; Ostacolo, C.; Novellino, E.; Pellicciari, R.; Gioiello, A. Continuous Flow Synthesis of Thieno [2,3-c]isoquinolin-5(4H)-one Scaffold: A Valuable Source of PARP-1 Inhibitors. Org. Process Res. Dev. 2014, 18, 1345-1353. [CrossRef]

10. Baumann, M.; Baxendale, I.R. The synthesis of active pharmaceutical ingredients (APIs) using continuous flow chemistry. Beilstein J. Org. Chem. 2015, 52, 1194-1219. [CrossRef] [PubMed]

11. Pellegatti, L.; Sedelmeier, J. Synthesis of Vildagliptin Utilizing Continuous Flow and Batch Technologies. Org. Process Res. Dev. 2015, 19, 551-554. [CrossRef]

12. Martin, A.D.; Siamaki, A.R.; Belecki, K.; Gupton, B.F. A flow-based synthesis of telmisartan. J. Flow Chem. 2015, 5, 145-147. [CrossRef]

13. Wegner, J.; Ceylan, S.; Kirschning, A. Ten key issues in modern flow chemistry. Chem. Commun. 2011, 47, 4583-4592. [CrossRef] [PubMed]

14. Nieves-Remacha, M.J.; Kulkarni, A.A.; Jensen, K.F. Hydrodynamics of liquid-liquid dispersion in an Advanced-Flow Reactor. Ind. Eng. Chem. Res. 2012, 51, 16251-16262. [CrossRef]

15. Ju, J.; Warrick, J. Passive micromixer using by convection and surface tension effects with air-liquid interface. BioChip J. 2013, 7, 361-366. [CrossRef] [PubMed]

16. Sinz, D.K.; Hanyak, M.; Darhuber, A.A. Self-induced surfactant transport along discontinuous liquid-liquid interfaces. J. Phys. Chem. Lett. 2013, 4, 1039-1043. [CrossRef] [PubMed]

17. Yamada, T.; Ono, N. A study on micromixing utilizing Marangoni effect induced on gas-liquid free interfaces. J. Micro Nano-Manuf. 2015, 3, 021003. [CrossRef]

18. Cheng, W.L.; Erbay, CV.; Sadr, R.; Han, H. Dynamic Flow Characteristics and Design Principles of Laminar Flow Microbial Fuel Cells. Micromachines 2018, 9, 479. [CrossRef] [PubMed]

19. Stroock, A.D.; Dertinger, S.K.W.; Ajdari, A.; Mezić, I.; Stone, H.A.; Whitesides, G.M. Chaotic Mixer for Microchannels. Science 2002, 295, 647. [CrossRef] [PubMed]

20. Abbas, Y.; Miwa, J.; Zengerle, R.; von Stetten, F. Active Continuous-Flow Micromixer Using an External Braille Pin Actuator Array. Micromachines 2013, 4, 80-89. [CrossRef]

21. Mavrogiannis, N.; Desmond, M.; Ling, K.; Fu, X.; Gagnon, Z. Microfluidic Mixing and Analog On-Chip Concentration Control Using Fluidic Dielectrophoresis. Micromachines 2016, 7, 214. [CrossRef] [PubMed]

22. Cai, Z.; Xiang, J.; Chen, H.; Wang, W. A Rapid Micromixer for Centrifugal Microfluidic Platforms. Micromachines 2016, 7, 89. [CrossRef] [PubMed]

23. Malgaretti, P.; Pagonabarraga, I.; Joanny, J.-F. Bistability, Oscillations, and Bidirectional Motion of Ensemble of Hydrodynamically Coupled Molecular Motors. Phys. Rev. Lett. 2017, 119, 168101. [CrossRef] [PubMed]

24. Eckert, K.; Grahn, A. Plume and finger regimes driven by an exothermic interfacial reaction. Phys. Rev. Lett. 1999, 82, 4436-4439. [CrossRef]

25. Bratsun, D.; De Wit, A. On Marangoni convective patterns driven by an exothermic chemical reaction in two-layer systems. Phys. Fluids 2004, 16, 1082-1096. [CrossRef]

26. Shi, Y.; Eckert, K. A novel Hele-Shaw cell design for the analysis of hydro-dynamic instabilities in liquid-liquid systems. Chem. Eng. Sci. 2008, 63, 3560-3563. [CrossRef]

27. Bratsun, D.; De Wit, A. Buoyancy-driven pattern formation in reactive immiscible two-layer systems. Chem. Eng. Sci. 2011, 66, 5723-5734. [CrossRef]

28. Eckert, K.; Acker, M.; Tadmouri, R.; Pimienta, V. Chemo-Marangoni convection driven by an interfacial reaction: Pattern formation and kinetics. Chaos Interdiscip. J. Nonlinear Sci. 2012, 22, 037112. [CrossRef] [PubMed]

29. Bratsun, D.A. On Rayleigh-Bénard mechanism of alignment of salt fingers in reactive immiscible two-layer systems. Microgravity Sci. 2014, 26, 293-303. [CrossRef] 
30. Schwarzenberger, K.; Eckert, K.; Odenbach, S. Relaxation oscillations between Marangoni cells and double diffusive fingers in a reactive liquid-liquid system. Chem. Eng. Sci. 2012, 68, 530-540. [CrossRef]

31. Eckert, K.; Shi, Y.; Schwarzenberger, K. Meniscus asymmetry and chemo-Marangoni convection in capillaries. Chem. Eng. Technol. 2017, 40, 2067-2074. [CrossRef]

32. Mizev, A. Influence of an adsorption layer on the structure and stability of surface tension driven flows. Phys. Fluids 2005, 17, 122107. [CrossRef]

33. Kostarev, K.; Shmyrov, A.; Zuev, A.; Viviani, A. Convective and diffusive surfactant transfer in multiphase liquid systems. Exp. Fluids 2011, 51, 457-470. [CrossRef]

34. Schwarzenberger, K.; Köllner, T.; Linde, H.; Boeck, T.; Odenbach, S.; Eckert, K. Pattern formation and mass transfer under stationary solutal Marangoni instability. Adv. Colloid Interface Sci., 2014, 206, 344-371. [CrossRef] [PubMed]

35. Birikh, R.V.; Denisova, M.O.; Kostarev, K.G. Development of concentration-capillary convection on an interfacial surface. Fluid. Dyn. 2015, 50, 361-370. [CrossRef]

36. Schwarzenberger, K.; Aland, S.; Domnick, H.; Odenbach, S.; Eckert, K. Relaxation oscillations of solutal Marangoni convection at curved interfaces. Colloids Surf. A Physicochem. Eng. Aspects 2015, 481, 633-643. [CrossRef]

37. Crank, J. The Mathematics of Diffusion; Clarendon Press: Oxford, UK, 1975; 414 p.

38. Grahn, A. Two-dimensional numerical simulations of Marangoni-Bénard instabilities during liquid-liquid mass transfer in a vertical gap. Chem. Eng. Sci. 2006, 61, 3586-3592. [CrossRef]

39. Boos, W.; Thess, A. Thermocapillary flow in a Hele-Shaw cell. J. Fluid Mech. 1997, 352, 305-330. [CrossRef]

40. Teigen, K.E.; Song, P.; Lowengrub, J.; Voigt, A. A diffuse-interface method for two-phase flows with soluble surfactants. J. Comput. Phys. 2011, 230, 375-393. [CrossRef] [PubMed]

(C) 2018 by the authors. Licensee MDPI, Basel, Switzerland. This article is an open access article distributed under the terms and conditions of the Creative Commons Attribution (CC BY) license (http:/ / creativecommons.org/licenses/by/4.0/). 


\title{
Article \\ Self-Lubricanting Slippery Surface with Wettability Gradients for Anti-Sticking of Electrosurgical Scalpel
}

\author{
Guang Liu ${ }^{1} @$, Pengfei Zhang ${ }^{2,3}$, Yang Liu ${ }^{1}$, Deyuan Zhang ${ }^{1,2}$ and Huawei Chen ${ }^{1,2, *}$ \\ 1 School of Mechanical Engineering and Automation, Beihang University, Beijing 100191, China; \\ liuguang0701@buaa.edu.cn (G.L.); liuyang168@buaa.edu.cn (Y.L.); zhangdy@buaa.edu.cn (D.Z.) \\ 2 Beijing Advanced Innovation Center for Biomedical Engineering, Beihang University, Beijing 100191, China; \\ pengfei89.zhang@gmail.com \\ 3 Department of Bioengineering and Therapeutic Sciences, University of California, \\ San Francisco, CA 94158, USA \\ * Correspondence: chenhw75@buaa.edu.cn; Tel.: +86-010-82-339-717
}

Received: 30 September 2018; Accepted: 8 November 2018; Published: 13 November 2018

\begin{abstract}
Soft tissue sticking on electrosurgical scalpels in minimally invasive surgery can increase the difficulty of operation and easily lead to medical malpractice. It is significant to develop new methods for anti-sticking of soft tissue on electrosurgical scalpels. Based on the characteristics of biomimetic ultra-slippery surface, a self-lubricating slippery surface with wettability gradients on electrosurgical scalpel was designed and fabricated. Non-uniformly distributed cylindrical micro pillars, which constitute the wettability gradients, were prepared by an electrolytic etching process and the theoretic of the spontaneous liquid spreading process was analyzed. The silicophilic property of wettability gradients surface was modified by octadecyltrichlorosilane (OTS) self-assembling coat with biocompatible liquid lubricant dimethyl silicone oil. The contact angle of gradient's surface at different temperatures was measured. The transportation behaviors of both water and dimethyl silicone oil on the wettability gradient's surface were investigated; the results illustrate that the wettability gradient's slippery surface can successfully self-lubricate from regions with low pillar density to regions with high pillar density, ascribed to the unbalanced Young's force. The anti-sticking capability of the electrosurgical scalpel with self-lubricating slippery surface was tested. Both the adhesion force and adhesion mass under different cycles were calculated. The results suggest that the as-prepared slippery surface has excellent anti-sticking ability associated with better durability.
\end{abstract}

Keywords: self-lubricating slippery surface; wettability gradient; electrosurgical scalpels; anti-sticking; soft tissue

\section{Introduction}

Minimally invasive surgery is becoming increasingly popular as it produces less pain, less trauma and rapid recovery for patients. However, soft tissue sticking always occurs on the surface of electrosurgical instruments, such as electrosurgical scalpels, monopole electrode, electrocoagulation and bipolar forceps [1-5]. Owing to the high operating temperature, soft tissue can easily char and adhere to the instrument surface, which may trigger failure of hemostasis or carbonized eschar; leading to unexpected surgical trauma and potential danger to patients [6-9]. Therefore, it is an urgent need to handle the problems caused by soft tissue sticking during surgical treatment. Several attempts have been made aiming to reduce sticking of soft tissue on electrosurgical scalpels. Based on the current research results, there are two main ways to alleviate tissue sticking: chemical approaches and physical methods. Chemical approaches include adopting low surface energy alloy coating or polymer coating, such as copper-doped diamond-like carbon [10-13], $\mathrm{CrN} \mathrm{[14],} \mathrm{TiO}_{2}$ [6,15-17], 
poly(l-lactic acid)-polyethylene glycol [18], polygalacturonic acid-1-ethyl-3-(3-dimethylaminopropyl) carbodiimide [19], polylactide-polyethylene glycol tri-block copolymer [20]. Physical methods mainly refer to the design and fabrication of micro/nanostructures on the surface of scalpels [16,21]. However, there are some deficiencies in both approaches such as the limited ability to resist tissue adhesion and the low reliability of alloy coatings that may decompose and release noxious substances under high temperatures. The strength of micro/nanostructures on the surface is insufficient and easily destroyed, failing to work effectively. Hence, it is significant to explore novel methods that promote anti-sticking of soft tissue on electrosurgical scalpels.

Liquid-infused surfaces have a wide range of applications, such as biosensors [22], blood-contacting medical devices [23,24], microfluidic devices [25] and so on [26]. Previous studies have demonstrated a marvelous anti-sticking capacity of the liquid-infused surface for biofouling, ice, frost and varieties of other liquids [27-32]. Recently, novel slippery anti-adhesion liquid-infused surfaces which are enhanced by the ultra-slippery property of the peristome of Nepenthes alata, have been fabricated by constructing a liquid/solid composite surface. Liquid-infused surfaces require the combination of a micro/nanostructure and the lubricant. The function of a micro/nanostructure is to hold the lubricant firmly, aiming to maintain sufficient and stable lubrication and thus making a liquid/object interface with an anti-sticking effect. Evidence suggests that an adequate and stable lubricant has an important role on the anti-sticking durability of liquid-infused surfaces [33-36]. Nevertheless, the research above did not fully clarify how to resolve the short term resistance of the lubricant.

Herein, a liquid-infused slippery surface with wettability gradients is proposed to enhance its anti-sticking function. Non-uniformly distributed cylindrical micro pillars on the surface of electrosurgical scalpels are designed to drive lubricant spreading spontaneously along the microstructure as shown in Figure 1. These wettability gradient microstructures are fabricated via a photolithography-assisted electrolytic etching process, and then a micro channel is generated by a laser process. Silicon oil is chosen as the lubricant for its outstanding biocompatibility and high-temperature resistance [17,37]. A thin layer of octadecyltrichlorosilane $\left(\mathrm{OTS}, \mathrm{CH}_{3}\left(\mathrm{CH}_{2}\right)_{16} \mathrm{CH}_{2} \mathrm{SiCl}_{3}\right)$, which has a few nanometer thickness and strong siloxane bond, is grafted on the wettability gradient's surface to strongly maintain the lubricant. Behavior of liquid transportation on the as-prepared surface is investigated, and finally the anti-sticking effects of the self-lubricating wettability gradient's surface are explored by adhesion force measurements and cycle tests.

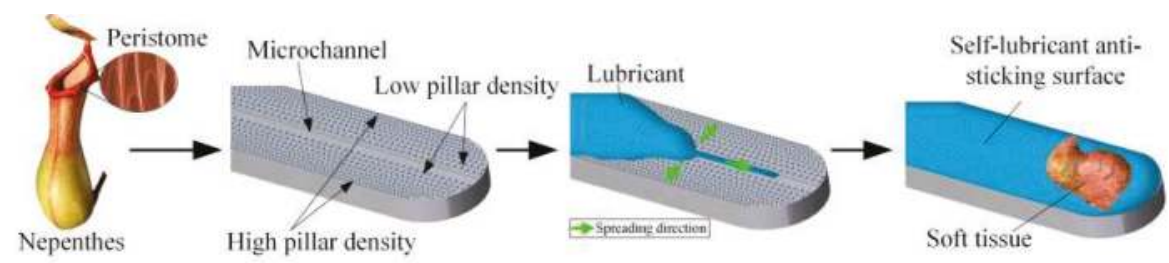

Figure 1. Nepenthes-inspired self-lubricating anti-sticking surface with wettability gradients.

\section{Materials and Methods}

Electrosurgical scalpels which are made of 316L stainless steel were commercially obtained from Beijing ENJOY Technologies Company Limited (Beijing, China) and were used as the typical electrosurgical instruments in this paper. Positive photoresist (BP212-37S) and its developer were purchased from Kempur Microelectronics Inc. (Beijing, China). Polydimethylsiloxane (PDMS, Dow Corning, Midland, MI, USA) was used as received. $\mathrm{NaOH}$ (Sodium hydroxide), $\mathrm{Na}_{2} \mathrm{CO}_{3}$ (Sodium carbonate), $\mathrm{H}_{3} \mathrm{PO}_{4}$ (Phosphoric acid), $\mathrm{HCl}$ (Hydrochloric acid), $\mathrm{FeCl}_{3}$ (Ferric chloride), $n$-hexane, acetone, ethanol and toluene were supplied by Beijing Chemical Works (Beijing, China). Octadecyltrichlorosilane (OTS) which was used as the silane coupling agent, was purchased from 
Chengdu Chemical Reagent (Chengdu, China). Dimethyl silicone oil with a viscosity of $100 \pm 5 \mathrm{~mm}^{2} / \mathrm{s}$, provided by Sinopharm Chemical Reagent (Shanghai, China), was used as the infused lubricant. Fresh resected pig liver was chosen as soft tissue materials to facilitate the measurement of adhesion force and tissue adhesion mass, for its relatively homogeneous composition.

Photolithography-assisted electrolytic etching process was applied to fabricate non-uniformly distributed cylindrical micro pillars on the surface of electrosurgical scalpels. Figure 2 shows the schematic of the manufacturing process. The scalpel was entirely immersed into alkaline solutions with a composition of $\mathrm{NaOH} 50 \mathrm{~g} / \mathrm{L}$ and $\mathrm{Na}_{2} \mathrm{CO}_{3} 40 \mathrm{~g} / \mathrm{L}$ for $30 \mathrm{~min}$ to first eliminate the oil stain. Then it was thoroughly cleaned by deionized water, $n$-hexane, acetone and ethanol for 10 minutes in proper sequence, respectively. It was subsequently completely dried by vacuum drying at $150{ }^{\circ} \mathrm{C}$ for approximately $30 \mathrm{~min}$ before spinning. The positive photoresist was spun onto the stainless steel at the speed of $700 \mathrm{rpm}$ for $9 \mathrm{~s}$ and $1500 \mathrm{rpm}$ for $15 \mathrm{~s}$ to maintain a consistency of thickness, which is about $10 \mu \mathrm{m}$. The scalpel was then placed on the hotplate to prebake at the temperature of $120^{\circ} \mathrm{C}$ for $3 \mathrm{~min}$. The photolithography process was followed by a contact aligner with ultraviolet (UV) light wavelength of $254 \mathrm{~nm}$ and light intensity of $13 \mathrm{~mW} / \mathrm{cm}^{2}$ for $25 \mathrm{~s}$. In this process, it is necessary to ensure that the central line of the mask and the symmetrical line of the scalpel coincides with each other, to make the wettability gradient's microstructure distribute perpendicularly to the symmetric line. Postbaking was exerted at $120^{\circ} \mathrm{C}$ for $2 \mathrm{~min}$ and afterward the scalpel was immersed with photoresist in the stripper for 10 minutes to develop. The electrolytic etching process was applied in an electrolytic cell with about $500 \mathrm{~mL}$ electrolyte with a composition of $\mathrm{FeCl}_{3} 400 \mathrm{~g} / \mathrm{L}$, Phosphoric acid $20 \mathrm{~g} / \mathrm{L}$ and Hydrochloric acid $100 \mathrm{~g} / \mathrm{L}$ for $30 \mathrm{~s}$ under a $0.5 \mathrm{~A}$ current. Following the electrolytic etching process the scalpel was removed and rinsed with deionized water. Then the gradient's microstructures were obtained after removing the photoresist from the scalpel, via ultrasonic cleaning in acetone for approximately $5 \mathrm{~min}$.
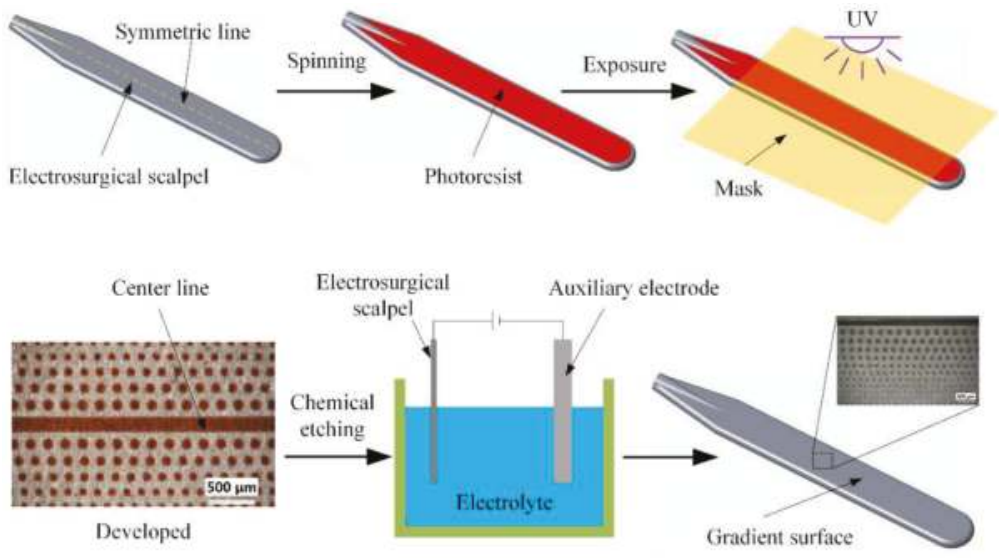

Figure 2. Schematic of non-uniformly distributed cylindrical micro pillars fabrication.

Silicon oil was selected as the lubricant for its outstanding biocompatibility and ability to withstand high temperatures. In order to construct a stable slippery wettability gradient surface, silicon oil should be firmly held in the surface. Thus, the property of the substrate must be functionalized to match the silicon oil closely. The silicophilic functionalized property can be realized via grafting a thin, self-assembling layer of OTS. It's few nanometer thickness, strong bond (siloxane bond) with the substrate $[17,37]$ and the schematic of grafting process, is shown in Figure 3a. Oxygen plasma treatment for $10 \mathrm{~min}$ with RF power $100 \mathrm{~W}$, pressure $100 \mu$ bar and flow rate $20 \mathrm{sccm}$ after the substrate was thoroughly cleaned, was used to increase the hydroxyl content of the surface; in order to facilitate the formation of OTS self-assembly in the next step. Next the substrate was dipped into anhydrous toluene solution with an OTS concentration of $1 \mathrm{mM} / \mathrm{L}$ for $4 \mathrm{~h}$. Then the substrate was taken out and 
the excess OTS was rinsed using anhydrous toluene. It was then dried under a nitrogen atmosphere. Figure $3 \mathrm{~b}$ shows the Attenuated Total Reflectance Fourier Transform Infrared (ATR-FTIR) spectra of the substrate before (red line) and after (dark line) the OTS graft. The position and broadening of the $\mathrm{Si}-\mathrm{O}$ and $\mathrm{Si}-\mathrm{Cl}$ group vary depending on how the OTS binds at the surface. In the ATR-FTIR spectra of both original substrate and OTS grafted substrate, defined peaks attributed to $v_{\mathrm{Si}-\mathrm{O}}=1181 \mathrm{~cm}^{-1}$ and $v_{\mathrm{Si}-\mathrm{Cl}}=687 \mathrm{~cm}^{-1}$ were presented. Compared with the spectrum of the original substrate, the grafted surface-bound films suggested a binding mode due to peaks at $v_{\mathrm{Si}-\mathrm{O}}=1181 \mathrm{~cm}^{-1}$ and $v_{\mathrm{C}-\mathrm{H}}=2923 \mathrm{~cm}^{-1}$ [38] which demonstrated that the self-assembled thin layer of OTS was successfully grafted onto the substrate.

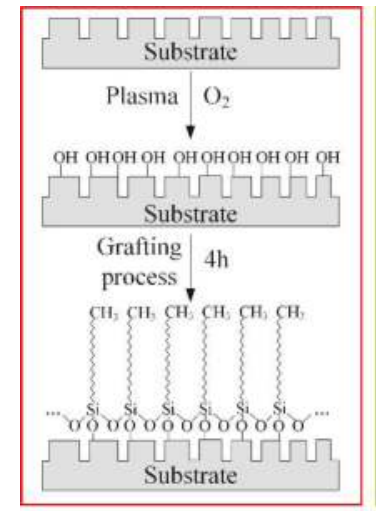

(a)

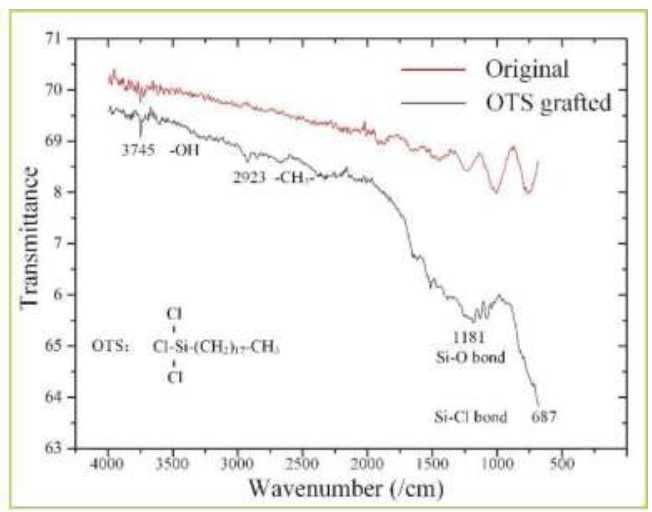

(b)

Figure 3. Surface modification of the substrate. (a) Schematic of self-assembly thin layer of octadecyltrichlorosilane (OTS) grafting process; (b) ATR-FTIR spectra of the substrate before (red line) and after (dark line) self-assembled thin layer of OTS grafts.

\section{Results and Discussion}

The wettability gradients can drive droplet transport direction due to the microstructures in the surface $[39,40]$. The diameters of non-uniformly distributed cylindrical micro pillars range from $70 \mu \mathrm{m}$ to $150 \mu \mathrm{m}$ with about $10 \mu \mathrm{m}$ height. The center-to-center space between pillars ranges from $195 \mu \mathrm{m}$ in the region with low pillar density, to $125 \mu \mathrm{m}$ in the region with high pillar density, over a span of $1.5 \mathrm{~mm}$ and symmetrically distributed on both sides of the symmetric line. The lubricant was delivered continuously along the microchannel in the center and spread spontaneously on the wettability gradients. These gradients, associated with the functionalized surface, promote an expected outstanding durability of the scalpel's self-lubricating anti-sticking ability. To explore the mechanisms of droplet spreading behaviors, we analyzed the law of droplet contact angle (CA) by adding a water droplet of $0.5 \mu \mathrm{L}$ on the dry functionalized gradient's surface, at temperatures from $25^{\circ} \mathrm{C}$ to $125^{\circ} \mathrm{C}$. The gradient substrate was placed on a hotplate before it was placed horizontally. The measurements of CA were taken with the help of a high-speed camera (I-speed LT, Olympus, Tokyo, Japan), which was mounted by a support and adjusted horizontally to match the surface of substrate [41,42]. The morphology of how the droplet initially contacted the surface was recorded. Figure 4 shows the static CA and asymmetric $C A$ profiles along the wettability gradient's orientation under different temperature conditions. The measured apparent contact angle of regions with high pillar density $\theta_{H P D}$ and low pillar density $\theta_{L P D}$ were $70 \pm 0.39^{\circ}$ and $82 \pm 0.88^{\circ}$ at $25^{\circ} \mathrm{C}, 81 \pm 0.3^{\circ}$ and $92 \pm 0.69^{\circ}$ at $50^{\circ} \mathrm{C}, 81 \pm 0.21^{\circ}$ and $94 \pm 0.71^{\circ}$ at $75^{\circ} \mathrm{C}, 88 \pm 0.16^{\circ}$ and $104 \pm 0.28^{\circ}$ at $100^{\circ} \mathrm{C}, 83 \pm 0.64^{\circ}$ and $101 \pm 0.7^{\circ}$ at $125^{\circ} \mathrm{C}$, respectively. According to Figure 4, the water droplet spread exhibited an asymmetric shape and the liquid CA $\theta_{H P D}$ in the region with high pillar density is obviously less than $\theta_{L P D}$ in the region with low pillar density. 
The underlying mechanism for droplet spreading was analyzed. Based on the CA, we determined that the droplet was in Wenzel state $[43,44]$. Under this condition, we can have:

$$
\cos \theta=R_{f} \cos \theta_{0}
$$

where $R_{f}$ represents the surface roughness, $\theta$ and $\theta_{0}$ represent the apparent and intrinsic contact angle of rough and smooth surfaces, respectively. The driving force generated by surface energy gradients owing to the difference of surface roughness can be depicted as [45]:

$$
F=\int_{L_{L P D}}^{L_{H P D}} \gamma\left(\cos \theta_{H P D}-\cos \theta_{L P D}\right) d l
$$

where $\gamma$ represents the surface tension of the droplet, $\theta_{H P D}$ and $\theta_{L P D}$ represent the advancing and receding angles of the droplet along the wettability gradient's direction respectively, $d l$ represents the integration variable from the region with low pillar density $L_{L P D}$ to the region with high pillar density $L_{H P D}$. As $\theta_{H P D}$ is more miner than $\theta_{L P D}$, we can have the unbalanced Young' force $F$ which, derived from the gradients, will exhibit the drifting trend of the droplet on the surface.

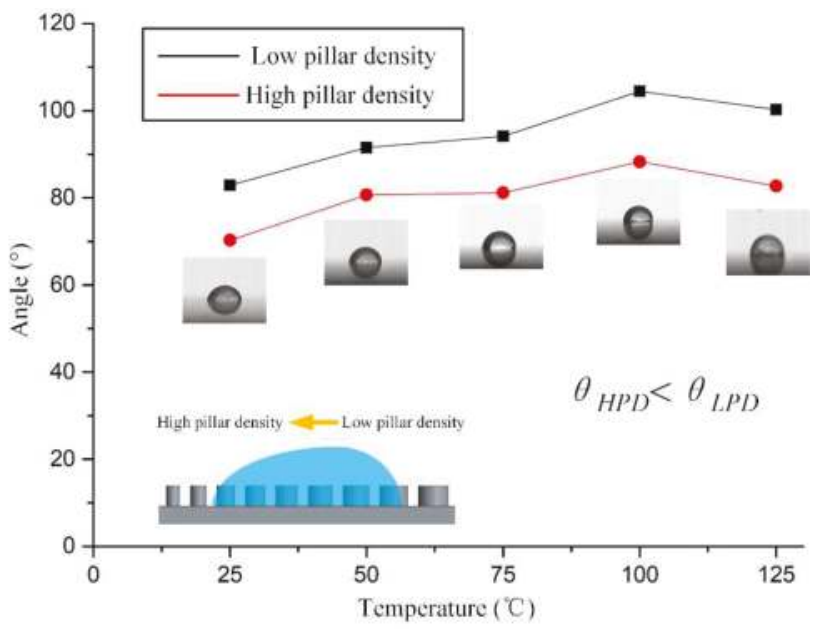

Figure 4. The static contact angle (CA) and asymmetric CA profiles along the wettability gradient's orientation on the dry surface, under different temperatures conditions.

The unidirectional liquid transport of the wettability gradient's surface was further investigated. For the silicophilic treated substrate of the scalpel, enough silicone oil was added to the gradient's surface and ensured that the volume of oil can almost cover the entire gradient's microstructures. In this procedure, the substrate should be maintained at a horizontal level in order to eliminate any effect of gravity. According to Figure 5a, when oil was added to the region with low pillar density, it could spread spontaneously and rapidly, moving to the region with high pillar density within $1.47 \mathrm{~s}$. While different from Figure 5a, oil stayed stationary when it was added to the region with high pillar density, shown in Figure 5b. This different spreading behavior is consistent with the above theoretical analysis indicating that the wettability gradient's surface is effective for self-lubrication. 

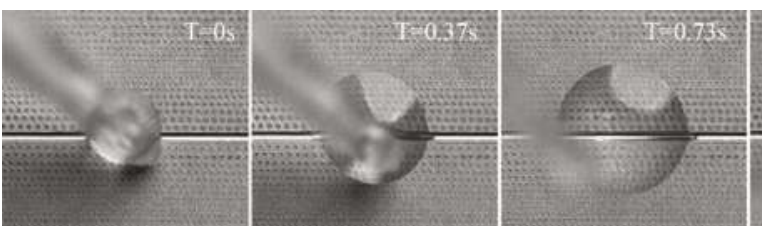

(a)
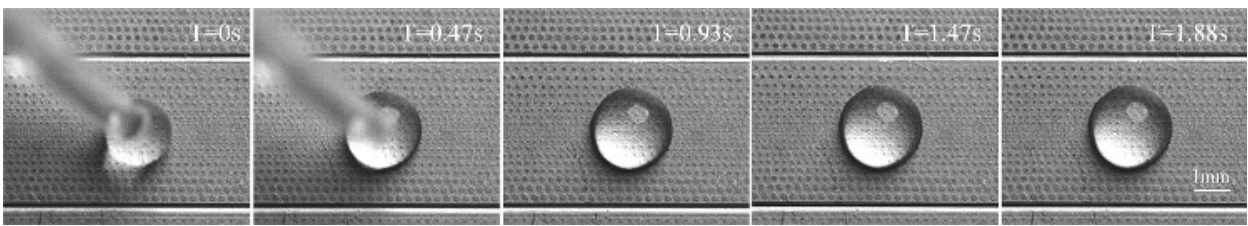

(b)

Figure 5. Silicone oil spreading morphology when added to different gradient regions on the functionalized surface: (a) Silicone oil spreading behavior when added to the region with low pillar density; (b) silicone oil spreading behavior when added to the region with high pillar density.

For the functionality of the wettability gradient's surface, the OTS self-assembled thin layer is critical for the spreading of silicone oil. The grafted $\mathrm{Si}-\mathrm{O}$ bonds make the surface chemical properties match the silicone oil's affinitive inducing silicophilic capability, to ensure the substrate is sufficiently lubricated. To examine the effect of an OTS thin layer, we investigated the silicone oil's spreading behaviors on an unmodified wettability surface and a functionalized wettability surface, as shown in Figure 6. Silicone oil with a volume of $2 \mu \mathrm{L}$ and viscosity of $100 \pm 5 \mathrm{~mm}^{2} / \mathrm{s}$ was added to the unmodified surface via pipette and it stayed originally even in the lowest pillar density region as seen in Figure 6a. Nevertheless, when the silicone oil was added on the OTS self-assembly wettability gradient's surface, it could stretch completely over the span of the gradient's distributed micro pillars along the microchannel; presented in Figure $6 \mathrm{~b}$. This indicates that the self-lubricating slippery wettability gradient's surface on the scalpel was successfully fabricated.
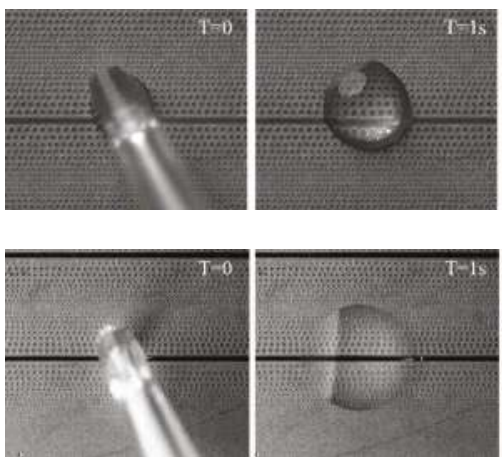

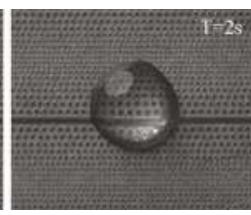

(a)

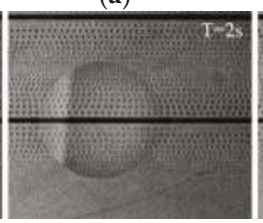

(b)
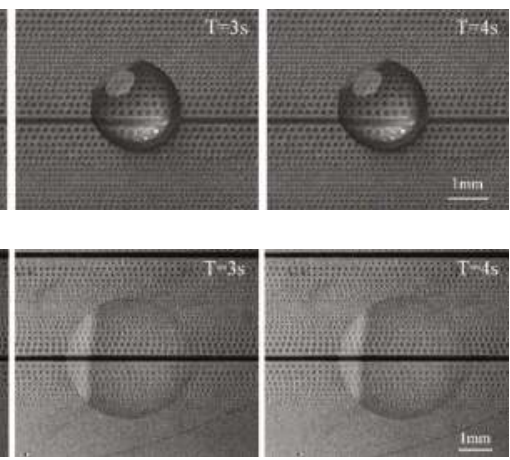

Figure 6. Spreading behavior of silicone oil on unmodified wettability surface and functionalized wettability surface: (a) Silicone oil spreading on unmodified wettability surface; (b) silicone oil spreading on functionalized wettability surface.

The amount of silicon oil stored on the surface may be consumed gradually during contact between the soft tissue and the gradient substrate. Consequently, a continuous supply of lubricant on the gradient's surface is crucial to sustain the anti-sticking capacity of the as-prepared surface. In order to vividly express continuous supply of lubricant on the gradient's surface, and make a 
distinct observation distinguished from the residue silicone oil, a water droplet was chosen as the alternative lubricant. Results show that when adding a water droplet to the oil-infused gradient's surface, it can easily slide; propelled by the unbalanced Young's force. Figure 7a illustrates the motion behavior of the droplet moving directionally on the slippery wettability gradient's surface and its optical photograph is shown in Figure $7 \mathrm{~b}$. For the slippery wettability gradient's surface, the water droplet gradually slid from the region with low pillar density to the region with high pillar density and ultimately remained stationary. The red-dashed lines labeled in Figure $7 \mathrm{c}$ represent the deviation distances center-to-center of the droplet. The deviation distance of the droplet was about $188 \pm 5 \mu \mathrm{m}$ within $1.43 \mathrm{~s}$ at a temperature of $25^{\circ} \mathrm{C}$ and $230 \pm 2.45 \mu \mathrm{m}$ within $3.93 \mathrm{~s}, 326 \pm 4.88 \mu \mathrm{m}$ within $1 \mathrm{~s}$, $452 \pm 2.86 \mu \mathrm{m}$ within $3.49 \mathrm{~s}$ at temperature of $50^{\circ} \mathrm{C}, 75^{\circ} \mathrm{C}$, and $100{ }^{\circ} \mathrm{C}$, respectively. The deviation values are shown in Table 1 and each came from three individual measurements.

Table 1. Deviation distance of droplet along the gradient's orientation.

\begin{tabular}{ccccc}
\hline Temperature $\left({ }^{\circ} \mathrm{C}\right)$ & 25 & 50 & 75 & 100 \\
\hline Deviation $(\mu \mathrm{m})$ & $188 \pm 5$ & $230 \pm 2.45$ & $326 \pm 4.88$ & $452 \pm 2.86$ \\
\hline
\end{tabular}

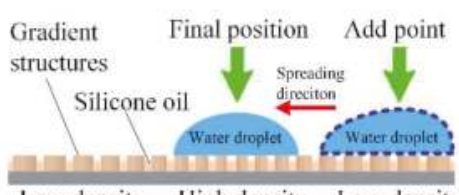

Low density High density Low density

(a)

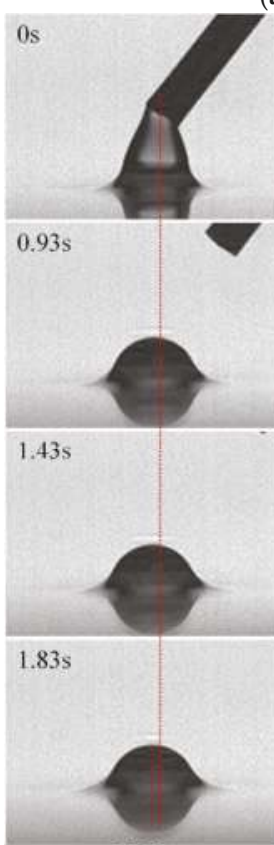

$25^{\circ} \mathrm{C}$ a)

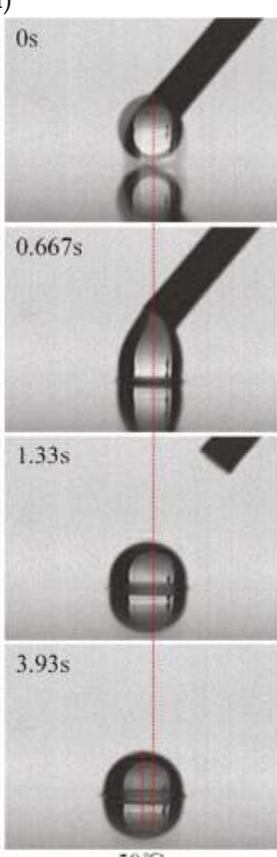

$50^{\circ} \mathrm{C}$

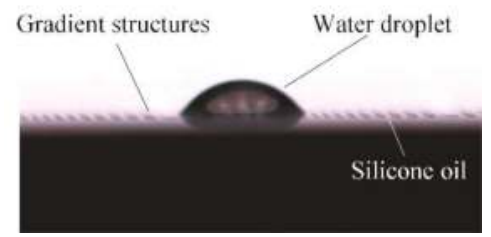

(b)

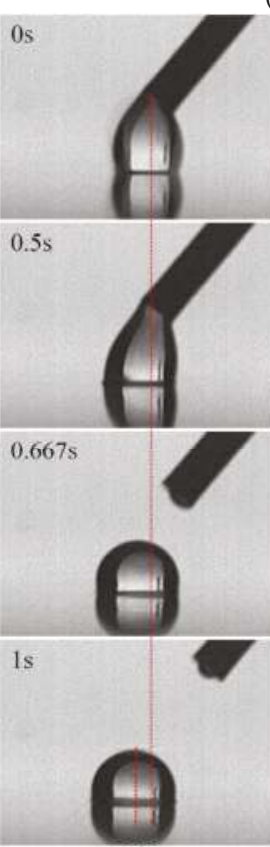

$75^{\circ} \mathrm{C}$

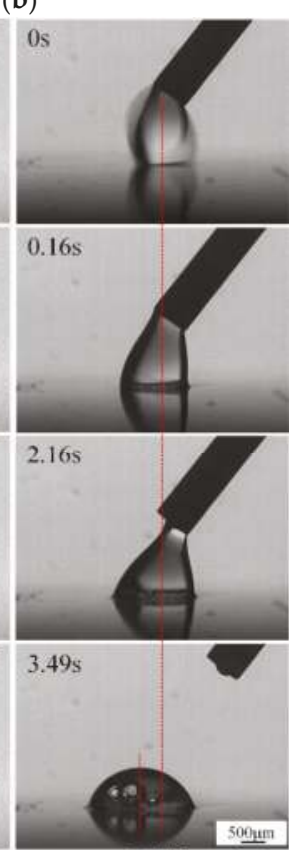

$100^{\circ} \mathrm{C}$

(c)

Figure 7. Motion behavior of a droplet on the slippery wettability gradient's surface: (a) Diagram of initial and steady position of the added liquid; (b) optical photograph of liquid morphology on the slippery wettability gradient's surface; (c) water spreading tests at different temperatures. 
Experiments of tissue cutting cycle tests were carried out to evaluate the anti-sticking effects of the as-prepared surface. In order to facilitate the operating process and precisely test the adhesion force and adhesion mass, fresh resected pig liver was cut into blocks with size of $3 \mathrm{~cm} \times 3 \mathrm{~cm}$, approximately $1 \mathrm{~cm}$ thickness and fixed on a digital force gauge (HANDPI-50, AIDEBAO, China) which was mounted on a mobile base controlled by a micromanipulator (MX7600R, SISKIYOU, Grants Pass, OR, USA). The test samples were fixed on a temperature-controlled hotplate. Before the tissue cutting test, the test sample was heated to 300 degrees [7] and held for five minutes to equalize the temperature. The weight of the test samples was measured with a high resolution electric scale before and after the tests to evaluate the adhesion mass. For the first test, the soft tissue was loaded on the test surface with $5 \mathrm{~N}$ and then unloaded at a speed of $500 \mu \mathrm{m} / \mathrm{s}$. The unloading pressures were recorded and seen as the adhesion forces. Each adhesion force and adhesion mass came from three individual measurements. Figure 8a shows the tissue sticking for the first time, during the 10th and 20th cycle test on the control smooth surface, respectively. Figure $8 \mathrm{~b}$ shows the tissue sticking for the first time, during the 10th and 20th cycle test on the dry gradient's surface, respectively. Due to direct contact with the dry substrate, soft tissue can easily stick for the first time and the sticking area will enlarge rapidly as experiments go on. As seen in Figure 8a,b, at the 20th cycle test, both kinds of surfaces were seriously stuck and greatly affected cutting functions. Tissue sticking on self-lubricating slippery wettability gradient's surface is shown in Figure 8c. Ascribed to the anti-sticking capability of the substrate, soft tissue rarely adhered to the surface for the first test and slightly stuck in the 10th cycle test. The lubricant stuck on the surface affected due to the anti-sticking durability of the surface. Microchannels on the self-lubricating slippery wettability gradient's surface can maintain the plenitudinous supply of lubricant and ensure better durability. Compared to the dry surface, the soft tissue sticking on the self-lubricating wettability surface was alleviated in the 20th cycle test, in accordance with Figure 8c.
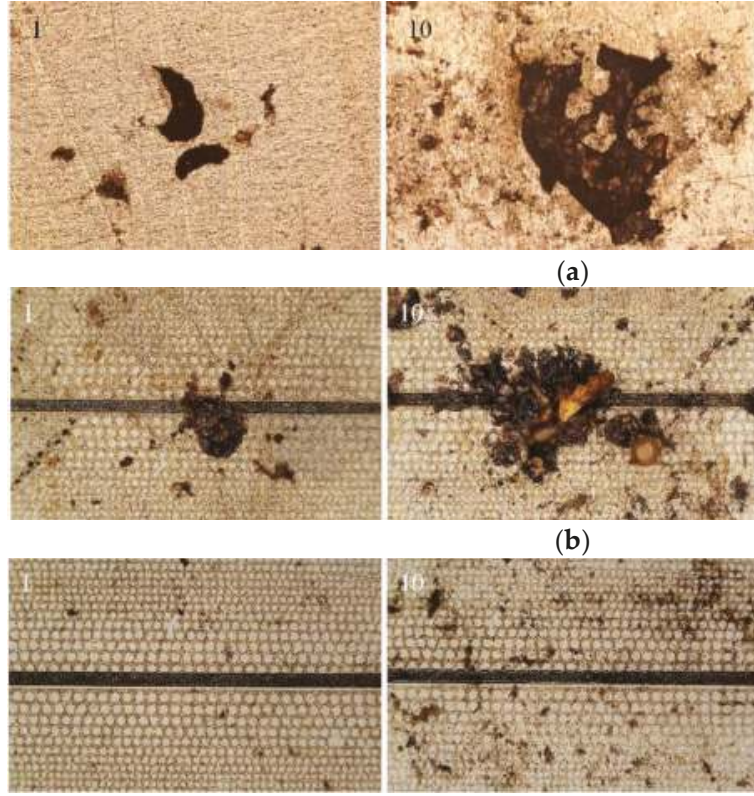

(c)

(a)

(b)
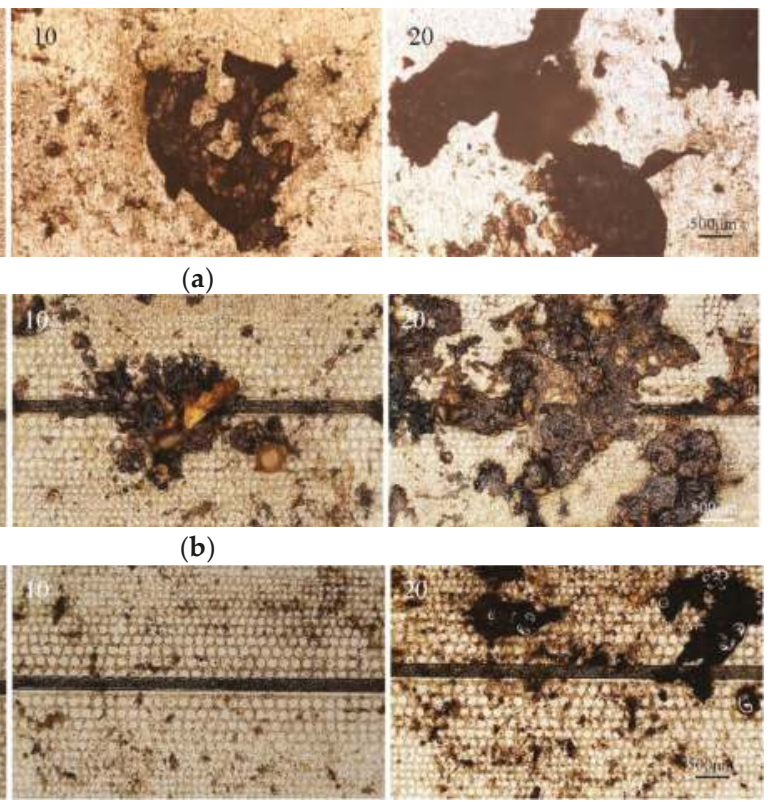

Figure 8. Soft tissue cutting cycle tests: (a) Tissue sticking on smooth surface at the first, 10th, 20th cycle tests; (b) tissue sticking on dry gradient surface at the first, 10th, and 20th cycle tests; (c) tissue sticking on self-lubricating slippery wettability gradient's surface at the first, 10th, and 20th cycle tests. 
The adhesion force and the adhesion mass were measured in the cycle tests. Based on Figure 9a, the adhesion force of the self-lubricating slippery wettability gradient's surface is about $0.51 \pm 0.07 \mathrm{~N}$ and significantly reduced by approximately $90 \%$ compared with the smooth and dry gradient surface. As the cycles increases, adhesion force on the smooth and dry gradient surfaces drastically increase and the smooth surface shows a larger adhesion force than the dry gradient surface, while the self-lubricating surface possessed relatively low adhesion force for the stable lubricant supply capability. Figure $9 \mathrm{~b}$ shows the adhesion mass of the cycle tests. The self-lubricating surface exhibited minute adhesion mass in contrast to smooth and dry gradient surfaces for the first cycle test, due to the excellent anti-sticking properties. The adhesion mass on the self-lubricating surface at 20th cycle was $9.9 \pm 0.26 \mathrm{mg}$, which was far less than that $40.7 \pm 1.26 \mathrm{mg}$ on the smooth surface and $29.6 \pm 0.85 \mathrm{mg}$ on the dry gradient's surface, which suggests a long durability for soft tissue sticking.

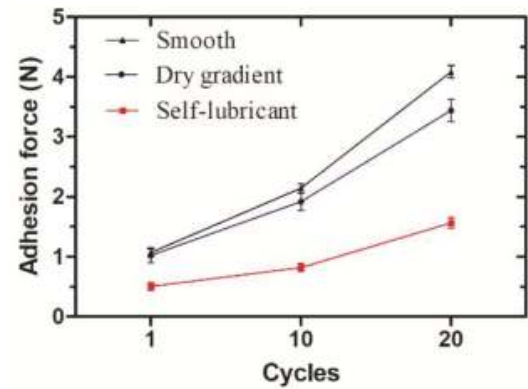

(a)

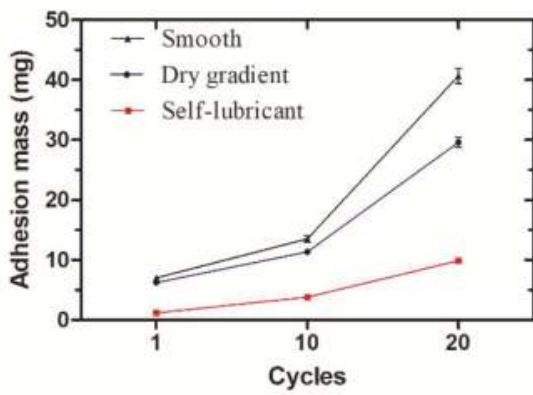

(b)

Figure 9. Contrast of anti-sticking stability between two kinds of surfaces: (a) Graph of adhesion force variance on the smooth, dry gradient and self-lubricating surface in cycle tests; (b) graph of adhesion mass accumulation of the smooth, dry gradient and self-lubricating surface in cycle tests.

\section{Conclusions}

In summary, we designed and fabricated surfaces with wettability gradients on the substrate of electrosurgical scalpels with a photolithography-assisted electrolytic etching process, which consisted of non-uniform cylindrical micro pillars distributed on both sides of a symmetric line. An OTS self-assembling thin layer was successfully grafted on the substrate and played an important role in silicone oil spreading on the functionalized surface. Experiments were conducted to explore the motion behavior of the wettability gradient's surface and we found that the liquid stretched from the region with low pillar density to the region with high pillar density; indicating that the self-lubricating surface was successfully prepared. The underlying mechanisms of water self-propulsion were further analyzed. Experiments of fresh resected pig liver cutting were carried out to test the anti-sticking ability of the as-prepared surface. Results revealed that the scalpel with a self-lubricating surface expressed a marvelous anti-sticking performance, the adhesion force was significantly reduced and the durability prolonged. We believe that this approach will provide new insight into constructing anti-sticking surfaces including industry, microfluidic system, biochips or other practical applications.

Author Contributions: Conceptualization, H.C., G.L., Y.L. and D.Z.; methodology, H.C. and G.L.; validation, H.C., G.L. and D.Z.; formal analysis, G.L.; investigation, H.C. and G.L.; resources, H.C. and D.Z.; data curation, G.L.; writing — original draft preparation, G.L.; writing—review and editing, H.C., G.L. and P.Z.; visualization, G.L.; supervision, H.C.; project administration, H.C.; funding acquisition, H.C.

Funding: This research was funded by National Science Fund for Distinguished Young Scholars. Grant No. 51725501.

Conflicts of Interest: The authors declare no conflicts of interest. 


\section{References}

1. Massarweh, N.N.; Cosgriff, N.; Slakey, D.P. Electrosurgery: History, principles, and current and future uses. J. Am. Coll. Surg. 2006, 202, 520-530. [CrossRef] [PubMed]

2. Zhang, P.; Liu, G.; Zhang, D.; Chen, H. Liquid-infused surfaces on electrosurgical instruments with exceptional antiadhesion and low-damage performances. ACS Appl. Mater. Interfaces 2018, 10, 33713-33720. [CrossRef] [PubMed]

3. Scott, J.E.; Swanson, E.A.; Cooley, J.; Wills, R.W.; Pearce, E.C. Healing of canine skin incisions made with monopolar electrosurgery versus scalpel blade. Vet. Surg. 2017, 46, 520-529. [CrossRef] [PubMed]

4. Choi, M.Y.; Koo, I.G.; Kim, P.Y.; Kang, S.K.; Kim, Y.-S.; Jung, J.-C.; Collins, G.J. Helium $/ \mathrm{H}_{2} \mathrm{O}_{2}$ atmospheric pressure plasma-assisted electrosurgery. Plasma Process. Polym. 2012, 9, 1015-1019. [CrossRef]

5. Kang, S.K.; Kim, P.Y.; Koo, I.G.; Kim, H.Y.; Jung, J.-C.; Choi, M.Y.; Lee, J.K.; Collins, G.J. Non-stick polymer coatings for energy-based surgical devices employed in vessel sealing. Plasma Process. Polym. 2012, 9, 446-452. [CrossRef]

6. Han, Z.; Fu, J.; Feng, X.; Niu, S.; Zhang, J.; Ren, L. Bionic anti-adhesive electrode coupled with maize leaf microstructures and $\mathrm{TiO}_{2}$ coating. RSC Adv. 2017, 7, 45287-45293. [CrossRef]

7. Zhang, P.; Chen, H.; Zhang, L.; Zhang, D. Anti-adhesion effects of liquid-infused textured surfaces on high-temperature stainless steel for soft tissue. Appl. Surf. Sci. 2016, 385, 249-256. [CrossRef]

8. Sutton, P.A.; Awad, S.; Perkins, A.C.; Lobo, D.N. Comparison of lateral thermal spread using monopolar and bipolar diathermy, the harmonic scalpel and the ligasure. Br. J. Surg. 2010, 97, 428-433. [CrossRef] [PubMed]

9. Sotiri, I.; Overton, J.C.; Waterhouse, A.; Howell, C. Immobilized liquid layers: A new approach to anti-adhesion surfaces for medical applications. Exp. Biol. Med. 2016, 241, 909-918. [CrossRef] [PubMed]

10. Han Yi Cheng, K.L.O. The application of advanced nanostructured film in electrosurgical device: Anti-sticking behavior and thermal injury. J. Nanomed. Nanotechnol. 2015, 6. [CrossRef]

11. Hsiao, W.T.; Lin, L.H.; Chiang, H.J.; Ou, K.L.; Cheng, H.Y. Biomedical electrosurgery devices containing nanostructure for minimally invasive surgery: Reduction of thermal injury and acceleration of wound healing for liver cancer. J. Mater. Sci. Mater. Med. 2015, 26, 77. [CrossRef] [PubMed]

12. Lin, L.-H.; Hsu, Y.-J.; Chiang, H.-J.; Cheng, H.-Y.; Wang, C.-S.; Ou, K.-L. The application of minimally invasive devices with nanostructured surface functionalization: Antisticking behavior on devices and liver tissue interface in rat. J. Nanomater. 2015, 2015, 1-9. [CrossRef]

13. Shen, Y.D.; Lin, L.H.; Chiang, H.J.; Ou, K.L.; Cheng, H.Y. Research of electrosurgical unit with novel antiadhesion composite thin film for tumor ablation: Microstructural characteristics, thermal conduction properties, and biological behaviors. J. Biomed. Mater. Res. B Appl. Biomater. 2016, 104, 96-105. [CrossRef] [PubMed]

14. Ou, K.L.; Chu, J.S.; Hosseinkhani, H.; Chiou, J.F.; Yu, C.H. Biomedical nanostructured coating for minimally invasive surgery devices applications: Characterization, cell cytotoxicity evaluation and an animal study in rat. Surg. Endosc. 2014, 28, 2174-2188. [CrossRef] [PubMed]

15. Wooh, S.; Butt, H.J. A photocatalytically active lubricant-impregnated surface. Angew. Chem. Int. Ed. Engl. 2017, 56, 4965-4969. [CrossRef] [PubMed]

16. Han, Z.; Fu, J.; Fang, Y.; Zhang, J.; Niu, S.; Ren, L. Anti-adhesive property of maize leaf surface related with temperature and humidity. J. Bionic. Eng. 2017, 14, 540-548. [CrossRef]

17. Phan, N.; Moronuki, N. Fabrication of high aspect ratio silicon micro-/nano-pore arrays and surface modification aiming at long lifetime liquid-infused-type self-cleaning function. J. Adv. Mech. Des. Syst. Manuf. 2017, 11, JAMDSM0013. [CrossRef]

18. Lee, J.H.; Go, A.K.; Oh, S.H.; Lee, K.E.; Yuk, S.H. Tissue anti-adhesion potential of ibuprofen-loaded PLLA-PEG diblock copolymer films. Biomaterials 2005, 26, 671-678. [CrossRef] [PubMed]

19. Lee, M.W.; Hung, C.L.; Cheng, J.C.; Wang, Y.J. A new anti-adhesion film synthesized from polygalacturonic acid with 1-ethyl-3-(3-dimethylaminopropyl)carbodiimide crosslinker. Biomaterials 2005, 26, 3793-3799. [CrossRef] [PubMed]

20. Yang, D.J.; Chen, F.; Xiong, Z.C.; Xiong, C.D.; Wang, Y.Z. Tissue anti-adhesion potential of biodegradable PELA electrospun membranes. Acta Biomater. 2009, 5, 2467-2474. [CrossRef] [PubMed] 
21. Lin, C.C.; Lin, H.J.; Lin, Y.H.; Sugiatno, E.; Ruslin, M.; Su, C.Y.; Ou, K.L.; Cheng, H.Y. Micro/nanostructured surface modification using femtosecond laser pulses on minimally invasive electrosurgical devices. J. Biomed. Mater. Res. B Appl. Biomater. 2017, 105, 865-873. [CrossRef] [PubMed]

22. Hosseini, A.; Villegas, M.; Yang, J.; Badv, M.; Weitz, J.I.; Soleymani, L.; Didar, T.F. Conductive electrochemically active lubricant-infused nanostructured surfaces attenuate coagulation and enable friction-less droplet manipulation. Adv. Mater. Interfaces 2018, 5, 1800617. [CrossRef]

23. Badv, M.; Jaffer, I.H.; Weitz, J.I.; Didar, T.F. An omniphobic lubricant-infused coating produced by chemical vapor deposition of hydrophobic organosilanes attenuates clotting on catheter surfaces. Sci. Rep. 2017, 7, 11639. [CrossRef] [PubMed]

24. Badv, M.; Imani, S.M.; Weitz, J.I.; Didar, T.F. Lubricant-infused surfaces with built-in functional biomolecules exhibit simultaneous repellency and tunable cell adhesion. ACS Nano 2018. [CrossRef] [PubMed]

25. Villegas, M.; Cetinic, Z.; Shakeri, A.; Didar, T.F. Fabricating smooth pdms microfluidic channels from low-resolution 3D printed molds using an omniphobic lubricant-infused coating. Anal. Chim. Acta 2018, 1000, 248-255. [CrossRef] [PubMed]

26. Zhao, Y.; Wang, H.; Zhou, H.; Lin, T. Directional fluid transport in thin porous materials and its functional applications. Small 2017, 13. [CrossRef]

27. Yong, J.; Chen, F.; Yang, Q.; Huo, J.; Hou, X. Superoleophobic surfaces. Chem. Soc. Rev. 2017, 46, 4168-4217. [CrossRef] [PubMed]

28. Xu, Q.; Zhang, W.; Dong, C.; Sreeprasad, T.S.; Xia, Z. Biomimetic self-cleaning surfaces: Synthesis, mechanism and applications. J. R. Soc. Interface 2016, 13. [CrossRef] [PubMed]

29. Zheng, Y.; Bai, H.; Huang, Z.; Tian, X.; Nie, F.Q.; Zhao, Y.; Zhai, J.; Jiang, L. Directional water collection on wetted spider silk. Nature 2010, 463, 640-643. [CrossRef] [PubMed]

30. Ju, J.; Bai, H.; Zheng, Y.; Zhao, T.; Fang, R.; Jiang, L. A multi-structural and multi-functional integrated fog collection system in cactus. Nat. Commun. 2012, 3, 1247. [CrossRef] [PubMed]

31. Muschi, M.; Brudieu, B.; Teisseire, J.; Sauret, A. Drop impact dynamics on slippery liquid-infused porous surfaces: Influence of oil thickness. Soft Matter 2018, 14, 1100-1107. [CrossRef] [PubMed]

32. Togasawa, R.; Tenjimbayashi, M.; Matsubayashi, T.; Moriya, T.; Manabe, K.; Shiratori, S. A fluorine-free slippery surface with hot water repellency and improved stability against boiling. ACS Appl. Mater. Interfaces 2018, 10, 4198-4205. [CrossRef] [PubMed]

33. Wong, T.S.; Kang, S.H.; Tang, S.K.; Smythe, E.J.; Hatton, B.D.; Grinthal, A.; Aizenberg, J. Bioinspired self-repairing slippery surfaces with pressure-stable omniphobicity. Nature 2011, 477, 443-447. [CrossRef] [PubMed]

34. Chen, H.; Zhang, P.; Zhang, L.; Liu, H.; Jiang, Y.; Zhang, D.; Han, Z.; Jiang, L. Continuous directional water transport on the peristome surface of Nepenthes alata. Nature 2016, 532, 85-89. [CrossRef]

35. Zhang, P.; Zhang, L.; Chen, H.; Dong, Z.; Zhang, D. Surfaces inspired by the nepenthes peristome for unidirectional liquid transport. Adv. Mater. 2017, 29. [CrossRef] [PubMed]

36. Juuti, P.; Haapanen, J.; Stenroos, C.; Niemelä-Anttonen, H.; Harra, J.; Koivuluoto, H.; Teisala, H.; Lahti, J.; Tuominen, M.; Kuusipalo, J.; et al. Achieving a slippery, liquid-infused porous surface with anti-icing properties by direct deposition of flame synthesized aerosol nanoparticles on a thermally fragile substrate. Appl. Phys. Lett. 2017, 110, 161603. [CrossRef]

37. Zhang, P.; Chen, H.; Zhang, L.; Zhang, Y.; Zhang, D.; Jiang, L. Stable slippery liquid-infused anti-wetting surface at high temperatures. J. Mater. Chem. A 2016, 4, 12212-12220. [CrossRef]

38. Heib, F.; Hempelmann, R.; Munief, W.M.; Ingebrandt, S.; Fug, F.; Possart, W.; Groß, K.; Schmitt, M. High-precision drop shape analysis (HPDSA) of quasistatic contact angles on silanized silicon wafers with different surface topographies during inclining-plate measurements: Influence of the surface roughness on the contact line dynamics. Appl. Surf. Sci. 2015, 342, 11-25. [CrossRef]

39. Liu, C.; Sun, J.; Li, J.; Xiang, C.; Che, L.; Wang, Z.; Zhou, X. Long-range spontaneous droplet self-propulsion on wettability gradient surfaces. Sci. Rep. 2017, 7, 7552. [CrossRef] [PubMed]

40. Zhou, K.; Zhu, X.G.; Li, Y.; Liu, J. Fabrication of PDMS micro through-holes using micromolding in open capillaries. RSC Adv. 2014, 4, 31988-31993. [CrossRef]

41. Schulze, R.D.; Possart, W.; Kamusewitz, H.; Bischof, C. Young's equilibrium contact angle on rough solid surfaces. Part I. An empirical determination. J. Adhes. Sci. Technol. 1989, 3, 39-48. [CrossRef] 
42. Koch, B.M.; Elliott, J.A.; Amirfazli, A. Study of model superoleophobic surfaces fabricated with a modified bosch etch method. Langmuir 2014, 30, 14039-14047. [CrossRef] [PubMed]

43. WENZEL, R.N. Resistance of solid surfaces to wetting by water. Ind. Eng. Chem. 1936, 28, 988-994. [CrossRef]

44. Wu, J.; Ma, R.; Wang, Z.; Yao, S. Do droplets always move following the wettability gradient? Appl. Phys. Lett. 2011, 98, 204104. [CrossRef]

45. Chaudhury, M.W.; Whitesides, G.M. How to make water uphill. Science 1992, 256, 1539-1541. [CrossRef] [PubMed]

(C) 2018 by the authors. Licensee MDPI, Basel, Switzerland. This article is an open access article distributed under the terms and conditions of the Creative Commons Attribution (CC BY) license (http:/ / creativecommons.org/licenses/by/4.0/). 
Article

\title{
Trapping a Hot Drop on a Superhydrophobic Surface with Rapid Condensation or Microtexture Melting
}

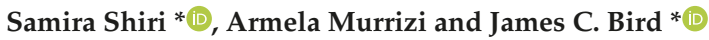 \\ Department of Mechanical Engineering, Boston University, Boston, MA 02215, USA; amurrizi@bu.edu \\ * Correspondence: sshiri@bu.edu (S.S.); jbird@bu.edu (J.C.B.); Tel.: +1-617-358-6929 (J.C.B.)
}

Received: 9 October 2018; Accepted: 30 October 2018; Published: 2 November 2018

\begin{abstract}
A water drop can bounce upon impacting a superhydrophobic surface. However, on certain superhydrophobic surfaces, a water drop will stick rather than bounce if it is sufficiently hot. Here, we aim to better understand the mechanisms that can lead to this bouncing-sticking transition. Specifically, we model two potential mechanisms in which a superhydrophobic surface could trap a sufficiently hot drop within milliseconds: melting of microtextured wax and condensation of the vapor within the superhydrophobic texture. We then test these mechanisms through systematic drop impact experiments in which we independently vary the substrate and drop temperatures on a waxy superhydrophobic Nasturtium leaf. We find that, whenever the surface or the drop is above a microtexture-melting temperature, the drop sticks. Below this temperature, a critical temperature threshold for bouncing can be predicted and controlled by considering the relative timescales between condensation growth and drop residence time. We envision that these results can provide insight into the design of a new class of superhydrophobic surfaces to act as a rapid thermal fuse to prevent drops that exceed a critical temperature from bouncing onto a thermally sensitive target.
\end{abstract}

Keywords: Nasturtium leaf; smart superhydrophobic surface; hot drop; condensation; microtexture melting

\section{Introduction}

For most surfaces, an impacting water drop will stick upon contact; however, for a superhydrophobic surface, an impacting water drop can spread out and recoil to such an extreme that it can completely bounce off of the surface [1-3]. The degree of superhydrophobicity can be tuned by modulating the chemistry and structure of the surface, thus enabling external control of whether a particular drop bounces or sticks [4-6]. For example, by modulating the superhydrophobic properties of the surface, a microdevice could be designed to prevent drops above a critical temperature from reaching a thermally sensitive region (Figure 1). A challenge with using external stimulants to modulate such a device is that the control would likely involve separate sensing, processing, and actuating steps. However, if a superhydrophobic surface can be designed to passively adjust its functionality in response to a drop property, then it can be used as a sensor or fuse. Here, we explore how one might design a smart superhydrophobic surface in which the surface can sense the drop temperature and act within milliseconds to selectively trap a drop that exceeds a critical threshold. In particular, we identify thermally-induced sticking mechanisms and predict the conditions that would be needed for them to act faster than the time it takes for the drop to bounce off the surface. A superhydrophobic surface combines chemical hydrophobicity with microscopic texture so that, in the Cassie-Baxter state, a water drop resides on top of the air-filled microtexture (Figure 1B) [7]. The air under the drop leads to a large effective contact angle and low contact friction, a combination that can enable the drop to bounce. By contrast, if the drop enters a Wenzel state, the water drop permeates the microtexture [8]. This attachment of water with surface roughness 
dampens the recoil following impact so that the drop sticks to the surface. Whether a drop adopts the Cassie-Baxter state or the Wenzel state strongly depends on the microtexture geometry and surface chemistry, as well as the surface tension of the liquid [9]. Indeed, attempts to create surfaces that can repel scalding water have been frustrated in part by drop sticking, a result that has been largely attributed to the lower surface tension associated with the hotter water [10].

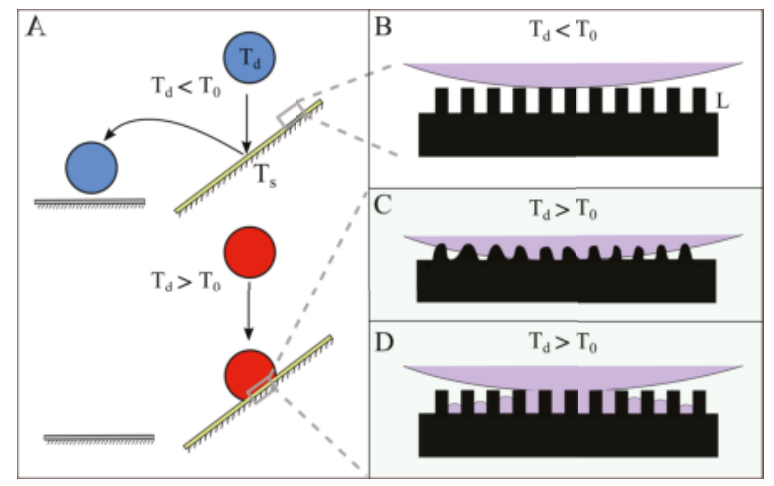

Figure 1. A schematic illustrating how a thermally responsive superhydrophobic material could act as a fuse to prevent water above a critical temperature from reaching a sensitive surface. (A) If the superhydrophobic surface that is at $T_{S}$ rapidly pinned drops above a critical temperature $T_{0}$, then a water drop with temperature $T_{d}$ below $T_{0}$ (top) would bounce off of the surface onto the target, whereas a water drop with temperature $T_{d}$ above $T_{0}$ (bottom) would stick, protecting the target; (B) surface microtexture with lengthscale $L$ can trap air beneath the drop (Cassie-Baxter state), enabling the drop to bounce; $(C)$ If heat from the drop melts the microstructure, the drop could enter a Wenzel state and stick; (D) Alternatively, if liquid from the drop evaporates and condenses within the microstructure, the drop could also enter a Wenzel state and stick.

There is evidence that surface tension is not the only mechanism by which a sufficiently hot drop could initiate a Cassie-Baxter to Wenzel state transition. Liu et al. [10] found that drops would stick to lotus leaves when the drop temperature exceeded $55^{\circ} \mathrm{C}$ and speculated that the waxy microtexture could be melting at these temperatures [10] (Figure 1C). Alternatively, if the surface is below the dew point, water vapor from the air can condense within the microtexture and drive the drop into a Wenzel state [11,12]. Even if the surface is above the dewpoint of the ambient air, the slight evaporation of the water drop residing on the microtexture can locally saturate the air, which can then condense on a slightly cooler microtexture (Figure 1D). In fact, experiments of static drops resting on superhydrophobic surfaces have shown that, when the surface temperature is lower than the drop temperature, then this evaporation-condensation process can transition the drop into a Wenzel state [13].

Past studies that have identified microtexture melting or evaporatation-condensation as processes that could initiate a Cassie-Baxter to Wenzel state transition have generally considered applications in which this transition is undesirable. In contrast, we focus on exploiting the transition, appreciating that these mechanisms must sufficiently modify the surface in a short enough time to trap the drop before it bounces. The time that a drop is in contact with a superhydrophobic surface is predominantly dictated by the inertia and surface tension of the drop with some additional tuning possible by exploiting the texture or geometry of the surface [14-19]. For a millimeter-sized water drop, this contact time is on the order of $10 \mathrm{~ms}$, which can be short enough to limit certain transport properties such as conductive heat exchange. Indeed, for these sized drops, it is expected that, if a drop were to bounce rather than stick, only around $1 \%$ of the heat would be transferred [20]. However, it is unclear under what conditions this heat or any evaporation and condensation would modify the surface significantly enough to trap the drop. To address this question, we combine experiments and modeling. We carry out drop impact 
experiments on Nasturtium leaves at different temperatures and compare the results to models in which we estimate the time it would take to change the surface conditions relative to the time the drop takes to bounce.

\section{Materials and Methods}

To explore the physics underlying the bouncing-sticking transition of a drop to a superhydrophobic surface, we carry out a series of experiments in which both drop and surface temperature are varied systematically. For each experiment, a single drop of water is released from a suspended needle, falls, and impacts a superhydrophobic Nasturtium leaf. The drop temperature $T_{d}$ is controlled with a water bath, and temperature of the leaf surface $T_{s}$ is controlled using a hot plate. To ensure the impact dynamics between experiments are similar, the height and gauge of the suspended needle are fixed to maintain a radius of $R \approx 1 \mathrm{~mm}$ and impact velocity of $V \approx 0.75 \mathrm{~ms}^{-1}$. The subsequent impact dynamics are recorded simultaneously with high speed and thermal cameras. High speed images are captured with a Photron Fastcame SA-X2 (made in Japan) at a frame rate of 8000 frames per second and a $200 \mathrm{~mm}$ Nikon lens (made in Japan). Thermographic images are simultaneously recorded from an inclined perspective using an FLIR A655sc thermal camera (made in Sweden) at frame rate of 200 frames per second with a close-up infrared (IR) camera lens (Figure 2A). These thermographic images are used to calculate $T_{d}$ and $T_{s}$.

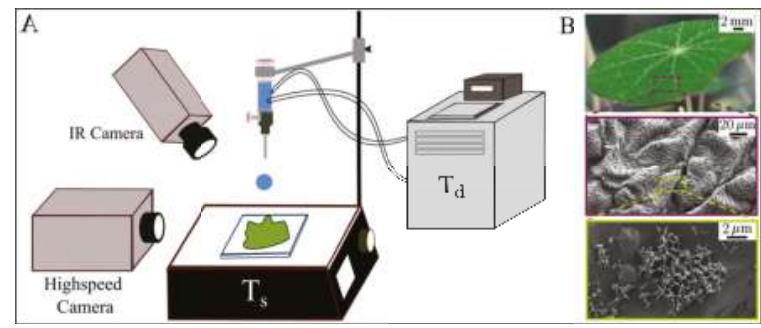

Figure 2. (A) a schematic of the experimental set. Cameras film a water drop at temperature $T_{d}$ as it falls from a suspended needle and impacts a Nasturtium leaf heated to temperature $T_{s} ;(\mathbf{B})$ images of a Nasturtium leaf taken at different magnifications illustrate the hierarchical microstructure.

Nasturtium leaves are ideal for these experiments for three reasons. First, these leaves are naturally superhydrophobic due to a waxy microstructure that can melt at temperatures below the boiling-point of water. Second, we find that condensation can form within the microstructure when the surface is cooled below the dew point. Finally, these plants are relatively simple to grow within in the lab, providing a reliable source of waxy superhydrophobic material. Prior to an experiment, a leaf is cut from the Nasturtium plant, secured to a glass slide, and placed on the hot plate. Images of one of our leaves reveal the shape and hierarchical surface structure responsible for the Nasturtium superhydrophobicity (Figure 2B).

\section{Results}

The interaction dynamics between a drop and Nasturtium leaf during an impact is demonstrated experimentally in Figure 3. In the first set of images (Figure 3A), a chilled water drop with radius $R=1 \mathrm{~mm}$ and temperature $T_{d}=298 \mathrm{~K}\left(25^{\circ} \mathrm{C}\right)$ falls and impacts a surface that is at room-temperature and measured to be $T_{d}=301 \mathrm{~K}$. The impact dynamics are captured simultaneously with both a high-speed from the side and thermal camera from an inclined perspective. The high speed images shows that, when the chilled drop contacts the superhydrophobic surface, it bounces. The time that the drop is in contact with surface, defined as the contact or residence time $t_{r}$, is approximately $10 \mathrm{~ms}$. Thermal images of this bounce highlight that the drop is at a lower temperature than the leaf. In the second set of images (Figure $3 \mathrm{~B}$ ), a hot water drop with temperature $T_{d}=323 \mathrm{~K}$ falls onto 
a Nasturtium leaf under nearly identical impact conditions. High speed images reveal that the hot drop is trapped by the leaf and sticks to it. Thermal images of the impact confirm that the drop is hotter than the ambient-temperature leaf.

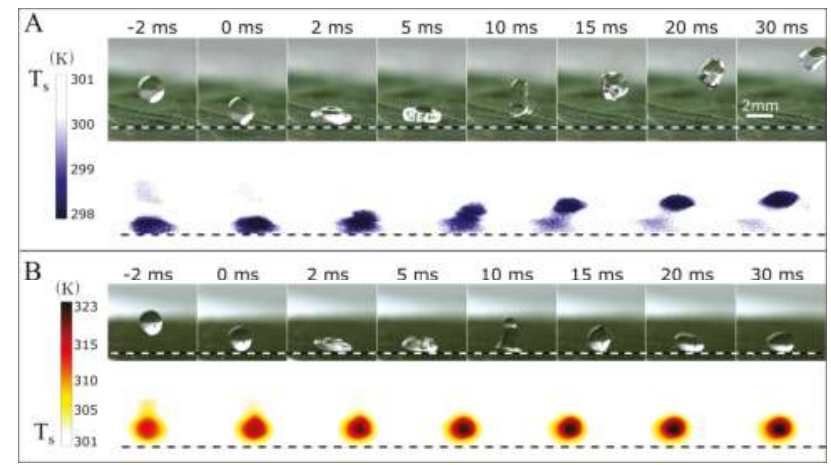

Figure 3. A superhydrophobic Nasturtium leaf that lets a cold drop bounce off of its surface traps a hot drop. (A) A cold water drop at temperatures $298 \mathrm{~K}$ impacts a Nasturtium leaf that is initially at ambient temperature $301 \mathrm{~K}$. High-speed images show that the cold water drop spreads, retracts and leaves the surface at a finite time $10 \mathrm{~ms}$. Simultaneously, thermal images show that the drop is at the lower temperature than the leaf in this experiment; (B) a hot drop at temperature $323 \mathrm{~K}$ at the same impact condition sticks to a Nasturtium leaf that is initially at ambient temperature $301 \mathrm{~K}$. Simultaneous thermographic images show a temperature map of the drop and substrate during impact. The dotted line shows the contact line between drop and leaf.

To evaluate the role of temperature in the trapping process, we extend the experiments highlighted in Figure 3 to a variety of drop temperatures that range from $T_{d}=295 \mathrm{~K}$ to $332 \mathrm{~K}$. Water drops that are colder than a threshold temperature of approximately $307 \mathrm{~K}$ bounce off of the surface (Figure 4, open circles), whereas water drops hotter than that threshold temperature stick to the surface (Figure 4, filled circles). The surface tension of many liquids, including water, decreases with increasing temperature [21]. This relationship can be approximated as $\gamma\left(T_{d}\right)=75.7-0.14 T_{d}$, where $\gamma\left(T_{d}\right)$ is the surface tension in units of $\mathrm{mN} / \mathrm{m}$ for a water drop with temperature $T_{d}$ specified in ${ }^{\circ} \mathrm{C}$ [22]. Thus the surface tension of water drops at the threshold temperature is calculated to be $\gamma \approx 70 \mathrm{mN} / \mathrm{m}$, although it is likely that this surface tension is slightly lower due to natural surfactants that can accumulate on the water interface.

To assess whether this critical temperature can be explained solely as a consequence of a critical surface tension, we compare the threshold surface tension for varying drop temperatures with the threshold surface tension for varying drop compositions (Figure 4). Specifically, we repeat the drop impact experiments at ambient conditions using drops that contain varying concentrations of ethanol and water. Due to the low surface tension of pure ethanol $(\gamma=22 \mathrm{mN} / \mathrm{m})$, adding a small amount of ethanol to water can dramatically lower the surface tension. Given that these experiments were carried out at room temperature, we approximate the drop temperatures as remaining constant at $T=301 \mathrm{~K}$ (Figure 4), and estimate the surface tension based on the concentration of ethanol in the drop following the empirical analysis by Khattab et al. [23]. At low ethanol concentrations, the drop bounces off of the Nasturtium leaf (Figure 4, open stars), whereas, at sufficiently high ethanol concentrations, the drop will stick on the surface (Figure 4, filled stars). The surface tension that corresponds to this transition is $\gamma \approx 43 \mathrm{mN} / \mathrm{m}$, a value that is far less than the surface tension of the water, even at the hottest temperatures. Therefore, it appears that the critical temperature for a water drop cannot be explained solely as a consequence of a critical surface tension, and instead relies on a mechanism in which the temperature modifies the surface during contact. 


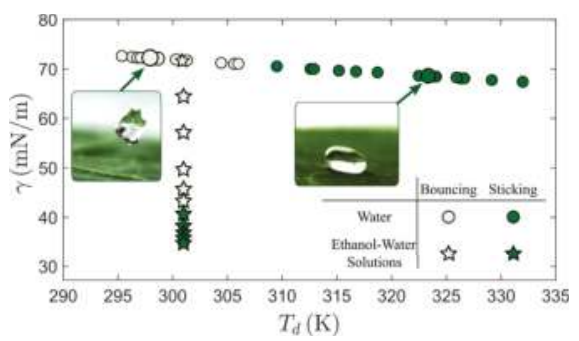

Figure 4. Effect of surface tension $\gamma$ and drop temperature $T_{d}$ on the bouncing-sticking transition for a Nasterium leaf at ambient conditions. For water drops, the transition between bouncing (open circles) and sticking (filled circles) occurs when the drop temperature $T_{d} \approx 307 \mathrm{~K}$, which corresponds to a surface tension $\gamma \approx 70 \mathrm{mN} / \mathrm{m}$. For drops of varying ethanol-water concentrations at ambient conditions, the transition between bouncing (open stars) and sticking (filled stars) occurs when the surface tension is at $\gamma \approx 43 \mathrm{mN} / \mathrm{m}$. This difference in surface tension suggests that surface tension alone cannot account for the transition. Note that the larger circles correspond to the specific drop illustrated in Figure 3.

Given the importance of surface temperature in both the microtexture melting mechanism and the evaporation-condensation sticking mechanism, experiments with the water at varying drop temperatures are repeated at higher surface temperatures by placing the leaf on a hotplate. The drop release height and drop size continue to be fixed to maintain nearly identical impact conditions. Figure 5 illustrates the results for ambient (circles), warm (squares), hot (diamonds), and scalding (triangles) surface temperatures. Here, we categorize the surface temperature as warm when it is between $T_{S}=313 \mathrm{~K}$ and $321 \mathrm{~K}$, hot when it is between $322 \mathrm{~K}$ and $333 \mathrm{~K}$, and scalding when it is above $336 \mathrm{~K}$. When the surface is heated above $T_{S} \approx 335 \mathrm{~K}$, the leaf begins to visibly deform and the drops stick for all drop temperatures $T_{d}$ (gray region). These results suggest that the surface geometry or chemistry could be changing, and we specify this transition as a microtexture melting temperature $T_{m}$. At lower temperatures, the results show that, when a water drop is cooler than the surface (purple area), the drop always bounces off of the surface. However, when the drop is hotter than surface, it may either bounce off (yellow region) or stick to the surface (white region). The drop temperature $T_{d}$ associated with the bouncing-sticking transition (denoted by a dashed guideline in Figure 5) increases with the surface temperature $T_{s}$ before appearing to level-off as the drop temperature approaches $T_{m}$.

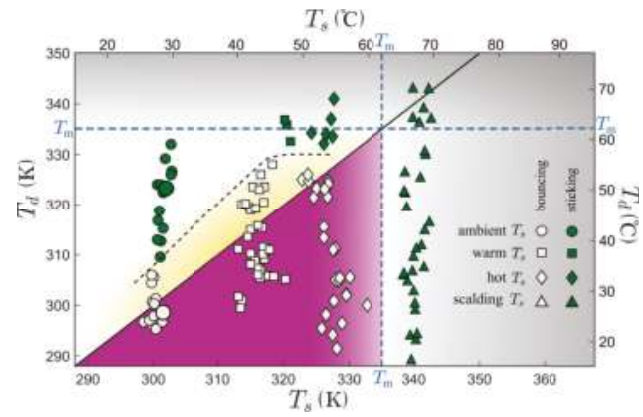

Figure 5. Effect of drop temperature $T_{d}$ and surface temperature $T_{S}$ on the bouncing-sticking transition on the leaf. Surface temperatures are separated into four groups for comparison: ambient (circles), warm (squares), hot (diamonds), and scalding (triangles). The transition between drop bouncing (open symbol) and sticking (closed symbol) is identified by a dashed line as a guide for the eye. The shaded gray region indicates temperatures above a surface melting temperature $T_{m}$. Below these temperatures, the purple region denotes where drops are colder than the surface, and the lighter yellow region highlights where drops bounce despite being warmer than the surface. 


\section{Discussion}

The results demonstrate that a Nasturtium leaf, and presumably any similar superhydrophobic surface, can selectively trap water drops that exceed a critical temperature while permitting colder drops to bounce. Furthermore, the experiments indicate that this phenomenon cannot be accounted for by a reduction surface tension alone. Specifically, the results show that a hot drop would stick on the superhydrophobic surface when, at the same surface tension, an ambient-temperature drop would bounce (Figure 4). Additionally, a heated drop that would stick on an ambient-temperature surface might bounce had the surface been heated (for example $T_{d}=320 \mathrm{~K}$ in Figure 5). Thus, the temperature of the drop and the surface both appear to be important parameters for the bouncing-sticking transition, independent of the surface tension. This result is noteworthy because it suggests that the temperature of the drop can significantly affect the properties of the superhydrophobic surface. Because the drop and surface only interact during the bounce, the mechanism required to stop a bounce must sufficiently modify the surface in the short time before the bounce is complete.

Drawing from past studies, we focus on two alternative mechanisms: melting of surface microtexture and evaporation-condensation within the superhydrophobic texture. The results in Figure 5 might appear inconsistent with both mechanisms. Specifically, if a drop at $T_{d}=310 \mathrm{~K}$ were able to melt the microtexture, then it would seem improbable that drops of any temperature would bounce if the surface were above this temperature $T_{s}=310 \mathrm{~K}$, yet the results illustrate that they do (thus we estimate $T_{m} \approx 335 \mathrm{~K}$ ). Similarly, the existence of the yellow region in Figure 5 is in contrast with previous works for static drops, which predicts sticking for a drop whenever its temperature is larger than that of the surface [13]. However, if the dynamics are considered, it might be possible that, depending on the conditions, there could be regimes in which these effects are present but insufficient to inhibit bouncing.

\subsection{Melting of the Surface Microtexture}

The height of surface microtexture is one of the physical conditions that can noticeably influence transition from the Cassie-Baxter state (necessary for drop bouncing) to the Wenzel state [9]. When the drop temperature is larger than the melting point of microtexture material, melting may shorten and smooth the microtexture surface and thus create conditions that promote the Wenzel state (Figure 1C). To model this melting process, we approximate the superhydrophobic surface as a semi-infinite body that undergoes phase change. We assume a uniform solid surface temperature $T_{S}$ that is cooler the material melting point $T_{m}$. Subsequently, at $t=0$, the drop contacts the superhydrophobic surface and resides on the surface over the period of the residence time $t_{r}$. If the drop temperature is cooler the substrate melting point $\left(T_{d}<T_{m}\right)$, then the substrate remains intact and the drop bounces. However, if the drop temperature exceeds the substrate melting point $\left(T_{d}>T_{m}\right)$, the energy transfer can induce a phase change. The amount of material that can melt will grow with the amount of time that that heat can transfer from the hot drop. The extent of this melting can be estimated by the position of the self-similar melting front $x_{m}$, which we refer to as the melted length. Thus, this moving boundary problem can be solved by using Neumann's solution for the melting of a semi-infinite body [24].

Based on Neumann's problem, when a solid-liquid interface forms as a result of melting, two regions can be defined that obey the following governing equations: a liquid region $\left(0<x<x_{m}\right)$ where $\frac{\partial^{2} T_{\ell}}{\partial x^{2}}=\frac{1}{\alpha_{\ell}} \frac{\partial T_{\ell}}{\partial t}$ and a solid region $\left(x_{m}<x\right)$ where $\frac{\partial^{2} T_{\mathscr{S}}}{\partial x^{2}}=\frac{1}{\alpha_{\mathscr{S}}} \frac{\partial T_{\mathscr{S}}}{\partial t}$. Applying the boundary conditions $T_{\ell}(0, t)=T_{d}$ and $T_{\ell}\left(x_{m}, t\right)=T_{m}$ for the liquid, $T_{\mathscr{S}}\left(x_{m}, t\right)=T_{m}$ and $T_{\mathscr{S}}(\infty, t)=T_{S}$ and the solid, and the initial conditions $T_{\mathscr{S}}(x, 0)=T_{S}, x_{m}(t=0)=0$ together with the interface energy equation, leads to:

$$
\frac{\exp \left(-\lambda^{2}\right)}{\operatorname{erf}(\lambda)}-\sqrt{\frac{\alpha_{\ell}}{\alpha_{\mathscr{S}}}} \frac{\kappa_{\mathscr{S}}}{\kappa_{\ell}} \frac{T_{m}-T_{S}}{T_{d}-T_{m}} \frac{\exp \left(-\alpha_{\ell} \lambda^{2} / \alpha_{\mathscr{S}}\right)}{1-\operatorname{erf}\left(\sqrt{\alpha_{\ell} / \alpha_{\mathscr{S}}}\right) \lambda}=\frac{\sqrt{\pi} \mathcal{L} \lambda}{c_{\ell}\left(T_{d}-T_{m}\right)} .
$$


Here, the subscripts $\ell$ and $\mathscr{S}$ denote the properties of the liquid and solid phase, respectively, $\kappa$ is the thermal conductivity, $c$ is the thermal capacity, $\alpha$ is the thermal diffusivity, $\rho$ is the density and $\mathcal{L}$ is the latent heat of fusion. This equation provides a value of $\lambda$ that can subsequently be used to calculate the melted length, noting $x_{m} \sim \lambda \sqrt{\alpha_{\ell} t_{r}}$. Therefore, if melting occurs over the entire time that the drop resides on the surface $\left(t_{r}\right)$, the length of surface microtexture would be $L-x_{m}$, which-depending on conditions-could transition the drop from the Cassie-Baxter to the Wenzel state.

According to the solution of Equation (1), the melting mechanism predicts that a drop would more readily melt the surface if the surface were warmer. Specifically, if melting of the surface microtexture were responsible for the observed bouncing-sticking transition, then the threshold drop temperature would be expected to decrease with increasing surface temperature $T_{s}$. This prediction is in stark contrast to what is observed; the threshold temperature increases with surface temperature, at least up to a point. Thus, it seems unlikely that melting of the microstucure would be responsible for the transition for $T_{d}<T_{m} \approx 335 \mathrm{~K}$ in these experiments (Figure 5).

\subsection{Condensation of the Vapor within the Superhydrophobic Texture}

Condensation is another mechanism that has been attributed to the transition from the Cassie-Baxter to the Wenzel state (Figure 1D). Through this mechanism, liquid evaporates from the drop to saturate the air within the microtexture and then condenses. If air pockets within the microtexture fill with water, then the drop transitions to the Wenzel state. Here, we estimate the timescale associated with this evaporation-condensation process. Specifically, we model the air within the microtexture between the surface and the drop, neglecting any fluxes to regions that are not covered by the drop. Noting that condensation occurs only when the relative humidity is maintained at $100 \%$, we first calculate the timescale for evaporation to fully saturate the air, and then we calculate the timescale for sufficient liquid to condense to fill up the air gap within the microstructure.

To estimate the time needed to saturate the microtexture air, we note that the mass flux across the liquid interface can be modeled as: $J=-D \frac{\partial C}{\partial x} \approx D \frac{C_{s}-C_{\infty}}{L} \approx \frac{D C_{s}}{L}\left(1-R_{H}\right)$. Here, $J$ is the evaporation rate, $D=2 \times 10^{-5} \mathrm{~m}^{2} / \mathrm{s}$ is the diffusion coefficient of water [25], $C_{s}$ is the concentration of the saturated vapor, $C_{\infty}=C_{s} R_{H}$ is the concentration far from the evaporating liquid, $R_{H}$ is relative humidity and $L$ is a characteristic lengthscale on order of the surface microtexture. By integrating the equation with respect to time, the relative humidity can be computed as a function of time: $R_{H}(t)=1-\left(1-R_{H 0}\right) e^{-t / \tau_{e}}$, where $R_{H 0}$ is the humidity of the ambient air and $\tau_{e}$ is the characteristic evaporation timescale. The value of $\tau_{e} \sim \frac{L V}{D A}=\frac{L^{2}}{D}$ where $V$ is the total volume of gap between microstructure and $A$ is its projected area. Thus, the time for the air within the microtexture to become saturated relative to the drop residence time is $t_{e} / t_{r} \approx \frac{L^{2} \gamma}{D\left(\rho R^{3}\right)^{1 / 2}}$. In the experiments, the evaporation time is estimated to be on the order of a microsecond. Because this timescale is more than a thousand times faster than the residence time, the air within the microstructure can be estimated as being fully saturated.

We next estimate the condensation time $t_{c}$, which we define as the characteristic time needed for condensate to grow and fill the superhydrophobic microtexture. If saturated air contacts a surface that is at slightly lower temperature, the saturated air will locally cool and condensation will occur. Condensation will continue as long as warmer saturated air is cooled on the surface. To estimate the condensation rate, we adopt a condensation model proposed by Kim et al. [26] in which the rate of condensation growth $\frac{d r}{d t}$ can be approximated as $\frac{d r}{d t} \approx h_{i}\left(\frac{T_{s a t}-T_{s}}{\rho_{\ell} H_{f g}}\right)$. Here, $h_{i}$ is the interfacial heat transfer coefficient, $T_{s a t}$ is the saturated air temperature, $T_{s}$ is the surface temperature, $H_{f g}$ is the latent heat of vaporization and $\rho_{\ell}$ is the water density. This expression can be further reduced by modeling the interfacial heat transfer coefficient $h_{i}=\left(\frac{2 \hat{\sigma}}{2-\hat{\sigma}}\right)\left(\frac{\rho_{v} H_{f g}^{2}}{T_{\text {sat }}}\right)\left(\frac{\bar{M}}{2 \pi \bar{R} T_{\text {sat }}}\right)^{1 / 2}$ in terms of 
the vapor properties [27]. Additionally, we estimate the time to fill a microtexture with characteristic lengthscale $L$ as $t_{c} \approx L\left(\frac{d r}{d t}\right)^{-1}$, so that this time is modeled in terms of the temperatures as:

$$
t_{c} \approx \frac{2-\hat{\sigma}}{2 \hat{\sigma}}\left(\frac{2 \pi \bar{R}}{\bar{M}}\right)^{1 / 2}\left(\frac{\rho_{\ell} L}{\rho_{v} H_{f g}}\right)\left(\frac{T_{s a t}^{3 / 2}}{T_{s a t}-T_{s}}\right), \quad T_{s a t}=\frac{\left(T_{d}+T_{s}\right)}{2} .
$$

Here, $\hat{\sigma}$ is the condensation accommodation coefficient [28], $\bar{R}$ is the universal gas constant, $\bar{M}$ is the the molecular weight, $\rho_{\ell}$ is the liquid density, $\rho_{v}$ is the vapor density, and $T_{s a t}$ is temperature of the saturated vapor within the microstructure, which is modeled to be halfway between the drop temperature $T_{d}$ and surface temperature $T_{s}$.

Figure 6 shows plots of the condensation time, predicted from Equation (2), as a function of the temperature difference between the water drop and surface. Here, the characteristic microtexture length is $L=10 \mu \mathrm{m}$, and the different curves represent three different surface temperatures. The results show that this condensation filling timescale $t_{c}$ is significantly larger than the evaporation timescale $t_{e}$, and therefore it is reasonable to assume that the drop evaporation keeps the microtexture air fully saturated as the condensate forms. Additionally, the results illustrate the importance of the temperature difference $T_{d}-T_{s}$, rather than the temperatures themselves, in determining the condensation filling timescale $t_{c}$.

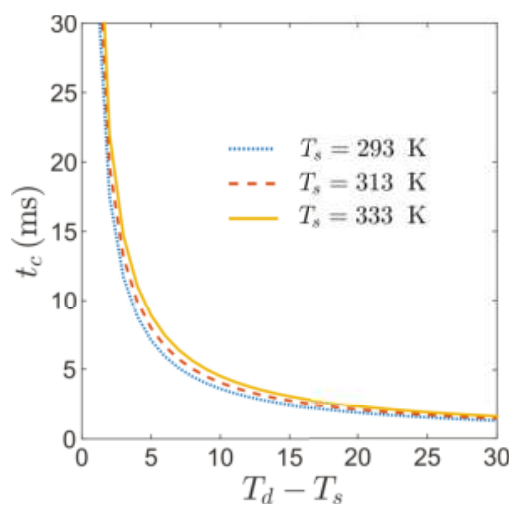

Figure 6. The time $t_{c}$ required for condensate to fill superhydrophobic microtexture is estimated and plotted as a function of temperature difference $T_{d}-T_{S}$ for three representative surface temperatures (curves). These values are calculated using Equation (2) assuming the properties of a water drop on a microtexture with a characteristic lengthscale of $L=10 \mu \mathrm{m}$.

For the evaporation-condensation mechanism to trap a drop, the condensate must sufficiently fill the microtexture air before the drop bounces off of the surface. Therefore, it is natural to expect this process to depend on the relative scales of the condensation time $t_{c}$ and the drop residence time $t_{r} \sim \sqrt{\rho_{\ell} R^{3} / \gamma}$. This ratio motivates a dimensionless grouping of parameters, which we define as $\beta$ :

$$
\frac{t_{c}}{t_{r}} \sim \beta \equiv\left(\frac{L}{R}\right)\left(\frac{\rho_{\ell}}{\rho_{v}}\right)\left(\frac{T_{s a t}}{T_{s a t}-T_{s}}\right)\left(\frac{\bar{R} T_{s a t} \gamma}{\bar{M} H_{f g}^{2} \rho_{\ell} R}\right)^{1 / 2}
$$

Here, we have dropped all dimensionless prefactors, as the focus is on the scaling relationship and the grouping of the dimensional parameters. Note that a condensation time $t_{c}$ requires $T_{\text {sat }}>T_{s}$; however, our definition for $\beta$ is valid regardless of the values of $T_{S}$ and $T_{\text {sat }}$, which we have defined as $T_{\text {sat }} \equiv\left(T_{d}+T_{s}\right) / 2$.

The relevance of the parameter $\beta$ in the drop trapping is illustrated in Figure 7 . Here, a value of $\beta$ is calculated for each experimental data point in Figure 5 and plotted in terms of the ratio $T_{d} / T_{S}$ 
(symbols in Figure 7). Small values of $\beta$ are associated with drop bouncing and large values of $\beta$ are associated with drop sticking. Furthermore, the mechanics motivating the parameters suggest the data fall in one of three regimes. When $T_{d} / T_{s}<1$, the surface is hotter than the drop, and therefore even when the gap air is completely saturated, it will be below saturation directly on the texture surface so that no condensation will form, the surface will remain dry, and drops will bounce (purple region). When $T_{s} / T_{d}>1$, condensation would be expected to form and, if $\beta$ is above a critical value, it would sufficiently fill the microtexture and trap the drop (white region). From the data, it appears that the critical value is around unity, although caution should be taken interpreting this value too closely, as estimates of certain parameters, such as the characteristic microtexture lengthscale of the Nasterium leaf, are less precise than others. Finally, our results indicate that if $\beta<1$, bouncing on the Nasterium leaf occur even when $T_{s} / T_{d}>1$ (yellow region). In our model, this region represents drops that bounce on superhydrophobic surfaces that are filled with insufficient condensate to transition the drop into a Wenzel state.

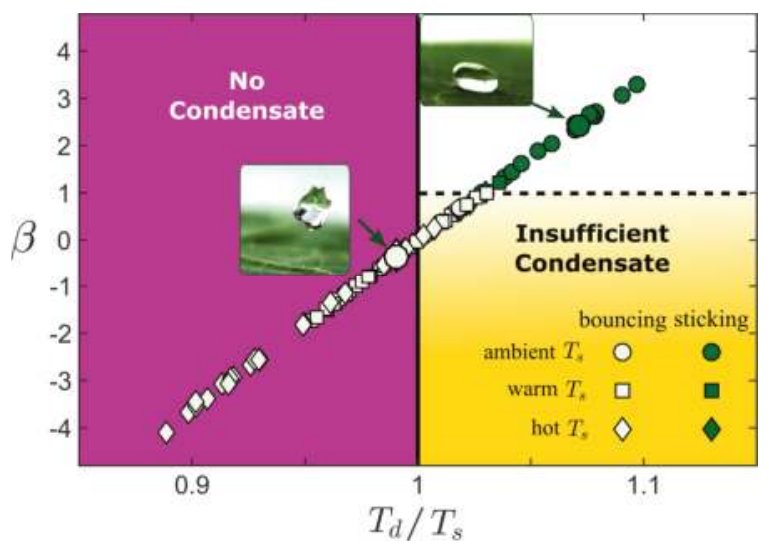

Figure 7. A phase plot illustrating the three condensate regimes. The experimental data from Figure 5 is replotted in terms of the temperature ratio $T_{d} / T_{s}$ and the proposed dimensionless group $\beta$ (Equation (3)). Here, only data below the melting temperature $T_{m}$ is considered. When no condensate is expected to form within the microtexture (purple region), the drop is expected to bounce, whereas, when significant condensation is expected within the microtexture, it is expected to stick (white region). The finite timescale of the bounce introduces a third regime (yellow region) in which condensate develops but can be insufficient to trap the drop.

\section{Conclusions}

We model two potential mechanisms in which a superhydrophobic surface could trap a sufficiently hot drop within milliseconds. The first is a mechanism by which the superhydrophobic texture can be melted by the heat exchanged from the droplet during contact. The second is a mechanism by which liquid evaporates from the drop and condenses within the microtexture during contact. Because both of these mechanisms require sufficient time to develop to a scale where they adequately influence the microtexture, we highlight the importance of the residence, or contact, time of the drop to propose regimes in which these mechanisms—-while present—are insufficient to prevent bouncing.

Of particular interest is the evaporation-condensation mechanism, as condensate can form under the drop whenever a drop is hotter than the superhydrophobic surface and this condensate can compromise the air pocket within the microtexture [11-13]. However, the experimental results in this study demonstrate that drops can bounce off of a superhydrophobic surface even when condensate is expected to form. Motivated by a balance of condensation and bouncing timescales, we propose a dimensionless group $\beta$, (Equation (3)), whose value indicates the importance of condensation as a trapping mechanism. When $\beta$ is positive, condensate would be expected to nucleate; however, 
only for $\beta$ greater than approximately 1 would trapping be expected. We anticipate that this criteria would extend to a wide range of superhydrophobic surfaces and could be relevant to a variety of applications, including the design of smart superhydrophobic surfaces that immediately trap drops that exceed a critical temperature.

Author Contributions: J.C.B. and S.S. conceived the research. S.S. and A.M. designed and fabricated the experimental setup and carried out the experiments. S.S. and J.C.B. contributed to the development of the theory, conducted the analyses, and wrote the manuscript together.

Funding: This research received no external funding.

Acknowledgments: S.S. acknowledges partial support from the Boston University's Dean's Catalyst Award. A.M. acknowledges partial support from the Boston University Undergraduate Research Opportunities Program (UROP).

Conflicts of Interest: The authors declare no conflict of interest.

\section{References}

1. Richard, D.; Clanet, C.; Quéré, D. Surface phenomena: Contact time of a bouncing drop. Nature 2002, 417, 811. [CrossRef] [PubMed]

2. Quéré, D. Non-sticking drops. Rep. Prog. Phys. 2005, 68, 2495. [CrossRef]

3. Yarin, A.L. Drop impact dynamics: Splashing, spreading, receding, bouncing... Annu. Rev. Fluid Mech. 2006, 38, 159-192. [CrossRef]

4. Yu, X.; Wang, Z.; Jiang, Y.; Shi, F.; Zhang, X. Reversible pH-responsive surface: From superhydrophobicity to superhydrophilicity. Adv. Mater. 2005, 17, 1289-1293. [CrossRef]

5. Xia, F.; Ge, H.; Hou, Y.; Sun, T.; Chen, L.; Zhang, G.; Jiang, L. Multiresponsive surfaces change between superhydrophilicity and superhydrophobicity. Adv. Mater. 2007, 19, 2520-2524. [CrossRef]

6. Li, B.; Zhang, J. Durable and self-healing superamphiphobic coatings repellent even to hot liquids. Chem. Comrefmun. 2016, 52, 2744-2747. [CrossRef] [PubMed]

7. Cassie, A.; Baxter, S. Wettability of porous surfaces. Trans. Faraday Soc. 1944, 40, 546-551. [CrossRef]

8. Wenzel, R.N. Resistance of solid surfaces to wetting by water. Ind. Eng. Chem. 1936, 28, 988-994. [CrossRef]

9. Koishi, T.; Yasuoka, K.; Fujikawa, S.; Ebisuzaki, T.; Zeng, X.C. Coexistence and transition between Cassie and Wenzel state on pillared hydrophobic surface. Proc. Natl. Acad. Sci. USA 2009, 106, 8435-8440. [CrossRef] [PubMed]

10. Liu, Y.; Chen, X.; Xin, J. Can superhydrophobic surfaces repel hot water? J. Mater. Chem. 2009, 19, 5602-5611. [CrossRef]

11. Cheng, Y.T.; Rodak, D.E. Is the lotus leaf superhydrophobic? Appl. Phys. Lett. 2005, 86, 144101. [CrossRef]

12. Yin, L.; Wang, Q.; Xue, J.; Ding, J.; Chen, Q. Stability of superhydrophobicity of lotus leaf under extreme humidity. Chem. Lett. 2010, 39, 816-817. [CrossRef]

13. Yu, Z.J.; Yang, J.; Wan, F.; Ge, Q.; Yang, L.L.; Ding, Z.L.; Yang, D.Q.; Sacher, E.; Isimjan, T.T. How to repel hot water from a superhydrophobic surface? J. Mater. Chem. A 2014, 2, 10639-10646. [CrossRef]

14. Bartolo, D.; Bouamrirene, F.; Verneuil, E.; Buguin, A.; Silberzan, P.; Moulinet, S. Bouncing or sticky droplets: Impalement transitions on superhydrophobic micropatterned surfaces. EPL 2006, 74, 299. [CrossRef]

15. Reyssat, M.; Richard, D.; Clanet, C.; Quéré, D. Dynamical superhydrophobicity. Faraday Discuss. 2010, 146, 19-33. [CrossRef] [PubMed]

16. Bird, J.C.; Dhiman, R.; Kwon, H.M.; Varanasi, K.K. Reducing the contact time of a bouncing drop. Nature 2013, 503, 385. [CrossRef] [PubMed]

17. Liu, Y.; Moevius, L.; Xu, X.; Qian, T.; Yeomans, J.M.; Wang, Z. Pancake bouncing on superhydrophobic surfaces. Nature Phys. 2014, 10, 515. [CrossRef] [PubMed]

18. Liu, Y.; Andrew, M.; Li, J.; Yeomans, J.M.; Wang, Z. Symmetry breaking in drop bouncing on curved surfaces. Nature Commun. 2015, 6, 10034. [CrossRef] [PubMed]

19. Patterson, C.J.; Shiri, S.; Bird, J.C. Macrotextured spoked surfaces reduce the residence time of a bouncing Leidenfrost drop. J. Phys. Condens. Matter 2017, 29, 064007. [CrossRef] [PubMed]

20. Shiri, S.; Bird, J.C. Heat exchange between a bouncing drop and a superhydrophobic substrate. Proc. Natl. Acad. Sci. USA 2017, 114, 6930-6935. [CrossRef] [PubMed] 
21. Sugden, S. VI.-The variation of surface tension with temperature and some related functions. J. Chem. Soc. Trans. 1924, 125, 32-41. [CrossRef]

22. Loglio, G.; Ficalbi, A.; Cini, R. A new evaluation of the surface tension temperature coefficients for water. J. Colloid Interface Sci. 1978, 64, 198. [CrossRef]

23. Khattab, I.S.; Bandarkar, F.; Fakhree, M.A.A.; Jouyban, A. Density, viscosity, and surface tension of water+ ethanol mixtures from 293 to 323K. Korean J. Chem. Eng. 2012, 29, 812-817. [CrossRef]

24. Rathjen, K.A.; Jiji, L.M. Heat conduction with melting or freezing in a corner. J. Heat Transf. 1971, 93, 101-109. [CrossRef]

25. Cussler, E.L. Diffusion: Mass Transfer in Fluid Systems; Cambridge University Press: New York, USA, 2009.

26. Kim, S.; Kim, K.J. Dropwise condensation modeling suitable for superhydrophobic surfaces. J. Heat Transf. 2011, 133, 081502. [CrossRef]

27. Rykaczewski, K. Microdroplet growth mechanism during water condensation on superhydrophobic surfaces. Langmuir 2012, 28, 7720-7729. [CrossRef] [PubMed]

28. Mills, A.; Seban, R. The condensation coefficient of water. Int. J. Heat Mass Transf. 1967, 10, $1815-1827$. [CrossRef]

(C) 2018 by the authors. Licensee MDPI, Basel, Switzerland. This article is an open access article distributed under the terms and conditions of the Creative Commons Attribution (CC BY) license (http:/ / creativecommons.org/licenses/by/4.0/). 


\title{
Actuation of Flexible Membranes via Capillary Force: Single-Active-Surface Experiments
}

\author{
Christina Barth and Carl Knospe *(B) \\ Department of Mechanical and Aerospace Engineering, University of Virginia, Charlottesville, VA 22904, USA; \\ cab5s@@irginia.edu \\ * Correspondence: knospe@virginia.edu; Tel.: +1-434-924-7424
}

Received: 20 September 2018; Accepted: 23 October 2018; Published: 25 October 2018

check for updates

\begin{abstract}
Conventional approaches to microscale actuation, such as electrostatic, have difficulty in achieving large motion at moderate voltages. Recently, actuators relying on the active control of capillary pressure have been demonstrated, with the pressure change caused by electrowetting on a pair of opposing surfaces. In this work, experimental results are presented from five prototype devices in which only a single active surface is used. The results demonstrate that pressure changes induced in a liquid bridge in this manner can produce large deflections $(15 \mu \mathrm{m})$ of a flexible membrane. Voltages employed in the tests were moderate $(\leq 25 \mathrm{~V})$. The influence of several design variables, such as membrane diameter and thickness, on the membrane deflection are examined. Theoretical predictions are also presented and generally follow the experimental values. Potential sources for the discrepancies between theory and experimental results are discussed. While deflections obtained using a single active surface are not as large as those obtained with two active surfaces, single-active-surface configurations offer a simple route to achieving adequate deflections for lab-on-a-chip microsystems.
\end{abstract}

Keywords: electrowetting; actuation; capillary pressure; lab-on-a-chip

\section{Introduction}

For the past decade there has been strong and growing interest in the development of portable lab-on-a-chip (LOC) devices containing tens or even hundreds of independently controlled valves [1]. Actuation of these membrane valves is typically achieved pneumatically, requiring an external pump and a large number of pneumatic tube connections. To relieve the undesirable manufacturing, size, weight, and power characteristics of this approach, researchers have turned to alternative actuation technologies that permit on-chip, electronic control of LOC valves. By and large, the alternative technologies have fallen short, either requiring large voltage/power or yielding inadequate valve deflection $(<5 \mu \mathrm{m})[2-5]$. The authors have recently presented results showing that large deflections of a flexible membrane, like that appearing in LOC devices, can be achieved via a new actuation technique based upon actively controlling capillary pressure [6]. In this paper we offer new experimental results demonstrating that a simpler actuator design can also achieve substantial membrane deflections, although not as large as those previously reported $(>50 \mu \mathrm{m})[6]$. The membranes tested herein have similar thicknesses and diameters as those reported in the literature. However, significantly larger membrane deflections are achieved at much lower voltages.

In both our previous work and that reported on here, membrane actuation is achieved by lowering the capillary pressure of a liquid droplet that is in contact with the membrane. This reduction in pressure is accomplished via electrowetting. In electrowetting, a droplet in contact with a dielectric-covered electrode undergoes a reduction in its contact angle when a voltage is applied between the droplet and the electrode. The change in contact angle alters the curvature of the droplet's 
free (i.e., liquid/air) interface and results in a decrease in capillary pressure. In our previous design, electrowetting occurred on two opposing electrodes (see Figure 1a), which we refer to as active surfaces. In this paper, we introduce an alternative membrane actuator containing only a single active surface (see Figure 1b). Such a configuration may offer easier integration into LOC systems since electrical connections only need to be supplied to a single substrate.
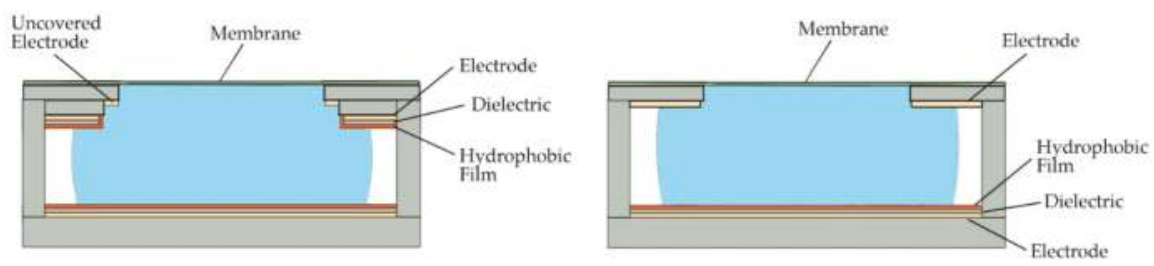

(a)

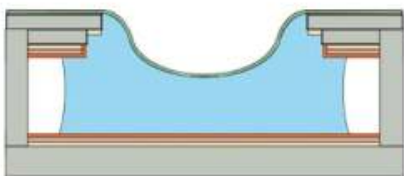

(b)

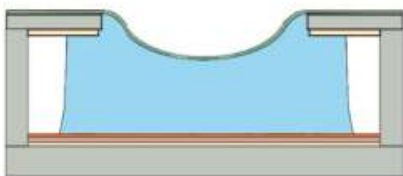

Figure 1. Capillary pressure actuation of a membrane: (a) previously investigated dual-active-surface device, before and after application of voltage; (b) single-active-surface device examined in this paper, before and after application of voltage. Electrowetting only occurs on the lower surface, not on the upper electrode. This bare electrode can alternatively be placed on the lower surface without changing actuator function.

\section{Background}

The change in contact angle with applied voltage during electrowetting is governed by the Lippmann-Young equation [7]

$$
\cos \left(\theta_{A}\right)=\cos \left(\theta_{A, 0}\right)+\frac{\varepsilon_{d}}{2 \sigma_{g l} t_{d}} V_{d}^{2}
$$

where $\varepsilon_{d}$ is the permittivity of the dielectric film, $t_{d}$ is the thickness of the dielectric film, $\theta_{A}$ is the contact angle when a voltage $V_{d}$ is applied across the dielectric film, $\theta_{A, 0}$ is the contact angle without an electric field, and $\sigma_{g l}$ is the surface tension of the gas/liquid interface. As Equation (1) indicates, greater changes in contact angle will occur during electrowetting when the permittivity is large and the film thickness is small.

Beyond a certain voltage $V_{s}$, known as the saturation voltage, the contact angle ceases to decrease during electrowetting $[7,8]$ and Equation (1) is no longer accurate. The minimum contact angle seen, $\theta_{s}$, will be referred to as the saturation angle. The saturation phenomenon is not fully understood at this time and several causes have been proposed [8]. The thin dielectric films used are exposed to very high electric fields during electrowetting and there may be significant charge injection at larger voltages, even those below the saturation voltage $[8,9]$.

In the membrane actuator considered (Figure 1b), the droplet spans between two surfaces, one of which carries the elastic membrane. The contact angles on the two surfaces will, in general, be different and will vary during actuator operation. These contact angles will be denoted $\theta_{A}$ (active) and $\theta_{P}$ (passive). Electrowetting occurs only on the active surface and the contact angle changes with voltage as described above. On the passive surface, the contact angle is determined by surface and liquid properties. Ideally, the angle on this surface, $\theta_{P}$, does not change $\left(\theta_{P}=\theta_{P, 0}\right)$ when electrowetting occurs on the active surface. In practice, $\theta_{P}$ will vary within a range as determined by the passive surface's contact angle hysteresis. 
The liquid bridge that spans between the surfaces will take on an axisymmetric shape that is among the Plateau sequence of shapes $[10,11]$; this shape is determined by the volume of the bridge, the distance between the surfaces (bridge height, $h$ ), and the contact angles [10]. If the radius $R_{1}$ at the bridge's waist is significantly greater than the bridge height, the profile of the axisymmetric bridge may be accurately approximated by a circular arc [10], whose radius of curvature is denoted $R_{2}$, see Figure 2. This radius of curvature can be expressed in terms of the contact angles [10] as:

$$
R_{2}=\frac{-h}{\cos \left(\theta_{A}\right)+\cos \left(\theta_{P}\right)}
$$

(A negative radius of curvature corresponds to a bridge profile that is concave.)

The Laplace equation describes the relationship between the bridge's radii of curvature and the capillary pressure difference $\left(P_{c}\right)$ across the liquid/air interface:

$$
P_{c}=\sigma_{g l}\left(\frac{1}{R_{1}}+\frac{1}{R_{2}}\right)
$$

Before voltage is applied, the contact angles of the active and passive surfaces are $\theta_{A, 0}$ and $\theta_{P, 0}$, respectively. From Equations (1) and (2), the capillary pressure without applied voltage is

$$
P_{c, 0}=\sigma_{g l}\left(\frac{1}{R_{1}}-\frac{\cos \left(\theta_{A, 0}\right)+\cos \left(\theta_{P, 0}\right)}{h}\right)
$$

After application of voltage to the device and the onset of electrowetting the bridge's capillary pressure will change to

$$
P_{c}=\sigma_{g l}\left(\frac{1}{R_{1}}-\frac{\cos \left(\theta_{A}\right)+\cos \left(\theta_{P}\right)}{h}\right)
$$

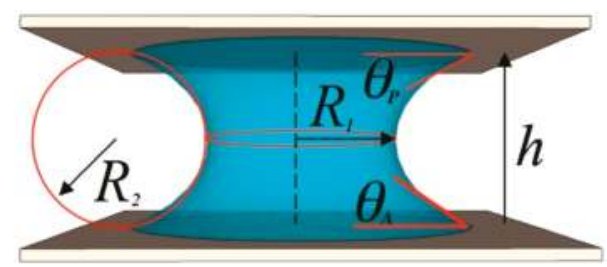

Figure 2. Capillary bridge between surfaces indicating radii of curvature and contact angles.

(Note that the radius of the bridge's waist, $R_{1}$, remains almost constant during electrowetting as it is much larger than the bridge's height. For this reason, Equations (4) and (5) use the same value of $R_{1}$ ) Thus, the change in capillary pressure due to the application of the voltage is given by

$$
\Delta P_{c}=\frac{\sigma_{g l}}{h}\left\{\left(\cos \left(\theta_{A, 0}\right)-\cos \left(\theta_{A}\right)\right)+\left(\cos \left(\theta_{P, 0}\right)-\cos \left(\theta_{P}\right)\right)\right\}
$$

When the contact angle on the active surface is lowered via electrowetting, the capillary pressure of the liquid is reduced $\left(\Delta P_{c}<0\right)$. This change in the liquid's pressure results in an inward deflection of the flexible membrane due to its contact with the liquid. The reader will note that Equation (6) predicts that larger changes in capillary pressure will occur for a given change in $\theta_{A}$ if the bridge height is made smaller.

The contact angle on the passive surface may change within the range dictated by the surface's contact angle hysteresis. Due to volume conservation for the bridge, if the passive surface contact line does not advance sufficiently when electrowetting occurs on the active surface, the passive surface contact angle will increase. This result is due to the requirement that the free interface of the liquid 
bridge must belong to the Plateau sequence of shapes. Therefore, the term $\left(\cos \left(\theta_{P, 0}\right)-\cos \left(\theta_{P}\right)\right)$ is typically positive and tends to counteract the decrease in pressure caused by the change in contact angle on the active surface. If there was no contact angle hysteresis on the passive surface, equilibrium of the forces acting upon the passive surface's contact line would only occur at a single angle, $\theta_{P, 0}$, no matter what angle existed on the active surface. In this (ideal) case, the change in capillary pressure would be

$$
\Delta P_{c}=\frac{\sigma_{g l}}{h}\left\{\cos \left(\theta_{A, 0}\right)-\cos \left(\theta_{A}\right)\right\}
$$

and the maximum possible membrane displacement would occur.

For the devices tested in this paper, the layout of components is as shown in Figure 1b. The flexible membrane is attached to the passive surface substrate. This substrate also carries a bare gold electrode that is in contact with the liquid bridge and is needed for completing the electrowetting circuit. The active surface lies opposite the passive surface. When electrowetting occurs, the pressure in the drop is reduced and the membrane is pulled toward the active surface. The reader should note that many configurations of the components are possible. For example, in microfabricated devices, it is desirable to avoid electrodes on both the substrates shown in Figure $1 \mathrm{~b}$ because of the difficulties associated with electrical interconnects. Since the location of the bare electrode has no impact upon device performance, this can be achieved by having the substrate that contains the active surface also carry the bare electrode. This was not done in this effort since it was simpler to modify an existing fabrication process than to develop one to permit both electrodes on one substrate. Another configuration of the device could have the active surface substrate carry the flexible membrane. The bare electrode could also be on this substrate or on the accompanying passive surface. Such configurations should have essentially the same performance as that seen in this paper.

\section{Materials and Methods}

\subsection{Fabrication of Electrowetting Surfaces}

E-beam evaporation (CHA Industries, Fremont, CA, USA) was used to deposit a 100 nanometer $(\mathrm{nm})$ thick aluminum (Al) film onto borosilicate glass slides (top plate, $120 \mu \mathrm{m}$ thick) or soda-lime glass slides (bottom plate, $1.59 \mathrm{~mm}$ thick). A $30.6 \mathrm{~nm}$ aluminum oxide $\left(\mathrm{Al}_{2} \mathrm{O}_{3}\right)$ film was grown by anodization (18 V) [12]. An ultrathin $(50.8 \mathrm{~nm})$ film of the hydrophobic fluoropolymer CYTOP-809 (Bellex International Corporation, Wilmington, DE, USA) was deposited on the oxide film via spin coating and the slides were then baked at $160^{\circ}$ for $1 \mathrm{~h}$ [13]. To complete the fabrication of the active surface, a silicon oil mixture (Dow Corning OS-10, OS-20, and OS-30, Krayden, Inc., Denver, CO, USA, 1:8:1 by weight), was applied to the CYTOP and then allowed to evaporate from the surface. This treatment was found to reduce contact angle hysteresis and yield more repeatable electrowetting [13,14]. (Contact angle hysteresis was typically $10^{\circ}$ after treatment).

\subsection{Device Components}

Proof-of-concept devices were fabricated using facile, low cost techniques that permitted ease in assembly, disassembly, retesting, and visual observation of the liquid bridge during operation. Each device was composed of a passive top plate with a polydimethylsiloxane (PDMS) membrane, a bottom plate with active surface, and a liquid bridge extending between them-see Figure $1 \mathrm{~b}$. While dimensions vary among the devices tested, the working components occupy a region of dimensions approximately $2 \mathrm{~mm} \times 2 \mathrm{~mm} \times 0.5 \mathrm{~mm}$. Devices with smaller dimensions should be equally effective according to theory [13] but were not examined in this initial investigation due to the design constraints imposed by simple fabrication procedures and liquid bridge observation. 


\subsection{Fabrication of Membranes}

The elastomeric membrane in each device was fabricated using a Sylgard 184 PDMS kit (Dow Corning, Midland, MI, USA). The base polymer and curing agent were mixed 10:1 by weight and allowed to degas in air for $90 \mathrm{~min}$. The PDMS solution was then deposited onto a poly(methyl methacrylate (PMMA) surface and spun for $60 \mathrm{~s}$ at 1100-2200 RPM to achieve the desired final thickness, typically 40 or $50 \mu \mathrm{m}$. The coated PMMA was then baked in an oven for $1 \mathrm{~h}$ at $70{ }^{\circ} \mathrm{C}$. The cured PDMS was then cut into $\sim 5 \mathrm{~mm}$ squares. A Dektak Profilometer system (Bruker, Billerica, MA, USA) was used to measure the thickness of PDMS membrane-see Table 1 for measured values. A titanium flake ( $100 \mathrm{~nm}$ thick) was transferred to the center of each PDMS square to provide a reflective surface for laser interferometric measurement of membrane displacement during testing.

Table 1. Dimensions and descriptions for the single-active-surface tests conducted.

\begin{tabular}{|c|c|c|c|c|c|c|}
\hline \multirow{2}{*}{ Test } & \multicolumn{2}{|c|}{ Bridge } & \multicolumn{2}{|c|}{ Membrane } & \multirow{2}{*}{ Max Voltage (V) } & \multirow{2}{*}{ Description } \\
\hline & Height $(\mu \mathrm{m})$ & Radius (mm) & Thickness ( $\mu \mathrm{m})$ & Radius ( $\mu \mathrm{m})$ & & \\
\hline 1 & 93 & 1.36 & 39 & 510 & 25 & $1 \mathrm{~V}$ step staircase \\
\hline 2 & 94 & 1.80 & 39 & 439 & 24 & $1 \mathrm{~V}$ step staircase \\
\hline 3 & 96 & 1.33 & 50 & 510 & 25 & $1 \mathrm{~V}$ step staircase \\
\hline 4 & 108 & 0.97 & 39 & 438 & 14 & $14 \mathrm{~V}$ square wave \\
\hline 5 & 111 & 1.23 & 40 & 441 & 14 & $14 \mathrm{~V}$ square wave \\
\hline
\end{tabular}

\subsection{Fabrication of Device Bottom Plate}

The bottom plate was a glass slide with an electrowetting surface fabricated as described above (see Fabrication of Electrowetting Surfaces section).

\subsection{Fabrication of Device Top Plate}

The top plate (passive surface) was composed of a borosilicate glass slide with a gold electrode to act as the cathode for electrowetting. Fabrication was achieved using the following procedure. Holes were cut into each slide via VersaLASER VLS3.50, $50 \mathrm{~W} \mathrm{CO}_{2}$ laser cutter (Universal Laser Systems, Scottsdale, AZ, USA). The hole defines the perimeter of the free-standing elastomeric membrane. After cutting and cleaning, a $10 \mathrm{~nm}$ thick layer of Au was deposited via e-beam evaporation, utilizing a $2.5 \mathrm{~nm}$ Ti adhesion layer. An ultrathin film of CYTOP was spin coated onto the Au layer, to allow the eased motion of the conducting drop on the passive surface. The CYTOP film was applied with one spin coat application (30 s, 2000 RPM) of either $0.5 \%$ wt. or $1 \% \mathrm{wt}$. CYTOP and then baked for $1 \mathrm{~h}$ at $160^{\circ} \mathrm{C}$. It was found in earlier testing that such ultrathin films of CYTOP by themselves provided essentially no resistance to the conduction of current, undergoing electrical breakdown as soon as any voltage was applied. Thus, the presence of the CYTOP does not affect the prototype's electrical performance and electrode prepared in this fashion can be considered 'bare' in spite of the CYTOP film. The CYTOP layer was then treated with silicon oil as described above. A PDMS piece was then applied to the top of the slide covering the hole with the reflective flake centered within the hole. The PDMS square adhered to the glass with suitable strength and did not require any additional treatment (e.g., oxygen plasma bonding).

Plastic microbeads (Polyscience, Warrington, PA, USA) were deposited from solution onto the lower surface of the top plate to act as spacers and thus establish the distance between the top and bottom plates (i.e., bridge height) in the final assembled device. The beads deposited were (nominally) $100 \mu \mathrm{m}$ in diameter $( \pm 10 \mu \mathrm{m})$.

\subsection{Device Assembly}

A "flip chip" assembly process was used to form the test articles. A $0.1 \mathrm{M} \mathrm{Cs}_{2} \mathrm{SO}_{4}$ aqueous solution was prepared with deionized (DI) water and $10 \mu \mathrm{Mol} / \mathrm{L}$ Fluorescein salt was added (chemicals from Sigma Aldrich, St. Louis, MO, USA). A 1.2-1.5 $\mu \mathrm{L}$ droplet of the solution was deposited within the 
hole in the top plate (beneath the PDMS membrane) by micropipette (Eppendorf, Hamburg, Germany). Variations in droplet volume will have very little effect on device performance as the capillary pressure is not strongly dependent on $R_{1}$ when $R_{1} \gg h$. The top plate was then placed on the bottom plate with tweezers. The assembled components were held in place by the attraction between a permanent magnet placed below the bottom plate and ferromagnetic foil pieces secured around the PDMS square on the upper surface of the top plate via tape. Due to variations in the size of beads used for spacers $( \pm 10 \mu \mathrm{m})$ and bow in the glass slides, the bridge height achieved after assembly could not be tightly controlled. Therefore, the height was measured via the side view camera to determine an accurate value for use in theoretical predictions. The side-view camera was also used to detect misalignments between plates. Since the variation in bead diameters was much less than the distance between bead locations, the alignment between the top and bottom plates was very good. When misalignment was detected, the device was reassembled to eliminate it.

\subsection{Device Testing}

A fiber optic extrinsic Fabry-Perot interferometer (Fiber Pro2, Luna Innovations, Roanoke, VA, USA) was employed to measure membrane displacement during testing. The fiber was centered over the reflective flake on the PDMS membrane, brought into range, and the interferometer settings were adjusted to maximize signal quality. The received signal typically temporarily lost quality when displacements were large, and changes were sudden. As a result, spurious spikes sometimes would appear in the displacement data.

The profile of the liquid bridge was imaged via a side view camera (Prosilica GC2450, Allied Vision Technologies, Stadtroda, Germany) paired with a long-distance microscope (K2/SC, Infinity-USA, Centennial, CO, USA) to achieve an effective magnification of $\sim 0.7 \mu \mathrm{m} / \mathrm{px}$. The inclusion of a fluorescent dye in the liquid allowed imaging of the bridge profile in spite of the close spacing between top and bottom plates of the device. The bridge's fluorescence was excited using a $405 \mathrm{~nm}$ wavelength laser pointer and a bandpass optical filter was placed in front of the aperture of the side view camera to attenuate light at the excitation wavelength.

Only direct current (DC) voltages were used in testing. As discussed below, the anodic oxide dielectric films employed in our devices fail under reversed polarity [12] and preclude alternating current $(\mathrm{AC})$ operation.

\section{Results}

\subsection{Description of Tests}

We highlight the results of five tests here; for further results see [13]. For all the prototypes discussed, bridge heights were set via the microbeads to be close to $100 \mu \mathrm{m}$. Membranes were constructed in two sizes, small diameter $(\sim 440 \mu \mathrm{m})$ and large diameter $(\sim 510 \mu \mathrm{m})$, and two ranges of thickness, thin $(\sim 40 \mu \mathrm{m})$ and thick $(\sim 50 \mu \mathrm{m})$. The relevant dimensions for each prototype device and the testing conditions are described in Table 1 . The measured data from these tests as well as calculated values based upon this data are provided in Table 2. The contact angles of the liquid bridge on the passive and active surfaces are given for the time before application of voltage ('initial') and for that when the voltage first reaches its peak value ('final'). Measurements of membrane displacements are also provided for these times. (The values reported are calculated from an average of 10 measurements taken after the transient has settled). The model relating capillary pressure to membrane deflection that was used in this research is contained in the Appendix of our previous work [6]. Theoretical values of membrane displacement were calculated using Equation (6) and are presented in Table 2. Note that these calculations are based upon the measured values of contact angle, not angles derived from the Lippmann-Young equation. Calculated values of the change in capillary pressure between the 'initial' state and the 'final' state are also displayed in the table. Two values are calculated: (1) a predicted pressure change based on the measured values of contact angle using Equation (6); and (2) a 
'no hysteresis' case where the pressure change is based on the measured initial and final contact angles on the active surface and the specification that the contact angle on the passive surface does not vary from its initial value (see Equation (7)).

Good agreement between theory and experiment occurred for most tests that were conducted. The expected trends were also apparent in the experimental results. Thin membranes, large diameter membranes, and higher voltages yielded larger membrane deflections. Each test is discussed in detail below.

Table 2. Results from single-active-surface tests-contact angles on active and passive surfaces as measured by side view camera; measured membrane deflection and theoretical value calculated from measured angles; values of change in capillary pressure as determined from measured angles (Equation (6)) and with a no hysteresis model (Equation (7)).

\begin{tabular}{|c|c|c|c|c|c|c|c|c|c|}
\hline \multirow{3}{*}{ Test } & \multicolumn{4}{|c|}{ Contact Angles $\left({ }^{\circ}\right)$} & \multirow{2}{*}{\multicolumn{3}{|c|}{ Maximum Membrane Deflection $(\mu \mathrm{m})$}} & \multirow{2}{*}{\multicolumn{2}{|c|}{ Calculated Capillary Pressure Change, $\Delta \mathbf{P}_{\mathrm{c}}(\mathrm{Pa})$}} \\
\hline & \multicolumn{2}{|c|}{ Initial } & \multicolumn{2}{|c|}{ Final * } & & & & & \\
\hline & $\theta_{A, 0}$ & $\theta_{P, 0}$ & $\theta_{A}$ & $\theta_{P}$ & Measured & Theory & Error $(\%)$ & Equation (6) & Equation (7) \\
\hline 1 & 107 & 58 & 89 & 64 & 15.2 & 13.8 & 10.1 & 160 & 227 \\
\hline 2 & 101 & 89 & 83 & 96 & 7.3 & 9.0 & 18.9 & 138 & 226 \\
\hline 3 & 104 & 86 & 80 & 94 & 9.2 & 12.3 & 25.2 & 196 & 294 \\
\hline 4 & 91 & 101 & 81 & 104 & 6.2 & 4.7 & 31.9 & 77 & 110 \\
\hline 5 & 103 & 94 & 80 & 98 & 13.4 & 12.2 & 9.8 & 202 & 244 \\
\hline
\end{tabular}

\subsection{Staircase Voltage Signal Tests}

In Test 1 , the prototype was a device with a thin, large diameter membrane. In the test, the voltage was increased in $1 \mathrm{~V}$ increments each second, stepping from $0 \mathrm{~V}$ up to $25 \mathrm{~V}$ (a 'staircase' signal). Figure 3 shows the measured membrane displacement and voltage during the test. The membrane reaches its maximum displacement, $15.2 \mu \mathrm{m}$, when $20 \mathrm{~V}$ is applied. For voltage increases beyond this value, the deflection remains essentially constant due to the onset of contact angle saturation. The reader will note that $13 \mu \mathrm{m}$ ( $85 \%$ of the final deflection) was achieved when only $14 \mathrm{~V}$ was applied. The measured deflection is quite close to that predicted from the measured contact angles, exceeding the theoretical prediction by just $10 \%$.

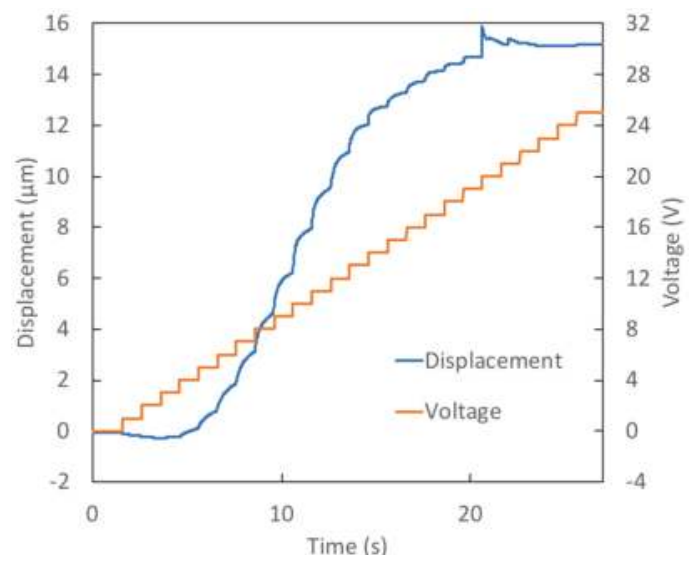

Figure 3. Test 1 -Response of prototype with a thin $(39 \mu \mathrm{m})$, large diameter $(510 \mu \mathrm{m})$ membrane to staircase voltage signal.

In Test 1 , the contact angle on the active surface decreased by $18^{\circ}$ between initial and final states. The contact angle on the passive surface, however, increased by $6^{\circ}$. This increase results in less curvature of the interface profile than would occur if the passive surface contact angle remained 
unchanged. As a result, $40 \%$ less change in the capillary pressure occurred in this test than would have taken place in the 'no hysteresis' case. This example shows the importance of the passive surface characteristics to achieving good performance.

Figure 4 shows the results from Test 2 where a thin membrane with a small diameter was used. The voltage applied was a staircase signal with $1 \mathrm{~V}$ steps occurring every $3 \mathrm{~s}$. The maximum voltage applied was $24 \mathrm{~V}$. The changes in the contact angles on the active and passive surfaces are quite similar to those seen in Test 1 . As a result, the changes in capillary pressure are also comparable. The reduced deflection seen in Test 2 is therefore primarily due to the smaller diameter membrane employed in this test. Membrane deflection is quite sensitive to this variable: a $14 \%$ reduction in membrane diameter results in more than a 50\% decrease in displacement.

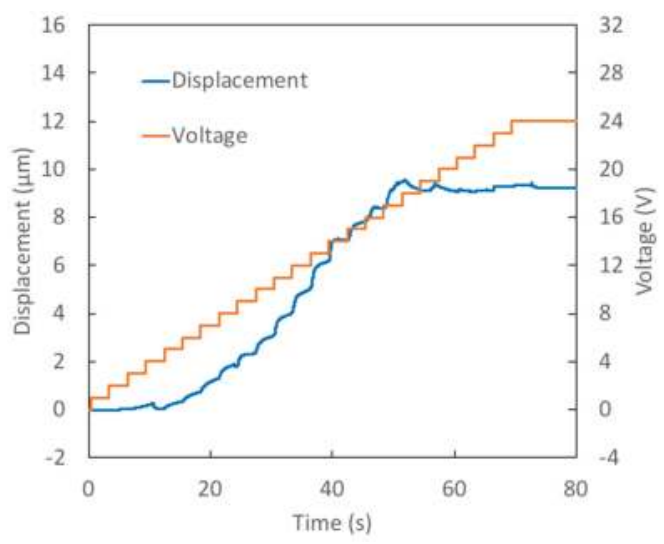

Figure 4. Test 2-Response of prototype with a thin $(39 \mu \mathrm{m})$, small diameter $(439 \mu \mathrm{m})$ membrane to staircase voltage signal.

In Test 3, the prototype examined contains a membrane with the same large diameter as used in Test 1 , but with $25 \%$ greater thickness than that test. The voltage applied was a staircase signal with a maximum voltage of $25 \mathrm{~V}$. The deflection observed in this test is $40 \%$ lower (see Figure 5) than that seen in Test 1, primarily due to the thicker membrane used. However, the measured deflection also significantly underperforms the theoretical value. It is likely that this is the result of contact angles that are not as favorable as those that were measured. This circumstance can occur if the bridge is not axisymmetric. The contact angles can vary along the contact line; the angles measured may not be representative of the actual surface curvatures and the capillary pressure that arise from them.

The reader will note that in all three tests the displacement for the most part reaches its maximum by $18 \mathrm{~V}$. This is due to contact angle saturation.

\subsection{Square Wave Voltage Signal Tests}

The prototypes examined in Test 4 and Test 5 are essentially the same as that studied in Test 2; they have a thin, small diameter membrane. The voltage applied in both tests was a square wave with a minimum of $0 \mathrm{~V}$ and a maximum of $14 \mathrm{~V}$. The period of the voltage signal is $6 \mathrm{~s}$. Figures 6 and 7 show the measured displacements for Tests 4 and 5, respectively. In spite of the nearly identical dimensions, the deflection seen in Test 4 is significantly less than that in Test 5 . The cause of this marked difference can be found in the data in Table 2: the initial contact angle on the active surface was much lower in Test $4\left(91^{\circ}\right)$ than in Test $5\left(103^{\circ}\right)$. Since the final (electrowetted) contact angle for both tests is essentially the same $\left(\sim 80^{\circ}\right)$, the angle change in Test 4 is much less than in Test 5 . Thus, the change in capillary pressure in Test 4 is less than half that seen in Test 5 . Since the active surface is coated with CYTOP and treated with silicon oil, we would not expect the sessile angle of the bridge to be as low as $91^{\circ}$. It is 
likely that there was some contamination in the manufacture of this surface. The result shows that this approach to actuation is quite sensitive to variations in these surfaces. For successful application of this actuator technology, careful attention must be paid to manufacturing processes to ensure quality and consistency.

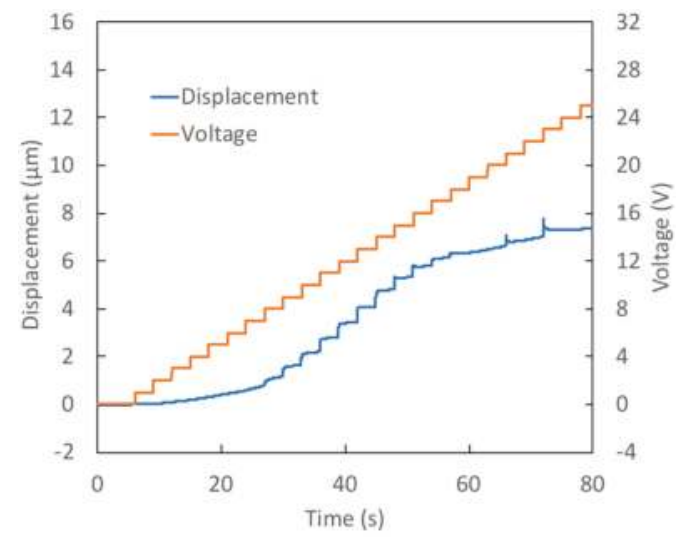

Figure 5. Test 3 -Response of prototype with a thick $(50 \mu \mathrm{m})$, large diameter $(510 \mu \mathrm{m})$ membrane to staircase voltage signal.

Note that in both Test 4 and Test 5, the contact angle on the passive surface changed very little from its initial value during electrowetting. As a result, the capillary pressure change achieved is not far from that which would occur in the ideal 'no hysteresis' case. Equation (6) shows that the value of the passive surface's sessile contact angle has very little effect on actuator performance [10]. The important factor for performance is whether this angle remains unchanged when electrowetting occurs on the active surface, or whether this angle increases.

As seen in Figures 6 and 7, the response time when changing membrane deflection is quite quick, with almost all of the deflection taking place in less than $0.2 \mathrm{~s}$.

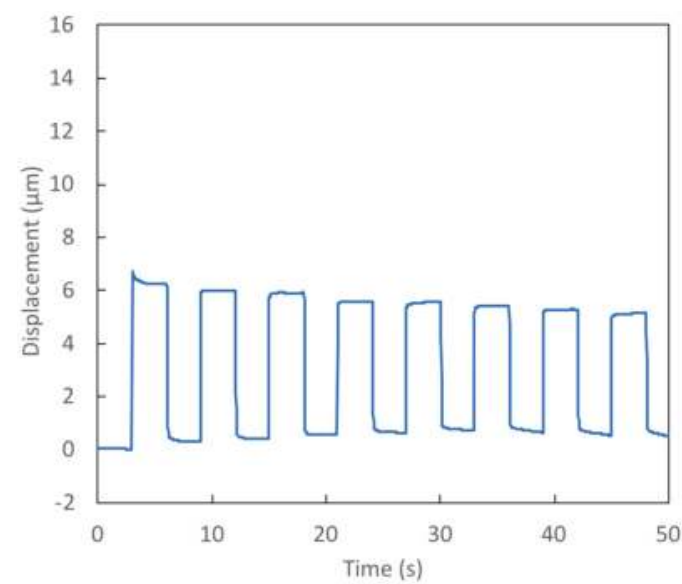

Figure 6. Test 4 -Response of prototype with a thin $(39 \mu \mathrm{m})$, small diameter $(438 \mu \mathrm{m})$ membrane to $14 \mathrm{~V}$ square wave voltage signal. 


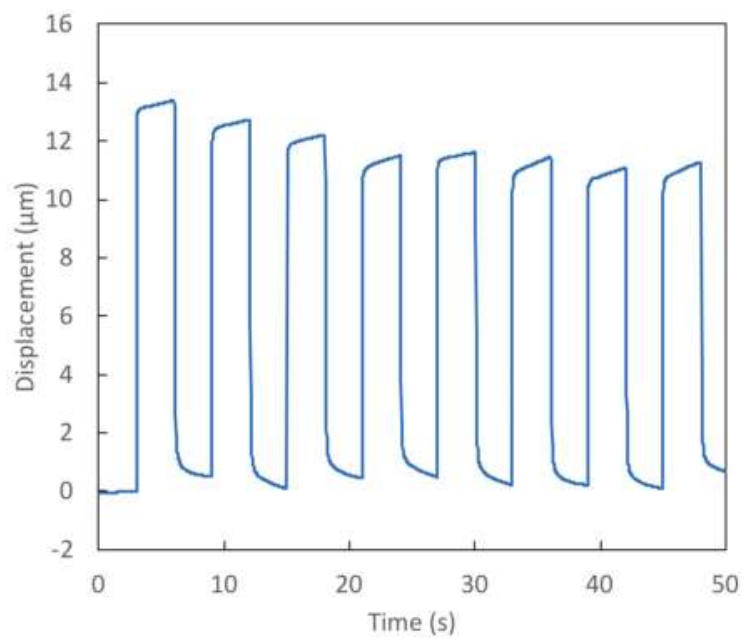

Figure 7. Test 5 - Response of prototype with a thin $(40 \mu \mathrm{m})$, small diameter $(441 \mu \mathrm{m})$ membrane to $14 \mathrm{~V}$ square wave voltage signal.

\section{Discussion}

As the results demonstrate, capillary pressure changes due to electrowetting are an effective means for the generation of membrane movement in microdevices, even when electrowetting occurs on only one surface and not that containing the membrane. The displacements obtained (5-15 $\mu \mathrm{m})$, like those found in a previous investigation of dual-active-surface devices [6], were significantly larger than those earlier achieved by electrostatic actuation using much higher voltage and power [4]. This paper's single-active-surface results opens up a range of possible variations in actuator design. Membranes can be attached to surfaces, either active or passive, in a variety of ways and can adjoin the $\operatorname{droplet}(\mathrm{s})$ in many arrangements [15].

The membrane displacements obtained for single-active-surface devices are about half of those obtained by their dual active surface counterparts [6], as predicted by theory [10]. Just as experiments with those actuators demonstrated, significant improvements in the deflection performance of single-active-surface devices can be achieved by decreasing bridge height. With a height of $40 \mu \mathrm{m}$, it should be possible to achieve $15 \mu \mathrm{m}$ of membrane displacement using only $10 \mathrm{~V}$. Thus, the range of motion desirable for LOC applications can be achieved with membranes of typical LOC diameter and thickness.

Experimental results show that contact angle hysteresis on the passive surface is an important factor in affecting actuation performance for single-active-surface devices. If the angle on the passive surface increases significantly as electrowetting occurs, the change in capillary pressure will be substantially reduced. It is therefore desirable to engineer the passive surface to have as little contact angle hysteresis as possible. This would be a fruitful area for further research.

Although a systematic investigation of long-term actuation behavior was not carried out, several comments on this aspect of performance during the square wave tests are warranted. There was some decrease in motion within the first few cycles. If the voltage was less than $14 \mathrm{~V}$, the displacement behavior settled into a steady pattern with an increasing number of cycles and further degradation was not noted. In this case, the magnitude of the displacement motion was between $60 \%$ and $80 \%$ of the initial displacement, depending upon the test. Charge injection was detected in tests where the voltage exceeded $16 \mathrm{~V}$. 
Author Contributions: C.K. and C.B. conceived and designed the experiments; C.B. performed the experiments; C.K. and C.B. analyzed the data; C.K. and C.B. wrote the paper.

Funding: This research received no external funding.

Acknowledgments: This research was supported by the University of Virginia.

Conflicts of Interest: The authors declare no conflict of interest.

\section{References}

1. Iannone, E. Labs on Chip: Principles, Design and Technology, Devices, Circuits, and Systems; CRC Press: Boca Raton, FL, USA, 2014.

2. Oh, K.W.; Ahn, C.H. A review of microvalves. J. Micromech. Microeng. 2006, 16, R13-R39. [CrossRef]

3. Desai, A.V.; Tice, J.D.; Apblett, C.A.; Kenis, P.J. Design considerations for electrostatic microvalves with applications in poly(dimethylsiloxane)-based microfluidics. Lab Chip 2012, 12, 1078-1088. [CrossRef] [PubMed]

4. Tice, J.D.; Rosheck, J.B.; Hamlin, C.D.; Apblett, C.A.; Kenis, P.J. Normally-closed electrostatic microvalve fabricated using exclusively soft-lithographic techniques and operated with portable electronics. J. MicroElectroMechan. Syst. 2013, 22, 1251-1253. [CrossRef]

5. Tice, J.D.; Desai, A.V.; Bassett, T.A.; Apblett, C.A.; Kenis, P.J. Control of pressure-driven components in integrated microfluidic devices using an on-chip electrostatic microvalve. RSC Adv. 2014, 4, 51593-51602. [CrossRef]

6. Barth, C.A.; Hu, X.; Mibus, M.; Reed, M.L.; Knospe, C.R. Large membrane deflection via capillary force actuation. J. Micromechan. Microeng. 2018, 28, 065008. [CrossRef]

7. Mugele, F.; Baret, J.C. Electrowetting: From basics to applications. J. Phys. Cond. Matter 2005, 17, R705-R744. [CrossRef]

8. Mugele, F. Fundamental challenges in electrowetting: From equilibrium shapes to contact angle saturation and drop dynamics. Soft Matter 2009, 5, 3377-3384. [CrossRef]

9. Mibus, M.; Hu, X.; Knospe, C.; Reed, M.; Zangari, G. Failure modes during low-voltage electrowetting. ACS Appl. Mater. Interfaces 2016, 8, 15767-15777. [CrossRef] [PubMed]

10. Nezamoddini, S.A. Design, Microfabrication and Characterization of Capillary Force Actuators. Ph.D. Thesis, University of Virginia, Charlottesville, VA, USA, 2008.

11. Knospe, C.; Nezamoddini, S.A. Capillary force actuation. J. Micro-Nano Mechatron. 2009, 5, 57-68. [CrossRef]

12. Mibus, M.; Jensen, C.; Hu, X.; Knospe, C.R.; Reed, M.L.; Zangari, G. Dielectric breakdown and failure of anodic aluminum oxide films for electrowetting systems. J. Appl. Phys. 2013, 114, 014901. [CrossRef]

13. Barth, C. Capillary Force Actuation for Achieving Large Deflections of an Elastomeric Membrane at low Voltages. Master's Thesis, University of Virginia, Charlottesville, VA, USA, August 2016.

14. Verheijen, H.; Prins, M. Reversible electrowetting and trapping of charge: Model and experiments. Langmuir 1999, 15, 6616-6620. [CrossRef]

15. Knospe, C.R.; Barth, C.A. Actuation of elastomeric micro devices via capillary forces. In Advanced Mechatronics and MEMS Devices II; Zhang, D., Wei, B., Eds.; Springer: Zurich, Switzerlands, 2017; pp. 1-18, ISBN 978-3-319-32180-6.

(C) 2018 by the authors. Licensee MDPI, Basel, Switzerland. This article is an open access article distributed under the terms and conditions of the Creative Commons Attribution (CC BY) license (http:/ / creativecommons.org/licenses/by/4.0/). 
Article

\title{
Numerical Demonstration of In-Tube Liquid-Column Migration Driven by Photoisomerization
}

\author{
Kei Nitta ${ }^{1}$ and Takahiro Tsukahara ${ }^{1,2, *(D)}$ \\ 1 Department of Mechanical Engineering, Tokyo University of Science, 2641 Yamazaki, \\ Noda, Chiba 278-8510, Japan; 7517651@ed.tus.ac.jp \\ 2 Water Frontier Science \& Technology Research Center (W-FST), Research Institute for Science \& Technology, \\ Tokyo University of Science, 1-3 Kagurazaka, Shinjuku-ku, Tokyo 162-8601, Japan \\ * Correspondence: tsuka@rs.tus.ac.jp; Tel.: +81-4-7122-9352
}

Received: 30 September 2018; Accepted: 17 October 2018; Published: 20 October 2018

check for updates

\begin{abstract}
Droplet manipulation by light-induced isomerization was numerically demonstrated and investigated regarding the driving mechanism. Such a non-invasive manipulation of a droplet in a microchannel can be realized, for example, by the use of watery solution of photoresponsive surfactant that exhibits the isomerization. Due to variable fluid properties between the cis and trans isomers, one-side light irradiation on a liquid column in a tube would lead to some kind of imbalance between the two ends of the liquid column and then drive droplet migration. The present numerical simulations of air-liquid two-phase flow and its scalar transport of the isomer, considering the variable static contact angle, agreed quantitatively with the experimental results in terms of the migration speed. This fact supports the contention that the droplet migration is more likely to be driven by an imbalance in the wettability, or the contact angle. The migration speed was found to be less dependent on the liquid-column length, but proportional to the tube diameter.
\end{abstract}

Keywords: computational fluid dynamics; droplet manipulation; lab-on-a-chip; microfluidics; non-invasive control; photochemical reaction; photoresponsible surfactant; surface tension; two-phase flow; wettability

\section{Introduction}

Droplet manipulation techniques for microfluidic devices and lab-on-a-chip have attracted much attention in various fields such as medicine, chemistry, and biology [1-5]. This is because such an analytic operation on a micro scale increases the surface-to-volume ratio, thus obtaining many advantages, for instance, minituarization of samples, high-speed reaction, and downsizing of devices. Hence, several researches have devised a method of fluid driving in a micro-scale channel by changing the surface tension and wettability, e.g., a plasma-etched polymer nanostructure that enhances the droplet mobility [6], dielectrophoresis that employs an electric dipole moment by immersing an electrocode in a channel [7], and EWOD (Electrowetting on Dielectric) that generates the wettability gradient due to static electricity [8]. However, the challenges related to those methods include not only the fabrication of the channel but also the contamination by contact and the difficultly of flexible and selective manipulation. An alternative method using light as an external stimulus has several advantages such as not requiring the fabrication of a channel, simple adjustment of the stimulus, flexible and selective manipulation, and less contamination due to the non-contact [9]. In addition, it can be utilized on a living body due to its non-invasiveness.

There exist various ways of driving liquid by light irradiation: optical tweezers [10,11], photothermal Marangoni flow [12-15], and photoisomerization [16-23]. Optical tweezers is a method that uses the radiation pressure of light, but its corresponding force is very small, in the order of 
pico-newtons. The use of photothermal Marangoni flows should be accompanied by heat, together with the light irradiation. This method is flexible and selectively operable, and the resulting force is sufficient for manipulation; however, it cannot be applied to a substance that is denatured by heat. On the other hand, the fluid manipulation by utilizing cis-trans photoisomerization can avoid the use of heat, although it is necessary to change the fluid properties in response to light.

As shown in Figure 1, the cis-trans photoisomerization is a property in which the cis and trans isomers are reversibly changed by light of a specific wavelength such as ultraviolet (UV) light [24]. The isomers are represented by the same molecular formula but different molecular structures. As the molecular structure changes, fluid properties such as the contact angle and surface tension are varied. Here, the reaction rate constant $k$ represents a rate at which the reactants increase or decrease in a chemical reaction. In the photoisomerization, $k$ represents the degree of photo-induced change between the cis isomer and the trans isomer. Azobenzene is a representative example [25-28]. In previous studies, droplets on a substrate were manipulated by performing photoisomerization on the substrate and changing wettability [16-20], or on photoresponsive surfactant [20-23]. Recently, Muto et al. [23] demonstrated the manipulation of a liquid column in a millimeter-scale glass tube. Its droplet manipulation was done by UV-light irradiation on a one-side surface of the liquid column generating differences in the contact angle and surface tension between both sides of the finite liquid column. The mobility of the migrated liquid column was reported to depend on the liquid column length. However, such an experimental demonstration might often suffer from a pinning effect that cannot be avoided, and its flow dynamics and developments of each isomer distribution are not fully understood, thus increasing the difficulty of experimental measurement. To the authors' knowledge, no numerical simulation of such a liquid driving has been performed.

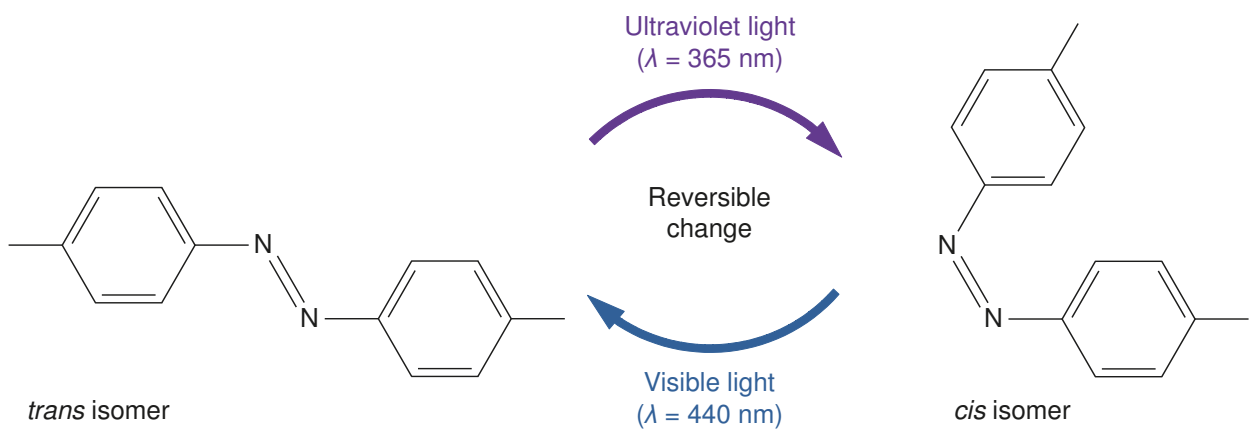

Figure 1. Schematic diagram of the photochemical isomerization, reproduced on the basis of Ref. [23]. The cis isomer irradiated with visible light should change into the trans isomer, while the trans isomer irradiated with ultraviolet (UV) light would change into the cis isomer. These two isomers may exhibit different fluid properties. The rate at which the number of isomers increases or decreases by photo-induced isomerization is expressed as a reaction rate constant $k$.

In order to reveal the light-induced droplet-migration phenomenon due to cis-trans isomerization in a watery solution of photoresponsive surfactant, we carried out a numerical study of in-tube liquid columns, considering the variable contact angle and surface tension. We used a framework of OpenFOAM ${ }^{\circledR}$ (version 2.3.1) [29], which is an open source software and has been verified by benchmark tests for multiphase flows [30-34]. The Volume-of-Fluid (VoF) method [35] is used as an interface-capturing method, and the Continuum-Surface-Force (CSF) model [36] is applied to calculate the surface tension on liquid-air interfaces. In addition, we have implemented the Continuous-Species-Transfer (CST) method [37] to express the cis/trans-isomer transports more accurately. We discuss a validation with the experiment [23], and numerically investigate the isomer distribution and the effects of the liquid column length and radius on the liquid-column migration. 


\section{Problem Setting: A Photoisomerizable Liquid Column in a Tube}

We focus on an experimental demonstration performed by Muto et al. [23] and employ a similar problem setting for our numerical analysis. Figure 2 shows the present analysis object, which is a liquid column given in an infinite straight tube with a constant radius $R$. The liquid column was initially placed at the center of the computational domain. A UV light is assumed to be irradiated on the right half of the domain: see Figure 2. The irradiation is started from the state of all trans isomers in the liquid of interest; that is, the initial $C_{c i s}$ was 0 in the entire domain. Since the tube is on the millimeter-scale, as tested by Muto et al. [23], the UV light is assumed not to decay throughout the liquid [38]. In the simulation, we used a wedge mesh which consisted of a single grid cell in the circumferential direction by assuming an axisymmetric flow with respect to the $z$ axis. This allows us to reduce the computational cost by keeping fine meshes in the other directions. While Muto et al. [23] used a sufficiently-long open tube for their measurement, it is practically difficult to simulate such a system rigorously. Two different boundary conditions in the $z$ direction were tested in this study: the periodic boundary condition (PBC) and the inlet/outlet boundary condition. On the tube surface, the no-slip boundary condition was applied. The initial length of the gas phase on both sides of the liquid column was set at $L_{g}=15 \mathrm{~mm}$, regardless of the liquid column length $L_{c}$.
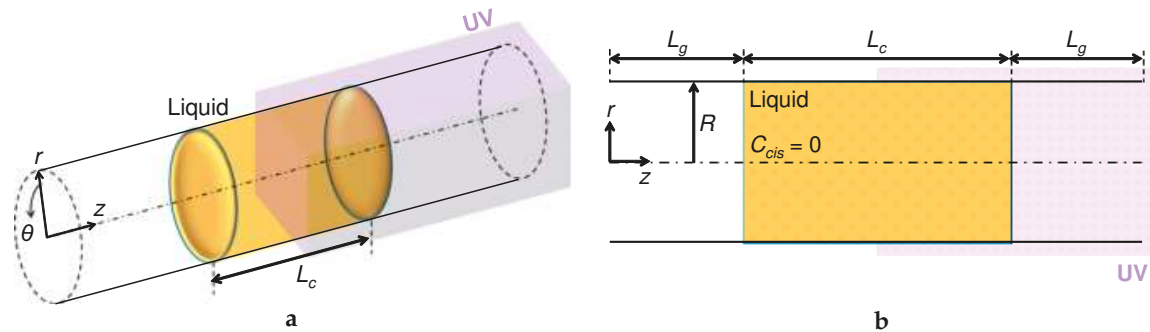

b

Figure 2. Coordinate system and configuration of an in-tube photoisomerizable liquid column to be irradiated partially with UV light. (a) Bird's eye view; (b) cross-sectional view. The liquid column is initially located at the center of the tube domain and in the form of a simple column shape, being surrounded by air. The length of the liquid column is denoted as $L_{c}$, while that of the gas phase is $L_{g}$.

Table 1 shows the fluid properties of our present targets. Since we simulated both the air and liquid phases simultaneously, the air properties at room temperature were given. Essentially important properties in the present problem are the contact angle $\vartheta$ and the surface tension $\sigma$, as explained in Section 1 . Those values vary depending on the isomer, and the liquid of our interest reveals rather hydrophilic features with the cis-isomer: the contact angle of purely cis-isomer liquid, $\vartheta_{c i s}$, is slightly lower than that for the trans-isomer, $\vartheta_{\text {trans }}$. We referred to the values of $\vartheta_{\text {cis }}$ and $\vartheta_{\text {trans }}$ measured experimentally by the extension/contraction method, and $\sigma_{c i s}$ and $\sigma_{\text {trans }}$ measured by the pendant drop method [39]. The reaction rate constant $k$ for the photoisomerization was identified by ${ }^{1} \mathrm{H}-\mathrm{NMR}$ measurement. The concentration diffusivity $D$ for the isomer diffusion in each fluid was chosen as a typical value: cf., Ref. [28].

Table 1. Fluid properties: $\rho$, density; $\mu$, viscosity; $D$, mass concentration diffusivity; $\vartheta$, static contact angle on the solid wall; $\sigma$, surface tension; and $k$, reaction rate constant.

\begin{tabular}{|c|c|c|c|c|c|c|c|c|c|c|}
\hline \multicolumn{2}{|c|}{$\rho\left(\mathrm{kg} / \mathrm{m}^{3}\right)$} & \multicolumn{2}{|c|}{$\mu(\mathrm{mPa} \cdot \mathrm{s})$} & \multicolumn{2}{|c|}{$D\left(\mathrm{~m}^{2} / \mathrm{s}\right)$} & \multicolumn{2}{|c|}{$\vartheta\left({ }^{\circ}\right)$} & \multicolumn{2}{|c|}{$\sigma(\mathrm{mN} / \mathrm{m})$} & \multirow[t]{2}{*}{$k\left(\mathrm{~s}^{-1}\right)$} \\
\hline Liquid & Air & Liquid & Air & Liquid & Air & Cis & Trans & Cis & Trans & \\
\hline 992.3 & 1.247 & 0.890 & 0.018 & $10^{-9}$ & $10^{-5}$ & 31.4 & 36.6 & 41.0 & 40.0 & 0.0225 \\
\hline
\end{tabular}




\section{Numerical Procedure}

\subsection{Governing Equations for Fluid Motions}

Although the actual system of our interest consists of incompressible liquid and compressible air contained in a tube, we considered the air phase as incompressible for simulating the fluid behavior. This assumption allows us to use the governing equations of incompressible and immiscible gas-liquid two-phase flows: the equation of continuity

$$
\nabla \cdot \mathbf{u}=0
$$

and the Navier-Stokes equation

$$
\rho\left\{\partial_{t} \mathbf{u}+(\mathbf{u} \cdot \nabla) \mathbf{u}\right\}=-\nabla p+\mu \nabla^{2} \mathbf{u}+\mathbf{f}_{\sigma},
$$

which includes a surface tension force $\mathbf{f}_{\sigma}$ that works on the liquid-air interfaces based on the Continuum-Surface-Force (CSF) model [36]. In the present study, all simulations were under the zero-gravity condition. We used the Volume-of-Fluid (VoF) method [35], which is a well-known way to capture an interface between two different fluids. In this method, the advection equation of the VoF function $\alpha$ can be written as

$$
\partial_{t} \alpha+(\mathbf{u} \cdot \nabla) \alpha=0
$$

The VoF function $\alpha$ describes the liquid fraction in each computational grid cell to determine the two-fluid allocation. In the present air-liquid two-phase flow, we defined it as

$$
\alpha= \begin{cases}1 & : \text { Liquid phase } \\ 0 & : \text { Air phase } \\ (0,1) & : \text { Interface }\end{cases}
$$

Instead of using simply Equation (3), one may compute the following equation with an artificial term with the aim of sharpening the liquid-air interface.

$$
\partial_{t} \alpha+(\mathbf{u} \cdot \nabla) \alpha+\nabla \cdot\left(\alpha(1-\alpha) \mathbf{u}_{\mathrm{r}}\right)=0
$$

The third term on the left hand side of Equation (5) represents the artificial interface compression term, in which the compression velocity $\mathbf{u}_{\mathrm{r}}$ is calculated, as follows:

$$
\mathbf{u}_{\mathrm{r}}=\mathbf{n}_{f} \min \left(C_{\alpha}\left|\frac{\phi}{S_{f}}\right|,\left|\frac{\phi}{S_{f}}\right|_{\max }\right) .
$$

Here, $\mathbf{n}_{f}$ is the normal vector of the interface in a grid-cell control volume, $\phi$ is the mass flux, $S_{f}$ is the area of the interface in the control volume, and $C_{\alpha}$ is an adjustable coefficient. While $C_{\alpha}$ can be changed arbitrary, we decided to set $C_{\alpha}=0$ in this study to make unavoidable spurious currents less pronounced, as discussed in Appendix A.1.

The local density $\rho$ and viscosity $\mu$ at each grid cell depend on $\alpha$ :

$$
\begin{aligned}
& \rho=\rho_{\mathrm{l}} \alpha+\rho_{\mathrm{g}}(1-\alpha), \\
& \mu=\mu_{1} \alpha+\mu_{\mathrm{g}}(1-\alpha) .
\end{aligned}
$$

The surface tension term in Equation (2) is expressed as

$$
\mathbf{f}_{\sigma}=\sigma \kappa \nabla \alpha
$$


in the CSF model [36]. The unit normal vector $\mathbf{n}$ and the curvature $\kappa$ of a local interface can be expressed by the VoF function $\alpha$, respectively, as

$$
\mathbf{n}=\frac{\nabla \alpha}{|\nabla \alpha|}, \quad \kappa=-\nabla \cdot \mathbf{n} .
$$

Note here that Equation (9) includes only the normal force on the liquid-air interface, neglecting the tangential force. The normal force induces the Laplace pressure, while the Marangoni convection may be triggered by tangential force on the interface. Compared to the normal force, the expected tangential force would be much smaller in this study, because the difference between $\sigma_{c i s}$ and $\sigma_{\text {trans }}$ is almost negligible relative to its absolute value, as given in Table 1 .

\subsection{Representation of Cis-/Trans-Isomer Liquid}

Since the driving force exerted on a liquid column by the cis-trans photoisomerization is induced by the imbalance of the surface tension and contact angle (or Laplace pressure), it is necessary to express numerically the two different states of either the cis or trans isomer and to model the exchange between them due to the photoisomerization. Then, let us introduce $C_{c i s}$, which is defined in each computational grid cell as the volume-fraction ratio of the cis isomer:

$$
C_{c i s}= \begin{cases}1 & : \text { All cis isomer } \\ 0 & : \text { All trans isomer } \\ (0,1) & : \text { Mix of cis \& trans isomers }\end{cases}
$$

Both the contact angle and surface tension were determined as a function of $C_{c i s}$ :

$$
\begin{gathered}
\vartheta=\vartheta_{c i s} C_{c i s}+\vartheta_{\text {trans }}\left(1-C_{c i s}\right), \\
\sigma=\sigma_{c i s} C_{c i s}+\sigma_{\text {trans }}\left(1-C_{c i s}\right) .
\end{gathered}
$$

A validation test was performed on a simple droplet on the flat wall with a variable contact angle that depends on $C_{c i s}$, and we confirmed a reasonable $C_{c i s}$-dependence of the contact angle as well as a response of the droplet wetting to UV-light irradiation, as presented in Appendix A.2.

\subsection{Transport of the Cis/Trans Isomer with the CST Method}

The transport equation of the isomer ratio $C_{c i s}$ can be written as follows:

$$
\partial_{t} C_{c i s}+(\mathbf{u} \cdot \nabla) C_{c i s}=D \nabla^{2} C_{c i s}+R,
$$

where $R$ is the source term due to the photochemical reaction and $D$ is the arithmetic mean diffusivity:

$$
D=D_{1} \alpha+D_{g}(1-\alpha)
$$

In the actual phenomenon, no transport of the isomer across the liquid-air interface should occur. However, unavoidable spurious currents, i.e., numerically-artificial flows, in simulation may lead to scalar transport across the interface. To suppress such a leakage of scalar (or $C_{c i s}$ in this study) across the liquid-air interface, we implemented the Continuous-Species-Transfer (CST) method [37]. Then, Equation (14) is reformulated into

$$
\partial_{t} C_{c i s}+(\mathbf{u} \cdot \nabla) C_{c i s}=\nabla \cdot\left(D \nabla C_{c i s}+\Phi\right)+R,
$$

where $\Phi$ is the discontinuity term and it works at the interface, as follows: 


$$
\begin{aligned}
\Phi=- & \left(D_{1}-D_{\mathrm{g}}\right) \alpha\left(\frac{1}{\alpha+(1-\alpha) / H}\right) \\
& +\frac{C_{c i s}}{\alpha+(1-\alpha) / H}\left\{\frac{1}{H} \frac{D_{1}-D_{\mathrm{g}}}{\alpha+(1-\alpha) / H}-\left(D_{1}-\frac{D_{\mathrm{g}}}{H}\right)\right\} \nabla \alpha
\end{aligned}
$$

The degree of suppression can be calibrated by the Henry coefficient $H$. We set $H=100$ : see also Appendix A.1.

It should be noted that, in the present simulation, only the change from the trans to cis photoisomerization by UV-light irradiation was expressed by

$$
R=\alpha\left\{k\left(1-C_{c i s}\right)\right\} \delta_{\mathrm{UV}}
$$

but the opposite change from the cis to trans photoisomerization (by visible light) was not considered. Because the latter photoisomerization would not provide any contribution to the liquid-droplet migration under the present condition. Here, $k$ in Equation (18) is the reaction rate constant of change from trans to $c i s$, and $\delta_{\mathrm{UV}}$ is

$$
\delta_{\mathrm{UV}}= \begin{cases}1 & : \text { UV-light irradiation part } \\ 0 & : \text { Others }\end{cases}
$$

Equation (18) expresses that the UV-photoisomerization occurs only in a liquid portion that is irradiated with UV light, by multiplying $\alpha$ and $\delta_{\mathrm{UV}}$. Basically, $C_{c i s}=0$ should be kept in the air and/or non-irradiated part.

\section{Results}

\subsection{Grid Resolution: Comparative Validation with Experimental Results}

Figure 3 shows our obtained migration distance $z_{m}$, normalized by the liquid column length $L_{c}$, from the initial position as a function of the irradiation time $T$. The instance of $T=0$ corresponds to the beginning of the simulation as well as that of the UV-light irradiation. The migration distance $z_{m}$ from the initial position $z_{0}$ was calculated from the axial shift of the center of gravity of the liquid column.

$$
z_{m}=\frac{\int_{V} \alpha \cdot z \mathrm{~d} V}{\int_{V} \alpha \mathrm{d} V}-z_{0}
$$

According to the experimental results shown also in Figure 3, the liquid column would be transported at a constant speed toward the UV light side (i.e., to the positive $z$ direction). As $z_{m} / L_{c}$ approaches 0.5 , the migration speed appears to decelerate and finally the liquid column stops moving. Note here that $z_{m} / L_{c}=0.5$ corresponds to the situation that the entire liquid column has just entered the UV area. To demonstrate such phenomena in our simulation, we first examined the required grid resolution, or the number of grids in the axial- $\left(N_{z}\right)$ and radial- $\left(N_{r}\right)$ directions. Our parametric study for $N_{z}=500-2000$ and $N_{r}=25-300$ revealed that a combination of $\left(N_{z}, N_{r}\right)=(512,256)$ was required for the case of $L_{c}=20 \mathrm{~mm}$, as shown in Figure 3a. The linear motion in the initial stage could be reproduced even with lower resolutions, but the migration speed was overestimated remarkably. Furthermore, regarding the $z$-direction boundary condition, the periodic boundary condition (PBC) was found to provide a better result, compared to the inlet/outlet condition. 


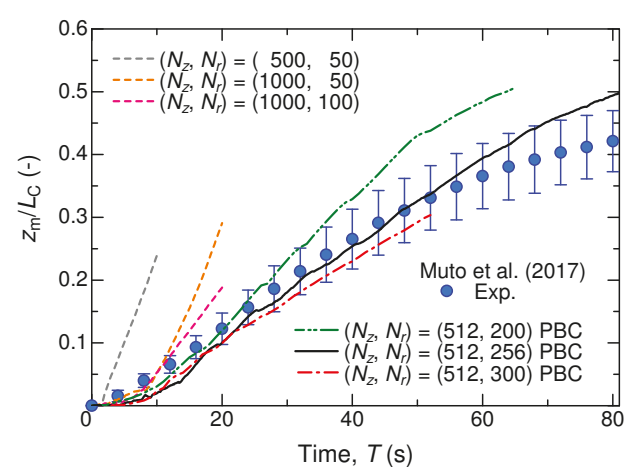

(a)

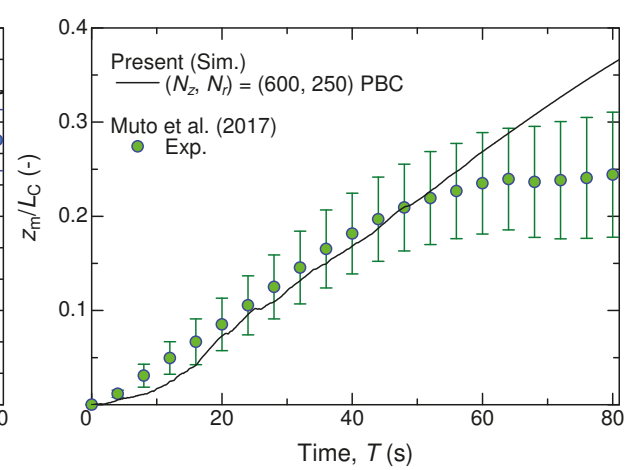

(b)

Figure 3. Temporal variations of the migration distance $z_{m}$ of the liquid column normalized by the liquid column length $L_{c}$, calculated by Equation (20), and comparison with the experimental results [23]. In the legend, 'PBC' represents a case with the periodic boundary condition in the $z$ direction. (a) $L_{\mathcal{C}}=20 \mathrm{~mm},(\mathbf{b}) L_{\mathcal{C}}=30 \mathrm{~mm}$. The tube radius is $R=1.25 \mathrm{~mm}$.

As for a longer liquid column of $L_{c}=30 \mathrm{~mm}$, we achieved good quantitative agreement with the experimental results, as shown in Figure $3 \mathrm{~b}$. Similar to the shorter case mentioned above, the liquid column exhibited an acceleration period until $T<20 \mathrm{~s}$, and it attained a constant migration speed at $T \approx 20 \mathrm{~s}$. Whereas the linear motion obviously terminated before reaching $z_{m} / L_{c}=0.5$ in the experiment, the present simulation shows a continuous linear motion until $z_{m} / L_{c}$ reaches 0.5 . Such a deviation in this late stage of the droplet migration must be attributed to the pinning effect that cannot be simulated with the present code. At least, in the initial stage including the linear motion, the photoisomerization-induced droplet migration has been reasonably demonstrated by our simulation.

Figure 4 shows the developing distribution of $C_{c i s}$ in the $z-r$ section of the domain including both the air and liquid phases. The top panel in the figure represents the initial condition. At $T=0.01 \mathrm{~s}$, the liquid-air interface is already concave due to the wettability with $\vartheta<90^{\circ}$ and surface tension. This implies that the timescale of surface deformation is much faster than the reaction rate of the photoisomerization of interest. From $T=20 \mathrm{~s}$, one can recognize that the isomers in the UV-light irradiation side changed gradually from trans to cis with time, but it can also be recognized that an axial shift of the liquid column already occurred. Figure 5 shows the temporal variation of the distribution of $C_{c i s}$ on the $z$-axis inside the liquid column, which is marked with a white dashed line in Figure 4. The horizontal axis represents the $z$ subtracted by the moving distance $z_{m}$ of the liquid column: $z-z_{m}=0$ corresponds to the center of the liquid column and the air-liquid interfaces locate at about $z-z_{m}= \pm 10 \mathrm{~mm}$ because $L_{c}=20 \mathrm{~mm}$. As seen in the figure, $C_{c i s}$ increases firstly on the UV-light irradiation side because of the photoisomerization. As time progresses, the plateau of the $C_{c i s}$ profile becomes high and narrow $\left(z-z_{m}=2-8 \rightarrow 6-7\right.$ as $\left.T=20 \rightarrow 80\right)$, while the values gradually start to increase also in the column that is half on the non-irradiated side. This is the result of competition between the photoisomerization reaction and the incoming trans-isomer liquid from the non-irradiated side. At $T=80 \mathrm{~s}$, the non-zero $C_{c i s}$ region reaches the interface on the non-irradiated side. This is to be expected due to the fact that the liquid column starts to decelerate its migration at about $T=80 \mathrm{~s}$. From this, we may draw the conclusion that the liquid column would stop when the cis isomer concentrations at both sides of the liquid column are comparable. 

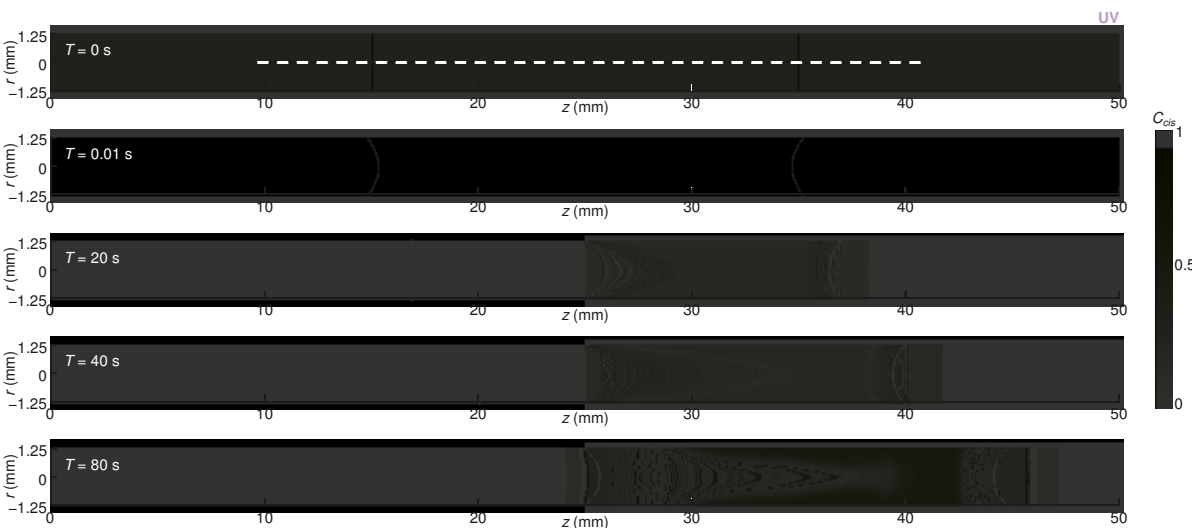

Figure 4. Cross-sectional views of the liquid column with a length of $L_{\mathcal{C}}=20 \mathrm{~mm}$ and the developing distribution of the ratio of the cis isomer, $C_{c i s}$, during the droplet migration driven by photoisomerization by a partial UV-light irradiation, at time instances of $0,0.01,20,40$, and $80 \mathrm{~s}$ (from top to bottom). The tube radius is $R=1.25 \mathrm{~mm}$. Contour shows the ratio of the cis isomer: $C_{c i s}=1$, all cis isomers; $C_{c i s}=0$, all trans isomers. Cyan solid lines indicate the liquid-air interface positions; purple-colored backgrounds (on the right half of the domain) represent the UV-light irradiated parts.

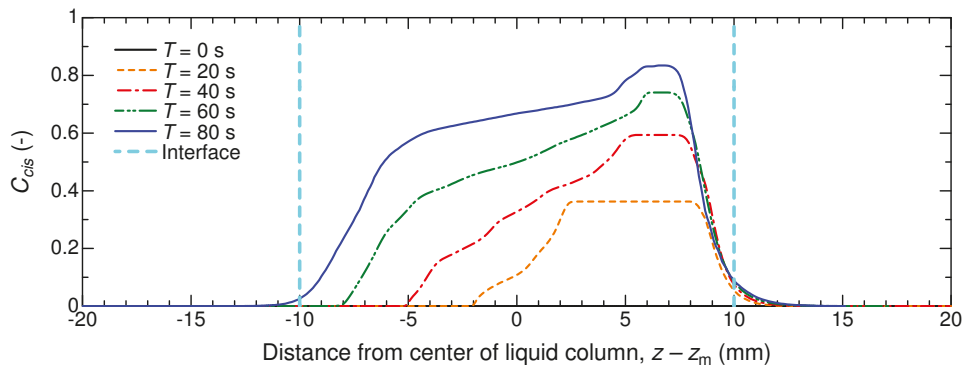

Figure 5. Temporal variation of $C_{c i s}$ along the white dashed line in Figure 4 for $L_{c}=20 \mathrm{~mm}$. The horizontal axis represents the relative position with respect to the center of the liquid column. The interface on the UV-light irradiation side is located at $z-z_{m} \approx 10 \mathrm{~mm}$, and the opposite one is at $z-z_{m} \approx-10 \mathrm{~mm}$.

\subsection{Influence of the Liquid Column Length and Tube Radius}

Furthermore, the dependence of the migration motion on the column length $L_{c}$ was investigated. Four column lengths of 5, 10, 20, and $30 \mathrm{~mm}$ were examined under the same system with a fixed tube radius $R=1.25 \mathrm{~mm}$, and the initial exposed column length was set to $L_{c} / 2$ for all cases. Figure 6 compares the four cases, where the profiles are plotted in a dimensional form of millimeter versus second. From the gradient of each profile, the migration speed can be determined. The present numerical results obviously reveal the independence of the migration speed on the column length, at least in the initial stage of the migration. This aspect contradicts the experimental observation [23], but this mismatch might be due to a pinning effect on an actual tube in the experiment. With the increasing $L_{c}$, the migration distance is elongated further. The constant linear motion is found to be at a speed of $\approx 0.15 \mathrm{~mm} / \mathrm{s}$, irrespective of $L_{c}$. This speed could be varied by changing the fluid properties as well as the tube geometry. Further investigation on the fluid-property dependence of the migration speed is potentially interesting for a practical application of the photoisomerization in a droplet manipulation. 


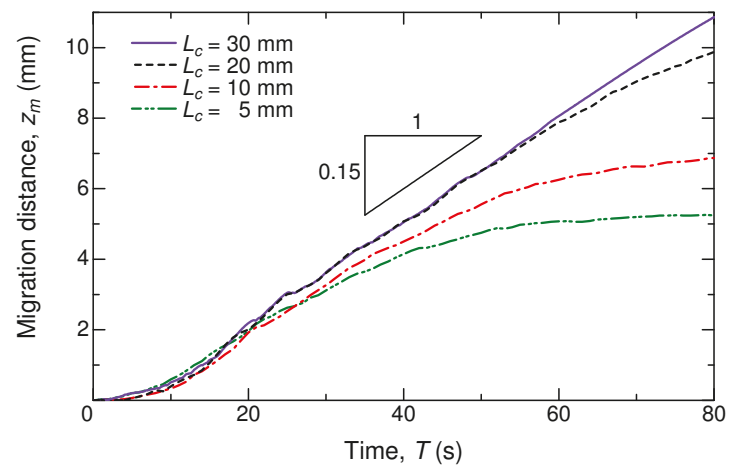

Figure 6. Time series of the migration distance of the liquid column for different liquid column lengths, without normalization. With $R=1.25 \mathrm{~mm}, N_{z}=350-600$, and $N_{r}=256$.

The dependence on the tube radius was not investigated experimentally [23], so we numerically examined it under the same fluid conditions but with various $R$. Figure 7 shows results for $R=0.25-2.5 \mathrm{~mm}$. The temporal evolution of the migration distance did not change qualitatively with a different $R$, but the migration speed was found to vary significantly. Normalized by each tube radius, the profiles are scaled well, as given in Figure $7 \mathrm{~b}$. According to the present numerical result, we conjecture that the migration speed is roughly proportional to the tube radius. This aspect is consistent with Muto et al. [23] with respect to their discussion on the net driving force $F$ that was exerted on the liquid column. They suggested a form of

$$
F=2 \pi R\left(\sigma_{\text {cis }} \cos \vartheta_{a-\text {-cis }}-\sigma_{\text {trans }} \cos \vartheta_{\text {r-trans }}\right),
$$

where $\vartheta_{a-c i s}$ and $\vartheta_{r \text {-trans }}$ denote the advancing contact angle of the cis isomer and the receding one of the trans isomer, respectively. Actually, Equation (21) does not relate to the column length $L_{c}$, but includes the tube radius $R$, supporting also the $L_{c}$-independency observed in Figure 6.

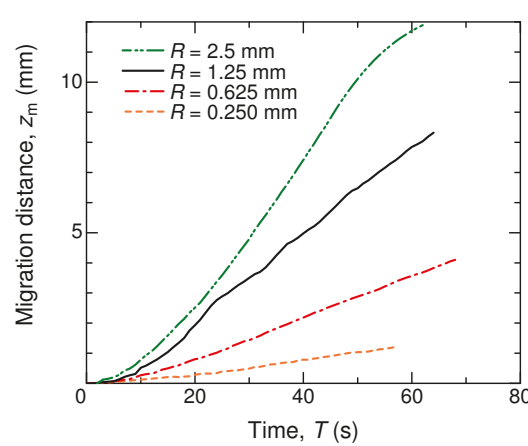

(a)

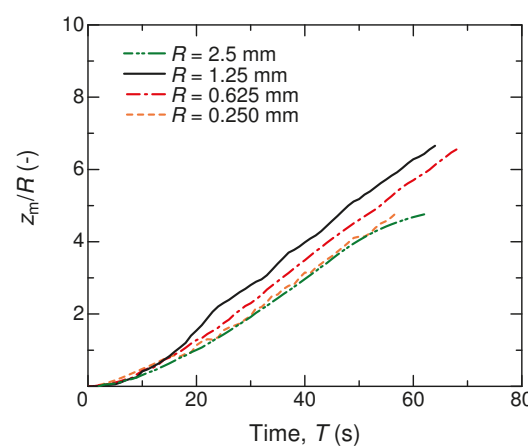

(b)

Figure 7. Time series of the migration distance of the liquid column for different tube radii in units of millimeter (a). In (b), the migration distance is normalized by the tube radius. With $L_{\mathcal{c}}=20 \mathrm{~mm}$, $N_{z}=512$, and $N_{r}=256$.

Last, but not least, we would argue that the main driving force of the liquid-column migration by photoisomerization is attributed to the difference between the static contact angles $\vartheta_{c i s}$ and $\vartheta_{\text {trans }}$. In the context of Equation (21), the given condition of $\vartheta_{c i s}<\vartheta_{\text {trans }}$ reasonably results in $F>0$. In terms of the surface tension, $\sigma_{c i s}$ that is slightly larger than $\sigma_{\text {trans }}$ might also contribute to the onset of a positive $F$, but a comparison such as $\cos \vartheta_{\text {cis }} / \cos \vartheta_{\text {trans }}>\sigma_{\text {cis }} / \sigma_{\text {trans }}$ (cf. Table 1) implies 
that the contact-angle variation would account for the imbalance between the two ends of the liquid column. On the other hand, Muto et al. [23] declared that the migration was induced by a difference in the surface tension rather than the contact angle, because they observed $\vartheta_{a \text {-cis }} \ll \vartheta_{r \text {-trans }}$; that is, $\cos \vartheta_{a-c i s}<\cos \vartheta_{\text {r-trans }}$ and this fact should demand $\sigma_{c i s} \gg \sigma_{\text {trans }}$ to derive the positive $F$ from Equation (21). However, they still suffered from difficulties in measuring the dynamics contact angle and surface tension and did not perform quantitative evaluation. Although our conclusion opposes their hypothesis, the quantitative agreement in the migration speed with the experimental result supports the present conclusion that the wettability, or the contact angle, is key to the driving force for the present droplet manipulation.

\section{Conclusions}

We performed numerical simulations to investigate the liquid-column migration driven by the cis-trans photoisomerization phenomenon that was experimentally demonstrated by Muto et al. [23]. The liquid-column migration should be induced by an imbalance in the wettability, or the contact angle and surface tension, between the two ends of the liquid column. To track the liquid-air interface, we employed the VoF method in conjunction with the CST model and the CSF model. In order to express the developing distribution of the cis / trans isomer, we also defined a volume-fraction ratio of the cis isomer, on which both the contact angle and surface tension were dependent. Neglecting the gravity, evaporation, thermocapillary, and dynamic contact angle, our simulation successfully demonstrated the photoisomerization-induced droplet migration and achieved good agreement with the experimental results. Our conclusion regarding the mechanism of the present droplet manipulation is that the driving force is caused mainly by the imbalance in the wettability, or the contact angle, between the two ends of liquid column rather than the surface tension. Through a numerical investigation of the cis isomer distribution, which is difficult to measure experimentally, we confirmed that the liquid-column migration terminated when the cis isomer distribution reached the non-irradiated region. We also found that the migration speed was less dependent on the liquid-column length and was proportional to the tube diameter.

Author Contributions: K.N. and T.T. conceived and designed the simulations; K.N. performed the numerical simulations and analyzed the data; K.N. and T.T. wrote the paper. All authors discussed the results and commented on the manuscript.

Funding: This research received no external funding.

Acknowledgments: We are particularly grateful to Masahiro Motosuke, Ken Yamamoto, and Masakazu Muto, at Tokyo University of Science, for their own experimental data and valuable discussions. Some simulations were performed using SX-ACE at Cybermedia Center, Osaka University. We also thank the reviewers for their insightful and constructive comments.

Conflicts of Interest: The authors declare no conflict of interest.

\section{Appendix A. Preliminary Tests}

The present simulations were executed in a framework of a customized opensourse solver in OpenFOAM ${ }^{\mathbb{R}}$ [29]. By tuning the solver code, we combined several schemes of the CST model and the CSF model to obtain sharp liquid-air interfaces and to suppress scalar mass flux across the interfaces. For those models, we should determine the optimal values of the relevant numerical parameters. In particular, the Henry coefficient $H$ in Equation (17) and $C_{\alpha}$ in Equation (6) are crucial parameters. As a preliminary test to examine $H$ and $C_{\alpha}$, which provide a reasonable result for the presently-given combination of fluids and scales, numerical simulations were carried out on a square droplet. In addition, the solver code was extended to simulate the photoisomerization in a part irradiated with UV light. To validate our code in terms of the modelling of the photoisomerization, we simulated a droplet on a flat plate, where the relationship between the wetting width and the contact angle is known theoretically. Figure A1 schematically illustrates the configurations of two preliminary simulations, the results of which will be reported in the following subsections. 


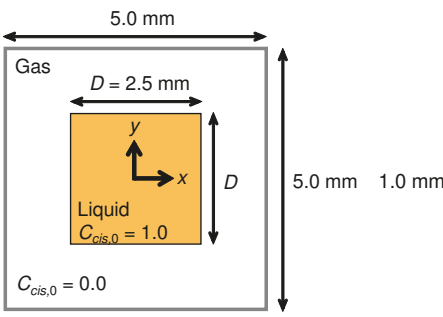

(a)

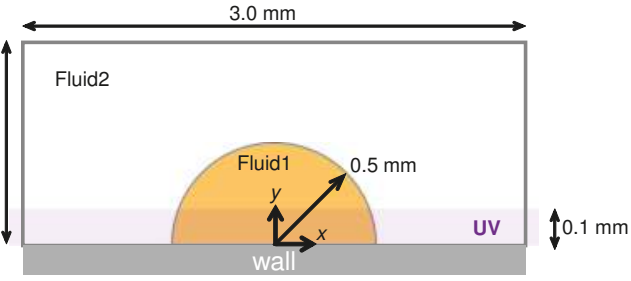

(b)

Figure A1. (a) Square liquid droplet surrounded by gas without gravity. Initially, the cis isomer $\left(C_{c i s}=1\right)$ dominates in the entire droplet. (b) Semisphere droplet on a flat wall to be partially irradiated with UV light that induces cis-trans photoisomerization. Initially, the trans isomer $\left(C_{c i s}=0\right)$ dominates in the entire domain. In this preliminary test, the trans-isomer liquid is assumed to have a contact angle of $\vartheta_{\text {trans }}=90^{\circ}$, and a UV-light irradiation area is limited only in the vicinity of the wall.

\section{Appendix A.1. Scalar Transport Near the Interface}

A square droplet with a length of $D=2.5 \mathrm{~mm}$ was given in the center of a computational domain: see also Figure A1a. The computational domain size and the number of grids were $L_{x}=L_{y}=5 \mathrm{~mm}$ and $N_{x}=N_{y}=100$, respectively. The surface tension was fixed at $\sigma_{\text {trans }}=40 \mathrm{mN} / \mathrm{m}$, since no UV-light irradiation was considered here. Other physical properties were the same as the liquid column driven by the photoisomerization, as given in Table 1 . By systematically changing the Henry coefficient to $H=0.01,1$, and 100, and the interface compression value to $C_{\alpha}=0,0.5$, and 1 , we investigated the degree of leakage of $C_{c i s}$ through the liquid-air interface and the influence of spurious currents occurring near the interface.

Figure A2 shows the cross-sectional views of the droplet, where the interface has been deformed into the circle shape by the surface tension. In the simulation without the CST model, we detected a severe leakage of the cis isomer from the droplet, as shown in (b). Figure A2c also shows a significant decrease in $C_{c i s}$ inside the droplet. By applying the CST model with a large Henry coefficient $H=100$, we successfully avoid an increase in $C_{c i s}$ outside the droplet, as visualized in (d)-(f). Non-zero $C_{\alpha}$ values may provide more suppression of variations in $C_{c i s}$ outside of the droplet, but the $C_{c i s}$ conservation inside the droplet would obviously suffer from the spurious currents that exist near the liquid-air interface, as shown in (e) and (f).

In order to confirm the suppression of leakage from the droplet by employing the CST model with $C_{\alpha}=0$ and $H=100$, we define an index representing the conservation of $C_{c i s}$ in the liquid. The following index $C_{\mathrm{cr}}$ is a ratio between an arbitrary time $T$ and the initial $t=0$, regarding the volume-integrated cis-isomer concentration:

$$
C_{\mathrm{cr}}=\frac{\left[\int_{A} C_{c i s} \cdot \alpha \mathrm{d} A\right]_{t=T}}{\left[\int_{A} C_{c i s} \cdot \alpha \mathrm{d} A\right]_{t=0}} .
$$

Here, $A$ represents the area of the computational domain, since the present simulation was performed in two-dimensional space. This ratio $C_{\mathrm{cr}}$ would be time-dependent in simulations, once a leakage of $C_{c i s}$ from the liquid to air phase occurs. In the ideal situation, $C_{\mathrm{cr}}$ should be 1 at any time. As shown in Figure A3, the case visualized also in Figure A2d indeed exhibits less deviation from the unity value. According to this result, we have chosen the CST model with $C_{\alpha}=0$ and $H=100$ for the main liquid-column simulation. 


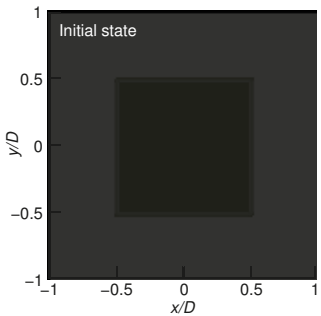

a

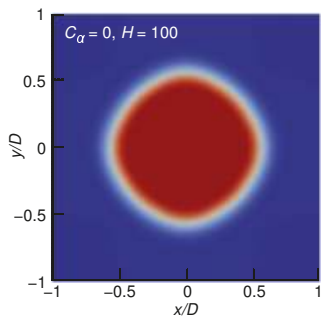

d

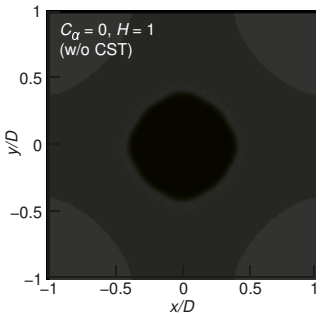

b

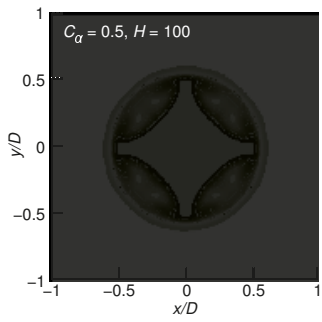

e

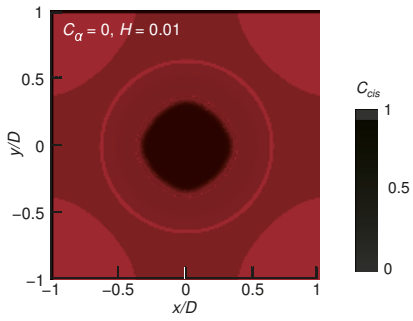

c

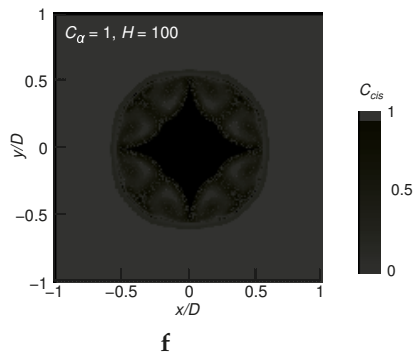

f

Figure A2. Contour of $C_{c i s}$ distribution obtained at $0.2 \mathrm{~s}$ (except for a) after the release of a free square droplet, for different values of $H$ and $C_{\alpha}$ and with/without the CST model. (a) Initial state of all cases at 0 s. (b) $C_{\alpha}=0, H=1$, without the CST method. (c) $C_{\alpha}=0, H=0.01$. (d) $C_{\alpha}=0, H=100$. (e) $C_{\alpha}=0.5, H=100$. (f) $C_{\alpha}=1, H=100$. Solid cyan line outlines the droplet shape.

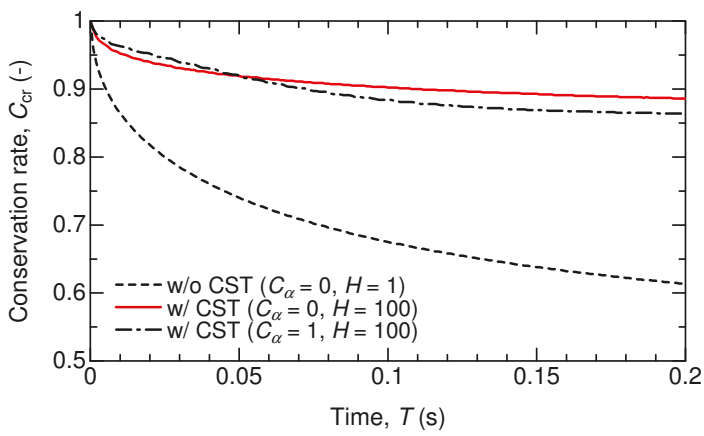

Figure A3. Comparison of the temporal variation of conservation of $C_{c i s}$ in the liquid.

\section{Appendix A.2. Changed Static Contact Angle by Photoisomerization}

An initially-hemispherical droplet with a radius of $R_{0}=0.5 \mathrm{~mm}$ was placed on the wall, as shown in Figure A1b. The computational domain size and the number of grids were $L_{x} \times L_{y}=3 \times 1 \mathrm{~mm}^{2}$ and $N_{x} \times N_{y}=150 \times 50$, respectively. The surface tension was fixed at $\sigma_{\text {trans }}=40 \mathrm{mN} / \mathrm{m}$ without any change due to photoisomerization, but the static contract angle ranged from $\vartheta_{\text {trans }}=90^{\circ}$ to $\vartheta_{\text {cis }}=30^{\circ}$. The reaction rate constant in this droplet simulation was set at a rather large value of $k=1 \mathrm{~s}^{-1}$. Other physical properties were the same as the main liquid-column simulation, as shown in Table 1.

Figure A4 shows the cross-sectional views of the droplet, where the contact angle between the liquid-air interface and the bottom solid wall can be observed to change gradually from the given $\vartheta_{\text {trans }}$ to $\vartheta_{\text {cis }}$. Such a decrease in the contact angle can be interpreted as an increase in the wettability of the liquid. 

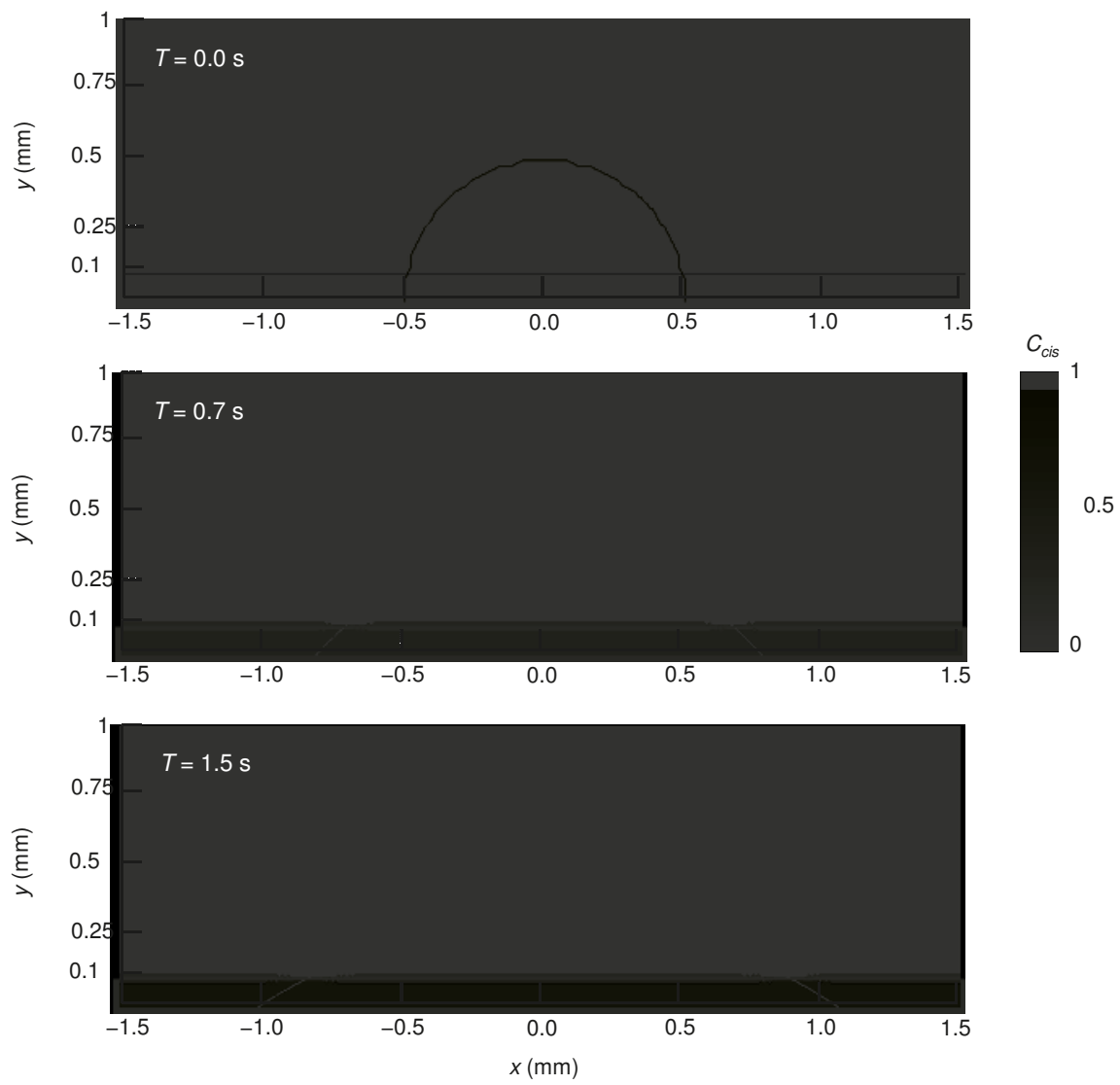

Figure A4. Temporal evolution of a droplet due to the photoisomerization-induced contact-angle change. Contour shows $C_{c i s}$. The contact angles for $C_{c i s}=0$ and 1 are $30^{\circ}$ and $90^{\circ}$, respectively. Solid cyan line outlines the droplet shape, and the purple-colored regions represent the UV-light irradiation area.

The droplet wet length $L$ can be derived theoretically from the initial droplet size $R_{0}$ and the contact angle $\vartheta$. By assuming that the inertia should be negligible compared to the surface tension and viscosity, the expression of

$$
L=2 R_{0} \sin \vartheta \sqrt{\frac{\pi}{2(\vartheta-\sin \vartheta \cos \vartheta)}}
$$

would be valid. In the present (preliminary) system, $\vartheta$ should be changed monotonically from that for the trans isomer to that for the cis isomer as a function of $C_{c i s}$, which is also time dependent in the UV-irradiated area and can be estimated mathematically on the basis of Equation (18). In Figure A5, the predicted evolution of the wet length is plotted with a dashed line. At $T=1 \mathrm{~s}, C_{c i s}$ in the light-irradiated liquid has attained $C_{c i s}=1$; that is, a state of all cis isomers in the vicinity of the wall. After that, no expansion of the wet length occurs and the droplet maintains the contact angle of $\vartheta=\vartheta_{c i s}$. Also shown in Figure A5 is the result obtained by the present simulation. It can be confirmed that the present simulation reasonably demonstrates the temporal variation of the wet length, implying the validity of our simulation code with respect to the photoisomerization and its accompanying variation of the wettability. 


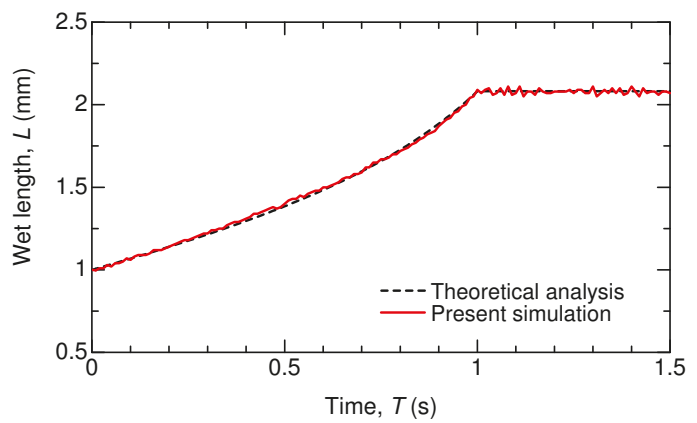

Figure A5. Temporal variation of the wet length of the droplet with the cis-trans photoisomerization by UV-light irradiation. The red line is a numerical result, while the black dashed line is a theoretical solution expressed by Equation (A2).

\section{References}

1. Mark, D.; Haeberle, S.; Roth, G.; Stetten, F.; Zengerle, R. Microfluidic lab-on-a-chip platforms: Requirements, characteristics and applications. Chem. Soc. Rev. 2010, 39, 1153-1182. [CrossRef] [PubMed]

2. Wang, Z.; Zhe, J. Recent advances in particle and droplet manipulation for lab-on-a-chip devices based on surface acoustic waves. Lab Chip 2011, 11, 1280-1285. [CrossRef] [PubMed]

3. Elvira, K.S.; Solvas, X.C.I.; Wootten, R.R.; de Mello, A.J. The past, present and potential for microfluidic reactor technology in chemical synthesis. Nat. Chem. 2013, 5, 905-915. [CrossRef] [PubMed]

4. Sackmann, E.K.; Fulton, A.L.; Beebe, D.J. The present and future role of microfluidics in biomedical reserch. Nature 2014, 507, 181-189. [CrossRef] [PubMed]

5. Pit, A.M.; Duits, M.H.; Mugele, F. Droplet manipulations in two phase flow microfluidics. Micromachines 2015, 6, 1768-1793. [CrossRef]

6. Du, K.; Jiang, Y.; Liu, Y.; Wathuthanthri, I.; Choi, C.H. Manipulation of the superhydrophobicity of plasma-etched polymer nanostructures. Micromachines 2018, 9, 304. [CrossRef]

7. Wu, T.; Suzuki, Y.; Kasagi, N. Low-voltage droplet manipulation using liquid dielectrophoresis on electret. J. Micromech. Microeng. 2010, 20, 085043. [CrossRef]

8. Lee, J.; Moon, H.; Fowler, J.; Schoellhammer, T.; Kim, C.-J. Electrowetting and electrowetting-on-dielectric for microscale liquid handling. Sens. Actuators A Phys. 2002, 95, 259-268. [CrossRef]

9. Baigl, D. Photo-actuation of liquids for light-driven microfluidics: State of the art and perspective. Lab Chip 2012, 12, 3637-3653. [CrossRef] [PubMed]

10. Ashkin, A.; Dziedzic, J.M. Radiation pressure on a free liquid surface. Phys. Rev. Lett. 1973, 30, 139-142. [CrossRef]

11. Kuo, S.C.; Sheetz, M.P. Force of single kinesin molecules measured with optical tweezers. Science 1993, 260, 232-234. [CrossRef] [PubMed]

12. Baroud, C.N.; Delville, J.P.; Gallaire, F.; Wunenburger, R. Thermocapillary valve for droplet production and sorting. Phys. Rev. E 2007, 75, 046302. [CrossRef] [PubMed]

13. Namura, K.; Nakajima, K.; Kimura, K.; Suzuki, M. Photothermally controlled Marangoni flow around a micro bubble. Appl. Phys. Lett. 2015, 106, 043101. [CrossRef]

14. Muto, M.; Yamamoto, M.; Motosuke, M. A noncontact picolitor droplet handling by photothermal control of interfacial flow. Anal. Sci. 2016, 32, 49-55. [CrossRef] [PubMed]

15. Kazuno, N.; Tsukahara, T.; Motosuke, M. Laplace pressure versus Marangoni convection in photothermal manipulation of micro droplet. Eur. Phys. J.-Spec. Top. 2017, 226, 1337-1348. [CrossRef]

16. Ichimura, K.I.; Oh, S.-K.; Nakagawa, M. Light-driven motion of liquids on a photoresponsive surface. Science 2000, 288, 1624-1626. [CrossRef] [PubMed]

17. Oh, S.-K.; Nakagawa, M.; Ichimura, K. Photocontrol of liquid motion on an azobenzene monolayer. J. Mater. Chem. 2002, 12, 2262-2269. [CrossRef]

18. Berna, J.; Leigh, D.A.; Lubomska, M.; Mendoza, S.M.; Perez, E.M.; Rudolf, P.; Teobaldi, G.; Zerbetto, F. Macroscopic transport by synthetic molecular machines. Nat. Mater. 2005, 4, 704-710. [CrossRef] [PubMed] 
19. Yang, D.; Piech, M.; Bell, N.S.; Gust, D.; Vail, S.; Garcia, A.A.; Schneider, J.; Park, C.-D.; Hayes, M.A.; Picraux, S.T. Photon control of liquid motion on reversibly photoresponsive surfaces. Langmuir 2007, 23, 10864-10872. [CrossRef] [PubMed]

20. Lv, J.; Liu, Y.; Wei, J.; Chen, E.; Qin, L.; Yu, Y. Photocontrol of fluid slugs in liquid crystal polymer microactuators. Nature 2016, 537, 179-184. [CrossRef] [PubMed]

21. Diguet, A.; Guillermic, R.-M.; Magome, N.; Saint-Jalmes, A.; Chen, Y.; Yoshikawa, K.; Baigl, D. Photomanipulation of a droplet by the chromocapillary effect. Angew. Chem. Int. Ed. 2009, 48, 9281-9284. [CrossRef] [PubMed]

22. Diguet, A.; Li, H.; Queyriaux, N.; Chen, Y.; Baigl, D. Photoreversible fragmentation of a liquid interface for micro-droplet generation by light actuation. Lab Chip 2011, 11, 2666-2669. [CrossRef] [PubMed]

23. Muto, M.; Ayako, Y.; Yamamoto, K.; Yamamoto, M.; Kondo, Y.; Motosuke, M. Photochemical migration of liquid column in a glass tube. Eur. Phys. J.-Spec. Top. 2017, 226, 1199-1205. [CrossRef]

24. Orihara, Y.; Matsumura, A.; Saito, Y.; Ogawa, N.; Saji, T.; Yamaguchi, A.; Sakai, H.; Abe, M. Reversible release control of an oily substance using photoresponsive micelles. Langmuir 2001, 17, 6072-6076. [CrossRef]

25. Shin, J.Y.; Abbott, N L. Using light to control dynamics surface tensions of aqueous solutions of water soluble surfactants. Langmuir 1999, 15, 4404-4410. [CrossRef]

26. Eastoe, J.; Dominguez, M.S.; Wyatt, P. Properties of a stilbene-containing gemini photosurfactant: Light-triggered changes in surface tension and aggregation. Langmuir 2002, 18, 7837-7844. [CrossRef]

27. Shang, T.; Smith, K.A.; Hatton, T.A. Photoresponsive surfactants exhibiting unusually large, reversible surface tension changes under varying illumination conditions. Langmuir 2003, 19, 10764-10773. [CrossRef]

28. Chevallier, E.; Mamane, A.; Srone, H.A.; Tribet, C.; Lequeux, F.; Monteux, C. Pumping-out photo-surfactants from an air-water interface using light. Soft Matter 2011, 7, 7866-7874. [CrossRef]

29. OpenFAOM. Available online: https:/ / www.openfoam.org/ (accessed on 30 September 2018).

30. Deshpande, S.S.; Anumolu, L.; Trujillo, M.F. Evaluating the performance of the two-phase flow solver interFoam. Comput. Sci. Discov. 2012, 5, 014016. [CrossRef]

31. Hoang, D.A.; Steijn, V.; Portela, L.M.; Kreutzer, M.T.; Kleijn, C.R. Benchmark numerical simulations of segmented two-phase flows in microchannels using the Volume of Fluid method. Comput. Fluids 2013, 86, 28-36. [CrossRef]

32. Klostermann, J.; Schaake, K.; Schwarze, R. Numerical simulation of a single rising bubble by VOF with surface compression. Int. J. Numer. Mech. Fluids 2013, 71, 960-982. [CrossRef]

33. Nieves-Remacha, M.J.; Yang, L.; Jensen, K.F. OpenFOAM computational fluid dynamic simulations of two-phase flow and mass transfer in an advanced-flow reactor. Ind. Eng. Chem. Res. 2015, 54, 6649-6659. [CrossRef]

34. Yang, L.; Nieves-Remacha, M.J.; Jensen, K.F. Simulations and analysis of multiphase transport and reaction in segmented flow microreactors. Chem. Eng. Sci. 2017, 169, 106-116. [CrossRef]

35. Hirt, C.W.; Nichols, B.D. Volume of fluid (VOF) method for the dynamics of free boundaries. J. Comput. Phys. 1981, 39, 201-225. [CrossRef]

36. Brackbill, J.U.; Kothe, D.B.; Zemach, C. A continuum method for modeling surface tension. J. Comput. Phys. 1992, 100, 335-354. [CrossRef]

37. Deising, D.; Marschall, H.; Bothe, D. A unified single-field model framework for Volume-Of-Fluid simulations of interfacial species transfer applied to bubble flows. Chem. Eng. Sci. 2016, 139, 173-195. [CrossRef]

38. Quickenden, T.I.; Irvin, J.A. The ultraviolet absorption spectrum of liquid water. J. Chem. Phys. 1980, 72, 4416. [CrossRef]

39. Motosuke, M.; Muto, M. (Tokyo University of Science, Tokyo, Japan). Personal communication, 2018. 


\title{
Article \\ Controlling Normal Stiffness in Droplet-Based Linear Bearings
}

\author{
Qi Ni ${ }^{1}$ and Nathan Crane ${ }^{2, *(D)}$ \\ 1 Mechanical Engineering Department, University of South Florida, Tampa, FL 33620, USA; qni@mail.usf.edu \\ 2 Mechanical Engineering Department, Brigham Young University, Provo, UT 84602, USA \\ * Correspondence: nbcrane@byu.edu; Tel.: +1-801-422-7731
}

Received: 25 September 2018; Accepted: 15 October 2018; Published: 17 October 2018

check for updates

\begin{abstract}
While capillary forces are negligible relative to gravity at the macroscale, they provide adequate force to effectively manipulate millimeter to micro meter objects. The fluidic actuation can be accomplished using droplets that also act as bearings. While rotary droplet bearings have been previously demonstrated, this paper addresses the positioning accuracy of a droplet-based bearing consisting of a droplet between a moving plate and a stationary substrate with constrained wetting region under a normal load. Key wetting cases are analyzed using both closed form analytical approximations and numerical simulations. The vertical force and stiffness characteristics are analyzed in relation to the wetting boundaries of the supporting surface. Case studies of different wetting boundaries are presented and summarized. Design strategies are presented for maximizing load carrying capability and stiffness. These results show that controlled wetting and opposing droplet configurations can create much higher stiffness fluidic bearings than simple droplets.
\end{abstract}

Keywords: surface tension; capillary; bearing; wetting

\section{Introduction}

Due to scaling relations, capillary force becomes dominant as the length scale decreases below the millimeter scale and can dominate from micrometer to millimeter scales [1]. The relatively large magnitude of the capillary force has been exploited to perform mechanical operations such as grasp and release [2,3], out-of-plane self-assembly [4], and vertical actuation via electrowetting [5]. A common gripper configuration utilizes the tensile force exerted by a capillary bridge between two wetting surfaces. When the surfaces are non-wetting (contact angle is greater than $90^{\circ}$ ), a repelling force is generated when the two surfaces are close together [6,7] as illustrated in Figure 1. Electrowetting, an apparent change in surface energy or contact angle due to electrostatic forces acting on a surface, can be used to modulate these forces [8,9].

A new class of bearing based on the capillary bridge was proposed by several groups and rotating micromachines were demonstrated in references [10-14]. The bearing surfaces were patterned by either surface coating and/or surface texture to control the location and shape of the droplet/surface contact. Single droplets, multiple drops, and fluid rings have been used to support vertical loads by surface tension. The advantages of this type of bearing are low friction, wear resistance and self-centering [10]. In addition, external force/torque can be applied either by external field to the rotating rotor [13] or through the droplet $[12,14]$ which could further reduce device size.

The same principle could be further extended to use droplets to support vertical loads in linear translational motion. With electrowetting actuation, the droplets can serve as both bearing and actuator. Moon and Kim demonstrated this concept by using electrowetting to move droplets which carried a solid platform for biochemical analysis [15]. In their demonstration, a voltage was applied to the electrodes on the actuation substrate which induced a shape change within the droplets. This 
propelled droplet translation and the droplets transferred the force to the solid platform. The force is transferred to the moving element through the surface tension. The droplet interface on the actuated object is constrained, by a defined wetting pattern created by a local coating and/or surface texture. For an aqueous actuation droplet, the surface inside the wetting region is hydrophilic and outside it is hydrophobic. Motion accuracy depends on maintaining a constant spatial relationship between the droplet/solid surfaces of the droplet. For integrated EW-actuated linear motion, the electrowetting effect actuates the droplet which is supporting the plate, so the bottom surface must be hydrophobic.
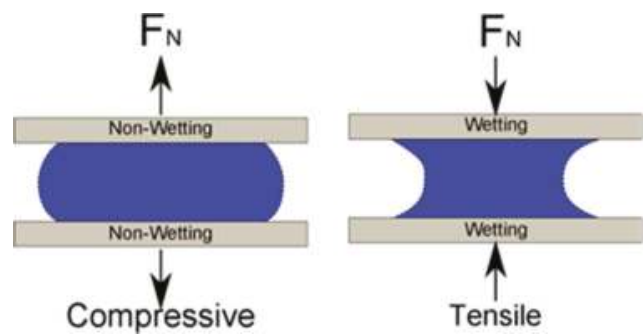

Figure 1. Tensile and compressive forces from a capillary bridge formed by two parallel surfaces.

For linear bearings, the stiffness and load carrying capacity are critical parameters. The vertical stiffness determines the precision of the bearing in the transverse direction under varying loads. For rotational bearings, it was noted in reference [10] that the maximum speed of the rotor was highly dependent on the thickness of the droplet due to the viscosity of the fluid. It is important to optimize the load and the fluid volume which have large influence on the resulting gap height. While valuable prior work has been done on the force of capillary bridges [5,16] and their dynamics [17], these do not consider their use as linear bearings. This paper develops static force and stiffness relations for circular droplets supporting normal loads between two parallel plates. These provide critical information about the load carrying capability of droplet-based bearings and the positioning accuracy at slow speeds. Analytical approximations are compared to numerical simulation of surface forces under varying surface constraints (wetting boundary and/or geometrical boundary). Depending on the wetting properties of the surfaces, different stiffness and load capability can be utilized for droplet based bearings. Based on these results, design strategies for droplet linear bearings are proposed. This work can serve as a design guideline to implement droplet based linear bearings in electrowetting driven application, as well as in designing droplet-based rotating machines.

\section{Materials and Methods}

In its simplest form, a droplet-based bearing consists of a capillary bridge formed between two parallel surfaces. The interfacial tensions act at the triple point where the liquid, solid, and ambient fluid meet. The angle between the liquid and solid is the contact angle. Lambert and co-workers have shown that both the Laplace approach and interfacial energy approach are equivalent [18]. In here, we will take the Laplace approach. Given the condition that the characteristic dimension is much less than the capillary length $\sqrt{\frac{\gamma}{\rho g}}$ ) (where $\gamma$ is the surface tension, $\rho$ the density difference between the droplet and the ambient, and $g$ the acceleration due to gravity), the effect of gravity on the fluid can be neglected. For water in an air ambient, the capillary length is approximately $2.7 \mathrm{~mm}$.

As shown in Figure 2, the bottom surface is fixed and the separation distance $(h)$ is known. The forces acting on the top plate are the surface tension force and the pressure force. The vertical component of the surface tension $\left(F_{T}\right)$ acting on the top plate is found by integrating around the interface. For the case of a circular droplet with constant wetting angle:

$$
F_{T}=2 \pi R_{\text {top }} \times \gamma \sin (\theta)
$$


where $\gamma$ is the interfacial tension of the liquid-ambient, $R_{\text {top }}$ is the radius of the contact area and $\theta$ is the contact angle of the liquid and top plate. The pressure force, which originates from the pressure jump across the liquid/ambient interface can be calculated using the Laplace pressure equation:

$$
F_{P}=\pi R_{\text {top }}^{2} \times \Delta P=\pi R_{\text {top }}^{2} \times \gamma\left(\frac{1}{R_{1}}+\frac{1}{R_{2}}\right)
$$

where, $R_{1}$ and $R_{2}$ are the principle radii of the curved interface. Giving the separation distance $(h)$ between the plates, one principle radius can be found by geometry:

$$
h=-R_{1} \times(\cos (\theta)+\cos (\phi))
$$

where the contact angle with the moving and stationary plates are $\theta$ and $\phi$, respectively. The pressure can be either positive or negative depending on the contact angles. When both angles are larger than $90^{\circ}$, the pressure is positive, which acts to push the plates apart. When both angles are less than $90^{\circ}$, the resulting negative pressure force will pull the plates together. If the radius of the capillary bridge is much larger than the separation distance $\left(R_{2}>h\right)$, the second principle radius can be approximated by the radius of the plate contact $\left(R_{2}=R_{t o p}\right)$. The top radius is used as it will be assumed to be prescribed while the other may vary with load. Then the normal force $\left(F_{N}\right)$ on the top plate is as follows:

$$
\begin{gathered}
F_{N}=F_{P}-F_{T}=\pi R_{\text {top }}^{2} \times \gamma\left(\frac{1}{R_{1}}+\frac{1}{R_{2}}\right)-2 \pi R_{\text {top }} \times \gamma \sin (\theta) \\
=\pi R_{\text {top }} \gamma \times\left[-\frac{R_{\text {top }}}{h}(\cos (\theta)+\cos (\phi))+1-2 \sin (\theta)\right]
\end{gathered}
$$

The equation is nondimensionalized by normalizing the height $\left(\bar{h}=h / 2 R_{\text {top }}\right)$ with the diameter of the drop $\left(D=2 R_{\text {top }}\right)$ to get the following:

$$
\frac{F_{N}}{\pi D_{\text {top }} \gamma}=\frac{-\cos (\theta)-\cos (\phi)}{4 \times \bar{h}}+0.5-\sin (\theta)
$$

For droplet-based bearings, the contact radius/diameter of the top surface can be controlled by either surface coating or roughness so that inside of the wetting region, the surface is hydrophilic. Outside of the region, the surface is hydrophobic with a contact angle greater than $90^{\circ}$. The key quantities of interest are the normal load capability and stiffness of droplets with known bottom contact angle, plate spacing, and droplet volume. In practice, the actual contact angles may not be known a priori at the boundary. If the contact line is at the boundary between two regions with different effective contact angles, the contact angle can assume any value between the interior and exterior wetting angles without moving the contact line. These relationships will be used to develop estimates of the forces, but are not suitable for calculating actual fluid reactions in many cases of practical interest.

The reaction forces in more general cases are calculated numerically using software such as Surface Evolver [19]. Surface Evolver calculates the total system energy by discretizing the surface and summing a series of surface and line integrals. The equilibrium shape is found by gradient-based energy minimization. In the simulation, a droplet with defined volume and contact angle condition on the top and bottom plate was constrained between two planes that represented the substrate (bottom) and moving plate (top). An example of the simulation boundaries is presented in Figure 3. The effect of gravity was not included since the desired operating range of the droplet based bearing is substantially less than the capillary length.

Once the equilibrium shape of the surface was found, a small displacement in the $\mathrm{z}$ direction $(50 \mu \mathrm{m})$ was applied and the energy change was calculated. The normal force was found using the principle of virtual work $\left(F_{z}=d E / d z\right)$ [20,21]. Results of surface evolver calculations are compared to the limiting closed form approximations (Equation (5)) for relevant wetting boundary conditions below. These numerical predictions of normal forces for a range of wetting conditions and droplet/gap 
ratios will be compared to simplified assumptions based on the analytical solutions. These results will be used to identify promising configurations for high stiffness fluidic bearings.

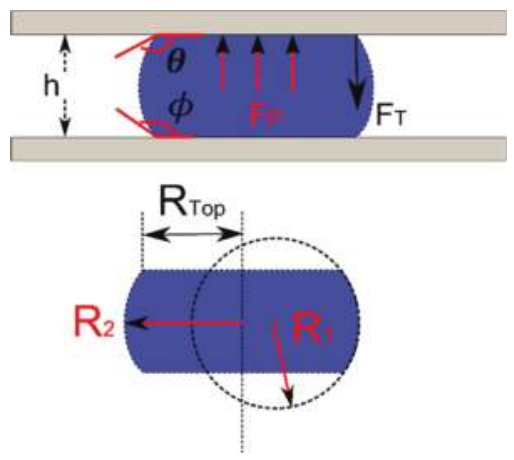

Figure 2. Capillary bridge between two parallel surfaces. When the top and bottom contact angles $(\theta$ and $\phi)$ are greater than $90^{\circ}$, the pressure force $\left(F_{p}\right)$ pushes the top plate upwards and the surface tension force $\left(F_{T}\right)$ acts downwards to pull the plates together. The two principle radii of curvature $\left(R_{1}\right.$ and $R_{2}$ ) can be are used to calculate the pressure difference across the interface and $R_{\text {Top }}$ is the contact radius of the droplet to the top plate.

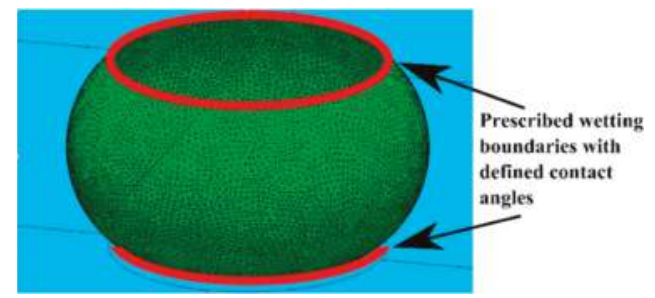

Figure 3. An example of the simulation boundaries. Inside the red lines, the surface energy is defined by low contact angle $\left(10^{\circ}\right)$. Outside the red lines, high surface energy is defined by large contact angle $\left(110^{\circ}-165^{\circ}\right)$.

\section{Results}

An effective fluid bearing must be able to support the normal loads applied and should also have a high stiffness to minimize the displacement caused by changes in applied forces. While the force and stiffness are linearly dependent on surface tension, the droplet diameter, the substrate/plate gap, and contact line constraints will nonlinearly impact the performance of the fluid bearing. This paper considers the impact of the droplet aspect ratio (gap height/diameter) for three different wetting arrangements in a simple fluid bearing for different contact line constraint conditions.

\subsection{Type 1-Uniform Wetting}

For axisymmetric wetting, without wetting boundaries, the contact angles of the top and bottom are the same $(\theta=\phi)$, Equation (5) reduces to:

$$
\frac{F_{N}}{\pi D_{\text {top }} \gamma}=\frac{-\cos (\theta)}{2 \times \bar{h}}+0.5-\sin (\theta)
$$

This case is used as a bench mark for the Surface Evolver simulation. The surface tension for all types was fixed at $0.072 \mathrm{~N} / \mathrm{m}$. The variables used are listed in Table 1. 
Table 1. Simulation parameters used.

\begin{tabular}{ll}
\hline Droplet volume & $5-50 \mu \mathrm{L}(5 \mu \mathrm{L}$ increments $)$ \\
\hline Contact angle (both top and bottom) & $165^{\circ}, 120^{\circ}, 110^{\circ}$ \\
Gap height & $0.5-3.0 \mathrm{~mm}$ \\
\hline
\end{tabular}

The normal force at each condition was extracted and the resulting forces and the top contact diameter were extracted from the models and the normalized values are summarized in Figure 4. When normalized, the force for a given contact angle collapses to a single curve for all droplet volumes. The simulation data (markers) agrees well with Equation (6) predictions (solid lines) for small values of aspect ratio for all contact angles. Good agreement is seen at larger aspect ratios and for contact angles closer to $90^{\circ}$ where the assumptions in Equation (6) are most accurate. For large contact angles $\left(165^{\circ}\right)$, Equation (6) over predicts compared to the simulation, especially at larger aspect ratios. The error arises from using the contact radius as a principle radius of curvature and assuming cylindrical droplet shape for calculating the diameter. However, Equation (6) gives a good approximation of forces over the design region of greatest interest (small aspect ratios) for all contact angles evaluated.

At large aspect ratios, the droplet force is relatively insensitive to the aspect ratio, but this equates to a low stiffness. However, when the aspect ratio is less than 0.1 the slope is much higher-creating a stiffer bearing in which position is less sensitive to applied loads. Alternatively, the higher normal force could allow the bearing diameter to be reduced for the same force/stiffness capacity.

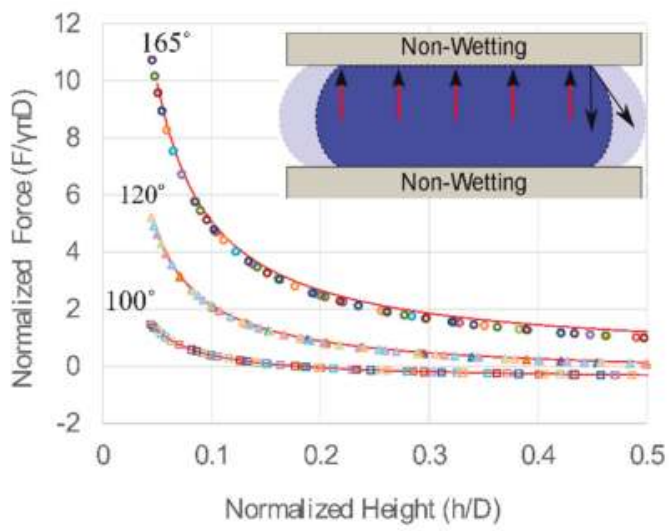

Figure 4. Normalized force vs. normalized height, Type 1. For a symmetric capillary bridge on a nonwetting surface, the contact angles are the same top and bottom, circular, triangle and square marks are the simulation results for contact angles of $165^{\circ}, 120^{\circ}$ and $100^{\circ}$, respectively. Each data set includes simulation results for 10 droplet volumes used $(5-50 \mu \mathrm{L})$. The solid lines are the calculated values from Equation (6).

For hydrophobic surfaces, the surface tension forces pull the moving plate toward the substrateopposite the pressure force. As the contact angle increases, the surface tension has a smaller normal components and acts to increase the pressure increases resulting in much larger normal forces. Figure 5 shows the calculated force per unit contact area on the top plate with a $50 \mu \mathrm{L}$ droplet at various contact angles from Equation (6). As the contact angles increase from $100^{\circ}$ to $165^{\circ}$, the force increases by a factor of 5 or more (see Figure 5). Higher contact angles and/or smaller aspect ratios should be used to support larger vertical loads. 


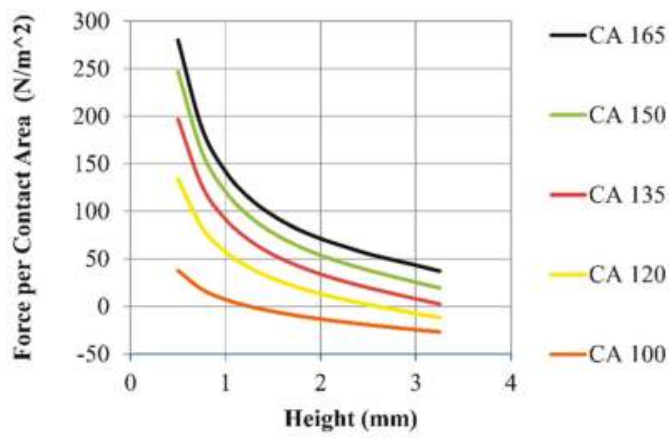

Figure 5. Force per unit area calculated from Equation (6). Each line indicates the different contact angles used for the top and bottom surfaces. The droplet's volume was fixed at $50 \mu \mathrm{L}$.

\subsection{Type 2-Defined Wetting Region}

In a practical bearing, the droplet contact line must be constrained relative to one of the plates while sliding across the other. This could be done by defining a specific wetting region on one plate while the other plate remains hydrophobic — breaking the symmetry of the droplet wetting. This non-symmetric wetting was simulated in Surface Evolver using a hydrophilic circular region on the top (moving) plate (contact angle $\left.=10^{\circ}\right)$ with different wetting radius $\left(R_{t o p}=2,3\right.$, and $4 \mathrm{~mm}$ ). The remainder of the top surface and the entire bottom were non-wetting (contact angle $=165^{\circ}$ ). Droplet volume was fixed at $50 \mu \mathrm{L}$. The vertical forces were extracted and then normalized by the top contact diameter and compared to Type 1 performance for various droplet heights. The result is presented in Figure 6.

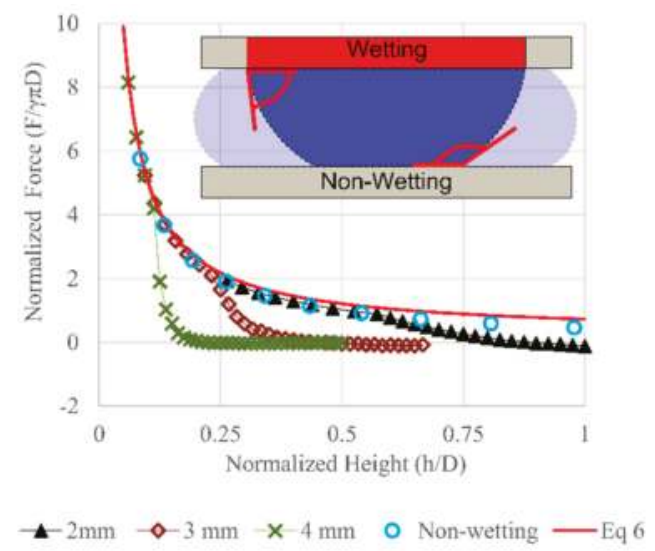

Figure 6. Normalized force vs. Normalized height with Type 2 wetting. The force were normalized by the product of the surface tension, $\pi$ and the top contact diameter. The circles are the simulation result from Type 1 (symmetric non-wetting, contact angle: $165^{\circ}$ ), the line is the prediction from Equation (6). The cross, square, and triangle marks are the simulation results for different wetting radius $(4,3,2 \mathrm{~mm})$ when the droplet's volume was fixed at $50 \mu \mathrm{L}$. The inset shows the wetting region on the top plate. As the gap height betweent the plate decrease, the droplet sperad out to the non-wetting region.

At small heights, the force follows the Type 1 curve until the wetting region on the top plate reaches the edge of the wetting region. As aspect ratio continues to increase (height grows), the force drops dramatically relative to the Type 1 case as the bottom plate contact area decreases while the 
top contact line remains stationary. While the lower normal force in this region is undesirable, the increased stiffness in the transition region is favorable for reducing variation in plate positioning with applied normal forces. For a given wetting area, this also allows the bearing to operate at a higher gap height with favorable stiffness. Larger gaps and smaller aspect ratios should reduce the drag introduced by the fluids in a linear actuator.

\subsection{Type 3-Constrained Top Wetting}

Physical constraints can also be added to the edge of the wetting region to reliably constrain the wetting boundary even beyond $180^{\circ}$ (measured relative to the horizontal surface). This could be accomplished using a protruding surface as illustrated in Figure 7. This effect was modeled in Surface Evolver model by constraining the interface so that it could not move beyond the wetting region. The resulting force increases at a much faster rate than Type 1 and 2 as seen in Figure 7.

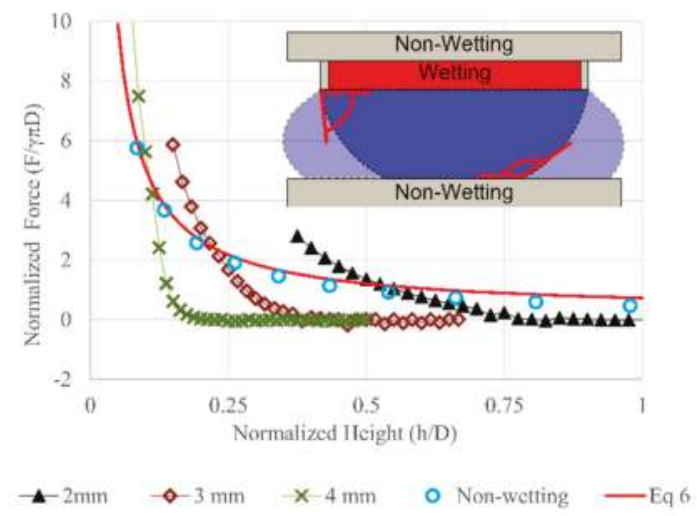

Figure 7. Normalized force vs. normalized height with Type 3 wetting. Results are compared to predictions from Equation (6), the simulation result from Type 1 (symmetric non-wetting, contact angle: $165^{\circ}$, droplet volume $50 \mu \mathrm{L}$ ). The inset shows the wetting region and geometrical constrain on the top plate.

The Type 3 design can increase the working range of the bearing from 0.1 aspect ratio $(h / D)$ to at least 0.25 . This would be the most favorable design for droplet based bearings. High stiffness and large force could be achieved with larger gap height when the contact line is constrained by both geometry and wetting. As discussed earlier, the viscous drag introduced by the droplet could impose a speed limit on the bearing. By operating at larger height, the drag would be reduced and the maximum speed of the bearing could be increased.

\section{Discussion}

This analysis provides insight into droplet and wetting arrangements that will provide the most accurate fluidic bearing in electrowetting and other applications. To achieve precise translational motion in the $x-y$ plane, the relative motion between the top plate and droplet interface on the substrate needs to be minimized. Type 2 and 3 limit the relative motion by constraining the contact line on the moving plate. For a given contact area, smaller droplet volumes would require less gap height to resist the same normal force. Stiffness increases significantly when the top edge is better constrained (Type 3).

For a typical application the size and weight of the plate being carried is fixed. Equation (6) indicates droplet-based bearings favor a large diameter or small gap height. Since the pressure force scales with the diameter squared, larger contact areas can support larger loads. However, a single droplet bearing does not provide much stiffness in rotation about the $\mathrm{x}$ and $\mathrm{y}$ axis [22] and would add additional uncertainty in plate placement [23]. When multiple droplets support the plate, rotations about the $\mathrm{x}$ and 
$y$-axes are resisted by the z-stiffness of the droplets. Since two droplets define a line and three droplets define a plane, three droplets would be an appropriate minimum. However, variation in the droplet volume could cause orientation errors which would be reduced by using additional droplets to average out random variation. Additionally, the $\mathrm{x}$ and $\mathrm{y}$ displacement stiffness is proportional to the droplet circumference which increases when more droplets are used.

Some case studies are used to demonstrate the tradeoffs when designing such bearing. All the calculations are based on Equation (6) (Although the equation is only applicable to Type 1 wetting described above, the calculated force should match closely to Type 2 and 3 at the design point where they intersect the unconstrained droplet force line. While Equation (6) does not predict the stiffness of Type $2 \& 3$ droplets, it does provide a lower bound for stiffness in these cases.

\subsection{Design Case 1-Fixed Gap Height}

In this first case study (Figure 8), the normal force and the gap height are held constant. The number of droplets and the droplet volume are the variables. The calculated force should be applicable for all types of wetting, but the calculated stiffness is for Type 1 only. From earlier analysis, the stiffness for Type 2 and 3 wetting should be higher than the idealized condition presented here.

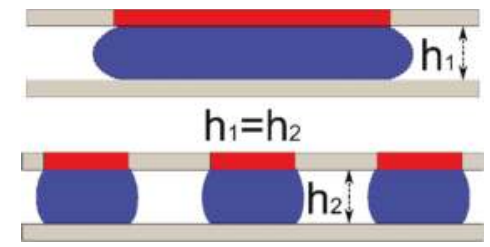

Figure 8. Case study 1: fixed gap height $(1 \mathrm{~mm})$ and fixed load $(1078 \mu \mathrm{N})$. The wetting areas (hydrophilic) are the shaded region. This case compares single vs. multiple droplets for bearing application.

For a square glass plate (density $2200 \mathrm{~kg} / \mathrm{m}^{3}$ ) with dimension of $10 \times 10 \times 0.5 \mathrm{~mm}$, the minimal force needed to support the weight of the plate is $1078 \mu \mathrm{N}$. Assuming an air ambient and water droplets with surface tension $0.072 \mathrm{~N} / \mathrm{m}$, and $110^{\circ}$ contact angle on a Cytop coating. At $1 \mathrm{~mm}$ gap height, a single $6.8 \mathrm{~mm}$ diameter droplet would provide sufficient force to support the plate. However, due to stability issues mentioned above, multiple droplets are preferred. Table 2 shows the impact on the aspect ratio, total droplet area, and the projected length (proportional to the magnitude of an EW actuation force) when using multiple droplets. The supporting force is calculated using Equation (6). This is most accurate for small values of aspect ratio and contact angles near $90^{\circ}$ and should be a good approximation for these cases $\left(h / D<0.3, C A=110^{\circ}\right)$. As the droplets are parallel springs, the total stiffness is the number of droplets multiplied by the stiffness of a single droplet.

Table 2. Case study of droplet(s) supporting a fixed load at constant gap height, the contact angle is $110^{\circ}$ top and bottom. The total supporting force is $1078 \mu \mathrm{N}$ and the gap height is $1 \mathrm{~mm}$.

\begin{tabular}{|c|c|c|c|c|c|c|}
\hline $\begin{array}{c}\text { \# of } \\
\text { Droplets }\end{array}$ & $\begin{array}{c}\text { Volume of Each } \\
\text { Droplet }(\mu \mathrm{L})\end{array}$ & $\begin{array}{c}\text { Aspect } \\
\text { Ratio (h/D) }\end{array}$ & $\begin{array}{c}\text { Diameter of } \\
\text { the Drop }(\mathrm{mm})\end{array}$ & $\begin{array}{c}\text { Total Stiffness } \\
(\mathrm{N} / \mathrm{m})\end{array}$ & $\begin{array}{l}\text { Minimum Total Area } \\
\text { Required }\left(\mathrm{mm}^{2}\right)\end{array}$ & $\begin{array}{c}\text { Total Projected } \\
\text { Length (mm) }\end{array}$ \\
\hline 1 & 36.3 & 0.147 & 6.8 & 1.8067 & 36.3 & 6.8 \\
\hline 3 & 16.6 & 0.217 & 4.6 & 2.48031 & 49.9 & 13.8 \\
\hline 5 & 12.6 & 0.25 & 4 & 3.12578 & 62.8 & 20 \\
\hline 7 & 10.8 & 0.27 & 3.7 & 3.7443 & 75.3 & 25.9 \\
\hline
\end{tabular}

The table above shows the same load could be supported by a number of possible configurations, depending on the design goal. If the normal force per unit area were the main concern, the larger droplet should be used. If the stiffness is the driving parameter, multiple small droplets could achieve higher stiffness. If the actuation force from electrowetting is to be maximized, maximum allowable number of droplet should be used due to the larger project length from multiple droplets. 


\subsection{Design Case 2-Fixed Stiffness and Droplet Diameter}

External disturbances such as gravity, shock and vibration will introduce positioning error. When the gap height is small, minor displacement in the vertical direction could cause a large droplet diameter change. This could cause the spacing between the droplets to decrease until they merge. To account for the external disturbance, the stiffness needs to be considered. This case study (Figure 9) uses the same loading $(1078 \mu \mathrm{N})$ and plate area $\left(100 \mathrm{~mm}^{2}\right)$ as above. Instead of fixing the gap height, the bearings are designed for a maximum displacement of the top plate of $\pm 10 \mu \mathrm{m}$ at $150 \%$ overloading. This can be translated to a design requirement of fixed stiffness $\left(\frac{1.5 \times 1078}{10} \frac{\mu N}{\mu m}=162 \frac{N}{m}\right)$. Other parameters such as the surface tension and contact angle remain the same $\left(0.072 \mathrm{~N} / \mathrm{m}, 110^{\circ}\right.$, respectively). The same droplet diameters were used. The gap height is adjusted by varying the volume of the droplet. The same calculation were performed using Equation (6). As seen in Table 3, the aspect ratio needs to be much smaller than the previous case in order to meet the high stiffness requirement. All other outcomes such as the total area required and the actuation force are unaffected due to the fixed wetting diameter. The force approximation is suitable for all wetting conditions. However, due to the higher stiffness of Type $2 / 3$ wetting, the gap height could be much larger for the same stiffness when Type 2/3 wetting is employed.

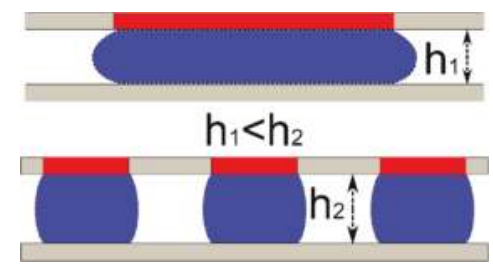

Figure 9. Case study 2: fixed stiffness $(162 \mathrm{~N} / \mathrm{m})$ and fixed droplet diameter. The wetting areas (hydrophilic) are the shaded region. This case evaluates the impact on droplet volume and gap height when designing for stiffness.

Table 3. Case study of droplet(s) with constant stiffness $(162 \mathrm{~N} / \mathrm{m})$, the contact angle is $110^{\circ}$ top and bottom. The diameter of the top wetting region is the same as in case study 1.

\begin{tabular}{|c|c|c|c|c|c|c|}
\hline $\begin{array}{c}\text { \# of } \\
\text { Droplets }\end{array}$ & $\begin{array}{c}\text { Volume of Each } \\
\text { Droplet }(\mu \mathrm{L})\end{array}$ & $\begin{array}{c}\text { Aspect } \\
\text { Ratio (h/D) }\end{array}$ & $\begin{array}{c}\text { Diameter of } \\
\text { the Drop }(\mathrm{mm})\end{array}$ & $\begin{array}{l}\text { Height of the } \\
\text { Gap (mm) }\end{array}$ & $\begin{array}{l}\text { Minimum Total Area } \\
\text { Required }\left(\mathrm{mm}^{2}\right)\end{array}$ & $\begin{array}{c}\text { Total Projected } \\
\text { Length }(\mathrm{mm})\end{array}$ \\
\hline 1 & 3.8 & 0.015452 & 6.8 & 0.105 & 36.3 & 6.8 \\
\hline 3 & 2.1 & 0.026764 & 4.6 & 0.123 & 49.9 & 13.8 \\
\hline 7 & 1.6 & 0.040883 & 3.7 & 0.151 & 75.3 & 25.9 \\
\hline
\end{tabular}

Another effect of the tradeoffs between design for force and stiffness can be seen in the rapid decrease in the volume of droplets. About one order of magnitude of reduction in volume is required for the increased stiffness. These small volumes could accentuate another error source-droplet volume variation could impact the alignment precision in the $x-y$ plane. This could be offset by increasing the number of droplets to average out random variations which has the benefit of increased gap height for the same force/stiffness values.

\subsection{Opposing Droplets}

For linear bearing application, the normal force and the stiffness under loading are the primary interests. Higher force enables a larger loading capability and high stiffness would improve the rigidity of the joint. For optimal stiffness and force in the vertical direction, the plate should have both wetting and geometrical constraints. In addition, a preload on the droplet would force the bearing to operate at a higher stiffness range (case 3). In the prior cases, the preload is applied by gravity which makes the plate position sensitive to orientation. Alternatively, we propose a symmetrical droplet bearing 
which uses opposing droplets to apply a preload to the plate, the resulting force/stiffness could be symmetrical around the middle point which could further increase the reliability of the device-either orientation around the $\mathrm{x}-\mathrm{y}$ plane is possible. The simplified case with no wetting contrast (Type 1) is analyzed below (Figure 10).

To illustrate the force-displacement relation, calculations based on Equation (6) were performed. The force is then normalized by the equilibrium diameter $\left(D^{\prime}\right)$ times $\pi \gamma$ and the change in height with respect to the middle point $(d z)$ was divided by the equilibrium diameter $\left(D^{\prime}\right)$. The results are presented in Figure 11. The main assumption is that the weight of the plate is counteracted by the buoyancy force (plate density $\approx$ ambient fluid density) and all the fluid contacts are non-wetting (Type 1). Implementation of Type $2 / 3$ wetting would significantly increase system stiffness but the trends would be similar. The contact angle values used in the calculation were $165^{\circ}$ for both top and bottom. The maximum allowable gap height is fixed at $1,0.5$ and $0.25 \mathrm{~mm}$ for both top and bottom gap (without the thickness of the middle plate, $h_{\text {Top }}=h_{\text {Bottom }}$ ). The droplet volume was $50 \mu \mathrm{L}$ each.

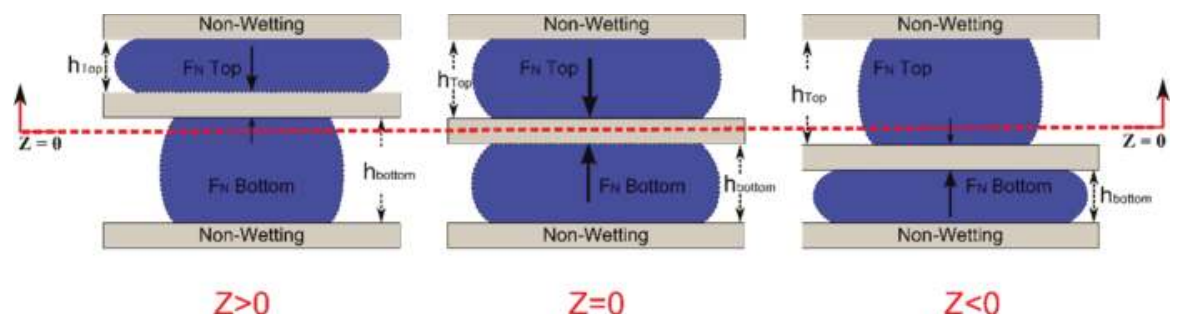

Figure 10. Opposing droplet configuration. Two identical droplets are used to provide support on either side of the center plate. Both the top and bottom plates are fixed. The resulting force and stiffness is symmetrical around the midpoint $(Z=0)$. The left and right illustrations shows the droplet shape when the center plate is displaced around the balance point.

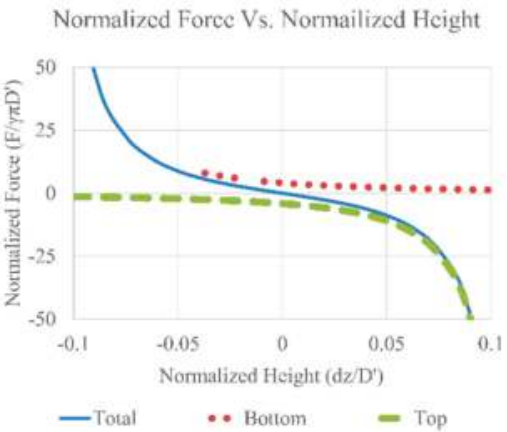

(A)
Normalized Force Vs. Normailized Height

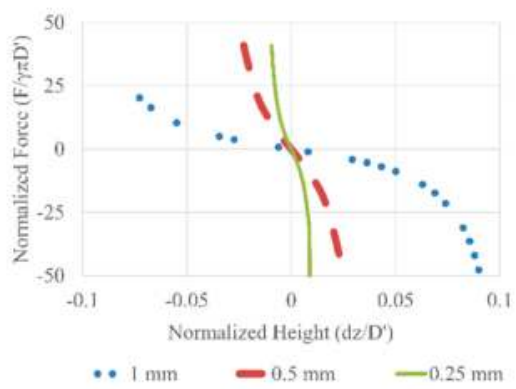

(B)

Figure 11. Opposing droplet configuration for optimal stiffness and precise z-location. (A) the normalized force from both the top and the bottom droplets are plotted against the normalized height (change in height divided by the nominal droplet diameter, $d h / D^{\prime}$ ), the equilibrium height is $1 \mathrm{~mm}$ for both top and bottom gap. (B) Fixed gap heights with changing diameters. The contact angle used in calculation was $165^{\circ}$ and the surface tension value used was $0.072 \mathrm{~N} / \mathrm{m}$.

As seen in Figure 11A, the top and the bottom droplets exert the same force but in opposite directions around the center (dotted lines), the total force follows a non-linear but symmetrical curve (solid line). When designing the bearing, the droplet contact area could be specified and the height can be tailored to target stiffness. Due to the symmetric force, the location of the plate and/or the stiffness in the $z$-direction could be fine-tuned by using either the volume of the droplet, the wetting 
pattern or the gap height, to change the preload on the bearing and further improve stiffness as seen in Figure 11B. As the total gap height decreases the droplets are preloaded and the effective stiffness of the droplet bearing increases.

\section{Conclusions}

The normal force of a capillary bridge between two parallel surfaces was analyzed using both Laplace-based calculation and simulation. The force-deflection response of three wetting conditionsuniform wetting, defined wetting region and constrained top-wetting were analyzed using numerical simulation and compared to a simplified closed form approximation. This approximation is shown to provide useful reference information for predicting key aspects of each wetting type. For all cases, smaller gaps create higher stiffness and larger forces. The peak force and stiffness are achieved with geometrical constraints on the top plate that enable effective contact angles above $180^{\circ}$ at the edge of the wetting boundary.

For ultimate precision, an opposing droplet configuration was proposed. Simplified force analysis showed a symmetrical stiffness response due to the preloading effect. The stiffness could be further fine-tuned by changing the total gap height. For linear droplet bearing actuated by electrowetting, a series of design cases were presented to demonstrate the advantage of using multiple droplets to support the same load. The larger actuation force capability of multiple droplets could improve the speed of the actuator. When designing such actuators, the total area and the gap height should be the driving parameters to meet required load and stiffness performance levels.

Author Contributions: Q.N.: methodology, software, investigation, visualization, writing —original draft preparation. N.C.: conceptualization, validation, writing - review and editing, project administration, funding acquisition.

Funding: This research was funded by the National Science Foundation, grant number CMMI-1130755.

Conflicts of Interest: The authors declare no conflict of interest. The funders had no role in the design of the study; in the collection, analyses, or interpretation of data; in the writing of the manuscript, or in the decision to publish the results.

\section{References}

1. Mastrangeli, M. The Fluid Joint: The Soft Spot of Micro- and Nanosystems. Adv. Mater. 2015, 27, 4254-4272. [CrossRef] [PubMed]

2. Lambert, P.; Seigneur, F.; Koelemeijer, S.; Jacot, J. Design of a Capillary Gripper for a Submillimetric Application. In Precision Assembly Technologies for Mini and Micro Products, Proceedings of the IFIP TC5 WG5.5 Third International Precision Assembly Seminar (IPAS '2006), Bad Hofgastein, Austria, 19-21 February 2006; Ratchev, S., Ed.; Springer: Boston, MA, USA, 2006; pp. 3-10.

3. Fantoni, G.; Hansen, H.N.; Santochi, M. A new capillary gripper for mini and micro parts. Cirp. Ann.Manuf. Technol. 2013, 62, 17-20. [CrossRef]

4. Syms, R.R.A.; Yeatman, E.M.; Bright, V.M.; Whitesides, G.M. Surface tension-powered self-assembly of microstructures-The state-of-the-art. J. Microelectromech. Syst. 2003, 12, 387-417. [CrossRef]

5. Knospe, C.R.; Nezamoddini, S.A. Capillary force actuation. J. Micro-Nano Mechatron. 2010, 5, 57-68. [CrossRef]

6. Carter, W.C. The Forces and Behavior of Fluids Constrained by Solids. Acta Metall. 1988, 36, $2283-2292$. [CrossRef]

7. Fortes, M.A. Axisymmetric Liquid Bridges between Parallel Plates. J. Colloid Interface Sci. 1982, 88, 338-352. [CrossRef]

8. Mugele, F.; Baret, J.C. Electrowetting: From basics to applications. J. Phys.-Condens. Matter 2005, 17, R705-R774. [CrossRef]

9. Ni, Q.; Capecci, D.E.; Crane, N.B. Electrowetting force and velocity dependence on fluid surface energy. Microfluid. Nanofluid. 2015, 19, 181-189. [CrossRef] 
10. Chan, M.L.; Yoxall, B.; Park, H.; Kang, Z.Y.; Izyumin, I.; Chou, J.; Megens, M.M.; Wu, M.C.; Boser, B.E.; Horsley, D.A. Design and characterization of MEMS micromotor supported on low friction liquid bearing. Sens. Actuators A Phys. 2012, 177, 1-9. [CrossRef]

11. Liu, T.L.; Sun, G.; Kim, J.J.; Yang, C.K.K.; Kim, C.J.C.J. Electrostatic bottom-driven rotary stage on multiple conductive liquid-ring bearings. In Proceedings of the 2013 IEEE 26th International Conference on Micro Electro Mechanical Systems (MEMS), Taipei, Taiwan, 20-24 January 2013; pp. 86-89.

12. Sun, G.Y.; Liu, T.Y.; Sen, P.; Shen, W.J.; Gudeman, C.; Kim, C.J. Electrostatic Side-Drive Rotary Stage on Liquid-Ring Bearing. J. Microelectromech. Syst. 2014, 23, 147-156. [CrossRef]

13. Yoxall, B.E.; Chan, M.L.; Harake, R.S.; Pan, T.R.; Horsley, D.A. Rotary Liquid Droplet Microbearing. J. Microelectromech. Syst. 2012, 21, 721-729. [CrossRef]

14. Takei, A.; Matsumoto, K.; Shomoyama, I. Capillary motor driven by electrowetting. Lab Chip 2010, 10, 1781-1786. [CrossRef] [PubMed]

15. Moon, I.; Kim, J. Using EWOD (electrowetting-on-dielectric) actuation in a micro conveyor system. Sens. Actuators A Phys. 2006, 130, 537-544. [CrossRef]

16. Nezamoddini, S.A. Capillary Force Actuators. Ph.D. Thesis, University of Virginia, Charlottesville, VA, USA, 2008.

17. Valsamis, J.B.; Mastrangeli, M.; Lambert, P. Vertical excitation of axisymmetric liquid bridges. Eur. J. Mech. $B$ Fluids 2013, 38, 47-57. [CrossRef]

18. Lambert, P.; Chau, A.; Delchambre, A.; Regnier, S. Comparison between two capillary forces models. Langmuir ACS J. Surfaces Colloids 2008, 24, 3157-3163. [CrossRef] [PubMed]

19. Brakke, K. The Surface Evolver. Exp. Math. 1992, 1, 141-165. [CrossRef]

20. Berthier, J.; Mermoz, S.; Brakke, K.; Sanchez, L.; Fretigny, C.; Cioccio, L.D. Capillary self-alignment of polygonal chips: A generalization for the shift-restoring force. Microfluid. Nanofluid. 2013, 14, 845-858. [CrossRef]

21. Josell, D.; Wallace, W.E.; Warren, J.A.; Wheeler, D.; Powell, A.C. Misaligned flip-chip solder joints: Prediction and experimental determination of force-displacement curves. Trans. ASME. J. Electron. Packag. 2002, 124, 227-233. [CrossRef]

22. Mastrangeli, M.; Valsamis, J.B.; Van Hoof, C.; Celis, J.P.; Lambert, P. Lateral capillary forces of cylindrical fluid menisci: A comprehensive quasi-static study. J. Micromech. Microeng. 2010, 20, 075041. [CrossRef]

23. Berthier, J.; Brakke, K.A.; Mermoz, S.; Fretigny, C.; Di Cioccio, L. Stabilization of the tilt motion during capillary self-alignment of rectangular chips. Sens. Actuators A Phys. 2015, 234, 180-187. [CrossRef]

(C) 2018 by the authors. Licensee MDPI, Basel, Switzerland. This article is an open access article distributed under the terms and conditions of the Creative Commons Attribution (CC BY) license (http:/ / creativecommons.org/licenses/by/4.0/). 


\title{
Article \\ Enabling Droplet Functionality on Anisotropic Ratchet Conveyors
}

\author{
Hal R. Holmes ${ }^{1}$ (D), Ana E. Gomez ${ }^{1}$ (D) and Karl F. Böhringer 1,2,* \\ 1 Department of Bioengineering, University of Washington, Seattle, WA 98105, USA; \\ hrholmes@uw.edu (H.R.H.); anag3@uw.edu (A.E.G.) \\ 2 Department of Electrical Engineering, University of Washington, Seattle, WA 98195, USA \\ * Correspondence: karlb@uw.edu
}

Received: 27 November 2017; Accepted: 14 December 2017; Published: 16 December 2017

\begin{abstract}
Anisotropic ratchet conveyors (ARCs) are a recently developed microfluidic platform that transports liquid droplets through a passive, microfabricated surface pattern and applied orthogonal vibrations. In this work, three new functionalities are presented for controlling droplet transport on the ARC system. These devices can pause droplet transport (ARC gate), decide between two pathways of droplet transport (ARC switch), and pass droplets between transport tracks (ARC delivery junction). All devices function solely through the modification of pinning forces acting on the transported droplet and are the first reported devices that can selectively control droplet timing and directionality without active (e.g., thermal, electrical, or magnetic) surface components.
\end{abstract}

Keywords: droplet transport; microfluidics; vibrations; contact line oscillation; asymmetric surfaces; anisotropic ratchet conveyor

\section{Introduction}

Anisotropic ratchet conveyors (ARCs) are a type of digital microfluidic (DMF) system that can transport an individual liquid droplet or many droplets in parallel through a passive micropatterned surface and applied orthogonal vibrations. The functionality of ARC devices comes from two primary features: (1) an anisotropic surface pattern of periodically occurring curved structures or "rungs," and (2) oscillation of the contact line or "footprint" of the droplet on the substrate, induced by the applied orthogonal vibrations [1]. The asymmetry of the surface pattern creates a difference in pinning forces between leading and trailing edges of the droplet. The applied vibrations cycle the contact line between wetting, de-wetting, and equilibrium phases. This combination produces a net force in the direction of the leading edge, which essentially causes the droplet to take a step through each vibration cycle (Figure 1) [1-3].

While ARCs do not offer the robust programmability available to electrowetting-on-dielectric (EWOD) or dielectrophoresis (DEP)-based DMF systems [4-6], this platform provides the ability to handle liquid droplets with a passive surface pattern and a globally applied sinusoidal vibration (e.g., a speaker). This configuration allows for droplets to be driven with a single signal source, which substantially simplifies the circuitry and programming required to operate an ARC system. Like EWOD and DEP systems, the ability of ARCs to handle liquid in the form of discrete droplets can reduce required sample volumes and reagent quantities compared to continuous flow devices. Droplets also provide a form of 'compartmentalization', wherein the contents of each droplet are individually isolated, preventing undesirable interactions between samples or reagents [4].

The simple microelectromechanical systems (MEMS)-based fabrication process allows for high-throughput manufacturing of ARC devices, which could provide for inexpensive ARC chips with integrated MEMS components or electronic sensors. Such a system could fill the niche for diagnostic 
or analytic applications that require more process control or measurement accuracy than paper-based or passive microfluidic systems [7,8]. The simplicity of this system makes it a good candidate for field-ready or point-of-contact (where the sample is first encountered in the field) tests, potentially enabling a point-of-contact platform with improved clinical utility [9-11], or for molecular (nucleic acid) assays that are less expensive and more easily deployable [12-14]. Furthermore, ARCs could also provide a useful research tool, such as in applications for automating protein [15] or nucleic acid [16] purification.

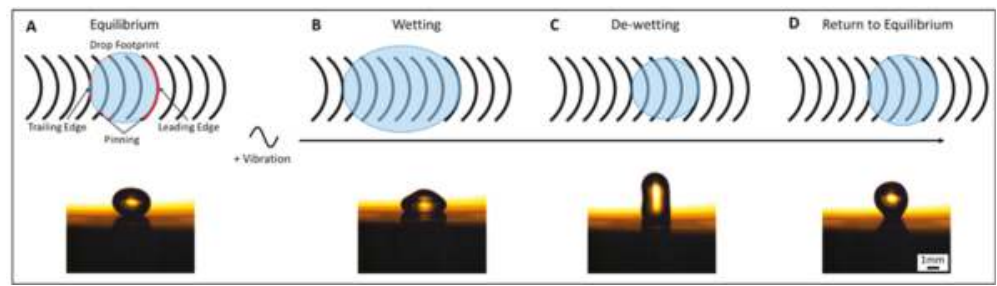

Figure 1. Principles of anisotropic ratchet conveyor (ARC) functionality. ARC systems transport droplets through an anisotropic surface pattern composed of periodically occurring curved rungs (black) defined by a hydrophobic background (white). This asymmetric geometry creates a difference in pinning between leading and trailing edges of the contact line or "footprint" of the droplet (A). Applied orthogonal vibrations induce the contact line to oscillate between wetting, de-wetting and equilibrium states (B-D). This combination results in a net force through each vibration cycle that transports droplets.

However, before any applications for an automated ARC platform can be realized, the functional toolbox available to ARC systems must be expanded. Thus, we have developed three new modules for the ARC system: (1) ARC gates that can selectively pause droplet transport, (2) ARC switches that can select the direction of droplet transport between two paths, and (3) ARC delivery junctions that can controllably deliver droplets on a convergent path. In electrowetting systems, these functions are innately enabled by the position of electrodes, with respect to the droplets, being activated $[4,17,18]$. On ARC systems, functionality is dictated by the design of the passive surface pattern. Therefore, each droplet function on ARC systems must be enabled with a specific design strategically placed on chip. The following sections will demonstrate how the design of the surface pattern on these ARC modules pairs with the applied vibrations to enable essential functions for automated liquid handling processes on ARC systems.

\section{Materials and Methods}

All ARC devices used in this work are fabricated by patterning an oxidized silicon wafer with photoresist. Exposed regions of the wafer are coated with fluorooctyltrichlorosilane (FOTS). The resist is then stripped with acetone, revealing hydrophilic (contact angle $<5^{\circ}$ [19]) silicon dioxide $\left(\mathrm{SiO}_{2}\right)$ rungs defined by the hydrophobic (contact angle of approximately $105^{\circ}$ [19]) FOTS background (Figure A1). At rest, both leading and trailing edges exhibit a contact angle near a native FOTS surface, but the contact angles at the two edges differ during forced vibration as a result of the asymmetric ARC pattern [20]. Images of ARC designs are captured prior to resist stripping and superimposed for clarity, as the final ARC devices in this work are invisible to the naked eye.

For all experiments in this work, $10 \mu \mathrm{L}$ droplets of deionized water $\left(\mathrm{diH}_{2} \mathrm{O}\right)$ were driven on ARC substrates with sinusoidal vibrations produced by a function generator and an electromagnetic motor. Target frequencies were applied through the programming of the function generator. The acceleration amplitude of applied vibrations was measured with a laser-Doppler vibrometer, and images of moving droplets were captured with a high-speed camera. Measurements of droplet edge displacement were 
performed in MATLAB (The MathWorks, Natick, MA, USA) using custom scripts. All numeric data is presented as mean \pm standard deviation.

\section{Results and Discussion}

ARC tracks are characterized by the minimum acceleration amplitude at which the substrate must be vibrated to initiate droplet transport (ARC threshold). This threshold is known to be dependent on volume and material properties of the droplet (e.g., surface tension) and the interaction of the droplet footprint with the ARC surface pattern $[19,20]$. Similarly, devices presented in this work are characterized for droplets $10 \mu \mathrm{L}$ in volume and different behaviors are to be expected if the size or liquid of the droplet are sufficiently altered. However, the scaling of droplet behavior on ARC devices is predictable [20], and it is expected that scaling of the feature size for these devices could in turn accommodate a wider range of sizes and liquid properties.

\subsection{Effects of Duty Cycle}

On ARC patterns used in this work, we define rung duty cycle as the width of the hydrophilic rung divided by the hydrophobic period (center to center distance) between rungs (Figure A1). ARC designs used here consisted of $10 \mu \mathrm{m}$ wide rungs with a radius of $1000 \mu \mathrm{m}$ and a period of $60 \mu \mathrm{m}$ or $120 \mu \mathrm{m}$, providing for a duty cycle of $16.6 \%$ or $8.3 \%$, respectively (Figure 2 ). The ARC threshold of the $\mathrm{SiO}_{2}$-FOTS tracks was first determined over a range from 60 to $100 \mathrm{~Hz}$ (Figure 2E). We observed that the ARC threshold profiles, although not identical, were relatively similar on tracks with both $8.3 \%$ and $16.6 \%$ duty cycles. Additionally, the transition from $8.3 \%$ to $16.6 \%$ duty cycle also demonstrated an overlapping ARC threshold profile. However, the ARC threshold for the transition from $16.6 \%$ to $8.3 \%$ duty cycle exhibited a unique profile with significantly higher vibration thresholds above $60 \mathrm{~Hz}$. The observed increase in ARC threshold is due to the combination of increased pinning on the higher duty cycle region (trailing edge-facing the direction opposite of transport) and increased slip (de-wetting) on the lower duty cycle region (leading edge-facing the direction of transport-Figure A2). Essentially, the high duty cycle region creates more drag on the trailing edge, requiring a larger vibration amplitude to initiate transport.

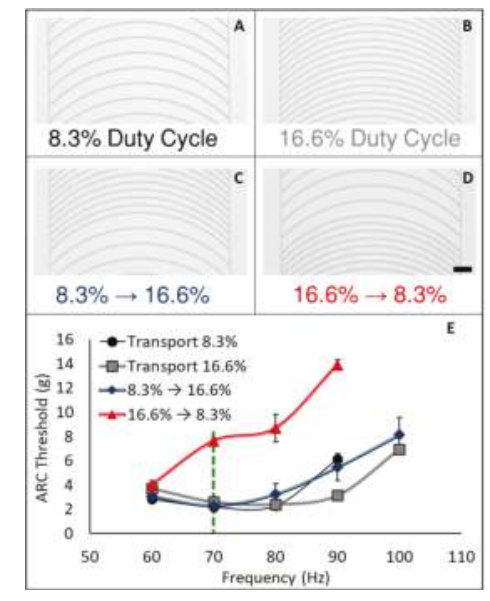

Figure 2. Rung duty cycle modulates ARC threshold. The ARC threshold for droplet transport $(10 \mu \mathrm{L}$ $\mathrm{diH}_{2} \mathrm{O}$ ) was measured on ARC tracks with $8.3 \%$ (A) and $16.6 \%$ (B) duty cycles, and transitions from $8.3 \%$ to $16.6 \%$ (C) and $16.6 \%$ to $8.3 \%$ (D). Only the transition from $16.6 \%$ to $8.3 \%$ required a significantly higher ARC threshold at frequencies above $60 \mathrm{~Hz}(\mathrm{E})$. The dotted green line indicates $70 \mathrm{~Hz}$ response used in subsequent experiments with ARC gates. Scale bar $=200 \mu \mathrm{m}$. 


\subsection{Anisotropic Ratchet Conveyor (ARC) Gates}

The effects of duty cycle transitions were then employed to enable "ARC gates", which can selectively pause droplet transport based on the signal of the applied vibrations. Droplet gates were developed by nesting a region with a higher $(16.6 \%)$ duty cycle within a track composed of a lower $(8.3 \%)$ duty cycle. Droplets driven by vibrations below the ARC threshold for the gate will pass through the transition from low to high duty cycle, but will pause on the transition from $16.6 \%$ to $8.3 \%$ duty cycle. When the vibration signal is increased above the ARC threshold for the gate, droplet transport will resume. Additionally, if a droplet is driven with a vibration above the ARC threshold for the gate before entering the gate, then it will pass through without stopping (Figure A3).

Stopping droplets on an ARC chip was previously achievable by turning off the vibration signal. However, this would stop all droplets being transported on a chip. ARC gates provide the ability to pause a single droplet without affecting the transport of other droplets on chip. For example, Figure 3 (also shown in Video S1) demonstrates how droplets with unique transport paths can be synchronized with ARC gates. On this chip, three droplets, each on a distinct ARC path, are transported by vibrations below the ARC threshold for the gate. The transport of each droplet will be paused once it reaches the gate. This allows for droplets on longer paths, such as the droplet on the left, or droplets that are performing processes elsewhere on chip to continue their transport. Once all three droplets have lined up on the gates, the vibration amplitude is increased, resuming the transport of all droplets in a tight distribution. In addition to synchronization, these gates can also be applied on an ARC system to hold droplets over a detection region or sensor, controllably mix droplets in the same transport path (Figure A4 and Video S2), and control the timing or sequencing of a droplet on chip.

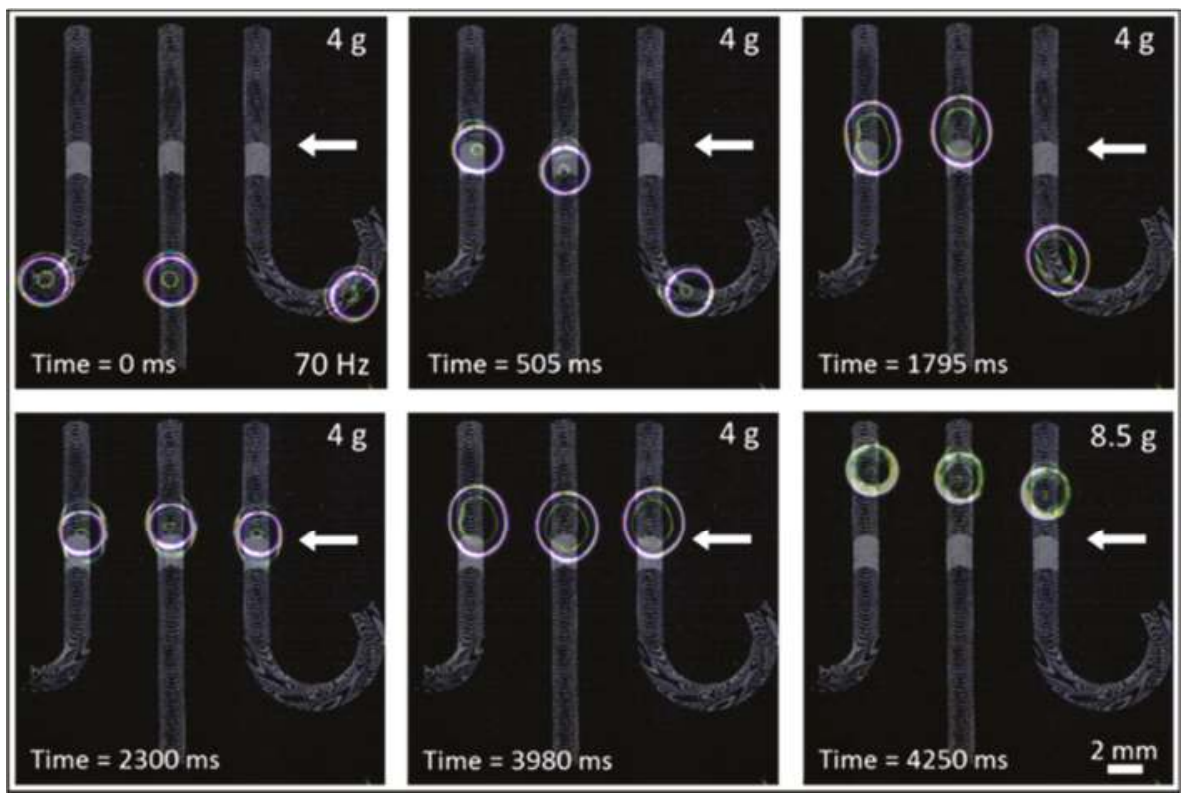

Figure 3. Droplet synchronization with ARC gates. Droplets transported on unique ARC paths with vibrations below the threshold of the ARC gate will pause at the transition from $16.6 \%$ to $8.3 \%$ duty cycle (indicated by the white arrow). Droplets will remain indefinitely at this position in the ARC gate, which allows droplets on all transport paths to line up (ARC patterns are superimposed in grey). Increasing the vibration signal above the gate threshold continues droplet transport in a tight distribution. 


\subsection{Anisotropic Ratchet Conveyor (ARC) Switches}

A transition in duty cycle changes the balance of pinning forces along one dimension of the droplet (between the leading and trailing edges). To understand how this balance of forces responds to changes in two dimensions, we added a second track perpendicular to the main track. In this case, pinning forces act on the leading and trailing edges of the droplet like a normal ARC track, but when the droplet reaches the perpendicular track, pinning forces also act on one side of the droplet. We found this simple combination provides an intersection, or switch, that can dictate the direction of droplet transport based on the applied vibration signal (Figure 4). Previously, switches on ARC systems had been realized through pairing with electrowetting [21], but the switches presented here are the first to provide the capability of controlling droplet directionality with no active surface components. The threshold profile for ARC switches was determined as previously discussed. However, switches have two thresholds-(1) the vibration required for a droplet to be transported through the intersection on the main track (straight-Video S3) and (2) the vibration required for the droplet to turn onto the perpendicular track (turn-Video S4). Expectedly, turning is more efficient when the duty cycle of the perpendicular track is higher than that of the main track (Figure A5).

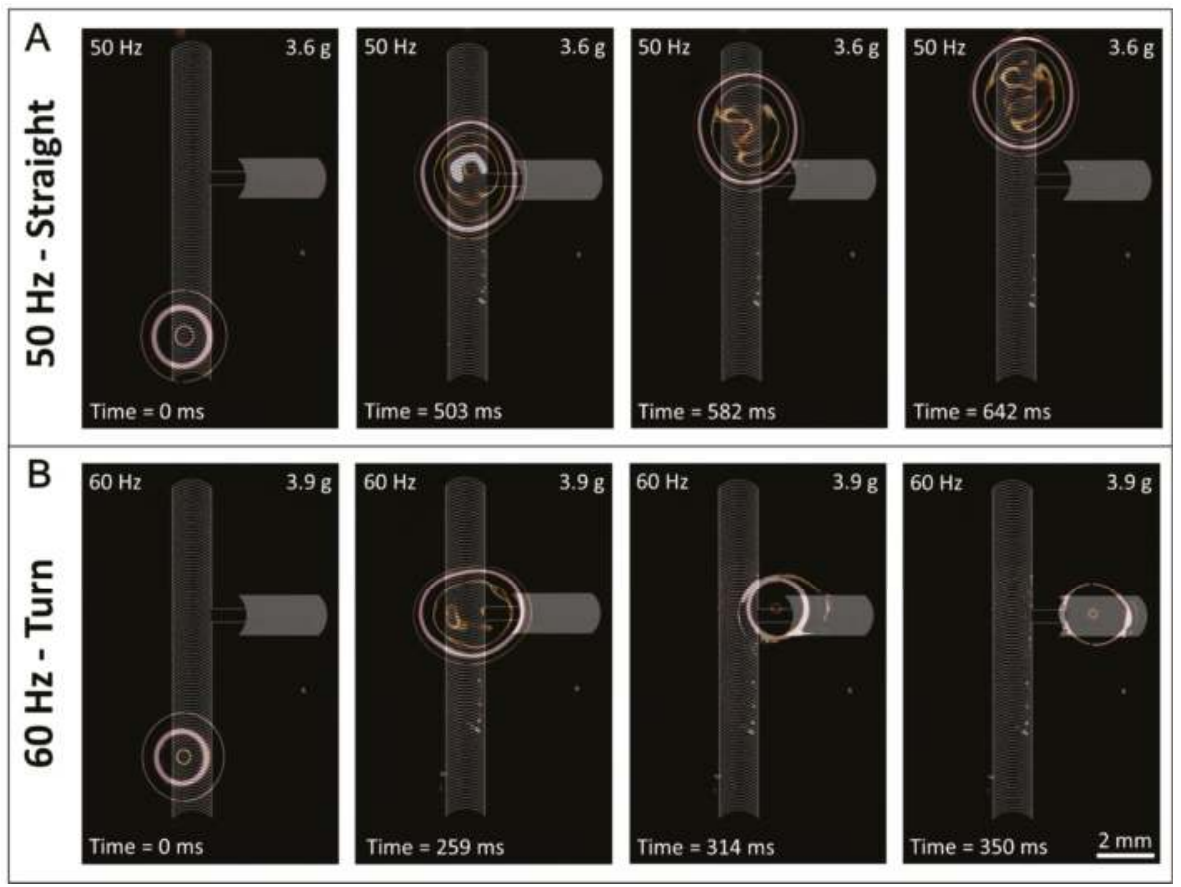

Figure 4. ARC switches can select direction of droplet transport. Image sequence shows droplets transported on an ARC switch having a main track of an $8.3 \%$ duty cycle with a perpendicular track of a $16.6 \%$ duty cycle. Droplets transported at $50 \mathrm{~Hz}$ and $3.6 \mathrm{~g}$ (A) contact the perpendicular track but move straight through the intersection. Vibrations of $60 \mathrm{~Hz}$ and $3.9 \mathrm{~g}$ (B) provide sufficient wetting and aspect ratio to turn the droplets at the intersection.

Data gathered from videos of droplets on the switches (shown in supplementary information) also indicate that two conditions must be met for turning to occur. The first condition is that the droplet edges must expand enough to contact the perpendicular track (Figure A6A). Although this condition is somewhat trivial, it suggests that these switches could innately sort droplets based on 
volume, as small droplets would be unable to sufficiently expand to contact the perpendicular track. The second condition is that the aspect ratio (length of the droplet in the direction of the main track divided by the width of the droplet perpendicular to the track) during wetting must be low enough (i.e., the droplet must be wide enough) for the pinning forces of the perpendicular track to dominate and change the direction of the droplet (Figure A6B). Furthermore, the shape of the droplets was always circular or elliptical and the formation of additional nodes was not observed. This indicates that a change in vibration mode $[22,23]$ is not responsible for these observations.

\subsection{Anisotropic Ratchet Conveyor (ARC) Delivery Junctions}

Passing droplets from one track to another is an important capability that is necessary for mixing droplets processed on alternate paths or derived from different sources. However, this function is non-trivial with ARC systems because simply merging two paths can create a local concentration of pinning forces (hydrophilic regions) that can impede transport through these regions (i.e., droplets get stuck). In order to provide this functionality to our ARC system, droplet junctions were developed that can deliver a droplet to an adjacent track without compromising transport of droplets on either track. This was accomplished by connecting the two tracks with hydrophilic guides. The spacing of these guides was adjusted to provide for delivery of a droplet from the terminating track without compromising the transport of droplets along the main track (i.e., if the guides are too close together droplets will get stuck at the junction, but if they are too far apart the droplet on the terminating track cannot be delivered to the main track). We hypothesize that the hydrophilic guides promote delivery from the terminated track by wicking or pulling the droplet edge towards the main track. When paired with the correct vibration signal, pinning forces on the main trail will overtake the droplet from the terminating track. Due to these interactions of droplets from different directions, two thresholds also exist for the delivery junctions: (1) Pass-the vibration amplitude required for the droplet to travel on the main track and pass the hydrophilic guides without getting stuck, and (2) Deliver-the vibration amplitude required to transfer a droplet from the terminating track onto the main track (Figure A7). Interestingly, the relationship of the pass and deliver thresholds appears quite similar to that for ARC gates.

Additionally, we discovered that these thresholds also provide distinct droplet behaviors when two droplets meet at this junction. Under vibration parameters that meet the conditions for passing the junction but not delivery, a droplet will be held at the junction while the droplet on the main track passes by. The droplets will remain separate, even though they appear to nearly touch (supplementary videos), and the droplet at the junction can be later delivered with appropriate vibration signal (Figure 5A and Video S5). However, if vibration parameters for delivery are applied, the droplets will merge at the junction, and the merged droplet will be subsequently delivered to the main track (Figure 5B and Video S6). Thus, this seemingly simple configuration presents a large variety of possible functions that can be performed through strategic selection of vibration parameters. 


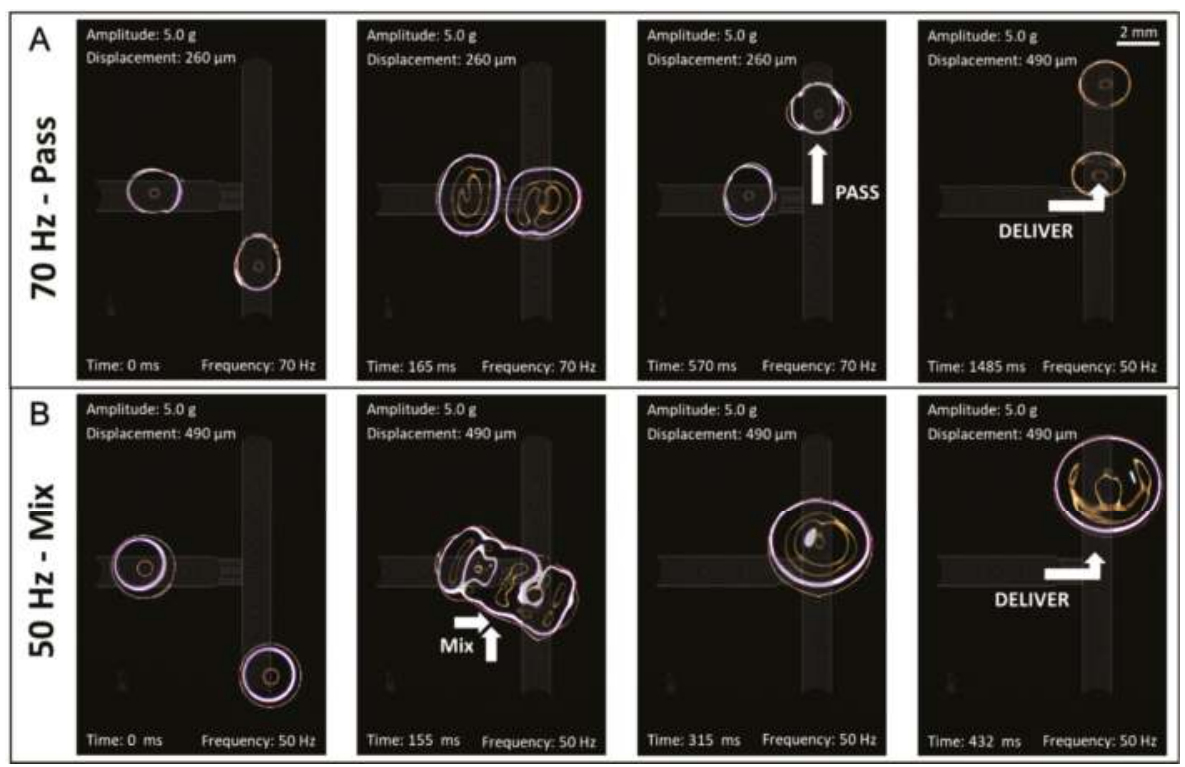

Figure 5. Delivery junctions can transfer and mix droplets. Droplets on a terminated track can be delivered to a main track with a delivery junction. At this intersection droplets can (A) pass by each other or (B) merge depending on the applied vibration signal. Most importantly, delivery junctions accomplish this functionality without impeding transport of droplets on the main track.

\section{Conclusions}

ARCs are a recently developed microfluidic platform that transports liquid droplets through a passive surface pattern and orthogonal vibrations. The facile fabrication and operation of ARC devices shows much potential to meet applications in low-cost diagnostic and analytic applications. In this work, we demonstrate new expansions to the ARC functional toolbox with the development of ARC gates, switches, and delivery junctions. All three modules derive their utility by changing the balance of pinning forces between edges of a transported droplet, either in one or two dimensions. ARC gates can controllably pause droplet transport through an increase in pinning forces at the trailing edge of a droplet, ARC switches provide control over droplet direction at an intersection by applying pinning forces at a side edge of the droplet, and ARC delivery junctions use hydrophilic guides to transfer droplets between tracks without impeding transport. In short, this system combines a simple hardware platform with a sequence of sine wave signals. Expanding on this work, future ARC devices could perform complex protocols that require conditional processing steps, or sequential regimens.

\section{Patents}

This work resulted in US Provisional Patent Applications No. 62/281,879 and No. 62/302,948 that have been converted into an International Patent Cooperation Treaty (PCT) Patent (PCT/US2017/014529) entitled "Contact-Line-Driven Microfluidic Devices and Methods".

Supplementary Materials: The following are available online at www.mdpi.com/2072-666X/8/12/363/s1, Video S1. Droplet synchronization on ARC gates; Video S2. Merging droplets on ARC gates; Video S3. ARC switch straight direction; Video S4. ARC switch turn direction; Video S5. Passing droplets on ARC delivery junctions; Video S6. Merging droplets on ARC delivery junctions.

Acknowledgments: The authors would like to thank Timothy A. Holmes for his help with design and fabrication of high-speed camera mounts. This work was supported by National Science Foundation grant ECCS-1308025 
“Droplet Ratchets: Low Cost Digital Microfluidics." Hal R. Holmes would like to acknowledge support by the Department of Defense (DoD) through the National Defense Science \& Engineering Graduate Fellowship (NDSEG) Program. Fabrication of ARCs was performed at the Washington Nanofabrication Facility (WNF), a National Nanotechnology Coordinated Infrastructure (NNCI) site at the University of Washington, which is supported in part by the National Science Foundation (awards 1542101, 1337840 and 0335765), the Washington Research Foundation, the M. J. Murdock Charitable Trust, Altatech, ClassOne Technology, Global Capital Equipment (GCE) Market, Google, and SPTS.

Author Contributions: Hal R. Holmes and Karl F. Böhringer conceived and designed the devices and experiments; Hal R. Holmes and Ana E. Gomez performed the experiments and analyzed the data; Hal R. Holmes wrote the paper.

Conflicts of Interest: The authors declare no conflict of interest.

\section{Appendix A}

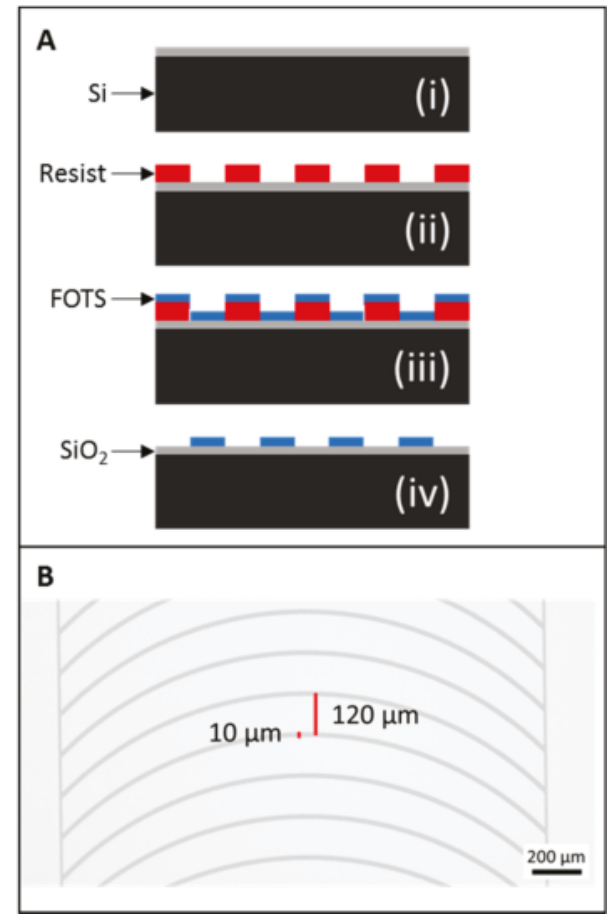

Figure A1. ARC fabrication and duty cycle. $\mathrm{SiO}_{2}$-FOTS ARCs are fabricated on a silicon wafer with an $\mathrm{SiO}_{2}$ surface layer (A-i). The ARC design is patterned with photoresist (A-ii) and the wafer is coated with FOTS (A-iii). Stripping the resist reveals the hydrophilic $\mathrm{SiO}_{2}$ pattern (A-iv). Rung duty cycle is defined as the width of the rungs $(10 \mu \mathrm{m})$ divided by the period between rungs. For example, a $120 \mu \mathrm{m}$ period (B) provides a duty cycle of $8.3 \%$. 

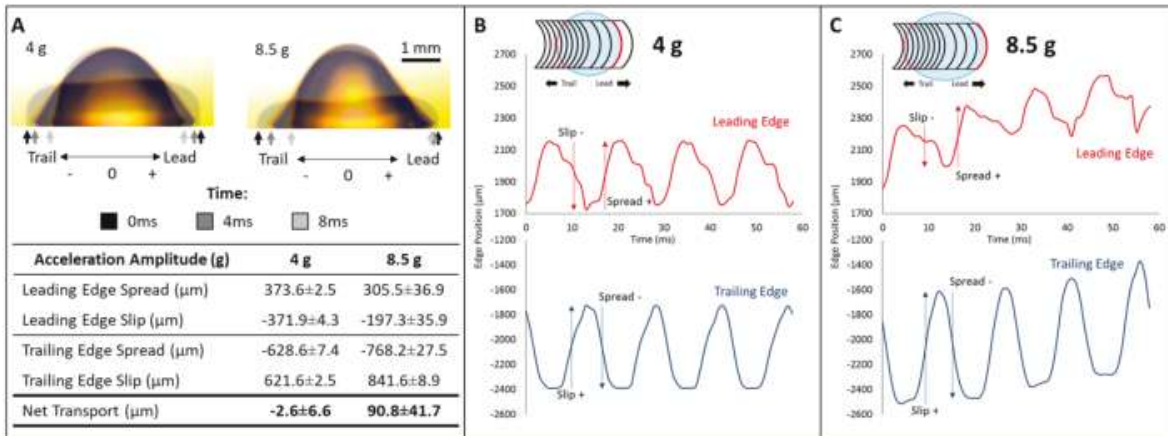

Figure A2. Increased trailing edge mobility reduces slip at leading edge. De-wetting sequence (A-figure overlay) demonstrates the difference in droplet response when vibrated on a $16.6 \%$ to $8.3 \%$ duty cycle transition at $4 \mathrm{~g}$ and $8.5 \mathrm{~g}$. Measurements of droplet edges (A-table) indicate slip (de-wetting) and spread (wetting) is the same for both edges at $4 \mathrm{~g}$. Raising the vibration amplitude to $8.5 \mathrm{~g}$ increased the spread of the trailing edge, but actually reduced the spread of the leading edge. However, this resulted in a lower slip at the leading edge and higher slip at the trailing edge (compared to spread), which provided for droplet transport. Real-time positions of droplet edges (with respect to the droplet center at 0 ) at $4 \mathrm{~g}(\mathrm{~B})$ and $8.5 \mathrm{~g}(\mathbf{C})$ demonstrate how these observed differences in slip and spread translate to net transport at $8.5 \mathrm{~g}$. Note that at the leading edge, spread is in the positive direction (direction of net transport) and slip is in the negative direction, conversely, at the trailing edge, spread is in the negative direction while slip is in the positive direction. Additionally, slip is defined as the distance from maximum to minimum wetting, while spread is the distance from minimum wetting to maximum wetting in the next half of the cycle.

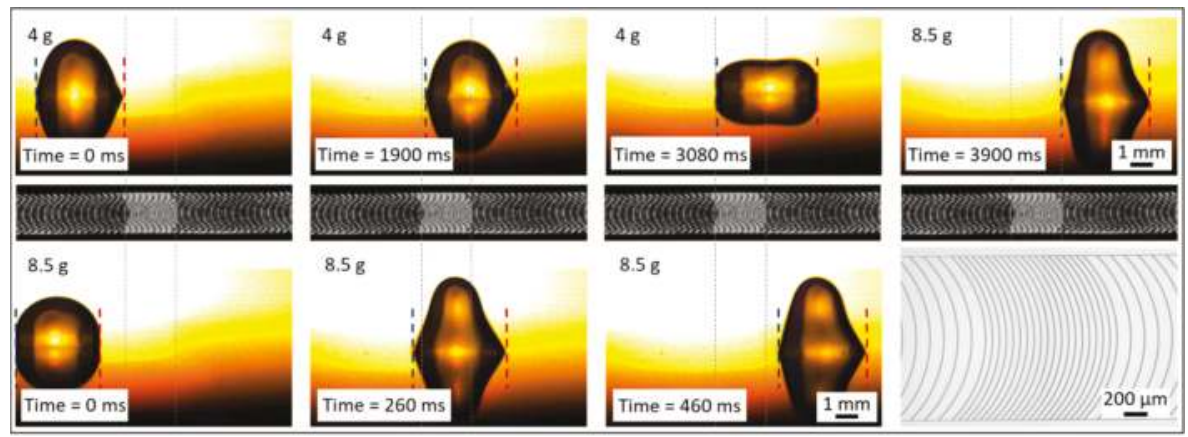

Figure A3. Droplet gate selectively passes droplets. Droplets $(10 \mu \mathrm{L})$ driven with a $70 \mathrm{~Hz}$ vibration of $4 \mathrm{~g}$ stopped at the gate (design-bottom right) until a vibration of $8.5 \mathrm{~g}$ was applied (top sequence). Droplets driven with a vibration of $8.5 \mathrm{~g}$ moved through the gate without stopping (bottom sequence). Center panels and gray dotted lines depict the track and gate with respect to the droplets. Red and blue dotted lines depict the leading and trailing edges of the droplets, respectively. 

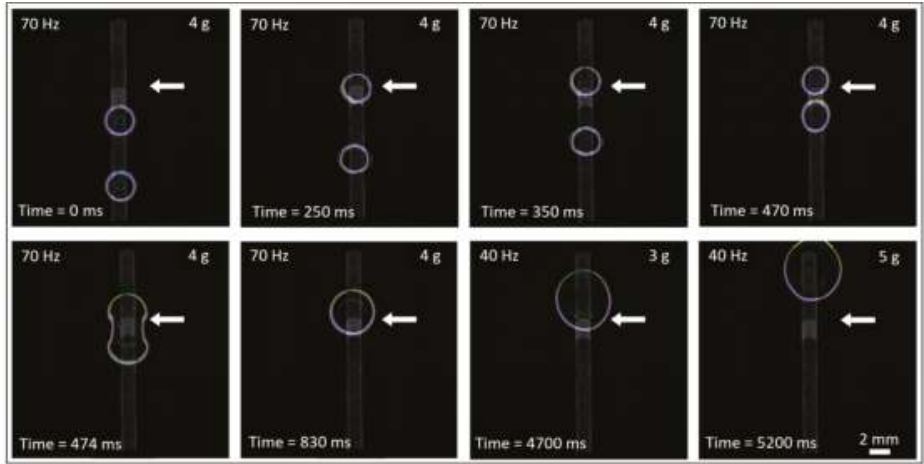

Figure A4. Droplet gates can mix droplets of the same volume. Two droplets (10 $\mu \mathrm{L})$ are transported on an ARC track with a droplet gate. Both droplets are transported, maintaining their initial separation, until the first droplet comes to the droplet gate. The gate holds this droplet in place until the second droplet comes in contact. The droplets spontaneously merge, forming a larger droplet. The larger droplet is then held in place by the droplet gate until sufficient vibration amplitude is applied (5 g). Note that the larger droplet has a lower natural frequency, which requires a lower vibration frequency for transport $(40 \mathrm{~Hz})$. The track design is depicted by the overlay (gray) and the white arrows indicate where the transition from the $16.6 \%$ to $8.3 \%$ duty cycle occurs.

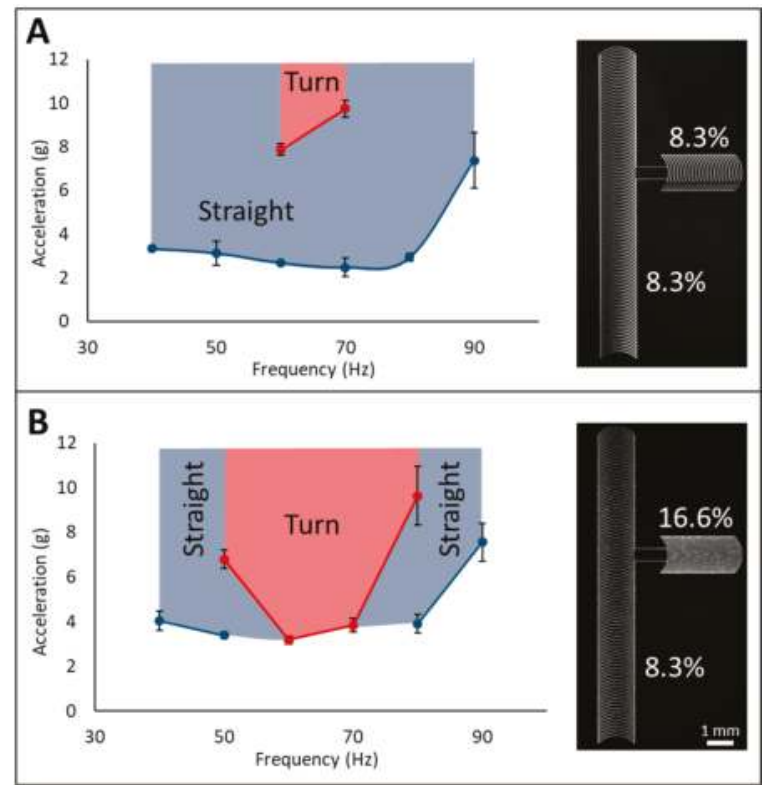

Figure A5. Perpendicular intersection enables ARC switch. The ARC thresholds for transporting droplets straight through or turning at the intersection were measured for switches having main and perpendicular tracks with $8.3 \%$ duty cycle (A) and a main track of $8.3 \%$ with a perpendicular track of $16.6 \%$ duty cycle (B). The increased pinning of the higher duty cycle perpendicular track enabled droplets to turn at much lower vibration amplitudes. Blue regions correspond to vibration parameters that drive the droplet straight through the intersection, while red regions correspond to parameters that turn the droplet at the intersection. 

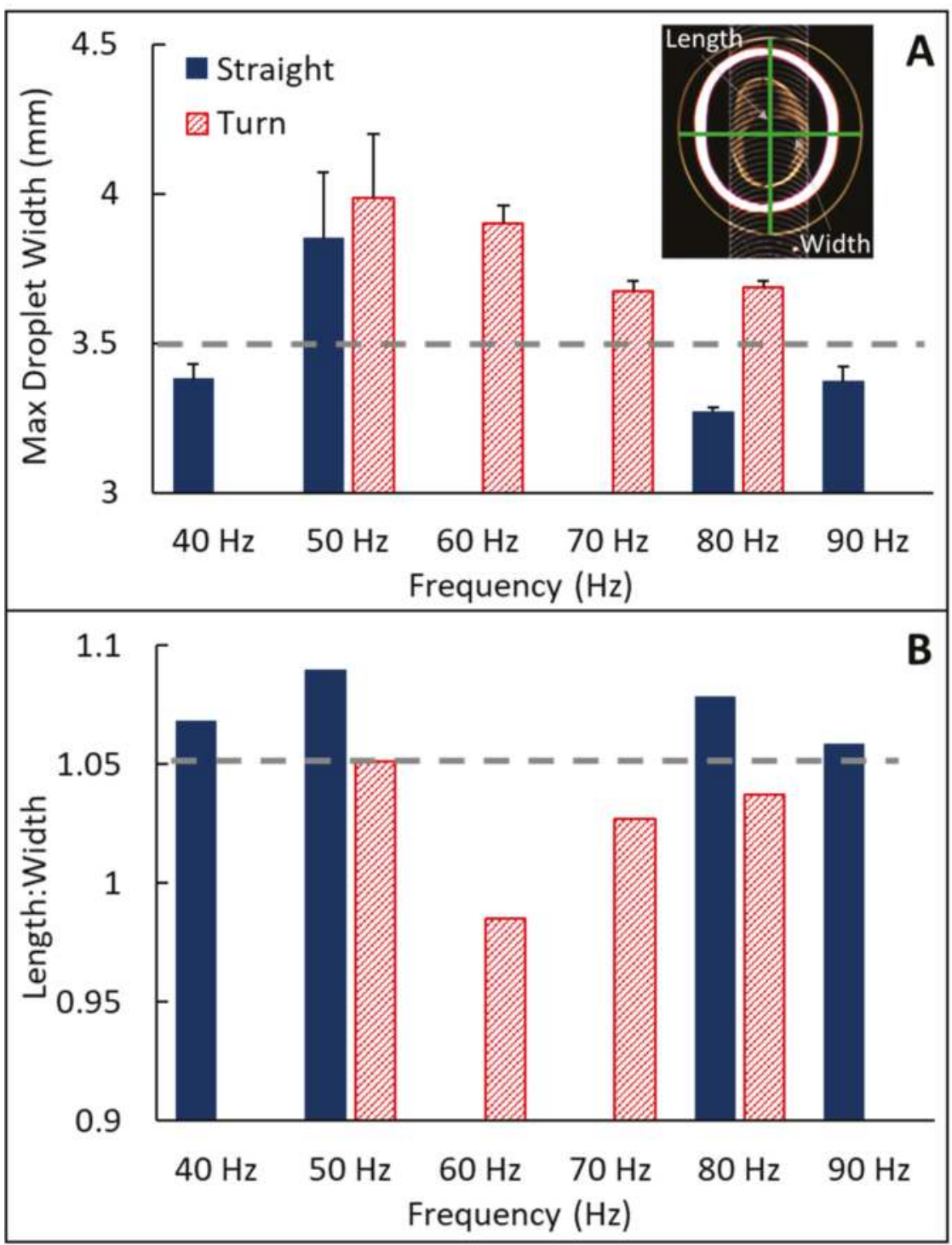

Figure A6. Turning droplets depends on droplet width and aspect ratio. The length and width (insert) of droplets during maximum wetting were measured for switches with a $16.6 \%$ duty cycle perpendicular track (8.3\% main track). These data indicate that two conditions must be met for a droplet to turn: the width of the droplet during wetting must be large enough to contact the perpendicular track (this distance is indicated by the dotted grey line-(A), and the aspect ratio (B) must be sufficient for pinning forces on the right edge of the droplet to dominate. 


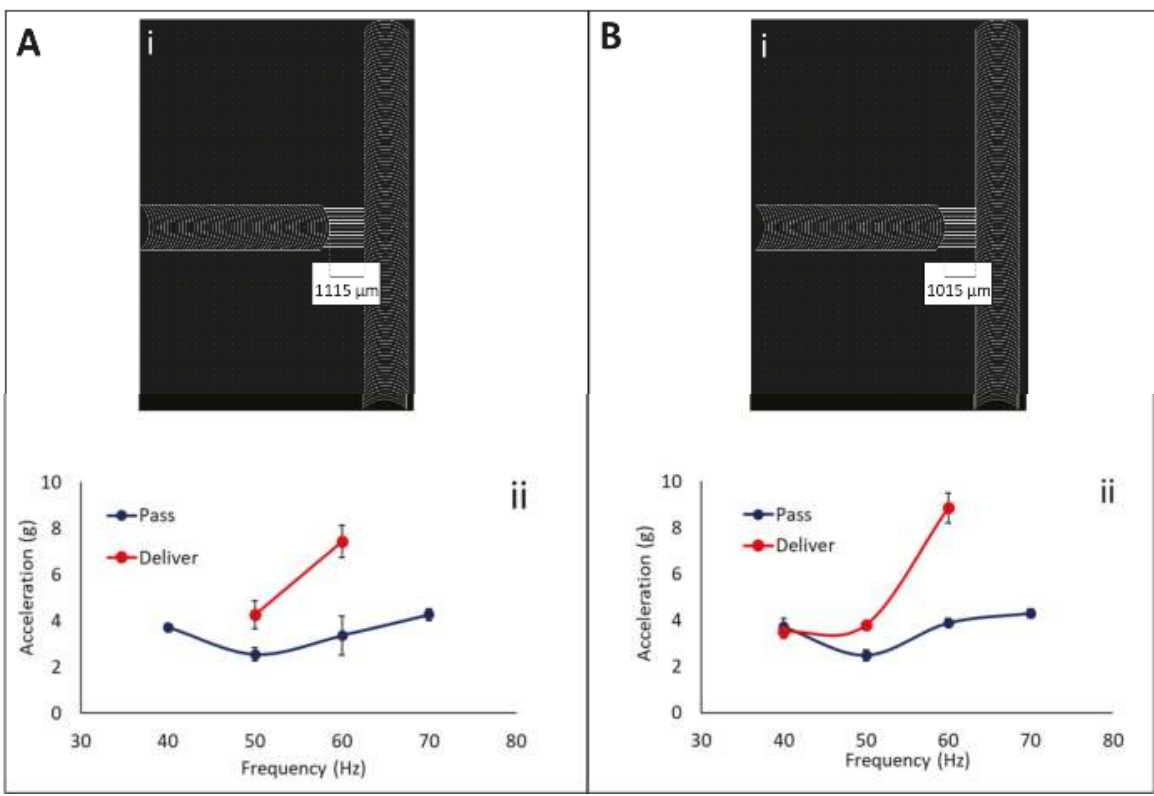

Figure A7. Delivery junctions can transfer droplets between tracks without impeding transport. Pass and delivery thresholds for delivery junctions with (A) $1115 \mu \mathrm{m}$ and (B) $1015 \mu \mathrm{m}$ separation between the terminating track and the main track were investigated. Thin hydrophilic guides (Ai,Bi) provide connectivity between the terminating track and main track without impeding transport of droplets on the main track. ARC thresholds (Aii,Bii) to pass a droplet on the main track by this junction and deliver a droplet across this junction were characterized for $10 \mu \mathrm{L}$ droplets.

\section{References}

1. Holmes, H.R.; Böhringer, K.F. Transporting droplets through surface anisotropy. Microsyst. Nanoeng. 2015, 1. [CrossRef]

2. Duncombe, T.A.; Erdem, E.Y.; Shastry, A.; Baskaran, R.; Böhringer, K.F. Controlling liquid drops with texture ratchets. Adv. Mater. 2012, 24, 1545-1550. [CrossRef] [PubMed]

3. Duncombe, T.A.; Parsons, J.F.; Böhringer, K.F. Directed drop transport rectified from orthogonal vibrations via a flat wetting barrier ratchet. Langmuir 2012, 28, 13765-13770. [CrossRef] [PubMed]

4. Abdelgawad, M.; Wheeler, A.R. The digital revolution: A new paradigm for microfluidics. Adv. Mater. 2009, 21, 920-925. [CrossRef]

5. Park, S.-Y.; Kalim, S.; Callahan, C.; Teitell, M.A.; Chiou, E.P. A light-induced dielectrophoretic droplet manipulation platform. Lab Chip 2009, 9, 3228-3235. [CrossRef] [PubMed]

6. Geng, H.; Feng, J.; Marie Stabryla, L.; Kwon Cho, S. Dielectrowetting manipulation for digital microfluidics: Creating, transporting, splitting, and merging of droplets. Lab Chip 2017, 17, 1060-1068. [CrossRef] [PubMed]

7. Fu, E.; Liang, T.; Spicar-Mihalic, P.; Houghtaling, J.; Ramachandran, S.; Yager, P. Two-dimensional paper network format that enables simple multistep assays for use in low-resource settings in the context of malaria antigen detection. Anal. Chem. 2012, 84, 4574-4579. [CrossRef] [PubMed]

8. Li, X.; Ballerini, D.R.; Shen, W. A perspective on paper-based microfluidics: Current status and future trends. Biomicrofluidics 2012, 6. [CrossRef] [PubMed]

9. Fu, E.; Liang, T.; Houghtaling, J.; Ramachandran, S.; Ramsey, S.A.; Lutz, B.; Yager, P. Enhanced sensitivity of lateral flow tests using a two-dimensional paper network format. Anal. Chem. 2011, 83, 7941-7946. [CrossRef] [PubMed] 
10. Fu, E.; Lutz, B.; Kauffman, P.; Yager, P. Controlled reagent transport in disposable 2D paper networks. Lab Chip 2010, 10, 918-920. [CrossRef] [PubMed]

11. Gervais, L.; Delamarche, E. Toward one-step point-of-care immunodiagnostics using capillary-driven microfluidics and PDMS substrates. Lab Chip 2009, 9, 3330-3337. [CrossRef] [PubMed]

12. Holland, C.A.; Kiechle, F.L. Point-of-care molecular diagnostic systems-past, present and future. Curr. Opin. Microbiol. 2005, 8, 504-509. [CrossRef] [PubMed]

13. Niemz, A.; Ferguson, T.M.; Boyle, D.S. Point-of-care nucleic acid testing for infectious diseases. Trends Biotechnol. 2011, 29, 240-250. [CrossRef] [PubMed]

14. Britton, S.; Cheng, Q.; McCarthy, J.S. Novel molecular diagnostic tools for malaria elimination: A review of options from the point of view of high-throughput and applicability in resource limited settings. Malar. J. 2016, 15, 88. [CrossRef] [PubMed]

15. Camper, D.V.; Viola, R.E. Fully automated protein purification. Anal. Biochem. 2009, 393, 176-181. [CrossRef] [PubMed]

16. Berensmeier, S. Magnetic particles for the separation and purification of nucleic acids. Appl. Microbiol. Biotechnol. 2006, 73, 495-504. [CrossRef] [PubMed]

17. Lee, J.; Moon, H.; Fowler, J.; Schoellhammer, T.; Kim, C.-J. Electrowetting and electrowetting-on-dielectric for microscale liquid handling. Sens. Actuators A Phys. 2002, 95, 259-268. [CrossRef]

18. Mugele, F.; Baret, J.-C. Electrowetting: From basics to applications. J. Phys. Condens. Matter 2005, 17, R705. [CrossRef]

19. Holmes, H.R.; Böhringer, K.F. Transport velocity of droplets on ratchet conveyors. Adv. Colloid Interface Sci. 2017. [CrossRef] [PubMed]

20. Dong, Y.; Holmes, H.R.; Böhringer, K.F. Converting vertical vibration of anisotropic ratchet conveyors into horizontal droplet motion. Langmuir 2017, 33, 10745-10752. [CrossRef] [PubMed]

21. Duncombe, T.A.; Kumemura, M.; Fujita, H.; Böhringer, K.F. Integrating EWOD with Surface Ratchets for Active Droplet Transport and Sorting. In Proceedings of the IEEE 22nd International Conference on Micro Electro Mechanical Systems, Sorrento, Italy, 25-29 January 2009; pp. 531-534.

22. Perez, M.; Brechet, Y.; Salvo, L.; Papoular, M.; Suery, M. Oscillation of liquid drops under gravity: Influence of shape on the resonance frequency. Europhys. Lett. 1999, 47, 189-195. [CrossRef]

23. McHale, G.; Elliott, S.J.; Newton, M.I.; Herbertson, D.L.; Esmer, K. Levitation-free vibrated droplets: Resonant oscillations of liquid marbles. Langmuir 2008, 25, 529-533. [CrossRef] [PubMed]

(C) 2017 by the authors. Licensee MDPI, Basel, Switzerland. This article is an open access article distributed under the terms and conditions of the Creative Commons Attribution (CC BY) license (http:/ / creativecommons.org/licenses/by/4.0/). 
Article

\title{
Laser-Assisted Mist Capillary Self-Alignment
}

\author{
Bo Chang ${ }^{1,2, *}$, Zhaofei Zhu ${ }^{1}$, Mikko Koverola ${ }^{3}$ and Quan Zhou ${ }^{3}$ \\ 1 College of Mechanical and Electrical Engineering, Shaanxi University of Science and Technology, \\ Xi'an 710021, China; zhuzhaofei@sust.edu.cn \\ 2 Department of Applied Physics, Aalto University, 02150 Espoo, Finland \\ 3 Department of Electrical Engineering and Automation, Aalto University, 02150 Espoo, Finland; \\ mikko.koverola@aalto.fi (M.K.); quan.zhou@aalto.fi (Q.Z.) \\ * Correspondence: bo.chang@aalto.fi; Tel.: +358-40-5546-333
}

Received: 9 November 2017; Accepted: 13 December 2017; Published: 15 December 2017

\begin{abstract}
This paper reports a method combining laser die transfer and mist capillary self-alignment. The laser die transfer technique is employed to feed selected microchips from a thermal release tape onto a receiving substrate and mist capillary self-alignment is applied to align the microchips to the predefined receptor sites on the substrate in high-accuracy. The parameters for a low-power laser die transfer process have been investigated and experimentally optimized. The acting forces during the mist-induced capillary self-alignment process have been analyzed and the critical volume enabling capillary self-alignment has been estimated theoretically and experimentally. We have demonstrated that microchips can be transferred onto receptor sites in 300-400 ms using a low-power laser $(100 \mathrm{~mW})$, and chips can self-align to the corresponding receptor sites in parallel with alignment accuracy of $1.4 \pm 0.8 \mu \mathrm{m}$. The proposed technique has great potential in high-throughput and high-accuracy assembly of micro devices. This paper is extended from an early conference paper (MARSS 2017).
\end{abstract}

Keywords: mist capillary self-alignment; laser die transfer; hydrophilic/superhydrophobic patterned surfaces; microasssembly

\section{Introduction}

High-throughput and high-accuracy microassembly is still a challenging task due to the undesired adhesion forces between micro parts and handling tools at the microscale. To tackle the challenge, microassembly driven by capillary forces [1-5], electrostatic forces [6], and magnetic forces [7] have been reported for various applications, such as assembly of radio frequency identification (RFID) [8], electrical networks [9], lighting emitting diodes [10], and solar cells [11]. Especially, capillary forces have been extensively exploited in fluidic self-assembly [1,11,12], droplet self-assembly $[1,13,14]$, and capillary self-folding $[15,16]$. In microassembly, micro parts need to be transported to the desired assembly sites. Hybrid microassembly techniques combining robotic pick-and-place and capillary self-alignment techniques have been reported to achieve fast transportation and accurate self-alignment [17]. Assembly methods, such as transfer printing and the laser die transfer technique, have also been reported for high-performance transportation of micro components onto the desired assembly sites. Transfer printing is a technique that transfers micro parts using a flexible stamp onto the target substrate [18]. Similar to the transfer printing technique, the laser die transfer technique uses a laser device to release microchips from their carrier and transfer towards a receiving substrate [19]. Both the transfer printing and laser die transfer techniques provide effective solutions for feeding microchips onto receiving receptor sites. However, the transfer printing technique requires a special stamp, and is mainly used for constructing thin structures and stretchable electronics [20,21]. On the other hand, the placement accuracy of laser die transfer is often very low and on the scale of tens of 
micrometers [19,22]. Moreover, laser die transfer often utilizes a high-power laser source, which may increase the cost of the assembly equipment.

In this paper, we propose a new hybrid microassembly technique to achieve high-throughput and high-accuracy microassembly. The proposed method combines non-contact laser die transfer and parallel mist capillary self-alignment. The laser die transfer technique is used for fast transportation of microchips onto receptor sites and the mist capillary self-assembly technique is applied to achieve parallel and high-accuracy alignment. To transport chips, a low-power and low-cost laser device was employed. Hydrophilic/superhydrophobic patterned surfaces were fabricated and served as receptor sites. Microscopic droplets in the form of water mist were introduced as the media for mist capillary self-alignment. Initial results of this work have been reported in a conference (MARSS 2017) [23]. In addition to the concept and experimental study, this paper reports detailed fabrication methods for the substrate, methods for estimating effective laser burning areas, analysis of the mist-alignment process, related critical volume and forces, and in-depth analysis of the experimental results.

\section{Methods and Materials}

\subsection{Materials}

In this work, microchips were fabricated by dicing 5 -inch silicon wafers using a dicing saw (Micro Ace 3, Micro Precision Engineering, Wisconsin, WI, USA) and the size of the diced chips is $500 \mu \mathrm{m} \times 500 \mu \mathrm{m} \times 300 \mu \mathrm{m}$. The diced silicon chips are shown in Figure 1a. The chips were attached to a thermal release tape (Graphene Supermarket, graphene transfer tape) which has similar adhesion as normal adhesive tape at room temperature and loses its adhesive property by heating up to $100{ }^{\circ} \mathrm{C}$. To achieve mist capillary self-alignment, water droplets should be confined inside the receptor sites and a large wetting contrast between the receptor sites and its surrounding is preferred [3]. A similar method [24] was used to fabricate hydrophilic receptor sites and superhydrophobic black silicon substrate, with a different final step where the fluoropolymer coating was removed from the receptor sites using lift-off in buffered hydrofluoric acid. The fabricated patterned surface consists of $500 \mu \mathrm{m} \times 500 \mu \mathrm{m}$ Si pads and black silicon background as shown in Figure 1b. The surface of the black silicon substrate has micro and nano structures. The measured water contact angles are $50^{\circ}$ and $170^{\circ}$ on Si receptor sites and the superhydrophobic black silicon substrate, respectively.
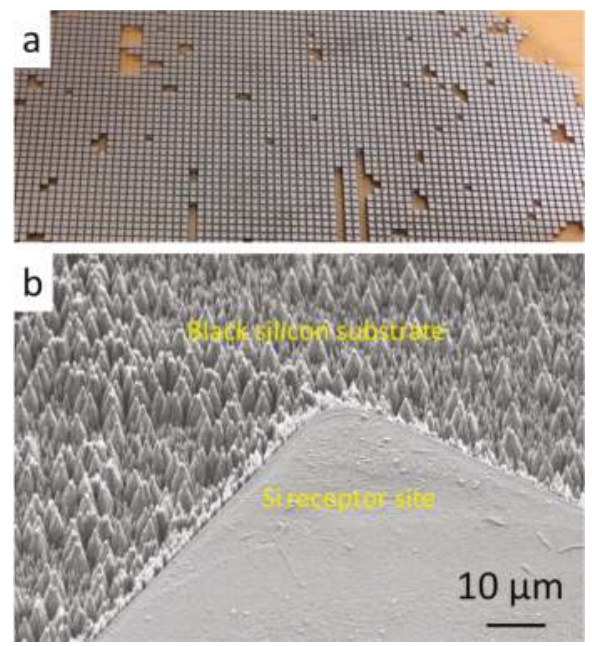

Figure 1. Silicon chips and fabricated receptor sites: (a) $500 \mu \mathrm{m} \times 500 \mu \mathrm{m} \times 300 \mu \mathrm{m}$ diced silicon chips; and (b) $500 \mu \mathrm{m} \times 500 \mu \mathrm{m}$ hydrophilic Si receptor sites with superhydrophobic black silicon substrate. 


\subsection{Method}

We propose a laser-assisted mist capillary self-alignment method, which combines laser die transfer technique and mist capillary self-alignment. The proposed method is illustrated in Figure 2, the microchips were firstly attached to a thermal release tape (Figure 2a); next the laser beam was pointed at the target chips and the laser fired; then the chips were released from the tape and landed on the corresponding receptor sites with an initial placement error when the curing temperature of the thermal tape was reached, as shown in Figure 2b. Next, water mist was introduced to the assembly sites. Finally, all the chips were self-aligned with the corresponding receptor sites.

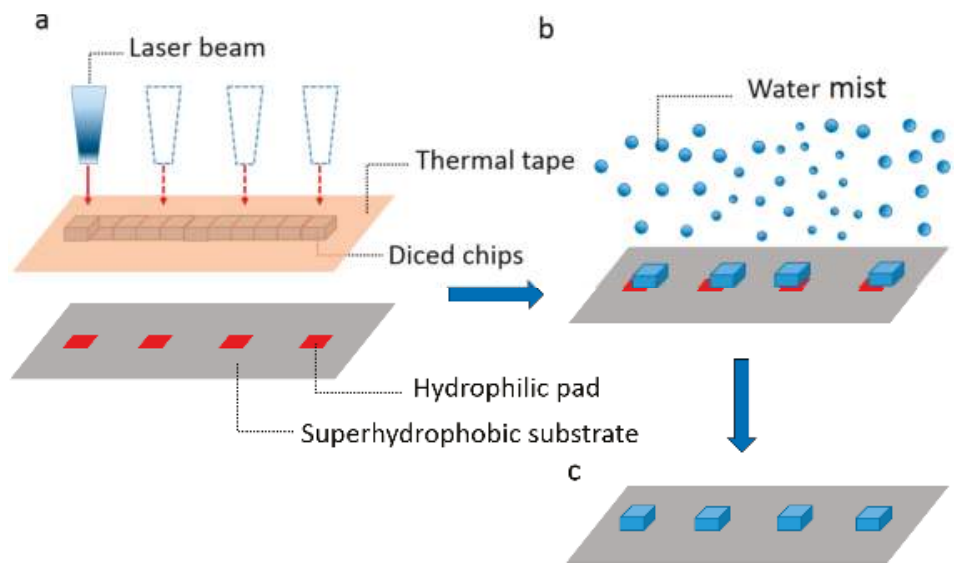

Figure 2. Procedure of laser-assisted mist capillary self-alignment: (a) chips were attached to a thermal release tape and a laser beam was pointed at the target chip; (b) the laser was fired and the target chips were released from the thermal tape and landed on the receiving receptor sites with an initial placement error; and (c) mist-induced micro droplets were delivered to the assembly sites and microchips were aligned to the corresponding receptor sites.

\subsection{Experimental Setup}

A robotic system has been set up for carrying out the laser die transfer experiments. The system consists of a laser device (Wicked Lasers, EVO, Euro Intlchoice Tech. Ltd), a sample carrier mounting on three motorized stages (Physik Instrumente M-122.2DD, M-404.8PD, and M-111.1DG, PI GmbH \& Co. KG, Karlsruhe, Germany) and two microscopes (InfiniTube and VZM 1000i, Edmund Optics, Barrington, NJ, USA) connected to two identical charge-coupled device (CCD) cameras, respectively (IGV-B1621, Imprex, Milwaukee, WI, USA). In our tests, we have selected a low-cost and low-power laser device with a wavelength of $532 \mathrm{~nm}$ and a power of $100 \mathrm{~mW}$. The laser operates in continuous mode. A focal lens was attached to the laser device to release the microchips from the thermal release tape. The motorized stages were used to move the test samples along three axis. The CCD cameras were used to calibrate the coordinates of each microchip relative to the laser beam coordinates and record the releasing process of microchips. Due to the high brightness of the laser beam, a protective glass was placed in front of the side view microscope to protect the reflected laser light damaging the side view CCD camera. Mist capillary self-alignment was carried out using an ultrasonic humidifier (model: Bionaire Ultrasonic Compact BU1300W-I, Bionaire Inc., Montreal, QC, Canada), which can generate a massive amount of microscopic droplets. To spread the mist evenly on the sample, two silicon tubes were connected to the exit of the humidifier and placed near to the sample carrier from two opposite directions. 


\section{Results}

\subsection{Laser Beam Diameter Estimation}

A stable release of a microchip from a thermal tape requires a powerful enough laser device, a large enough laser beam diameter, and a uniform laser beam intensity distribution [25]. Ideally, the laser beam should cover the whole surface area of the wafer die at the laser beam focal point. An estimation of the laser beam diameter was performed as shown in Figure 3. To further increase the burning power, a focal lens was attached to the laser device and the focal length is roughly $5 \mathrm{~cm}$, as indicated in Figure 3a. A piece of thermal release tape was attached to the sample holder and one reference mark was marked on the thermal release tape. The sample holder was mounted on the sample carrier and moved roughly $5 \mathrm{~cm}$ from the focal lens. The test was repeated three times, where the reference mark was burned with the focused laser beam for $2 \mathrm{~s}$. After the reference mark was burned with laser, the burn mark was inspected and calibrated with a microscope (SZ-STU2, Olympus, Tokyo, Japan) as shown in Figure 4b.

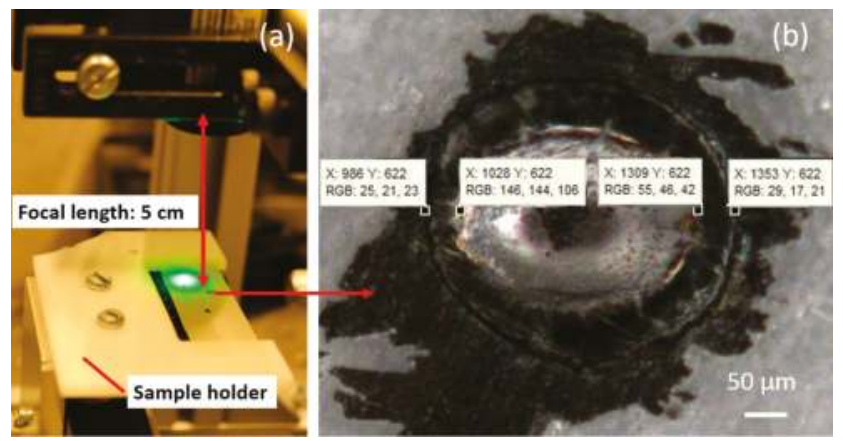

Figure 3. Laser beam diameter estimation: (a) the tape is mounted roughly $5 \mathrm{~cm}$ away from the focal lens and a reference mark is used to estimate the laser beam diameter at its maximum intensity; and (b) zoomed image of a laser beam burn with the inner and outer diameter in pixels.

The inner and outer diameters of the laser beam burn marks are presented in micrometers, as shown in Table 1. The inner diameter represents presumably the diameter of the laser beam when its intensity is at its maximum. This estimation was done to obtain an idea of the scale of the laser beam diameters and it was used to narrow down the size of microchips used in die release tests.

Table 1. Inner and outer diameters of the laser beam.

\begin{tabular}{ccc}
\hline Test & Inner Diameter $(\mu \mathrm{m})$ & Outer Diameter $(\mu \mathrm{m})$ \\
\hline$\# 1$ & 240.54 & 316.22 \\
$\# 2$ & 253.15 & 330.63 \\
$\# 3$ & 263.06 & 315.32 \\
\hline Average & 252.58 & 320.72 \\
\hline
\end{tabular}

\subsection{Mist Capillary Self-Alignment Process}

Mist capillary self-alignment is based on the principle of surface energy minimization, where the gradient of potential drives the chip to align with the receptor site. In our case, the alignment process involves drops merging and liquid meniscus formation. The schematic and force configuration of the mist capillary self-alignment process is shown in Figure 4 , in which $G, N, F_{\text {max }}, F_{\text {Adhesion }}, F_{\text {friction }}, F_{\gamma}$ and $E_{\gamma}$ represents the gravity of the chip, normal force, static friction force, adhesion force, friction force, surface tension force, and surface energy, respectively. 
(a)

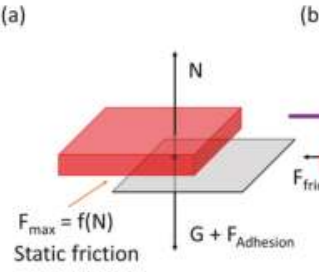

Dry contact (b)

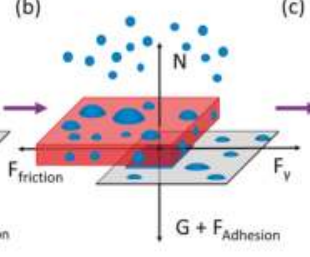

Partial wetting

$F_{y}=F_{\text {friction }}<F_{\max }$

(c)

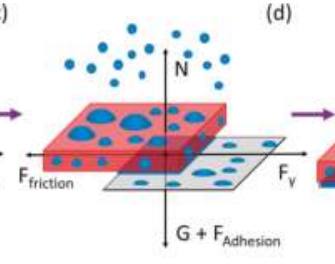

Self-aligning
$F_{y}>F_{\max }$ (d)

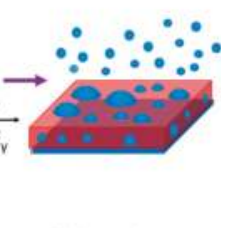

Self-aligned

$E_{\mathrm{v}}=\min \left(E_{\mathrm{y}}\right)$

Figure 4. Schematic and force configuration of mist capillary self-alignment process: (a) a chip is placed on the top of a receptor site and dry contact forms in between; (b) mist induced droplets are delivered onto the assembly site and partially wet the gap between the chip and receptor site; (c) droplets wet the whole gap and overcome the static friction force while driving the chip to self-align with the receptor site; and (d) the surface energy of the meniscus in the gap is minimized and the chip is aligned with the receptor site.

Firstly, a chip is transferred onto a receptor site using the laser die transfer technique and the chip is in dry contact with the receptor site, the normal force $N$ equals the sum of the gravity $G$ and adhesion force $F_{\text {Adhesion, }}$ and the magnitude of the static friction force depends on the normal force (Figure $4 \mathrm{a}$ ). Then water mist is delivered to the assembly site and droplets start to accumulate and merge with each other on both the chip and the receptor site; gradually, a water meniscus is formed in the gap between the chip and the receptor site and partially wets the gap (Figure $4 \mathrm{~b}$ ). The surface tension of the meniscus $F_{\gamma}$ overcomes the dry friction force $F_{\text {max }}$ and pulls the chip towards the receptor site when the volume of the meniscus in the gap increases to a critical volume $V_{c}$ (Figure 4c). Finally, the chip is aligned to the receptor site where the surface energy of the meniscus $E_{\gamma}$ is minimized (Figure 4d). The droplet accumulation process is a linear process [14], where the volume of droplets increase linearly with the accumulation time; therefore, the critical volume $V_{c}$ to enable self-alignment can be estimated based on the droplet accumulation rate and time on the receptor site. We recorded the droplet accumulation process with a video camera and the volume of the droplets was calculated frame by frame. Figure 5 shows the volume of drops accumulated on a $0.2 \mathrm{~mm}^{2}$ receptor site as the function of accumulation time and the relationship can be represented with a linear equation $V=0.13 t-0.031$, where $V$ is the volume of the droplets accumulated on the $0.2 \mathrm{~mm}^{2}$ receptor site and $t$ is the accumulation time. We recorded the size of the gap between the chip and the receptor site and the accumulation time for self-alignment from the captured video (Supplementary Video S1). Based on the accumulation time and the size of the gap, the critical volume for capillary self-alignment can be calculated and estimated, which is about $0.7 \mathrm{~nL}$.

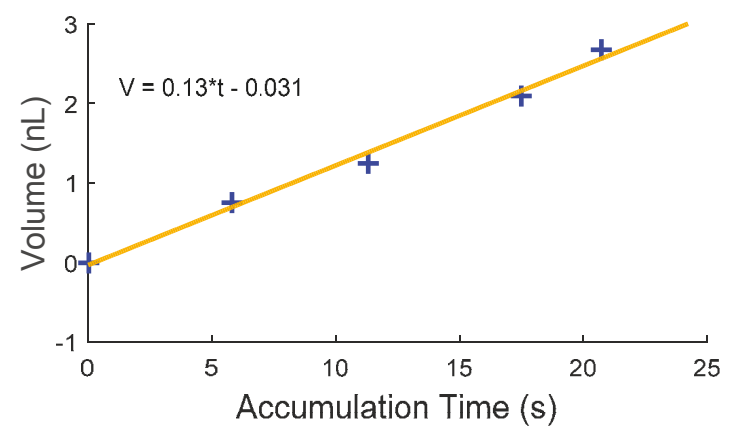

Figure 5. Linear water accumulation process. 
We simulated the mist capillary self-alignment process for $500 \mu \mathrm{m} \times 500 \mu \mathrm{m} \times 300 \mu \mathrm{m}$ chips on the same sized receptor sites using Surface Evolver [26] which finds the static equilibrium for a liquid meniscus by evolving the surface using the gradient descent method. Surface Evolver breaks the surface of the liquid meniscus into smaller elements and minimizes the surface energy of each element by optimizing the location of each vertex. In the simulation, the contact angles of the chip, receptor site, and background were $80^{\circ}, 50^{\circ}, 170^{\circ}$, respectively. The volume of the liquid used is the estimated critical volume of $0.7 \mathrm{~nL}$. Figure 6 shows the surface energy and the restoring force versus the misalignment bias. The $x$ bias is defined as the placement error between the chip and receptor site along the $x$-axis and the restoring force is the driven force for the chip to align with the receptor site. The results show that the surface energy of the water meniscus is minimized as the $x$ bias reaches zero, which is where the chip is aligned with the receptor site. The restoring force is in the range of $15-25 \mu \mathrm{N}$ which should be in the same scale as the static friction force.

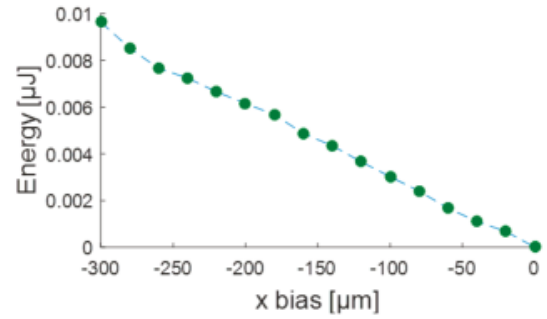

(a)

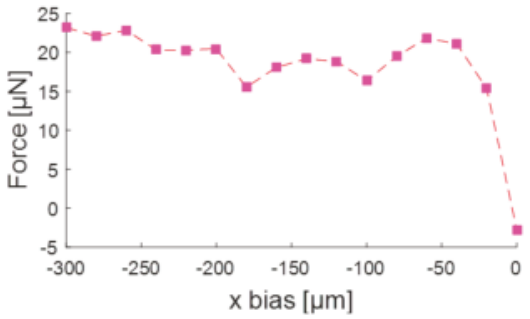

(b)

Figure 6. Surface energy (a) and restoring force (b) as a function of $x$ bias.

\subsection{Laser Die Releasing Procedure}

We have developed a procedure to carry out laser die releasing tests. The procedure is illustrated in Figure 7: firstly, a black dot was marked as a reference mark on the tape with attached microchips; then, the reference mark was moved under the laser beam and the laser beam was focused at the reference mark to make a burn (Figure 7a,d); meanwhile, the coordinates of the laser beam focal point were stored; after this, the reference mark was moved under the top view microscope and the center of the reference mark burn was stored and highlighted with a crosshair (Figure $7 \mathrm{~b}, \mathrm{e}$ ); next, the center of the chip was moved under the top view microscope and their coordinates were stored separately. By subtracting the relative distances between the center of the reference mark burn and the centers of the chips from the previously-stored coordinates of the laser beam focal point, the control script could calculate the correct position of the microchips so that the laser beam focal point is aligned with the center of each chip (Figure 7c,f). These coordinates were stored by the control script and the tape with microchips could be moved to the coordinates by pushing a button on the gamepad. Each of the chips was moved under the laser beam focal point sequentially and the laser beam was fired to release the chip from the thermal release tape. The releasing process was observed and recorded by the side view microscope.

We have carried out the die releasing tests using $500 \mu \mathrm{m} \times 500 \mu \mathrm{m} \times 300 \mu \mathrm{m}$ silicon chips. The chips were placed manually on a thermal tape and the target receptor sites were mounted between the sample holder and the sample carrier, with the distance between the chips and the receptor sites being about $0.5 \mathrm{~mm}$. Examples of releasing tests are shown in Figure 8, as captured images from Supplementary Video S2. Immediately after the laser was fired, the target area was deformed and the chip was detached from the tape, as shown in Figure 8b,c. The release time was measured to be 340 and $389 \mathrm{~ms}$ respectively. The release time of the chip was calculated from the moment the laser was triggered to the moment the chip was fully released from the thermal release tape. The releasing tests were repeated five times, where in each test, the tape with five chips was heated with a continuous laser with power of $100 \mathrm{~mW}$. In total, 25 experiments were performed. Table 2 summarizes the success rate 
of releasing and the average releasing time from each test, which shows the chips can be released with the releasing time in the range of 300-400 ms. The difference in releasing time may be caused by the following factors: (1) certain randomness due to the material composition and the quality of the initial contact between each chip and the tape; and (2) randomness in the time required to reduce the adhesion force to the threshold of releasing. In a few cases, the chips were partially released where one corner or one edge of the chip was adhered to the thermal tape. This is mainly caused by the misalignment between the laser focal point and the center of the chip. Another cause could be the non-uniform intensity distribution of the laser beam. The reliability of releasing could be improved using a more powerful or larger laser beam with a more uniform spatial laser beam intensity distribution or using a thermal tape with a lower curing temperature. In addition, we also tested $300 \mu \mathrm{m} \times 300 \mu \mathrm{m}$ microchips, which were difficult to release. We attribute the phenomena to two factors: (1) the laser beam causes topographical changes to the thermal tape which leads to similar effective contact area for both $300 \mu \mathrm{m}$ and $500 \mu \mathrm{m}$ chips; and (2) the gravity of $500 \mu \mathrm{m}$ chip is greater, therefore, $500 \mu \mathrm{m}$ chips are easier to detach.

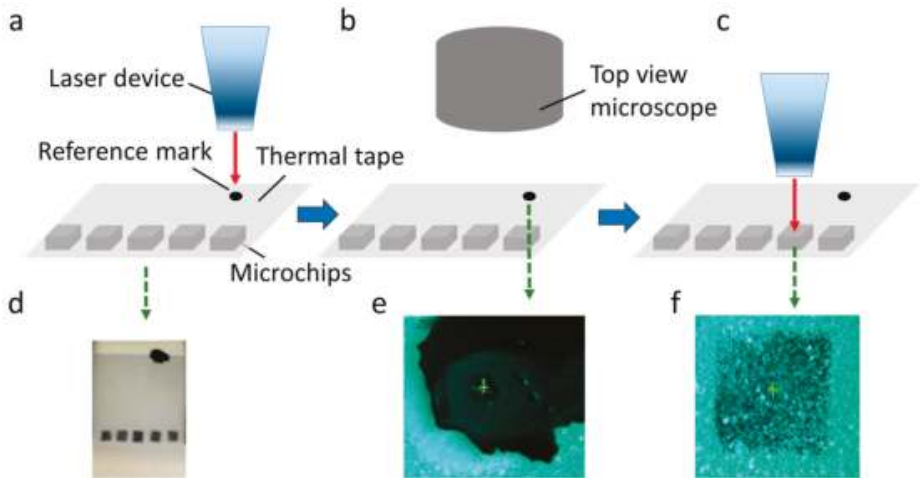

Figure 7. Laser die releasing procedure: $(\mathbf{a}, \mathbf{d})$ a reference mark was moved under the laser beam and the laser was fired at the mark to make a burn; $(\mathbf{b}, \mathbf{e})$ the reference burn mark was moved under a top view microscope and the center of the burn was stored and highlighted with a cross hair; $(\mathbf{c}, \mathbf{f})$ the laser beam focal point was aligned with the center of the chip and the laser was ready to fire to release the chip from the thermal release tape.
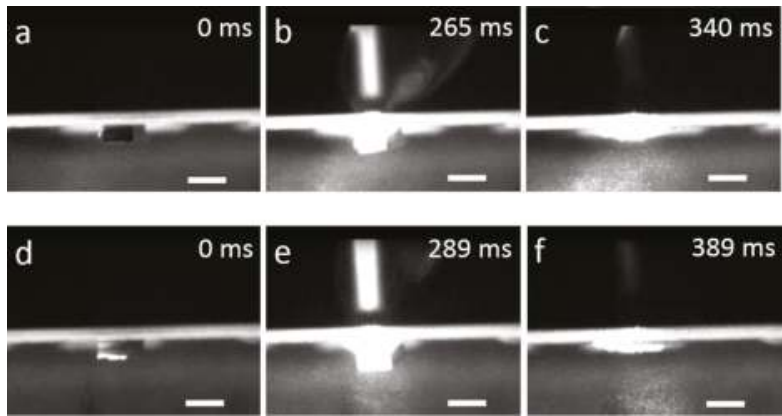

Figure 8. Releasing of chips from a thermal tape by firing a laser beam. (a,d) $500 \mu \mathrm{m} \times 500 \mu \mathrm{m} \times 300 \mu \mathrm{m}$ chips were attached to a thermal release tape; $(\mathbf{b}, \mathbf{e})$ the chips were moved under the laser beam and the laser beam was fired at the chips; $(\mathbf{c}, \mathbf{f})$ the tape was heated to its curing temperature by the laser beam and the chips were released from the thermal release tape (Supplementary Video S2). Scale bar: $500 \mu \mathrm{m}$. 
Table 2. Releasing time and success rate.

\begin{tabular}{ccc}
\hline Test & Average Releasing Time & Success Rate \\
\hline$\# 1$ & $390.3 \mathrm{~ms}$ & $4 / 5$ \\
$\# 2$ & $380.4 \mathrm{~ms}$ & $5 / 5$ \\
$\# 3$ & $398.1 \mathrm{~ms}$ & $3 / 5$ \\
$\# 4$ & $300.2 \mathrm{~ms}$ & $5 / 5$ \\
$\# 5$ & $357.3 \mathrm{~ms}$ & $4 / 5$ \\
\hline
\end{tabular}

\subsection{Mist Capillary Self-Alignment}

Immediately after the chips were released from the thermal tape and landed on the corresponding receptor sites, we applied the mist capillary self-alignment method to correct the initial placement error between the chip and the receptor site. Figure 9 shows the typical process of the mist capillary self-alignment. Firstly, a chip landed on the top of a receptor site with an initial placement error, and then water mist was delivered to the assembly site using a commercial ultrasonic humidifier; meanwhile, microscopic droplets accumulated at the assembly site; and, finally, the chip was self-aligned with the receptor site when the volume of the droplets between the gap of the chip and the receptor site increases to the critical volume. Detailed description about the droplets' accumulation process is described in Section 3.2. We have reported earlier that the alignment accuracy of capillary self-alignment is significantly better than the manufacturing precision of the chips and receptor sites, and the accuracy can be better than $1 \mu \mathrm{m}$ when the edges of the chips and the receptor sites are well defined [17]. In this work, 10 out of 10 microchips were successfully self-aligned with the corresponding receptor sites, and the alignment accuracy was inspected using an optical microscope. The results show that the alignment accuracy is $1.4 \pm 0.8 \mu \mathrm{m}$, which is consistent with our earlier results [17]. Mist capillary self-alignment is an entirely parallel process and, in principle, it could be introduced to thousands to millions of assembly sites and realize massive self-alignments simultaneously.
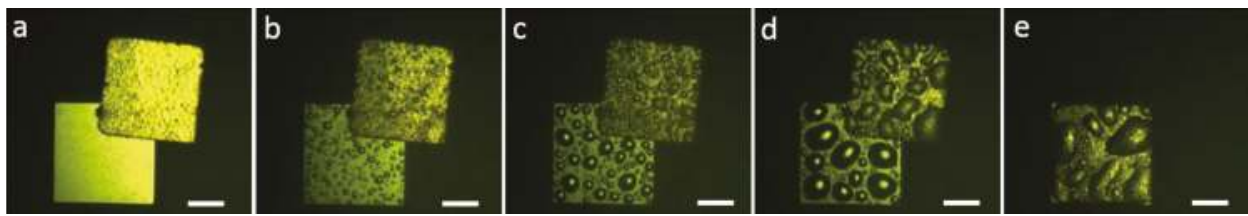

Figure 9. Mist capillary self-alignment: (a) a $500 \mu \mathrm{m} \times 500 \mu \mathrm{m} \times 300 \mu \mathrm{m}$ chip landed on a receptor site with an initial placement error; (b) water mist was delivered to the assembly site; (c,d) microscopic droplets accumulated at the assembly site; and (e) the chip was self-aligned with the corresponding receptor site (Supplementary Video S1). Scale bar: $200 \mu \mathrm{m}$.

\section{Conclusions}

In this paper, we reported a laser-assisted mist capillary self-alignment method for the assembly of microchips on hydrophilic/superhydrophobic receptor sites. We have demonstrated that laser die transfer can transport microchips from a thermal release tape onto the receptor sites within an accuracy that the mist capillary self-alignment technique can successively apply to achieve high assembly accuracy. Microchips can be released in a non-contact manner from a thermally-curable adhesive tape with a low-cost and low-power commercial laser device. The releasing tests suggest that the release time below $400 \mathrm{~ms}$ is achievable using a laser device (100 $\mathrm{mW}$ power) for $500 \mu \mathrm{m} \times 500 \mu \mathrm{m} \times 300 \mu \mathrm{m}$ silicon chips. In comparison with the hybrid method combining robotic pick-and-place and capillary self-alignment proposed by us earlier, this method is especially suitable for fragile micro components, where non-contact handling is preferred. Furthermore, the method can be potentially applied to ultra-thin chips by employing the laser ablation die transfer technique. The mist capillary 
self-alignment has been studied through theoretical analysis and experiments. The acting forces for capillary self-alignment have been analyzed and the critical volume enabling capillary self-alignment has been estimated. We have demonstrated that microchips self-align to the corresponding receptor site in parallel with an alignment accuracy of $1.4 \pm 0.8 \mu \mathrm{m}$. The proposed technique has great potential for high-throughput and high-accuracy assembly of micro devices. Furthermore, since water droplets will eventually evaporate and disappear shortly after the capillary self-alignment, it is possible to integrate the proposed technology with bonding techniques, such as thermal bonding, as reported in our earlier work [27]. The proposed technique can also be applied to microchips containing asymmetric patterns and micro bumps [8]. In future work, more powerful lasers will be investigated to improve the reliability and efficiency of the chips releasing.

Supplementary Materials: The following are available online at www.mdpi.com/2072-666X/8/12/361/s1, Video S1: Water mist-induced capillary self-alignment, Video S2: Laser die transfer.

Acknowledgments: The work is supported by the National Natural Science Foundation of China (61703255) and the Academy of Finland (275117).

Author Contributions: Bo Chang and Quan Zhou conceived and designed the experiments; Bo Chang and Mikko Koverola performed the experiments; Bo Chang and Zhaofei Zhu analyzed the data; and Bo Chang wrote the paper.

Conflicts of Interest: The authors declare no conflict of interest.

\section{References}

1. Fang, J.; Böhringer, K.F. Parallel micro component-to-substrate assembly with controlled poses and high surface coverage. J. Micromech. Microeng. 2006, 16, 721-730. [CrossRef]

2. Park, S.C.; Fang, J.; Biswas, S.; Mozafari, M.; Stauden, T.; Jacobs, H.O. A first implementation of an automated reel-to-reel fluidic self-assembly machine. Adv. Mater. 2014, 26, 5942-5949. [CrossRef] [PubMed]

3. Chang, B.; Shah, A.; Zhou, Q.; Ras, R.H.A.; Hjort, K. Self-transport and self-alignment of microchips using microscopic rain. Sci. Rep. 2015, 5, 14966. [CrossRef] [PubMed]

4. Mastrangeli, M.; Zhou, Q.; Sariola, V.; Lambert, P. Surface tension-driven self-alignment. Soft Matter 2017, 13, 304-327. [CrossRef] [PubMed]

5. Lambert, P.; Mastrangeli, M.; Valsamis, J.B.; Degrez, G. Spectral analysis and experimental study of lateral capillary dynamics for flip-chip applications. Microfluid. Nanofluid. 2010, 9, 797-807. [CrossRef]

6. Boote, J.J.; Critchley, K.; Evans, S.D. Surfactant mediated assembly of gold nanowires on surfaces. J. Exp. Nanosci. 2006, 1, 125-142. [CrossRef]

7. Whitesides, G.M.; Grzybowski, B.A.; Stone, H.A. Dynamic self-assembly of magnetized, millimetre-sized objects rotating at a liquid-air interface. Nature 2000, 405, 1033-1036.

8. Chang, B.; Routa, I.; Sariola, V.; Zhou, Q. Self-alignment of RFID dies on four-pad patterns with water droplet for sparse self-assembly. J. Micromech. Microeng. 2011, 21, 095024. [CrossRef]

9. Gracias, D.H. Forming Electrical Networks in Three Dimensions by Self-Assembly. Science 2000, 289, 1170-1172. [CrossRef] [PubMed]

10. Jacobs, H.O. Fabrication of a Cylindrical Display by Patterned Assembly. Science 2002, 296, 323-325. [CrossRef] [PubMed]

11. Knuesel, R.J.; Jacobs, H.O. Self-assembly of microscopic chiplets at a liquid-liquid-solid interface forming a flexible segmented monocrystalline solar cell. Proc. Natl. Acad. Sci. USA 2010, 107, 993-998. [CrossRef] [PubMed]

12. Chung, S.E.; Park, W.; Shin, S.; Lee, S.A.; Kwon, S. Guided and fluidic self-assembly of microstructures using railed microfluidic channels. Nat. Mater. 2008, 7, 581-587. [CrossRef] [PubMed]

13. Sato, K.; Hata, S.; Shimokohbe, A. Self-Alignment for Microparts Assembly Using Water Surface Tension. In Proceedings of the Device and Process Technologies for MEMS and Microelectronics, Gold Coast, Australia, 1 October 1999; Volume 3892, pp. 321-329.

14. Chang, B.; Sariola, V.; Jääskeläinen, M.; Zhou, Q. Self-alignment in the stacking of microchips with mist-induced water droplets. J. Micromech. Microeng. 2011, 21, 015016. [CrossRef] 
15. Leong, T.G.; Lester, P.A.; Koh, T.L.; Call, E.K.; Gracias, D.H. Surface tension-driven self-folding polyhedra. Langmuir 2007, 23, 8747-8751. [CrossRef] [PubMed]

16. Gracias, D.H.; Kavthekar, V.; Love, J.C.; Paul, K.E.; Whitesides, G.M. Fabrication of micrometer-scale, patterned polyhedra by self-assembly. Adv. Mater. 2002, 14, 235-238. [CrossRef]

17. Routa, I.; Chang, B.; Shah, A.; Zhou, Q. Surface tension-driven self-alignment of microchips on low-precision receptors. J. Microelectromech. Syst. 2014, 23, 819-828. [CrossRef]

18. Meitl, M.A.; Zhu, Z.-T.; Kumar, V.; Lee, K.J.; Feng, X.; Huang, Y.Y.; Adesida, I.; Nuzzo, R.G.; Rogers, J.A. Transfer printing by kinetic control of adhesion to an elastomeric stamp. Nat. Mater. 2006, 5, 33-38. [CrossRef]

19. Karlitskaya, N.S. Study of laser die release by Q-switched Nd:YAG laser pulses. In Proceedings of the Society of Photo-Optical Instrumentation Engineers, Taos, NM, USA, 20 September 2004; Volume 5448, pp. 935-943.

20. Rogers, J.A.; Someya, T.; Huang, Y. Materials and mechanics for stretchable electronics. Science 2010, 327, 1603-1607. [CrossRef] [PubMed]

21. Xu, S.; Zhang, Y.; Jia, L.; Mathewson, K.E.; Jang, K.-I.; Kim, J.; Fu, H.; Huang, X.; Chava, P.; Wang, R.; et al. Soft Microfluidic Assemblies of Sensors, Circuits, and Radios for the Skin. Science 2014, 344, 70-74. [CrossRef] [PubMed]

22. Marinov, V.R.; Swenson, O.; Atanasov, Y.; Schneck, N. Laser-assisted ultrathin die packaging: Insights from a process study. Microelectron. Eng. 2013, 101, 23-30. [CrossRef]

23. Chang, B.; Koverola, M.; Ge, Z.; Zhou, Q. Hybrid Microassembly combining Laser Die Transfer and Capillary Self-alignment. In Proceedings of the 2017 International Conference on Manipulation, Automation and Robotics at Small Scales (MARSS), Montreal, QC, Canada, 17-21 July 2017; pp. 38-41.

24. Shah, A.; Chang, B.; Suihkonen, S.; Zhou, Q.; Lipsanen, H. Surface-Tension-Driven Self-Alignment of Microchips on Black-Silicon-Based Hybrid Template in Ambient Air. J. Microelectromech. Syst. 2013, 22, 739-746. [CrossRef]

25. Karlitskaya, N. Laser Die Transfer: Laser-Induced Transfer of Microcomponents; University of Twente: Enschede, The Netherlands, 2011.

26. Brakke, K.A. The Surface Evolver. Exp. Math. 1992, 1, 141-165. [CrossRef]

27. Zhou, Q.; Liimatainen, V.; Routa, I. Microsystem integration using hybrid microassembly. In Proceedings of the 2014 5th Electronics System-Integration Technology Conference ESTC, Helsinki, Finland, 16-18 September 2014; pp. 4-9.

(C) 2017 by the authors. Licensee MDPI, Basel, Switzerland. This article is an open access article distributed under the terms and conditions of the Creative Commons Attribution (CC BY) license (http:/ / creativecommons.org/licenses/by/4.0/). 

MDPI

St. Alban-Anlage 66

4052 Basel

Switzerland

Tel. +41616837734

Fax +41 613028918

www.mdpi.com

Micromachines Editorial Office

E-mail: micromachines@mdpi.com www.mdpi.com/journal/micromachines

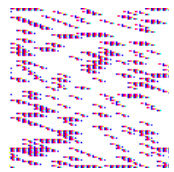



MDPI

St. Alban-Anlage 66

4052 Basel

Switzerland

Tel: +41 616837734

Fax: +41 613028918

www.mdpi.com 